Cochrane Database of Systematic Reviews

\title{
Cognitive behavioural therapy for anxiety disorders in children and adolescents (Review)
}

James AC, Reardon T, Soler A, James G, Creswell C

James AC, Reardon T, Soler A, James G, Creswell C.

Cognitive behavioural therapy for anxiety disorders in children and adolescents.

Cochrane Database of Systematic Reviews 2020, Issue 11. Art. No.: CD013162.

DOI: 10.1002/14651858.CD013162.pub2.

www.cochranelibrary.com 
TABLE OF CONTENTS

HEADER

ABSTRACT

PLAIN LANGUAGE SUMMARY

SUMMARY OF FINDINGS

BACKGROUND

OBJECTIVES

METHODS

RESULTS

Figure 1.

Figure 2.

Figure 3.

Figure 4.

Figure 5.

Figure 6.

Figure 7.

Figure 8.

DISCUSSION

AUTHORS' CONCLUSIONS

ACKNOWLEDGEMENTS

REFERENCES

CHARACTERISTICS OF STUDIES

DATA AND ANALYSES

Analysis 1.1. Comparison 1: CBT versus waitlist/no treatment, Outcome 1: Remission of primary anxiety diagnosis posttreatment (ITT)

Analysis 1.2. Comparison 1: CBT versus waitlist/no treatment, Outcome 2: Subgroup analysis: remission of primary anxiety diagnosis post-treatment (ITT) (individual vs group)

Analysis 1.3. Comparison 1: CBT versus waitlist/no treatment, Outcome 3: Subgroup analysis: remission of primary anxiety diagnosis post-treatment (ITT) (amount of therapist contact time)

Analysis 1.4. Comparison 1: CBT versus waitlist/no treatment, Outcome 4: Subgroup analysis: remission of primary anxiety diagnosis post-treatment (ITT) (age)

Analysis 1.5. Comparison 1: CBT versus waitlist/no treatment, Outcome 5: Subgroup analysis: remission of primary anxiety diagnosis post-treatment (ITT) (ASD vs non-ASD)

Analysis 1.6. Comparison 1: CBT versus waitlist/no treatment, Outcome 6: Remission of primary anxiety diagnosis posttreatment (completers)

Analysis 1.7. Comparison 1: CBT versus waitlist/no treatment, Outcome 7: Acceptability (number of participants lost to posttreatment assessment)

Analysis 1.8. Comparison 1: CBT versus waitlist/no treatment, Outcome 8: Remission of all anxiety diagnoses post-treatment (ITT)

Analysis 1.9. Comparison 1: CBT versus waitlist/no treatment, Outcome 9: Subgroup analysis: remission of all anxiety diagnoses post-treatment (ITT) (individual vs group)

Analysis 1.10. Comparison 1: CBT versus waitlist/no treatment, Outcome 10: Subgroup analysis: remission of all anxiety diagnoses post-treatment (ITT) (amount of therapist contact time)

Analysis 1.11. Comparison 1: CBT versus waitlist/no treatment, Outcome 11: Subgroup analysis: remission of all anxiety diagnoses post-treatment (ITT) (age)

Analysis 1.12. Comparison 1: CBT versus waitlist/no treatment, Outcome 12: Remission of all anxiety diagnoses post-treatment (completers)

Analysis 1.13. Comparison 1: CBT versus waitlist/no treatment, Outcome 13: Reduction in anxiety symptoms (child report) posttreatment

Analysis 1.14. Comparison 1: CBT versus waitlist/no treatment, Outcome 14: Sensitivity analysis: reduction in anxiety symptoms (child report) post-treatment (broad anxiety measures)

Analysis 1.15. Comparison 1: CBT versus waitlist/no treatment, Outcome 15: Subgroup analysis: reduction in anxiety symptoms (child report) post-treatment (individual vs group)

Analysis 1.16. Comparison 1: CBT versus waitlist/no treatment, Outcome 16: Subgroup analysis: reduction in anxiety symptoms (child report) post-treatment (amount of therapist contact time) 
Analysis 1.17. Comparison 1: CBT versus waitlist/no treatment, Outcome 17: Subgroup analysis: reduction in anxiety symptoms (child report) post-treatment (age)

Analysis 1.18. Comparison 1: CBT versus waitlist/no treatment, Outcome 18: Subgroup analysis: reduction in anxiety symptoms (child report) post-treatment (ASD vs non-ASD)

Analysis 1.19. Comparison 1: CBT versus waitlist/no treatment, Outcome 19: Reduction in anxiety symptoms (parent report) post-treatment

Analysis 1.20. Comparison 1: CBT versus waitlist/no treatment, Outcome 20: Sensitivity analysis: reduction in anxiety symptoms (parent report) post-treatment (broad anxiety measures only)

Analysis 1.21. Comparison 1: CBT versus waitlist/no treatment, Outcome 21: Subgroup analysis: reduction in anxiety symptoms (parent report) post-treatment (individual vs group)

Analysis 1.22. Comparison 1: CBT versus waitlist/no treatment, Outcome 22: Subgroup analysis: reduction in anxiety symptoms (parent report) post-treatment (amount of therapist contact time)

Analysis 1.23. Comparison 1: CBT versus waitlist/no treatment, Outcome 23: Subgroup analysis: reduction in anxiety symptoms (parent report) post-treatment (age)

Analysis 1.24. Comparison 1: CBT versus waitlist/no treatment, Outcome 24: Subgroup analysis: reduction in anxiety symptoms (parent report) post-treatment (ASD vs non-ASD)

Analysis 1.25. Comparison 1: CBT versus waitlist/no treatment, Outcome 25: Reduction in depressive symptoms posttreatment

Analysis 1.26. Comparison 1: CBT versus waitlist/no treatment, Outcome 26: Improvement in global functioning posttreatment

Analysis 1.27. Comparison 1: CBT versus waitlist/no treatment, Outcome 27: Remission of primary anxiety diagnosis at followup (ITT)

Analysis 1.28. Comparison 1: CBT versus waitlist/no treatment, Outcome 28: Remission of primary anxiety diagnosis at followup (completers)

Analysis 1.29. Comparison 1: CBT versus waitlist/no treatment, Outcome 29: Remission of all anxiety diagnoses at follow-up (ITT)

Analysis 1.30. Comparison 1: CBT versus waitlist/no treatment, Outcome 30: Remission of all anxiety diagnoses at follow-up (completers)

Analysis 1.31. Comparison 1: CBT versus waitlist/no treatment, Outcome 31: Reduction in anxiety symptoms (child report) at follow-up

Analysis 1.32. Comparison 1: CBT versus waitlist/no treatment, Outcome 32: Sensitivity analysis: remission of primary anxiety diagnosis post-treatment (ITT) (only studies where sequence generation is clear)

Analysis 1.33. Comparison 1: CBT versus waitlist/no treatment, Outcome 33: Sensitivity analysis: remission of primary anxiety diagnosis post-treatment (ITT) (only studies where blind assessors used)

Analysis 2.1. Comparison 2: CBT versus treatment as usual, Outcome 1: Remission of primary anxiety diagnosis post-treatment (ITT)

Analysis 2.2. Comparison 2: CBT versus treatment as usual, Outcome 2: Subgroup analysis: remission of primary anxiety diagnosis post-treatment (ITT) (ASD vs non-ASD)

Analysis 2.3. Comparison 2: CBT versus treatment as usual, Outcome 3: Remission of primary anxiety diagnosis post-treatment (completers)

Analysis 2.4. Comparison 2: CBT versus treatment as usual, Outcome 4: Acceptability (number of participants lost to posttreatment assessment)

Analysis 2.5. Comparison 2: CBT versus treatment as usual, Outcome 5: Remission of all anxiety diagnoses post-treatment (ITT)

Analysis 2.6. Comparison 2: CBT versus treatment as usual, Outcome 6: Remission of all anxiety diagnoses post-treatment (completers)

Analysis 2.7. Comparison 2: CBT versus treatment as usual, Outcome 7: Reduction in anxiety symptoms (child report) posttreatment

Analysis 2.8. Comparison 2: CBT versus treatment as usual, Outcome 8: Sensitivity analysis: reduction in anxiety symptoms (child report) post-treatment (broad anxiety measures only)

Analysis 2.9. Comparison 2: CBT versus treatment as usual, Outcome 9: Reduction in anxiety symptoms (parent report) posttreatment

Analysis 2.10. Comparison 2: CBT versus treatment as usual, Outcome 10: Sensitivity analysis: reduction in anxiety symptoms (parent report) post-treatment (broad anxiety measures only)

Analysis 3.1. Comparison 3: CBT versus attention control, Outcome 1: Remission of primary anxiety diagnosis post-treatment (ITT) 
Analysis 3.2. Comparison 3: CBT versus attention control, Outcome 2: Subgroup analysis: remission of primary anxiety diagnosis post-treatment (ITT) (individual vs group)

Analysis 3.3. Comparison 3: CBT versus attention control, Outcome 3: Remission of primary anxiety diagnosis post-treatment (completers)

Analysis 3.4. Comparison 3: CBT versus attention control, Outcome 4: Acceptability (number of participants lost to posttreatment assessment)

Analysis 3.5. Comparison 3: CBT versus attention control, Outcome 5: Remission of all anxiety diagnoses post-treatment (ITT) . Analysis 3.6. Comparison 3: CBT versus attention control, Outcome 6: Remission of all anxiety diagnoses post-treatment (completers)

Analysis 3.7. Comparison 3: CBT versus attention control, Outcome 7: Reduction in anxiety symptoms (child report) posttreatment

Analysis 3.8. Comparison 3: CBT versus attention control, Outcome 8: Sensitivity analysis: reduction in anxiety symptoms (child report) post-treatment (broad anxiety measures only)

Analysis 3.9. Comparison 3: CBT versus attention control, Outcome 9: Reduction in anxiety symptoms (parent report) posttreatment

Analysis 3.10. Comparison 3: CBT versus attention control, Outcome 10: Sensitivity analysis: reduction in anxiety symptoms (parent report) post-treatment (broad anxiety measures only)

Analysis 3.11. Comparison 3: CBT versus attention control, Outcome 11: Reduction in depressive symptoms post-treatment .. Analysis 3.12. Comparison 3: CBT versus attention control, Outcome 12: Remission of primary anxiety diagnosis at follow-up (ITT)

Analysis 3.13. Comparison 3: CBT versus attention control, Outcome 13: Remission of primary anxiety diagnosis at follow-up (completers)

Analysis 3.14. Comparison 3: CBT versus attention control, Outcome 14: Remission of all anxiety diagnoses at follow-up (ITT) .

Analysis 3.15. Comparison 3: CBT versus attention control, Outcome 15: Remission of all anxiety diagnoses at follow-up (completers)

Analysis 3.16. Comparison 3: CBT versus attention control, Outcome 16: Reduction in anxiety symptoms (child report) at followup

Analysis 3.17. Comparison 3: CBT versus attention control, Outcome 17: Reduction in anxiety symptoms (parent report) at follow-up

Analysis 4.1. Comparison 4: CBT versus alternative treatment, Outcome 1: Acceptability (number of participants lost to posttreatment assessment)

Analysis 4.2. Comparison 4: CBT versus alternative treatment, Outcome 2: Remission of all anxiety diagnoses post-treatment (ITT)

Analysis 4.3. Comparison 4: CBT versus alternative treatment, Outcome 3: Remission of all anxiety diagnoses post-treatment (completers)

Analysis 4.4. Comparison 4: CBT versus alternative treatment, Outcome 4: Reduction in anxiety symptoms (child report) posttreatment

Analysis 4.5. Comparison 4: CBT versus alternative treatment, Outcome 5: Sensitivity analysis: reduction in anxiety symptoms (child report) post-treatment (broad anxiety measures only)

Analysis 4.6. Comparison 4: CBT versus alternative treatment, Outcome 6: Reduction in anxiety symptoms (parent report) posttreatment

ADDITIONAL TABLES

APPENDICES

WHAT'S NEW

HISTORY

CONTRIBUTIONS OF AUTHORS

DECLARATIONS OF INTEREST

SOURCES OF SUPPORT

DIFFERENCES BETWEEN PROTOCOL AND REVIEW

252

253 
[Intervention Review]

\section{Cognitive behavioural therapy for anxiety disorders in children and adolescents}

Anthony C James 1,2 , Tessa Reardon 1,3,4, Angela Soler², Georgina James ${ }^{5}$, Cathy Creswell1,3,4

1Department of Psychiatry, University of Oxford, Oxford, UK. 2Highfield Unit, Warneford Hospital, Oxford, UK. 3Department of Experimental Psychology, University of Oxford, Oxford, UK. ${ }^{4}$ School of Psychology \& Clinical Language Sciences, University of Reading, Reading, UK. ${ }^{5}$ Sunderland Royal Hospital, Sunderland, UK

Contact address: Tessa Reardon, tessa.reardon@psy.ox.ac.uk.

Editorial group: Cochrane Common Mental Disorders Group.

Publication status and date: New, published in Issue 11, 2020.

Citation: James AC, Reardon T, Soler A, James G, Creswell C. Cognitive behavioural therapy for anxiety disorders in children and adolescents. Cochrane Database of Systematic Reviews 2020, Issue 11. Art. No.: CD013162. DOI: 10.1002/14651858.CD013162.pub2.

Copyright (c) 2020 The Cochrane Collaboration. Published by John Wiley \& Sons, Ltd.

\section{A B S T R A C T}

\section{Background}

Previous Cochrane Reviews have shown that cognitive behavioural therapy (CBT) is effective in treating childhood anxiety disorders. However, questions remain regarding the following: up-to-date evidence of the relative efficacy and acceptability of CBT compared to waiting lists/no treatment, treatment as usual, attention controls, and alternative treatments; benefits across a range of outcomes; longerterm effects; outcomes for different delivery formats; and amongst children with autism spectrum disorders (ASD) and children with intellectual impairments.

\section{Objectives}

To examine the effect of CBT for childhood anxiety disorders, in comparison with waitlist/no treatment, treatment as usual (TAU), attention control, alternative treatment, and medication.

\section{Search methods}

We searched the Cochrane Common Mental Disorders Controlled Trials Register (all years to 2016), the Cochrane Central Register of Controlled Trials (CENTRAL), MEDLINE, Embase, and PsycINFO (each to October 2019), international trial registries, and conducted grey literature searches.

\section{Selection criteria}

We included randomised controlled trials of CBT that involved direct contact with the child, parent, or both, and included nonCBT comparators (waitlist/no treatment, treatment as usual, attention control, alternative treatment, medication). Participants were younger than age 19, and met diagnostic criteria for an anxiety disorder diagnosis. Primary outcomes were remission of primary anxiety diagnosis post-treatment, and acceptability (number of participants lost to post-treatment assessment), and secondary outcomes included remission of all anxiety diagnoses, reduction in anxiety symptoms, reduction in depressive symptoms, improvement in global functioning, adverse effects, and longer-term effects.

\section{Data collection and analysis}

We used standard methodological procedures as recommended by Cochrane. We used GRADE to assess the quality of the evidence.

\section{Main results}

We included 87 studies and 5964 participants in quantitative analyses. 
Compared with waitlist/no treatment, CBT probably increases post-treatment remission of primary anxiety diagnoses (CBT: 49.4\%, waitlist/no treatment: $17.8 \%$; OR 5.45, 95\% confidence interval (Cl) 3.90 to $7.60 ; n=2697,39$ studies, moderate quality); NNTB 3 (95\% Cl 2.25 to 3.57 ) and all anxiety diagnoses (OR $4.43,95 \% \mathrm{Cl} 2.89$ to $6.78 ; \mathrm{n}=2075,28$ studies, moderate quality).

Low-quality evidence did not show a difference between CBT and TAU in post-treatment primary anxiety disorder remission (OR 3.19, 95\% $\mathrm{Cl} 0.90$ to $11.29 ; n=487,8$ studies), but did suggest CBT may increase remission from all anxiety disorders compared to TAU (OR 2.74, 95\% $\mathrm{Cl} 1.16$ to $6.46 ; \mathrm{n}=203,5$ studies).

Compared with attention control, CBT may increase post-treatment remission of primary anxiety disorders $(\mathrm{OR} 2.28,95 \% \mathrm{Cl} 1.33$ to 3.89 ; $\mathrm{n}=822,10$ studies, low quality) and all anxiety disorders (OR $2.75,95 \% \mathrm{Cl} 1.22$ to $6.17 ; \mathrm{n}=378,5$ studies, low quality).

There was insufficient available data to compare CBT to alternative treatments on post-treatment remission of primary anxiety disorders, and low-quality evidence showed there may be little to no difference between these groups on post-treatment remission of all anxiety disorders (OR $0.89,95 \% \mathrm{Cl} 0.35$ to $2.23 ; \mathrm{n}=401,4$ studies)

Low-quality evidence did not show a difference for acceptability between CBT and waitlist/no treatment (OR $1.09,95 \% \mathrm{Cl} 0.85$ to 1.41 ; $\mathrm{n}=3158,45$ studies), treatment as usual (OR 1.37, 95\% Cl 0.73 to $2.56 ; \mathrm{n}=441,8$ studies), attention control (OR $1.00,95 \% \mathrm{Cl} 0.68$ to $1.49 ; \mathrm{n}$ $=797,12$ studies) and alternative treatment (OR $1.58,95 \% \mathrm{Cl} 0.61$ to $4.13 ; \mathrm{n}=515,7$ studies).

No adverse effects were reported across all studies; however, in the small number of studies where any reference was made to adverse effects, it was not clear that these were systematically monitored.

Results from the anxiety symptom outcomes, broader outcomes, longer-term outcomes and subgroup analyses are provided in the text.

We did not find evidence of consistent differences in outcomes according to delivery formats (e.g. individual versus group; amount of therapist contact time) or amongst samples with and without ASD, and no studies included samples of children with intellectual impairments.

\section{Authors' conclusions}

CBT is probably more effective in the short-term than waiting lists/no treatment, and may be more effective than attention control. We found little to no evidence across outcomes that CBT is superior to usual care or alternative treatments, but our confidence in these findings are limited due to concerns about the amount and quality of available evidence, and we still know little about how best to efficiently improve outcomes.

\section{PLAIN LANGUAGE SUMMARY}

\section{Cognitive behavioural therapy for anxiety in children and young people}

\section{Why is this review important?}

Many children and young people experience problems with anxiety. Children and young people with anxiety disorders are more likely than their peers to have difficulty with friendships, family life, and school, and to develop mental health problems later in life. Therapies such as cognitive behavioural therapy (CBT) can help children and young people to overcome difficulties with anxiety by using new ways of thinking and facing their fears.

\section{Who will be interested in this review?}

Parents, children, and young people; people working in education and mental health services for children and young people; and general practitioners.

\section{What questions does this review aim to answer?}

This review updates and replaces previous Cochrane Reviews from 2005 and 2015, which showed that CBT is an effective treatment for children and young people with anxiety disorders.

This review aimed to answer the following questions:

- Is CBT more effective than a waiting list or no treatment?

- Is CBT more effective than other treatments and medication?

- Does CBT help to reduce anxiety for children and young people in the longer term?

-Are some types of CBT more effective than others? (e.g. individual versus group therapy)

- Is CBT effective for specific groups? (e.g. children with autism) 


\section{Which studies were included in the review?}

We searched the databases to find all studies of CBT for anxiety disorders in children and young people published up to October 2019. In order to be included in the review, studies had to be randomised controlled trials (a type of study in which participants are assigned to one of two or more treatment groups using a random method) and had to include young people under 19 years of age with an anxiety disorder diagnosis. We included 87 studies with a total of 5964 participants in the analysis.

\section{What does the evidence from the review tell us?}

We rated the overall quality of the evidence as 'moderate' or 'low'. There is evidence that CBT is more effective than a waiting list or no treatment in reducing anxiety in children and young people, although the findings did vary across studies. There is no clear evidence that CBT is more effective than other treatments. A small number of studies looked at outcomes six months after CBT was given and showed that reductions in anxiety continued. We found no clear evidence that one way of providing CBT is more effective than another (e.g. in a group, longer treatments, with parents) or that CBT is more or less effective for any specific group of children (e.g. children with autism spectrum disorders).

\section{What should happen next?}

Future research should compare CBT to alternative treatments and medication; identify who does and does not benefit from CBT and what those who do not benefit need; establish how to make CBT more accessible; and give far more consideration to neglected populations, including children and young people from low- and middle-income countries. 


\section{SUMMARY OF FINDINGS}

\section{Summary of findings 1. CBT compared with waitlist for children and adolescents with anxiety disorders}

CBT compared with waitlist for children and adolescents with anxiety disorders

Patient or population: children and adolescents with anxiety disorders

Settings: outpatient clinics/schools

Intervention: CBT

Comparison: waitlist/no treatment

\begin{tabular}{|c|c|c|c|c|c|c|}
\hline \multirow[t]{3}{*}{ Outcomes } & \multicolumn{2}{|c|}{$\begin{array}{l}\text { Illustrative comparative risks* } \\
(95 \% \mathrm{CI})\end{array}$} & \multirow{3}{*}{$\begin{array}{l}\text { Relative } \\
\text { effect } \\
(95 \% \mathrm{CI})\end{array}$} & \multirow{3}{*}{$\begin{array}{l}\text { No. of } \\
\text { partici- } \\
\text { pants } \\
\text { (stud- } \\
\text { ies) }\end{array}$} & \multirow{3}{*}{$\begin{array}{l}\text { Qual- } \\
\text { ity of } \\
\text { the evi- } \\
\text { dence } \\
\text { (GRADE) }\end{array}$} & \multirow[t]{3}{*}{ Comments } \\
\hline & Assumed risk & $\begin{array}{l}\text { Corresponding } \\
\text { risk }\end{array}$ & & & & \\
\hline & Waitlist ${ }^{1}$ & CBT & & & & \\
\hline $\begin{array}{l}\text { Remission of pri- } \\
\text { mary anxiety } \\
\text { diagnosis post- } \\
\text { treatment (ITT) }\end{array}$ & 178 per 1000 & $\begin{array}{l}\mathbf{5 4 1} \text { per } \mathbf{1 0 0 0} \\
\text { (458 to } 622 \text { ) }\end{array}$ & $\begin{array}{l}\text { OR } 5.45 \\
(3.90 \text { to } \\
7.60)\end{array}$ & $\begin{array}{l}2697 \\
\text { (39 stud- } \\
\text { ies) }\end{array}$ & $\begin{array}{l}\oplus \oplus \oplus \ominus \\
\text { moder- } \\
\text { ate } 2\end{array}$ & $\begin{array}{l}\text { Subgroup analyses: } \\
\text { - Difference in outcomes for different delivery formats (child-focused, child } \\
\text { and parent, parent only) }\left(\mathrm{Chi}^{2}=8.57, \mathrm{df}=2, \mathrm{P}=0.01, \mathrm{I}^{2}=76.7 \%\right) \\
\text { - No difference in outcomes for individual versus group formats }\left(\mathrm{Chi}^{2}=0.90 \text {, }\right. \\
\left.\mathrm{df}=1, \mathrm{P}=0.34 ; \mathrm{I}^{2}=0 \%\right) \\
\text { - No difference in outcomes for interventions with different amounts of } \\
\text { therapist contact }\left(\mathrm{Chi} \mathrm{i}^{2}=0.52, \mathrm{df}=2, \mathrm{P}=0.77 ; \mathrm{I}^{2}=0 \%\right) \\
\text { - No difference in outcomes for participants with and without ASD }\left(\mathrm{Chi}^{2}=\right. \\
\left.2.25, \mathrm{df}=1, \mathrm{P}=0.13 ; \mathrm{I}^{2}=55.5 \%\right)\end{array}$ \\
\hline $\begin{array}{l}\text { Remission of all } \\
\text { anxiety diagnoses } \\
\text { post-treatment } \\
\text { (ITT) }\end{array}$ & 191 per 1000 & $\begin{array}{l}\mathbf{5 1 2} \text { per } \mathbf{1 0 0 0} \\
(406 \text { to } 616)\end{array}$ & $\begin{array}{l}\text { OR } 4.43 \\
(2.89 \text { to } \\
6.78)\end{array}$ & $\begin{array}{l}2075 \\
\text { (28 stud- } \\
\text { ies) }\end{array}$ & $\begin{array}{l}\oplus \oplus \oplus \ominus \\
\text { moder- } \\
\text { ate } 2\end{array}$ & $\begin{array}{l}\text { Subgroup analyses: } \\
\text { - Difference in outcomes for different delivery formats (child-focused, child } \\
\text { and parent, parent only) }\left(\mathrm{Chi}^{2}=8.14, \mathrm{df}=2, \mathrm{P}=0.02, \mathrm{I}^{2}=75.4 \% \text { ) }\right. \\
\text { - No difference in outcomes for individual versus group formats }\left(\mathrm{Chi}^{2}=0.35 \text {, }\right. \\
\left.\mathrm{df}=1, \mathrm{P}=0.56 ; \mathrm{I}^{2}=0 \%\right)\end{array}$ \\
\hline
\end{tabular}




\begin{tabular}{|c|c|c|c|c|c|}
\hline 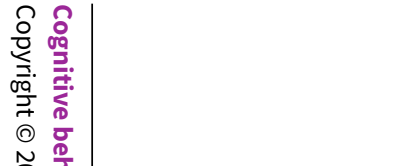 & & & & & $\begin{array}{l}\text { - Difference in outcomes for interventions with different amounts of thera- } \\
\text { pist contact }\left(\mathrm{Chi}^{2}=10.75, \mathrm{df}=2, \mathrm{P}=0.005 ; \mathrm{I}^{2}=81.4 \%\right) \\
\text { - Insufficient studies for subgroup analyses examining outcomes for partic- } \\
\text { ipants with and without ASD }\end{array}$ \\
\hline $\begin{array}{l}\text { Reduction in anx- } \\
\text { iety symptoms } \\
\text { (child report) } \\
\text { post-treatment }\end{array}$ & $\begin{array}{l}\text { The mean anxiety symptoms } \\
\text { (child report) in the CBT groups } \\
\text { was } \mathbf{0 . 6 7} \text { standard deviations } \\
\text { lower ( } 0.88 \text { to } 0.47 \text { lower). }\end{array}$ & $\begin{array}{l}\text { Moder- } \\
\text { ate ef- } \\
\text { fect size }\end{array}$ & $\begin{array}{l}2831 \\
\text { (45 stud- } \\
\text { ies) }\end{array}$ & $\begin{array}{l}\oplus \oplus \ominus \odot \\
\text { low } 4\end{array}$ & $\begin{array}{l}\text { Subgroup analyses: } \\
\text { - Difference in outcomes for different delivery formats (child-focused, child } \\
\text { and parent, parent only) }\left(\mathrm{Chi}^{2}=14.67, \mathrm{df}=2, \mathrm{P}<0.001, \mathrm{I}^{2}=86.4 \%\right) \\
\text { - Difference in outcomes for individual versus group formats }\left(\mathrm{Chi}^{2}=6.47 \text {, df }\right. \\
\left.=1, \mathrm{P}=0.01 ; \mathrm{I}^{2}=84.5 \%\right) \\
\text { - No difference in outcomes for interventions with different amounts of } \\
\text { therapist contact }\left(\mathrm{Chi} \mathrm{i}^{2}=3.33, \mathrm{df}=2, \mathrm{P}=0.19 ; \mathrm{I}^{2}=39.9 \%\right) \\
\text { - No difference in outcomes for participants with and without } \mathrm{ASD}\left(\mathrm{Chi}^{2}=\right. \\
\left.0.02, \mathrm{df}=1, \mathrm{P}=0.88 ; \mathrm{I}^{2}=0 \%\right)\end{array}$ \\
\hline $\begin{array}{l}\text { Reduction in anx- } \\
\text { iety symptoms } \\
\text { (parent report) } \\
\text { post-treatment }\end{array}$ & $\begin{array}{l}\text { The mean anxiety symptoms } \\
\text { (parent report) in the CBT groups } \\
\text { was } \mathbf{0 . 7 0} \text { standard deviations } \\
\text { lower ( } 0.90 \text { to } 0.51 \text { lower). }\end{array}$ & $\begin{array}{l}\text { Moder- } \\
\text { ate ef- } \\
\text { fect size }\end{array}$ & $\begin{array}{l}2137 \\
\text { (35 stud- } \\
\text { ies) }\end{array}$ & $\begin{array}{l}\oplus \oplus \odot \ominus \\
\text { low } 4\end{array}$ & $\begin{array}{l}\text { Subgroup analyses: } \\
\text { - No difference in outcomes for different delivery formats (child-focused, } \\
\text { child and parent, parent only) }\left(\mathrm{Chi}^{2}=3.43, \mathrm{df}=2, \mathrm{P}=0.18, \mathrm{I}^{2}=41.8 \%\right) \\
\text { - Difference in outcomes for individual versus group formats }\left(\mathrm{Chi}^{2}=6.79 \text {, df }\right. \\
\left.=1, \mathrm{P}=0.009, \mathrm{I}^{2}=85.3 \%\right) \\
\text { - No difference in outcomes for interventions with different amounts of } \\
\text { therapist contact }\left(\mathrm{Chi} \mathrm{i}^{2}=3.77, \mathrm{df}=2, \mathrm{P}=0.15 ; \mathrm{I}^{2}=46.9 \%\right) \\
\text { - No difference in outcomes for participants with and without } \mathrm{ASD}\left(\mathrm{Chi}^{2}=\right. \\
\left.1.42, \mathrm{df}=1, \mathrm{P}=0.23 ; \mathrm{I}^{2}=29.8 \%\right)\end{array}$ \\
\hline $\begin{array}{l}\text { Reduction in de- } \\
\text { pressive symp- } \\
\text { toms post-treat- } \\
\text { ment }\end{array}$ & $\begin{array}{l}\text { The mean depressive symptoms } \\
\text { in the CBT groups was } \mathbf{0 . 3 4} \text { stan- } \\
\text { dard deviations lower ( } 0.51 \text { to } \\
0.17 \text { lower). }\end{array}$ & $\begin{array}{l}\text { Small ef- } \\
\text { fect size }\end{array}$ & $\begin{array}{l}1157 \\
(17 \text { stud- } \\
\text { ies) }\end{array}$ & $\begin{array}{l}\oplus \oplus \oplus \odot \\
\text { moder- } \\
\text { ate } 2\end{array}$ & \\
\hline $\begin{array}{l}\text { Improvement in } \\
\text { global functioning } \\
\text { post-treatment }\end{array}$ & $\begin{array}{l}\text { The mean global functioning in } \\
\text { the CBT groups was } 1.03 \text { stan- } \\
\text { dard deviations higher ( } 0.68 \text { to } \\
1.38 \text { higher). }\end{array}$ & $\begin{array}{l}\text { Large ef- } \\
\text { fect size }\end{array}$ & $\begin{array}{l}557 \\
\text { (11 stud- } \\
\text { ies) }\end{array}$ & $\begin{array}{l}\oplus \oplus \ominus \ominus \\
\text { low } 5\end{array}$ & \\
\hline $\begin{array}{l}\text { Adverse events } \\
\text { (randomisation to } \\
\text { post-treatment) }\end{array}$ & See comment See comment & $\begin{array}{l}\text { Not es- } \\
\text { timable }\end{array}$ & - & $\begin{array}{l}\text { See } \\
\text { com- } \\
\text { ment }\end{array}$ & $\begin{array}{l}\text { No study reported adverse events in both CBT and waitlist/no treatment } \\
\text { groups }\end{array}$ \\
\hline
\end{tabular}

pist contact $\left(\mathrm{Chi}^{2}=10.75, \mathrm{df}=2, \mathrm{P}=0.005 ; \mathrm{I}^{2}=81.4 \%\right)$

$\begin{array}{llllll}\text { iety symptoms } & \text { (child report) in the CBT groups } & \text { ate ef- } & \text { (45 stud- } & \text { low } 4 & \text {. Difference in outcomes for different delivery formats (child-focused, child } \\ \text { (child report) } & \text { was } \mathbf{0 . 6 7} \text { standard deviations } & \text { fect size } & \text { ies) } & & \text { ies }\end{array}$

- Difference in outcomes for individual versus group formats $\left(\mathrm{Chi}^{2}=6.47 \mathrm{df}\right.$ $=1, \mathrm{P}=0.01 ; \mathrm{I}^{2}=84.5 \%$ )

No difference in outcomes for interventions with different amounts of 列 $\left.{ }^{2}=3.33, \mathrm{df}=2, \mathrm{P}=0.19 ; \mathrm{I}^{2}=39.9 \%\right)$

No difference in outcomes for participants with and without ASD $\left(\mathrm{Chi}^{2}=\right.$

*The basis for the assumed risk (e.g. the median control group risk across studies) is provided in footnotes. The corresponding risk (and its $95 \%$ confidence interval) is based on the as sumed risk in the comparison group and the relative effect of the intervention (and its 95\% $\mathrm{Cl})$. 


\section{GRADE Working Group grades of evidence}

High quality: Further research is very unlikely to change our confidence in the estimate of ef-

fect.

Moderate quality: Further research is likely to have an important impact on our confidence

in the estimate of effect and may change the estimate.

Low quality: Further research is very likely to have an important impact on our confidence in

the estimate of effect and is likely to change the estimate.

Very low quality: We are very uncertain about the estimate.

${ }^{1}$ Control group risk estimates come from pooled estimates of waitlist groups.

2Downgraded one level due to moderate heterogeneity (inconsistency).

${ }^{3}$ Downgraded two levels due large variation in treatment effects across studies (inconsistency) and wide confidence intervals (imprecision).

${ }^{4}$ Downgraded two levels due to substantial heterogeneity (inconsistency).

5Downgraded two levels due to substantial heterogeneity (inconsistency) and assessed and reported in small number of eligible studies (study limitations).

\section{Summary of findings 2. CBT compared with treatment as usual for anxiety disorders in children and adolescents}

\section{CBT compared with treatment as usual for anxiety disorders in children and adolescents}

Patient or population: children and adolescents with anxiety disorders

\section{Settings: outpatient clinics/schools}

Intervention: $\mathrm{CBT}$

Comparison: treatment as usual

\begin{tabular}{|c|c|c|c|c|c|c|}
\hline \multirow[t]{3}{*}{ Outcomes } & \multicolumn{2}{|c|}{ Illustrative comparative risks* $(95 \% \mathrm{Cl})$} & \multirow{3}{*}{$\begin{array}{l}\text { Relative effect } \\
(95 \% \mathrm{CI})\end{array}$} & \multirow{3}{*}{$\begin{array}{l}\text { No. of partic- } \\
\text { ipants } \\
\text { (studies) }\end{array}$} & \multirow{3}{*}{$\begin{array}{l}\text { Quality of the } \\
\text { evidence } \\
\text { (GRADE) }\end{array}$} & \multirow[t]{3}{*}{ Comments } \\
\hline & Assumed risk ${ }^{1}$ & Corresponding risk & & & & \\
\hline & Treatment as usual & CBT & & & & \\
\hline $\begin{array}{l}\text { Remission of primary anxi- } \\
\text { ety diagnosis post-treatment } \\
\text { (ITT) }\end{array}$ & 408 per 1000 & $\begin{array}{l}\mathbf{6 8 7} \text { per } \mathbf{1 0 0 0} \\
\text { (383 to } 886 \text { ) }\end{array}$ & $\begin{array}{l}\text { OR } 3.19 \text { ( } 0.90 \text { to } \\
11.29 \text { ) }\end{array}$ & $\begin{array}{l}487 \\
\text { (8 studies) }\end{array}$ & $\begin{array}{l}\oplus \oplus \ominus \ominus \\
\text { low } 2\end{array}$ & $\begin{array}{l}\text { Subgroup analyses: } \\
\text { - Difference in outcomes for } \\
\text { different delivery formats } \\
\text { (child-focused versus child } \\
\text { and parent) }\left(\text { Chi }^{2}=10.90, \mathrm{df}=\right. \\
\left.1, \mathrm{P}<0.001, \mathrm{I}^{2}=90.8 \%\right)\end{array}$ \\
\hline
\end{tabular}




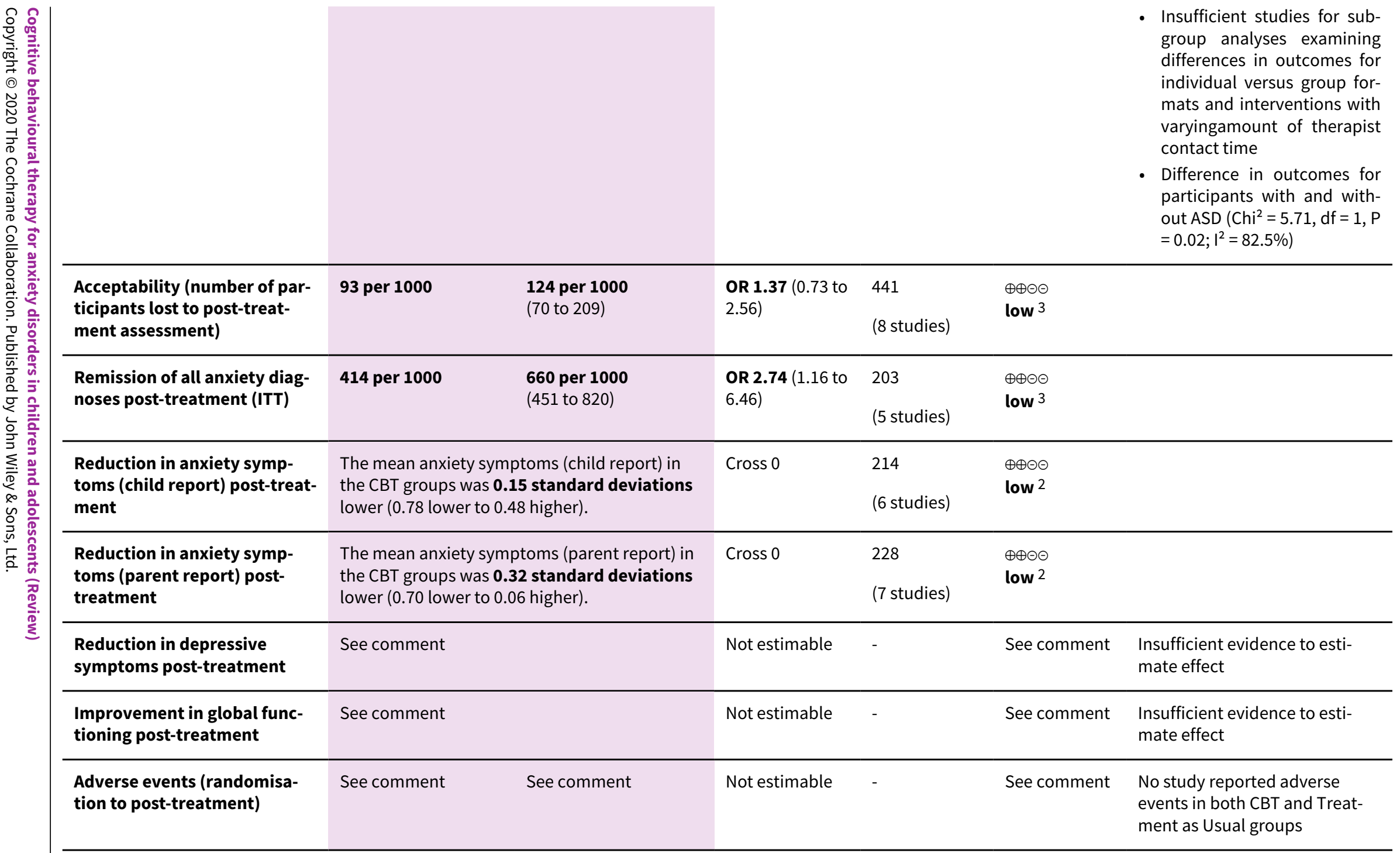

*The basis for the assumed risk (e.g. the median control group risk across studies) is provided in footnotes. The corresponding risk (and its $95 \%$ confidence interval) is based on the assumed risk in the comparison group and the relative effect of the intervention (and its $95 \% \mathrm{Cl}$ ).

CBT: cognitive behavioural therapy; Cl: confidence interval; ITT: intention-to-treat; OR: odds ratio

GRADE Working Group grades of evidence

High quality: Further research is very unlikely to change our confidence in the estimate of effect. 
Moderate quality: Further research is likely to have an important impact on our confidence in the estimate of effect and may change the estimate.

Low quality: Further research is very likely to have an important impact on our confidence in the estimate of effect and is likely to

change the estimate.

Very low quality: We are very uncertain about the estimate.

${ }^{1}$ Control group risk estimates come from pooled estimates of treatment as usual groups.

2Downgraded two levels due to at least moderate heterogeneity (inconsistency) and wide confidence intervals and small number of events or participants (imprecision).

${ }^{3}$ Downgraded two levels due to large variation in treatment effects across studies (inconsistency) and wide confidence intervals and small number of events (imprecision)

\section{Summary of findings 3. CBT compared with attention control for anxiety disorders in children and adolescents}

\section{CBT compared with attention control for anxiety disorders in children and adolescents}

Patient or population: children and adolescents with anxiety disorders

Settings: outpatient clinics/schools

Intervention: $\mathrm{CBT}$

Comparison: attention control

\begin{tabular}{|c|c|c|c|c|c|c|}
\hline \multirow[t]{3}{*}{ Outcomes } & \multicolumn{2}{|c|}{ Illustrative comparative risks ${ }^{\star}(95 \% \mathrm{CI})$} & \multirow{3}{*}{$\begin{array}{l}\text { Relative effect } \\
(95 \% \mathrm{CI})\end{array}$} & \multirow{3}{*}{$\begin{array}{l}\text { No. of partic- } \\
\text { ipants } \\
\text { (studies) }\end{array}$} & \multirow{3}{*}{$\begin{array}{l}\text { Quality of the } \\
\text { evidence } \\
\text { (GRADE) }\end{array}$} & \multirow[t]{3}{*}{ Comments } \\
\hline & Assumed risk & Corresponding risk & & & & \\
\hline & Attention control $\mathbf{1}$ & CBT & & & & \\
\hline $\begin{array}{l}\text { Remission of primary anxi- } \\
\text { ety diagnosis post-treatment } \\
\text { (ITT) }\end{array}$ & 293 per 1000 & $\begin{array}{l}\mathbf{4 8 6} \text { per } \mathbf{1 0 0 0} \\
\text { (355 to } 617)\end{array}$ & $\begin{array}{l}\text { OR } 2.28 \text { ( } 1.33 \text { to } \\
3.89 \text { ) }\end{array}$ & $\begin{array}{l}822 \\
\text { (10 studies) }\end{array}$ & $\begin{array}{l}\oplus \oplus \odot \ominus \\
\text { low 2, }\end{array}$ & $\begin{array}{l}\text { Subgroup analyses: } \\
\text { - Difference in outcomes } \\
\text { for different delivery for- } \\
\text { mats (child-focused ver- } \\
\text { sus child and parent) } \\
\left(\mathrm{Chi}^{2}=7.65, \mathrm{df}=1, \mathrm{P}=\right. \\
\left.0.006 ; \mathrm{I}^{2}=86.9 \%\right) \\
\text { - No difference in out- } \\
\text { comes for individual ver- } \\
\text { sus group formats }\left(\mathrm{Chi}^{2}=\right. \\
1.22, \mathrm{df}=1, \mathrm{P}=0.27 ; \mathrm{I}^{2}= \\
17.7 \%) \\
\text { - Insufficient studies for } \\
\text { subgroup analyses exam- } \\
\text { ining outcomes for in- }\end{array}$ \\
\hline
\end{tabular}




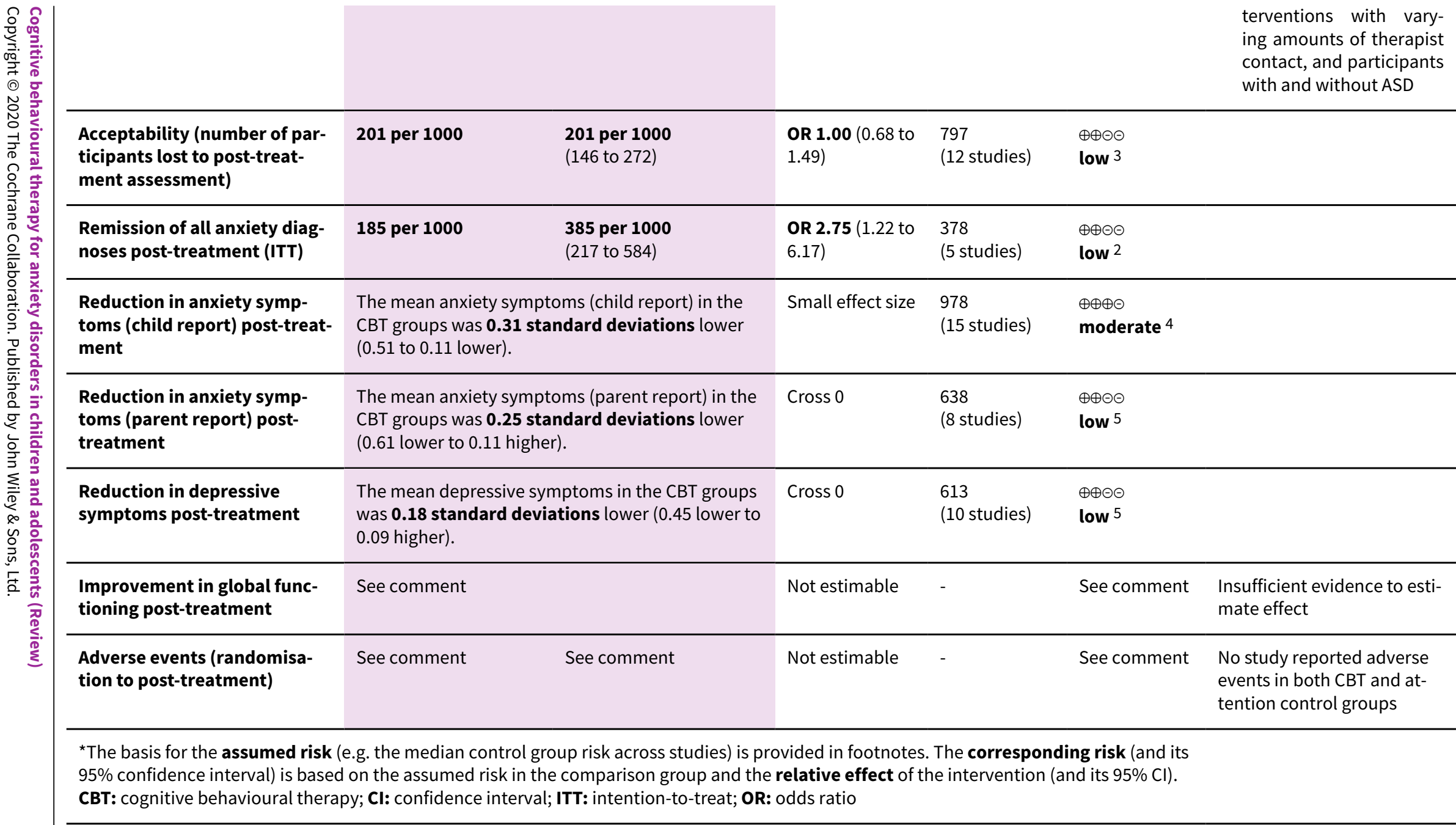

\section{GRADE Working Group grades of evidence}

High quality: Further research is very unlikely to change our confidence in the estimate of effect.

Moderate quality: Further research is likely to have an important impact on our confidence in the estimate of effect and may change the es-

timate.

Low quality: Further research is very likely to have an important impact on our confidence in the estimate of effect and is likely to change

the estimate.

Very low quality: We are very uncertain about the estimate.

${ }^{1}$ Control group risk estimates come from pooled estimates of attention control groups. 


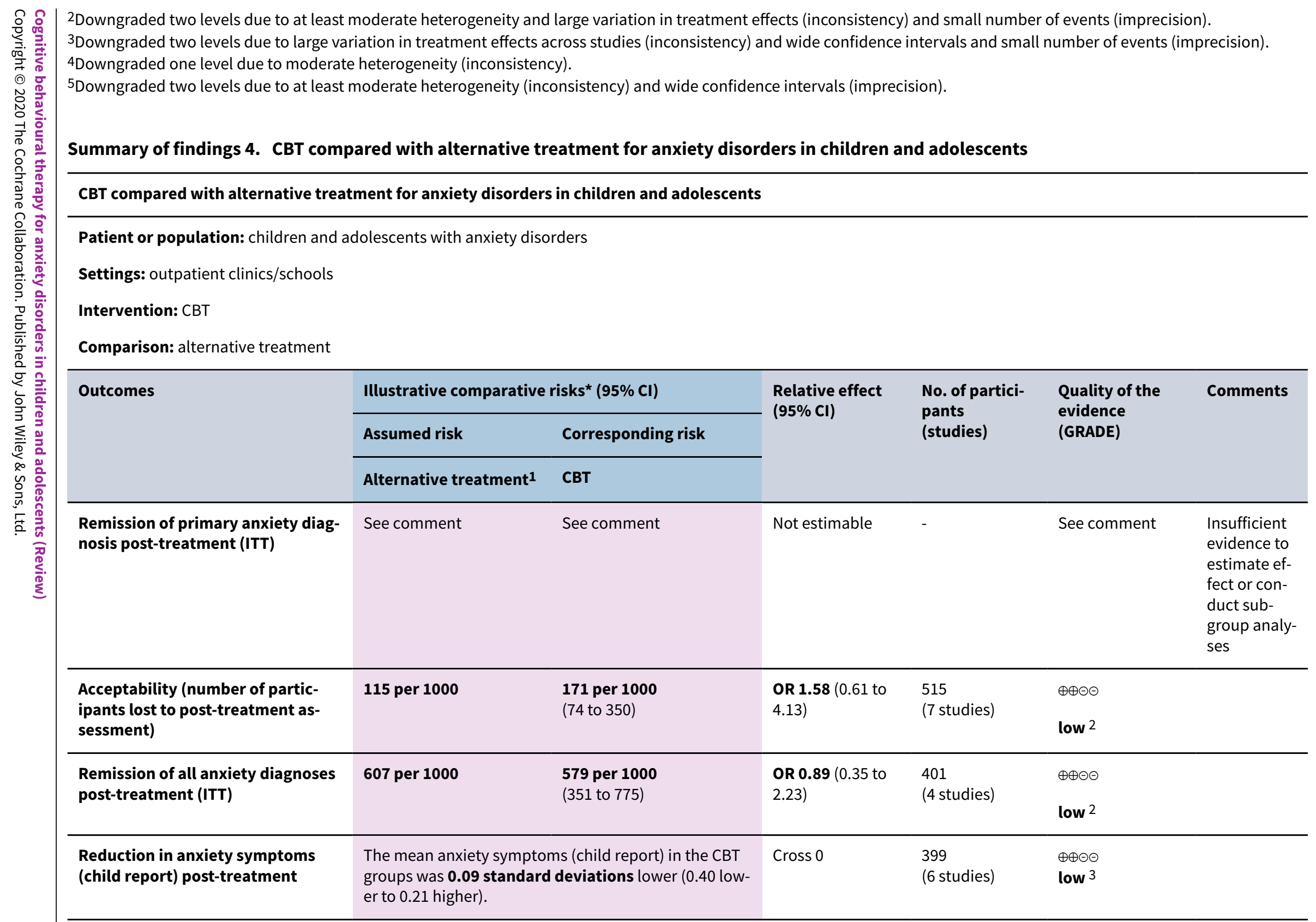




\begin{tabular}{|c|c|c|c|c|c|c|}
\hline \multirow{2}{*}{\multicolumn{2}{|c|}{ 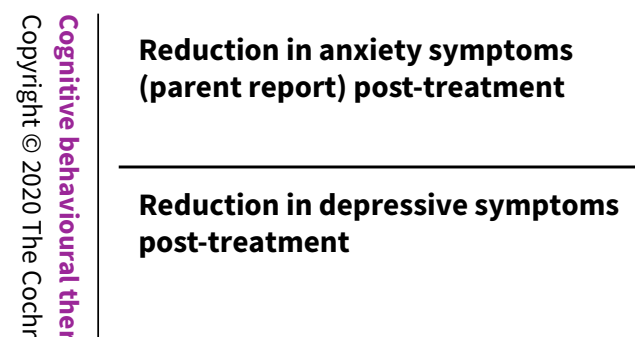 }} & $\begin{array}{l}\text { The mean anxiety symptoms (child report) in the CBT } \\
\text { groups was } \mathbf{0 . 1 3} \text { standard deviations lower ( } 0.33 \text { low- } \\
\text { er to } 0.06 \text { higher). }\end{array}$ & Cross 0 & $\begin{array}{l}423 \\
\text { (6 studies) }\end{array}$ & $\begin{array}{l}\oplus \oplus \ominus \ominus \\
\text { low } 4\end{array}$ & \\
\hline & & Not estimable & Not estimable & - & See comment & $\begin{array}{l}\text { Insufficient } \\
\text { evidence to } \\
\text { estimate ef- } \\
\text { fect }\end{array}$ \\
\hline 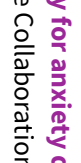 & $\begin{array}{l}\text { Improvement in global functioning } \\
\text { post-treatment }\end{array}$ & Not estimable & Not estimable & - & See comment & $\begin{array}{l}\text { No evidence } \\
\text { available to } \\
\text { estimate ef- } \\
\text { fect }\end{array}$ \\
\hline 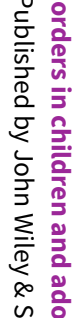 & $\begin{array}{l}\text { Adverse events (randomisation to } \\
\text { post-treatment) }\end{array}$ & See comment & Not estimable & - & See comment & $\begin{array}{l}\text { No study re- } \\
\text { ported ad- } \\
\text { verse events } \\
\text { in both } \\
\text { CBT and } \\
\text { Treatment } \\
\text { as Usual } \\
\text { groups }\end{array}$ \\
\hline 范管 & \multicolumn{6}{|c|}{$\begin{array}{l}\text { *The basis for the assumed risk (e.g. the median control group risk across studies) is provided in footnotes. The corresponding risk (and its } 95 \% \text { confi- } \\
\text { dence interval) is based on the assumed risk in the comparison group and the relative effect of the intervention (and its } 95 \% \mathrm{CI} \text { ). } \\
\text { CBT: cognitive behavioural therapy; CI: confidence interval; ITT: intention-to-treat; OR: odds ratio }\end{array}$} \\
\hline$\underline{\underline{\omega}}$ & \multicolumn{6}{|c|}{$\begin{array}{l}\text { GRADE Working Group grades of evidence } \\
\text { High quality: Further research is very unlikely to change our confidence in the estimate of effect. } \\
\text { Moderate quality: Further research is likely to have an important impact on our confidence in the estimate of effect and may change the estimate. } \\
\text { Low quality: Further research is very likely to have an important impact on our confidence in the estimate of effect and is likely to change the estimate. } \\
\text { Very low quality: We are very uncertain about the estimate. }\end{array}$} \\
\hline
\end{tabular}

${ }^{1}$ Control group risk estimates come from pooled estimates of alternative treatment groups.

2Downgraded two levels due to at least moderate heterogeneity (inconsistency) and wide confidence intervals and small number of events (imprecision).

${ }^{3}$ Downgraded two levels due to moderate heterogeneity (inconsistency) and wide confidence intervals (imprecision).

${ }^{4}$ Downgraded two levels due to large variation in treatment effects across studies (inconsistency) and wide confidence intervals (imprecision). 


\section{B A C K G R O U N D}

\section{Description of the condition}

Anxiety disorders are amongst the most common psychiatric disorders, occurring in $6.5 \%$ of all children and adolescents (Polanczyk 2015). One of the diagnostic challenges in children and adolescents involves distinguishing normal, developmentally appropriate worries, fears, and shyness from anxiety disorders. For example, primary school-age children commonly have worries about injury and natural events, whereas older children and adolescents typically have worries and fears related to school performance, social competence, and health issues (Beesdo 2009). Distinguishing features of pathological anxiety include severity, persistence, and associated impairment.

The International Classification of Diseases (ICD), WHO 1992, and Diagnostic and Statistical Manual of Mental Disorders (DSM-5), APA 2013, diagnostic systems distinguish various types of anxiety disorders, including generalised anxiety disorder, panic disorder, social anxiety disorder, separation anxiety disorder, agoraphobia, specific phobias, and selective mutism. These anxiety disorders are often associated with significant impairment in personal, social, and academic functioning (Pine 2009). Comorbidities are common, in particular with other anxiety disorders in children and adolescents (Leyfer 2013), and depression in adolescents (Essau 2003).

The presentation of anxiety disorders varies with age. Separation anxiety disorders are more common in younger children than in adolescents, and difficulties with social anxiety are typically associated with greater disturbance in adolescence (Waite 2014). Anxiety disorders with an onset in childhood often persist into adolescence and early adulthood (Copeland 2014), and are also associated with adverse academic, health, and social functioning in adulthood (Copeland 2014; Essau 2014). Yet these often remain untreated, with an average delay of 9 to 23 years before anxiety disorders are first treated (Wang 2005). It is clear that anxiety disorders in this age group present serious ongoing health issues, therefore effective and readily accessible treatments are needed.

\section{Description of the intervention}

Current treatments for anxiety disorders in this age group include behavioural therapy, cognitive behavioural therapy (CBT), or medication, or a combination of some or all these. National Institute for Health and Care Excellence (NICE) guidelines are available for the treatment of social anxiety disorder, which recommend CBT that is specifically focused on social anxiety (NICE 2013). Given the prevalence of these disorders, the age of onset, and public views on the acceptability of pharmacological treatments, psychological treatments are often preferred as first-line therapy (Brown 2007; Young 2006). CBT is a collaborative psychological treatment that can be delivered in various formats, individually or in groups, and with varying levels of parent or family involvement.

One of the first manualised CBT programmes was Coping Cat (Kendall 1994), which consists of psycho-education, modification of negative cognitions, exposure, social competence training, coping behaviour, and self-reinforcement sessions. Others have followed, including the Cool Kids programme (Hudson 2009; Rapee 2006), the Coping Koala programme (Barrett 1996), Skills for Academic and Social Success (SASS) (Masia-Warner 2005),
ACTION (Waters 2009), Intervention With Adolescents With Social Phobia (IAFS) (Sanchez-Garcia 2009), the TAPS (Warner 2011), and Building Confidence programme (Galla 2012). Alternative programmes involve providing direct support to parents alone, guiding them to implement CBT strategies with their child (Guided Parent-Delivered CBT (GPD-CBT) (Lyneham 2006; Thirlwall 2013; Waters 2009). CBT programmes have typically adopted a generic approach and target a range of anxiety disorders (e.g. Coping Cat), but some are disorder-specific, targeting, for example, social anxiety disorder (e.g. SASS). CBT programmes have been modified in various ways to make them appropriate for children with autism spectrum disorders (ASD), such as by including social stories, social coaching, visual aids, and structured worksheets (Ung 2015). Such programmes include the Multimodal Anxiety and Social Skills Intervention (MASSI) programme (White 2013), TAFF (Schneider 2011), Behavioural Interventions for Anxiety in Children with Autism (BIACA) (Wood 2009), and Facing Your Fears (FYF) (Reaven 2012).

\section{How the intervention might work}

CBT for anxiety disorders in children and adolescents typically involves helping the child to recognise anxious feelings and bodily or somatic reactions to anxiety, identify thoughts or cognitions in anxiety-provoking situations (e.g. unrealistic or negative attributions and expectations), and modifying these anxiety-provoking cognitions (e.g. testing out predictions based on anxious thoughts, modifying anxious self-talk into coping self-talk). A key CBT procedure is exposure (Creswell 2020b), which typically involves testing out and 'facing' fears, often in a gradually increasing hierarchy. Behavioural training strategies such as modelling and role playing are often applied as opportunities for developing coping skills (e.g. problem-solving skills, social skills, relaxation training).

CBT for anxiety disorders in children and adolescents has traditionally begun with six to nine face-to-face sessions of anxiety management strategies (emotion identification, relaxation training, cognitive strategies), followed by exposure work (Barrett 1996; Kendall 2006). In one meta-analysis (Reynolds 2012), this traditional format of anxiety management sessions followed by exposure was observed in 93\% of studies. However, Ale 2015 found that treatment outcomes in CBT treatment trials for child and adolescent anxiety disorders were not related to the use of relaxation strategies or the timing of exposure work, and therefore suggested that relaxation training may not be an essential ingredient of CBT, and that it may not be necessary to delay exposure until after anxiety management sessions. Moreover, there is also some preliminary evidence that introducing exposure early in treatment, without any prior anxiety management sessions, could improve outcomes whilst requiring fewer appointments (Whiteside 2015).

Indeed, questions remain about the mechanism of change within CBT. Cognitive restructuring and exposure tasks have each been found to make substantial contributions to improvement in child and adolescent anxiety in line with CBT theory (Peris 2015). More time devoted to exposure has been linked to better outcomes, and greater time spent on more difficult exposure tasks has been shown to predict better outcomes (Peris 2017). Change in coping efficacy, but not anxious self-talk, has been found to mediate change in anxiety symptoms associated with CBT, medication (sertraline), and their combination, compared to placebo control 
(Kendall 2016). Furthermore, therapists' ratings of child compliance and mastery also predict better outcomes (Peris 2017). Cognitive development also plays an important role, and whilst targeting behavioural avoidance appears to be crucial for both children and adolescents, treatment that directly addresses interpretation biases may be particularly beneficial for adolescents, but less so for younger children (Waite 2015).

CBT has been adapted to include family and parents in treatment sessions; however, parents/family members have been included in a wide variety of ways, including, for example, providing education and information on the child-focused CBT, and/or modifying parents' beliefs and behaviours, and/or addressing parental anxiety. These different approaches are based on different conceptualisations of the mechanisms of change in child anxiety through parental involvement. It is important to note, however, that changes in parent and child responses are likely to be bidirectional. For example, one study found that child-focused anxiety treatments resulted in improvements in non-targeted parent symptoms and family functioning, particularly when children responded successfully to treatment (Keeton 2013).

It is generally assumed that CBT can be applied only after the child has reached a certain level of cognitive development. Kendall 1993 argued that the ability to measure a thought or belief against the notion of a rational standard and the ability to understand that a thought or belief can cause a person to behave and feel in a certain way were central to its proper use. The question arises: at what age does a child have the cognitive capacities to undertake these cognitive operations? One study reported positive effects of CBT in children younger than six years of age (Hirshfeld-Becker 2010); however, it is not clear whether children this age are able to use the cognitive strategies that are included in traditional CBT protocols. In line with this, research suggests that young children may be more responsive to the behavioural than the cognitive elements of this approach (Essau 2004). With younger children, parental involvement appears particularly important. Indeed, the treatment of anxiety disorders in young children can be effective by applying CBT principles through working directly with parents alone (Cartwright-Hatton 2011), although there are inconsistent findings in relation to whether child-parent delivery format is superior to parent-only or not (Monga 2015; Waters 2009).

\section{Why it is important to do this review}

Anxiety disorders in children and adolescents represent a considerable source of morbidity and are associated with later adult psychopathology and greater cost than any other mental health disorder (Fineberg 2013). However, despite high prevalence and substantial morbidity, anxiety disorders in childhood and adolescence can be difficult to diagnose, and may be underrecognised (NICE 2013), and therefore undertreated (Pine 2009). It is widely reported that only a minority of children and adolescents with mental health problems receive treatment (Green 2004; Merikangas 2011); a recent UK survey reported that fewer than $40 \%$ of children with anxiety disorders receive any professional support, and less than $3 \%$ receive CBT (Reardon 2020). Limited service provision represents a key barrier to treatment access (Reardon 2017), highlighting the importance of maximising the efficiency of treatment delivery to help ensure that effective treatment is more readily available to children and young people when they need it.
The evidence base for the treatment of anxiety disorders in children and adolescents is growing. Initial trials of CBT were positive (Barrett 1996; Kendall 1994; Kendall 1997), and further randomised controlled trials and reviews followed. Several reviews suggest that CBT for anxiety disorders in this age group is effective (Ale 2015; Crowe 2017; James 2015; Reynolds 2012; Silverman 2008), including an overview of systematic reviews and a network metaanalysis (Bennett 2016; Zhou 2019). Overall there was a moderate response rate (e.g. 59\%; James 2015), and a recent review indicated that benefits extend to broader outcomes, including depressive symptoms and general functioning (Kreuze 2018). However, CBT has not been shown to be superior to active controls or treatment as usual (James 2015; Southam-Gerow 2010). In some previous studies, the effect sizes associated with CBT for childhood anxiety disorders did not differentiate it from attention placebo, although CBT has been found to be more effective than waiting list control (Ale 2015). It remains unclear whether CBT is superior to alternative treatment approaches.

This review updates and replaces previous Cochrane Reviews of CBT for anxiety disorders in children and adolescents (James 2005; James 2015). The current review aims to provide comprehensive and up-to-date evidence on the efficacy and acceptability of CBT in the treatment of anxiety disorders in children and adolescents, with varying amounts of therapist contact time and differing delivery formats, including individual, group, with/without family/parent involvement, and parent-led. Furthermore, we planned to examine the efficacy of CBT relative to treatment as usual, attention control, and alternative treatments. The question of the comparative efficacy of medication versus CBT and the combination of CBT and medication was also to be addressed.

A recent development is the issue of the reporting of remission of all anxiety diagnoses, as well as the primary anxiety diagnosis (Warwick 2016). Given the high level of comorbid anxiety disorders, this is an important issue, but surprisingly has been previously overlooked. Indeed, focusing solely on recovery from the primary anxiety diagnosis means that children with comorbid anxiety disorders that are present following treatment are often still classed as 'recovered'. As such, this review distinguishes between and examines outcomes based on both the absence of the "primary anxiety disorder' and the absence of all anxiety disorders. As well as providing evidence on anxiety outcomes (diagnoses and symptoms), we also planned to examine benefits in relation to broader outcomes, including depressive symptoms and global functioning.

We also aimed to assess whether treatment effects of CBT are maintained beyond post-treatment and at longer-term follow-up. Whilst it would not be possible to determine the youngest age at which a child can benefit from CBT without individual patient data, we would identify the age of the youngest participants in trials of CBT for child and adolescent anxiety disorders. It is recognised that children and adolescents with ASDs have high rates of anxiety disorders (van Steensel 2013); however, studies of CBT for anxiety disorders in ASD have had mixed outcomes (Murphy 2017; Ung 2015). Furthermore, it is unclear how anxiety disorders are recognised or, indeed, treated in those with intellectual impairments, indicating a pressing need for work in this area. We planned to examine the efficacy of CBT in children and adolescents with ASD and those with intellectual impairments. 
Previous Cochrane Reviews used a cutoff of nine sessions of CBT, based on the practice and thinking at the time (James 2005; James 2015). However, since the last Cochrane Review (James 2015), there have been several developments in the delivery of CBT for anxiety disorders. These include briefer or shorter interventions, in terms of not only the number of sessions, but also the total duration of treatment. As such, we would not set a threshold for the minimum number of sessions, and would explore how treatment effects differ with treatment duration. It is important to note that one potentially efficient means to deliver CBT is online or via digital devices (e.g. computerised (CBT). But as online and digital CBT interventions for child anxiety disorders have been reviewed elsewhere (e.g. Pennant 2015), this review focused on face-to-face delivery models that include direct contact with either the child or parent alone, or the child and parent together.

\section{O B JE C T IVES}

- To carry out a meta-analysis of identified studies to determine whether CBT leads to remission of 1 ) the primary child/ adolescent anxiety disorder and 2) all anxiety diagnoses, and/ or 3) a clinically significant reduction in anxiety symptoms in comparison with waiting list/no treatment, treatment as usual, attention control, alternative treatment, or medication.

- To determine the comparative efficacy of CBT alone, and the combination of CBT and medication, versus drug placebo.

- To determine if CBT is an acceptable treatment, relative to waiting list/no treatment, treatment as usual, attention control, alternative treatment, and medication.

- To determine whether CBT for anxiety leads to a clinically significant reduction in depressive symptoms and/or improvements in global functioning.

- To identify any adverse effects associated with CBT, relative to waiting list/no treatment, treatment as usual, attention control, alternative treatment, and medication.

- To determine whether post-treatment gains of CBT are maintained at longer-term follow-up.

- To describe the age range of participants included in CBT trials in order to determine the age of the youngest participants.

- To carry out subgroup analyses of different types of CBT according to 1) amount of therapist contact time; and 2) delivery format (individual and group; child-focused, child and parent/ family, and parent-only).

- To carry out a subgroup analysis of CBT for children and adolescents with ASD and for children and adolescents with intellectual impairments.

\section{METHO D S}

\section{Criteria for considering studies for this review}

\section{Types of studies}

We included randomised controlled trials (RCTs), cross-over trials, and cluster-randomised trials.

\section{Types of participants}

\section{Participant characteristics}

Children and adolescents younger than 19 years.

\section{Diagnosis}

We included participants meeting diagnostic criteria of the DSM (DSM III, III-R, IV, IV-TR, 5), APA 1980; APA 1987; APA 1994; APA 2000; APA 2013, or the ICD-9 and ICD-10, WHO 1978; WHO 1992, for an anxiety disorder.

Diagnoses had to be made by reliable and valid structured interviews for DSM or ICD child and adolescent anxiety disorders. Disorders classified as anxiety disorders vary across different versions of the DSM, and we included participants meeting diagnostic criteria for one or more of the following disorders: generalised anxiety disorder or overanxious disorder, separation anxiety disorder, social phobia or social anxiety disorder, panic disorder, agoraphobia, simple or specific phobias, or selective mutism.

\section{Comorbidity}

We included all comorbidities allowable for anxiety disorders under the rules of DSM or ICD, such as ASD, intellectual impairment, depressive disorders, and physical disorders.

\section{Settings}

We included all settings, such as research settings (i.e. university outpatient clinics, inpatient services, community clinics, and schools).

\section{Exclusion criteria}

We excluded studies that only included participants with posttraumatic stress disorder or obsessive compulsive disorder, or both, as they are covered by separate Cochrane Reviews (Gillies 2016; O'Kearney 2006), and are no longer classified as 'anxiety disorders' in the DSM-5 (APA 2013).

\section{Types of interventions}

\section{Experimental intervention}

The intervention had to be manualised CBT, or modular CBT, alone or in combination with medication, following a documented, written protocol and provided by trained therapists. Since the last review (James 2015), where the number of sessions was arbitrarily fixed at nine, there are now several studies indicating that shorter treatment, in terms of number of sessions or duration of sessions, or both, may be effective. We therefore did not include a minimum number of sessions or duration of sessions as a requirement.

CBT had to be administered according to standard principles as a psychological model of treatment involving helping the child to recognise anxious feelings and somatic reactions to anxiety; identify cognitions in anxiety-provoking situations; modify these anxiety-provoking cognitions; and respond to behavioural training strategies with exposure in vivo or by imagination.

CBT could be delivered to children (child-focused), to children and parents/family members (child and parent), or to parents alone (parent-only). In child-focused CBT, the child had direct face-toface contact with a therapist, and the intervention was delivered to children only, with minimal or no parental involvement. CBT with direct parent/family involvement (child and parent) included face-to-face child sessions and conjoint or separate sessions with parents/family that included providing psychoeducation for parents or teaching parents to be co-therapists. 
Parent-delivered CBT (parent-only) only involved direct face-toface contact with parents, and provided support for parents to help them to implement CBT strategies in their child's day-today life. Child-focused, child and parent, and parent-only CBT could be delivered individually or in a group format. We did not include CBT interventions delivered online or via digital devices (e.g. computerised CBT).

Where studies included medication for the treatment of anxiety (in combination with CBT or alone), no concurrent medication for the treatment of anxiety was to be administered naturalistically. Where studies did not include medication for the treatment of anxiety, any medications administered naturalistically needed to be stable before and during the study.

\section{Comparator interventions}

\section{Where CBT was delivered alone}

- Waitlist and no treatment for anxiety during that period

- Treatment as usual (usual treatment or care; where usual care includes therapy, it does not include elements of CBT)

- Attention control (attention only, e.g. support or education, but with no elements of CBT)

- Alternative treatment (one specific non-pharmacological intervention for the treatment of anxiety that followed a documented protocol and did not include CBT elements)

- Medication for the treatment of anxiety

- Drug placebo

Where CBT was delivered in combination with medication for the treatment of anxiety

- Drug placebo

\section{Types of outcome measures}

We included studies that met the above inclusion criteria and reported or provided data on at least one of the following outcomes.

\section{Primary outcomes}

- Remission: the absence of the primary diagnosis of an anxiety disorder post-treatment, made by reliable and valid structured interviews for DSM or ICD child and adolescent anxiety disorders, such as:

* Anxiety Disorder Interview Schedule for Children - Child and Parent (ADIS-C/P) (Silverman 1987);

* Anxiety Disorder Interview Schedule for Children - Child (ADIS-C) (Silverman 1987);

* Anxiety Disorder Interview Schedule for Children - Parent (ADIS-P) (Silverman 1987);

* Diagnostic Interview Schedule for Children, Adolescents and Parents (DISCAP) (Holland 1995).

- Acceptability: loss of participants to post-treatment assessment

\section{Secondary outcomes}

- Remission: defined as the absence of all diagnoses of an anxiety disorder post-treatment, made by reliable and valid structured interviews for DSM or ICD child and adolescent anxiety disorders.
- Reduction in anxiety symptoms post-treatment: measured using psychometrically robust measures of anxiety symptoms that yield symptom scores on continuous scales (Myers 2002), such as:

* Screen for Child Anxiety Related Emotional Disorders (SCARED) (Birmaher 1999);

* Spence Children's Anxiety Scale (SCAS) (Spence 1997);

* Revised Children's Anxiety and Depression Scale (RCADS) Anxiety Scale (Chorpita 2000);

* Revised Children's Manifest Anxiety Scale (RCMAS) (Reynolds 1985);

* Multidimensional Anxiety Scale for Children (MASC) (March 1997);

* State-Trait Anxiety Inventory for Children (STAI-C) (Spielberger 1973);

* Social Phobia and Anxiety Inventory for Children (SPAI-C) (Beidel 1995);

* Social Anxiety Scale for Adolescents (SAS-A) (La Greca 1998).

These scales could be self-report or completed by a parent or an independent rater. Multiple reporters are often used, but the reliability of each reporter is likely to vary with the child's age (Evans 2017). We therefore determined reduction in anxiety symptoms separately for 1 ) self-reported and 2) parent-reported or independent rater, or both. Multiple measures are also often reported, and we included the most validated, best recognised, or most frequently used measures in the analysis.

A crucial issue is how well these measures discriminate between clinical and non-clinical levels of anxiety. We prioritised symptom measures that were closely aligned with diagnostic categories, with strong discriminant validity (e.g. RCADS, SCAS, SCARED). We also prioritised broad measures of anxiety symptoms (e.g. SCAS, SCARED, MASC), rather than disorder-specific symptom measures (e.g. SPAI-C, SAS-A). (See Appendix 1)

- Reduction in depressive symptoms post-treatment: measured using psychometrically robust measures of depressive symptoms that yielded symptom scores on continuous scales, such as:

* Children's Depression Inventory (Kovacs 1989);

* Beck Depression Inventory (Beck 1996);

* Revised Children's Anxiety and Depression Scale (RCADS) Depression Scale (Chorpita 2000);

* Mood and Feelings Questionnaire (Angold 1995).

If multiple depressive symptom measures/reporters were used, we included the most validated, best recognised, or most frequently used measures in the analysis.

- Improvement in global functioning post-treatment: measured using psychometrically robust measures of global functioning that yield symptom scores on continuous scales, such as:

* Children's Global Assessment Scale (CGAS) (Shaffer 1983).

If multiple global functioning measures/reporters were used, we included the most validated, best recognised, or most frequently used measures in the analysis.

- Adverse events: we determined adverse events outcomes by the number and type of reported adverse events during the 
trial from randomisation to post-treatment assessment (e.g. deterioration in anxiety symptoms, deterioration in global functioning, rates of self-harm, suicide attempts).

- Remission defined by the absence of the primary anxiety disorder diagnosis at a series of follow-up time points $(\leq 6$ months post-treatment, $>6$ months post-treatment but $\leq 12$ months post-treatment, and $>12$ months post-treatment).

- Remission defined as the absence of all diagnoses of an anxiety disorder at a series of follow-up time points ( $\leq 6$ months posttreatment, $>6$ months post-treatment but $\leq 12$ months posttreatment, and $>12$ months post-treatment).

- Reduction in anxiety symptoms at a series of follow-up time points ( $\leq 6$ months post-treatment, $>6$ months post-treatment but $\leq 12$ months post-treatment, and $>12$ months posttreatment).

\section{Search methods for identification of studies}

We identified eligible studies (RCTs) of CBT for anxiety disorders in children and adolescents from the Cochrane Common Mental Disorders Controlled Trials Register (CCMDCTR; all years to 2016) (Appendix 2).

\section{Electronic searches}

We also ran searches on the following databases using relevant keywords, subject headings (controlled vocabularies), and search syntax, appropriate to each resource (Appendix 3). Searches were initially conducted on 21 November 2018 and updated 10 October 2019.

- Cochrane Central Register of Controlled Trials (CENTRAL; Issue 10 of 12, 2019) in the Cochrane Library

- Ovid MEDLINE (2016 to 10 October 2019)

- Ovid Embase (2016 to 2019 Week 40)

- Ovid PsycINFO (all years to October Week 40 2019)

We applied no restriction on language or publication status to the searches.

We also searched the international trial registries (10 October 2019) (including US National Institutes of Health Ongoing Trials Register ClinicalTrials.gov (clinicaltrials.gov) and the World Health Organization International Clinical Trials Registry Platform (apps.who.int/trialsearch/)) to identify additional ongoing and unpublished studies.

\section{Searching other resources}

\section{Grey literature}

We searched the grey literature for dissertations and theses (all years to 14 October 2019) (Appendix 4), as follows.

- Electronic Theses Online Service (EThOS) - British Library (ethos.bl.uk/Home.do)

- DART - Europe e-theses Portal (www.dart-europe.eu/basicsearch.php)

- Networked Digital Library of Theses and Dissertations (NDLTD) (search.ndltd.org/)

- PQDT Open - open access dissertations and theses (pqdtopen.proquest.com/search.html)
- Proquest Dissertations \& Theses Global (search.proquest.com/ pqdtglobal/dissertations/)

\section{Reference lists}

We checked the reference lists of all included studies and relevant systematic reviews to identify additional studies missed from the original electronic searches (e.g. unpublished or in-press citations).

\section{Correspondence}

We contacted study authors and subject experts for information on unpublished or ongoing studies, or to request additional data. Where studies included some eligible participants (i.e. some participants were younger than 19 years, or some participants met the diagnostic criteria for an anxiety disorder), we contacted the authors to request data on eligible participants.

\section{Data collection and analysis}

\section{Selection of studies}

Two review authors (AJ, TR) independently screened the titles and abstracts of all studies identified as a result of the search, coding them as 'retrieve' (eligible or potentially eligible/unclear) or 'do not retrieve'. We retrieved the full-text study reports/publications, and two review authors (AJ, TR) independently screened the full texts to identify studies for inclusion, and identified and recorded reasons for exclusion of the ineligible studies. Any disagreements were resolved through discussion or by consulting a third review author (GJ, AS, CC) if required. We identified and excluded duplicate records and collated multiple reports that related to the same study so that each study, rather than each report, was the unit of interest in the review. We recorded the selection process in sufficient detail to complete a PRISMA flow diagram and 'Characteristics of excluded studies' table (Moher 2015). We used Covidence software for the screening process (Covidence).

\section{Data extraction and management}

We used a data collection form created in Covidence to extract study characteristics and outcome data that we had piloted on two studies in the review. Two review authors (AJ, TR) extracted study characteristics and outcome data from the included studies. We extracted the following study characteristics.

- Methods: study design, total duration of study, details of any 'run-in' period, number of study centres and location, study setting, withdrawals, and date of study

- Participants: number, mean age, age range, gender, severity of condition, diagnostic criteria, comorbid conditions, inclusion criteria, and exclusion criteria

- Interventions: intervention, comparison, concomitant medications, excluded medications, delivery format, therapist contact time, who delivers intervention

- Outcomes: primary and secondary outcomes specified and collected, and time points reported

- Notes: funding for study, and notable conflicts of interest of study authors

We noted in the 'Characteristics of included studies' table if outcome data were not reported in a useable way. Any disagreements were resolved by consensus or by involving a third review author (GJ, AS, CC). One review author (TR) transferred data into the Review Manager 5 file (Review Manager 2014). We double- 
checked that data were entered correctly by comparing the data presented in the systematic review with the study reports. A second review author (AJ) spot-checked study characteristics for accuracy against the study report.

\section{Main comparisons}

- $\mathrm{CBT}$ compared with waiting list and no treatment controls

- CBT compared with treatment as usual

- CBT compared with attention control

- CBT compared with alternative treatments

- CBT compared with medication or drug placebo

- CBT and medication combination compared with drug placebo

\section{Assessment of risk of bias in included studies}

Two review authors (AJ, TR) independently assessed risk of bias for each study using the criteria outlined in the Cochrane Handbook for Systematic Reviews of Interventions (Higgins 2017). Any disagreements were resolved by discussion or by involving another review author (GJ, CC). We assessed risk of bias according to the following domains.

- Random sequence generation

- Allocation concealment

- Blinding of participants and personnel

- Blinding of outcome assessment

- Incomplete outcome data

- Selective outcome reporting

- Other bias, including therapy integrity

We judged each potential source of bias as low, high, or unclear and provided a supporting quotation from the study report together with a justification for our judgement in the 'Risk of bias' table. We summarised the 'Risk of bias' judgements across different studies for each of the domains listed.

Selection bias: we assessed the adequacy of the randomisation process in terms of the description of adequacy of sequence generation and the concealment of treatment group allocation.

Performance bias: given the nature of psychological interventions, blinding of either participants or personnel delivering the treatments could only be possible in studies involving CBT versus treatment as usual, attention control, or alternative treatment, therefore we were only able to assess attempts at blinding in those studies.

Detection bias: we evaluated whether study personnel carrying out outcome assessments were blinded to the treatment status of participants.

Attrition bias: we determined whether studies provided a description of withdrawals and dropouts.

Other bias: we also assessed therapy integrity, including therapist competence and adherence to treatment protocol, for all included studies.

We did not exclude studies from meta-analysis on the basis of the 'Risk of bias' assessment. We conducted sensitivity analyses for the primary outcome, excluding trials with high or unclear risk of bias ratings for allocation concealment, and excluding trials with high or unclear risk of bias for blinding of outcome assessment if appropriate. We reported the remainder of the 'Risk of bias' assessments for these trials, and included discussion of this assessment in the Results and Discussion sections.

\section{Measures of treatment effect}

In order to assess post-treatment outcomes, we used dichotomous data on remission of primary anxiety diagnosis and all anxiety diagnoses, and continuous data on anxiety symptoms, depressive symptoms, and global functioning, with the use of standardised measures. We used data from the assessment administered immediately after treatment (or the assessment closest to the end of treatment) to assess post-treatment outcomes. We also used these measures to evaluate the maintenance of treatment effects at a series of follow-up time points ( $\leq 6$ months post-treatment, $>6$ months post-treatment but $\leq 12$ months post-treatment, and $>12$ months post-treatment). Where studies reported follow-up data at multiple time points within one category (e.g. one-month and three-month follow-up), we used data from the longer followup period. To assess acceptability, we used frequency data on the numbers of participants who were lost to post-treatment assessment. Adverse events were determined by the number and type of adverse events during the trial, from randomisation to the post-treatment assessment.

\section{Dichotomous data}

We analysed dichotomous data as odds ratios (OR) and 95\% confidence intervals $(\mathrm{Cl})$.

\section{Continuous data}

We analysed continuous data as mean difference (MD) or standardised mean difference (SMD). We entered data presented as a scale with a consistent direction of effect.

We narratively described skewed data reported as medians and interquartile ranges.

\section{Unit of analysis issues}

\section{Cluster-randomised trials}

We included cluster-randomised controlled trials based in schools. Cluster-randomised trials can, in principle, be combined with individually randomised trials in the same meta-analysis (Deeks 2017). We did not anticipate that there would be many clusterrandomised trials, therefore we included identified clusterrandomised trials in the meta-analyses and sensitivity analyses that we planned to undertake to investigate the robustness of any conclusions drawn. To correct the influence of any cluster trials, we used an average intraclass correlation coefficient of 0.02 (Health Services Research Unit 2004).

The effective sample size of a single intervention group in a clusterrandomised trial is its original sample size divided by the 'design effect'. The design effect is $1+(M-1)$ ICC, where $M$ is the average cluster size and ICC is the intracluster correlation coefficient (Rao 1992). For dichotomous data, we divided both the number of participants and the number experiencing the event by the same design effect. For continuous data, only the sample size was reduced; we did not alter means and standard deviations (SDs). 


\section{Cross-over trials}

We did not anticipate that there would be many cross-over trials, and the data required to include a paired analysis in a metaanalysis is often not reported (Higgins 2011). We therefore planned to include any identified cross-over trials in the meta-analysis, but only data from the first trial period (i.e. prior to the 'cross-over').

\section{Studies with multiple treatment groups}

Where multiple trial arms were reported in a single trial, we included only the relevant arms. Studies with more than two intervention arms can pose analytical problems in pair-wise metaanalysis. Where studies had two or more relevant active treatment arms to be compared against controls, we managed data as follows.

\section{Continuous data}

We divided the control group equally into two or more groups to compare the means and SDs of these groups against the means and SDs of the two treatment arms.

\section{Dichotomous data}

For trials with two or more active treatment arms and a control group, we split participants in the control arm group equally between the active treatment arms.

\section{Dealing with missing data}

We contacted investigators or study sponsors in order to verify key study characteristics and to obtain missing numerical outcome data where possible (e.g. when we identified a study as abstract only, when a study included a post-treatment diagnostic assessment but did not report the required remission outcomes, or when a study did not report standard errors or SDs). We documented all correspondence with study authors and reported which study authors responded to our queries.

\section{Missing statistics}

In the first instance, we attempted to contact the original researchers for any missing data. If the study only reported standard errors, we calculated SDs.

\section{Missing participants}

We undertook intention-to-treat (ITT) analyses. When analysing dichotomous data, we assumed that all non-completers in the CBT group were treatment failures, and non-completers in the control group were treatment successes, thereby yielding the most conservative treatment estimate.

For dichotomous outcomes, we also undertook completer analysis, using only data from participants who completed post-treatment assessments.

We did not use any statistical or other methods to impute missing data for continuous outcomes, as we did not have access to raw data.

\section{Assessment of heterogeneity}

We assessed clinical heterogeneity by comparing differences in the distribution of important participant factors between studies (e.g. age, gender, specific diagnosis, duration and severity of disorder, associated comorbidities). We assessed methodological heterogeneity by comparing trial factors (randomisation, concealment, blinding of outcome assessment, losses to followup). We used the Chi ${ }^{2}$ test, Deeks 2017, and the $\mathrm{I}^{2}$ statistic, Higgins 2003 , to assess heterogeneity. We set significance at $\mathrm{P}<0.1$. The Cochrane Handbook for Systematic Reviews of Interventions recommends using a range for the $\mathrm{I}^{2}$ statistic and a guide to interpretation (Deeks 2017). If we found either moderate heterogeneity $\left(I^{2}\right.$ in the range of $30 \%$ to $\left.60 \%\right)$ or substantial heterogeneity $\left(\mathrm{I}^{2}\right.$ in the range of $60 \%$ to $\left.90 \%\right)$, we performed subgroup and sensitivity analyses where possible.

\section{Assessment of reporting biases}

Where a minimum of 10 studies were included, we investigated publication bias using funnel plots, Sterne 2017, and subjected any asymmetry found to statistical investigation using Egger's test (Stata 2012).

\section{Data synthesis}

We undertook meta-analyses only where this was meaningful, that is if the treatments, participants, and the underlying clinical question were similar enough for pooling to make sense. We undertook ITT and completer analyses.

We planned to carry out separate analyses to identify whether CBT was more effective post-treatment than waiting list/no treatment; treatment as usual, attention controls, alternative treatments, medication; and drug placebo; and also to identify whether CBT in combination with medication was more effective than drug placebo.

We used follow-up data for each comparison to assess maintenance of treatment gains. If it was meaningful to do so, we pooled data separately for each follow-up time point ( $\leq 6$ months posttreatment, $>6$ months post-treatment but $\leq 12$ months posttreatment, and $>12$ months post-treatment). Where studies reported follow-up data at multiple time points within one category (e.g. 9-month and 12-month follow-up), we included data from the longer follow-up period.

\section{Dichotomous data}

We used ORs and $95 \% \mathrm{Cls}$ based on the random-effects model, with pooling of data via the inverse variance method of weighting. We set significance at $P<0.05$. Where available, we used combined data from an interview with the child or adolescent and the parent; otherwise we used data from one interview (child/adolescent or parent interview). We calculated the number needed to treat for an additional beneficial outcome (NNTB) with 95\% Cls (Stata 2012). We calculated a summary statistic of all those responding to treatment as a percentage of the total number of participants for each comparison.

\section{Continuous data}

We conducted analysis of continuous data based on the randomeffects model, with pooling of data via the inverse variance method of weighting. We used the SMD to pool continuous data measured in different ways across studies but conceptually the same (i.e. measuring anxiety or depressive symptoms or global functioning). For continuous data measuring anxiety symptoms, we pooled child/adolescent report and parental/clinician reports separately. Where both endpoint and change data were available for the same outcome, we presented the endpoint. We set significance at $\mathrm{P}<$ 0.05 . 


\section{Tables and figures}

We transferred data into Review Manager 5 (Review Manager 2014), and presented them graphically, so that the area to the left of the line of no effect (or right for remission outcomes) indicated a favourable outcome for CBT. We used tables to display characteristics of the included studies. We presented a brief list of excluded studies in a table with their reasons for exclusion. We summarised risk of bias in the included studies in a figure, and included a PRISMA flow chart (Moher 2015).

\section{Subgroup analysis and investigation of heterogeneity}

The critical need to improve access to treatment for child anxiety disorders means that it is particularly important to explore the efficacy of approaches that may help maximise treatment efficiency, including alternative delivery formats and briefer interventions that involve less therapist contact time than traditional approaches. As outlined above, there are also unanswered questions in relation to the benefits of CBT for children and adolescents with ASD and intellectual impairments. We therefore set out to explore the efficacy of different delivery formats and of briefer and shorter interventions, and to examine treatment effects amongst children and adolescents with ASD and those with intellectual impairment, using subgroup analyses. Specifically, where it was possible and meaningful to do so, we undertook subgroup analyses to examine differences between:

- delivery formats (child-focused, child and parent, and parentonly; individual and group);

- interventions with a varying amount of therapist contact time (< 10 hours, $\geq 10$ hours and $<20$ hours, $\geq 20$ hours);

- children and adolescents with and without ASDs;

- children and adolescents with and without intellectual impairments.

To examine differences between age groups, we also undertook post hoc subgroup analyses evaluating the differences between studies where all participants were age 12 or younger ( $\leq 12$ years); all participants were age 12 or older ( $\geq 12$ years); and studies that included participants under and over age $12(<12$ years and $\geq 12$ years).

We undertook subgroup analyses for the primary outcome (remission of primary anxiety disorder) across comparisons. For the main comparison with the largest number of studies (CBT versus waitlist/no treatment), we also undertook subgroup analyses for the most frequently reported secondary outcomes (remission of all anxiety diagnoses post-treatment and reduction in childand parent-reported anxiety symptoms post-treatment). To ensure subgroup analyses were meaningful, we only undertook these analyses where there were data from at least three studies for each subgroup.

We assessed statistical heterogeneity for all analyses and between groups with the $\mathrm{Chi}^{2}$ test and the $\mathrm{I}^{2}$ statistic, and set significance at $\mathrm{P}<0.1$.

\section{Sensitivity analysis}

Sensitivity analysis is the study of how the uncertainty in the output of an analysis can be apportioned to different sources of uncertainty in its inputs. Sensitivity analyses can therefore be carried out to test the robustness of decisions made in the review process. We carried out sensitivity analyses where there was evidence of the following:

- significant heterogeneity: we inspected forest plots and examined each study in turn to determine the source of any significant heterogeneity;

- selection bias: we excluded those studies judged to be at high risk of selection bias from the main analysis;

- allocation concealment: we excluded those studies judged to be at high risk of bias for allocation concealment from the main analysis.

Where appropriate, we undertook all of the above sensitivity analyses for the ITT and completer analyses.

Where meta-analyses examining anxiety symptoms included broad measures of anxiety symptoms and disorder-specific symptom measures, we also undertook sensitivity analyses excluding studies that only reported disorder-specific symptom measures.

\section{GRADE and 'Summary of findings' table}

We created a 'Summary of findings' table including the following primary outcomes:

- remission of primary anxiety diagnosis post-treatment;

- acceptability in terms of dropouts from randomisation to the post-treatment assessment;

and the following secondary outcomes:

- remission of all anxiety diagnoses post-treatment;

- reduction in anxiety symptoms (self-reported and parentreported) post-treatment;

- reduction in depressive symptoms post-treatment;

- improvement in global functioning post-treatment;

- adverse events from randomisation to the post-treatment assessment.

We used the five GRADE considerations (study limitations, consistency of effect, imprecision, indirectness, and publication bias) to assess the quality of a body of evidence as it relates to the studies that contribute data to the meta-analyses for the prespecified primary and secondary outcomes. Two review authors (AJ, TR) independently assessed risk of bias, and in case of disagreement sought consensus between four review authors (AJ, TR, GJ, CC). We used the methods and recommendations described in Section 8.5, Higgins 2017, and Chapter 12, Schünemann 2017, of the Cochrane Handbook for Systematic Reviews of Interventions, employing GRADEpro GDT software (GRADEpro GDT). We justified all decisions to downgrade or upgrade the quality of studies using footnotes, and made comments to aid the reader's understanding of the review where necessary. We considered whether there was any additional outcome information that could not be incorporated into the meta-analyses, noting this in the comments and stating if it supported or contradicted the information from the meta-analyses.

\section{RE S U L T S}

\section{Description of studies}

See Characteristics of included studies and Table 1. 


\section{Results of the search}

The initial database search (21 November 2018) identified 6354 records, and an updated database search (10 October 2019) identified a further 2081 records. A search of the grey literature identified a further 473 records, and an additional 38 records were identified from searching reference lists and correspondence with study authors and experts in the field.

After removing duplicates, we screened a total of 5488 titles/ abstracts, and excluded 4746 records. We then assessed 742 fulltexts for eligibility for inclusion in the review, and identified 88 eligible studies (130 articles) (Figure 1). 
Figure 1. Study flow diagram.

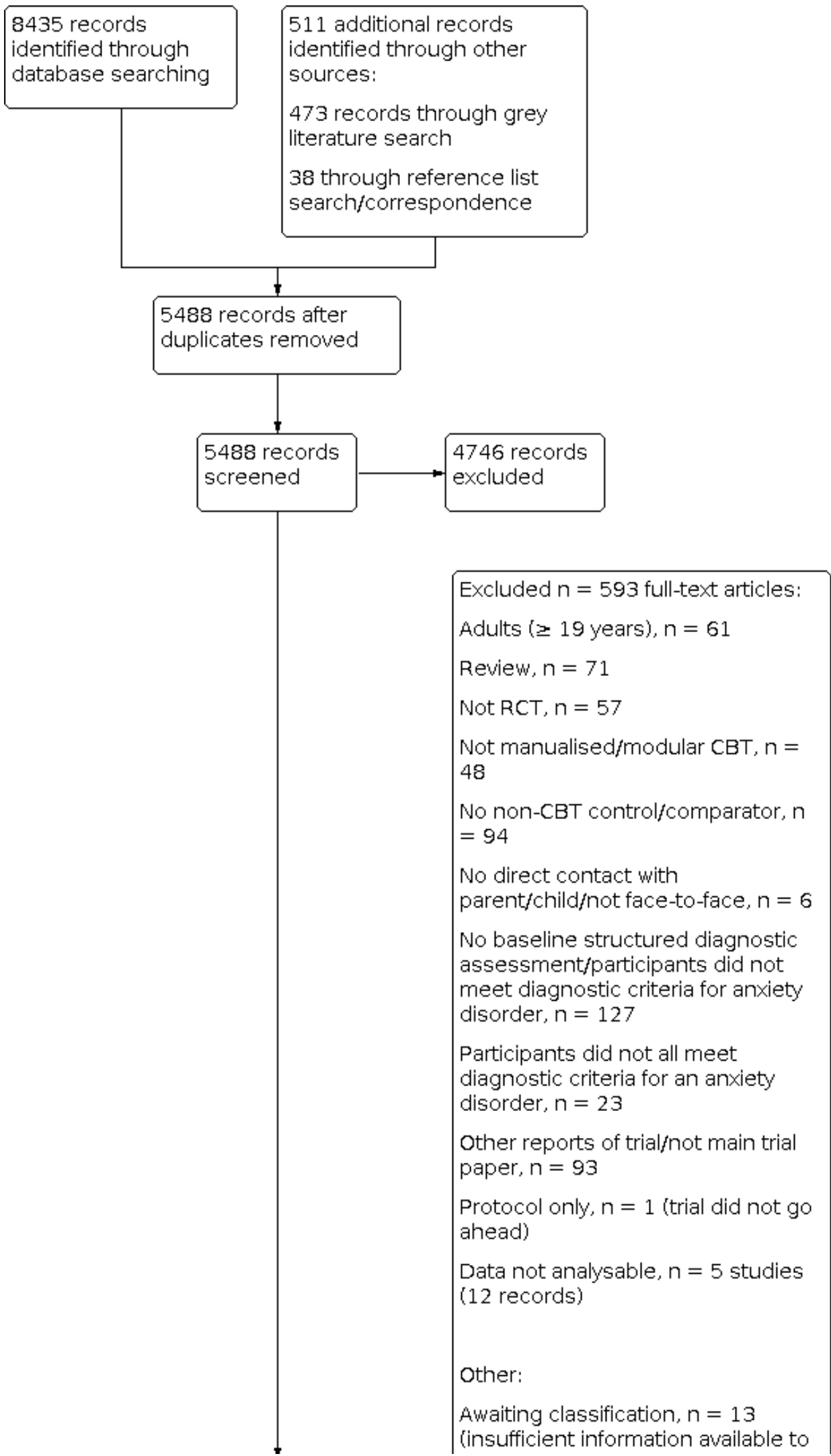


Figure 1. (Continued)

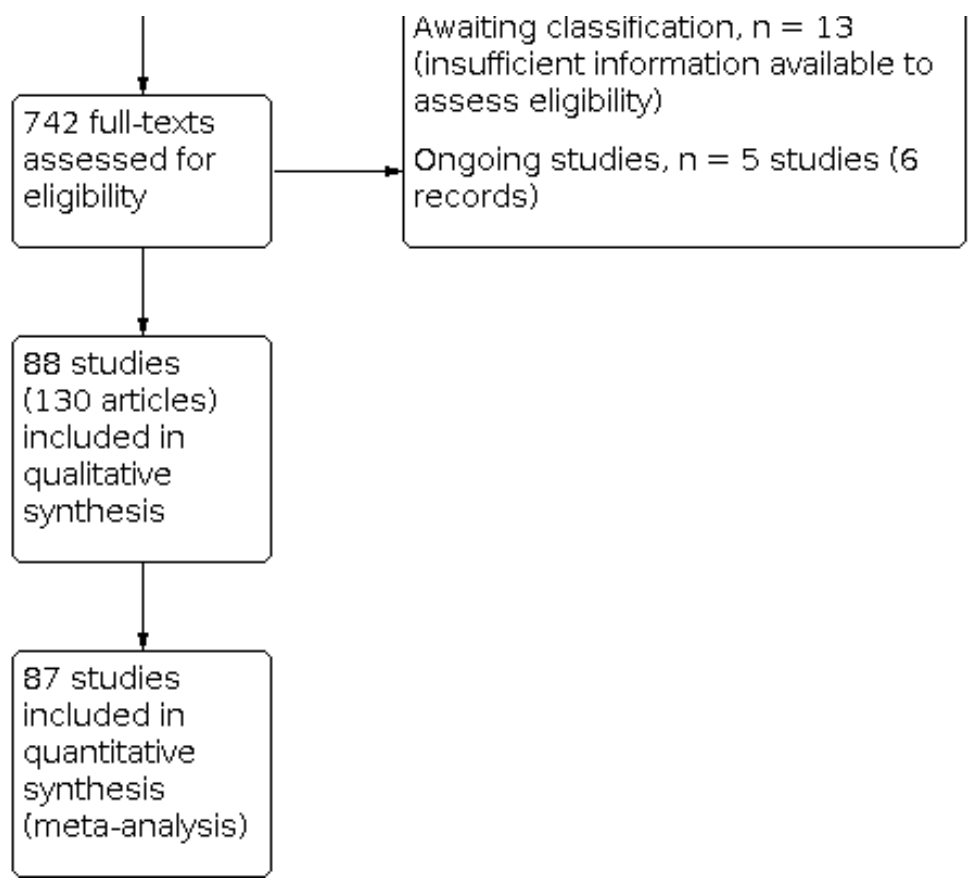

We contacted 58 study authors for clarification or additional data, or both, and 16 study authors provided relevant outcome data for 20 of the included studies.

\section{Included studies}

Eighty-eight studies met the criteria for inclusion in the review, and 87 studies were included in meta-analyses.

\section{Design}

All included studies were RCTs. Two studies used a clusterrandomised design, where schools, Dadds 1997, and schoolbased clinicians, Ginsburg 2019a, were randomised, rather than individual participants. No included studies used a cross-over design.

Amongst the studies included in meta-analyses, 20 studies had two CBT arms (Afshari 2014; Barrett 1996; Barrett 1998; Cheung 2016; Flannery Schroeder 2000; Hancock 2018; Herbert 2009; Ingul 2013; Kendall 2008; Masia Warner 2016; Olivares 2014; Olivares 2019; Ost 2001; Salum 2018; Simon 2011; Spence 2000; Thirlwall 2013; Villabo 2018; Waters 2009; Wergeland 2014), including one factorial design (Salum 2018), and another three studies included multiple comparison arms (Cheung 2016; Ollendick 2009; Rosa Alcazar 2009).

Fifty-seven studies were included in CBT versus waitlist/no treatment comparisons (Afshari 2014; Arendt 2016; Barrett 1996; Barrett 1998; Berge 2017; Cartwright Hatton 2011; Chalfant 2007; Cheung 2016; Chiu 2013; Cobham 2017; Cornacchio 2019; Dadds 1997; Flannery Schroeder 2000; Gallagher 2004; Hancock 2018; Hirshfeld Becker 2010; Holmes 2014; Ishikawa 2019; Kendall 1994; Kendall 1997; Kennedy 2009; Kidd 2018; Lau 2010; Lau 2017; Leutgeb 2012; Masia Warner 2005; Masia Warner 2011; McConachie 2014; McNally Keehn 2013; Melfsen 2011; Olivares 2005; Olivares 2014; Olivares 2019; Ollendick 2009; Ost 2001; Perrin 2019; Rapee 2005; Rapee 2006; Rosa Alcazar 2009; Salari 2018; Sanchez Garcia
2009; Santucci 2013; Schneider 2011; Shortt 2001; Silverman 1999b; Simon 2011; Smith 2014; Spence 2000; Spence 2006; Spence 2011; Thirlwall 2013; Villabo 2018; Waters 2009; Wergeland 2014; White 2013; Wood 2009; Wood 2015). With the exception of three studies (Dadds 1997; Rapee 2005; Simon 2011), participants assigned to the control arm were offered CBT after a waitlist period. Seven studies included eligible follow-ups that included CBT and waitlist/ no treatment participants. Follow-up assessments were conducted between 3 and 24 months post-treatment.

Twelve studies compared CBT to treatment as usual (TAU) (Barrington 2005; Fujii 2013; Ginsburg 2012; Ginsburg 2019a; O'Brien 2007; Reaven 2012; Sciberras 2018; Shahnavaz 2016; Sharma 2017; Southam Gerow 2010; Storch 2013; Storch 2015). TAU varied considerably across studies, ranging from eclectic nonCBT therapeutic approaches or strategies that were offered as part of routine care (Barrington 2005; Ginsburg 2012; Ginsburg 2019a; Southam Gerow 2010), to allowing participants to continue and seek any psychosocial or pharmacological interventions (Fujii 2013; Reaven 2012; Storch 2013; Storch 2015). Four CBT versus TAU studies included 6-month, 12-month, or both 6-and 12-month follow-ups.

Fifteen studies compared CBT to an attention control (Ginsburg 2002; Herbert 2009; Hudson 2009; Ingul 2013; Kendall 2008; Khanna 2010; Last 1998; Masia Warner 2007; Masia Warner 2016; Muris 2002; Ollendick 2009; Pincus 2010; Rosa Alcazar 2009; Salum 2018; Silverman 1999a), which was designed to act as an attention condition, and often included a psychoeducation component. Six CBT versus attention control studies included follow-ups up to 12 months.

Seven studies included a CBT versus an alternative treatment comparison (Cheung 2016; Creswell 2017; Lebowitz 2019; Reigada 2015; Salum 2018; Silk 2018). Alternative treatments designed to target children's anxiety difficulties included: solution-focused 
therapy (Creswell 2017), the parent intervention SPACE (Supportive Parenting for Anxious Childhood Emotions) (Lebowitz 2019), childcentred therapy (Silk 2018), counselling (Murphy 2017), nondirective supportive therapy (Reigada 2015), and ABMT (Attention Bias Modification Treatment) (Cheung 2016; Salum 2018). Four CBT versus alternative treatment studies included follow-ups up to 12 months post-treatment.

Only one study compared CBT to medication, and CBT in combination with medication to drug placebo (Walkup 2008), therefore it was not possible to conduct meta-analyses for these comparisons.

\section{Sample size}

The 87 studies included in meta-analyses involved a total of 3492 CBT participants and 2472 controls (waitlist/no treatment controls, $\mathrm{N}=1537$; TAU, $\mathrm{N}=260$; attention control, $\mathrm{N}=441$; alternative treatment, $\mathrm{N}=234$ ).

Study sample sizes ranged from 12 participants, Ginsburg 2002; Sciberras 2018, to 206 participants (corrected sample size; Ginsburg 2019a).

\section{Setting}

Two-thirds of the included studies were conducted in either the USA or Australia. The remaining studies were conducted in the UK, Spain, Norway, the Netherlands, Germany, Sweden, Iran, Brazil, Hong Kong, India, Japan, Denmark, and Ireland.

Most studies were conducted in clinical settings, either university research clinics (specified in 41 studies), community mental health clinics (specified in 12 studies), or dental clinics (Berge 2017; Shahnavaz 2016). Sixteen studies recruited participants through schools (Chiu 2013; Dadds 1997; Ginsburg 2002; Ginsburg 2012; Ginsburg 2019a; Ingul 2013; Masia Warner 2005; Masia Warner 2007; Masia Warner 2016; Muris 2002; Olivares 2005; Olivares 2014; Olivares 2019; Rosa Alcazar 2009; Sanchez Garcia 2009; Simon 2011), and two through preschools (Lau 2017; Rapee 2005). No studies were conducted in inpatient settings.

\section{Participants}

The included studies involved participants of varied ages. Sixteen studies focused on adolescents and only included participants aged 12 years or over (Ginsburg 2002; Herbert 2009; Ingul 2013; Kidd 2018; Masia Warner 2005; Masia Warner 2007; Masia Warner 2016; Murphy 2017; Olivares 2005; Olivares 2014; Olivares 2019; Pincus 2010; Rosa Alcazar 2009; Spence 2011; White 2013; Wood 2015). Twenty-five studies included participants up to age 12 years (Cartwright Hatton 2011; Cheung 2016; Chiu 2013; Cornacchio 2019; Creswell 2017; Fujii 2013; Gallagher 2004; Hirshfeld Becker 2010; Holmes 2014; Kennedy 2009; Lau 2010; Lau 2017; Lebowitz 2019; Muris 2002; Rapee 2005; Rapee 2006; Salari 2018; Salum 2018; Santucci 2013; Schneider 2011; Sciberras 2018; Shortt 2001; Storch 2013; Thirlwall 2013; Waters 2009), including 5 studies where participants were all under 8 years of age (Hirshfeld Becker 2010; Kennedy 2009; Lau 2017; Rapee 2005; Schneider 2011). The youngest participants in the included studies were two to three years of age (Cartwright Hatton 2011; Kennedy 2009; Lau 2017; Rapee 2005). The remaining studies included participants both under and over 12 years.
All participants met the diagnostic criteria for at least one anxiety disorder. In most studies, any anxiety disorder, or a subset of anxiety disorders (e.g. generalised anxiety disorder (GAD), social anxiety disorder, or separation anxiety disorder) was required for inclusion. In five studies (Cartwright Hatton 2011; Creswell 2017; Dadds 1997; Rapee 2005; Simon 2011), not all study participants met the diagnostic criteria for an anxiety disorder, but only those who did were included in this review.

Twenty-four studies focused on a specific anxiety disorder where participants all met the diagnostic criteria for the target anxiety disorder. Disorder-specific studies included participants with social anxiety disorder (Gallagher 2004; Herbert 2009; Ingul 2013; Masia Warner 2005; Masia Warner 2007; Masia Warner 2016; Melfsen 2011; Olivares 2005; Olivares 2014; Olivares 2019; Rosa Alcazar 2009; Sanchez Garcia 2009; Spence 2000), specific phobia (Berge 2017; Leutgeb 2012; Ollendick 2009; Ost 2001; Shahnavaz 2016), separation anxiety disorder (Santucci 2013; Schneider 2011), GAD (Holmes 2014; Perrin 2019), panic disorder with/without agoraphobia (Pincus 2010), and selective mutism (Cornacchio 2019).

In some studies comorbid conditions were required for inclusion. Twelve studies included participants with an ASD (Chalfant 2007; Fujii 2013; Kidd 2018; McConachie 2014; McNally Keehn 2013; Murphy 2017; Reaven 2012; Storch 2013; Storch 2015; White 2013; Wood 2009; Wood 2015). Other comorbidities required for inclusion in individual studies included attention-deficit/ hyperactivity disorder (ADHD) (Sciberras 2018), school refusal (Last 1998), headaches (Sharma 2017), functional physical complaints (Masia Warner 2011), and inflammatory bowel disease (IBD) (Reigada 2015). No studies included samples of children with intellectual impairments.

\section{Intervention}

The most commonly used or adapted treatment manuals were Coping Cat (Kendall 1994), and Cool Kids (Hudson 2009; Rapee 2006). Studies targeting specific anxiety disorders used disorderspecific interventions (e.g. OST (One-Session Treatment) for specific phobias, IAFS (Intervention in Adolescents with Social Phobia) for social anxiety disorder), and studies targeting children with ASD (or another comorbidity) used adapted interventions developed specifically for the target population (e.g. BIACA (Behavioral Interventions for Anxiety in Children with Autism), TAPS (Treatment of Anxiety and Physical Symptoms)).

Delivery formats varied across the included studies, including childfocused interventions (45 studies), interventions delivered to both children and parents ( 40 studies), and interventions delivered only to parents (10 studies). Approximately half of the CBT interventions involved a group format, including child-focused interventions (e.g. Gallagher 2004; Ginsburg 2002; Olivares 2005), interventions delivered to children and parents (e.g. Hudson 2009; McConachie 2014; Waters 2009), and parent-only interventions (e.g. Cartwright Hatton 2011; Cobham 2017; Rapee 2005).

The amount of therapist contact time also varied considerably in the included studies, ranging from under 10 hours (interventions in 16 studies, e.g. Creswell 2017; Ishikawa 2019; Ollendick 2009) to more than 20 hours (interventions in 23 studies, e.g. Herbert 2009; Sharma 2017; Storch 2015). The remaining interventions involved between 10 and 20 hours of therapist contact time. 


\section{Measures}

The majority of included studies (70 out of 87 ) included in meta-analyses used a post-treatment diagnostic assessment, and reported (or provided) data on remission from primary and/or all anxiety disorders. In most cases, the Anxiety Disorder Interview Schedule for Children (ADIS-C) was used, although there was variation in how it was administered, including either separate child and parent interviews (e.g. Spence 2011; Thirlwall 2013; Wergeland 2014), joint child and parent interviews (Barrington 2005; Ishikawa 2019; Khanna 2010), only child interviews (e.g. Ginsburg 2002; Olivares 2005; Sanchez Garcia 2009), or only parent interviews (e.g. Lau 2017; McNally Keehn 2013; Waters 2009). Alternative post-treatment diagnostic assessments included: the Kinder-DIPS (Melfsen 2011; Schneider 2011), Kiddie Schedule for Affective Disorders and Schizophrenia (K-SADS) (Hirshfeld Becker 2010; Last 1998; Lau 2010; Silk 2018), Diagnostic Interview Schedule for Children, Adolescents and Parents (DISCAP) (Shortt 2001), Diagnostic Interview Schedule for Children (Southam Gerow 2010), an adapted version of the Development and Well-Being Assessment (DAWBA) (Shahnavaz 2016), and the Mini-KID (Sharma 2017).

Anxiety symptoms were assessed using a range of child- and parentreported questionnaire measures of child anxiety symptoms, including the Spence Children's Anxiety Scale (SCAS-C/P) (or the parent-reported Preschool Anxiety Scale for younger children), Multidimensional Anxiety Scale for Children (MASC-C/P), the Screen for Children Anxiety Related Emotional Disorders (SCARED-C/ P), State Trait Anxiety Inventory-Trait Scale (STAIC-T), the childreported Revised Children's Manifest Anxiety Scale (RCMAS), the child-reported Revised Child Anxiety and Depression ScaleAnxiety Scale (RCADS-A), the parent-reported Child Behavior Checklist-Anxiety Scale (CBCL-A), and the parent-reported Child and Adolescent Symptom Inventory-4 ASD Anxiety Scale. Where broad measures of anxiety symptoms were not reported, the following disorder-specific symptom questionnaire measures were used in the main analyses: Social Anxiety Scale for Adolescents (SAS-A) (Herbert 2009; Masia Warner 2005; Masia Warner 2007; Olivares 2014; Rosa Alcazar 2009), Social Phobia and Anxiety Inventory for Children (SPAI) (Herbert 2009; Masia Warner 2005; Masia Warner 2007; Masia Warner 2016; Melfsen 2011; Olivares 2005; Rosa Alcazar 2009; Sanchez Garcia 2009), IBD-Specific Anxiety Scale (IBD-SAS) (Reigada 2015), Intra Oral Injection Fear Scale (IOIFs) (Berge 2017), and Child Survey Fear Schedule: Dental Subscale (Shahnavaz 2016).

Depressive symptoms were most frequently assessed using selfreported questionnaires, and where parent-report was used it was always used alongside self-report, so only self-report depressive symptom measures were used in the analyses. The most frequently used measure of depressive symptoms was the Children's Depression Inventory; other measures included the
Short Mood and Feelings Questionnaire (Arendt 2016; Kidd 2018; Thirlwall 2013; Wergeland 2014), Mood and Feelings Questionnaire (Perrin 2019), Revised Child Anxiety and Depression ScaleDepression scale (RCADS-D) (Muris 2002), and Beck Depression Inventory-II (BDII) (Masia Warner 2007).

The included studies that assessed post-treatment global functioning all used the clinician-rated Children's Global Assessment Scale (CGAS) or adapted, White 2013, or translated versions of the CGAS (Melfsen 2011).

\section{Excluded studies}

We excluded 593 full-text articles; the reasons for exclusion are detailed in Characteristics of excluded studies.

We excluded studies if participants were not fully randomised to conditions (Bodden 2008a; Cobham 2012; Hayward 2000; Kujawa 2019; Mendlowitz 1999; Nauta 2003), or if the study did not use a standardised structured interview to determine the presence of baseline anxiety disorders (e.g. Gil Bernal 2009; Jansen 2012; Kerns 2016; Sevi Tok 2016; Sung 2011). We excluded studies where only some participants were under 19 years of age (e.g. Salzer 2018), or only some participants met the diagnostic criteria for anxiety disorder (e.g. Bernstein 2005; Ginsburg 2019b; Pereira 2014; Weisz 2012), if the study authors did not provide data on eligible participants. We also excluded studies if the intervention did not include both cognitive and behavioural elements (e.g. Beidel 2000; Beidel 2007; Bergman 2013; Cotton 2019; Ebrahiminejad 2016; Oerbeck 2014; Ollendick 2018; Ost 2015; Ozyurt 2018; Rudy 2017; Storch 2019); there were no face-to-face CBT sessions (e.g. Lyneham 2006); or where the control intervention included elements of CBT (e.g. Clementi 2019).

We excluded five studies because useable data were not provided for any relevant outcomes (Asbrand; Asbrand 2019; Baer 2005; Flatt 2010; NCT00576719).

\section{Ongoing studies}

We identified five ongoing studies that meet our inclusion criteria (see Characteristics of ongoing studies).

\section{Studies awaiting classification}

We identified trial registrations for 13 studies where insufficient information was available to determine eligibility, and either the trial was ongoing/not started or the trial data were not available (see Characteristics of studies awaiting classification).

\section{Risk of bias in included studies}

A summary of the risk of bias in the included studies is provided in Figure 2 and Figure 3. 
Figure 2. Risk of bias summary: review authors' judgements about each risk of bias item for each included study.

Arendt 2016

Barrett 1996

Barrett 1998

Barrington 2005

Berge 2017

Cartwright Hatton 2011

Chalfant 2007

Cheung 2016

Chiu 2013

Cobham 2017

Cornacchio 2019

Creswell 2017

Dadds 1997

Flannery Schroeder 2000

Fujii 2013

Gallagher 2004

Ginsburg 2002

Ginsburg 2012

Ginsburg 2019a

Hancock 2018

Herbert 2009

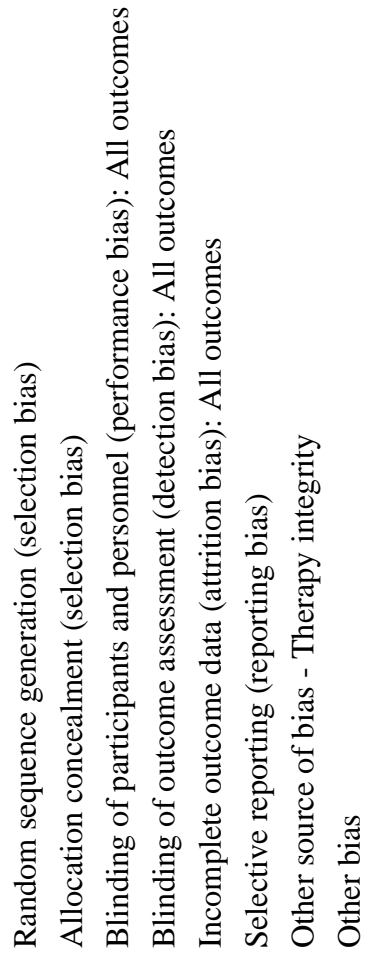

\begin{tabular}{|l|l|l|l|l|l|l|l|}
\hline$?$ & $?$ & + & + & - & + & $?$ & $?$ \\
\hline
\end{tabular}

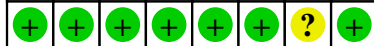

\begin{tabular}{llllllllll}
\hline$?$ & $?$ & + & $\oplus$ & $?$ & + & + & + \\
\hline
\end{tabular}

\begin{tabular}{llllllllll}
\hline & $?$ & $†$ & $\dagger$ & $?$ & $\oplus$ & + & + \\
\hline
\end{tabular}

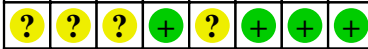

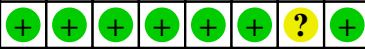

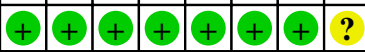

\begin{tabular}{lllllllllll}
\hline & $?$ & $†$ & $?$ & $?$ & $†$ & $?$ & $†$ \\
\hline
\end{tabular}

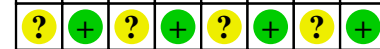

\begin{tabular}{llllllllll}
\hline$?$ & $?$ & + & + & + & + & + & + \\
\hline
\end{tabular}

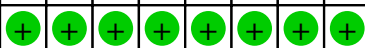

\begin{tabular}{lllllllll}
\hline $\boldsymbol{2}$ & $\boldsymbol{2}$ & + & + & + & + & + & + \\
\hline
\end{tabular}

\begin{tabular}{llllllll}
\hline+ & + & + & + & + & + & + \\
\hline
\end{tabular}

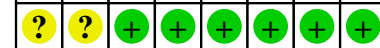

\begin{tabular}{lllllllllll}
\hline$?$ & $?$ & $\dagger$ & $?$ & $\dagger$ & + & + & $\ddots$ \\
\hline
\end{tabular}

\begin{tabular}{lllllllll}
\hline$?$ & $?$ & + & + & + & + & + & $\odot$ \\
\hline
\end{tabular}

\begin{tabular}{llllllllll}
\hline$?$ & $?$ & + & $\oplus$ & $?$ & + & + & + \\
\hline
\end{tabular}

\begin{tabular}{ccc|cccccc}
\hline & $?$ & $?$ & + & + & + & + & + \\
\hline
\end{tabular}

$+\uparrow+?$ ? + † + ?

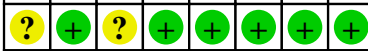

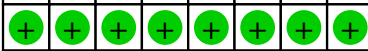

\begin{tabular}{lllllllll}
+ & $?$ & $?$ & + & + & + & + & + \\
\hline
\end{tabular}

Hirshfeld Becker 2010 
Figure 2. (Continued)

Herbert 2009

Hirshfeld Becker 2010

Holmes 2014

Hudson 2009

Ingul 2013

Ishikawa 2019

Kendall 1994

Kendall 1997

Kendall 2008

Kennedy 2009

Khanna 2010

Kidd 2018

Last 1998

Lau 2010

Lau 2017

Lebowitz 2019

Leutgeb 2012

Masia Warner 2005

Masia Warner 2007

Masia Warner 2011

Masia Warner 2016

McConachie 2014

McNally Keehn 2013

Melfsen 2011

Muris 2002

Murphy 2017

O'Brien 2007

Olivares 2005

Olivares 2014

Olivares 2019

Ollendick 2009

Ost 2001

Perrin 2019

Pincus 2010

Rapee 2005

Rapee 2006

Reaven 2012

Reigada 2015

Rosa Alcazar 2009

Salari 2018

Salum 2018

Sanchez Garcia 2009

Santucci 2013

Schneider 2011

Sciberras 2018

Shahnavaz 2016

Sharma 2017

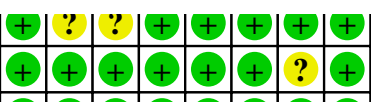

\begin{tabular}{|cccccccc}
\hline & + & + & + & + & + & + & + \\
\hline
\end{tabular}

\begin{tabular}{llllllll}
\hline & $?$ & + & $?$ & + & + & + & + \\
\hline
\end{tabular}

\begin{tabular}{lllllllll}
+ & $?$ & $?$ & + & $?$ & + & $?$ & + \\
\hline
\end{tabular}

$+\oplus+\oplus+\odot+$

\begin{tabular}{lllllllll}
\hline$?$ & $?$ & + & $?$ & $?$ & + & + & \\
\hline
\end{tabular}

\begin{tabular}{llllllll} 
? & ? & + & $?$ & ? & + & + & - \\
\hline
\end{tabular}

$+\odot$ ? $+\odot$ +

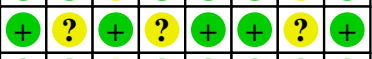

\begin{tabular}{llllllll}
\hline & + & $?$ & + & + & + & + & + \\
\hline
\end{tabular}

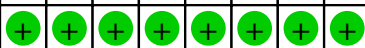

\begin{tabular}{llllllll}
\hline ? & ? & ? & ? & - & + & + & + \\
\hline
\end{tabular}

\begin{tabular}{lllllllll}
\hline$?$ & $?$ & + & + & + & + & + & + \\
\hline
\end{tabular}

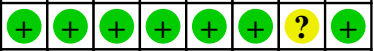

\begin{tabular}{lllllll}
\hline & + & + & + & + & + & + \\
\hline
\end{tabular}

\begin{tabular}{|lllllllll}
\hline$?$ & $?$ & + & + & + & + & $?$ & + \\
\hline
\end{tabular}

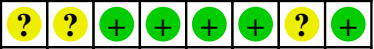

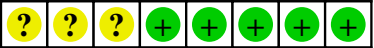

\begin{tabular}{llllllll}
\hline & $?$ & + & + & + & ? & - \\
\hline
\end{tabular}

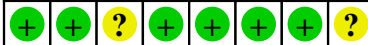

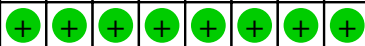

+ ? $++\oplus+\oplus$

$+++\odot+\leftarrow+$

\begin{tabular}{llllllll}
\hline & $?$ & $?$ & + & + & + & $?$ & +
\end{tabular}

\begin{tabular}{llllllll}
\hline & + & $?$ & + & + & + & + & + \\
\hline
\end{tabular}

\begin{tabular}{llllllllll}
\hline ? & $?$ & + & + & + & ? & + & + \\
\hline
\end{tabular}

\begin{tabular}{llllllllll}
\hline$?$ & $?$ & + & + & + & + & $?$ & + \\
\hline
\end{tabular}

\begin{tabular}{|lll|l|l|l|l|l|}
\hline$?$ & $?$ & + & $?$ & $?$ & + & + & + \\
\hline
\end{tabular}

\begin{tabular}{llllllllll}
\hline$?$ & $?$ & + & + & ? & + & ? & + \\
\hline
\end{tabular}

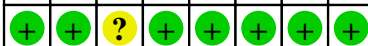

\begin{tabular}{lllllllll}
\hline ? & ? & + & + & + & + & ? & + \\
\hline
\end{tabular}

\begin{tabular}{lllllll}
\hline & + & + & + & + & + & + \\
\hline
\end{tabular}

\begin{tabular}{lllllllll}
\hline$?$ & $?$ & + & $?$ & $?$ & + & + & $\odot$ \\
\hline
\end{tabular}

\begin{tabular}{lllllllll}
\hline$?$ & $?$ & + & + & + & + & $?$ & + \\
\hline
\end{tabular}

\begin{tabular}{llllllll}
\hline & + & + & + & + & + & ? & +
\end{tabular}

\begin{tabular}{llllllll}
\hline & + & + & + & + & + & + & +
\end{tabular}

\begin{tabular}{llllllll}
\hline & $?$ & $?$ & + & + & + & + & + \\
\hline
\end{tabular}

\begin{tabular}{|ll|lllllll}
\hline$?$ & $?$ & $?$ & $?$ & + & + & $?$ & + \\
\hline
\end{tabular}

\begin{tabular}{|l|l|llllll}
\hline ? & ? & + & + & + & + & ? & + \\
\hline
\end{tabular}

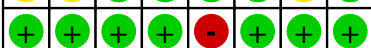

\begin{tabular}{llllllllll}
\hline$?$ & $?$ & + & $?$ & $?$ & + & $?$ & ? \\
\hline
\end{tabular}

\begin{tabular}{llllllllll}
\hline$?$ & $?$ & + & + & + & + & $?$ & + \\
\hline
\end{tabular}

\begin{tabular}{llllllll}
\hline & + & + & + & + & + & + & + \\
\hline
\end{tabular}

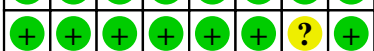

\begin{tabular}{|cccccccc|}
\hline & + & + & + & + & + & + & + \\
\hline
\end{tabular} 
Figure 2. (Continued)

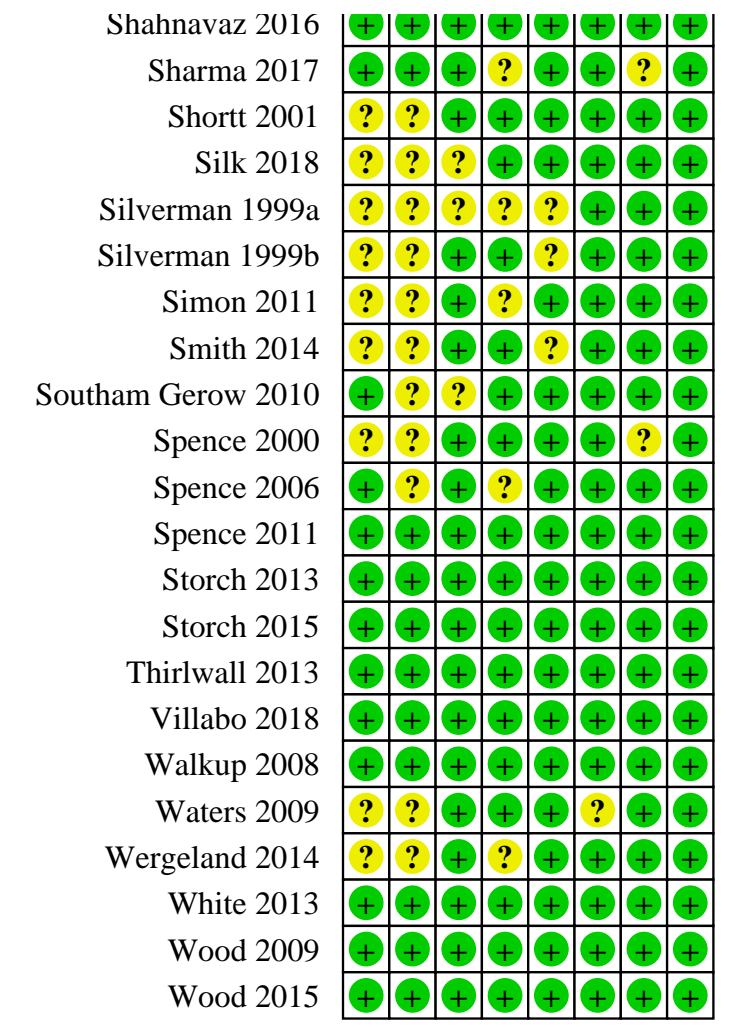

Figure 3. Risk of bias graph: review authors' judgements about each risk of bias item presented as percentages across all included studies.

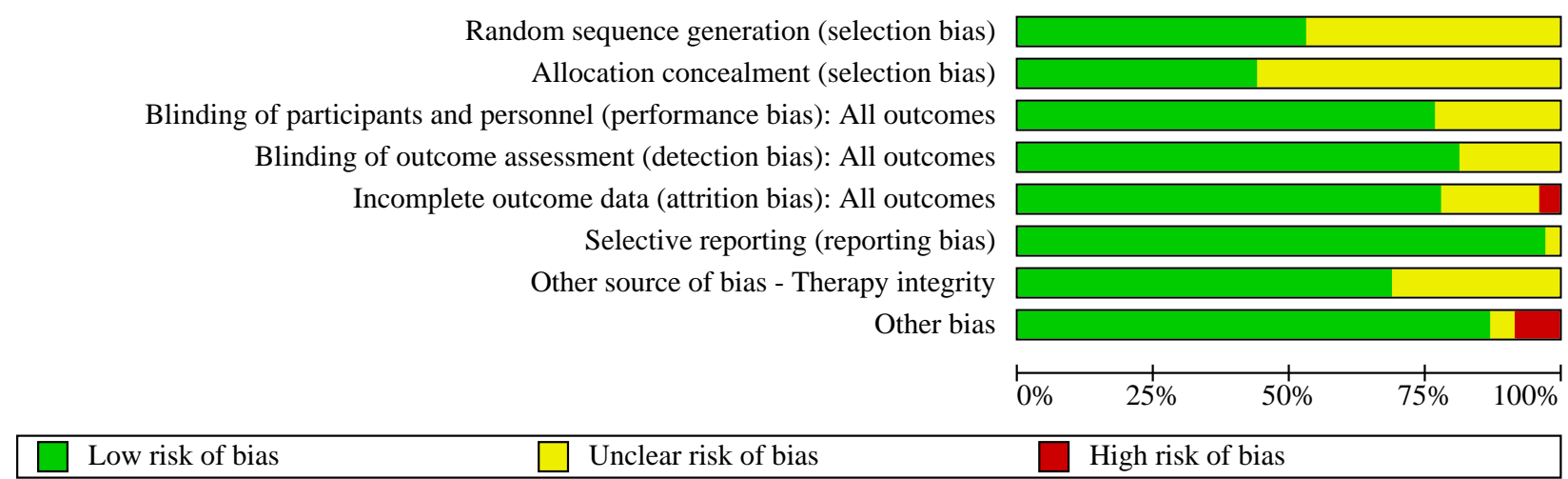

\section{Allocation}

Over half of the included studies (47 out of 88 ) clearly described an adequate process of sequence generation, and just under half (39 out of 88 ) clearly described an adequate process of allocation concealment. In remaining studies, insufficient or no information about the sequence generation process was provided.

We performed a sensitivity analysis for the primary outcome (remission of primary anxiety disorder post-treatment) amongst CBT versus waitlist controls, using only those studies that clearly described sequence generation, and found similar findings to the main analysis (odds ratio (OR) 4.84, 95\% confidence interval (CI) 3.03 to $7.73, \mathrm{Z}=6.61, \mathrm{P}<0.001$ ) (Analysis 1.32).

\section{Blinding}

The vast majority of studies (72 out of 88 ) either clearly detailed using blind assessors to administer post-treatment diagnostic interviews, or only used a parent/child report questionnaire, therefore we judged the risk of bias from not blinding to be low. 
We performed a sensitivity analysis examining remission of primary anxiety disorder diagnosis amongst CBT versus waitlist controls, where studies that did not specify using blind assessors were removed. The findings were similar to the main analysis (OR 5.54, $95 \% \mathrm{Cl} 3.76$ to $8.18, \mathrm{Z}=8.63, \mathrm{P}<0.001$ ) (Analysis 1.33).

Blinding of participants and personnel was only possible in some CBT versus TAU, active control, or alternative treatment studies. These studies described measures to ensure comparability of interventions (e.g. amount of contact time); however, few studies specifically detailed attempts to conceal 'test' versus 'control' intervention from participants.

\section{Incomplete outcome data}

Most studies described information on dropouts and missing outcome data. The amount of missing data was small, with a description of appropriate methods to manage missing data provided. Sixteen studies provided insufficient information on missing data, and in three studies missing data were not balanced across groups, with more missing data in the CBT group than in the control group (Afshari 2014; Last 1998; Salum 2018).

\section{Selective reporting}

With the exception of two studies where post-treatment anxiety symptoms outcomes were not reported for CBT and controls (O'Brien 2007; Waters 2009), the study reports included expected outcomes relevant to this review.

Notably, however, six studies included a post-treatment diagnostic assessment and reported outcomes from this assessment (e.g. remission of a subset of anxiety disorders, reduction in the number of anxiety disorders), but did not report or provide data on remission of primary anxiety disorder or remission of all anxiety disorders (Ginsburg 2012; Hancock 2018; Murphy 2017; Pincus 2010; Walkup 2008; Wood 2009), so these studies were not included in our analyses of diagnostic outcomes.

\section{Other potential sources of bias}

Therapy integrity, including therapist competence and adherence, was assessed across all studies. In the majority of studies (61 out of 88), trained and supervised therapists were used, and adherence was measured and reported. In the remaining studies insufficient information was provided on competence or adherence, or both.
The most frequently identified other potential source of bias related to the timing of the post-treatment assessment. In seven studies (Flannery Schroeder 2000; Fujii 2013; Ishikawa 2019; Kendall 1994; Kendall 1997; Masia Warner 2011; Pincus 2010), the timing of the post-treatment assessment varied across groups, with a shorter waitlist or control period than treatment period.

\section{Effects of interventions}

See: Summary of findings 1 CBT compared with waitlist for children and adolescents with anxiety disorders; Summary of findings 2 CBT compared with treatment as usual for anxiety disorders in children and adolescents; Summary of findings 3 CBT compared with attention control for anxiety disorders in children and adolescents; Summary of findings 4 CBT compared with alternative treatment for anxiety disorders in children and adolescents

\section{Comparison 1: CBT versus waitlist/no treatment}

See Summary of findings 1.

\section{Primary outcomes}

\subsection{Remission of primary anxiety diagnosis post-treatment}

We included 39 studies in the analyses examining remission of primary anxiety disorder diagnosis post-treatment (Arendt 2016; Cartwright Hatton 2011; Cobham 2017; Cornacchio 2019; Dadds 1997; Flannery Schroeder 2000; Gallagher 2004; Hirshfeld Becker 2010; Holmes 2014; Ishikawa 2019; Kendall 1994; Kendall 1997; Kidd 2018; Lau 2010; Masia Warner 2005; Masia Warner 2011; McConachie 2014; McNally Keehn 2013; Melfsen 2011; Olivares 2005; Olivares 2014; Olivares 2019; Ollendick 2009; Ost 2001; Perrin 2019; Rapee 2006; Rosa Alcazar 2009; Sanchez Garcia 2009; Santucci 2013; Schneider 2011; Silverman 1999b; Spence 2000; Spence 2006; Spence 2011; Thirlwall 2013; Villabo 2018; Waters 2009; Wergeland 2014; Wood 2015).

Moderate-quality evidence from ITT analysis revealed a response rate for remission of primary anxiety diagnosis of $49.4 \%$ (802 out of 1623 ) for CBT versus $17.8 \%$ (191 out of 1074) for controls (OR 5.45, $95 \% \mathrm{Cl} 3.90$ to $7.60, \mathrm{Z}=9.96, \mathrm{P}<0.001$ ) (Analysis 1.1 ). Over half of the studies (59\%; 23 out of 39$)$ and comparisons (52\%; 25 out of 48$)$ showed a clear benefit for CBT (95\% Cl does not cross 1) (Figure 4), with evidence of moderate heterogeneity $\left(\mathrm{Chi}^{2}=97.73, \mathrm{df}=47, \mathrm{P}<\right.$ $\left.0.001 ; 1^{2}=52 \%\right)$. 
Figure 4. Forest plot of comparison: 1 CBT vs waitlist/no treatment, outcome: 1.1 Remission of primary anxiety diagnosis post-treatment (ITT).

\begin{tabular}{ccccccc} 
& \multicolumn{2}{c}{ CBT } & Wait list / no treatment & & Odds Ratio & Odds Ratio \\
Study or Subgroup & Events & Total & Events & Total & Weight & IV, Random, 95\% CI
\end{tabular}

1.1.1 Child focused

Dadds 1997

Flannery Schroeder 2000

Flannery Schroeder 2000

Gallagher 2004

Kendall 1994

Kendall 1997

Lau 2010

Masia Warner 2005

Masia Warner 2011

McNally Keehn 2013

Olivares 2005

Olivares 2014

Olivares 2014

Olivares 2019

Olivares 2019

Ollendick 2009

Ost 2001

Perrin 2019

Rosa Alcazar 2009

Sanchez Garcia 2009

Spence 2000

Villabo 2018

Villabo 2018

Subtotal (95\% CI)

Total events:

Events To

Events

Total

Weight

V, Random, 95\% CI

IV, Random, $95 \%$ CI

$\begin{array}{rrrrr}21 & 23 & 28 & 33 & 2.1 \% \\ 9 & 18 & 2 & 7 & 1.9 \% \\ 6 & 13 & 1 & 7 & 1.4 \% \\ 5 & 12 & 0 & 11 & 1.0 \% \\ 17 & 40 & 1 & 20 & 1.6 \% \\ 32 & 69 & 17 & 49 & 3.8 \% \\ 14 & 21 & 0 & 17 & 1.0 \% \\ 12 & 21 & 5 & 21 & 2.7 \% \\ 9 & 20 & 1 & 20 & 1.6 \% \\ 7 & 12 & 0 & 10 & 1.0 \% \\ 10 & 17 & 0 & 17 & 1.0 \% \\ 11 & 39 & 2 & 19 & 2.2 \% \\ 20 & 39 & 2 & 20 & 2.3 \% \\ 8 & 36 & 0 & 18 & 1.0 \% \\ 13 & 36 & 0 & 18 & 1.0 \% \\ 47 & 85 & 1 & 41 & 1.7 \% \\ 18 & 21 & 0 & 10 & 1.0 \% \\ 16 & 20 & 0 & 20 & 1.0 \% \\ 12 & 20 & 0 & 20 & 1.0 \% \\ 21 & 28 & 0 & 25 & 1.0 \% \\ 11 & 19 & 1 & 7 & 1.5 \% \\ 29 & 55 & 4 & 27 & 3.0 \% \\ 36 & 55 & 4 & 28 & 2.9 \% \\ & \mathbf{7 1 9} & & \mathbf{4 6 5} & \mathbf{3 8 . 7 \%}\end{array}$

69

$2.1 \%$

$1.0 \%$

$1.6 \%$

$3.8 \%$

$.6 \%$

$.0 \%$

$.2 \%$

$.0 \%$

$1.0 \%$

$1.7 \%$

$1.0 \%$

$1.0 \%$

$1.0 \%$

$.5 \%$

$2.9 \%$

$38.7 \%$
$1.88[0.33,10.63]$

$2.50[0.38,16.42]$

$5.14[0.48,55.64]$

$16.87[0.81,351.79]$

$14.04[1.71,115.41]$

$1.63[0.77,3.46]$

67.67 [3.56, 1287.81]

$4.27[1.13,16.05]$

$15.55[1.73,139.65]$

$28.64[1.37,600.41]$

$49.00[2.53,948.62]$

$9.47[1.93,46.46]$

$11.04[0.60,202.89]$

$21.26[1.18,381.56]$

$111.00[5.21,2363.64]$

$150.33[7.54,2997.83]$

$60.29[3.20,1137.79]$

$146.20[7.89,2709.82]$

$8.25[0.82,82.67]$

$6.41[1.96,21.01]$

$11.37[3.44,37.58]$

$10.42[5.84,18.58]$
$3.34[0.66,16.92]$

$49.47[6.50,376.64]$

Heterogeneity: $\mathrm{Tau}^{2}=0.85 \cdot \mathrm{Chi}^{2}=43.41, \mathrm{df}=22(\mathrm{P}=0.004) ; \mathrm{I}^{2}=49 \%$

Test for overall effect: $\mathrm{Z}=7.94(\mathrm{P}<0.00001)$

\subsubsection{Child and parent}

Arendt 2016

Cornacchio 2019

Hirshfeld Becker 2010

Holmes 2014

Ishikawa 2019

Kidd 2018

McConachie 2014

Melfsen 2011

Ost 2001

Rapee 2006

Santucci 2013

Schneider 2011

Silverman 1999b

Spence 2000

Spence 2006

Spence 2011

Waters 2009

Wergeland 2014

Wergeland 2014

Wood 2015

Subtotal $(95 \%$ CI $)$

Total events:

Heterogeneity: $\mathrm{Tau}^{2}=0.28 ; \mathrm{Chi}^{2}=29.75, \mathrm{df}=19(\mathrm{P}=0.06) ; \mathrm{I}^{2}=36 \%$

Test for overall effect: $\mathrm{Z}=6.79(\mathrm{P}<0.00001)$

\subsubsection{Parent only}

Cartwright Hatton 2011

Cobham 2017

$\mid$

$15.25[5.53,42.05]$

$1.08[0.06,19.05]$

$2.40[0.87,6.57]$

$5.18[1.15,23.29]$

$3.85[0.99,14.89]$

$2.92[0.91,9.44]$

$0.28[0.01,7.31]$

$5.25[0.95,29.05]$

27.94 [1.43, 546.98]

$3.94[2.01,7.71]$

$11.38[1.17,110.42]$

$14.40[3.29,63.08]$

$2.13[0.64,7.16]$

$32.67[2.85,374.13]$

$9.63[2.19,42.37]$

$2.41[0.70,8.36]$

$4.86[0.49,48.57]$

$2.98[0.79,11.26]$

$2.88[0.77,10.77]$

$0.62[0.15,2.58]$

$4.08[2.72,6.11]$

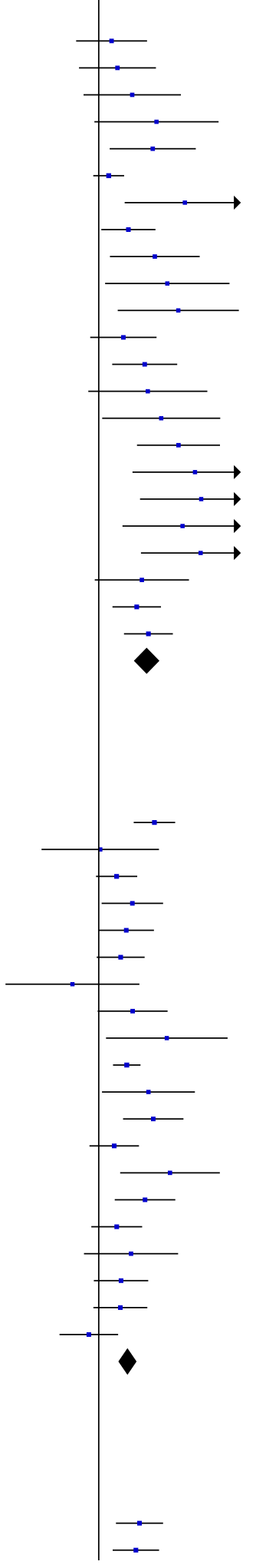


Figure 4. (Continued)

\begin{tabular}{|c|c|c|c|}
\hline Cartwright Hatton 2011 & 21 & 37 & 5 \\
\hline Cobham 2017 & 20 & 33 & 6 \\
\hline Thirlwall 2013 & 18 & 61 & 11 \\
\hline Thirlwall 2013 & 25 & 64 & 11 \\
\hline Waters 2009 & 21 & 38 & 1 \\
\hline Subtotal $(95 \%$ CI $)$ & & 233 & \\
\hline Total events: & 105 & & 34 \\
\hline \multirow{2}{*}{\multicolumn{4}{|c|}{$\begin{array}{l}\text { Heterogeneity: } \mathrm{Tau}^{2}=0.74 ; \mathrm{Chi}^{2}=13.17, \mathrm{df}=4(\mathrm{P}=0.01) ; \mathrm{I}^{2}=70 \% \\
\text { Test for overall effect: } \mathrm{Z}=2.20(\mathrm{P}=0.03)\end{array}$}} \\
\hline & & & \\
\hline Total $(95 \%$ CI $)$ & & 1623 & \\
\hline Total events: & 802 & & 191 \\
\hline \multirow{2}{*}{\multicolumn{4}{|c|}{$\begin{array}{l}\text { Heterogeneity: } \mathrm{Tau}^{2}=0.61 ; \mathrm{Chi}^{2}=97.73, \mathrm{df}=47(\mathrm{P}<0.0001) ; \mathrm{I}^{2}=52 \% \\
\text { Test for overall effect: } \mathrm{Z}=9.96(\mathrm{P}<0.00001)\end{array}$}} \\
\hline & & & \\
\hline \multicolumn{4}{|c|}{ Test for subgroup differences: $\mathrm{Chi}^{2}=8.57, \mathrm{df}=2(\mathrm{P}=0.01), \mathrm{I}^{2}=76.7 \%$} \\
\hline
\end{tabular}

\section{Subgroup analyses}

We found a difference in outcomes for the different delivery formats (child-focused, child and parent, parent only) (Chi ${ }^{2}=$ $8.57, \mathrm{df}=2, \mathrm{P}=0.01$ ), with substantial subgroup heterogeneity $\left(I^{2}=76.7 \%\right)$ (Analysis 1.1). Inspection of the forest plot and the summary statistics suggest that studies evaluating child-focused CBT produced greater remission of primary anxiety diagnosis in the CBT arms (OR 10.42, 95\% Cl 5.84 to 18.58) compared to waitlist/no treatment controls than studies evaluating CBT delivered to both children and parents (OR $4.08,95 \% \mathrm{Cl} 2.72$ to 6.11 ) or parents only (OR $2.83,95 \% \mathrm{Cl} 1.12$ to 7.16 ). Notably, however, there was also evidence of moderate heterogeneity amongst studies evaluating child-focused CBT $\left(\mathrm{Chi}^{2}=43.41, \mathrm{df}=22, \mathrm{P}=0.004 ; \mathrm{I}^{2}=49 \%\right)$.

We found no differences in remission of primary anxiety diagnosis outcomes for individual- versus group-delivered CBT $\left(\mathrm{Chi}^{2}=0.90\right.$, $\mathrm{df}=1, \mathrm{P}=0.34 ; \mathrm{I}^{2}=0 \%$ ); interventions with different amounts of therapist contact time ( $<10$ hours, $\geq 10$ and $<20$ hours, $\geq 20$ hours) $\left(\mathrm{Chi}^{2}=0.52, \mathrm{df}=2, \mathrm{P}=0.77 ; \mathrm{I}^{2}=0 \%\right)$; or studies with participants in different age groups ( $\leq 12$ years, $\geq 12$ years, $<12$ years and $\geq 12$ years) $\left(\mathrm{Chi}^{2}=1.59, \mathrm{df}=2, \mathrm{P}=0.45 ; \mathrm{I}^{2}=0 \%\right.$ ) (Analysis 1.2; Analysis 1.3; Analysis 1.4).

Subgroup analyses revealed no significant difference in outcomes between studies including participants with and without ASD $\left(\mathrm{Chi}^{2}\right.$ $=2.25, \mathrm{df}=1, \mathrm{P}=0.13 ; \mathrm{I}^{2}=55.5 \%$ ) (Analysis 1.5 ); however, only four studies included children/adolescents with ASD, compared to 35 studies with participants without ASD. The four studies of children/ adolescents with ASD revealed no significant difference between CBT and waitlist/no treatment controls in rates of remission of primary anxiety disorder diagnosis (OR $1.79,95 \% \mathrm{Cl} 0.39$ to $8.16, \mathrm{Z}$ $\left.=0.76, P=0.45, n=136,\left.\right|^{2}=59 \%\right)$.

\section{Completer analysis}

The completer analysis included 1422 CBT participants and 984 controls, with a response rate for remission of primary anxiety diagnosis of $56.4 \%$ for CBT versus $11.7 \%$ for controls (OR 11.55 , 95\% $\mathrm{Cl} 8.41$ to $15.86, \mathrm{Z}=15.11, \mathrm{P}<0.001$ ) (Analysis 1.6). Most of the studies (89\%, 34 out of 38 ) and comparisons ( $81 \%$; 38 out of 47 ) showed a clear benefit for CBT ( $95 \% \mathrm{Cl}$ does not cross 1$)$. There was no heterogeneity across studies $\left(\mathrm{Chi}^{2}=61.53, \mathrm{df}=46, \mathrm{P}=0.06 ; \mathrm{I}^{2}=\right.$ $25 \%)$.

$\begin{array}{rrr}33 & 3.0 \% & 7.35[2.32,23.27] \\ 30 & 3.1 \% & 6.15[1.98,19.14] \\ 34 & 3.5 \% & 0.88[0.35,2.16] \\ 35 & 3.6 \% & 1.40[0.58,3.35] \\ 6 & 1.5 \% & 6.18[0.66,58.03] \\ \mathbf{1 3 8} & \mathbf{1 4 . 7 \%} & \mathbf{2 . 8 3}[\mathbf{1 . 1 2}, \mathbf{7 . 1 6}]\end{array}$

$074 \quad 100.0 \%$

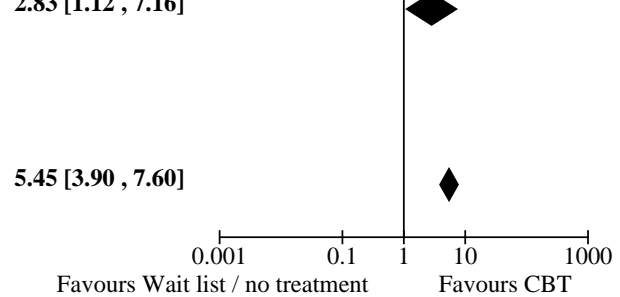

\subsection{Acceptability: loss of participants to post-treatment assessment}

Low-quality evidence showed no difference between CBT and waitlist/no treatment controls in the rate of those lost to posttreatment assessment (Analysis 1.7), indicating a similar degree of acceptability. The rate of loss to post-treatment assessment was $13.5 \%$ (260 out of 1919 ) in the CBT group versus $10.4 \%$ ( 129 out of 1239 ) in the control group (OR $1.09,95 \% \mathrm{Cl} 0.85$ to $1.41, \mathrm{Z}=0.69, \mathrm{P}$ $\left.=0.49, \mathrm{n}=3158, \mathrm{k}=45, \mathrm{I}^{2}=4 \%\right)$.

\section{Secondary outcomes}

\subsection{Remission for all anxiety disorders post-treatment}

We included 28 studies in the analyses examining post-treatment remission of all anxiety disorder diagnoses (Arendt 2016; Barrett 1996; Barrett 1998; Cartwright Hatton 2011; Chalfant 2007; Chiu 2013; Cobham 2017; Dadds 1997; Hirshfeld Becker 2010; Ishikawa 2019; Kennedy 2009; Lau 2017; McConachie 2014; Melfsen 2011; Olivares 2005; Perrin 2019; Rapee 2005; Rapee 2006; Rosa Alcazar 2009; Sanchez Garcia 2009; Shortt 2001; Simon 2011; Spence 2000; Spence 2006; Spence 2011; Thirlwall 2013; Villabo 2018; Waters 2009).

The ITT analysis included 1213 CBT participants and 862 controls, with a response rate for remission of all anxiety disorder diagnoses of $46.8 \%$ for CBT versus $19.1 \%$ for waitlist/no treatment controls (OR 4.43, 95\% Cl 2.89 to $6.78, \mathrm{Z}=6.85, \mathrm{P}<0.001$ ) (Analysis 1.8). Sixty per cent of included studies (17 out of 28 ) and $51 \%$ of comparisons (18 out of 35 ) reported findings showing a clear benefit for CBT (95\% $\mathrm{Cl}$ does not cross 1 ). There was evidence of moderate heterogeneity $\left(\mathrm{Chi}^{2}=84.49, \mathrm{df}=34, \mathrm{P}<0.001 ; \mathrm{I}^{2}=60 \%\right)$, and thus moderatequality evidence overall for the benefit of CBT compared to waitlist/ no treatment controls for remission of all anxiety disorders.

\section{Subgroup analyses}

We found a difference in outcomes for different delivery formats (child-focused, child and parent, parent-only) $\left(\mathrm{Chi}^{2}=8.14\right.$, $\mathrm{df}$ $=2, \mathrm{P}=0.02)$, with substantial subgroup heterogeneity $\left(\mathrm{I}^{2}=\right.$ $75.4 \%)$. There was evidence of substantial heterogeneity amongst studies evaluating child-focused CBT $\left(1^{2}=70 \%\right)$, but we found that overall these studies produced greater remission of all anxiety disorder diagnoses following CBT compared to waitlist/no treatment controls (OR $8.52,95 \% \mathrm{Cl} 2.97$ to 24.39), than studies evaluating child-and-parent delivered CBT (OR 5.19, 95\% Cl 3.26 to 
8.27), or studies evaluating parent-delivered CBT (OR 1.89, 95\% Cl crosses 1$)$.

We found no differences in remission of all anxiety disorder diagnoses outcomes for individual- versus group-delivered CBT $\left(\mathrm{Chi}^{2}=0.35, \mathrm{df}=1, \mathrm{P}=0.56 ; \mathrm{I}^{2}=0 \%\right.$ ) (Analysis 1.9); however, there was variation in these outcomes amongst interventions with differing amounts of therapist contact time $\left(\mathrm{Chi}^{2}=10.75, \mathrm{df}=2\right.$, $P=0.005 ; I^{2}=81.4 \%$ ) (Analysis 1.10). Only five studies evaluated interventions involving $<10$ hours therapist contact time (Cobham 2017; Ishikawa 2019; Rapee 2005; Simon 2011; Thirlwall 2013), and did not indicate a benefit for CBT compared to waitlist/no treatment control groups for remission of all anxiety disorders (OR $1.42,95 \% \mathrm{Cl} 0.68$ to $2.96, \mathrm{Z}=0.93, \mathrm{P}=0.35$ ), whilst interventions with 10 to 20 hours or $\geq 20$ hours contact time both showed a clear benefit for CBT (OR 6.59, 95\% Cl 3.62 to 12.01 and OR 5.03, 95\% Cl 2.55 to 9.93 , respectively).

Subgroup analyses did not show differences in remission of all anxiety disorder diagnoses outcomes amongst studies with participants in different age groups $\left(\mathrm{Chi}^{2}=0.80, \mathrm{df}=2, \mathrm{P}=0.67 ; \mathrm{I}^{2}\right.$ $=0 \%$ ) (Analysis 1.11), and as only two studies included participants with ASD, meaningful subgroup analyses comparing studies with and without participants with ASD was not possible.

\section{Completer analysis}

The completer analysis included 1074 CBT participants and 797 controls, with a response rate for remission of any anxiety disorder diagnosis of $52.9 \%$ for CBT versus $14.2 \%$ for controls (OR 9.13, 95\% $\mathrm{Cl} 5.78$ to $14.41, \mathrm{Z}=9.49, \mathrm{P}<0.001$ ) (Analysis 1.12). More than threequarters of included studies (21 out of 27 ) and $65 \%$ of comparisons (22 out of 34 ) reported findings showing a clear benefit for CBT (95\% $\mathrm{Cl}$ does not cross 1 ). There was evidence of moderate heterogeneity $\left(\mathrm{Chi}^{2}=68.75, \mathrm{df}=33, \mathrm{P}<0.001 ; \mathrm{I}^{2}=52 \%\right)$.

\subsection{Reduction in anxiety symptoms (child report) post-treatment}

We included 45 studies in the analyses examining post-treatment child-reported anxiety symptoms (Afshari 2014; Arendt 2016; Barrett 1996; Berge 2017; Chalfant 2007; Cheung 2016; Chiu 2013; Cobham 2017; Dadds 1997; Flannery Schroeder 2000; Gallagher 2004; Hancock 2018; Holmes 2014; Ishikawa 2019; Kendall 1994; Kendall 1997; Kidd 2018; Lau 2010; Leutgeb 2012; Masia Warner 2005; McConachie 2014; McNally Keehn 2013; Melfsen 2011; Olivares 2005; Olivares 2014; Ollendick 2009; Ost 2001; Perrin 2019; Rapee 2006; Rosa Alcazar 2009; Salari 2018; Sanchez Garcia 2009; Santucci 2013; Schneider 2011; Shortt 2001; Silverman 1999b; Simon 2011; Smith 2014; Spence 2000; Spence 2006; Spence 2011; Thirlwall 2013; Villabo 2018; Wergeland 2014; Wood 2009). The standardised mean difference (SMD) between CBT groups and waitlist/no treatment controls was $-0.67(95 \% \mathrm{Cl}-0.88$ to -0.47 , $\mathrm{Z}=6.36, \mathrm{P}<0.001, \mathrm{n}=2831$ ) (Analysis 1.13; Figure 5). Substantial heterogeneity $\left(\mathrm{Chi}^{2}=352.54, \mathrm{df}=56, \mathrm{P}<0.001 ; \mathrm{I}^{2}=84 \%\right)$ reduced our confidence in this result, and we rated this evidence as low-quality. 
Figure 5. Forest plot of comparison: 1 CBT vs waitlist/no treatment, outcome: 1.13 Reduction in anxiety symptoms (child report) post-treatment.

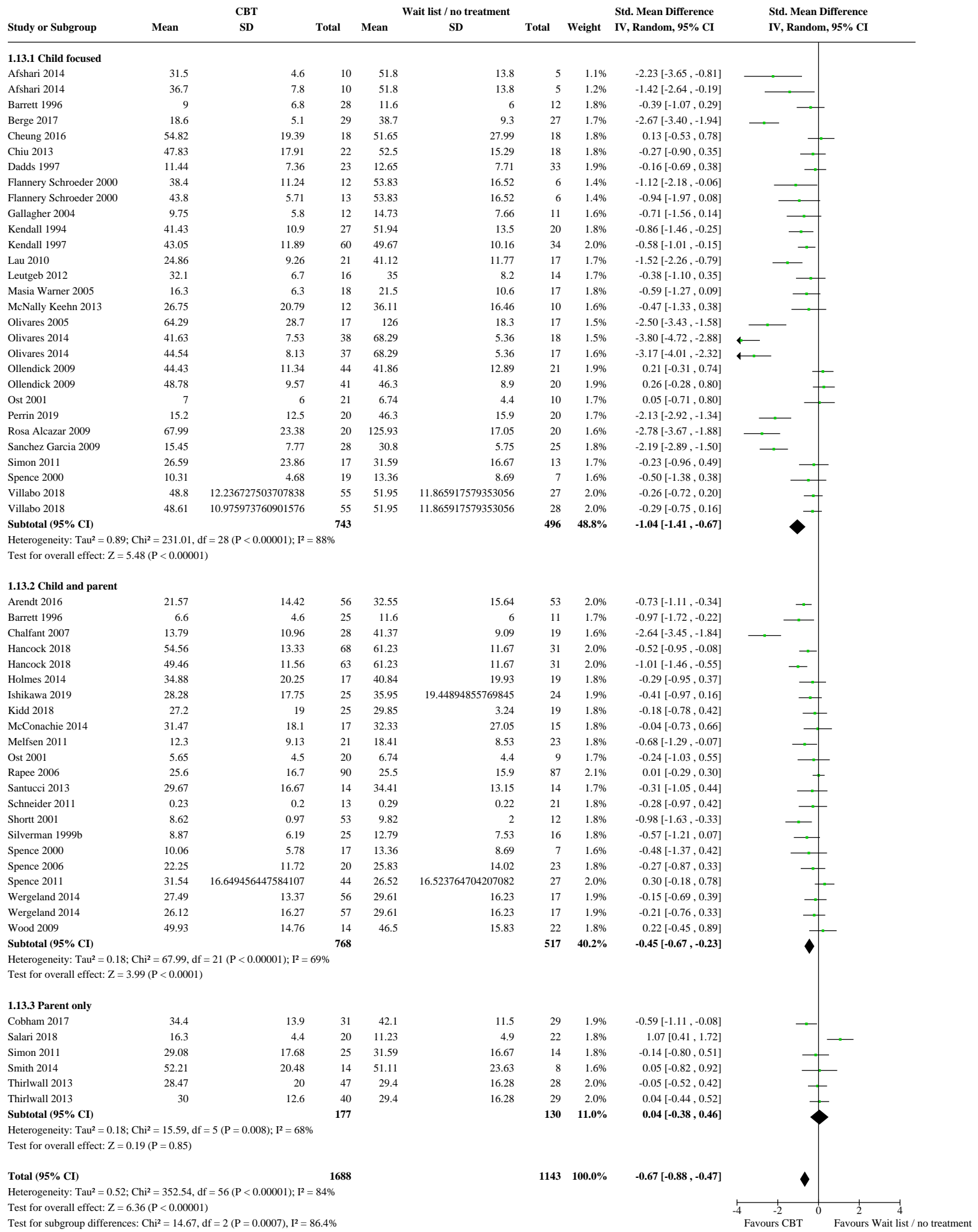

Indeed, including only studies that used a broad measure of childreported anxiety symptoms (38 studies, 2459 participants), the SMD between CBT groups and waitlist controls was reduced to -0.41 $\left(95 \% \mathrm{Cl}-0.57\right.$ to $\left.-0.25, \mathrm{Z}=5.13, \mathrm{P}<0.001, \mathrm{I}^{2}=68 \%\right)$ (Analysis 1.14). 


\section{Subgroup analyses}

We found a significant difference between child-focused, child-andparent, and parent-only $\mathrm{CBT}\left(\mathrm{Chi}^{2}=14.67, \mathrm{df}=2, \mathrm{P}<0.001\right)$ on childreported anxiety symptom outcomes, with considerable subgroup heterogeneity $\left(I^{2}=86.4 \%\right)$. There was no significant difference between CBT and waitlist/no treatment control groups in posttreatment child-reported anxiety symptoms for parent-delivered CBT (SMD 0.04, $95 \% \mathrm{Cl}-0.38$ to $0.46, \mathrm{Z}=0.19, \mathrm{P}=0.85,5$ studies). The forest plot and summary statistics indicated that child-focused CBT produced a greater reduction on child-reported anxiety symptoms amongst CBT groups compared to waitlist/no treatment controls (SMD -1.04, 95\% Cl -1.41 to $-0.67, \mathrm{Z}=5.48, \mathrm{P}<0.001,24$ studies) than child-and-parent delivered CBT (SMD $-0.45,95 \% \mathrm{Cl}-0.67$ to $-0.23, Z=3.99, P<0.001,20$ studies), but with substantial heterogeneity within each of these subgroups (child-focused: $I^{2}=$ $88 \%$; child-and-parent: $I^{2}=69 \%$ ).

Subgroup analyses also revealed a significance difference between individual- and group-focused CBT $\left(\mathrm{Chi}^{2}=6.47, \mathrm{df}=1, \mathrm{P}=0.01 ; \mathrm{I}^{2}\right.$ $=84.5 \%$ ) (Analysis 1.15). The SMD in post-treatment child-reported anxiety symptoms between CBT and waitlist/no treatment control groups was $-0.91(95 \% \mathrm{Cl}-1.22$ to -0.60$)$ in studies evaluating group-focused CBT compared to $-0.39(95 \% \mathrm{Cl}-0.64$ to -0.15$)$ in studies evaluating individual-focused CBT.

We found no differences between interventions with different amounts of therapist contact time $(<10$ hours, $\geq 10$ and $<20$ hours, $\geq 20$ hours) $\left(\mathrm{Chi}^{2}=3.33, \mathrm{df}=2, \mathrm{P}=0.19 ; \mathrm{I}^{2}=39.9 \%\right.$ ) (Analysis 1.16), but child-reported anxiety symptoms outcomes did vary amongst different age groups $\left(\mathrm{Chi}^{2}=8.27, \mathrm{df}=2, \mathrm{P}=0.02\right)$, with substantial subgroup heterogeneity $\left(I^{2}=75.8 \%\right)$ (Analysis 1.17). Amongst studies of children aged $\geq 12$ years, the SMD between CBT and waitlist/no treatment controls was $-1.78(95 \% \mathrm{Cl}-3.01$ to -0.56$)$ compared to $-0.62(95 \% \mathrm{Cl}-0.83$ to -0.41$)$ where studies included participants over and under 12 years of age, and -0.23 ( $95 \% \mathrm{Cl}$ cross 0 ) amongst studies of children $\leq 12$ years, but with variation within each of these age groups $\left(I^{2}=71 \%\right.$ to $\left.95 \%\right)$.

We did not find differences between studies including participants with and without ASD on post-treatment child-reported anxiety symptoms outcomes $\left(\mathrm{Chi}^{2}=0.02, \mathrm{df}=1, \mathrm{P}=0.88 ; \mathrm{I}^{2}=0 \%\right.$ ) (Analysis 1.18).

\subsection{Reduction in anxiety symptoms (parent report) post-treatment}

We included 35 studies in analyses examining post-treatment parent-reported anxiety symptoms (Afshari 2014; Arendt 2016; Chalfant 2007; Cheung 2016; Chiu 2013; Cobham 2017; Flannery Schroeder 2000; Holmes 2014; Ishikawa 2019; Kendall 1994; Kendall 1997; Kennedy 2009; Kidd 2018; Lau 2010; Lau 2017; Masia Warner 2005; McConachie 2014; McNally Keehn 2013; Olivares 2014; Perrin 2019; Rapee 2005; Rapee 2006; Rosa Alcazar 2009; Santucci 2013; Schneider 2011; Silverman 1999b; Smith 2014; Spence 2006; Spence 2011; Thirlwall 2013; Villabo 2018; Wergeland 2014; White 2013; Wood 2009; Wood 2015). The SMD between CBT and waitlist/ no treatment control groups was $-0.70(95 \% \mathrm{Cl}-0.90$ to $-0.51, \mathrm{Z}$ $=7.01, \mathrm{P}<0.001, \mathrm{n}=2137$, low-quality evidence), with substantial heterogeneity $\left(\mathrm{Chi}^{2}=171.97, \mathrm{df}=40, \mathrm{P}<0.001 ; \mathrm{I}^{2}=77 \%\right.$ ) (Analysis 1.19; Figure 6. 
Figure 6. Forest plot of comparison: 1 CBT vs waitlist/no treatment, outcome: 1.19 Reduction in anxiety symptoms (parent report) post-treatment.

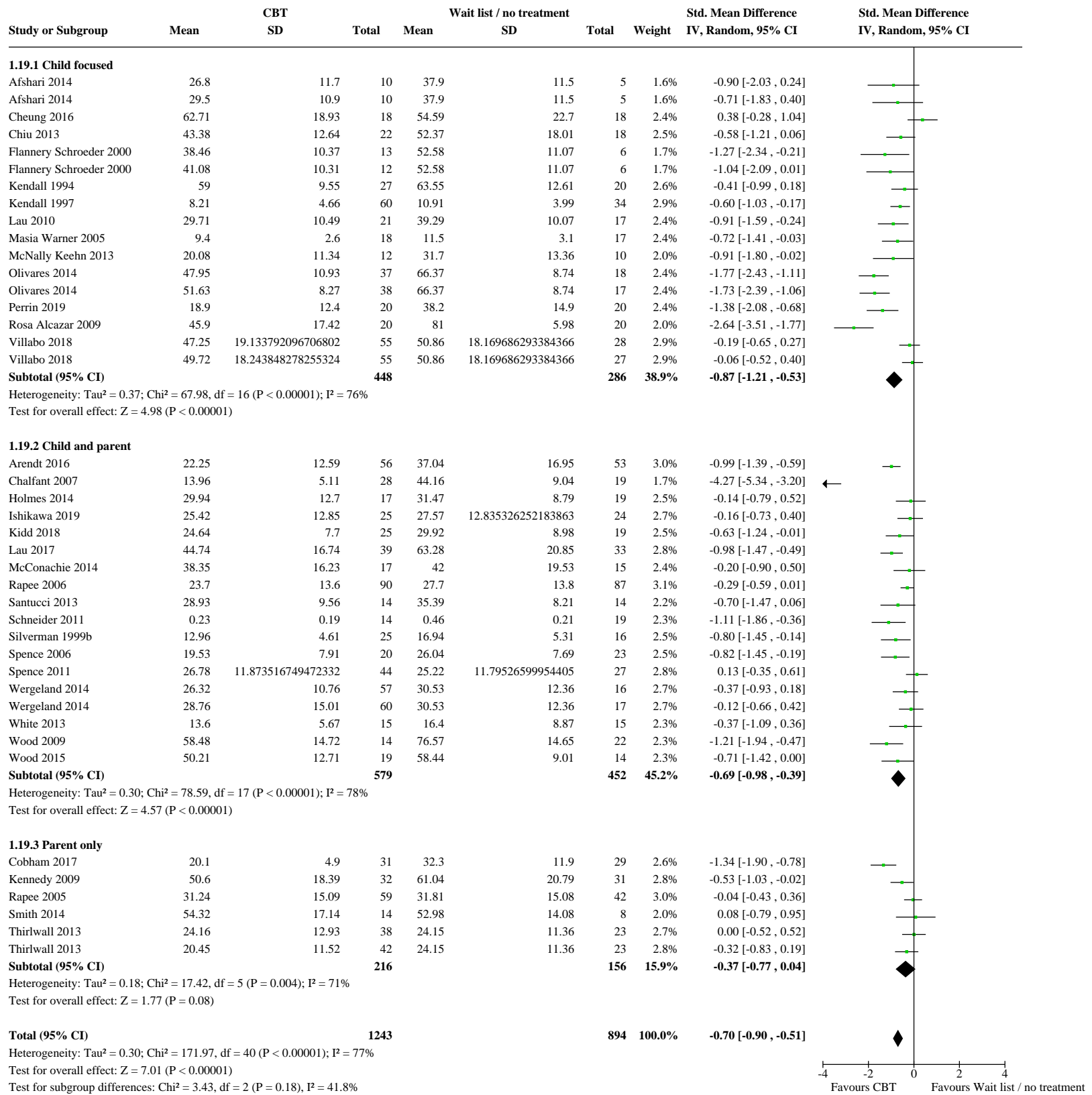

Including only studies that used a broad measure of parentreported anxiety symptoms (32 studies, 1952 participants), the SMD between CBT and waitlist groups was reduced to $-0.59(95 \% \mathrm{Cl}$ -0.77 to $-0.41, \mathrm{Z}=6.33, \mathrm{P}<0.001, \mathrm{I}^{2}=71 \%$ ) (Analysis 1.20 ).

\section{Subgroup analyses}

Subgroup analyses revealed no significant differences between child-focused, child and parent, and parent-only on parentreported anxiety symptom outcomes $\left(\mathrm{Chi}^{2}=3.43, \mathrm{df}=2, \mathrm{P}=\right.$ 0.18 ) (Analysis 1.19). However, there was evidence of a difference amongst individual compared to group interventions $\left(\mathrm{Chi}^{2}=6.79\right.$, $\mathrm{df}=1, \mathrm{P}=0.009)$, with substantial subgroup heterogeneity $\left(\mathrm{I}^{2}\right.$ $=85.3 \%$ ) (Analysis 1.21). The SMD between CBT and waitlist/ no treatment post-treatment parent-reported anxiety symptoms was $-0.92(95 \% \mathrm{Cl}-1.21$ to -0.62$)$ amongst group interventions, compared to $-0.43(95 \% \mathrm{Cl}-0.65$ to -0.21$)$ for individual interventions.

We did not find differences in parent-reported anxiety symptom outcomes across interventions involving varying amount of therapist contact time $\left(\mathrm{Chi}^{2}=3.77, \mathrm{df}=2, \mathrm{P}=0.15\right.$; Analysis 1.22). On the other hand, there was evidence of a difference in these outcomes amongst different age groups $\left(\mathrm{Chi}^{2}=5.87, \mathrm{df}=2, \mathrm{P}=0.05\right)$, with substantial subgroup heterogeneity $\left(\mathrm{I}^{2}=65.9 \%\right.$ ) (Analysis 1.23). Amongst studies that included participants aged $\geq 12$ years, the SMD between CBT and waitlist/no treatment groups was -1.07 ( $95 \% \mathrm{Cl}-1.78$ to -0.37$)$, compared to $-0.76(95 \% \mathrm{Cl}-1.04$ to -0.49$)$ 
in studies that included participants under and over 12 years of age, and $-0.40(95 \% \mathrm{Cl}-0.63$ to -0.17$)$ where participants were $\leq 12$ years.

We found no significant differences in parent-reported anxiety symptom outcomes amongst samples with and without ASD $\left(\mathrm{Chi}^{2}\right.$ $=1.42, \mathrm{df}=1, \mathrm{P}=0.23$; Analysis 1.24).

\subsection{Reduction in depressive symptoms post-treatment}

Seventeen studies reported post-treatment depressive symptoms (Arendt 2016; Barrett 1996; Flannery Schroeder 2000; Gallagher 2004; Ishikawa 2019; Kendall 1994; Kendall 1997; Kidd 2018; Masia Warner 2005; Melfsen 2011; Ollendick 2009; Ost 2001; Perrin 2019; Salari 2018; Silverman 1999b; Thirlwall 2013; Wergeland 2014). The SMD between CBT and waitlist/no treatment control groups was $-0.34(95 \% \mathrm{Cl}-0.51$ to $-0.17, \mathrm{Z}=3.96, \mathrm{P}<0.001, \mathrm{n}=1157$, moderatequality evidence), with moderate heterogeneity $\left(\mathrm{Chi}^{2}=39.62, \mathrm{df}=\right.$ $22, \mathrm{P}=0.01 ; \mathrm{I}^{2}=44 \%$ ) (Analysis 1.25).

\subsection{Improvement in global functioning post-treatment}

Data pooled from 11 studies that reported post-treatment global functioning produced an SMD between CBT and waitlist/no treatment control groups of $1.03(95 \% \mathrm{Cl} 0.68$ to $1.38, \mathrm{Z}=$ $5.83, P<0.001, n=557$, low-quality evidence), with substantial heterogeneity $\left(\mathrm{Chi}^{2}=37.41, \mathrm{df}=11, \mathrm{P}<0.001 ; \mathrm{I}^{2}=71 \%\right.$ ) (Analysis 1.26) (Cornacchio 2019; Holmes 2014; Masia Warner 2005; Masia Warner 2011; Melfsen 2011; Perrin 2019; Salari 2018; Santucci 2013; Spence 2011; Villabo 2018; White 2013).

\subsection{Adverse events}

No included study reported adverse events in both CBT and waitlist/no treatment groups, so meta-analysis was not possible.

\section{Remission of primary anxiety disorder diagnosis at follow-up}

Four studies reported remission of primary anxiety disorder at follow-up assessments $\leq 6$ months post-treatment (Dadds 1997; Gallagher 2004; Olivares 2005; Rosa Alcazar 2009), with ITT analysis showing a rate for remission of primary anxiety diagnosis of $66.7 \%$ (48 out of 72 ) for CBT groups versus $34.6 \%$ (28 out of 81 ) for waitlist/no treatment controls (OR $10.94,95 \% \mathrm{Cl} 2.33$ to 51.41 , $Z=3.03, P=0.002, I^{2}=40 \%$ ) (Analysis 1.27). Only 3 studies (166 participants) reported remission of primary anxiety disorder 12 months post-treatment (Cartwright Hatton 2011; Dadds 1997; Rosa Alcazar 2009); pooled data did not suggest a benefit for CBT compared to waitlist/no treatment control groups (OR 2.80, 95\% 0.24 to $\left.33.19, \mathrm{Z}=0.82, \mathrm{P}=0.41, \mathrm{I}^{2}=83 \%\right)$.

Only one study reported remission of primary anxiety disorder $>12$ months post-treatment, so meta-analysis was not possible.

Completer analyses produced similar findings, with evidence of an intervention effect at follow-up assessments $\leq 6$ months posttreatment but not at 12-month follow-up (Analysis 1.28)

\section{Remission of all anxiety disorder diagnoses at follow-up}

Five studies reported data on remission of all anxiety disorders at follow-up assessments (Cartwright Hatton 2011; Dadds 1997; Olivares 2005; Rosa Alcazar 2009; Simon 2011), including $\leq 6$ months post-treatment (Dadds 1997; Olivares 2005; Rosa Alcazar 2009), 12 months post-treatment (Cartwright Hatton 2011; Dadds 1997; Rosa Alcazar 2009), and 24 months post-treatment (Dadds 1997; Simon
2011). Neither ITT analyses (Analysis 1.29) nor completer analyses (Analysis 1.30) showed a significant difference between CBT and waitlist/no treatment at any of these follow-up time points.

\section{Reduction in anxiety symptoms at follow-up}

Six studies reported data on child-reported anxiety symptoms at follow-up assessments (Analysis 1.31) (Afshari 2014; Dadds 1997; Gallagher 2004; Olivares 2005; Rosa Alcazar 2009; Simon 2011). Child-reported anxiety symptoms at $\leq 6$ months post-treatment produced an SMD of $-1.92(95 \% \mathrm{Cl}-2.95$ to $-0.89, \mathrm{Z}=3.65, \mathrm{P}<0.001$, $\mathrm{n}=179$ ) between CBT and waitlist/no treatment groups (Afshari 2014; Dadds 1997; Gallagher 2004; Olivares 2005; Rosa Alcazar 2009), with substantial heterogeneity across studies $\left(\mathrm{Chi}^{2}=35.35\right.$, $\left.\mathrm{df}=5, \mathrm{P}<0.001 ; \mathrm{I}^{2}=86 \%\right)$. Only two studies reported corresponding outcomes at 12-month follow-up (Dadds 1997; Rosa Alcazar 2009), and only one study at 24-month follow-up (Simon 2011), so metaanalyses were not possible for these time points.

As only two studies reported data on parent-reported anxiety symptoms at 6 months post-treatment (Afshari 2014; Rosa Alcazar 2009), and one with corresponding data at 12 months posttreatment (Rosa Alcazar 2009), meta-analyses were not performed using these data.

\section{Comparison 2: CBT versus treatment as usual}

See Summary of findings 2.

\section{Primary outcomes}

\subsection{Remission of primary anxiety diagnosis post-treatment}

Eight studies provided uncertain evidence on post-treatment remission of primary anxiety disorder diagnosis for CBT versus TAU (Fujii 2013; Ginsburg 2019a; Reaven 2012; Shahnavaz 2016; Sharma 2017; Southam Gerow 2010; Storch 2013; Storch 2015).

The ITT analysis included 281 CBT participants and 206 TAU participants, with no evidence of a significant difference in the rate of remission of primary anxiety diagnosis post-treatment $(44.1 \%$ and $40.8 \%$ respectively; OR $3.19,95 \% \mathrm{Cl} 0.90$ to $11.29, \mathrm{Z}=1.80, \mathrm{P}$ $=0.07$, low-quality evidence) (Analysis 2.1). There was evidence of substantial heterogeneity $\left(\mathrm{Chi}^{2}=28.93, \mathrm{df}=7, \mathrm{P}<0.001 ; \mathrm{I}^{2}=76 \%\right)$.

\section{Subgroup analyses}

There was a significant difference in remission of primary anxiety disorder outcomes amongst studies evaluating child-focused CBT compared to child-and-parent delivered CBT $\left(\mathrm{Chi}^{2}=10.90, \mathrm{df}=1, \mathrm{P}\right.$ $<0.001 ; \mathrm{I}^{2}=90.8 \%$ ). Data pooled from five studies (172 participants) that evaluated a child-and-parent delivered intervention showed a clear benefit for CBT compared to TAU (OR $8.56,95 \% \mathrm{Cl} 3.10$ to 23.66, $Z=4.14, P<0.001$ ), whereas data from three studies (315 participants) evaluating child-focused interventions did not (OR $0.61,95 \% \mathrm{Cl}$ cross 1$)$. None of the studies that compared CBT to TAU evaluated a parent-only intervention.

Planned subgroup analyses comparing individual versus group interventions, interventions with varying amount of therapist contact time, and different age groups were not possible due to an insufficient number of studies. Only two studies evaluated group $\mathrm{CBT}$; only one study used an intervention with $<10$ hours therapist contact time; and five (out of eight) studies included pre-adolescent and adolescent participants. 
Subgroup analyses also indicated a difference in remission outcomes in studies with participants with ASD compared to studies with participants without $\mathrm{ASD}\left(\mathrm{Chi}^{2}=5.71, \mathrm{df}=1, \mathrm{P}=0.02 ; \mathrm{I}^{2}\right.$ $=82.5 \%$ ) (Analysis 2.2). Pooled data from four ASD samples showed a higher rate of remission of primary anxiety disorder amongst CBT groups compared to TAU groups (OR $11.25,95 \% \mathrm{Cl} 3.11$ to 40.79 , Z $\left.=3.68, P<0.001, n=142, I^{2}=0 \%\right)$, but corresponding data from four samples without ASD showed no significant difference between CBT and TAU groups (OR 1.14, 95\% $\mathrm{Cl} 0.29$ to $4.47, \mathrm{Z}=0.18, \mathrm{P}=0.85$, $\left.\mathrm{n}=345, \mathrm{I}^{2}=75 \%\right)$.

\section{Completer analysis}

The completer analysis included 248 CBT participants and 192 TAU participants, with a response rate for remission of primary anxiety diagnosis of $50.4 \%$ for CBT versus $36.5 \%$ for controls (OR 4.87, $95 \%$ $\mathrm{Cl} 1.51$ to $15.72, \mathrm{Z}=2.65, \mathrm{P}=0.008$ ) (Analysis 2.3). Similar to the ITT analysis, there was substantial heterogeneity across the eight studies $\left(\mathrm{Chi}^{2}=21.30, \mathrm{df}=7, \mathrm{P}=0.003 ; \mathrm{I}^{2}=67 \%\right)$.

\subsection{Acceptability: loss of participants to post-treatment assessment}

We found no evidence of a difference between CBT and TAU in rate of those lost to post-treatment assessment, illustrating a comparable degree of acceptability (OR $1.37,95 \% \mathrm{Cl} 0.73$ to 2.56 , Z $=0.98, P=0.33, I^{2}=0 \%, k=8, n=441$, low-quality evidence) (Analysis 2.4).

\section{Secondary outcomes}

\subsection{Remission of all anxiety disorders post-treatment}

Five studies provided uncertain evidence on remission of all anxiety disorder diagnoses post-treatment for CBT versus TAU (Barrington 2005; Sciberras 2018; Sharma 2017; Storch 2013; Storch 2015).

The ITT analyses showed a rate of remission of all anxiety diagnoses of $55.8 \%$ for CBT versus $41.4 \%$ for TAU (OR $2.74,95 \% \mathrm{Cl} 1.16$ to 6.46 , $\mathrm{Z}=2.30, \mathrm{P}=0.02, \mathrm{k}=5, \mathrm{n}=203$, low-quality evidence) (Analysis 2.5). There was no evidence of significant heterogeneity $\left(\mathrm{Chi}^{2}=3.92, \mathrm{df}\right.$ $\left.=4, \mathrm{P}=0.42 ;\left.\right|^{2}=0 \%\right)$.

Completer analyses showed a similar benefit for CBT versus TAU (OR 2.78, 95\% Cl 1.18 to $6.55, \mathrm{Z}=2.33, \mathrm{P}=0.02, \mathrm{k}=5, \mathrm{n}=201, \mathrm{I}^{2}=$ $0 \%)$ (Analysis 2.6).

\subsection{Reduction in anxiety symptoms (child-report) post-treatment}

We included six studies in the analyses examining post-treatment child-reported anxiety symptoms (Barrington 2005; Ginsburg 2012; Sciberras 2018; Shahnavaz 2016; Sharma 2017; Southam Gerow 2010). We found no difference between CBT and TAU in childreported anxiety symptoms post-treatment (SMD $-0.15,95 \% \mathrm{Cl}$ -0.78 to $0.48, Z=0.46, P=0.64, n=214$, low-quality evidence), although there was substantial heterogeneity $\left(\mathrm{Chi}^{2}=23.55, \mathrm{df}=5\right.$, $\mathrm{P}<0.001 ; \mathrm{I}^{2}=79 \%$ ) (Analysis 2.7). This finding was replicated after removal of the one study that used a disorder-specific symptoms measure (SMD $0.07,95 \% \mathrm{Cl}-0.54$ to $0.68, \mathrm{Z}=0.22, \mathrm{P}=0.83, \mathrm{n}=187$, $I^{2}=74 \%$ ) (Analysis 2.8).

\subsection{Reduction in anxiety symptoms (parent-report) post-treatment}

Pooled data from seven studies showed no difference between CBT and TAU in post-treatment parent-reported anxiety symptoms (SMD -0.32, 95\% Cl -0.70 to $0.06, \mathrm{Z}=1.64, \mathrm{P}=0.10, \mathrm{n}=228$, lowquality evidence) (Barrington 2005; Ginsburg 2002; Sciberras 2018; Shahnavaz 2016; Southam Gerow 2010; Storch 2013; Storch 2015), with moderate heterogeneity across studies $\left(\mathrm{Chi}^{2}=11.66, \mathrm{df}=6, \mathrm{P}\right.$ $=0.07 ; 1^{2}=49 \%$ ) (Analysis 2.9). Removal of one study that used a disorder-specific measure did not change this finding (SMD -0.16 , $95 \% \mathrm{Cl}-0.44$ to $0.11, \mathrm{Z}=1.16, \mathrm{P}=0.25, \mathrm{n}=202, \mathrm{I}^{2}=0 \%$ ) (Analysis 2.10).

\subsection{Reduction in depressive symptoms post-treatment}

Only one study reported depressive symptoms post-treatment for CBT versus TAU (O'Brien 2007), so meta-analysis was not possible.

\subsection{Improvement in global functioning post-treatment}

Only one study reported post-treatment global functioning for CBT versus TAU (Sharma 2017), so meta-analysis was not possible.

\subsection{Adverse events}

No included study reported adverse events in both CBT and TAU groups, so meta-analysis was not possible.

\subsection{Follow-up outcomes}

There was an insufficient number of studies to conduct meta-analyses examining longer-term diagnostic or symptom outcomes. Only two studies reported remission from primary anxiety disorders at 12 months post-treatment (Ginsburg 2019a; Shahnavaz 2016), and one study reported remission from all anxiety disorders at 6 and 12 months (Barrington 2005). Similarly, only three studies reported child-reported and parentreported anxiety symptoms at 6-month and/or 12-month follow-up (Barrington 2005; Ginsburg 2012; Shahnavaz 2016).

\section{Comparison 3: CBT versus attention control}

See Summary of findings 3.

\section{Primary outcomes}

\subsection{Remission of primary anxiety diagnosis post-treatment}

Ten studies evaluating CBT versus attention control provided data on post-treatment remission of primary anxiety disorder diagnosis (Ginsburg 2002; Hudson 2009; Kendall 2008; Khanna 2010; Last 1998; Masia Warner 2007; Masia Warner 2016; Ollendick 2009; Rosa Alcazar 2009; Silverman 1999a).

The ITT analysis (484 CBT participants; 338 control participants) provided uncertain evidence for a greater rate of remission of primary anxiety diagnosis post-treatment for CBT compared to attention controls ( $48.3 \%$ versus $29.3 \%$; OR $2.28,95 \% \mathrm{Cl} 1.33$ to $3.89, Z=3.00, P=0.003$, low-quality evidence), with moderate heterogeneity across studies $\left(\mathrm{Chi}^{2}=28.77, \mathrm{df}=12, \mathrm{P}=0.004 ; \mathrm{I}^{2}=\right.$ 58\%) (Analysis 3.1).

\section{Subgroup analyses}

None of the studies that evaluated CBT versus attention controls used a parent-only intervention, but there was a difference in the pattern of results for remission of primary anxiety disorder outcomes amongst studies evaluating child-focused CBT and childand-parent delivered CBT when each was compared to attention controls $\left(\mathrm{Chi}^{2}=7.65, \mathrm{df}=1, \mathrm{P}=0.006 ; \mathrm{I}^{2}=86.9 \%\right.$ ) (Analysis 3.1). Summary statistics indicated a benefit for CBT compared to attention controls amongst child-focused interventions (OR 3.58, $95 \% \mathrm{Cl} 1.92$ to $6.65, \mathrm{k}=7, \mathrm{n}=509$ ), but not amongst child-and-parent delivered interventions (OR $1.12,95 \% \mathrm{Cl}$ cross $1, \mathrm{k}=4, \mathrm{n}=313$ ). 
Subgroup analyses revealed no significant difference in remission of primary anxiety disorder outcomes in studies evaluating individual CBT versus group CBT $\left(\mathrm{Chi}^{2}=1.22, \mathrm{df}=1, \mathrm{P}=0.27 ; \mathrm{I}^{2}=\right.$ 17.7\%) $\left(\mathrm{Chi}^{2}=0.48, \mathrm{df}=1, \mathrm{P}=0.49 ; \mathrm{I}^{2}=0 \%\right)$ (Analysis 3.2$)$.

Further subgroup analyses were not possible because there were fewer than three studies in at least one group for each planned subgroup analysis.

\section{Completer analysis}

In the completer analysis, $53.1 \%$ of CBT participants compared to $23.2 \%$ of attention control participants were free of their primary anxiety disorder diagnosis post-treatment, with a significant intervention effect (OR 3.88, 95\% Cl 2.52 to 5.97, $\mathrm{Z}=6.17, \mathrm{P}<0.001$, $\mathrm{k}=10, \mathrm{n}=752, \mathrm{I}^{2}=21 \%$ ) (Analysis 3.3 ).

\subsection{Acceptability: loss of participants to post-treatment assessment}

Low-quality evidence indicated no difference between CBT and attention controls in rate of those lost to post-treatment assessment, suggesting a similar degree of acceptability (OR 1.00, $95 \% \mathrm{Cl}$ cross $1, \mathrm{Z}=0.01, \mathrm{P}=0.99, \mathrm{n}=797, \mathrm{k}=12, \mathrm{I}^{2}=0 \%$ ) (Analysis 3.4).

\section{Secondary outcomes}

\subsection{Remission of all anxiety disorders post-treatment}

We included five studies in analyses comparing CBT versus attention controls for remission of all anxiety disorder diagnoses post-treatment (Ginsburg 2002; Hudson 2009; Kendall 2008; Masia Warner 2007; Rosa Alcazar 2009).

Both ITT analyses and completer analyses indicated a benefit for CBT compared to attention control conditions (ITT: OR 2.75, 95\% CI 1.22 to $6.17, Z=2.45, P=0.01, n=378, I^{2}=49 \%$, low-quality evidence; completers: $O R 3.88,95 \% \mathrm{Cl} 1.58$ to $9.51, \mathrm{Z}=2.96, \mathrm{P}=0.003, \mathrm{n}=359$, $\mathrm{I}^{2}=48 \%$ ) (Analysis 3.5; Analysis 3.6).

\subsection{Reduction in anxiety symptoms (child-report) post-treatment}

Fifteen studies provided moderate-quality evidence for posttreatment child-reported anxiety symptoms for CBT and attention control groups (Ginsburg 2002; Herbert 2009; Hudson 2009; Ingul 2013; Kendall 2008; Khanna 2010; Last 1998; Masia Warner 2007; Masia Warner 2016; Muris 2002; Ollendick 2009; Pincus 2010; Rosa Alcazar 2009; Salum 2018; Silverman 1999a). The SMD between CBT and attention controls for child-reported anxiety symptoms was $-0.31(95 \% \mathrm{Cl}-0.51$ to $-0.11, \mathrm{Z}=3.09, \mathrm{P}=0.002, \mathrm{n}=978)$ (Analysis 3.7). There was evidence of moderate heterogeneity across studies $\left(\mathrm{Chi}^{2}=40.95, \mathrm{df}=20, \mathrm{P}=0.004 ; \mathrm{I}^{2}=51 \%\right)$.

After removal of studies that used a disorder-specific symptom measure, the SMD between CBT and attention controls was reduced to $-0.18\left(95 \% \mathrm{Cl}-0.34\right.$ to $-0.02, \mathrm{Z}=2.26, \mathrm{P}=0.02, \mathrm{n}=685, \mathrm{k}=11, \mathrm{I}^{2}$ $=0 \%$ ) (Analysis 3.8).

\subsection{Reduction in anxiety symptoms (parent-report) post-treatment}

Eight studies provided data on post-treatment parent-reported anxiety symptoms for CBT and attention control groups (Herbert 2009; Hudson 2009; Kendall 2008; Masia Warner 2007; Masia Warner 2016; Rosa Alcazar 2009; Salum 2018; Silverman 1999a). The analyses indicated no significant differences between CBT and attention controls (SMD $-0.25,95 \% \mathrm{Cl}-0.61$ to $0.11, \mathrm{Z}=1.37$, $\mathrm{P}=0.17, \mathrm{n}=638$, low-quality evidence), but with substantial heterogeneity across studies $\left(\mathrm{Chi}^{2}=47.61, \mathrm{df}=11, \mathrm{P}<0.001 ; \mathrm{I}^{2}=\right.$ $77 \%$ ) (Analysis 3.9). Removal of studies that used disorder-specific measures did not change this finding (SMD $-0.04,95 \% \mathrm{Cl}-0.26$ to $0.18, \mathrm{Z}=0.36, \mathrm{P}=0.72, \mathrm{n}=345, \mathrm{k}=4, \mathrm{I}^{2}=0 \%$ ) (Analysis 3.10).

\subsection{Reduction in depressive symptoms post-treatment}

Ten studies reported post-treatment depressive symptoms for CBT versus attention controls (Ingul 2013; Kendall 2008; Khanna 2010; Last 1998; Masia Warner 2007; Muris 2002; Ollendick 2009; Pincus 2010; Salum 2018; Silverman 1999a), finding no between-group difference (SMD $-0.18,95 \% \mathrm{Cl}-0.45$ to $0.09, \mathrm{Z}=1.32, \mathrm{P}=0.19, \mathrm{n}=$ 613 , low-quality evidence) (Analysis 3.11). There was evidence of moderate heterogeneity across studies $\left(\mathrm{Chi}^{2}=29.19, \mathrm{df}=12, \mathrm{P}=\right.$ $\left.0.004 ;\left.\right|^{2}=59 \%\right)$.

\subsection{Improvement in global functioning post-treatment}

Only two studies reported post-treatment global functioning for CBT versus attention controls (Khanna 2010; Masia Warner 2007), so meta-analysis was not possible.

\subsection{Adverse events}

No study reported adverse events in both CBT and attention control groups, so meta-analysis was not possible.

\section{Remission of primary anxiety disorder diagnoses at follow-up}

Four studies provided data on remission of primary anxiety disorder at $\leq 6$ months follow-up (Hudson 2009; Masia Warner 2007; Masia Warner 2016; Rosa Alcazar 2009). ITT analyses showed a greater rate of remission for CBT groups compared to attention controls (OR 3.06, 95\% $\mathrm{Cl} 1.22$ to $7.65, \mathrm{Z}=2.39, \mathrm{P}=0.02, \mathrm{n}=341$ ), but with substantial heterogeneity across studies $\left(\mathrm{Chi}^{2}=12.62, \mathrm{df}=5, \mathrm{P}\right.$ $=0.02 ; I^{2}=60 \%$ ) (Analysis 3.12).

The intervention effect increased in the completer analysis (OR $6.52,95 \% \mathrm{Cl} 2.58$ to $16.43, \mathrm{Z}=3.97, \mathrm{P}<0.001, \mathrm{k}=4, \mathrm{n}=302, \mathrm{I}^{2}=44 \%$ ) (Analysis 3.13).

Only one study reported remission of primary anxiety disorder at 12-month follow-up, so meta-analysis was not possible for this time point.

\section{Remission of all anxiety disorder diagnoses at follow-up}

Three studies reported remission from all anxiety disorder diagnoses at $\leq 6$ months post-treatment (Hudson 2009; Masia Warner 2007; Rosa Alcazar 2009). ITT analyses and completer analyses indicated a greater rate of remission of all anxiety disorders for CBT groups compared to attention controls (ITT: OR 5.44, $95 \% \mathrm{Cl} 1.00$ to $29.60, \mathrm{Z}=1.96, \mathrm{P}=0.05, \mathrm{n}=205$; completer analysis: $\mathrm{OR} 11.19,95 \% \mathrm{Cl} 1.94$ to $64.51, \mathrm{Z}=2.70, \mathrm{P}=0.007, \mathrm{n}=182$ ), but with substantial heterogeneity across studies (ITT: $I^{2}=72 \%$, completer analysis: $\mathrm{I}^{2}=62 \%$ ) (Analysis 3.14; Analysis 3.15).

Only one study reported remission of all anxiety disorder diagnoses at 12 months (Rosa Alcazar 2009), so meta-analysis was not possible for this time point.

\section{Reduction in anxiety symptoms at follow-up}

Six studies reported child-reported anxiety symptoms at $\leq 6$ months post-treatment (Herbert 2009; Hudson 2009; Masia Warner 2007; Masia Warner 2016; Rosa Alcazar 2009; Silverman 1999a). Pooled data from these studies indicated a benefit for CBT compared to 
attention controls (SMD $-0.44,95 \% \mathrm{Cl}-0.79$ to $-0.08, \mathrm{Z}=2.40, \mathrm{P}$ $=0.02, \mathrm{n}=434$ ), but there was substantial heterogeneity across studies $\left(\mathrm{Chi}^{2}=23.08, \mathrm{df}=8, \mathrm{P}=0.003 ; \mathrm{I}^{2}=65 \%\right)$ (Analysis 3.16$)$.

The same six studies provided parent-reported anxiety symptoms at $\leq 6$ months post-treatment; analyses showed no significant difference between CBT and attention controls (SMD $-0.63,95 \% \mathrm{Cl}$ cross $0, n=434, I^{2}=90 \%$ ) (Analysis 3.17)

Only two studies reported child-reported anxiety symptoms and parent-reported anxiety symptoms at 12 months follow-up (Rosa Alcazar 2009; Silverman 1999a), so meta-analyses for these outcomes were not possible for this time point.

\section{Comparison 4: CBT versus alternative treatment}

See Summary of findings 4 .

\section{Primary outcomes}

\subsection{Remission of primary anxiety diagnosis post-treatment}

Only two studies that compared CBT to an alternative treatment reported post-treatment remission of primary anxiety disorder (Creswell 2017; Reigada 2015), therefore data were insufficient to conduct meaningful meta-analysis or planned subgroup analyses for this outcome.

\subsection{Acceptability: loss of participants to post-treatment assessment}

Low-quality evidence showed no difference between CBT and alternative treatments in rate of those lost to post-treatment assessment (OR 1.58, 95\% Cl crosses $1, \mathrm{Z}=0.94, \mathrm{P}=0.35, \mathrm{k}=7, \mathrm{n}$ $\left.=515, I^{2}=53 \%\right)$, suggesting no difference in degree of acceptability (Analysis 4.1).

\section{Secondary outcomes}

\subsection{Remission of all anxiety disorders post-treatment}

Pooled data from four studies that reported remission of all anxiety disorder diagnoses post-treatment showed no difference between CBT and alternative treatment groups in either ITT analyses (OR $0.89,95 \% \mathrm{Cl}$ crosses $1, \mathrm{Z}=0.25, \mathrm{P}=0.80, \mathrm{n}=401, \mathrm{I}^{2}=73 \%$, low-quality evidence) or completer analyses (OR 1.52, 95\% Cl crosses $1, Z=1.06$, $\mathrm{P}=0.29, \mathrm{n}=348, \mathrm{I}^{2}=57 \%$ ) (Analysis 4.2; Analysis 4.3) (Creswell 2017; Lebowitz 2019; Reigada 2015; Silk 2018).

\subsection{Reduction in anxiety symptoms (child-report) post-treatment}

Low-quality evidence showed no differences between CBT and alternative treatment groups in post-treatment child-reported anxiety symptoms (SMD $-0.09,95 \% \mathrm{Cl}$ crosses $0, \mathrm{Z}=0.58, \mathrm{P}=0.56, \mathrm{k}$ $=6, n=399, I^{2}=51 \%$ ) (Analysis 4.4). Removal of one study that used a disorder-specific symptom measure did not change this finding (Analysis 4.5).

\subsection{Reduction in anxiety symptoms (parent-report) post-treatment}

We also found no significant differences between CBT and alternative treatment groups in post-treatment parent-reported anxiety symptoms (SMD $-0.13,95 \% \mathrm{Cl}-0.33$ to $0.06, \mathrm{Z}=1.31, \mathrm{P}$ $=0.19, \mathrm{k}=6, \mathrm{n}=423,6$ studies, $\mathrm{I}^{2}=0 \%$, low-quality evidence) (Analysis 4.6). The studies all used a broad-based anxiety measure, so planned sensitivity analysis was not required.

\subsection{Reduction in depressive symptoms post-treatment}

Only only study reported post-treatment depressive symptoms (Salum 2018), so meta analysis was not possible.

\subsection{Improvement in global functioning post-treatment}

No studies reported post-treatment global functioning for CBT versus alternative treatments.

\subsection{Adverse events}

No included study reported adverse events in both CBT and alternative treatment groups, so meta-analysis was not possible.

\subsection{Follow-up outcomes}

For each of the longer-term outcomes, data were only available from a maximum of two studies, so meta-analyses were not possible.

\section{Publication bias}

We investigated publication bias in relation to remission of the primary anxiety disorder post-treatment for CBT versus waitlist controls, and CBT versus attention controls.

\section{CBT versus waitlist/no treatment}

There was clear evidence of publication bias from inspection of the funnel plot (Figure 7), which was asymmetric, and the Egger's test, which was highly significant $(t=5.52, P<0.000)$. Furthermore, a contour funnel plot (not included here) indicated that the missing studies were in the areas of low significance, more compatible with publication bias than other explanations of funnel plot asymmetry such as variable study design (Peters 2008). 
Figure 7. Funnel plot of comparison: 1 CBT vs waitlist/no treatment, outcome: 1.1 Remission of primary anxiety diagnosis post-treatment (ITT).

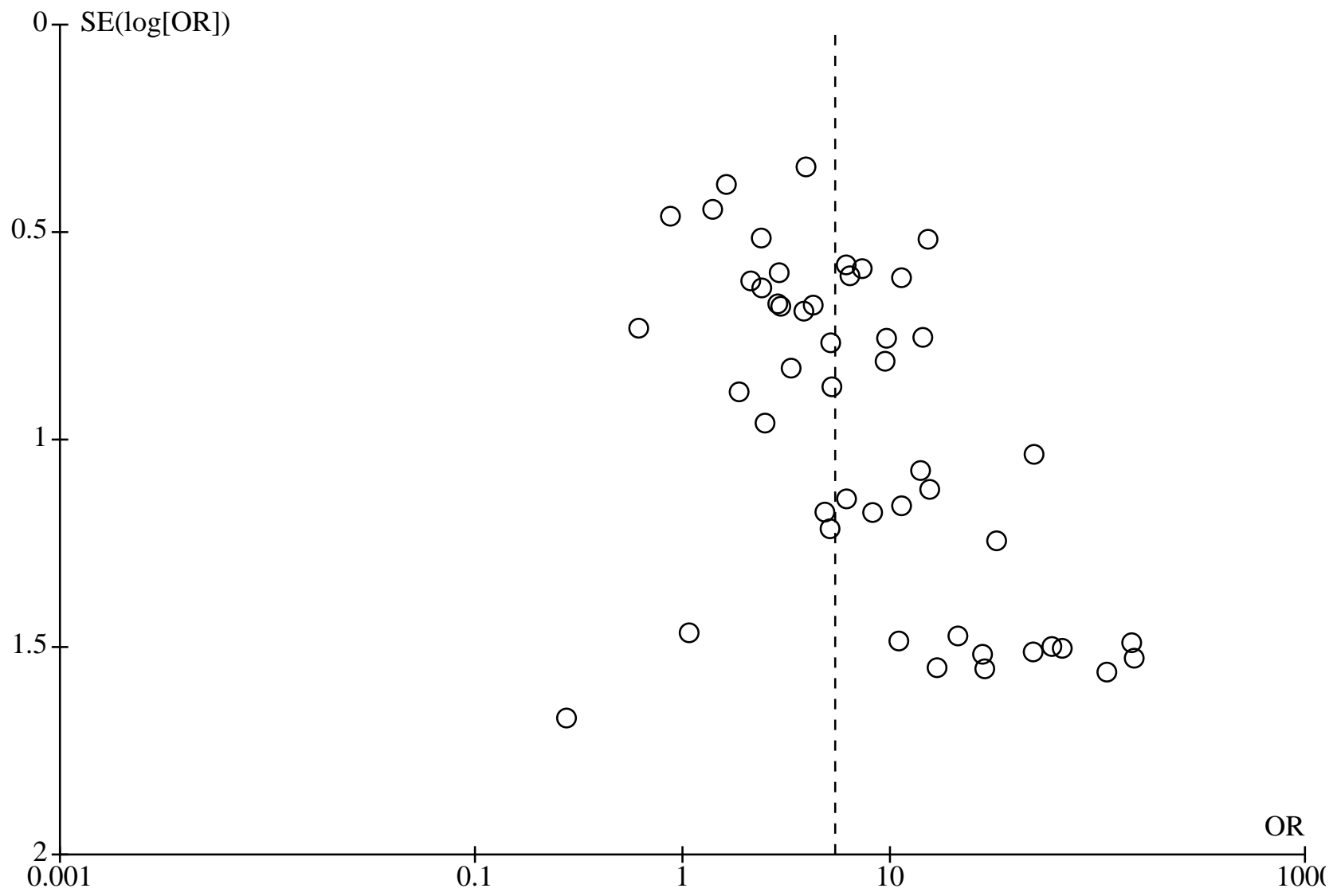

\section{CBT versus attention control}

There was no evidence of publication bias with a symmetrical funnel plot (Figure 8) and non-significant Egger's test $(\mathrm{t}=1.99, \mathrm{P}=$ 0.072). 
Figure 8. Funnel plot of comparison: 3 CBT vs attention control, outcome: 3.1 Remission of primary anxiety diagnosis post-treatment (ITT).

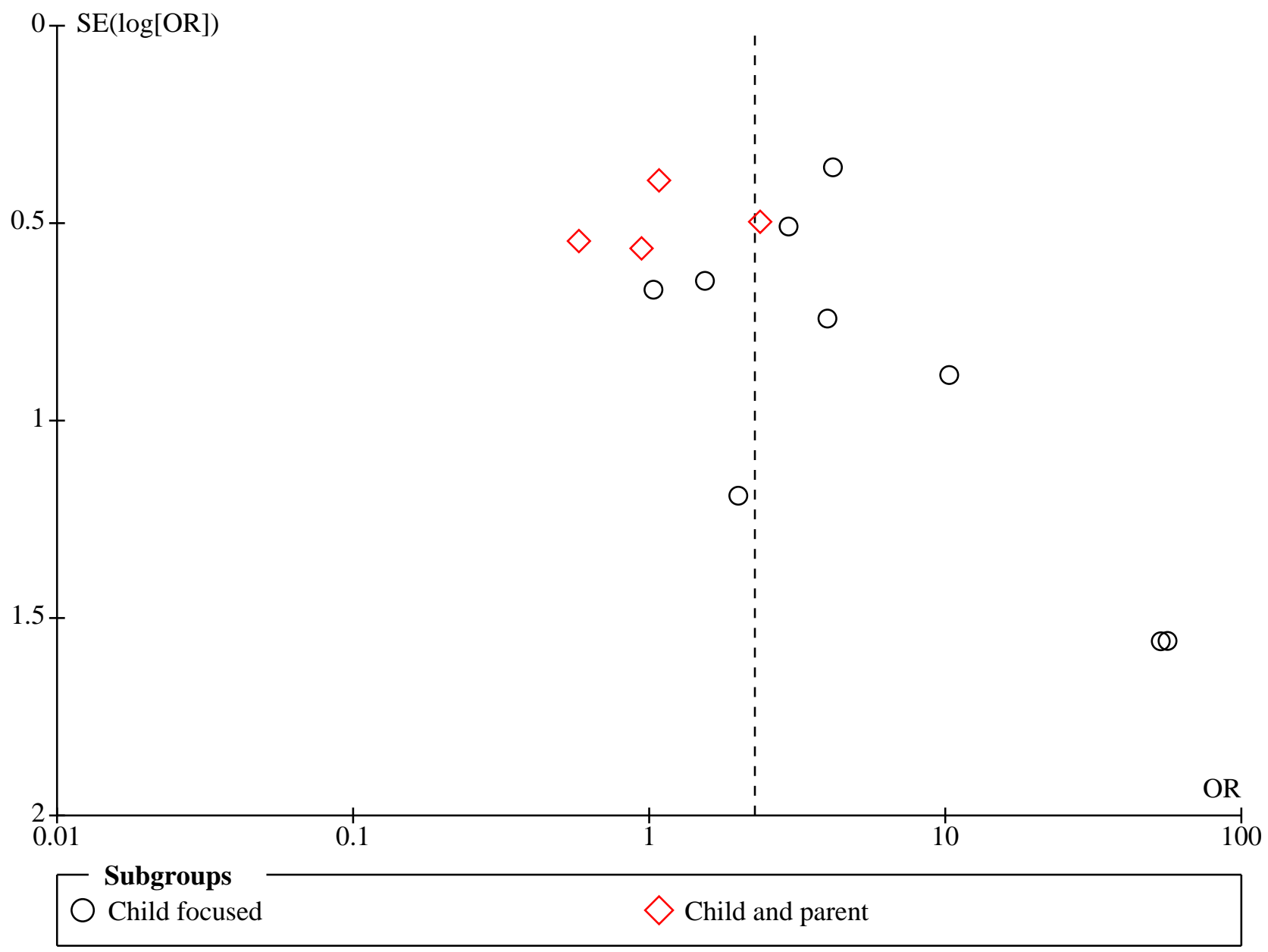

\section{DISCUSSION}

\section{Summary of main results}

Since the last Cochrane Review, James 2015, there has been a considerable increase in the number of studies available for review, from 41 to 88 , with more than three times the total number of participants. We found moderate-quality evidence that CBT leads to greater remission of primary and all anxiety disorders than waitlist/no treatment in the short term. Using conservative ITT criteria, we found a remission rate of $49 \%$ for the primary anxiety disorder for CBT versus $18 \%$ for waitlist/no treatment controls (OR $5.45,95 \% \mathrm{Cl} 3.90$ to 7.60 ). Although this indicates a slight attenuation in effects from the last Cochrane Review, this is not uncommon as studies accumulate over time. Consistent with the last review, the number needed to treat for an additional beneficial outcome (NNTB) using these conservative ITT data was $3(95 \% \mathrm{Cl}$ 2.25 to 3.57). Notably, the benefit of CBT compared to waitlist/no treatment controls was shown across all post-treatment outcomes, albeit with uncertain evidence due to variation in reductions in child- and parent-reported anxiety symptoms, and depressive symptoms, and limited data related to global functioning.
Unfortunately, despite the general increase in studies, there remains a relatively small number of studies comparing CBT to 'active' controls, and where this has been done the control arms included a broad range of interventions (from undefined and variable 'treatment as usual' to attention controls, and in a small number of cases treatments that might credibly be delivered in practice). Due to insufficient data, there was lowquality evidence for each of these comparisons across most outcomes. Encouragingly, the available data did indicate that CBT may be associated with a higher rate of remission of the primary anxiety disorder and all anxiety disorders and a greater reduction in child-reported anxiety symptoms, compared to attention controls. However, the limited data did not reveal an advantage of CBT compared to treatment as usual across most outcomes, or indicate a benefit of CBT compared to specified alternative treatments. Furthermore, we were unable to compare CBT to medication or CBT in combination with medication to drug placebo due to a lack of available studies.

Unsurprisingly given the high frequency of waitlist control designs, there also continues to be only a very small number of studies that have controlled outcomes at longer-term follow-up points. Where outcomes beyond post-treatment were available, the studies 
had mixed results, but in terms of remission of the primary anxiety disorder, the current findings support an advantage of CBT compared to waitlist/no treatment and attention controls six months after treatment. However, there is as yet no evidence that this advantage of CBT continues at later time points, and there were insufficient data to determine whether any longer-term benefits hold up against treatment as usual or alternative treatments.

Previous Cochrane Reviews on this topic have only included studies that involved children older than four years of age; however, in the current review we removed this restriction in order to identify the age of the youngest participants in RCTs of CBT for child anxiety disorders, and to explore relative outcomes for different age groups. We identified studies that included children as young as two years of age. Notably, studies with younger children typically took a parent-led approach in which parents were supported by a clinician to implement CBT principles in their child's day-to-day life (e.g. Cartwright Hatton 2011).

Given the low number of children with anxiety disorders who access evidence-based treatments for anxiety disorders (e.g. Reardon 2020), we specifically looked at outcomes for subgroups of studies categorised by the amount of therapist contact time. Relatively few studies involved less than 10 hours of therapist contact time, limiting the amount of data available. However, primary anxiety disorder and anxiety symptom outcomes for CBT compared to waitlist/no treatment did not differ from the more lengthy treatments. The currently available data therefore suggests that services could provide CBT programmes that involve relatively low levels of therapist contact time without a negative impact on posttreatment outcomes.

The wide variation across trials in relation to a range of study and sample characteristics limits our ability to draw clear conclusions about particular treatment formats or age groups. Amongst waitlist/no treatment comparisons, we found stronger effects for child-focused CBT than child-and-parent and parent-only interventions. However, parent involvement in trials is related to potentially confounding factors (e.g. child's age, study setting), and even within the child-focused CBT studies there was considerable heterogeneity in outcomes and variation across study characteristics. Indeed, although we also found variation in outcomes amongst different age groups for CBT versus wait list/no treatment comparisons, this differed according to outcome and may indicate age-related measurement issues, rather than differences in true effects. We found stronger effects amongst samples of adolescents than samples of children or mixed child/adolescent samples on child- and parent-reported anxiety symptoms, but not in terms of remission of primary or all anxiety disorders. We found a similar picture when looking at subgroups of studies on the basis of whether they delivered CBT with individuals or groups. For example, there was no evidence of different results on diagnostic outcomes, but outcomes for group formats were stronger than individual formats when parentand child-reported symptoms were assessed (in the waitlist/no treatment comparison). Again, various potential confounders are likely to have influenced these outcomes, including, for example, participant age, study setting (e.g. school versus clinic), and target disorders. Consequently, on the basis of findings relating to treatment format, the currently available literature does not give clear and consistent evidence that one format is better or worse than any other.
Finally, we also set out to assess outcomes specifically for children with autism spectrum disorders (ASD) and intellectual disabilities. Twelve studies evaluated CBT for the treatment of anxiety disorders in children with ASD, with mixed findings across comparisons and outcomes. Notably, the four studies that compared CBT to treatment as usual for children with ASD (where 'treatment as usual' typically meant participants could continue to seek alternative interventions only) did find a consistent advantage of CBT in relation to remission of primary anxiety disorder. However, amongst waitlist/no treatment control comparisons, we only found evidence of a benefit for CBT in parent-reported anxiety symptoms, not child-reported symptoms or diagnostic outcomes. No RCTs evaluated the effectiveness of CBT for anxiety disorders amongst children with intellectual disabilities.

\section{Overall completeness and applicability of evidence}

As in the previous Cochrane Review, almost all included studies involved participants of both sexes. Whilst a number of countries are represented in this review, it is notable that they were almost exclusively high-income countries (with two-thirds of the included studies having been conducted either in the USA or Australia).

Although the overall age of participants ranged from 2 to 18 years, less than $30 \%$ of studies focused specifically on children up to age 12 (with less than $6 \%$ focusing on children under 8 years of age), and less than $20 \%$ focused specifically on adolescents, with the majority including children and adolescents across broad age ranges. This made it difficult to draw conclusions on the basis of specific child ages.

We included participants with comorbid disorders such as depression, other anxiety disorders, ADHD, and conduct disorders, permitting generalised conclusions that appear to be applicable to clinical practice. However, the majority of studies were conducted in outpatient settings, with 41 studies conducted in universitybased research settings. We considered other settings, including primary care and schools; notably, however, no studies were conducted with the most severe cases who receive treatment as day- or inpatients, which is perhaps a reflection of the challenges associated with running trials in these settings. Despite the continued growth in studies evaluating CBT for anxiety disorders in general, there were few additional studies that targeted children with ASD since the last Cochrane Review, and the lack of evaluation of CBT for anxiety disorders, including parent-only approaches, amongst children with intellectual disabilities remains a considerable limitation.

It is important to note that we conducted separate analyses for different diagnostic and child/parent report measures; this meant that given the high levels of inconsistency in measures used and how they were reported, only a subset of studies were included in any one analysis. The most consistent diagnostic outcome measure was remission of the primary diagnosis (included in about two-thirds of the CBT versus waitlist/no treatment comparisons), whereas less than half of the studies reported remission of all anxiety disorder diagnoses. Some studies did not report remission of anxiety disorders in either of these ways, instead, for example, reporting remission from particular groups of diagnoses, or changes in the total number of different diagnoses. As a result, these studies were not included in our analyses of diagnostic outcomes. Similarly, relevant studies were identified that did not 
provide data on any of the target outcomes and were therefore not included in the review (e.g. Baer 2005; Flatt 2010; NCT00576719).

Encouragingly, the evidence of the acceptability of CBT was consistent across comparisons, with no difference in the number of CBT participants retained to post-treatment assessments compared to each of the control groups. However, it is important to acknowledge that rates of attrition may in part reflect acceptability of the research process, and alternative measures of acceptability (e.g. participant perceptions of the intervention) were not assessed in this review.

As in previous Cochrane Reviews of CBT for child anxiety disorders, the number of follow-up studies remains very limited (four studies reported on our primary outcome at follow-up assessments), making it difficult to draw conclusions about the medium- to longterm benefits of CBT. The available findings from controlled studies suggest that there is evidence that CBT provides an advantage over waitlist/no treatment six months after treatment, but there is currently no evidence to support longer-term benefits. However, the recent Child/Adolescent Anxiety Multimodal Study (CAMS) highlights the importance of controlled outcome data at longerterm follow-up, based on their findings that over two-thirds of patients that showed initial treatment gains relapsed over the following four years (Ginsburg 2018).

A major weakness found across studies was the absence of systematic reporting of adverse effects. No adverse effects were reported across all studies; however, in the small number of studies where any reference was made to adverse effects, it was not clear that these were systematically monitored. This issue is not limited to trials of CBT for child anxiety disorders; for example, Duggan 2014 reported that none of the 72 trials of psychological interventions considered in their systematic review mentioned the occurrence of adverse events in their final report, despite the recognition that psychological treatments can introduce a range of negative effects (Barlow 2010).

Given the limited data for treatment as usual, attention control, and alternative treatment comparisons, not all analyses were possible across all comparisons, and many of our subgroup analyses were limited to waitlist/no treatment comparisons.

\section{Quality of the evidence}

This review included 87 studies in the quantitative synthesis, with a reasonably large number of study participants $(n=5964)$. As noted above, we distinguished between baseline and outcome measures using tight criteria in order to ensure we were comparing like with like as far as possible. For example, we distinguished between remission in the primary anxiety disorder and remission of all anxiety disorders, and selected symptom measures that were most consistently reported, prioritising measures of broad anxiety symptoms where possible.

We rated evidence as moderate or low quality across comparisons and outcomes. There was consistent evidence for the benefit of CBT compared to waitlist/no treatment controls, but with moderate to substantial heterogeneity across outcomes, reducing certainty in the results. We assessed the evidence for CBT compared to treatment as usual, attention controls, and alternative treatments mostly as low quality, primarily due to the limited amount of available data.
We noted methodological shortcomings in some studies, although this typically reflected inadequate reporting, rather than evidence of high risk of bias, and was more common amongst older studies. Participant blinding is not possible in research on psychological interventions, which is an obvious potential source of bias; however, the evaluations were typically carried out blind. Nonetheless, we found similar outcomes when we removed studies with weaknesses related to allocation and blinding, supporting the robustness of the results.

Given the increase in number of studies available since previous reviews, we took the opportunity for a stricter approach to our inclusion criteria. We only included studies with robust randomisation procedures, and excluded studies included in other reviews where, for example, children who might be considered at risk were automatically enrolled in the intervention arm (e.g. Nauta 2003). However, we did include studies where assessment points differed between treatment arms, and, in some cases, the period between randomisation and assessment was twice as long in the intervention arm compared to the control arm (e.g. Fujii 2013; Kendall 1994; Kendall 1997), which clearly introduces the potential for substantial bias. The timing of the post-treatment assessment (the assessment closest to the end of the treatment) also varied across trials.

There was evidence of publication bias (asymmetric funnel plot, significant Egger's test and contour funnel plot), with a marked absence of non-significant studies for both CBT versus waitlist/no treatment controls. This is an area that has been richly debated, and remains a significant area of concern when interpreting the results of meta-analyses, as it is likely that, with the lack of published negative trials, any estimate of the effectiveness of an intervention (CBT in this case) is likely to be inflated. We welcome the call for journals to publish negative trials, and for chief investigators of all registered trials to publish findings, especially negative ones, online within a set time of the trial end date.

\section{Potential biases in the review process}

We were able to obtain all the referenced papers; we searched grey literature and reference lists; and made contact with leading researchers in the field. We also included studies that were not in the English language. However, meta-analyses are limited by the robustness of any search method. The electronic search was thorough and large in scale with broad parameters. Of note, we broadened certain parameters since the last Cochrane Review on this topic, for example removing a restriction on the minimum number of sessions, minimum child age, and including CBT delivered via parents. Nonetheless, there are some limitations to the review. For example, whilst we set a fairly broad definition of 'cognitive behaviour therapy', some studies were excluded where the description of the intervention did not include all the core stated components of CBT despite the treatments appearing similar in many ways (e.g. social effectiveness therapy; Beidel 2000; Beidel 2007; behaviour therapy; Rudy 2017; Storch 2019), and it is possible that the brief descriptions of treatments provided in papers may not fully reflect how the treatment is being delivered in practice. We also required some in-person treatment delivery, and therefore did not include CBT interventions delivered online or purely over the telephone, despite these interventions often being very similar in content. Indeed there is evidence supporting the effectiveness of these remotely delivered approaches (e.g. Lyneham 2006; Pennant 2015). 
The analysis of studies with three arms - CBT in two formats versus waiting list controls or active controls - is problematic in that double counting of any group is wrong. We chose a conservative approach to managing this by splitting the control samples into two equal halves between the CBT groups; however, this means that in these cases the odds ratios and confidence intervals appear smaller despite recovery rates that are similar to those found in other studies (e.g. Flannery Schroeder 2000; Thirlwall 2013). We also prioritised ITT analyses and adopted a conservative approach in which drop out in the treatment group was treated as if the participant did not recover, but drop out in the control group was treated as recovery. This conservative approach may have underestimated treatment effects; unsurprisingly, treatment effects were larger in the follow-up completer analyses. Whilst we took a strict approach to measures that were included (only using specific measures of anxiety symptoms and not, for example, combined measures of emotional and behavioural symptoms), and prioritised broad measures of anxiety symptoms to promote consistency, this may have come at a disadvantage for studies that focused on treating specific disorders, where only a subset of the items on a broad measure might apply to the target of treatment.

\section{Agreements and disagreements with other studies or reviews}

Two previous Cochrane Reviews have been conducted on this topic. The first review identified 18 studies with 498 participants and 311 controls, and found a response rate for remission of any measure of anxiety diagnosis using an ITT analysis of $56 \%$ for CBT versus $28.2 \%$ for controls with the NNTB of $3.0(95 \% \mathrm{Cl} 2.5$ to 4.5$)$ (James 2005). The second review identified 41 studies with 1806 participants, with a rate of remission of any measure of anxiety disorder of $58.9 \%$ for CBT versus $16 \%$ for the waitlist (James 2015). In the current review, we identified a further substantial increase in studies, including 81 studies with 5964 participants. We made a number of changes, most notably distinguishing between remission of the primary anxiety disorder and remission of all anxiety disorders; however, the findings were fairly consistent, with $49.4 \%$ of participants being free of their primary anxiety disorder following CBT (versus $17.8 \%$ for waitlist controls), and $46.8 \%$ being free of all anxiety disorders (versus $19.1 \%$ for waitlist controls). It is important to note that studies presenting remission based on primary diagnosis and all anxiety diagnoses only partly overlapped, so these outcomes should not be compared directly. Despite the differences in outcome measures, and our broader inclusion criteria for CBT formats, length, and participant ages, the NNTB has been consistent at NNTB $=3$ across all three Cochrane Reviews. These effects are consistent with other meta-analyses of CBT for anxiety disorders in children and young people (e.g. Cartwright-Hatton 2004; Compton 2004; Reynolds 2012; Seligman 2011; Silverman 2008; Wang 2017; Warwick 2016).

Our findings are also consistent with previous reviews in terms of the limited available data for comparisons with treatment as usual/ alternative treatments (e.g. Wang 2017). Indeed, like others, we have concluded that there is no clear evidence of an advantage of CBT over treatment as usual or treatments that might credibly be used within mental health settings. However, we did find more consistent evidence of an advantage of CBT over attention controls, although the available data are still limited.

However, our conclusions differed from those of a recent network meta-analysis of psychotherapies for anxiety disorders in children and young people, which concluded that group CBT was more effective that other forms of psychotherapy (including other CBT formats included in this review) (Zhou 2019). Notably, this network meta-analysis considered outcomes based on symptom questionnaires (prioritising child self-report). Indeed, when we consider our subgroup analyses, amongst waitlist/no treatment comparisons, we also found stronger effects of group CBT than individual CBT on child- and parent-reported anxiety symptoms; however we did not find significant differences between these subgroups based on our primary outcome (remission of primary anxiety diagnosis) or on our other diagnostic outcome (free of all anxiety diagnoses). Furthermore, studies that differed in terms of treatment delivery format also differed on a number of other characteristics that would likely be associated with outcome (including differences in the study setting, population, and nature of outcome measures). For example, most (5 out of 7) studies that only reported disorder-specific symptom measures were studies that evaluated treatments delivered in groups, so differences between group versus individual formats may reflect a measurement issue rather than a true difference in effect sizes. As such, we do not feel that the evidence provides clear and consistent support for group CBT having an advantage over other delivery formats.

The same conclusion applies to treatment delivery with children, children and parents, or parents alone. Like previous reviews (e.g. Breinholst 2012; Reynolds 2012; Silverman 2008), we did not find clear evidence of an advantage of any of these delivery formats. Although we found the strongest effects for child-focused CBT among waitlist/no treatment comparisons, there was considerable variation in findings amongst child-focused CBT studies, and the treatment delivery subgroups also often differed on other key study and sample characteristics (e.g. child age, setting, comorbidities, treatment duration). Notably, there were relatively few studies that delivered treatment through parents only, and these tended to be more recent studies and included children of younger ages than studies that took a child-only or child-and-parent approach.

Where there were sufficient studies, we generally found fairly consistent findings regardless of whether children in the studies had ASD or not, supporting the evidence from previous metaanalyses suggesting that CBT can be an effective treatment of anxiety for children with ASD (e.g. Perihan 2019; Ung 2015). Notably, recent reviews have suggested that parental involvement may be particularly important for treating childhood anxiety disorders in the context of ASD, and that longer-term interventions were associated with larger effects (Perihan 2019). These questions are beyond the scope of the current review; however, it is noteworthy that neither of these sets of findings were replicated in our broader sample, highlighting the need to consider the specific conditions that are needed to optimise CBT approaches amongst particular subgroups of children and young people with anxiety disorders.

\section{AUTHORS' CONCLUSIONS}

\section{Implications for practice}

This review reinforces conclusions from previous reviews finding moderate-quality evidence that cognitive behavioural therapy (CBT) is more effective in improving diagnostic outcomes in the short term than a waiting list or no treatment. The advantage of CBT compared to waitlist/no treatment is evident across a range of other short-term outcomes, although variation in findings or 
limited available data, or both, reduces certainty in these results. Clear and consistent evidence of differences between the formats of individual, group, and parent/family CBT continues to be lacking. Available, low-quality evidence supports a benefit of CBT compared to attention control, but the evidence base remains small, and there continues to be insufficient and inclusive evidence that CBT is superior to either treatment as usual or alternative treatments.

Pharmacological interventions are typically not recommended as a first-line treatment due to patient preference (e.g. Brown 2007), relative high rates of attrition, and concerns about potential side effects (Wang 2017). However, we know very little about the comparative efficacy of pharmacological interventions and CBT, with evidence still limited to one, albeit large, multicentre study (Walkup 2008). Clearly further work is needed to guide practice about the circumstances in which pharmacological approaches might be most acceptable and effective for the treatment of anxiety disorders in children and young people.

A major challenge for clinical practice is the very limited access that children with anxiety disorders have to evidence-based psychological interventions, such as CBT. In a recent study, only $2 \%$ of children with anxiety disorders identified in the community in England had received CBT (Reardon 2020). These findings are consistent with previous studies indicating low rates of service use in the UK, Australia, and the USA (Green 2004; Lawrence 2015; Merikangas 2011). Of importance to this clinical challenge is our finding that there is currently no evidence of differences in outcome on the basis of therapist contact time, with similar effects being achieved when therapists provided less than 10 hours of clinical contact compared to higher amounts of clinical contact in terms of remission of the primary anxiety disorder and childand parent-reported anxiety symptoms. Notably, results were less strong in terms of remission of all anxiety disorders; however, there is evidence to suggest that benefits might generalise to other disorders beyond the end of (brief) treatment (e.g. Ollendick 2010). These findings indicate that access could be improved through the provision of stepped-care models of treatment delivery in which relatively brief interventions are offered as a first-line approach, and more intensive interventions are saved for those who need them (Bower 2005). A recent systematic evaluation of stepped-care treatment for child anxiety disorders provided promising evidence for a benefit of a stepped-care approach in terms of clinical time saved and reduced societal costs (Chatterton 2019).

\section{Implications for research}

There is now sufficient evidence to conclude that CBT is an effective treatment for anxiety disorders in children and young people when compared to waiting lists/no treatment.

A number of reviews have concluded that overall about $50 \%$ of children and young people recover from their primary anxiety disorder following a course of CBT, but still very little is known about how these outcomes can be improved and what the other $50 \%$ of patients need. A better understanding of what the core mechanisms of effective interventions are and how this varies according to individual characteristics or contexts is needed. For example, currently very little is known about how treatments should best be delivered according to child age and developmental status, the presence of comorbid conditions, or the nature of the anxiety disorders experienced. Almost three-quarters of the studies in this review included children with a broad range of anxiety disorders, and those that focused on specific disorders were spread across studies of, for example, social anxiety disorder, selective mutism, panic disorder, generalised anxiety disorder, and specific phobia, thereby limiting our ability to draw firm conclusions about any particular approach for any particular disorder, let alone how that might vary according to, for example, child age. Larger studies that are sufficiently powered to examine predictors, mediators, and moderators of treatment outcomes are now needed to identify how to improve treatment outcomes and develop more personalised models of care.

Individual patient data meta-analyses may be useful to help us better understand the extensive heterogeneity between study outcomes; however, meaningful conclusions will rely on clear and consistent reporting across studies so that data can be linked in sensible ways. In this review we focused on remission of the primary and all anxiety diagnoses, but a number of studies were excluded from these analyses as they reported other kinds of data from diagnostic assessments. This issue was highlighted in a recent position paper which set out a number of recommendations for reporting and terminology to promote greater consistency going forwards (Creswell 2020a). It is important to note a number of other areas where the field would benefit from greater clarity and consistency in reporting by researchers. For example, in treatment as usual studies, it was not always clear what 'treatment as usual' actually consisted of and to what extent it differed from the index treatment or a no treatment control. Indeed, categorising the format of any treatment typically relied on only a brief description, which may have led to faulty conclusions. We encourage researchers to make good use of online supplementary materials to ensure clear descriptions of the content and format of interventions, using consistent terminology (Creswell 2020a).

There continues to be a lack of evidence that CBT is superior to other credible alternative treatments, so it will be essential to identify what the core components of effective treatments are, for example through experimental and treatment dismantling studies (e.g. Whiteside 2015), so that outcomes can be optimised and treatments can be targeted to maximise their efficiency. Head-to-head comparisons of different active (and credible) interventions will be required, but these will inevitably require larger sample sizes, as they will need to be set up to detect smaller effects than those found with waitlist/no treatment comparisons; however, these designs will also enable studies to compare longer-term outcomes between groups. Given the risks for longer-term adjustment of anxiety disorders in childhood and adolescence, controlled studies with longer-term outcomes are essential to ensure that children's life trajectories are being optimally supported through early intervention.

As noted above, increasing access to effective treatments is an urgent challenge for our field, and it will be essential that studies consider accessibility of treatment and cost-effectiveness going forwards. We did not consider outcomes in terms of costeffectiveness in this review; however, it is clear that to date very few studies have taken cost-effectiveness into account when evaluating CBT for child anxiety disorders. We anticipate that cost-effectiveness data will have a marked impact on the clinical implications that can be drawn from the available evidence, particularly given our finding that clinician contact time did not seem to be related to treatment outcome. Equally, it is often assumed that group treatments may be cost-effective; however, 
this has not been supported when evaluated in other contexts (e.g. Mavranezouli 2015), and some of the group treatments identified in this review involved very high levels of therapist involvement and supported activities. Future studies would benefit from including a cost-effectiveness analysis, and considering the costs and benefits to both the health service and broader society including costs related to the child themselves and family members, whose work and home lives are often heavily impacted by child anxiety problems (e.g. Bodden 2008b).

Finally, it was disappointing to see that, whilst the number of trials evaluating CBT for childhood anxiety disorders has increased substantially, these have not extended to children in low- or middle-income countries, children with intellectual disabilities or physical health conditions, the most severely affected children in inpatient settings, and there are still very few studies that have focused specifically on either young children or (strikingly given the prevalence of anxiety disorders) adolescents.

\section{ACKNOWLE DGEMENTS}

Oxford Health Foundation NHS Trust, Guy's and St Thomas' NHS Foundation Trust, National Institute for Health Research (NIHR), the University of Reading, and the University of Oxford provided support to the review authors to complete this review.

We are extremely grateful to the Cochrane Common Mental Disorders Group (CCMD) for their guidance, and in particular to Sarah Dawson (CCMD Information Specialist) for her input.

The authors and the Cochrane Common Mental Disorders Editorial Team are grateful to the protocol peer reviewers and Copy Edit Support for their time and comments. Peer reviewers included: Georgina Cox, Lina Gega, David Marshall, Ruth Simmonds, and Gill Worthy.

Cochrane review group funding acknowledgement: the NIHR is the largest single funder of Cochrane Common Mental Disorders.

Disclaimer: the views expressed are those of the author(s) and not necessarily those of the NHS, the NIHR, or the Department of Health and Social Care. 


\section{R E F E R E N C E S}

\section{References to studies included in this review}

Afshari 2014 \{published data only (unpublished sought but not used)\}

Afshari A, Neshat-Doost HT, Maracy M, Ahmady MK, Amiri S. The effective comparison between emotion-focused cognitive behavioral group therapy and cognitive behavioral group therapy in children with separation anxiety disorder. Journal of Research in Medical Sciences 2014;19(3):221-7.

\section{Arendt 2016 \{published data only (unpublished sought but not} used)\}

Arendt K, Thastum M, Hougaard E. Efficacy of a Danish version of the Cool Kids program: a randomized wait-list controlled trial. Acta Psychiatrica Scandinavica 2016;133(2):109-21. [DOI: 10.1111/acps.12448]

Barrett 1996 \{published data only (unpublished sought but not used)\}

Barrett PM, Dadds MR, Rapee RM. Family treatment of childhood anxiety: a controlled trial. Journal of Consulting and Clinical Psychology 1996;64(2):333-42.

Barrett 1998 \{published data only (unpublished sought but not used)\}

Barrett PM. Evaluation of cognitive-behavioral group treatments for childhood anxiety disorders. Journal of Clinical Child Psychology 1998;27(4):459-68. [DOI: 10.1207/ s15374424jccp2704_10]

Barrington 2005 \{published data only (unpublished sought but not used)\}

Barrington J, Prior M, Richardson M, Allen K. Effectiveness of CBT versus standard treatment for childhood anxiety disorders in a community clinic setting. Behaviour Change 2005;22(1):29-43. [DOI: 10.1375/bech.22.1.29.66786]

\section{Berge 2017 \{published data only\}}

* Berge KG, Agdal ML, Vika M, Skeie MS. Treatment of intraoral injection phobia: a randomized delayed intervention controlled trial among Norwegian 10- to 16-year-olds. Acta Odontologica Scandinavica 2017;75(4):294-301. [DOI: 10.1080/00016357.2017.1297849]

NCT02083432. Cognitive behavioural treatment of intra-oral Injection-phobia in 10-16 year old children and adolescents. clinicaltrials.gov/show/nct02083432 (first received 11 March 2014).

Cartwright Hatton 2011 \{published data only (unpublished sought but not used)\}

Cartwright-Hatton S, McNally D, Field AP, Rust S, Laskey B, Dixon $B$, et al. A new parenting-based group intervention for young anxious children: results of a randomized controlled trial. Journal of the American Academy of Child and Adolescent Psychiatry 2011;50(3):242-51. [10.1016/j.jaac.2010.12.015 ]
Chalfant 2007 \{published data only (unpublished sought but not used)\}

Chalfant AM, Rapee R, Carroll L. Treating anxiety disorders in children with high functioning autism spectrum disorders: a controlled trial. Journal of Autism and Developmental Disorders 2007;37(10):1842-57. [DOI: 10.1007/s10803-006-0318-4]

\section{Cheung 2016 \{published data only\}}

Cheung MC. Effectiveness of Attention Bias Modification Treatment for Children With Social Anxiety Problem [Thesis]. Pokfulam, Hong Kong: The University of Hong Kong, 2016. [hub.hku.hk/handle/10722/239961]

Chiu 2013 \{published data only (unpublished sought but not used)\}

* Chiu AW, Langer DA, McLeod BD, Har K, Drahota A, Galla BM, et al. Effectiveness of modular CBT for child anxiety in elementary schools. School Psychology Quarterly 2013;28(2):141-53. [DOI: 10.1037/spq0000017]

NCT00569829. Modular cognitive behavioral therapy for the treatment of child anxiety disorders in elementary school settings. clinicaltrials.gov/show/nct00569829 (first received 7 December 2007).

\section{Cobham 2017 \{published data only\}}

Cobham VE, Filus A, Sanders MR. Working with parents to treat anxiety-disordered children: a proof of concept RCT evaluating Fear-less Triple P. Behaviour Research and Therapy 2017;95:128-38. [DOI: 10.1016/j.brat.2017.06.004]

Cornacchio 2019 \{published data only (unpublished sought but not used)\}

* Cornacchio D, Furr JM, Sanchez AL, Hong N, Feinberg LK, Tenenbaum R, et al. Intensive group behavioral treatment (IGBT) for children with selective mutism: a preliminary randomized clinical trial. Journal of Consulting and Clinical Psychology 2019;87(8):720-33. [DOI: 10.1037/ccp0000422]

NCT03612102. Evaluating intensive group behavioral treatment for children with selective mutism. clinicaltrials.gov/show/ nct03612102 (first received 2 August 2018).

Creswell 2017 \{published and unpublished data\}

* Creswell C, Violato M, Fairbanks H, White E, Parkinson M, Abitabile $\mathrm{G}$, et al. Clinical outcomes and cost-effectiveness of brief guided parent-delivered cognitive behavioural therapy and solution-focused brief therapy for treatment of childhood anxiety disorders: a randomised controlled trial. Lancet Psychiatry 2017;4(7):529-39. [DOI: 10.1016/ S2215-0366(17)30149-9]

ISRCTN07627865. Guided self-help for childhood anxiety problems: a comparison with usual care. www.isrctn.com/ ISRCTN07627865 (first received 13 March 2012).

\section{Dadds 1997 \{published and unpublished data\}}

Dadds MR, Holland DE, Laurens KR, Mullins M, Barrett PM, Spence SH. Early intervention and prevention of anxiety disorders in children: results at 2-year follow-up. Journal of Consulting and Clinical Psychology 1999;67(1):145-50. 
* Dadds MR, Spence SH, Holland DE, Barrett PM, Laurens KR. Prevention and early intervention for anxiety disorders: a controlled trial. Journal of Consulting and Clinical Psychology 1997;65(4):627-35.

Flannery Schroeder $\mathbf{2 0 0 0}$ \{published data only (unpublished sought but not used)\}

* Flannery-Schroeder EC, Kendall PC. Group and individual cognitive-behavioral treatments for youth with anxiety disorders: a randomized clinical trial. Cognitive Therapy and Research 2000;24(3):251-78. [DOI: 10.1023/A:1005500219286]

Fujii 2013 \{published data only (unpublished sought but not used)\} Fujii C, Renno P, McLeod BD, Lin CE, Decker K, Zielinski K, et al. Intensive cognitive behavioral therapy for anxiety disorders in school-aged children with autism: a preliminary comparison with treatment-as-usual. School Mental Health 2013;5(1):25-37. [DOI: 10.1007/s12310-012-9090-0]

Gallagher 2004 \{published data only (unpublished sought but not used)\}

* Gallagher HM, Rabian BA, McCloskey MS. A brief group cognitive-behavioral intervention for social phobia in childhood. Journal of Anxiety Disorders 2004;18(4):459-79. [DOI: 10.1016/S0887-6185(03)00027-6]

\section{Ginsburg 2002 \{published data only\}}

Ginsburg GS, Drake KL. School-based treatment for anxious African-American adolescents: a controlled pilot study. Journal of the American Academy of Child and Adolescent Psychiatry 2002;41(7):768-75. [DOI: 10.1097/00004583-200207000-00007]

Ginsburg 2012 \{published data only (unpublished sought but not used)\}

* Ginsburg GS, Becker KD, Drazdowski TK, Tein J. Treating anxiety disorders in inner city schools: results from a pilot randomized controlled trial comparing CBT and usual care. Child and Youth Care Forum 2012;41(1):1-19. [DOI: 10.1007/ s10566-011-9156-4 ]

NCT00536094. School-based treatment for anxious children. clinicaltrials.gov/show/nct00536094 (first received 27 September 2007).

Ginsburg 2019a \{published data only (unpublished sought but not used)\}

* Ginsburg GS, Pella JE, Pikulski PJ, Tein JY, Drake KL. SchoolBased Treatment for Anxiety Research Study (STARS): a randomized controlled effectiveness trial. Journal of Abnormal Child Psychology 2019;48(3):407-17. [10.1007/ s10802-019-00596-5 ]

NCT01761396. School-based Treatment of Anxiety Research Study (STARS). clinicaltrials.gov/show/nct01761396 (first received 4 January 2013).

\section{Hancock 2018 \{published data only (unpublished sought but not used)\}}

ACTRN12611001280998. Efficacy of acceptancemindfulness therapy in children with anxiety: a controlled trial. www.who.int/trialsearch/Trial2.aspx?
TrialID=ACTRN12611001280998 (first received 13 December 2011).

* Hancock KM, Swain J, Hainsworth CJ, Dixon AL, Koo S, Munro K. Acceptance and commitment therapy versus cognitive behavior therapy for children with anxiety: outcomes of a randomized controlled trial. Journal of Clinical Child and Adolescent Psychology 2018;47(2):296-311. [DOI: 10.1080/15374416.2015.1110822]

Swain J, Hancock K, Dixon A, Koo S, Bowman J. Acceptance and commitment therapy for anxious children and adolescents: study protocol for a randomized controlled trial. Trials 2013;14:140. [DOI: 10.1186/1745-6215-14-140]

Swain J, Hancock K, Hainsworth C, Bowman J. Mechanisms of change: exploratory outcomes from a randomised controlled trial of acceptance and commitment therapy for anxious adolescents. Journal of Contextual Behavioral Science 2015;4(1):56-67. [DOI: 10.1016/j.jcbs.2014.09.001]

Herbert 2009 \{published data only (unpublished sought but not used)\}

Herbert JD, Gaudiano BA, Rheingold AA, Moitra E, Myers VH, Dalrymple KL, et al. Cognitive behavior therapy for generalized social anxiety disorder in adolescents: a randomized controlled trial. Journal of Anxiety Disorders 2009;23(2):167-77. [DOI: 10.1016/j.janxdis.2008.06.004]

\section{Hirshfeld Becker 2010 \{published and unpublished data\}}

* Hirshfeld-Becker DR, Masek B, Henin A, Blakely LR, PollockWurman RA, McQuade J, et al. Cognitive behavioural therapy for 4- to 7-year old children with anxiety disorders: a randomized clinical trial. Journal of Consulting and Clinical Psychology 2010;78(4):498-510. [DOI: 10.1037/a0019055]

NCT00865306. Early intervention for children at risk for anxiety. clinicaltrials.gov/show/nct00865306 (first received 19 March 2009).

Holmes 2014 \{published data only (unpublished sought but not used)\}

Holmes MC, Donovan CL, Farrell LJ, March S. The efficacy of a group-based, disorder-specific treatment program for childhood GAD - a randomized controlled trial. Behaviour Research and Therapy 2014;61:122-35. [DOI: 10.1016/ j.brat.2014.08.002]

\section{Hudson 2009 \{published data only\}}

Hudson JL, Rapee RM, Deveney C, Schniering CA, Lyneham HJ, Bovopoulos N. Cognitive-behavioral treatment versus an active control for children and adolescents with anxiety disorders: a randomized trial. Journal of the American Academy of Child and Adolescent Psychiatry 2009;48(5):533-44. [DOI: 10.1097/ $\mathrm{CHI} .0 \mathrm{~b} 013 \mathrm{e} 31819 \mathrm{c} 2401]$

\section{Ingul 2013 \{published data only\}}

Ingul JM, Aune T, Nordahl HM. A randomized controlled trial of individual cognitive therapy, group cognitive behaviour therapy and attentional placebo for adolescent social phobia. Psychotherapy and Psychosomatics 2013;83(1):54-61. [DOI: 10.1159/000354672] 
Ishikawa 2019 \{published data only\}

Ishikawa S, Kikuta K, Sakai M, Mitamura T, Motomura N, Hudson JL. A randomized controlled trial of a bidirectional cultural adaptation of cognitive behavior therapy for children and adolescents with anxiety disorders. Behaviour Research and Therapy 2019;120:103432. [DOI: 10.1016/j.brat.2019.103432]

\section{Kendall 1994 \{published and unpublished data\}}

Kendall PC. Treating anxiety disorders in youth: results of a randomized clinical trial. Journal of Consulting and Clinical Psychology 1994;62(1):100-10.

Kendall 1997 \{published data only (unpublished sought but not used)\}

Kendall PC, Flannery-Schroeder E, Panichelli-Mindel SM, Southam-Gerow M, Henin A, Warman M. Therapy for youths with anxiety disorders: a second randomized clinical trial. Journal of Consulting and Clinical Psychology 1997;65(3):366-80.

\section{Kendall 2008 \{published and unpublished data\}}

* Kendall PC, Hudson JL, Gosch E, Flannery-Schroeder E, Suveg C. Cognitive-behavioral therapy for anxiety disordered youth: a randomized clinical trial evaluating child and family modalities. Journal of Consulting and Clinical Psychology 2008;76(2):282-97. [DOI: 10.1037/0022-006X.76.2.282]

NCT00081406. Individual and family therapy for children with anxiety disorders. clinicaltrials.gov/show/nct00081406 (first received 12 April 2004).

Suveg CH, Hudson JL, Brewer G, Flannery-Schroeder E, Gosch E, Kendall PC. Cognitive-behavioral therapy for anxiety-disordered youth: secondary outcomes from a randomized clinical trial evaluating child and family modalities. Journal of Anxiety Disorders 2009;23(3):341-9. [DOI: 10.1016/j.janxdis.2009.01.003]

Kennedy 2009 \{published data only (unpublished sought but not used)\}

Kennedy SJ, Rapee RM, Edwards SL. A selective intervention program for inhibited preschool-aged children of parents with an anxiety disorder: effects on current anxiety disorders and temperament. Journal of the American Academy of Child and Adolescent Psychiatry 2009;48(6):602-9. [DOI: 10.1097/ CHI.0b013e31819f6fa9]

\section{Khanna 2010 \{published data only (unpublished sought but not} used)\}

Khanna MS, Kendall PC. Computer-assisted cognitive behavioral therapy for child anxiety: results of a randomized clinical trial. Journal of Consulting and Clinical Psychology 2010;78(5):737-45. [DOI: 10.1037/a0019739]

\section{Kidd 2018 \{published data only\}}

ACTRN12610001014044. Assessing the efficacy and social validity of a manualised, family-based group Cognitive Behavioural Therapy for adolescents with high-functioning autism and comorbid anxiety disorder. www.who.int/ trialsearch/Trial2.aspx?TrialID=ACTRN12610001014044 (first received 19 November 2010)

* Kidd TC. Treating Anxiety in Adolescents With Autism Spectrum Disorder Using Group Cognitive Behaviour
Therapy: A Randomised Controlled Trial [Doctoral thesis]. Perth, Western Australia: Curtin University, 2018. [hdl.handle.net/20.500.11937/73526]

\section{Last 1998 \{published data only\}}

Last CG, Hansen C, Franco N. Cognitive-behavioral treatment of school phobia. Journal of the American Academy of Child and Adolescent Psychiatry 1998;37(4):404-11. [DOI: 10.1097/00004583-199804000-00018]

Lau 2010 \{published and unpublished data\}

Lau WY, Chan CK, Li JC, Au TK. Effectiveness of group cognitivebehavioral treatment for childhood anxiety in community clinics. Behaviour Research and Therapy 2010;48(11):1067-77. [DOI: 10.1016/j.brat.2010.07.007]

Lau 2017 \{published data only (unpublished sought but not used)\} Lau EX, Rapee RM, Coplan RJ. Combining child social skills training with a parent early intervention program for inhibited preschool children. Journal of Anxiety Disorders 2017;51:32-8. [DOI: 10.1016/j.janxdis.2017.08.007]

Lebowitz 2019 \{published data only (unpublished sought but not used)\}

* Lebowitz ER, Marin C, Martino A, Shimshoni Y, Silverman WK. Parent-based treatment as efficacious as cognitive-behavioral therapy for childhood anxiety: a randomized noninferiority study of supportive parenting for anxious childhood emotions. Journal of the American Academy of Child and Adolescent Psychiatry 2019 Mar 7 [Epub ahead of print]. [DOI: 10.1016/ j.jaac.2019.02.014]

Lebowitz ER, Marin C, Martino A, Shimshoni Y, Silverman WK. Parent-based treatment as efficacious as cognitive-behavioral therapy for childhood anxiety: a randomized noninferiority study of supportive parenting for anxious childhood emotions. Journal of the American Academy of Child and Adolescent Psychiatry 2020;59(3):362-72. [DOI: 10.1016/j.jaac.2019.02.014]

NCT02310152. Explanatory clinical trial of a novel parent intervention for childhood anxiety (SPACE). clinicaltrials.gov/ show/nct02310152 (first received 5 December 2014).

\section{Leutgeb 2012 \{published data only\}}

Leutgeb V, Schienle A. Changes in facial electromyographic activity in spider-phobic girls after psychotherapy. Journal of Psychiatric Research 2012;46(6):805-10. [DOI: 10.1016/ j.jpsychires.2012.02.017]

Masia Warner 2005 \{published data only (unpublished sought but not used)\}

Masia Warner C, Klein RG, Dent HC, Fisher PH, Alvir J, Albano AM, et al. School-based intervention for adolescents with social anxiety disorder: results of a controlled study. Journal of Abnormal Child Psychology 2005;33(6):707-22. [DOI: 10.1007/s10802-005-7649-z]

Masia Warner 2007 \{published data only\}

Masia Warner C, Fisher PH, Shrout PE, Rathor S, Klein RG. Treating adolescents with social anxiety disorder in school: an attention control trial. Journal of Child Psychology and 
Psychiatry and Allied Disciplines 2007;48(7):676-86. [DOI: 10.1111/j.1469-7610.2007.01737.x]

Masia Warner 2011 \{published data only (unpublished sought but not used)\}

Masia Warner C, Colognori D, Kim RE, Reigada LC, Klein RG, Browner-Elhanan KJ, et al. Cognitive-behavioral treatment of persistent functional somatic complaints and pediatric anxiety: an initial controlled trial. Depression and Anxiety 2011;28(7):551-9. [DOI: 10.1002/da.20821]

Masia Warner 2016 \{published data only (unpublished sought but not used)\}

* Masia Warner C, Colognori D, Brice C, Herzig K, Mufson L, Lynch C, et al. Can school counselors deliver cognitivebehavioral treatment for social anxiety effectively? A randomized controlled trial. Journal of Child Psychology and Psychiatry and Allied Disciplines 2016;57(11):1229-38. [DOI: 10.1111/jcpp.12550]

NCT01320800. CBT for social anxiety disorder delivered by school counselors. clinicaltrials.gov/show/nct01320800 (first received 22 March 2011).

\section{McConachie 2014 \{published data only\}}

ISRCTN11219568. Effective therapy for anxiety in young people with autism spectrum disorder. www.isrctn.com/ ISRCTN11219568 (first received 12 May 2010).

* McConachie H, McLaughlin E, Grahame V, Taylor H, Honey E, Tavernor L, et al. Group therapy for anxiety in children with autism spectrum disorder. Autism 2014;18(6):723-32. [DOI: 10.1177/1362361313488839]

McNally Keehn 2013 \{published data only (unpublished sought but not used)\}

McNally Keehn RH, Lincoln AJ, Brown MZ, Chavira DA. The Coping Cat program for children with anxiety and autism spectrum disorder: a pilot randomized controlled trial. Journal of Autism and Developmental Disorders 2013;43(1):57-67. [DOI: 10.1007/s10803-012-1541-9]

\section{Melfsen 2011 \{published and unpublished data\}}

Melfsen S, Kuhnemund M, Schwieger J, Warnke A, Stadler C, Poustka F, et al. Cognitive behavioral therapy of socially phobic children focusing on cognition: a randomised wait-list control study. Child and Adolescent Psychiatry and Mental Health 2011;5:ArtID2. [DOI: 10.1186/1753-2000-5-5]

\section{Muris 2002 \{published data only\}}

Muris P, Meesters C, van Melick M. Treatment of childhood anxiety disorders: a preliminary comparison between cognitivebehavioral group therapy and a psychological placebo intervention. Journal of Behavior Therapy and Experimental Psychiatry 2002;33(3-4):143-58.

\section{Murphy 2017 \{published data only (unpublished sought but not used)\}}

Murphy SM, Chowdhury U, White SW, Reynolds L, Donald L, $\mathrm{Gahan} \mathrm{H}$, et al. Cognitive behaviour therapy versus a counselling intervention for anxiety in young people with highfunctioning autism spectrum disorders: a pilot randomised controlled trial. Journal of Autism and Developmental Disorders 2017;47(11):3446-57. [DOI: 10.1007/s10803-017-3252-8]

O'Brien 2007 \{published data only (unpublished sought but not used)\}

O'Brien F, Olden N, Migone M, Dooley B, Atkins L, Ganter K, et al. Group cognitive behavioural therapy for children with anxiety disorder - an evaluation of the 'Friends for Youth' programme. Irish Journal of Psychological Medicine 2007;24(1):5-12.

Olivares 2005 \{published and unpublished data\}

Olivares J, Rosa-Alcazar AI, Piqueras JA. Early detection and treatment of adolescents with generalized social phobia. Psicothema 2005;17(1):1-8.

\section{Olivares 2014 \{published data only\}}

Olivares J, Olivares-Olivares PJ, Rosa-Alcazar Al, Montesinos L, Macia D. The contribution of the therapist's competence in the treatment of adolescents with generalized social phobia. Psicothema 2014;26(4):483-9.

Olivares 2019 \{published data only\}

Olivares-Olivares PJ, Ortiz-González PF, Olivares J. Role of social skills training in adolescents with social anxiety disorder. International Journal of Clinical and Health Psychology 2019;19(1):41-8.

\section{Ollendick 2009 \{published data only\}}

Ollendick TH, Ost LG, Reuterskiold L, Costa N, Cederlund R, Sirbu $\mathrm{C}$, et al. One-session treatment of specific phobias in youth: a randomized clinical trial in the United States and Sweden. Journal of Consulting and Clinical Psychology 2009;77(3):504-16. [DOI: 10.1037/a0015158]

Ost 2001 \{published and unpublished data\}

Ost LG, Svensson L, Hellstrom K, Lindwall R. One-session treatment of specific phobias in youths: a randomized clinical trial. Journal of Consulting and Clinical Psychology 2001;69(5):814-24. [DOI: 10.1037/0022-006X.69.5.814]

Perrin 2019 \{published data only\} ISRCTN50951795. Cognitive therapy for generalised anxiety in youth. www.isrctn.com/ISRCTN50951795 (first received 30 June 2010).

* Perrin S, Bevan D, Payne S, Bolton D. GAD-specific cognitive behavioral treatment for children and adolescents: a pilot randomized controlled trial. Cognitive Therapy and Research 2019;43(6):1051-64. [DOI: 10.1007/s10608-019-10020-3]

Pincus 2010 \{published data only (unpublished sought but not used)\}

NCT00705380. Effectiveness of cognitive behavioral therapy with panic control treatment for adolescents with panic disorder. clinicaltrials.gov/show/nct00705380 (first received 25 June 2008)

* Pincus DB, May JE, Whitton SW, Mattis SG, Barlow DH. Cognitive-behavioral treatment of panic disorder in adolescence. Journal of Clinical Child and Adolescent Psychology 2010;39(5):638-49. [DOI: 10.1080/15374416.2010.501288] 
Rapee 2005 \{published and unpublished data\}

* Rapee RM, Kennedy S, Ingram M, Edwards S, Sweeney L. Prevention and early intervention of anxiety disorders in inhibited preschool children. Journal of Consulting and Clinical Psychology 2005;73(3):488-97. [DOI: 10.1037/0022-006X.73.3.488]

Rapee RM, Kennedy SJ, Ingram M, Edwards SL, Sweeney L. Altering the trajectory of anxiety in at-risk young children. American Journal of Psychiatry 2010;167(12):1518-25. [DOI: 10.1176/appi.ajp.2010.09111619]

Rapee RM. The preventative effects of a brief, early intervention for preschool-aged children at risk for internalising: followup into middle adolescence. Journal of Child Psychology \& Psychiatry 2013;54(7):780-8. [DOI: 10.1111/jcpp.12048]

\section{Rapee 2006 \{published and unpublished data\}}

Rapee RM, Abbott MJ, Lyneham HJ. Bibliotherapy for children with anxiety disorders using written materials for parents: a randomized controlled trial. Journal of Consulting and Clinical Psychology 2006;74(3):436-44. [DOI: 10.1037/0022-006X.74.3.436]

\section{Reaven 2012 \{published data only\}}

Reaven J, Blakeley-Smith A, Culhane-Shelburne K, Hepburn S. Group cognitive behavior therapy for children with highfunctioning autism spectrum disorders and anxiety: a randomized trial. Journal of Child Psychology and Psychiatry and Allied Disciplines 2012;53(4):410-9.

\section{Reigada 2015 \{published and unpublished data\}}

Reigada LC, Polokowski AR, Walder DJ, Szigethy EM, Benkov KJ, Bruzzese JM, et al. Treatment for comorbid pediatric gastrointestinal and anxiety disorders: a pilot study of a flexible health sensitive cognitive-behavioral therapy program. Clinical Practice in Pediatric Psychology 2015;3(4):314-26. [DOI: 10.1037/ cpp0000116]

\section{Rosa Alcazar 2009 \{published and unpublished data\}}

Rosa-Alcazar AI, Olivares-Olivares PJ, Olivares J. The role of nonspecific effects in the psychological treatment of adolescents with social phobia. Anuario de Psicologia 2009;40(1):43-61.

\section{Salari 2018 \{published and unpublished data\}}

NCT01513915. A parent-only group cognitive behavioral intervention for children with anxiety disorders: a control group study. clinicaltrials.gov/show/nct01513915 (first received 20 January 2012).

* Salari E, Shahrivar Z, Mahmoudi-Gharaei J, Shirazi E, Sepasi M. Parent-only group cognitive behavioral intervention for children with anxiety disorders: a control group study. Journal of the Canadian Academy of Child and Adolescent Psychiatry 2018;27(2):130-6.

\section{Salum 2018 \{published data only\}}

NCT01687764. Combination of active or placebo Attentional Bias Modification Treatment (ABMT) to either Cognitive Behavioral Group Therapy (CBGT) or Psychoeducational Control Intervention (PCI) for anxiety disorders in children: a randomized clinical trial. https://www.clinicaltrials.gov/ct2/ show/NCT01687764 19 September 2012.

* Salum GA, Petersen CS, Jarros RB, Toazza R, DeSousa D, Borba LN, et al. Group cognitive behavioral therapy and attention bias modification for childhood anxiety disorders: a factorial randomized trial of efficacy. Journal of Child and Adolescent Psychopharmacology 2018;28(9):620-30. [DOI: 10.1089/cap.2018.0022]

\section{Sanchez Garcia 2009 \{published and unpublished data\}}

Sanchez-Garcia R, Olivares J. Effectiveness of a program for early detection/intervention in children/adolescents with generalized social phobia. Anales de Psicologia 2009;25(2):241-9.

Santucci 2013 \{published data only (unpublished sought but not used)\}

Santucci LC, Ehrenreich-May J. A randomized controlled trial of the child anxiety multi-day program (CAMP) for separation anxiety disorder. Child Psychiatry and Human Development 2013;44(3):439-51. [DOI: 10.1007/s10578-012-0338-6]

Schneider 2011 \{published data only (unpublished sought but not used)\}

Schneider S, Blatter-Meunier J, Herren C, Adornetto C, InAlbon T, Lavallee K. Disorder-specific cognitive-behavioral therapy for separation anxiety disorder in young children: a randomized waiting-list-controlled trial. Psychotherapy and Psychosomatics 2011;80(4):206-15. [DOI: 10.1159/000323444]

Sciberras 2018 \{published data only (unpublished sought but not used)\}

ISRCTN33930984. Does the identification and treatment of comorbid anxiety disorders in children with attention deficit hyperactivity disorder (ADHD) improve outcomes? www.isrctn.com/ISRCTN33930984 (first received 2 October 2013).

* Sciberras E, Mulraney M, Anderson V, Rapee RM, Nicholson JM, Efron D, et al. Managing anxiety in children with ADHD using cognitive-behavioral therapy: a pilot randomized controlled trial. Journal of Attention Disorders 2018;22(5):515-20. [DOI: 10.1177/1087054715584054]

\section{Shahnavaz 2016 \{published data only\}}

NCT01798355. Children and adolescents with dental anxiety randomized controlled study of cognitive behavioral therapy. clinicaltrials.gov/show/nct01798355 (first received 25 February 2013).

* Shahnavaz S, Hedman E, Grindefjord M, Reuterskiold L, Dahllof G. Cognitive behavioral therapy for children with dental anxiety: a randomized controlled trial. JDR Clinical and Translational Research 2016;1(3):234-43. [DOI: 10.1177/2380084416661473]

\section{Sharma 2017 \{published and unpublished data\}}

Sharma P, Mehta M, Sagar R. Efficacy of transdiagnostic cognitive-behavioral group therapy for anxiety disorders and headache in adolescents. Journal of Anxiety Disorders 2017;46:78-84. [DOI: 10.1016/j.janxdis.2016.11.001] 
Shortt 2001 \{published data only\}

Shortt AL, Barrett PM, Fox TL. Evaluating the FRIENDS program: a cognitive-behavioral group treatment for anxious children and their parents. Journal of Clinical Child and Adolescent Psychology 2001;30(4):525-35. [DOI: 10.1207/ S15374424JCCP3004_09]

\section{Silk 2018 \{published and unpublished data\}}

NCT00774150. Transdisciplinary studies of CBT for anxiety in youth: child anxiety treatment study. clinicaltrials.gov/show/ nct00774150 (first received 17 October 2008).

* Silk JS, Tan PZ, Ladouceur CD, Meller S, Siegle GJ, McMakin DL, et al. A randomized clinical trial comparing individual cognitive behavioral therapy and child-centered therapy for child anxiety disorders. Journal of Clinical Child and Adolescent Psychology 2018;47(4):542-54. [DOI: 10.1080/15374416.2016.1138408]

Silverman 1999a \{published data only (unpublished sought but not used)\}

Silverman WK, Kurtines WM, Ginsburg GS, Weems CF, Rabian B, Serafini LT. Contingency management, self-control, and education support in the treatment of childhood phobic disorders: a randomized clinical trial. Journal of Consulting and Clinical Psychology 1999;67(5):675-87.

Silverman 1999b \{published data only (unpublished sought but not used)\}

Silverman WK, Kurtines WM, Ginsburg GS, Weems CF, Lumpkin PW, Carmichael DH. Treating anxiety disorders in children with group cognitive-behavioral therapy: a randomized clinical trial. Journal of Consulting and Clinical Psychology 1999;67(6):995-1003.

\section{Simon 2011 \{published and unpublished data\}}

NTR1143. Prevention of childhood anxiety disorders via the parent or via the child? www.who.int/trialsearch/Trial2.aspx? TrialID=NTR1143 (first received 27 November 2007).

* Simon E, Bogels SM, Voncken JM. Efficacy of child-focused and parent-focused interventions in a child anxiety prevention study. Journal of Clinical Child and Adolescent Psychology 2011;40(2):204-19. [DOI: 10.1080/15374416.2011.546039]

Smith 2014 \{published data only (unpublished sought but not used)\}

* Smith AM, Flannery-Schroeder EC, Gorman KS, Cook N Parent cognitive-behavioral intervention for the treatment of childhood anxiety disorders: a pilot study. Behaviour Research and Therapy 2014;61:156-61.

Smith AM. Cognitive-behavioral treatment of childhood anxiety: examining a parent consultation model. Dissertation Abstracts International: Section B: The Sciences and Engineering 2014;74:8-B(E)

Southam Gerow 2010 \{published data only (unpublished sought but not used)\}

Southam-Gerow MA, Weisz JR, Chu BC, McLeod BD, Gordis EB, Connor-Smith JK. Does cognitive behavioral therapy for youth anxiety outperform usual care in community clinics? An initial effectiveness test. Journal of the American Academy of Child and Adolescent Psychiatry 2010;49(10):1043-52. [DOI: 10.1016/ j.jaac.2010.06.009]

Spence 2000 \{published and unpublished data\}

Spence SH, Donovan C, Brechman-Toussaint M. The treatment of childhood social phobia: the effectiveness of a social skills training-based, cognitive-behavioural intervention, with and without parental involvement. Journal of Child Psychology and Psychiatry and Allied Disciplines 2000;41(6):713-26.

Spence 2006 \{published data only\}

Spence SH, Holmes JM, March S, Lipp OV. The feasibility and outcome of clinic plus internet delivery of cognitivebehavior therapy for childhood anxiety. Journal of Consulting and Clinical Psychology 2006;74(3):614-21. [DOI: 10.1037/0022-006X.74.3.614]

Spence 2011 \{published data only\}

Spence SH, Donovan CL, March S, Gamble A, Anderson RE, Prosser $\mathrm{S}$, et al. A randomized controlled trial of online versus clinic-based CBT for adolescent anxiety. Journal of Consulting and Clinical Psychology 2011;79(5):629-42. [DOI: 10.1037/ a0024512]

\section{Storch 2013 \{published data only\}}

NCT01178385. Cognitive-behavioral treatment for anxiety disorders in children with autism spectrum disorders. clinicaltrials.gov/show/nct01178385 (first received 10 August 2010).

* Storch EA, Arnold EB, Lewin AB, Nadeau JM, Jones AM, De Nadai AS, et al. The effect of cognitive-behavioral therapy versus treatment as usual for anxiety in children with autism spectrum disorders: a randomized, controlled trial. Journal of the American Academy of Child and Adolescent Psychiatry 2013;52(2):132-42.e2. [DOI: 10.1016/j.jaac.2012.11.007]

\section{Storch 2015 \{published data only\}}

NCT01563003. Cognitive behavioral therapy for anxiety disorders in adolescents with autism. clinicaltrials.gov/show/ nct01563003 (first received 26 March 2012).

* Storch EA, Lewin AB, Collier AB, Arnold E, De Nadai AS, Dane $B F$, et al. A randomized controlled trial of cognitivebehavioral therapy versus treatment as usual for adolescents with autism spectrum disorders and comorbid anxiety. Depression and Anxiety 2015;32(3):174-81. [DOI: 10.1002/ da.22332]

Thirlwall 2013 \{published data only\}

Thirlwall K, Cooper PJ, Karalus J, Voysey M, Willetts L, Creswell $C$. Treatment of child anxiety disorders via guided parent-delivered cognitive-behavioural therapy: randomised controlled trial. British Journal of Psychiatry 2013;203(6):436-44. [DOI: 10.1192/bjp.bp.113.126698]

\section{Villabo 2018 \{published data only\}}

NCT00735995. Effectiveness of cognitive behavioral therapy (CBT) for child anxiety disorders in community clinics in Norway. clinicaltrials.gov/show/nct00735995 (first received 15 August 2008). 
* Villabo MA, Narayanan M, Compton SN, Kendall PC, Neumer SP. Cognitive-behavioral therapy for youth anxiety: an effectiveness evaluation in community practice. Journal of Consulting and Clinical Psychology 2018;86(9):751-64. [DOI: $10.1037 / \mathrm{ccp} 0000326]$

Walkup 2008 \{published data only (unpublished sought but not used)\}

Albano AM, Comer JS, Compton SN, Piacentini J, Kendall PC, Birmaher B, et al. Secondary outcomes from the Child/ Adolescent Anxiety Multimodal Study: implications for clinical practice. Evidence-Based Practice in Child and Adolescent Mental Health 2018;3(1):30-41. [DOI: 10.1080/23794925.2017.1399485]

Compton SN, Walkup JT, Albano AM, Piacentini JC, Birmaher B, Sherrill JT, et al. Child/Adolescent Anxiety Multimodal Study (CAMS): rationale, design, and methods. Child and Adolescent Psychiatry and Mental Health 2010;4:1. [DOI: 10.1186/1753-2000-4-1]

Ginsburg GS, Becker EM, Keeton CP, Sakolsky D, Piacentini J, Albano AM, et al. Naturalistic follow-up of youths treated for pediatric anxiety disorders. JAMA Psychiatry 2014;71(3):310-8. [DOI: 10.1001/jamapsychiatry.2013.4186]

Ginsburg GS, Becker-Haimes EM, Keeton C, Kendall PC, Lyengar S, Sakolsky D, et al. Results from the Child/Adolescent Anxiety Multimodal Extended Long-Term Study (CAMELS): primary anxiety outcomes. Journal of the American Academy of Child and Adolescent Psychiatry 2018;57(7):471-80. [DOI: 10.1016/j.jaac.2018.03.017]

Ginsburg GS, Kendall PC, Sakolsky D, Compton SN, Piacentini J, Albano AM, et al. Remission after acute treatment in children and adolescents with anxiety disorders: findings from the CAMS. Journal of Consulting and Clinical Psychology 2011;79(6):806-13. [DOI: 10.1037/a0025933]

NCT00052078. Child and Adolescent Anxiety Disorders (CAMS). clinicaltrials.gov/ct2/show/NCT00052078 (first received 23 January 2003).

Piacentini J, Bennett S, Compton SN, Kendall PC, Birmaher B, Albano AM, et al. 24- and 36-week outcomes for the Child/ Adolescent Anxiety Multimodal Study (CAMS). Journal of the American Academy of Child and Adolescent Psychiatry 2014;53(3):297-310. [DOI: 10.1016/j.jaac.2013.11.010]

Rynn MA, Walkup JT, Compton SN, Sakolsky DJ, Sherrill JT, Shen S, et al. Child/Adolescent Anxiety Multimodal Study: evaluating safety. Journal of the American Academy of Child and Adolescent Psychiatry 2015;54(3):180-90. [DOI: 10.1016/ j.jaac.2014.12.015]

* Walkup JT, Albano AM, Piacentini J, Birmaher B, Compton SN, Sherrill JT, et al. Cognitive behavioural therapy, sertraline or a combination in childhood anxiety. New England Journal of Medicine 2008;359(26):2753-66. [DOI: 10.1056/NEJMoa0804633]

\section{Waters 2009 \{published data only\}}

Waters AM, Ford LA, Wharton TA, Cobham VE. Cognitivebehavioural therapy for young children with anxiety disorders: comparison of a child + parent condition versus a parent only condition. Behaviour Research and Therapy 2009;47(8):654-62. [DOI: 10.1016/j.brat.2009.04.008]

Wergeland 2014 \{published and unpublished data\}

NCT00586586. Effectiveness study of CBT for anxiety in children. clinicaltrials.gov/show/nct00586586 (first received 4 January 2008).

* Wergeland GJ, Fjermestad KW, Marin CE, Haugland BS, Bjaastad JF, Oeding K, et al. An effectiveness study of individual vs. group cognitive behavioral therapy for anxiety disorders in youth. Behaviour Research and Therapy 2014;57:1-12. [DOI: 10.1016/j.brat.2014.03.007]

\section{White 2013 \{published data only\}}

NCT00926471. Social skills and anxiety reduction treatment for children and adolescents with autism spectrum disorders. clinicaltrials.gov/show/nct00926471 (first received 23 June 2009).

* White SW, Ollendick T, Albano AM, Oswald D, Johnson C, Southam-Gerow MA, et al. Randomized controlled trial: Multimodal Anxiety and Social Skill Intervention for adolescents with autism spectrum disorder. Journal of Autism and Developmental Disorders 2013;43(2):382-94. [DOI: 10.1007/ s10803-012-1577-x]

Wood 2009 \{published data only (unpublished sought but not used)\}

Wood JJ, Drahota A, Sze K, Har K, Chiu A, Langer DA. Cognitive behavioral therapy for anxiety in children with autism spectrum disorders: a randomized, controlled trial. Journal of Child Psychology and Psychiatry and Allied Disciplines 2009;50(3):224-34. [DOI: 10.1111/j.1469-7610.2008.01948.x]

Wood 2015 \{published data only (unpublished sought but not used)\}

NCT01177969. CBT for anxiety disorders in autism: adapting treatment for adolescents. clinicaltrials.gov/ct2/show/ nct01177969 (first received 9 August 2010).

* Wood JJ, Ehrenreich-May J, Alessandri M, Fujii C, Renno P, Laugeson E, et al. Cognitive behavioral therapy for early adolescents with autism spectrum disorders and clinical anxiety: a randomized, controlled trial. Behavior Therapy 2015;46(1):7-19. [DOI: 10.1016/j.beth.2014.01.002]

\section{References to studies excluded from this review}

\section{Asbrand \{unpublished data only\}}

Asbrand J. Exposure-based cognitive behavior therapy for children and adolescents with social anxiety disorder: a randomized controlled group treatment trial. Manuscript provided by author (on 21 May 2019).

\section{Asbrand 2019 \{published data only\}}

Asbrand J, Heinrichs N, Nitschke K, Wolf OT, Schmidtendorf S, Tuschen-Caffier B. Repeated stress leads to enhanced cortisol stress response in child social anxiety disorder but this effect can be prevented with CBT. Psychoneuroendocrinology 2019;109:104352. [DOI: 10.1016/j.psyneuen.2019.06.003] 
Asbrand J, Heinrichs N, Schmidtendorf S, Nitschke K, TuschenCaffier B. Experience versus report: where are changes seen after exposure-based cognitive-behavioral therapy? A randomized controlled group treatment of childhood social anxiety disorder. Child Psychiatry and Human Development 2020;51(3):427-41. [DOI: 10.1007/s10578-019-00954-w]

* Asbrand J, Schmitz J, Kramer M, Nitschke K, Heinrichs N, Tuschen-Caffier B. Effects of group-based CBT on postevent processing in children with social anxiety disorder following an experimental social stressor. Journal of Abnormal Child Psychology 2019;47(12):1945-56. [DOI: 10.1007/ s10802-019-00558-x]

\section{Baer 2005 \{published data only\}}

Baer S, Garland EJ. Pilot study of community-based cognitive behavioral group therapy for adolescents with social phobia. Journal of the American Academy of Child and Adolescent Psychiatry 2005;44(3):258-64. [DOI: 10.1097/00004583-200503000-00010]

\section{Beidel 2000 \{published data only\}}

Beidel DC, Turner SM, Morris TL. Behavioral treatment of childhood social phobia. Journal of Consulting and Clinical Psychology 2000;68(6):1072-80.

Beidel DC, Turner SM, Young B, Paulson A. Social effectiveness therapy for children: three-year follow-up. Journal of Consulting and Clinical Psychology 2005;73(4):721-5.

Beidel DC, Turner SM, Young BJ. Social effectiveness therapy for children: five years later. Behavior Therapy 2006;37(4):416-25.

\section{Beidel 2007 \{published data only\}}

* Beidel DC, Turner SM, Sallee FR, Ammerman RT, Crosby LA, Pathak S. SET-C versus fluoxetine in the treatment of childhood social phobia. Journal of the American Academy of Child and Adolescent Psychiatry 2007;46(12):1622-32. [DOI: 10.1097/ chi.0b013e318154bb57]

NCT00043537. Treatment of childhood social phobia. clinicaltrials.gov/ct2/show/NCT00043537 (first received 12 August 2002).

\section{Bergman 2013 \{published data only\}}

Bergman RL, Gonzalez A, Piacentini J, Keller ML. Integrated Behavior Therapy for Selective Mutism: a randomized controlled pilot study. Behaviour Research and Therapy 2013;51(10):680-9. [DOI: 10.1016/j.brat.2013.07.003]

NCT00458198. Integrated behavioral therapy for treating children with selective mutism. clinicaltrials.gov/ct2/show/ NCT00458198 (first received 9 April 2007).

Bernstein 2005 \{published data only (unpublished sought but not used)\}

Bernstein GA, Bernat DH, Victor AM, Layne AE. School-based interventions for anxious children: 3-, 6-, and 12-month follow-ups. Journal of the American Academy of Child and Adolescent Psychiatry 2008;47(9):1039-47. [DOI: 10.1097/ CHI.ob013e31817eecco]
Bernstein GA, Layne AE, Egan EA, Tennison DM. School-based interventions for anxious children. Journal of the American Academy of Child and Adolescent Psychiatry 2005;44(11):1118-27. [DOI: 10.1097/01.chi.0000177323.40005.a1]

Lee SS, Victor AM, James MG, Roach LE, Bernstein GA. Schoolbased interventions for anxious children: long-term follow-up. Child Psychiatry and Human Development 2016;47(2):183-93. [DOI: 10.1007/s10578-015-0555-x]

\section{Bodden 2008a \{published data only\}}

Bodden DH, Bogels SM, Nauta MH, De Haan E, Ringrose J, Appelboom C, et al. Child versus family cognitive-behavioral therapy in clinically anxious youth: an efficacy and partial effectiveness study. Journal of the American Academy of Child and Adolescent Psychiatry 2008;47(12):1384-94. [DOI: 10.1097/ $\mathrm{CHI} .0 \mathrm{~b} 013 \mathrm{e} 318189148 \mathrm{e}]$

Bodden DHM, Dirksen CD, Bogels SM, Nauta MH, De Haan E, Ringrose J, et al. Costs and cost-effectiveness of family CBT versus individual CBT in clinically anxious children. Clinical Child Psychology and Psychiatry 2008;13(4):543-64. [DOI: 10.1177/1359104508090602]

\section{Clementi 2019 \{published data only\}}

Clementi MA. Efficacy of an integrated sleep and anxiety intervention for anxious children: a pilot randomized controlled trial. Dissertation Abstracts International: Section B: The Sciences and Engineering 2019;80:7-B(E).

\section{Cobham 2012 \{published data only\}}

Cobham V. Do anxiety-disordered children need to come into the clinic for efficacious treatment? Journal of Consulting and Clinical Psychology 2012;80(3):465-76.

\section{Cotton 2019 \{published data only\}}

Cotton S, Kraemer KM, Sears RW, Strawn JR, Wasson RS, McCune N, et al. Mindfulness-based cognitive therapy for children and adolescents with anxiety disorders at-risk for bipolar disorder: a psychoeducation waitlist controlled pilot trial. Early Intervention in Psychiatry 2019;14(2):211-9.

\section{Ebrahiminejad 2016 \{published data only\}}

Ebrahiminejad S, Poursharifi H, Bakhshiour Roodsari A, Zeinodini Z, Noorbakhsh S. The effectiveness of mindfulnessbased cognitive therapy on Iranian female adolescents suffering from social anxiety. Iranian Red Crescent Medical Journal 2016;18(11):e25116. [DOI: 10.5812/ircmj.25116]

\section{Flatt 2010 \{published data only\}}

Flatt N, King N. Brief psycho-social interventions in the treatment of specific childhood phobias: a controlled trial and a 1-year follow-up. Behaviour Change 2010;27(3):130-53.

\section{Gil Bernal 2009 \{published data only\}}

Gil-Bernal F, Hernandez-Guzman L. Cognitive-behavioural treatment in Mexican children with social phobia. Anuario de Psicologia 2009;40(1):89-104. 
Ginsburg 2019b \{published data only (unpublished sought but not used)\}

Ginsburg GS, Drake KL, Muggeo MA, Stewart CE, Pikulski PJ, Zheng D, et al. A pilot RCT of a school nurse delivered intervention to reduce student anxiety. Journal of Clinical Child and Adolescent Psychology 2019 Aug 2 [Epub ahead of print]. [DOI: 10.1080/15374416.2019.1630833]

\section{Hayward 2000 \{published data only\}}

Hayward C, Varady S, Albano AM, Thienemann M, Henderson L, Schatzberg AF. Cognitive-behavioral group therapy for social phobia in female adolescents: results of a pilot study. Journal of the American Academy of Child and Adolescent Psychiatry 2000;39(6):721-6. [DOI: 10.1097/00004583-200006000-00010]

\section{Jansen 2012 \{published data only\}}

Jansen M, van Doorn MM, Lichtwarck-Aschoff A, Kuijpers RC, Theunissen $\mathrm{H}$, Korte M, et al. Effectiveness of a cognitivebehavioral therapy (CBT) manualized program for clinically anxious children: study protocol of a randomized controlled trial. BMC Psychiatry 2012;12(1):16. [DOI: 10.1186/1471-244X-12-16]

\section{Kerns 2016 \{published data only\}}

* Kerns CM, Wood JJ, Kendall PC, Renno P, Crawford EA, Mercado RJ, et al. The Treatment of Anxiety in Autism Spectrum Disorder (TAASD) study: rationale, design and methods. Journal of Child and Family Studies 2016;25(6):1889-902. [DOI: 10.1007/ s10826-016-0372-2]

NCT02028247. Psychotherapy for Anxiety in Children With Autism Spectrum Disorder (TAASD). clinicaltrials.gov/show/ NCT02111395 (first received 7 January 2014).

\section{Kujawa 2019 \{published data only\}}

Kujawa A, Burkhouse KL, Karich SR, Fitzgerald KD, Monk CS, Phan KL. Reduced reward responsiveness predicts change in depressive symptoms in anxious children and adolescents following treatment. Journal of Child and Adolescent Psychopharmacology 2019;29(5):378-85.

\section{Lyneham 2006 \{published data only\}}

Lyneham HJ, Rapee RM. Evaluation of therapist-supported parent-implemented CBT for anxiety disorders in rural children. Behaviour Research and Therapy 2006;44(9):1287-300. [DOI: 10.1016/j.brat.2005.09.009]

\section{Mendlowitz 1999 \{published data only\}}

Mendlowitz SL, Manassis K, Bradley S, Scapillato D, Miezitis S, Shaw BF. Cognitive-behavioral group treatments in childhood anxiety disorders: the role of parental involvement. Journal of the American Academy of Child and Adolescent Psychiatry 1999;38(10):1223-9.

\section{Nauta 2003 \{published data only\}}

Nauta MH, Scholing A, Emmelkamp PM, Minderaa RB. Cognitive-behavioral therapy for children with anxiety disorders in a clinical setting: no additional effect of a cognitive parent training. Journal of the American Academy of Child and Adolescent Psychiatry 2003;42(11):1270-8. [DOI: 10.1097/01.chi.0000085752.71002.93]

\section{NCT00576719 \{published data only\}}

Elkins RM, Gallo KP, Pincus DB, Comer JS. Moderators of intensive cognitive behavioral therapy for adolescent panic disorder: the roles of fear and avoidance. Child and Adolescent Mental Health 2016;21(1):30-6. [DOI: 10.1111/camh.12122]

Gallo KP, Chan PT, Buzzella BA, Whitton SW, Pincus DB. The impact of an 8-day intensive treatment for adolescent panic disorder and agoraphobia on comorbid diagnoses. Behavior Therapy 2012;43(1):153-9. [DOI: 10.1016/j.beth.2011.05.002]

Gallo KP, Cooper-Vince CE, Hardway CL, Pincus DB, Comer JS. Trajectories of change across outcomes in intensive treatment for adolescent panic disorder and agoraphobia. Journal of Clinical Child and Adolescent Psychology 2014;43(5):742-50.

Hardway CL, Pincus DB, Gallo KP, Comer JS. Parental involvement in intensive treatment for adolescent panic disorder and its impact on depression. Journal of Child and Family Studies 2015;24(11):3306-17. [DOI: 10.1007/ s10826-015-0133-7]

* NCT00576719. Effectiveness of intensive cognitive behavioral therapy in treating adolescent panic disorder and agoraphobia. clinicaltrials.gov/show/nct00576719 (first received 19 December 2007).

Pincus DB, Whitton SW, Gallo KP, Weiner CL, Chow C, Hardway C. Intensive treatment of adolescent panic disorder: results of a randomized controlled trial with short-term maintenance. Citation.

\section{Oerbeck 2014 \{published data only\}}

Oerbeck B, Overgaard KR, Stein MB, Pripp AH, Kristensen H. Treatment of selective mutism: a 5-year follow-up study. European Child \& Adolescent Psychiatry 2018;27(8):997-1009. [DOI: 10.1007/s00787-018-1110-7]

Oerbeck B, Stein MB, Pripp AH, Kristensen H. Selective mutism: follow-up study 1 year after end of treatment. European Child \& Adolescent Psychiatry 2015;24(7):757-66. [DOI: 10.1007/ s00787-014-0620-1]

Oerbeck B, Stein MB, Wentzel-Larsen T, Langsrud O, Kristensen $\mathrm{H}$. A randomized controlled trial of a home and school-based intervention for selective mutism - defocused communication and behavioural techniques. Child and Adolescent Mental Health 2014;19(3):192-8. [DOI: 10.1111/ camh.12045]

\section{Ollendick 2018 \{published data only\}}

Ollendick TH, White SW, Richey J, Kim-Spoon J, Ryan SM, Wieckowski AT, et al. Attention bias modification treatment for adolescents with social anxiety disorder. Behavior Therapy 2018;50(1):126-39. [DOI: 10.1016/j.beth.2018.04.002]

Ost 2015 \{published data only\}

Öst LG, Cederlund R, Reuterskiöld L. Behavioral treatment of social phobia in youth: does parent education training improve the outcome? Behaviour Research and Therapy 2015;67:19-29. [DOI: 10.1016/j.brat.2015.02.001] 


\section{Ozyurt 2018 \{published data only\}}

Ozyurt G, Gencer O, Ozturk Y, Ozbek A. Is Triple P effective in childhood anxiety disorder? A randomized controlled study. Psychiatry and Clinical Psychopharmacology 2019;29(4):570-8. [DOI: 10.1080/24750573.2018.1483790]

Pereira 2014 \{published data only (unpublished sought but not used)\}

Pereira Al, Marques T, Russo V, Barros L, Barrett P. Effectiveness of the friends for life program in Portuguese schools: study with a sample of highly anxious children. Psychology in the Schools 2014;51(6):647-57. [DOI: 10.1002/pits.21767]

\section{Rudy 2017 \{published data only\}}

NCT02051192. A brief behavioral treatment for anxiety in young children. clinicaltrials.gov/ct2/show/NCT02051192 (first received 31 January 2014).

Rudy BM, Zavrou S, Johnco C, Storch EA, Lewin AB. Parent-led exposure therapy: a pilot study of a brief behavioral treatment for anxiety in young children. Journal of Child and Family Studies 2017;26(9):2475-84. [DOI: 10.1007/s10826-017-0772-y]

Salzer 2018 \{published data only (unpublished sought but not used)\}

Dams J, Kronmüller KT, Leibing E, Steil R, Henningsen P, Leichsenring F, et al. Direct costs of social phobia in adolescents and cost-effectiveness of psychotherapy. Psychiatrische Praxis 2019;46(3):148-55. [DOI: 10.1055/a-0733-4999]

ISRCTN22752528. Cognitive therapy (CT-A) versus short-term psychodynamic psychotherapy (STPP-A) for social phobia (SP) in adolescents. www.isrctn.com/ISRCTN22752528 (first received 17 February 2011)

* Salzer S, Stefini A, Kronmuller KT, Leibing E, Leichsenring F, Henningsen P, et al. Cognitive-behavioral and psychodynamic therapy in adolescents with social anxiety disorder: a multicenter randomized controlled trial. Psychotherapy and Psychosomatics 2018;87(4):223-33. [DOI: 10.1159/000488990]

Sevi Tok 2016 \{published data only\}

Sevi Tok ES, Arkar H, Bildik T. The effectiveness of cognitive behavioral therapy, medication, or combined treatment for childhood anxiety disorders. Turk Psikiyatri Dergisi [Turkish Journal of Psychiatry] 2016;27(2):1-8.

\section{Storch 2019 \{published data only\}}

NCT01919970. Exposure-focused family-based CBT for youth with ASD and comorbid anxiety. clinicaltrials.gov/ct2/show/ NCT01919970 (first received 9 August 2013).

* Storch EA, Schneider SC, De Nadai AS, Selles RR, McBride NM, Grebe SC, et al. A pilot study of family-based exposure-focused Treatment for Youth with Autism Spectrum Disorder and Anxiety. Child Psychiatry \& Human Development 2019 Sep 6 [Epub ahead of print]. [DOI: 10.1007/s10578-019-00923-3]

\section{Sung 2011 \{published data only\}}

NCT01031511. Effect of cognitive behavioural therapy (CBT) for children with autistic spectrum disorders. clinicaltrials.gov/ show/nct01031511 (first received 14 December 2009).
* Sung M, Ooi YP, Goh TJ, Pathy P, Fung DS, Ang RP, et al. Effects of cognitive-behavioral therapy on anxiety in children with autism spectrum disorders: a randomized controlled trial. Child Psychiatry and Human Development 2011;42(6):634-49. [DOI: 10.1007/s10578-011-0238-1]

Weisz 2012 \{published data only\}

Chorpita BF, Weisz JR, Daleiden EL, Schoenwald SK, Palinkas LA, Miranda J, et al. Long-term outcomes for the Child STEPs randomized effectiveness trial: a comparison of modular and standard treatment designs with usual care. Journal of Consulting and Clinical Psychology 2013;81(6):999-1009. [DOI: 10.1037/a0034200]

NCT01178554. The Clinic Treatment Project (CTP). clinicaltrials.gov/ct2/show/nct01178554 (first received 10 August 2010).

* Weisz JR, Chorpita BF, Palinkas LA, Schoenwald SK, Miranda J, Bearman SK, et al. Testing standard and modular designs for psychotherapy treating depression, anxiety, and conduct problems in youth: a randomized effectiveness trial. Archives of General Psychiatry 2012;69(3):274-82. [DOI: 10.1001/ archgenpsychiatry.2011.147]

\section{References to studies awaiting assessment}

\section{ACTRN12613000217707 \{unpublished data only\}}

ACTRN12613000217707. A multi-centre randomised controlled trial of cognitive behavioural therapy for managing anxiety in adolescents with acquired brain injury. www.who.int/ trialsearch/Trial2.aspx?TrialID=ACTRN12613000217707 (first received 25 February 2013).

\section{ACTRN12615000730505 \{unpublished data only\}}

ACTRN12615000730505. Cognitive behaviour therapy for comorbid anxiety and sleep disorders: a randomized controlled trial. www.who.int/trialsearch/Trial2.aspx? TrialID=ACTRN12615000730505 (first received 15 July 2015).

ACTRN12619000619145 \{unpublished data only\} ACTRN12619000619145. SCAN Study - Self Compassion \& Cognitive Behavioural Therapy (CBT): Early family intervention for children with Type 1 diabetes experiencing anxiety. www.who.int/trialsearch/Trial2.aspx? TrialID=ACTRN12619000619145 (first received 26 April 2019).

\section{DRKS00012823 \{unpublished data only\}}

DRKS00012823. Short-term, long-term and cost-effectiveness of treating depression and anxiety disorders in children and adolescents a randomized controlled trial. www.who.int/ trialsearch/Trial2.aspx?TrialID=DRKS00012823 (first received 27 September 2017).

\section{IRCT20181006041250N \{unpublished data only\}} IRCT20181006041250N1. Effectiveness of coping cat-based cognitive-behavioral therapy (CBT) in group format on treatment of anxiety in children. www.who.int/trialsearch/ Trial2.aspx?TrialID=IRCT20181006041250N1 (first received 18 October 2018). 
ISRCTN59518816 \{unpublished data only\}

ISRCTN59518816. Does the treatment of anxiety in children with ADHD improve outcomes? www.who.int/trialsearch/Trial2.aspx? TrialID=ISRCTN59518816 (first received 29 September 2015).

\section{JPRN-UMIN000008724 \{unpublished data only\}}

JPRN-UMIN000008724. Japanese Anxiety Children/Adolescents CBT study. www.who.int/trialsearch/Trial2.aspx?TrialID=JPRNUMIN000008724 (first received 27 August 2012).

\section{JPRN-UMIN000032275 \{unpublished data only\}}

JPRN-UMIN000032275. Transdiagnostic intervention for anxiety and depression in children and adolescents. www.who.int/ trialsearch/Trial2.aspx?TrialID=JPRN-UMIN000032275 (first received 19 April 2018).

\section{JPRN-UMIN000033218 \{unpublished data only\}}

JPRN-UMIN000033218. The efficacy of group CBT for children with autism spectrum disorder to reduce anxiety symptoms. www. who.int/trialsearch/Trial2.aspx?TrialID=JPRNUMIN000033218 (first received 1 July 2018).

\section{NCT02810171 \{unpublished data only\}}

NCT02810171. Dimensional brain behavior predictors of CBT outcomes in pediatric anxiety. clinicaltrials.gov/show/ nct02810171 (first received 20 June 2016).

\section{NCT02977962 \{unpublished data only\}}

NCT02977962. Treatment of anxiety in late adolescents with autism. clinicaltrials.gov/show/nct02977962 (first received 28 November 2016).

\section{NCT03412227 \{unpublished data only\}}

NCT03412227. Transdiagnostic individual behavioral activation and exposure therapy. clinicaltrials.gov/show/nct03412227 (first received 15 October 2017).

\section{NCT03899948 \{unpublished data only\}}

NCT03899948. Teacher anxiety program for elementary students. clinicaltrials.gov/show/NCT03899948 (first received 28 March 2019).

\section{References to ongoing studies}

\section{ACTRN12616001552471 \{published data only\}}

ACTRN12616001552471. Effectiveness of school group-based acceptance and commitment therapy for children with anxiety: a randomised controlled trial. www.who.int/trialsearch/ Trial2.aspx?TrialID=ACTRN12616001552471 (first received 10 November 2016).

\section{NCT02725619 \{published data only\}}

NCT02725619. CBT for anxiety in children with autism. clinicaltrials.gov/show/nct02725619 (first received 1 April 2016).

\section{NCT02908321 \{published data only\}}

Kilburn TR, Sorensen MJ, Thastum M, Rapee RM, Rask CU, Arendt KB, et al. Rationale and design for cognitive behavioral therapy for anxiety disorders in children with autism spectrum disorder: a study protocol of a randomized controlled trial. Trials 2018;19:210. [DOI: 10.1186/s13063-018-2591-x]

* NCT02908321. CBT for anxiety disorder in children with comorbid ASD. clinicaltrials.gov/show/nct02908321 (first received 20 September 2016).

NCT03279471 \{published data only\}

NCT03279471. Specifying and treating anxiety in autism research. clinicaltrials.gov/show/nct03279471 (first received 12 September 2017).

\section{NCT03585010 \{published data only\}}

NCT03585010. Brain response associated with parent-based treatment for childhood anxiety disorders. clinicaltrials.gov/ct2/ show/NCT03585010 (first received 12 July 2018).

\section{Additional references}

\section{Ale 2015}

Ale CM, McCarthy DM, Rothschild LM, Whiteside SP. Components of cognitive behavioral therapy related to outcome in childhood anxiety disorders. Clinical Child and Family Psychology Review 2015;18(3):240-51.

\section{Angold 1995}

Angold A, Costello EJ, Messer SC, Pickles A, Winder F, Silver $\mathrm{D}$. The development of a short questionnaire for use in epidemiological studies of depression in children and adolescents. International Journal of Methods in Psychiatric Research 1995;5:237-49.

\section{APA 1980}

American Psychiatric Association. Diagnostic and Statistical Manual of Mental Disorders (DSM-III). 3rd edition. Washington, DC: American Psychiatric Association, 1980.

\section{APA 1987}

American Psychiatric Association. Diagnostic and Statistical Manual of Mental Disorders (DSM-III-R). 3rd revised edition. Washington, DC: American Psychiatric Association, 1987.

\section{APA 1994}

American Psychiatric Association. Diagnostic and Statistical Manual of Mental Disorders (DSM-IV). 4th edition. Washington, DC: American Psychiatric Association, 1994.

\section{APA 2000}

American Psychiatric Association. Diagnostic and Statistical Manual of Mental Disorders (DSM-IV-TR). 4th text revision edition. Washington, DC: American Psychiatric Association, 2000.

\section{APA 2013}

American Psychiatric Association. Diagnostic and Statistical Manual of Mental Disorders. 5th edition. Washington, DC: American Psychiatric Association, 2013. 


\section{Barlow 2010}

Barlow DH. Negative effects from psychological treatments: a perspective. American Psychologist 2010;65(1):13-20. [DOI: 10.1037/a0015643]

\section{Beck 1996}

Beck A, Steer RA, Brown GK. Beck Depression Inventory Section Edition Manual. San Antonio: The Psychological Corporation, 1996.

\section{Beesdo 2009}

Beesdo K, Knappe S, Pine DS. Anxiety and anxiety disorders in children and adolescents: developmental issues and implications for DSM-V. Psychiatric Clinics 2009;32(3):483-524.

\section{Beidel 1995}

Beidel DC, Turner SM, Morris TL. A new instrument to assess childhood social anxiety and phobia: the Social Phobia and Anxiety Inventory for Children. Psychological Assessment 1995;10:73-9.

\section{Bennett 2016}

Bennett K, Manassis K, Duda S, Bagnell A, Bernstein GA, Garland EJ, et al. Treating child and adolescent anxiety effectively: overview of systematic reviews. Clinical Psychology Review 2016;50:80-94.

\section{Birmaher 1999}

Birmaher B. Screen for Child Anxiety Related Emotional Disorders (SCARED). Pittsburgh, PA: Western Psychiatric Institute and Clinic, 1999.

\section{Bodden 2008b}

Bodden DH, Dirksen CD, Bögels SM. Societal burden of clinically anxious youth referred for treatment: a cost-of-illness study. Journal of Abnormal Child Psychology 2008;36(4):487-97.

\section{Bower 2005}

Bower P, Gilbody S. Stepped care in psychological therapies: access, effectiveness and efficiency - narrative literature review. British Journal of Psychiatry 2005;186:11-7.

\section{Breinholst 2012}

Breinholst S, Esbjorn BH, Reinholdt-Dunne ML, Stallard P. CBT for the treatment of child anxiety disorders: a review of why parental involvement has not enhanced outcomes. Journal of Anxiety Disorders 2012;26(3):416-24.

\section{Brown 2007}

Brown AM, Deacon BJ, Abramowitz JS, Dammann J, Whiteside SP. Parents' perceptions of pharmacological and cognitive-behavioral treatments for childhood anxiety disorders. Behaviour Research and Therapy 2007;45(4):819-28.

\section{Cartwright-Hatton 2004}

Cartwright-Hatton S, Roberts C, Chitsabesan P, Fothergill C, Harrington R. Systematic review of the efficacy of cognitivebehaviour therapies for childhood and adolescent anxiety disorders. British Journal of Clinical Psychology 2004;43:421-36.

\section{Cartwright-Hatton 2011}

Cartwright-Hatton S, McNally D, Field AP, Rust S, Laskey B, Dixon C, et al. A new parenting-based group intervention for young anxious children: results of a randomized controlled trial. Journal of the American Academy of Child and Adolescent Psychiatry 2011;50(3):242-51.e6.

\section{Chatterton 2019}

Chatterton ML, Rapee RM, Catchpool M, Lyneham HJ, Wuthrich V, Hudson JL, et al. Economic evaluation of stepped care for the management of childhood anxiety disorders: results from a randomised trial. Australian and New Zealand Journal of Psychiatry 2019;53:673-82.

\section{Chorpita 2000}

Chorpita BF, Yim L, Moffitt C, Umemoto LA, Francis SE. Assessment of symptoms of DSM-IV anxiety and depression in children: a revised child anxiety and depression scale. Behaviour Research and Therapy 2000;38(8):835-55.

\section{Compton 2004}

Compton SN, March JS, Brent D, Albano AM, Weering R, Curry J. Cognitive-behavioral psychotherapy for anxiety and depressive disorders in children and adolescents: an evidence-based medicine review. Journal of the American Academy Child and Adolescent Psychiatry 2004;43:930-59.

\section{Copeland 2014}

Copeland WE, Angold A, Shanahan L, Costello EJ. Longitudinal patterns of anxiety from childhood to adulthood: the Great Smoky Mountains Study. Journal of the American Academy of Child and Adolescent Psychiatry 2014;53(1):21-33.

\section{Covidence [Computer program]}

Veritas Health Innovation Covidence. Version Accessed 3 December 2018. Melbourne, Australia: Veritas Health Innovation. Available at covidence.org.

\section{Creswell 2020a}

Creswell C, Nauta M, Hudson J, March S, Reardon T, Arendt K, et al. Recommendations for reporting on treatment trials for child and adolescent anxiety disorders: an international consensus statement. Journal of Child Psychology and Psychiatry 2020 Jul 19 [Epub ahead of print]. [DOI: 10.1111/jcpp.13283]

\section{Creswell 2020b}

Creswell C, Waite P, Hudson J. Practitioner Review: Anxiety disorders in children and young people - assessment and treatment. Journal of Child Psychology and Psychiatry 2020;61(6):628-43. [DOI: 10.1111/jcpp.13186]

\section{Crowe 2017}

Crowe K, McKay D. Efficacy of cognitive-behavioral therapy for childhood anxiety and depression. Journal of Anxiety Disorders 2017;49:76-87.

\section{Deeks 2017}

Deeks JJ, Higgins JP, Altman DG, editor(s) on behalf of the Cochrane Statistical Methods Group. Chapter 9: Analysing data and undertaking meta-analyses. In: Higgins JPT, Churchill R, Chandler J, Cumpston MS, editor(s). Cochrane 
Handbook for Systematic Reviews of Interventions version 5.2.0 (updated June 2017). Cochrane, 2017. Available from www.training.cochrane.org/handbook.

\section{Duggan 2014}

Duggan C, Parry G, McMurran M, Davidson K, Dennis J. The recording of adverse events from psychological treatments in clinical trials: evidence from a review of NIHR-funded trials. Trials 2014;15:335.

\section{Essau 2003}

Essau CA. Comorbidity of anxiety disorders in adolescents. Depression and Anxiety 2003;18(1):1-6. [DOI: 10.1002/da.10107]

\section{Essau 2004}

Essau CA, Conradt J, Ederer EM. Anxiety prevention among school children. Versicherungsmedizin 2004;56:123-30.

\section{Essau 2014}

Essau CA, Lewinsohn PM, Olaya B, Seeley JR. Anxiety disorders in adolescents and psychosocial outcomes at age 30. Journal of Affective Disorders 2014;163:125-32.

\section{Evans 2017}

Evans R, Thirlwall K, Cooper P, Creswell C. Using symptom and interference questionnaires to identify recovery among children with anxiety disorders. Psychological Assessment 2017;29(7):835-43.

\section{Fineberg 2013}

Fineberg NA, Haddad PM, Carpenter L, Gannon B, Sharpe R, Young $\mathrm{AH}$, et al. The size, burden and cost of disorders of the brain in the UK. Journal of Psychopharmacology (Oxford, England) 2013;27(9):761-70.

\section{Galla 2012}

Galla BM, Wood JJ, Chiu AW, Langer DA, Jacobs J, Ifekwunigwe M, et al. One year follow-up to modular cognitive behavioral therapy for the treatment of pediatric anxiety disorders in an elementary school setting. Child Psychiatry and Human Development 2012;43(2):219-26.

\section{Gillies 2016}

Gillies D, Maiocchi L, Bhandari AP, Taylor F, Gray C, O'Brien L. Psychological therapies for children and adolescents exposed to trauma. Cochrane Database of Systematic Reviews 2016, Issue 10. Art. No: CD012371. [DOI: 10.1002/14651858.CD012371]

\section{Ginsburg 2018}

Ginsburg GS, Becker-Haimes EM, Keeton C, Kendall PC, Lyengar S, Sakolsky D, et al. Results from the Child/Adolescent Anxiety Multimodal Extended Long-Term Study (CAMELS): primary anxiety outcomes. Journal of the American Academy of Child and Adolescent Psychiatry 2018;57(7):471-80. [DOI: 10.1016/j.jaac.2018.03.017]

\section{GRADEpro GDT [Computer program]}

McMaster University (developed by Evidence Prime) GRADEpro GDT. Version (accessed 4 October 2018). Hamilton (ON): McMaster University (developed by Evidence Prime). Available at gradepro.org.

\section{Green 2004}

Green H, McGinnity A, Meltzer H, Ford T, Goodman R. Mental health of children and young people in Great Britain, 2004. no-pa.uk/wp-content/uploads/2015/02/Mental-health-ofchildren.pdf (accessed 4 October 2018).

\section{Health Services Research Unit 2004}

Health Services Research Unit. Database of ICCs: spreadsheet (empirical estimates of ICCs from changing professional practice studies). https://www.abdn.ac.uk/hsru/what-we-do/ tools/index.php (accessed 1 September 2020).

\section{Higgins 2003}

Higgins JP, Thompson SG, Deeks JJ, Altman DG. Measuring inconsistency in meta-analyses. BMJ 2003;327:557-60.

\section{Higgins 2011}

Higgins JP, Deeks JJ, Altman DG, editor(s). Chapter 16: Special topics in statistics. In: Higgins JPT, Green S, editor(s). Cochrane Handbook for Systematic Reviews of Interventions Version 5.1.0 (updated March 2011). The Cochrane Collaboration, 2011. Available from handbook.cochrane.org.

\section{Higgins 2017}

Higgins JP, Altman DG, Sterne JA, editor(s). Chapter 8: Assessing risk of bias in included studies. In: Higgins JPT, Churchill R, Chandler J, Cumpston MS, editor(s). Cochrane Handbook for Systematic Reviews of Interventions version 5.2.0 (updated June 2017). Cochrane, 2017. Available from www.training.cochrane.org/handbook.

\section{Hirshfeld-Becker 2010}

Hirshfeld-Becker DR, Masek B, Henin A, Blakely LR, PollockWurman RA, McQuade J, et al. Cognitive behavioral therapy for 4- to 7-year-old children with anxiety disorders: a randomized clinical trial. Journal of Consulting and Clinical Psychology 2010;78(4):498-510.

\section{Holland 1995}

Holland D, Dadds M. Diagnostic Interview Schedule for Children, Adolescents and Parents. Brisbane: Griffith University, 1995.

\section{Keeton 2013}

Keeton CP, Ginsburg GS, Drake KL, Sakolsky D, Kendall PC, Birmaher B, et al. Benefits of child-focused anxiety treatments for parents and family functioning. Depression and Anxiety 2013;30(9):865-72.

\section{Kendall 1993}

Kendall PC. Cognitive-behavioral therapies with youth: guiding theory, current status, and emerging developments. Journal of Consulting and Clinical Psychology 1993;61(2):235-47.

\section{Kendall 2006}

Kendall PC, Hedtke KA. Cognitive-Behavioral Therapy for Anxious Children: Therapist Manual. 1st edition. Ardmore, PA: Workbook Publishing, 2006.

\section{Kendall 2016}

Kendall PC, Cummings CM, Villabo MA, Narayanan MK, Treadwell K, Birmaher B, et al. Mediators of change in the Child/ 
Adolescent Anxiety Multimodal Treatment Study. Journal of Consulting and Clinical Psychology 2016;84(1):1-14.

\section{Kovacs 1989}

Kovacs M, Gatsonis C, Paulauskas S, Richards C. Depressive disorders in childhood. IV. A longitudinal study of comorbidity with and the risk for anxiety disorders. Archives of General Psychiatry 1989;46:776-82.

\section{Kreuze 2018}

Kreuze LJ, Pijnenborg GHM, de Jonge YB, Nauta MH. Cognitivebehavior therapy for children and adolescents with anxiety disorders: a meta-analysis of secondary outcomes. Journal of Anxiety Disorders 2018;60:43-57.

\section{La Greca 1998}

La Greca A. Manual for Social Anxiety Scales for Children and Adolescents. Coral Gables, FL: University of Miami, 1998.

\section{Lawrence 2015}

Lawrence D, Johnson S, Hafekost J, de Haan KB, Sawyer M, Ainley J, et al. The Mental Health of Children and Adolescents. Report on the Second Australian Child and Adolescent Survey of Mental Health and Wellbeing. www1.health.gov.au/internet/main/publishing.nsf/ Content/9DA8CA21306FE6EDCA257E2700016945/\$File/ child2.pdf (accessed 4 October 2020).

\section{Leyfer 2013}

Leyfer O, Gallo K, Cooper-Vince C, Pincus DB. Patterns and predictors of comorbidity of DSM-IV anxiety disorders in a clinical sample of children and adolescents. Journal of Anxiety Disorders 2013;27(3):306-11.

\section{March 1997}

March JS, Parker JD, Sullivan K, Stallings P, Conners CK. The Multidimensional Anxiety Scale for Children (MASC): factor structure, reliability, and validity. Journal of the American Academy of Child and Adolescent Psychiatry 1997;36(4):554-65.

\section{Masia-Warner 2005}

Masia-Warner C, Klein RG, Dent HC, Fisher PH, Alvir J, Albano AM, et al. School-based intervention for adolescents with social anxiety disorder: results of a controlled study. Journal of Abnormal Child Psychology 2005;33(6):707-22.

\section{Mavranezouli 2015}

Mavranezouli I, Mayo-Wilson E, Dias S, Kew K, Clark DM, Ades AE, et al. The cost effectiveness of psychological and pharmacological interventions for social anxiety disorder: a model-based economic analysis. PLOS ONE 2015;10(10):e0140704. [DOI: 10.1371/journal.pone.0140704]

\section{Merikangas 2011}

Merikangas KR, He JP, Burstein M, Swendsen J, Avenevoli S, Case B, et al. Service utilization for lifetime mental disorders in U.S. adolescents: results of the National Comorbidity SurveyAdolescent Supplement (NCS-A). Journal of the American Academy of Child and Adolescent Psychiatry 2011;50(1):32-45.

\section{Moher 2015}

Moher D, Shamseer L, Clarke M, Ghersi D, Liberati A, Petticrew M, et al, PRISMA-P Group. Preferred reporting items for systematic review and meta-analysis protocols (PRISMA-P) 2015 statement. Systematic Review 2015;4:1. [DOI: 10.1186/2046-4053-4-1]

\section{Monga 2015}

Monga S, Rosenbloom BN, Tanha A, Owens M, Young A. Comparison of child-parent and parent-only cognitivebehavioral therapy programs for anxious children aged 5 to 7 years: short- and long-term outcomes. Journal of the American Academy of Child and Adolescent Psychiatry 2015;54(2):138-46.

\section{Myers 2002}

Myers K, Winters NC. Ten year review of rating scales II: scales for internalising disorders. Journal of the American Academy of Child and Adolescent Psychiatry 2002;41:634-59.

\section{NICE 2013}

National Institute for Health and Care Excellence. Social anxiety disorder: recognition, assessment and treatment: Clinical guideline [CG159]. www.nice.org.uk/guidance/cg159 (accessed 4 October 2020).

\section{O'Kearney 2006}

O'Kearney RT, Anstey KJ, von Sanden C. Behavioural and cognitive behavioural therapy for obsessive compulsive disorder in children and adolescents. Cochrane Database of Systematic Reviews 2006, Issue 4. Art. No: CD004856. [DOI: 10.1002/14651858.CD004856.pub2]

\section{Ollendick 2010}

Ollendick TH, Öst LG, Reuterskiöld L, Costa N. Comorbidity in youth with specific phobias: impact of comorbidity on treatment outcome and the impact of treatment on comorbid disorders. Behaviour Research and Therapy 2010;48(9):827-31.

\section{Pennant 2015}

Pennant ME, Loucas CE, Whittington C, Creswell C, Fonagy P, Fuggle $\mathrm{P}$, et al. Computerised therapies for anxiety and depression in children and young people: a systematic review and meta-analysis. Behaviour Research and Therapy 2015;67:1-18.

\section{Perihan 2019}

Perihan C, Burke M, Bowman-Perrott L, Bicer A, Gallup J, Thompson J, et al. Effects of cognitive behavioral therapy for reducing anxiety in children with high functioning ASD: a systematic review and meta-analysis. Journal of Autism and Developmental Disorders 2019;27:1-5. [DOI: 10.1007/ s10803-019-03949-7]

\section{Peris 2015}

Peris TS, Compton SN, Kendall PC, Birmaher B, Sherrill J, March J, et al. Trajectories of change in youth anxiety during cognitive-behavior therapy. Journal of Consulting and Clinical Psychology 2015;83(2):239-52. 


\section{Peris 2017}

Peris TS, Caporino NE, O'Rourke S, Kendall PC, Walkup JT, Albano AM, et al. Therapist-reported features of exposure tasks that predict differential treatment outcomes for youth with anxiety. Journal of the American Academy of Child and Adolescent Psychiatry 2017;56(12):1043-52.

\section{Peters 2008}

Peters JL, Sutton AJ, Jones DR, Abrams KR, Rushton L. Contour-enhanced meta-analysis funnel plots help distinguish publication bias from other causes of asymmetry. Journal of Clinical Epidemiology 2008;61(10):991-6.

\section{Pine 2009}

Pine DS, Helfinstein SM, Bar-Haim Y, Nelson E, Fox NA. Challenges in developing novel treatments for childhood disorders: lessons from research on anxiety. Neuropsychopharmacology 2009;34(1):213-28.

\section{Polanczyk 2015}

Polanczyk GV, Salum GA, Sugaya LS, Caye A, Rohde LA. Annual research review: a meta-analysis of the worldwide prevalence of mental disorders in children and adolescents. Journal of Child Psychology and Psychiatry and Allied Disciplines 2015;56(3):345-65.

\section{Rao 1992}

Rao JN, Scott AJ. A simple method for the analysis of clustered binary data. Biometrics 1992;48(2):577-85.

\section{Reardon 2017}

Reardon T, Harvey K, Baranowska M, O'Brien D, Smith L, Creswell $C$. What do parents perceive are the barriers and facilitators to accessing psychological treatment for mental health problems in children and adolescents? A systematic review of qualitative and quantitative studies. European Child \& Adolescent Psychiatry 2017;26(6):623-47.

\section{Reardon 2020}

Reardon T, Harvey K, Creswell C. Seeking and accessing professional support for child anxiety in a community sample. European Child and Adolescent Psychiatry 2020;29:649-64. [DOI: 10.1007/s00787-019-01388-4]

\section{Review Manager 2014 [Computer program]}

Nordic Cochrane Centre, The Cochrane Collaboration Review Manager 5 (RevMan 5). Version 5.3. Copenhagen: Nordic Cochrane Centre, The Cochrane Collaboration, 2014.

\section{Reynolds 1985}

Reynolds CR, Richmond BO. Revised Children's Manifest Anxiety Scale (RCAMS) Manual. Los Angeles: Western Psychological Services, 1985

\section{Reynolds 2012}

Reynolds S, Wilson C, Austin J, Hooper L. Effects of psychotherapy for anxiety in children and adolescents: a metaanalytic review. Clinical Psychology Review 2012;32(4):251-62.

\section{Sanchez-Garcia 2009}

Sanchez-Garcia R, Olivares J. Effectiveness of a program for early detection/intervention in children/adolescents with generalized social phobia. Anales de Psicologia 2009;25:241-9.

\section{Schünemann 2017}

Schünemann HJ, Oxman AD, Vist GE, Higgins JP, Deeks JJ, Glasziou P, et al, on behalf of the Cochrane Applicability and Recommendations Methods Group. Chapter 12: Interpreting results and drawing conclusions. In: Higgins JPT, Churchill R, Chandler J, Cumpston MS, editor(s). Cochrane Handbook for Systematic Reviews of Interventions version 5.2.0 (updated June 2017). Cochrane, 2017. Available from www.training.cochrane.org/handbook.

\section{Seligman 2011}

Seligman L, Ollendick T. Cognitive behavioral therapy for anxiety disorders in youth. Child \& Adolescent Psychiatric Clinics of North America 2011;20(2):217-38.

\section{Shaffer 1983}

Shaffer D, Gould MS, Brasic J, Ambrosini P, Fisher P, Bird H, et al. A children's global assessment scale (CGAS). Archives of General Psychiatry 1983;40(11):1228-31.

\section{Silverman 1987}

Silverman WK. Anxiety Disorder Interview for Children (ADIC). New York, NY: Graywind Publications, 1987.

\section{Silverman 2008}

Silverman WK, Pina AA, Viswesvaran C. Evidencebased psychosocial treatments for phobic and anxiety disorders in children and adolescents. Journal of Clinical Child and Adolescent Psychology 2008;37:105-30.

\section{Silverman 2009}

Silverman WK, Kurtines WM, Jaccard J, Pina AA. Directionality of change in youth anxiety treatment involving parents: an initial examination. Journal of Consulting and Clinical Psychology 2009;77:474-85.

\section{Southam-Gerow 2010}

Southam-Gerow MA, Weisz JR, Chu BC, McLeod BD, Gordis EB, Connor-Smith JK. Does cognitive behavioral therapy for youth anxiety outperform usual care in community clinics? An initial effectiveness test. Journal of the American Academy of Child and Adolescent Psychiatry 2010;49(10):1043-52.

\section{Spence 1997}

Spence S. Stucture of anxiety symptoms amongst children: a confirmatory factor-analytic study. Journal of Abnormal Psychology 1997;106:280-97.

\section{Spielberger 1973}

Spielberger C, Edwards C, Montuori J, Lushene R. State-Trait Anxiety Inventory for Children. Palto Alto, CA: Consulting Psychologist Press, 1973.

\section{Stata 2012 [Computer program]}

Stata. Version Stata/IC 12.1. College Station, TX: StataCorp, 2012. Available at www.stata.com. 


\section{Sterne 2017}

Sterne JA, Egger M, Moher D, Boutron I, editor(s). Chapter 10: Addressing reporting biases. In: Higgins JPT, Churchill R, Chandler J, Cumpston MS, editor(s). Cochrane Handbook for Systematic Reviews of Interventions version 5.2.0 (updated June 2017). Cochrane, 2017. Available from www.training.cochrane.org/handbook.

\section{Suveg 2006}

Suveg C, Kendall PC, Comer JS, Robin J. Emotion-focused cognitive-behavioral therapy for anxious youth: a multiplebaseline evaluation. Journal of Contemporary Psychotherapy 2006;36(2):77-85.

\section{Ung 2015}

Ung D, Selles R, Small BJ, Storch EA. A systematic review and meta-analysis of cognitive-behavioral therapy for anxiety in youth with high-functioning autism spectrum disorders. Child Psychiatry and Human Development 2015;46(4):533-47.

\section{van Steensel 2013}

van Steensel F, Bogels S, Perrin S. Anxiety disorders in children and adolescents with autistic spectrum disorders: a meta-analysis. Clinical Child and Family Psychology Review 2011;14:302-17.

\section{Waite 2014}

Waite P, Creswell C. Children and adolescents referred for treatment of anxiety disorders: differences in clinical characteristics. Journal of Affective Disorders 2014;167:326-32.

\section{Waite 2015}

Waite P, Codd J, Creswell C. Interpretation of ambiguity: differences between children and adolescents with and without an anxiety disorder. Journal of Affective Disorders 2015;188:194-201.

\section{Wang 2005}

Wang PS, Berglund P, Olfson M, Pincus HA, Wells KB, Kessler RC. Failure and delay in initial treatment contact after first onset of mental disorders in the National Comorbidity Survey Replication. Archives of General Psychiatry 2005;62(6):603-13.

\section{Wang 2017}

Wang Z, Whiteside S, Sim L, Farah W, Morrow AS, Alsawas M, et al. Comparative effectiveness and safety of cognitive behavioral therapy and pharmacotherapy for childhood anxiety disorders: a systematic review and meta-analysis. JAMA Pediatrics 2017;171(11):1049-56. [DOI: 10.1001/jamapediatrics.2017.3036]

\section{Warner 2011}

Warner CM, Colognori D, Kim RE, Reigada LC, Klein RG, BrownerElhanan KJ, et al. Cognitive-behavioral treatment of persistent functional somatic complaints and pediatric anxiety: an initial controlled trial. Depression and Anxiety 2011;28(7):551-9.

\section{Warwick 2016}

Warwick H, Reardon T, Cooper P, Murayama K, Reynolds S, Wilson $\mathrm{C}$, et al. Complete recovery from anxiety disorders following cognitive behavior therapy in children and adolescents: a meta-analysis. Clinical Psychology Review 2016;52:77-91.

\section{Whiteside 2015}

Whiteside SP, Ale CM, Young B, Dammann JE, Tiede MS, Biggs BK. The feasibility of improving CBT for childhood anxiety disorders through a dismantling study. Behaviour Research and Therapy 2015;73:83-9.

\section{WHO 1978}

World Health Organization. The ICD-9 Classification of Mental and Behavioural Disorders: Clinical Description and Diagnostic Guidelines. Geneva: World Health Organization, 1978.

\section{WHO 1992}

World Health Organization. The ICD-10 Classification of Mental and Behavioural Disorders: Clinical Description and Diagnostic Guidelines. Geneva: World Health Organization, 1992.

\section{Young 2006}

Young BJ, Beidel DC, Turner SM, Ammerman RT, McGraw K, Coaston SC. Pretreatment attrition and childhood social phobia: parental concerns about medication. Journal of Anxiety Disorders 2006;20(8):1133-47.

\section{Zhou 2019}

Zhou X, Zhang Y, Furukawa TA, Cuijpers P, Pu J, Weisz JR, et al. Different types and acceptability of psychotherapies for acute anxiety disorders in children and adolescents: a network meta-analysis. JAMA Psychiatry 2019;76(1):41-50. [DOI: 10.1001/ jamapsychiatry.2018.3070]

\section{References to other published versions of this review \\ James 2005}

James AA, Soler A, Weatherall RR. Cognitive behavioural therapy for anxiety disorders in children and adolescents. Cochrane Database of Systematic Reviews 2005, Issue 4. Art. No: CD004690. [DOI: 10.1002/14651858.CD004690]

\section{James 2015}

James AC, James G, Cowdrey FA, Soler A, Choke A. Cognitive behavioural therapy for anxiety disorders in children and adolescents. Cochrane Database of Systematic Reviews 2015, Issue 2. Art. No: CD004690. [DOI: 10.1002/14651858.CD004690.pub4]

\section{James 2018}

James AC, Reardon T, Soler A, James G, Creswell C. Cognitive behavioural therapy for anxiety disorders in children and adolescents. Cochrane Database of Systematic Reviews 2018, Issue 10. Art. No: CD013162. [DOI: 10.1002/14651858.CD013162]

* Indicates the major publication for the study 


\section{CHARACTERISTICS OF STUDIES}

Characteristics of included studies [ordered by study ID]

Afshari 2014

\section{Study characteristics}

Study design: randomised controlled trial
Total duration of study: 10 - to 12 -week treatment, 3-month follow-up
Number of study centres and location: 1 , Iran
Study setting: private clinic
Date of study: October 2011 to August 2012
N $=\mathbf{3 4}$ (CBT: $\mathrm{N}=12 ; \mathrm{CBT}$ (emotion-focused): $\mathrm{N}=12 ;$ no treatment control: $\mathrm{N}=10)$
Mean age (SD): 10.57 (2.27)
Age range: 9 to 13 years
Inclusion criteria: separation anxiety disorder diagnosis (based on the ADIS-C/P), not receiving med-
ication or stopped using drugs for at least 1 month before pretest
Exclusion criteria: IQ < 80 , learning disorders, psychosis, serious mental or physical problems, low in-
terest in continuing the study

\begin{tabular}{|c|c|}
\hline \multirow[t]{15}{*}{ Interventions } & CBT \\
\hline & Intervention: Coping Cat \\
\hline & Concomitant/excluded medications: no medication or stopped for at least 1 month before pretest \\
\hline & Delivery format: child-only sessions, does not specify if parent sessions included; group format \\
\hline & Therapist contact time: 10 hours ( $10 \times 1$ hour) \\
\hline & Who delivers the intervention: advanced PhD students in psychology \\
\hline & CBT (emotion-focused) \\
\hline & Intervention: emotion-focused CBT (ECBT) (Suveg 2006) \\
\hline & Concomitant/excluded medications: no medication or stopped for at least 1 month before pretest \\
\hline & Delivery format: child-only sessions, and 2 parent sessions; group format \\
\hline & Therapist contact time: 12 hours (child: $10 \times 1$; parent: $2 \times 1$ ) \\
\hline & Who delivers the intervention: advanced PhD students in psychology \\
\hline & Wait list/no treatment \\
\hline & Intervention: no treatment \\
\hline & Concomitant/excluded medications: no medication or stopped for at least 1 month before pretest \\
\hline \multirow[t]{2}{*}{ Outcomes } & Reduction in anxiety symptoms (child report) post-treatment and 3-month follow-up: SCARED-C \\
\hline & Reduction in anxiety symptoms (parent report) post-treatment and 3-month follow-up: SCARED-P \\
\hline
\end{tabular}


Afshari 2014 (Continued)

Risk of bias

\begin{tabular}{|c|c|c|}
\hline Bias & Authors' judgement & Support for judgement \\
\hline $\begin{array}{l}\text { Random sequence genera- } \\
\text { tion (selection bias) }\end{array}$ & Unclear risk & Judgement Comment: No detail given \\
\hline $\begin{array}{l}\text { Allocation concealment } \\
\text { (selection bias) }\end{array}$ & Unclear risk & Judgement Comment: No detail given \\
\hline $\begin{array}{l}\text { Blinding of participants } \\
\text { and personnel (perfor- } \\
\text { mance bias) } \\
\text { All outcomes }\end{array}$ & Low risk & $\begin{array}{l}\text { Judgement Comment: Even though blinding was not possible, we judged that } \\
\text { it was unlikely this led to a departure from the intended intervention }\end{array}$ \\
\hline $\begin{array}{l}\text { Blinding of outcome as- } \\
\text { sessment (detection bias) } \\
\text { All outcomes }\end{array}$ & Low risk & $\begin{array}{l}\text { Judgement Comment: No detail given but child/parent report questionnaires } \\
\text { only }\end{array}$ \\
\hline $\begin{array}{l}\text { Incomplete outcome data } \\
\text { (attrition bias) } \\
\text { All outcomes }\end{array}$ & High risk & $\begin{array}{l}\text { Judgement Comment: Reason for missing outcome data (low interest in at- } \\
\text { tending sessions) likely to be related to outcome }\end{array}$ \\
\hline $\begin{array}{l}\text { Selective reporting (re- } \\
\text { porting bias) }\end{array}$ & Low risk & Judgement Comment: Outcomes for each measure reported \\
\hline $\begin{array}{l}\text { Other source of bias - } \\
\text { Therapy integrity }\end{array}$ & Unclear risk & $\begin{array}{l}\text { Judgement Comment: Therapists PhD students. No detail on any attempt to } \\
\text { maintain or measure therapy integrity }\end{array}$ \\
\hline Other bias & Unclear risk & $\begin{array}{l}\text { Judgement Comment: Timings for post-treatment assessment not detailed. } \\
\text { Treatment period different for } 2 \text { treatment groups }\end{array}$ \\
\hline
\end{tabular}

Arendt 2016

\section{Study characteristics}

\begin{tabular}{ll}
\hline Sethods & Study design: randomised controlled trial \\
Total duration of study: non-controlled 3-month and 12-month follow-up \\
Number of study centres and location: 1, in Denmark \\
Study setting: university research clinic \\
Date of study: October 2011 to August 2012 \\
N $=\mathbf{1 0 9}$ (CBT: $\mathrm{N}=56 ;$ waitlist: $\mathrm{N}=53)$ \\
Mean age (SD): 11.78 (2.74) \\
Age range: 7 to 16 \\
Gender: 62 (57\%) female \\
Inclusion criteria: primary anxiety disorder diagnosis (based on the ADIS-C/P) \\
Exclusion criteria: psychosis, untreated attention deficit hyperactivity disorder (ADHD), intellectual \\
disability, and severe behaviour disorders
\end{tabular}


Arendt 2016 (Continued)

Notes: funded by TrygFonden (grant ID n10691). Authors declare no competing interests

CBT
Interventions
Concomitant/excluded medications: encouraged not to change medication during treatment period
Delivery format: child and parent sessions; group
Therapist contact time: 20 hours (10 x 2 hours), plus 1-hour booster
Who delivers the intervention: psychologists, plus graduate psychologists as assistants
Waitlist/no treatment
Intervention: 3-month waitlist
Concomitant/excluded medications: encouraged not to change medication during treatment period
Remission of primary anxiety diagnosis post-treatment: ADIS-C/P
Remission of all anxiety disorder diagnoses post-treatment: ADIS-C/P
Reduction in anxiety symptoms (child report) post-treatment: SCAS-C
Reduction in anxiety symptoms (parent report) post-treatment: SCAS-P (mother report)
Reduction in depressive symptoms post-treatment: SMFQ-C

Notes

\section{Risk of bias}

\begin{tabular}{|c|c|c|}
\hline Bias & Authors' judgement & Support for judgement \\
\hline $\begin{array}{l}\text { Random sequence genera- } \\
\text { tion (selection bias) }\end{array}$ & Low risk & $\begin{array}{l}\text { Judgement Comment: Block randomisation, computer-generated list. Good } \\
\text { detail }\end{array}$ \\
\hline $\begin{array}{l}\text { Allocation concealment } \\
\text { (selection bias) }\end{array}$ & Low risk & $\begin{array}{l}\text { Judgement Comment: Sequence list and allocation concealed from re- } \\
\text { searchers and therapists until start of treatment }\end{array}$ \\
\hline $\begin{array}{l}\text { Blinding of participants } \\
\text { and personnel (perfor- } \\
\text { mance bias) } \\
\text { All outcomes }\end{array}$ & Low risk & $\begin{array}{l}\text { Judgement Comment: Even though blinding was not possible, we judged that } \\
\text { it was unlikely this led to a departure from the intended intervention }\end{array}$ \\
\hline $\begin{array}{l}\text { Blinding of outcome as- } \\
\text { sessment (detection bias) } \\
\text { All outcomes }\end{array}$ & Low risk & Judgement Comment: ADIS assessors blind to group and prior diagnosis \\
\hline $\begin{array}{l}\text { Incomplete outcome data } \\
\text { (attrition bias) } \\
\text { All outcomes }\end{array}$ & Low risk & $\begin{array}{l}\text { Judgement Comment: Missing data reported in consort and have been imput- } \\
\text { ed using appropriate methods. Small amount of missing data }\end{array}$ \\
\hline $\begin{array}{l}\text { Selective reporting (re- } \\
\text { porting bias) }\end{array}$ & Low risk & $\begin{array}{l}\text { Judgement Comment: Outcomes for planned measures all reported, and } \\
\text { where collected and not reported acknowledged }\end{array}$ \\
\hline $\begin{array}{l}\text { Other source of bias - } \\
\text { Therapy integrity }\end{array}$ & Unclear risk & $\begin{array}{l}\text { Judgement Comment: Therapists trained and supervised. No assessment of } \\
\text { adherence }\end{array}$ \\
\hline
\end{tabular}


Arendt 2016 (Continued)

Other bias Low risk Judgement Comment: The study appears to be free of other sources of bias

Barrett 1996

\section{Study characteristics}

\begin{tabular}{ll}
\hline Methods & Study design: randomised controlled trial \\
Total duration of study: non-controlled 6-month, 12-month, and 6-year follow-up \\
Number of study centres and location: 1, in Australia \\
Study setting: university research clinic \\
\hline
\end{tabular}

Participants $\quad \mathbf{N}=\mathbf{8 5}($ CBT-child: $\mathrm{N}=31$; CBT-family: $\mathrm{N}=27$; waitlist: $\mathrm{N}=27$ )

Mean age (SD): CBT-child: 9.7 (2.5); CBT-family: 10.1 (1.9); waitlist: 8.2 (1.9)

Age range: 7 to 14

Gender: $34(43 \%)$ female

Inclusion criteria: principal diagnosis of overanxious disorder, separation anxiety disorder, or social phobia (based on ADIS-C/P)

Exclusion criteria: intellectual or physical disabilities, anti-anxiety or depression medication, parents involved in acute marital breakdown

Interventions

\section{CBT- child}

Intervention: Coping Koala (adaptation of Coping Cat)

Concomitant/excluded medications: anti-anxiety and depression medication excluded

Delivery format: child-only sessions; individual

Therapist contact time: 12 to 16 hours ( $12 \times 60$ to 80 minutes)

Who delivers the intervention: registered clinical psychologists

CBT- family

Intervention: Coping Koala (adaptation of Coping Cat), plus Family Anxiety Management (FAM)

Concomitant/excluded medications: anti-anxiety and depression medication excluded

Delivery format: child-only sessions, and parent and child sessions (FAM); individual

Therapist contact time: 12 to 16 hours ( $12 \times 60$ to 80 minutes)

Who delivers the intervention: registered clinical psychologists

\section{Waitlist/no treatment}

Intervention: 12-week waitlist

Concomitant/excluded medications: anti-anxiety and depression medication excluded 
Barrett 1996 (Continued)

Reduction in depressive symptoms post-treatment: $C D I$

\section{Notes}

\section{Risk of bias}

\begin{tabular}{|c|c|c|}
\hline Bias & Authors' judgement & Support for judgement \\
\hline $\begin{array}{l}\text { Random sequence genera- } \\
\text { tion (selection bias) }\end{array}$ & Unclear risk & $\begin{array}{l}\text { Judgement Comment: Lacks details; randomisation to treatment groups/ther- } \\
\text { apists unclear and refers to "alternating" }\end{array}$ \\
\hline $\begin{array}{l}\text { Allocation concealment } \\
\text { (selection bias) }\end{array}$ & Unclear risk & Judgement Comment: No detail given \\
\hline $\begin{array}{l}\text { Blinding of participants } \\
\text { and personnel (perfor- } \\
\text { mance bias) } \\
\text { All outcomes }\end{array}$ & Low risk & $\begin{array}{l}\text { Judgement Comment: Even though blinding was not possible, we judged that } \\
\text { it was unlikely this led to a departure from the intended intervention }\end{array}$ \\
\hline $\begin{array}{l}\text { Blinding of outcome as- } \\
\text { sessment (detection bias) } \\
\text { All outcomes }\end{array}$ & Low risk & Judgement Comment: Blinded assessors \\
\hline $\begin{array}{l}\text { Incomplete outcome data } \\
\text { (attrition bias) } \\
\text { All outcomes }\end{array}$ & Unclear risk & $\begin{array}{l}\text { Judgement Comment: Dropouts reported, but number randomised vs com- } \\
\text { pleters not clear and not reported for individual measures }\end{array}$ \\
\hline $\begin{array}{l}\text { Selective reporting (re- } \\
\text { porting bias) }\end{array}$ & Low risk & Judgement Comment: Outcomes for all measures reported \\
\hline $\begin{array}{l}\text { Other source of bias - } \\
\text { Therapy integrity }\end{array}$ & Low risk & $\begin{array}{l}\text { Judgement Comment: Protocol adherence assessed and confirmed for } 60 \% \\
\text { sessions by blind clinician }\end{array}$ \\
\hline Other bias & Low risk & Judgement Comment: The study appears to be free of other sources of bias \\
\hline
\end{tabular}

\section{Barrett 1998}

\section{Study characteristics}

\begin{tabular}{ll}
\hline Study design: randomised controlled trial \\
Total duration of study: non-controlled 12-month follow-up \\
Number of study centres and location: 1 , in Australia \\
Study setting: clinic \\
N $=\mathbf{6 0}$ (CBT-child: $\mathrm{N}=23$; CBT-family: $\mathrm{N}=17$; waitlist: $\mathrm{N}=20)$ \\
Age range: 7 to 14 \\
Gender: 28 (46.7\%) female \\
Inclusion criteria: principal diagnosis of overanxious disorder, separation anxiety disorder, or social \\
phobia (based on ADIS-C/P)
\end{tabular}


Barrett 1998 (Continued)

Exclusion criteria: principal diagnosis of simple phobia, intellectual or physical disabilities, currently taking anti-anxiety or depression medication, parents involved in acute marital breakdown

\begin{tabular}{|c|c|c|}
\hline Interventions & $\begin{array}{l}\text { CBT- child } \\
\text { Intervention: Coping } \\
\text { Concomitant/exclud } \\
\text { Delivery format: chil } \\
\text { Therapist contact tin } \\
\text { Who delivers the int } \\
\text { CBT- family } \\
\text { Intervention: Coping } \\
\text { Concomitant/exclud } \\
\text { Delivery format: chil } \\
\text { Therapist contact tin } \\
\text { Who delivers the int } \\
\text { Waitlist/no treatmen } \\
\text { Intervention: } 12 \text {-wee } \\
\text { Concomitant/exclud }\end{array}$ & $\begin{array}{l}\text { Koala (adaptation of Coping Cat) and Group Family Anxiety Management } \\
\text { d medications: anti-anxiety and depression medication excluded } \\
\text { and parent sessions; group } \\
\text { e: } 24 \text { hours ( } 12 \times 2 \text { hours) } \\
\text { vention: registered clinical psychologists } \\
\text { waitlist } \\
\text { d medications: anti-anxiety and depression medication excluded }\end{array}$ \\
\hline Outcomes & \multicolumn{2}{|c|}{ Remission of all anxiety disorder diagnoses post-treatment: $A D I S-C / P$} \\
\hline \multicolumn{3}{|l|}{ Notes } \\
\hline \multicolumn{3}{|l|}{ Risk of bias } \\
\hline Bias & Authors' judgement & Support for judgement \\
\hline $\begin{array}{l}\text { Random sequence genera- } \\
\text { tion (selection bias) }\end{array}$ & Unclear risk & $\begin{array}{l}\text { Judgement Comment: No detail given. Refers to matching across conditions } \\
\text { but does not detail procedure }\end{array}$ \\
\hline $\begin{array}{l}\text { Allocation concealment } \\
\text { (selection bias) }\end{array}$ & Unclear risk & Judgement Comment: No detail given \\
\hline $\begin{array}{l}\text { Blinding of participants } \\
\text { and personnel (perfor- } \\
\text { mance bias) } \\
\text { All outcomes }\end{array}$ & Low risk & $\begin{array}{l}\text { Judgement Comment: Even though blinding was not possible, we judged that } \\
\text { it was unlikely this led to a departure from the intended intervention }\end{array}$ \\
\hline $\begin{array}{l}\text { Blinding of outcome as- } \\
\text { sessment (detection bias) } \\
\text { All outcomes }\end{array}$ & Low risk & Judgement Comment: Blind assessors \\
\hline $\begin{array}{l}\text { Incomplete outcome data } \\
\text { (attrition bias) } \\
\text { All outcomes }\end{array}$ & Unclear risk & $\begin{array}{l}\text { Judgement Comment: Insufficient detail on completers vs number ran- } \\
\text { domised for each measure or how missing data were managed }\end{array}$ \\
\hline $\begin{array}{l}\text { Selective reporting (re- } \\
\text { porting bias) }\end{array}$ & Low risk & Judgement Comment: Reported outcomes for all measures \\
\hline
\end{tabular}


Barrett 1998 (Continued)

Other source of bias - Low risk_ Judgement Comment: Blind clinician assessed therapy integrity of random Therapy integrity sessions; trained therapists delivered treatment

Other bias Low risk Judgement Comment: The study appears to be free of other sources of bias

Barrington 2005

\section{Study characteristics}

\begin{tabular}{|c|c|}
\hline Methods & $\begin{array}{l}\text { Study design: randomised controlled trial } \\
\text { Total duration of study: } 12 \text {-month follow-up } \\
\text { Number of study centres and location: Australia } \\
\text { Study setting: clinic }\end{array}$ \\
\hline Participants & $\begin{array}{l}\text { N = } 54 \text { (CBT: } N=28 ; \text { TAU: } N=26) \\
\text { Mean age (SD): CBT: } 9.8 \text { (2.29); TAU: } 10.2(1.75) \\
\text { Age range: } 7 \text { to } 14 \\
\text { Gender: } 35 \text { (64.8\%) female } \\
\text { Inclusion criteria: referrals with predominantly anxiety-related problems (100\% primary anxiety disor- } \\
\text { der diagnosis, based on ADIS-C/P, parent and child interviewed together) } \\
\text { Exclusion criteria: predominant high levels of aggressive behaviour, intellectual disability, focus of } \\
\text { court procedures, exposed to recent trauma }\end{array}$ \\
\hline Interventions & $\begin{array}{l}\text { CBT } \\
\text { Intervention: based on CBT guidelines and CBT toolkit } \\
\text { Delivery format: child-only sessions and parent-only/child and parent together sessions; individual } \\
\text { Therapist contact time: } 12 \text { hours (approximate) } \\
\text { Who delivers the intervention: experienced therapists, masters/doctoral level, CBT trained } \\
\text { TAU } \\
\text { Intervention: variety of treatment modalities, including family therapy, play therapy, supportive psy- } \\
\text { chotherapy, psychodynamic psychotherapy, non-CBT eclectic approaches } \\
\text { Delivery format: child-only sessions and parent-only/child and parent together sessions; individual } \\
\text { Therapist contact time: } 12 \text { hours (approximate) } \\
\text { Who delivers the intervention: experienced therapists, masters/doctoral level, CBT trained }\end{array}$ \\
\hline Outcomes & $\begin{array}{l}\text { Remission of all anxiety disorder diagnoses post-treatment, 6-month follow-up and 12-month fol- } \\
\text { low-up: ADIS-C/P (child and parent interviewed together) } \\
\text { Reduction in anxiety symptoms (child report) post-treatment, 6-month follow-up and 12-month } \\
\text { follow-up: RCMAS } \\
\text { Reduction in anxiety symptoms (parent report) post-treatment, 6-month follow-up and 12-month } \\
\text { follow-up: SCAS-P }\end{array}$ \\
\hline
\end{tabular}


Barrington 2005 (Continued)

Notes Number randomised not clear in paper. Report SCAS-C z score, so prioritise RCMAS

\section{Risk of bias}

\begin{tabular}{|c|c|c|}
\hline Bias & Authors' judgement & Support for judgement \\
\hline $\begin{array}{l}\text { Random sequence genera- } \\
\text { tion (selection bias) }\end{array}$ & Unclear risk & Judgement Comment: No detail given \\
\hline $\begin{array}{l}\text { Allocation concealment } \\
\text { (selection bias) }\end{array}$ & Unclear risk & Judgement Comment: No detail given \\
\hline $\begin{array}{l}\text { Blinding of participants } \\
\text { and personnel (perfor- } \\
\text { mance bias) } \\
\text { All outcomes }\end{array}$ & Unclear risk & $\begin{array}{l}\text { Judgement Comment: No detail given about whether participants were blind- } \\
\text { ed to condition }\end{array}$ \\
\hline $\begin{array}{l}\text { Blinding of outcome as- } \\
\text { sessment (detection bias) } \\
\text { All outcomes }\end{array}$ & Low risk & Judgement Comment: Assessor blind to treatment condition and therapist \\
\hline $\begin{array}{l}\text { Incomplete outcome data } \\
\text { (attrition bias) } \\
\text { All outcomes }\end{array}$ & Unclear risk & $\begin{array}{l}\text { Judgement Comment: Missing data for questionnaires detailed, but number } \\
\text { randomised vs completers not clear }\end{array}$ \\
\hline $\begin{array}{l}\text { Selective reporting (re- } \\
\text { porting bias) }\end{array}$ & Low risk & Judgement Comment: Outcomes reported for all measures \\
\hline $\begin{array}{l}\text { Other source of bias - } \\
\text { Therapy integrity }\end{array}$ & Low risk & Judgement Comment: Adherence monitored and independently assessed \\
\hline Other bias & Low risk & Judgement Comment: The study appears to be free of other sources of bias \\
\hline
\end{tabular}

Berge 2017

\section{Study characteristics}

\section{Methods}

Study design: randomised controlled trial

Total duration of study: non-controlled 12-month follow-up

Number of study centres and location: 1 , in Norway

Study setting: odontophobia clinic

Date of study: 2013 to 2016
$\mathbf{N}=\mathbf{6 7}$ (CBT: $\mathrm{N}=34$; waitlist: $\mathrm{N}=33$ )

Mean age (SD): $12.2(2.0)$

Age range: 10 to 16

Gender: 39 (58.2\%) female

Inclusion criteria: 10 to 16 years, primary diagnosis of intra-oral injection phobia (I-OIP) (DSM-5 criteria, using modified ADIS), other phobias as either secondary diagnoses or co-primary diagnoses with I- 
Berge 2017 (Continued)

OIP allowed, willingness to try exposure treatment; willingness to participate in the study for a period of 1 year

Exclusion criteria: primary depression, drug or alcohol abuse, intellectual disabilities, psychotic symptoms

\begin{tabular}{ll}
\hline Interventions & CBT \\
Intervention: One Session Treatment (OST), modified for 5 sessions \\
Concomitant/excluded medications: no detail given \\
Delivery format: child only; individual \\
Therapist contact time: 5 hours (5 x 1 hour) \\
Who delivers the intervention: dentists, trained and accredited in OST \\
Waitlist/no treatment \\
Intervention: 5 -week waitlist \\
Concomitant/excluded medications: no detail given \\
Reduction in anxiety symptoms (child report) post-treatment: Intra Oral Injection Fear Scale (IOIF- \\
\hline S) \\
\hline Autcomes
\end{tabular}

\section{Risk of bias}

\begin{tabular}{|c|c|c|}
\hline Bias & Authors' judgement & Support for judgement \\
\hline $\begin{array}{l}\text { Random sequence genera- } \\
\text { tion (selection bias) }\end{array}$ & Low risk & Judgement Comment: Computer-generated random number \\
\hline $\begin{array}{l}\text { Allocation concealment } \\
\text { (selection bias) }\end{array}$ & Low risk & $\begin{array}{l}\text { Judgement Comment: Opaque and sealed envelopes were put in order ac- } \\
\text { cording to the randomisation }\end{array}$ \\
\hline $\begin{array}{l}\text { Blinding of participants } \\
\text { and personnel (perfor- } \\
\text { mance bias) } \\
\text { All outcomes }\end{array}$ & Low risk & $\begin{array}{l}\text { Judgement Comment: Even though blinding was not possible, we judged that } \\
\text { it was unlikely this led to a departure from the intended intervention }\end{array}$ \\
\hline $\begin{array}{l}\text { Blinding of outcome as- } \\
\text { sessment (detection bias) } \\
\text { All outcomes }\end{array}$ & Low risk & Judgement Comment: Outcome assessment questionnaires only \\
\hline $\begin{array}{l}\text { Incomplete outcome data } \\
\text { (attrition bias) } \\
\text { All outcomes }\end{array}$ & Low risk & $\begin{array}{l}\text { Judgement Comment: Dropouts detailed, and where reasons known this infor- } \\
\text { mation was provided; missing data for each measure reported }\end{array}$ \\
\hline $\begin{array}{l}\text { Selective reporting (re- } \\
\text { porting bias) }\end{array}$ & Low risk & Judgement Comment: Outcomes for all measures reported \\
\hline $\begin{array}{l}\text { Other source of bias - } \\
\text { Therapy integrity }\end{array}$ & Unclear risk & $\begin{array}{l}\text { Judgement Comment: Dentists trained and accredited in the OST delivered in- } \\
\text { tervention, but no detail provided on adherence }\end{array}$ \\
\hline Other bias & Low risk & Judgement Comment: The study appears to be free of other sources of bias \\
\hline
\end{tabular}




\section{Study characteristics}

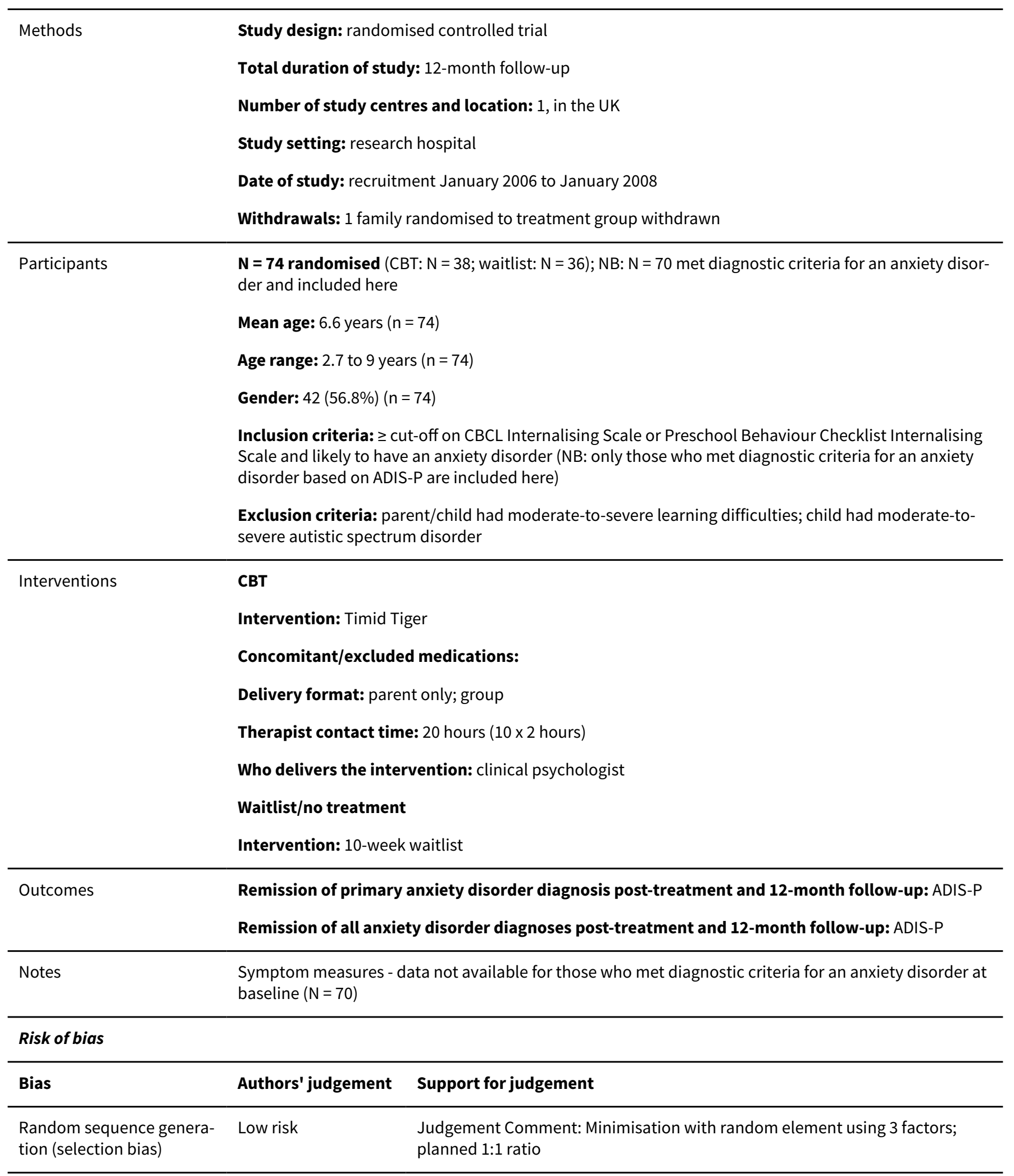


Cartwright Hatton 2011 (Continued)

$\begin{array}{ll}\begin{array}{l}\text { Allocation concealment } \\ \text { (selection bias) }\end{array} & \text { Low risk Judgement Comment: Telephone, with concealed allocation by independent } \\ \text { agency }\end{array}$

Blinding of participants Low risk and personnel (performance bias)

All outcomes

$\begin{array}{ll}\text { Blinding of outcome as- } & \text { Low risk Judgement Comment: Assessors blind to group allocation } \\ \text { sessment (detection bias) } & \end{array}$

All outcomes

Judgement Comment: Even though blinding was not possible, we judged that it was unlikely this led to a departure from the intended intervention

\begin{tabular}{lll}
\hline $\begin{array}{l}\text { Incomplete outcome data } \\
\text { (attrition bias) } \\
\text { All outcomes }\end{array}$ & Low risk & $\begin{array}{l}\text { Judgement Comment: Missing outcome data balanced in numbers across in- } \\
\text { tervention groups, and where possible imputed with appropriate method }\end{array}$ \\
\hline $\begin{array}{l}\text { Selective reporting (re- } \\
\text { porting bias) }\end{array}$ & Low risk & $\begin{array}{l}\text { Judgement Comment: Outcomes for all measures reported (with the excep- } \\
\text { tion of teacher reports, which is justified) }\end{array}$ \\
\hline $\begin{array}{l}\text { Other source of bias - } \\
\text { Therapy integrity }\end{array}$ & Low risk & $\begin{array}{l}\text { Judgement Comment: Therapist qualifications evidenced, and therapy adher- } \\
\text { ence detailed }\end{array}$ \\
\hline Other bias & Unclear risk & $\begin{array}{l}\text { Judgement Comment: Long-term follow-up data: } 38 \% \text { controls reported to } \\
\text { have received additional intervention vs 7\% in the CBT group }\end{array}$ \\
\hline
\end{tabular}

Chalfant 2007

\section{Study characteristics}

\begin{tabular}{ll}
\hline Study design: randomised controlled trial \\
Total duration of study: treatment period 5.5 months \\
Number of study centres and location: 1 , in Australia \\
Study setting: clinic \\
\hline $\mathbf{N}=\mathbf{5 1}$ (CBT: $\mathrm{N}=32 ;$ waitlist: $\mathrm{N}=19)$ \\
Mean age: $10.8(1.35)(\mathrm{n}=47)$ \\
Age range: 8 to 13 \\
Gender: 12 (25.5\%) ( $\mathrm{n}=47)$ \\
Inclusion criteria: documentation of diagnosis of high functioning autistic disorder or Asperger's dis- \\
order. Received primary anxiety disorder diagnosis (based on ADIS-C/P) \\
Exclusion criteria: intellectual delay, physical disability, currently taking anti-anxiety or anti-depres- \\
sant medication, presented with marked externalising difficulties (e.g. conduct disorder or opposition- \\
al defiant disorder), parents experiencing acute marital breakdown
\end{tabular}

Interventions

CBT

Intervention: adaptation of Cool Kids

Concomitant/excluded medications: currently taking anti-anxiety and anti-depressant medication excluded 
Delivery format: child and parent concurrent sessions; group

Therapist contact time: 24 hours ( $12 \times 2$ hours)

Who delivers the intervention: clinical psychologist

\section{Waitlist/no treatment}

Intervention: waitlist, approximately 5.5 months

Concomitant/excluded medications: currently taking anti-anxiety and anti-depressant medication excluded

\begin{tabular}{ll}
\hline Outcomes & Remission of all anxiety disorder diagnoses post-treatment: ADIS-C/P \\
& Reduction in anxiety symptoms (child report) post-treatment: SCAS-C \\
& Reduction in anxiety symptoms (parent report) post-treatment: SCAS-P \\
\hline Notes & $\begin{array}{l}\text { Total N randomised not clear. } \\
\text { Author confirmed not able to provide additional diagnostic outcome data. }\end{array}$
\end{tabular}

Sample with ASD

\section{Risk of bias}

\begin{tabular}{|c|c|c|}
\hline Bias & Authors' judgement & Support for judgement \\
\hline $\begin{array}{l}\text { Random sequence genera- } \\
\text { tion (selection bias) }\end{array}$ & Unclear risk & Judgement Comment: No detail on sequence generation given \\
\hline $\begin{array}{l}\text { Allocation concealment } \\
\text { (selection bias) }\end{array}$ & Unclear risk & Judgement Comment: No detail on allocation given \\
\hline $\begin{array}{l}\text { Blinding of participants } \\
\text { and personnel (perfor- } \\
\text { mance bias) } \\
\text { All outcomes }\end{array}$ & Low risk & $\begin{array}{l}\text { Judgement Comment: Even though blinding was not possible, we judged that } \\
\text { it was unlikely this led to a departure from the intended intervention }\end{array}$ \\
\hline $\begin{array}{l}\text { Blinding of outcome as- } \\
\text { sessment (detection bias) } \\
\text { All outcomes }\end{array}$ & Unclear risk & $\begin{array}{l}\text { Judgement Comment: Clinicians who collected post-treatment data not blind } \\
\text { to study aims. Not specified if blind to intervention group }\end{array}$ \\
\hline $\begin{array}{l}\text { Incomplete outcome data } \\
\text { (attrition bias) } \\
\text { All outcomes }\end{array}$ & Unclear risk & $\begin{array}{l}\text { Judgement Comment: Detailed number of dropouts, but not clear if dropouts } \\
\text { included in analyses. Did not provide } \mathrm{n} \text { for individual measures or any detail } \\
\text { on how missing data were managed }\end{array}$ \\
\hline $\begin{array}{l}\text { Selective reporting (re- } \\
\text { porting bias) }\end{array}$ & Low risk & Judgement Comment: Data reported for all outcome measures \\
\hline $\begin{array}{l}\text { Other source of bias - } \\
\text { Therapy integrity }\end{array}$ & Unclear risk & $\begin{array}{l}\text { Judgement Comment: Therapy delivered by clinical psychologists, no other } \\
\text { detail on training/supervision or adherence }\end{array}$ \\
\hline Other bias & Low risk & Judgement Comment: The study appears to be free of other sources of bias \\
\hline
\end{tabular}

\section{Study characteristics}


Cheung 2016 (Continued)

Methods

Study design: randomised controlled trial

Total duration of study: non-controlled 6-month follow-up

Number of study centres and location: 1, in Hong Kong

Study setting: Clinic-Child Assessment Service

Date of study: 2014 to 2015

Participants

$\mathbf{N}=\mathbf{8 1}$ (CBT: 22; active control (Attention Bias Modification Training (ABM)): 20; CBT + ABM: 20; waitlist: 19)

Mean age: 7.37

Age range: 6 to 12

Gender: $48(64 \%)(n=75)$

Inclusion criteria: diagnostic criteria for social anxiety disorder (based on ADIS-P)

Exclusion criteria: sensory impairments or other physical disabilities, ASD, received psychotherapy (such as CBT) or medication for anxiety or mood problems within the previous 6 months

\begin{tabular}{|c|c|}
\hline Interventions & $\begin{array}{l}\text { CBT } \\
\text { Intervention: adapted version of Coping Cat } \\
\text { Delivery format: child-only sessions, parent join some sessions } \\
\text { Therapist contact time: } 18 \text { hours ( } 9 \times 2 \text { hours) } \\
\text { Who delivers the intervention: clinical psychologist } \\
\text { Active control (ABM) } \\
\text { Intervention: Attention Bias Modification Training ( } 8 \text { sessions computerised ABM training) } \\
\text { CBT (CBT + ABM) } \\
\text { Intervention: adapted version of Coping Cat and ABM ( } 8 \text { sessions computerised ABM training) } \\
\text { Delivery format: child-only sessions, parent join some sessions } \\
\text { Therapist contact time: } 18 \text { hours ( } 9 \times 2 \text { hours) } \\
\text { Who delivers the intervention: clinical psychologist } \\
\text { Waitlist/no treatment } \\
\text { Intervention: } 2 \text {-month waitlist }\end{array}$ \\
\hline Outcomes & $\begin{array}{l}\text { Reduction in anxiety symptoms (child report) post-treatment: SCAS-C } \\
\text { Reduction in anxiety symptoms (parent report) post-treatment: SCAS-P }\end{array}$ \\
\hline Notes & $\begin{array}{l}\text { Comparisons: } C B T \text { vs waitlist; } C B T+A B M \text { vs active control (ABM) } \\
\text { Active control }(A B M)=\text { alternative treatment } \\
\text { Used post-treatment diagnostic assessment but did not report remission outcomes } \\
\text { Thesis }\end{array}$ \\
\hline
\end{tabular}


Cheung 2016 (Continued)

Risk of bias

\begin{tabular}{|c|c|c|}
\hline Bias & Authors' judgement & Support for judgement \\
\hline $\begin{array}{l}\text { Random sequence genera- } \\
\text { tion (selection bias) }\end{array}$ & Unclear risk & $\begin{array}{l}\text { Judgement Comment: Computerised random number generator, but unclear } \\
\text { allocation of additional children recruited after dropouts from CBT group }\end{array}$ \\
\hline $\begin{array}{l}\text { Allocation concealment } \\
\text { (selection bias) }\end{array}$ & Low risk & Judgement Comment: Computer based, so unlikely to foresee assignment \\
\hline $\begin{array}{l}\text { Blinding of participants } \\
\text { and personnel (perfor- } \\
\text { mance bias) } \\
\text { All outcomes }\end{array}$ & Unclear risk & Judgement Comment: No detail \\
\hline $\begin{array}{l}\text { Blinding of outcome as- } \\
\text { sessment (detection bias) } \\
\text { All outcomes }\end{array}$ & Low risk & Judgement Comment: Blind assessors \\
\hline $\begin{array}{l}\text { Incomplete outcome data } \\
\text { (attrition bias) } \\
\text { All outcomes }\end{array}$ & Unclear risk & Judgement Comment: No ITT analyses reported \\
\hline $\begin{array}{l}\text { Selective reporting (re- } \\
\text { porting bias) }\end{array}$ & Low risk & Judgement Comment: Outcomes for relevant measures reported \\
\hline $\begin{array}{l}\text { Other source of bias - } \\
\text { Therapy integrity }\end{array}$ & Unclear risk & Judgement Comment: No detail \\
\hline Other bias & Low risk & Judgement Comment: The study appears to be free of other sources of bias \\
\hline
\end{tabular}

\section{Chiu 2013}

\section{Study characteristics}

Methods Study design: randomised controlled trial

Total duration of study: treatment period 3 months. Non-controlled 1-year follow-up

Number of study centres and location: 2 schools in the USA

Study setting: elementary schools

Date of study: recruitment January 2005 to April 2008

Participants $\quad \mathbf{N}=\mathbf{4 0}(\mathrm{CBT}: \mathrm{N}=22$; waitlist: $\mathrm{N}=18)$

Mean age: $8.51(1.74)$

Age range: 5 to 12

Gender: $18(45 \%)$ female

Inclusion criteria: primary diagnosis of separation anxiety disorder, social phobia, or generalised anxiety disorder (DSM-IV) (based on ADIS-C/P); no psychiatric medication use or stable use of psychiatric medication for at least 1 month and agree to maintain that dose throughout the study

Exclusion criteria: child was currently in psychotherapy 
Chiu 2013 (Continued)

Interventions

\section{CBT}

Intervention: Building Confidence

Concomitant/excluded medications: no psychiatric medication use or stable use

Delivery format: child only, or if parent opted to attend session divided into child only, parent alone, parent and child together; individual

Therapist contact time: up to 16 hours (up to $16 \times 1$ hour)

Who delivers the intervention: clinical/educational psychology doctoral students

\section{Waitlist/no treatment}

Intervention: 3-month waitlist

Concomitant/excluded medications: no psychiatric medication use or stable use

$\begin{array}{ll}\text { Outcomes } & \text { Remission of all anxiety disorder diagnoses post-treatment: ADIS-C/P } \\ & \text { Reduction in anxiety symptoms (child report) post-treatment: MASC-Child report } \\ \text { Reduction in anxiety symptoms (parent report) post-treatment: MASC-Parent report }\end{array}$

Notes

\section{Risk of bias}

\begin{tabular}{|c|c|c|}
\hline Bias & Authors' judgement & Support for judgement \\
\hline $\begin{array}{l}\text { Random sequence genera- } \\
\text { tion (selection bias) }\end{array}$ & Unclear risk & $\begin{array}{l}\text { Judgement Comment: Did not detail sequence generation, description of } \\
\text { stratification method unclear }\end{array}$ \\
\hline $\begin{array}{l}\text { Allocation concealment } \\
\text { (selection bias) }\end{array}$ & Unclear risk & Judgement Comment: No detail given \\
\hline $\begin{array}{l}\text { Blinding of participants } \\
\text { and personnel (perfor- } \\
\text { mance bias) } \\
\text { All outcomes }\end{array}$ & Low risk & $\begin{array}{l}\text { Judgement Comment: Even though blinding was not possible, we judged that } \\
\text { it was unlikely this led to a departure from the intended intervention }\end{array}$ \\
\hline $\begin{array}{l}\text { Blinding of outcome as- } \\
\text { sessment (detection bias) } \\
\text { All outcomes }\end{array}$ & Low risk & Judgement Comment: Assessors blind to treatment condition \\
\hline $\begin{array}{l}\text { Incomplete outcome data } \\
\text { (attrition bias) } \\
\text { All outcomes }\end{array}$ & Low risk & $\begin{array}{l}\text { Judgement Comment: Described method for managing missing data. Small } \\
\text { amount of missing data, only } 1 \text { dropout }\end{array}$ \\
\hline $\begin{array}{l}\text { Selective reporting (re- } \\
\text { porting bias) }\end{array}$ & Low risk & Judgement Comment: Data for each measure reported \\
\hline $\begin{array}{l}\text { Other source of bias - } \\
\text { Therapy integrity }\end{array}$ & Low risk & $\begin{array}{l}\text { Judgement Comment: Trained clinicians, regular supervision. Blind assessors } \\
\text { rated random-selection therapy sessions for fidelity }\end{array}$ \\
\hline Other bias & Low risk & Judgement Comment: The study appears to be free of other sources of bias \\
\hline
\end{tabular}


Cobham 2017

\section{Study characteristics}

\begin{tabular}{ll}
\hline Methods & Study design: randomised controlled trial \\
& Total duration of study: non-controlled 12-month follow-up \\
& Number of study centres and location: 1 , in Australia \\
& Study setting: university clinic \\
\hline N $=\mathbf{6 3}(\mathrm{CBT}: \mathrm{N}=33 ;$ waitlist: $\mathrm{N}=30)$ \\
Mean age: $9.3(2.0)(\mathrm{n}=60)$ \\
Age range: 7 to 14 \\
Gender: 30 (49.2\%) female \\
Inclusion criteria: child aged 7 to 14 years; child meets diagnostic criteria for a primary DSM-IV anxiety \\
diagnosis (based on ADIS-C/P); parent able to attend treatment \\
Exclusion criteria: child currently in psychotherapy
\end{tabular}

Interventions $\quad$ CBT

Intervention: Fear-less Triple $\mathrm{P}$

Concomitant/excluded medications: medication for anxiety excluded

Delivery format: parent only; group

Therapist contact time: 9 hours ( $6 \times 1.5$ hours)

Who delivers the intervention: postgraduate clinical psychology interns

\section{Waitlist/no treatment}

Intervention: 6-week waitlist

Concomitant/excluded medications: medication for anxiety excluded

\begin{tabular}{|c|c|c|}
\hline Outcomes & \multicolumn{2}{|c|}{$\begin{array}{l}\text { Remission of primary anxiety disorder diagnoses post-treatment: ADIS-C/P } \\
\text { Remission of all anxiety disorder diagnoses post-treatment: ADIS-C/P } \\
\text { Reduction in anxiety symptoms (child report) post-treatment: SCAS-C } \\
\text { Reduction in anxiety symptoms (parent report) post-treatment: SCAS-P }\end{array}$} \\
\hline \multicolumn{3}{|l|}{ Notes } \\
\hline \multicolumn{3}{|l|}{ Risk of bias } \\
\hline Bias & Authors' judgement & Support for judgement \\
\hline $\begin{array}{l}\text { Random sequence genera- } \\
\text { tion (selection bias) }\end{array}$ & Low risk & Judgement Comment: Computer-generated, block randomisation \\
\hline $\begin{array}{l}\text { Allocation concealment } \\
\text { (selection bias) }\end{array}$ & Low risk & Judgement Comment: Computer generated, so unlikely to foresee assignment \\
\hline
\end{tabular}


Cobham 2017 (Continued)

$\begin{array}{ll}\text { Blinding of participants } & \text { Low risk } \\ \text { and personnel (perfor- } & \text { Judgement Comment: Even though blinding was not possible, we judged that } \\ \text { it was unlikely this led to a departure from the intended intervention }\end{array}$
mance bias)

All outcomes

\begin{tabular}{|c|c|c|}
\hline $\begin{array}{l}\text { Blinding of outcome as- } \\
\text { sessment (detection bias) } \\
\text { All outcomes }\end{array}$ & Low risk & Judgement Comment: Assessors blind to treatment condition \\
\hline $\begin{array}{l}\text { Incomplete outcome data } \\
\text { (attrition bias) } \\
\text { All outcomes }\end{array}$ & Low risk & $\begin{array}{l}\text { Judgement Comment: Attrition/withdrawals detailed, with reasons provided. } \\
\text { Few dropouts. Detail related to missing data provided, with evidence missing } \\
\text { at random and appropriate imputation methods used }\end{array}$ \\
\hline $\begin{array}{l}\text { Selective reporting (re- } \\
\text { porting bias) }\end{array}$ & Low risk & Judgement Comment: Outcomes reported for all measures \\
\hline $\begin{array}{l}\text { Other source of bias - } \\
\text { Therapy integrity }\end{array}$ & Low risk & $\begin{array}{l}\text { Judgement Comment: Intervention delivered by trained therapists, weekly su- } \\
\text { pervision. Treatment integrity assessed and confirmed by blind researcher }\end{array}$ \\
\hline Other bias & Low risk & Judgement Comment: The study appears to be free of other sources of bias \\
\hline
\end{tabular}

\section{Cornacchio 2019}

\section{Study characteristics}

\begin{tabular}{|c|c|}
\hline Methods & $\begin{array}{l}\text { Study design: randomised controlled trial } \\
\text { Total duration of study: non-controlled school year follow-up } \\
\text { Number of study centres and location: } 1 \text {, in the USA } \\
\text { Study setting: selective mutism treatment centre }\end{array}$ \\
\hline Participants & $\begin{array}{l}\mathbf{N}=29 \text { (CBT: } N=14 \text {; waitlist: } N=15) \\
\text { Mean age: } 6.6(1.3) \\
\text { Age range: } 5 \text { to } 9 \\
\text { Gender: } 22(75.9 \%) \text { female } \\
\text { Inclusion criteria: age } 5 \text { to } 9 \text {, meet DSM- } 5 \text { criteria for selective mutism (based on ADIS-P) } \\
\text { Exclusion criteria: identified as having any mental health condition more impairing than selective } \\
\text { mutism; or they were non-verbal with both of their parents }\end{array}$ \\
\hline Interventions & $\begin{array}{l}\text { CBT } \\
\text { Intervention: intensive group CBT programme centred around graded exposure to verbal communica- } \\
\text { tion that draws on parent-child interaction therapy } \\
\text { Concomitant/excluded medications: medication for anxiety excluded } \\
\text { Delivery format: child-only and parent-only sessions; group } \\
\text { Therapist contact time: } 30 \text { to } 40 \text { hours ( } 5 \times 6 \text { to } 8 \text { hours, } 5 \text { consecutive days) } \\
\text { Who delivers the intervention: counsellor and masters-level therapist } \\
\text { Waitlist/no treatment }\end{array}$ \\
\hline
\end{tabular}


Cornacchio 2019 (Continued)

Intervention: 4-week waitlist, 1-page psychoeducation information brochure on selective mutism

Concomitant/excluded medications: medication for anxiety excluded

\begin{tabular}{ll}
\hline Outcomes & Remission of primary anxiety disorder diagnoses post-treatment: ADIS-P \\
& Improvement in global functioning post-treatment: CGAS \\
\hline Notes & Report CBCL-Anxiety t score
\end{tabular}

\section{Risk of bias}

\begin{tabular}{|c|c|c|}
\hline Bias & Authors' judgement & Support for judgement \\
\hline $\begin{array}{l}\text { Random sequence genera- } \\
\text { tion (selection bias) }\end{array}$ & Unclear risk & Judgement Comment: Not detailed \\
\hline $\begin{array}{l}\text { Allocation concealment } \\
\text { (selection bias) }\end{array}$ & Unclear risk & Judgement Comment: Not detailed \\
\hline $\begin{array}{l}\text { Blinding of participants } \\
\text { and personnel (perfor- } \\
\text { mance bias) } \\
\text { All outcomes }\end{array}$ & Low risk & $\begin{array}{l}\text { Judgement Comment: Even though blinding was not possible, we judged that } \\
\text { it was unlikely this led to a departure from the intended intervention }\end{array}$ \\
\hline $\begin{array}{l}\text { Blinding of outcome as- } \\
\text { sessment (detection bias) } \\
\text { All outcomes }\end{array}$ & Low risk & Judgement Comment: Masked assessors \\
\hline $\begin{array}{l}\text { Incomplete outcome data } \\
\text { (attrition bias) } \\
\text { All outcomes }\end{array}$ & Low risk & $\begin{array}{l}\text { Judgement Comment: Retention detailed, and procedure for managing miss- } \\
\text { ing data appropriate }\end{array}$ \\
\hline $\begin{array}{l}\text { Selective reporting (re- } \\
\text { porting bias) }\end{array}$ & Low risk & Judgement Comment: Outcomes reported for relevant measures \\
\hline $\begin{array}{l}\text { Other source of bias - } \\
\text { Therapy integrity }\end{array}$ & Low risk & Judgement Comment: Trained staff, and fidelity assessed and reported \\
\hline Other bias & Low risk & Judgement Comment: The study appears to be free of other sources of bias \\
\hline
\end{tabular}

Creswell 2017

\section{Study characteristics}

\begin{tabular}{ll}
\hline Methods & Study design: randomised controlled trial \\
Total duration of study: 6 -month follow-up \\
Number of study centres and location: 4 , in the UK \\
Study setting: CAMHS \\
Date of study: recruitment March 2012 to March 2014 \\
\hline Participants \\
ety disorder and were included here (based on ADIS-C/P)
\end{tabular}




\section{Age range: 5 to 12}

Gender: $63(51.6 \%)$ female $(n=122)$

Inclusion criteria: child aged 5 to 12 years with anxiety associated with clinical impairment as the primary presenting problem. NB: we only included those who met diagnostic criteria $(90 \%$ original sample)

Exclusion criteria: children prescribed psychotropic medication, and parents or children with little understanding of English or with physical or intellectual impairment (including autism spectrum disorder) that would interfere with their ability to participate in assessments or treatment

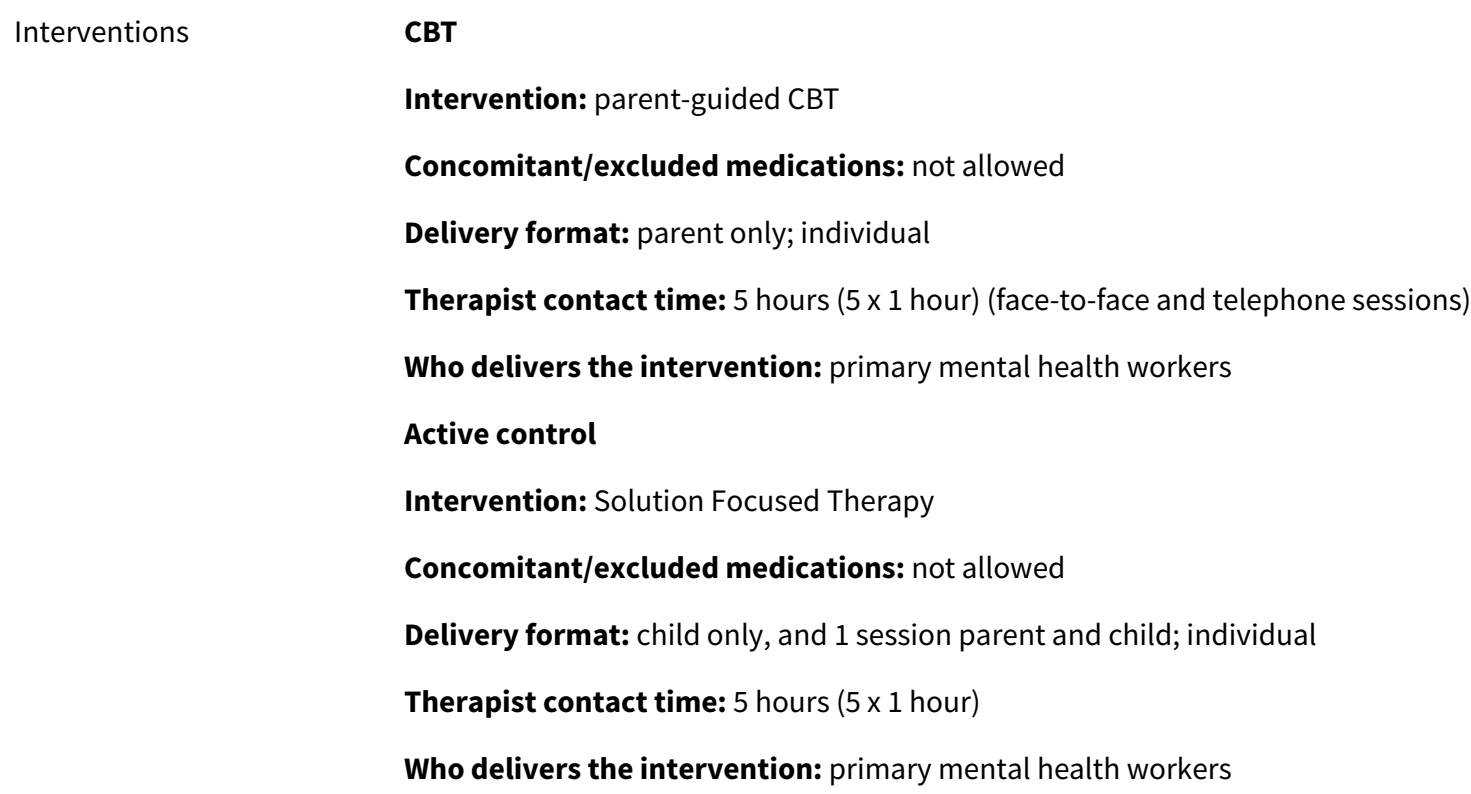

\begin{tabular}{|c|c|c|}
\hline Notes & \multicolumn{2}{|c|}{ Active control = alternative treatment } \\
\hline \multicolumn{3}{|l|}{ Risk of bias } \\
\hline Bias & Authors' judgement & Support for judgement \\
\hline $\begin{array}{l}\text { Random sequence genera- } \\
\text { tion (selection bias) }\end{array}$ & Low risk & Judgement Comment: Secure online minimisation tool \\
\hline $\begin{array}{l}\text { Allocation concealment } \\
\text { (selection bias) }\end{array}$ & Low risk & $\begin{array}{l}\text { Judgement Comment: Allocation sequence was retained on the secure online } \\
\text { minimisation program, only accessible to the principal investigator and the } \\
\text { clinical supervisor who allocated participants to clinicians }\end{array}$ \\
\hline $\begin{array}{l}\text { Blinding of participants } \\
\text { and personnel (perfor- } \\
\text { mance bias) } \\
\text { All outcomes }\end{array}$ & Low risk & $\begin{array}{l}\text { Judgement Comment: Even though blinding was not possible, we judged that } \\
\text { it was unlikely this led to a departure from the intended intervention }\end{array}$ \\
\hline
\end{tabular}


Creswell 2017 (Continued)

Blinding of outcome as- Low risk Judgement Comment: Research staff who obtained outcome measurements sessment (detection bias) were masked to group allocation

All outcomes

$\begin{array}{ll}\text { Incomplete outcome data } & \text { Low risk } \\ \text { (attrition bias) } & \text { manage missing data }\end{array}$

All outcomes manage missing data

Selective reporting (re- Low risk Judgement Comment: Outcomes reported/provided for all measures
porting bias)

Other source of bias - Low risk Judgement Comment: Trained, supervised and adherence measured
Therapy integrity

Other bias Low risk Judgement Comment: The study appears to be free of other sources of bias

\section{Study characteristics}

\begin{tabular}{|c|c|}
\hline Methods & $\begin{array}{l}\text { Study design: cluster-randomised controlled trial } \\
\text { Total duration of study: 6-, 12-, and 24-month follow-up } \\
\text { Number of study centres and location: } 8 \text { primary schools in Australia } \\
\text { Study setting: primary schools }\end{array}$ \\
\hline Participants & $\begin{array}{l}\mathbf{N}=128 \text { randomised (CBT: } N=61 \text {; active control: } N=68)(N B: N=64 \text { met diagnostic criteria for an anxi- } \\
\text { ety disorder and were included here (based on ADIS-P)) } \\
\text { Age range: } 7 \text { to } 14 \text { years } \\
\text { Inclusion criteria: children met diagnostic criteria for anxiety disorder or features of anxiety disorder. } \\
\text { We only included those who met diagnostic criteria (based on ADIS-P, CSR }=4+\text { ) } \\
\text { Exclusion criteria: Clinical Severity Rating } 6 \text { to } 8\end{array}$ \\
\hline
\end{tabular}

Interventions CBT

Intervention: Coping Koala (Australian modification of Coping Cat)

\section{Concomitant/excluded medications:}

Delivery format: child only, parent only; group

Therapist contact time: 10 to 23 hours ( $10 \times 1$ to 2 hours, plus 3 parent sessions)

Who delivers the intervention: clinical psychologists

\section{Waitlist/no treatment}

Intervention: monitoring, no intervention

Outcomes

Remission of primary anxiety disorder diagnosis post-treatment and 6-, 12-, and 24-month follow-up: ADIS-P

Remission of all anxiety disorder diagnoses post-treatment and 6-, 12-, and 24-month follow-up: ADIS-P 
Dadds 1997 (Continued)

Reduction in anxiety symptoms (child report) post-treatment and 6-, 12-, and 24-month follow-up: RCMAS

\begin{tabular}{|c|c|}
\hline \multirow[t]{2}{*}{ Notes } & $\begin{array}{l}\text { Author provided data on outcomes for participants who met diagnostic criteria for an anxiety disorder } \\
(\text { CSR 4+) at baseline. }\end{array}$ \\
\hline & Cluster trial - adjustments for analysis as per protocol \\
\hline
\end{tabular}

\section{Risk of bias}

\begin{tabular}{|c|c|c|}
\hline Bias & Authors' judgement & Support for judgement \\
\hline $\begin{array}{l}\text { Random sequence genera- } \\
\text { tion (selection bias) }\end{array}$ & Unclear risk & $\begin{array}{l}\text { Judgement Comment: Schools matched for size, sociodemographics, and } \\
\text { were randomly allocated to condition. No further detail }\end{array}$ \\
\hline $\begin{array}{l}\text { Allocation concealment } \\
\text { (selection bias) }\end{array}$ & Unclear risk & Judgement Comment: No detail \\
\hline $\begin{array}{l}\text { Blinding of participants } \\
\text { and personnel (perfor- } \\
\text { mance bias) } \\
\text { All outcomes }\end{array}$ & Low risk & $\begin{array}{l}\text { Judgement Comment: Even though blinding was not possible, we judged that } \\
\text { it was unlikely this led to a departure from the intended intervention }\end{array}$ \\
\hline $\begin{array}{l}\text { Blinding of outcome as- } \\
\text { sessment (detection bias) } \\
\text { All outcomes }\end{array}$ & Low risk & Judgement Comment: Blind assessors \\
\hline $\begin{array}{l}\text { Incomplete outcome data } \\
\text { (attrition bias) } \\
\text { All outcomes }\end{array}$ & Low risk & Judgement Comment: Author provided data \\
\hline $\begin{array}{l}\text { Selective reporting (re- } \\
\text { porting bias) }\end{array}$ & Low risk & Judgement Comment: Outcomes reported/provided \\
\hline $\begin{array}{l}\text { Other source of bias - } \\
\text { Therapy integrity }\end{array}$ & Low risk & $\begin{array}{l}\text { Judgement Comment: Regular supervision, integrity monitored, and no signif- } \\
\text { icant departures from protocol reported }\end{array}$ \\
\hline Other bias & Low risk & Judgement Comment: The study appears to be free of other sources of bias \\
\hline
\end{tabular}

Flannery Schroeder 2000

\section{Study characteristics}

Methods

Study design: randomised controlled trial

Total duration of study: non-controlled 3-month and 1-year follow-up

Number of study centres and location: 1 , in the USA

Study setting: university clinic

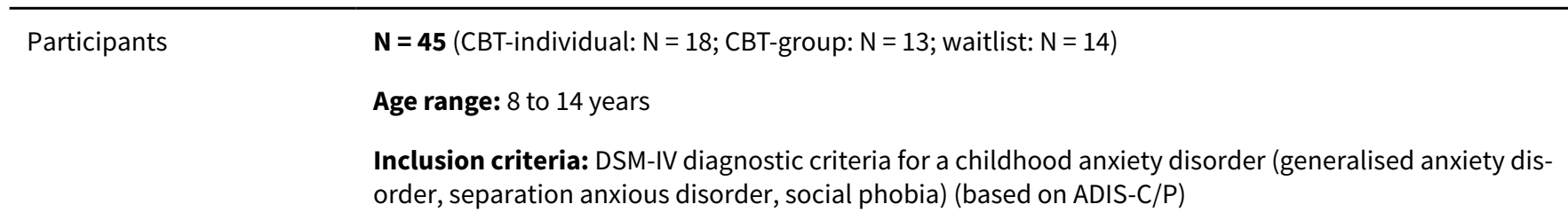


Flannery Schroeder 2000 (Continued)

Exclusion criteria: a disabling physical condition, psychotic symptoms or current use of anti-anxiety or anti-depressant medication; primary diagnosis of simple phobia

Interventions

\section{CBT-individual}

Intervention: Coping Cat

Concomitant/excluded medications: current use of anti-anxiety or anti-depressant medication excluded

Delivery format: child-only sessions, with additional parent meetings; individual

Therapist contact time: 15 to 18 hours ( $18 \times 50$ to 60 minutes)

Who delivers the intervention: trained therapists, doctoral candidates

CBT-group

Intervention: Adapted Coping Cat for groups

Concomitant/excluded medications: current use of anti-anxiety or anti-depressant medication excluded

Delivery format: child-only sessions, with additional parent meetings; group

Therapist contact time: 27 hours ( $18 \times 90$ minutes)

Who delivers the intervention: trained therapists, doctoral candidates

Waitlist/no treatment

Intervention: 9-week waitlist

Concomitant/excluded medications: current use of anti-anxiety or anti-depressant medication excluded
Outcomes
Remission of primary anxiety disorder diagnosis post-treatment: ADIS-C/P
Reduction in anxiety symptoms (child report) post-treatment: RCMAS
Reduction in anxiety symptoms (parent report) post-treatment: STAIC-trait (mother report)
Reduction in depressive symptoms post-treatment: CDI

Notes

\begin{tabular}{|c|c|c|}
\hline Bias & Authors' judgement & Support for judgement \\
\hline $\begin{array}{l}\text { Random sequence genera- } \\
\text { tion (selection bias) }\end{array}$ & Unclear risk & $\begin{array}{l}\text { Judgement Comment: Block randomisation used to assign to group CBT. No } \\
\text { detail on sequence generation }\end{array}$ \\
\hline $\begin{array}{l}\text { Allocation concealment } \\
\text { (selection bias) }\end{array}$ & Unclear risk & $\begin{array}{l}\text { Judgement Comment: No detail given (only detail on teachers blind to condi- } \\
\text { tion) }\end{array}$ \\
\hline $\begin{array}{l}\text { Blinding of participants } \\
\text { and personnel (perfor- } \\
\text { mance bias) } \\
\text { All outcomes }\end{array}$ & Low risk & $\begin{array}{l}\text { Judgement Comment: Even though blinding was not possible, we judged that } \\
\text { it was unlikely this led to a departure from the intended intervention }\end{array}$ \\
\hline
\end{tabular}


Flannery Schroeder 2000 (Continued)

All outcomes

$\begin{array}{ll}\begin{array}{l}\text { Incomplete outcome data } \\ \text { (attrition bias) }\end{array} & \text { Low risk }\end{array}$

All outcomes

Judgement Comment: Detail on dropouts. Reported ITT similar pattern as

Judgement Comment: Measures reported

Selective reporting (re- Low risk Judgement Comment: Measures reported
porting bias)

\begin{tabular}{ll}
\hline $\begin{array}{l}\text { Other source of bias - } \\
\text { Therapy integrity }\end{array}$ & Low risk \\
\end{tabular}

\begin{tabular}{ll}
\hline Other bias High risk Judgement Comment: Waitlist duration shorter than treatment \\
\hline
\end{tabular}

Fujii 2013

\section{Study characteristics}

\begin{tabular}{|c|c|}
\hline Methods & $\begin{array}{l}\text { Study design: randomised controlled trial } \\
\text { Total duration of study: } 32 \text {-week treatment period } \\
\text { Number of study centres and location: } 1 \text {, in the USA } \\
\text { Study setting: clinic }\end{array}$ \\
\hline Participants & $\begin{array}{l}\mathbf{N}=16(C B T: N=10 ; \text { TAU: } N=6) \\
\text { Mean age (SD): } 8.80(1.60), n=12 \\
\text { Age range: } 7 \text { to } 11 \\
\text { Gender: } 3(25 \%) \text { female }(n=12) \\
\text { Inclusion criteria: research criteria for ASD and at least } 1 \text { anxiety disorder, CSR } 5+\text { (based on ADIS-C/P) } \\
\text { Exclusion criteria: verbal IQ }<70 \text {, primary diagnosis other than anxiety }\end{array}$ \\
\hline Interventions & $\begin{array}{l}\text { CBT } \\
\text { Intervention: Building Confidence CBT Program } \\
\text { Concomitant/excluded medications: psychiatric medication, if used, was maintained at a stable dose } \\
\text { for at least } 1 \text { month prior to intake and throughout the duration of the trial } \\
\text { Delivery format: child and parent (part of each session individually, and part together); individual } \\
\text { Therapist contact time: } 48 \text { hours ( } 32 \times 90 \text { minutes) } \\
\text { Who delivers the intervention: graduate/postdocs in psychology or psychiatry } \\
\text { TAU } \\
\text { Intervention: free to seek any kind of treatment for period of } 16 \text { weeks } \\
\text { Concomitant/excluded medications: psychiatric medication, if used, was maintained at a stable dose } \\
\text { for at least } 1 \text { month prior to intake. Allowed to alter medication during TAU period, as long as stable for } \\
\text { at least } 1 \text { month prior to post-assessment }\end{array}$ \\
\hline
\end{tabular}


Fujii 2013 (Continued)

Notes Sample with ASD

\section{Risk of bias}

\begin{tabular}{|c|c|c|}
\hline Bias & Authors' judgement & Support for judgement \\
\hline $\begin{array}{l}\text { Random sequence genera- } \\
\text { tion (selection bias) }\end{array}$ & Unclear risk & $\begin{array}{l}\text { Judgement Comment: Blocked randomised by sex and age. No detail on se- } \\
\text { quence generation }\end{array}$ \\
\hline $\begin{array}{l}\text { Allocation concealment } \\
\text { (selection bias) }\end{array}$ & Unclear risk & Judgement Comment: No detail given \\
\hline $\begin{array}{l}\text { Blinding of participants } \\
\text { and personnel (perfor- } \\
\text { mance bias) } \\
\text { All outcomes }\end{array}$ & Low risk & $\begin{array}{l}\text { Judgement Comment: Even though blinding was not possible, we judged that } \\
\text { it was unlikely this led to a departure from the intended intervention }\end{array}$ \\
\hline $\begin{array}{l}\text { Blinding of outcome as- } \\
\text { sessment (detection bias) } \\
\text { All outcomes }\end{array}$ & Low risk & $\begin{array}{l}\text { Judgement Comment: All assessments were conducted by independent evalu- } \\
\text { ators blind }\end{array}$ \\
\hline $\begin{array}{l}\text { Incomplete outcome data } \\
\text { (attrition bias) } \\
\text { All outcomes }\end{array}$ & Low risk & Judgement Comment: Attrition detailed, data reported \\
\hline $\begin{array}{l}\text { Selective reporting (re- } \\
\text { porting bias) }\end{array}$ & Low risk & $\begin{array}{l}\text { Judgement Comment: Reported diagnostic outcomes. No mention of other } \\
\text { measures (but reasonable given small sample, population) }\end{array}$ \\
\hline $\begin{array}{l}\text { Other source of bias - } \\
\text { Therapy integrity }\end{array}$ & Low risk & $\begin{array}{l}\text { Judgement Comment: Trained and supervisors therapists. Adherence as- } \\
\text { sessed and reported }\end{array}$ \\
\hline Other bias & High risk & Judgement Comment: TAU period shorter than treatment period \\
\hline
\end{tabular}

Gallagher 2004

\section{Study characteristics}

\begin{tabular}{ll}
\hline Methods & Study design: randomised controlled trial \\
Total duration of study: 3-week follow-up (3 weeks after end of treatment) \\
Number of study centres and location: 1 in the USA \\
Study setting: clinic \\
\hline N $=\mathbf{2 3}$ (CBT: $\mathrm{N}=12 ;$ waitlist: $\mathrm{N}=11)$ \\
Age range: 8 to 11 \\
Gender: 12 (52.2\%) female \\
Inclusion criteria: meet diagnostic criteria for social phobia (based on ADIS-C/P) \\
Exclusion criteria: Attention Deficit Hyperactivity Disorder, conduct disorder, Oppositional Defiant \\
Disorder, bipolar, suicidal ideation, mental retardation \\
CBT
\end{tabular}


Intervention: brief group intervention for preadolescents with social phobia

Delivery format: child only (plus initial child + parent session); group

Therapist contact time: 9 hours $(3 \times 180$ minutes)

Who delivers the intervention: primary investigator and graduate students

\section{Waitlist/no treatment}

Intervention: 6-week waitlist

\begin{tabular}{|c|c|c|}
\hline Outcomes & \multicolumn{2}{|c|}{$\begin{array}{l}\text { Remission of primary anxiety disorder diagnosis post-treatment: ADIS-P } \\
\text { Reduction in anxiety symptoms (child report) post-treatment: RCMAS } \\
\text { Reduction in depressive symptoms post-treatment: } C D I\end{array}$} \\
\hline Notes & \multicolumn{2}{|c|}{ Report parent and child ADIS data separately; given age of sample, use ADIS-P data here } \\
\hline \multicolumn{3}{|l|}{ Risk of bias } \\
\hline Bias & Authors' judgement & Support for judgement \\
\hline $\begin{array}{l}\text { Random sequence genera- } \\
\text { tion (selection bias) }\end{array}$ & Unclear risk & Judgement Comment: No detail given on sequence generation \\
\hline $\begin{array}{l}\text { Allocation concealment } \\
\text { (selection bias) }\end{array}$ & Unclear risk & $\begin{array}{l}\text { Judgement Comment: Separation of therapists from initial screening, but allo- } \\
\text { cation concealment not detailed }\end{array}$ \\
\hline $\begin{array}{l}\text { Blinding of participants } \\
\text { and personnel (perfor- } \\
\text { mance bias) } \\
\text { All outcomes }\end{array}$ & Low risk & $\begin{array}{l}\text { Judgement Comment: Even though blinding was not possible, we judged that } \\
\text { it was unlikely this led to a departure from the intended intervention }\end{array}$ \\
\hline $\begin{array}{l}\text { Blinding of outcome as- } \\
\text { sessment (detection bias) } \\
\text { All outcomes }\end{array}$ & Low risk & $\begin{array}{l}\text { Judgement Comment: Procedure somewhat unclear, but sufficient detail to } \\
\text { determine post-treatment assessments carried out by blind assessors }\end{array}$ \\
\hline $\begin{array}{l}\text { Incomplete outcome data } \\
\text { (attrition bias) } \\
\text { All outcomes }\end{array}$ & Unclear risk & $\begin{array}{l}\text { Judgement Comment: } \mathrm{N} \text { given for diagnostic outcomes, but attrition not spec- } \\
\text { ified. Refer to missing parent data, but not detailed by group }\end{array}$ \\
\hline $\begin{array}{l}\text { Selective reporting (re- } \\
\text { porting bias) }\end{array}$ & Low risk & Judgement Comment: Report outcomes for measures \\
\hline $\begin{array}{l}\text { Other source of bias - } \\
\text { Therapy integrity }\end{array}$ & Low risk & $\begin{array}{l}\text { Judgement Comment: Treatment administered by doctoral students and au- } \\
\text { thor of manual. Adherence assessed and reported }\end{array}$ \\
\hline Other bias & Low risk & Judgement Comment: The study appears to be free of other sources of bias \\
\hline
\end{tabular}

Ginsburg 2002

\section{Study characteristics}

Methods Study design: randomised controlled trial

Number of study centres and location: 1 , in the USA 
Ginsburg 2002 (Continued)

Study setting: urban high school

Participants $\mathbf{N}$ (CBT: $N=6$; active control: $\mathrm{N}=6)$
Mean age (SD): 15.6
Age range: 14 to 17
Inclusion criteria: DSM-IV criteria for a primary (i.e. most severe) anxiety diagnosis (excluding Obses-
sive Compulsive Disorder and Post Traumatic Stress Disorder), with CSR $\geq 4$ (based on ADIS-C); not cur-
rently participating in psychiatric or psychosocial treatment aimed at reducing anxiety symptoms
Exclusion criteria: psychiatric condition contradicting study treatment (e.g. suicidal intent) or needed
more immediate or alternative treatment

CBT
Interventions
Concomitant/excluded medications: currently participating in any psychiatric treatment excluded
Delivery format: child only; group
Therapist contact time: 7.5 hours (10 x 45 minutes)
Who delivers the intervention: 2 advanced graduate students trained in CBT
Active control
Intervention: attention-support control. Sharing and discussing experiences related to anxiety. Avo id-
ed components of CBT
Concomitant/excluded medications: currently participating in any psychiatric treatment excluded
Delivery format: child only; group
Therapist contact time: 7.5 hours (10 45 minutes)

\begin{tabular}{ll}
\hline Outcomes & Remission of primary anxiety disorder diagnosis post-treatment: ADIS-C \\
& Remission of all anxiety disorder diagnoses post-treatment: ADIS-C \\
& Reduction in anxiety symptoms (child report) post-treatment: SCARED-C \\
\hline Notes & Active control = attention control \\
\hline
\end{tabular}

\section{Risk of bias}

\begin{tabular}{lll}
\hline Bias & Authors' judgement & Support for judgement \\
\hline $\begin{array}{l}\text { Random sequence genera- } \\
\text { tion (selection bias) }\end{array}$ & Low risk & Judgement Comment: Used random number table \\
\hline $\begin{array}{l}\text { Allocation concealment } \\
\text { (selection bias) }\end{array}$ & Unclear risk & Judgement Comment: No detail given \\
\hline $\begin{array}{l}\text { Blinding of participants } \\
\begin{array}{l}\text { and personnel (perfor- } \\
\text { mance bias) }\end{array}\end{array}$ & Unclear risk & Judgement Comment: No detail given about blinding participants \\
All outcomes & \\
\hline
\end{tabular}

\begin{tabular}{|c|c|c|}
\hline $\begin{array}{l}\text { Blinding of outcome as- } \\
\text { sessment (detection bias) }\end{array}$ & Low risk & Judgement Comment: ADIS assessors unaware of treatment condition \\
\hline
\end{tabular}


Ginsburg 2002 (Continued)

All outcomes

\begin{tabular}{|c|c|c|}
\hline $\begin{array}{l}\text { Incomplete outcome data } \\
\text { (attrition bias) }\end{array}$ & Low risk & $\begin{array}{l}\text { Judgement Comment: Attrition reported and similar across groups, and rea- } \\
\text { son reported where available ("no longer need group") }\end{array}$ \\
\hline
\end{tabular}

All outcomes

Judgement Comment: Attrition reported and similar across groups, and rea-

\begin{tabular}{lll}
$\begin{array}{l}\text { Selective reporting (re- } \\
\text { porting bias) }\end{array}$ & Low risk & Judgement Comment: Outcomes reported for all relevant included measures \\
\hline $\begin{array}{l}\text { Other source of bias - } \\
\text { Therapy integrity }\end{array}$ & Low risk & $\begin{array}{l}\text { Judgement Comment: Therapists trained and adherence monitored and ap- } \\
\text { proach justified }\end{array}$ \\
\hline
\end{tabular}

\begin{tabular}{ll}
\hline Other bias Low risk Judgement Comment: The study appears to be free of other sources of bias \\
\hline
\end{tabular}

Ginsburg 2012

\section{Study characteristics}

\begin{tabular}{|c|c|}
\hline Methods & $\begin{array}{l}\text { Study design: randomised controlled trial } \\
\text { Total duration of study: } 1 \text {-month follow-up } \\
\text { Number of study centres and location: } 14 \text { schools in the USA } \\
\text { Study setting: school-based mental health clinics }\end{array}$ \\
\hline Participants & $\begin{array}{l}\text { N = } 32 \text { (CBT: N = 17; TAU: N = 15) } \\
\text { Mean age (SD): CBT: } 11.12 \text { (2.75); TAU: } 9.33(2.06) \\
\text { Age range: } 7 \text { to } 17 \\
\text { Gender: } C B T: 12(70.6 \%) \text { female; TAU: } 8(53.3 \%) \text { female } \\
\text { Inclusion criteria: age } 7 \text { to 17, presence of GAD, social anxiety disorder, separation anxiety disorder, } \\
\text { specific phobia, or anxiety disorder not otherwise stated (based on ADIS-C/P), no medical or psychiatric } \\
\text { conditions contraindicating study intervention, not currently receiving anxiety treatment }\end{array}$ \\
\hline
\end{tabular}

Exclusion criteria: failed a previous trial of CBT for anxiety

Intervention: modular CBT adapted from CBT manuals

Concomitant/excluded medications: stable medication for another psychiatric disorder allowed

Delivery format: child only, encouraged to involve parents in at least 3 sessions; individual

Therapist contact time: 6 hours ( $8 \times 45$ minutes over 12 weeks)

Who delivers the intervention: novice CBT school-based clinicians

\section{TAU}

Intervention: therapeutic intervention that did not include CBT strategies. Therapy represented usual care for that therapist (e.g. art, play, or supportive therapy), and provided with attention control manual to use if they desired

Concomitant/excluded medications: stable medication for another psychiatric disorder allowed

Delivery format: child only, encouraged to involve parents in at least 3 sessions; individual 
Therapist contact time: 6 hours ( $8 \times 45$ minutes over 12 weeks)

Who delivers the intervention: novice CBT school-based clinician

Outcomes Reduction in anxiety symptoms (child report) post-treatment and follow-up ( $\leq 6$ months):

SCARED-C

Reduction in anxiety symptoms (parent report) post-treatment and follow-up ( $\leq 6$ months): SCARED-P

\begin{tabular}{|c|c|c|}
\hline Notes & Post-treatment diagnc & tic assessment included, but did not report required remission outcomes. \\
\hline \multicolumn{3}{|l|}{ Risk of bias } \\
\hline Bias & Authors' judgement & Support for judgement \\
\hline $\begin{array}{l}\text { Random sequence genera- } \\
\text { tion (selection bias) }\end{array}$ & Low risk & Judgement Comment: Sequence online and separate for each clinician \\
\hline $\begin{array}{l}\text { Allocation concealment } \\
\text { (selection bias) }\end{array}$ & Low risk & $\begin{array}{l}\text { Judgement Comment: Use website to generate number, so unlikely to foresee } \\
\text { assignment }\end{array}$ \\
\hline $\begin{array}{l}\text { Blinding of participants } \\
\text { and personnel (perfor- } \\
\text { mance bias) } \\
\text { All outcomes }\end{array}$ & Unclear risk & Judgement Comment: No detail given \\
\hline $\begin{array}{l}\text { Blinding of outcome as- } \\
\text { sessment (detection bias) } \\
\text { All outcomes }\end{array}$ & Low risk & $\begin{array}{l}\text { Judgement Comment: Independent assessors, scores reviewed by senior clini- } \\
\text { cian blind to condition }\end{array}$ \\
\hline $\begin{array}{l}\text { Incomplete outcome data } \\
\text { (attrition bias) } \\
\text { All outcomes }\end{array}$ & Low risk & $\begin{array}{l}\text { Judgement Comment: Attrition detailed, appropriate imputation for dealing } \\
\text { with missing data. } 11 \% \text { outcome data missing }\end{array}$ \\
\hline $\begin{array}{l}\text { Selective reporting (re- } \\
\text { porting bias) }\end{array}$ & Low risk & Judgement Comment: Outcomes reported \\
\hline $\begin{array}{l}\text { Other source of bias - } \\
\text { Therapy integrity }\end{array}$ & Unclear risk & $\begin{array}{l}\text { Judgement Comment: Adherence assessed and reported; there was some evi- } \\
\text { dence that TAU participants received elements of CBT, which could potentially } \\
\text { have influenced outcomes }\end{array}$ \\
\hline Other bias & Low risk & Judgement Comment: The study appears to be free of other sources of bias \\
\hline
\end{tabular}

Ginsburg 2019a

\title{
Study characteristics
}

Methods

\author{
Study design: cluster-randomised controlled trial \\ Total duration of study: 1-year follow-up \\ Number of study centres and location: USA
}

Study setting: schools

Date of study: 2012 to 2017 
Ginsburg 2019a (Continued)

\section{Participants}

$\mathbf{N}=\mathbf{2 1 6}(\mathrm{CBT}:$ 148; TAU: 68)

Mean age (SD): 10.87 (3.27)

Age range: 6 to 18

Gender: $48.6 \%$ female

Inclusion criteria: age 6 to 18, met DSM-IV diagnostic criteria for primary anxiety disorder (based on ADIS-C/P)

Excluded criteria: medical or psychiatric condition contraindicating study treatment (e.g. suicidality) and needed immediate or alternative treatment, were receiving psychosocial treatment for anxiety and/or were in the custody of state social services

Intervention: modular CBT. Adapted from empirically supported manuals. Included: psychoeducation, exposure, rewards, cognitive restructuring, problem solving, somatic/relaxation skills, relapse prevention, optional parental psychoeducation module

Concomitant/excluded medications: stable dose allowed

Delivery format: child only, optional parent; individual

Therapist contact time: 6 to 8 hours ( $12 \times 30$ to 40 minutes)

Who delivers the intervention: school-based clinicians

\section{TAU}

Intervention: therapeutic strategies clinicians would typically provide (e.g. supportive therapy)

Concomitant/excluded medications: stable dose allowed

Delivery format: child only; individual

Therapist contact time: 6 to 8 hours ( $12 \times 30$ to 40 minutes)

Who delivers the intervention: school-based clinicians

Cluster trial - reported data adjusted as per protocol

\section{Risk of bias}

\begin{tabular}{lll}
\hline Bias & Authors' judgement & Support for judgement \\
\hline $\begin{array}{l}\text { Random sequence genera- } \\
\text { tion (selection bias) }\end{array}$ & Unclear risk & $\begin{array}{l}\text { Judgement Comment: Web-based randomisation plan generator, but change } \\
\text { in randomisation ratios post onset }\end{array}$ \\
\hline $\begin{array}{l}\text { Allocation concealment } \\
\text { (selection bias) }\end{array}$ & Low risk & Judgement Comment: Web based, so unlikely to foresee assignment \\
\hline $\begin{array}{l}\text { Blinding of participants } \\
\begin{array}{l}\text { and personnel (perfor- } \\
\text { mance bias) }\end{array}\end{array}$ & Unclear risk & Judgement Comment: Not specified \\
All outcomes & \\
\hline
\end{tabular}


Ginsburg 2019a (Continued)

Blinding of outcome as- Low risk_ Judgement Comment: Blind assessors sessment (detection bias)

All outcomes

\begin{tabular}{lll}
\hline $\begin{array}{l}\text { Incomplete outcome data } \\
\text { (attrition bias) } \\
\text { All outcomes }\end{array}$ & Low risk & $\begin{array}{l}\text { Judgement Comment: Attrition reported and appropriate method for manag- } \\
\text { ing missing data }\end{array}$ \\
\hline $\begin{array}{l}\text { Selective reporting (re- } \\
\text { porting bias) }\end{array}$ & Low risk & Judgement Comment: Outcomes reported \\
\hline $\begin{array}{l}\text { Other source of bias - } \\
\text { Therapy integrity }\end{array}$ & Low risk & Judgement Comment: Adherence measured \\
\hline Other bias & Low risk & Judgement Comment: The study appears to be free of other sources of bias \\
\hline
\end{tabular}

Hancock 2018

\section{Study characteristics}

Methods

Study design: randomised controlled trial

Total duration of study: 3-month follow-up for treatment groups only

Number of study centres and location: 1 in Australia

Study setting: university hospital

Participants 193 (CBT (Acceptance and Commitment Therapy): N=68; CBT: N = 63; waitlist: $N=62$ )
Mean age (SD): 11.2 (2.76)
Age range: 7 to 17
Gender: 111 (58\%) female
Inclusion criteria: DSM-IV anxiety disorder (based on ADIS-C/P), age 7 to 17
Exclusion criteria: non-English speaker, complex mental health problems (e.g. psychotic symptoms,
major depression primary disorder) or medical conditions, attention deficit disorder with hyperactivi-
ty that was not well controlled, taking psychotropic medication for less than 2 months, post-traumat-
ic stress disorder (due to specialised treatment required), or academically more than 1 grade behind
peers

Interventions

CBT (ACT)

Intervention: ACT programme. Included mindfulness, psychoeducation, cognitive defusion, exposure, cognitive coping, problem solving, social skills

Concomitant/excluded medications: medication-free or stabilised on anti-depressants for at least 2 months

Delivery format: child and parent; group

Therapist contact time: 15 hours ( $10 \times 90$ minutes)

Who delivers the intervention: clinical psychologists

CBT (Cool Kids/Chilled) 
Intervention: Cool Kids/Chilled programme for children and adolescents

Concomitant/excluded medications: medication-free or stabilised on anti-depressants for at least 2 months

Delivery format: child and parent; group

Therapist contact time: 15 hours ( $10 \times 90$ minutes)

Who delivers the intervention: clinical psychologists

Waitlist

Intervention: 10 weeks waitlist

Concomitant/excluded medications: medication-free or stabilised on anti-depressants for at least 2 months

\begin{tabular}{|c|c|c|}
\hline Outcomes & \multicolumn{2}{|c|}{ Reduction in anxiety symptoms (child report) post-treatment: MASC-C } \\
\hline Notes & \multicolumn{2}{|c|}{ Use post-treatment diagnostic assessment, but did not report remission outcomes } \\
\hline \multicolumn{3}{|l|}{ Risk of bias } \\
\hline Bias & Authors' judgement & Support for judgement \\
\hline $\begin{array}{l}\text { Random sequence genera- } \\
\text { tion (selection bias) }\end{array}$ & Low risk & Judgement Comment: Clear details of sequence allocation \\
\hline $\begin{array}{l}\text { Allocation concealment } \\
\text { (selection bias) }\end{array}$ & Low risk & $\begin{array}{l}\text { Judgement Comment: Participants' enrolment into the study was conducted } \\
\text { by an intake officer. They were subsequently assessed by researchers masked } \\
\text { to the treatment condition, with both parties informed of the group allocation } \\
\text { after assessment. }\end{array}$ \\
\hline $\begin{array}{l}\text { Blinding of participants } \\
\text { and personnel (perfor- } \\
\text { mance bias) } \\
\text { All outcomes }\end{array}$ & Low risk & $\begin{array}{l}\text { Judgement Comment: Even though blinding was not possible, we judged that } \\
\text { it was unlikely this led to a departure from the intended intervention }\end{array}$ \\
\hline $\begin{array}{l}\text { Blinding of outcome as- } \\
\text { sessment (detection bias) } \\
\text { All outcomes }\end{array}$ & Low risk & $\begin{array}{l}\text { Judgement Comment: Blind assessors and subset interviews rated by clini- } \\
\text { cians blind to original diagnosis and group }\end{array}$ \\
\hline $\begin{array}{l}\text { Incomplete outcome data } \\
\text { (attrition bias) } \\
\text { All outcomes }\end{array}$ & Low risk & $\begin{array}{l}\text { Judgement Comment: Attrition detailed and reasons provided. Some of rea- } \\
\text { sons for attrition were potentially related to group allocation, but appropriate } \\
\text { imputation methods were used to manage missing data (ITT analyses under- } \\
\text { taken). }\end{array}$ \\
\hline $\begin{array}{l}\text { Selective reporting (re- } \\
\text { porting bias) }\end{array}$ & Low risk & $\begin{array}{l}\text { Judgement Comment: Outcomes for planned relevant measures reported. } \\
\text { Some measures reported elsewhere. }\end{array}$ \\
\hline $\begin{array}{l}\text { Other source of bias - } \\
\text { Therapy integrity }\end{array}$ & Low risk & $\begin{array}{l}\text { Judgement Comment: Therapist competence and adherence assessed and re- } \\
\text { ported }\end{array}$ \\
\hline Other bias & Low risk & Judgement Comment: The study appears to be free of other sources of bias \\
\hline
\end{tabular}


Herbert 2009

\section{Study characteristics}

Study design: randomised controlled trial
Total duration of study: 6 -month follow-up
Number of study centres and location: 1 in the USA
Study setting: university clinic

Participants $\quad$ N = 73 (CBT-group: $N=23$; CBT-individual: $N=24$; control: $N=26$ )

Mean age (SD): CBT-group: 14.6 (2.8); CBT-individual: 14.3 (2.1); control: 15.1 (1.4)

Age range: 12 to 17

Gender: 42 (57.5\%) female

Inclusion criteria: primary social anxiety disorder, generalised subtype (based on ADIS-C); aged 12 to 17; literacy in English

Excluded criteria: history of mental retardation, pervasive developmental disorder, organic mental disorder, bipolar disorder, a psychotic disorder, or borderline or schizotypal personality disorder, imminent suicidal risk, substance abuse or dependence within the past year, or a previous trial of behaviour or CBT for social anxiety disorder

\section{CBT (group)}

Intervention: psychoeducation, breathing retraining, cognitive restructuring, simulated and in vivo exposure to phobic stimuli, and social skills training

Concomitant/excluded medications: stabilised (at least 3 months with no change in medication or dosage) allowed

Delivery format: child only; group

Therapist contact time: 24 hours ( $12 \times 2$ hours)

Who delivers the intervention: advanced doctoral candidates in clinical psychology

\section{CBT (individual)}

Intervention: same content as group CBT

Concomitant/excluded medications: stabilised (at least 3 months with no change in medication or dosage) allowed

Delivery format: child only; individual

Therapist contact time: 12 hours ( $12 \times 1$ hour)

Who delivers the intervention: advanced doctoral candidates in clinical psychology

\section{Active control}

Intervention: psychoeducational supportive therapy. Discussions around various topics relevant to social anxiety disorder. Therapists offered support but did not provide specific advice, teach skills, problem-solve, or assign exposure exercises.

Concomitant/excluded medications: stabilised (at least 3 months with no change in medication or dosage) allowed

Delivery format: child only; group

Therapist contact time: 24 hours ( $12 \times 2$ hours) 
Herbert 2009 (Continued)

Who delivers the intervention: advanced doctoral candidates in clinical psychology

\begin{tabular}{ll} 
Outcomes & Reduction in anxiety symptoms (child report) post-treatment and follow-up (6 months): SPAI-C \\
& Reduction in anxiety symptoms (parent report) post-treatment and follow-up (6 months): SAS-P \\
\hline Notes & Post-treatment diagnostic assessment used but required remission outcomes not reported. \\
& Active control = attention control
\end{tabular}

\section{Risk of bias}

\begin{tabular}{|c|c|c|}
\hline Bias & Authors' judgement & Support for judgement \\
\hline $\begin{array}{l}\text { Random sequence genera- } \\
\text { tion (selection bias) }\end{array}$ & Low risk & Judgement Comment: Block randomisation \\
\hline $\begin{array}{l}\text { Allocation concealment } \\
\text { (selection bias) }\end{array}$ & Unclear risk & Judgement Comment: No detail \\
\hline $\begin{array}{l}\text { Blinding of participants } \\
\text { and personnel (perfor- } \\
\text { mance bias) } \\
\text { All outcomes }\end{array}$ & Unclear risk & Judgement Comment: No detail \\
\hline $\begin{array}{l}\text { Blinding of outcome as- } \\
\text { sessment (detection bias) } \\
\text { All outcomes }\end{array}$ & Low risk & Judgement Comment: Blind outcome assessment \\
\hline $\begin{array}{l}\text { Incomplete outcome data } \\
\text { (attrition bias) } \\
\text { All outcomes }\end{array}$ & Low risk & $\begin{array}{l}\text { Judgement Comment: Attrition detailed, differences between completers and } \\
\text { dropouts examined and reported; details for managing missing data reported }\end{array}$ \\
\hline $\begin{array}{l}\text { Selective reporting (re- } \\
\text { porting bias) }\end{array}$ & Low risk & Judgement Comment: Outcomes for planned measures reported \\
\hline $\begin{array}{l}\text { Other source of bias - } \\
\text { Therapy integrity }\end{array}$ & Low risk & $\begin{array}{l}\text { Judgement Comment: Trained therapists, regular supervisor to monitor ad- } \\
\text { herence, measures of fidelity given }\end{array}$ \\
\hline Other bias & Low risk & Judgement Comment: The study appears to be free of other sources of bias \\
\hline
\end{tabular}

Hirshfeld Becker 2010

\section{Study characteristics}

\begin{tabular}{ll}
\hline Methods & Study design: randomised controlled trial \\
Total duration of study: 1 -year non-controlled follow-up \\
Number of study centres and location: 1 in the USA \\
Study setting: research clinic \\
Date of study: February 2001 to January 2004 \\
\hline Participants \\
Mean age (CBD): $5.4(1.0)$
\end{tabular}

Cognitive behavioural therapy for anxiety disorders in children and adolescents (Review)

Copyright ๑ 2020 The Cochrane Collaboration. Published by John Wiley \& Sons, Ltd. 
Hirshfeld Becker 2010 (Continued)

\section{Age range: 4 to 7}

Gender: 34 (53\%) female

Inclusion criteria: current DSM-IV anxiety disorder (based on K-SADS)

Exclusion criteria: active psychosis, suicidality, or substance abuse in a parent; mental retardation in the child; current psychiatric treatment or past CBT; if a consensus of 2 senior clinicians judged the child to be too unco-operative or distractible to take part in the trial or too severely symptomatic to wait 6 months to receive treatment, based on suicidal ideation, serious impairment in eating or sleeping habits, severe social isolation, severe impairment in school function or attendance, or severe OCD

Interventions

\section{CBT}

Intervention: Being Brave: A Program for Coping with Anxiety for Young Children and Their Parents. Loosely modelled after the manualised Coping Cat programme

\section{Concomitant/excluded medications:}

Delivery format: parent only, and child and parent sessions; individual

Therapist contact time: up to 20 sessions over 6 months

Who delivers the intervention: psychologists and postdoctoral fellow

\section{Waitlist/no treatment}

Intervention: 6-month waitlist

$\begin{array}{ll}\text { Outcomes } & \text { Remission of primary anxiety disorder diagnosis post-treatment: K-SADS-E } \\ \text { Remission of all anxiety disorder diagnoses post-treatment: } K-S A D S-E\end{array}$

Notes

\section{Risk of bias}

\begin{tabular}{lll}
\hline Bias & Authors' judgement & Support for judgement \\
\hline $\begin{array}{l}\text { Random sequence genera- } \\
\text { tion (selection bias) }\end{array}$ & Low risk & $\begin{array}{l}\text { Judgement Comment: Block randomisation; the allocation sequences were } \\
\text { generated in advance for the } 2 \text { cells (presence or absence of parental anxiety } \\
\text { disorders) by the study co-ordinator }\end{array}$ \\
\hline
\end{tabular}

\begin{tabular}{ll}
\hline $\begin{array}{l}\text { Allocation concealment } \\
\text { (selection bias) }\end{array}$ & $\begin{array}{l}\text { Judgement Comment: Concealed in a computer file from all other staff (in- } \\
\text { cluding the clinicians who enrolled the participants, the assessors and clini- } \\
\text { cians who conducted the assessments, and the clinicians who determined the } \\
\text { eligibility of each child) }\end{array}$ \\
\hline
\end{tabular}

\begin{tabular}{ll}
\hline $\begin{array}{l}\text { Blinding of participants } \\
\text { and personnel (perfor- }\end{array}$ & Low risk \\
Judgement Comment: Even though blinding was not possible, we judged that \\
it was unlikely this led to a departure from the intended intervention
\end{tabular}
mance bias)

All outcomes

\begin{tabular}{lll}
\hline $\begin{array}{l}\text { Blinding of outcome as- } \\
\text { sessment (detection bias) } \\
\text { All outcomes }\end{array}$ & Low risk & Judgement Comment: Blind assessors \\
\hline $\begin{array}{l}\text { Incomplete outcome data } \\
\text { (attrition bias) }\end{array}$ & Low risk & $\begin{array}{l}\text { Judgement Comment: Attrition fully reported, and for all measures. Quite high } \\
\text { level of missing data for CBCL-I, which is potentially related to outcome but } \\
\text { not used here }\end{array}$ \\
\hline
\end{tabular}


Hirshfeld Becker 2010 (Continued)

Selective reporting (re- Low risk Judgement Comment: Outcomes reported for all measures
porting bias)

\begin{tabular}{|c|c|c|}
\hline $\begin{array}{l}\text { Other source of bias - } \\
\text { Therapy integrity }\end{array}$ & Unclear risk & $\begin{array}{l}\text { Judgement Comment: Intervention delivered by psychologists/postdoctoral } \\
\text { fellow, but no detail given on assessment of adherence and competence }\end{array}$ \\
\hline
\end{tabular}

Other bias Low risk Judgement Comment: The study appears to be free of other sources of bias

Holmes 2014

\section{Study characteristics}

\begin{tabular}{|c|c|}
\hline Methods & $\begin{array}{l}\text { Study design: randomised controlled trial } \\
\text { Total duration of study: non-controlled 3-month follow-up } \\
\text { Number of study centres and location: } 1 \text { in Australia }\end{array}$ \\
\hline Participants & $\begin{array}{l}\mathbf{N}=42 \text { (CBT: } N=20 \text {; waitlist: } N=22 \text { ) } \\
\text { Mean age (SD): } 9.64 \text { (1.41) } \\
\text { Age range: } 7 \text { to } 12 \\
\text { Gender: } 28(66.7 \%) \text { female } \\
\text { Inclusion criteria: aged } 7 \text { to 12, primary diagnosis GAD (based on ADIS-C/P) } \\
\text { Exclusion criteria: pervasive developmental disorder, intellectual handicap or learning disability, be- } \\
\text { havioural problems more impairing than anxiety, substance abuse, self-harm or suicidal ideation; cur- } \\
\text { rently receiving psychological assistance or medical treatment }\end{array}$ \\
\hline Interventions & $\begin{array}{l}\text { CBT } \\
\text { Intervention: No Worries - target intolerance of uncertainty, negative beliefs about worry, negative } \\
\text { problem orientation, cognitive avoidance } \\
\text { Delivery format: child and parent; group } \\
\text { Therapist contact time: } 15 \text { hours ( } 10 \times 90 \text { minutes) } \\
\text { Who delivers the intervention: postgraduate students receiving advanced clinical training, super- } \\
\text { vised } \\
\text { Waitlist/no treatment } \\
\text { Intervention: } 12 \text {-week waitlist }\end{array}$ \\
\hline Outcomes & $\begin{array}{l}\text { Remission of primary anxiety disorder diagnosis post-treatment: ADIS-C/P } \\
\text { Reduction in anxiety symptoms (child report) post-treatment: SCAS-C } \\
\text { Reduction in anxiety symptoms (parent report) post-treatment: SCAS-P } \\
\text { Improvement in global functioning post-treatment: CGAS }\end{array}$ \\
\hline
\end{tabular}

Notes

\section{Risk of bias}

Cognitive behavioural therapy for anxiety disorders in children and adolescents (Review)

Copyright $\odot 2020$ The Cochrane Collaboration. Published by John Wiley \& Sons, Ltd. 
Holmes 2014 (Continued)

\begin{tabular}{|c|c|c|}
\hline Bias & Authors' judgement & Support for judgement \\
\hline $\begin{array}{l}\text { Random sequence genera- } \\
\text { tion (selection bias) }\end{array}$ & Low risk & Judgement Comment: Computer-generated, blocked randomisation list \\
\hline $\begin{array}{l}\text { Allocation concealment } \\
\text { (selection bias) }\end{array}$ & Low risk & Judgement Comment: Computer generated, so unlikely to foresee assignment \\
\hline $\begin{array}{l}\text { Blinding of participants } \\
\text { and personnel (perfor- } \\
\text { mance bias) } \\
\text { All outcomes }\end{array}$ & Low risk & $\begin{array}{l}\text { Judgement Comment: Even though blinding was not possible, we judged that } \\
\text { it was unlikely this led to a departure from the intended intervention }\end{array}$ \\
\hline $\begin{array}{l}\text { Blinding of outcome as- } \\
\text { sessment (detection bias) } \\
\text { All outcomes }\end{array}$ & Low risk & $\begin{array}{l}\text { Judgement Comment: Assessors blind to both experimental condition and } \\
\text { client history }\end{array}$ \\
\hline $\begin{array}{l}\text { Incomplete outcome data } \\
\text { (attrition bias) } \\
\text { All outcomes }\end{array}$ & Low risk & $\begin{array}{l}\text { Judgement Comment: Dropouts and reasons for them detailed and clear. } \\
\text { Completer analysis reported, and compared to ITT. }\end{array}$ \\
\hline $\begin{array}{l}\text { Selective reporting (re- } \\
\text { porting bias) }\end{array}$ & Low risk & Judgement Comment: Outcomes for all measures reported. \\
\hline $\begin{array}{l}\text { Other source of bias - } \\
\text { Therapy integrity }\end{array}$ & Low risk & $\begin{array}{l}\text { Judgement Comment: Adherence rated and reported; delivered by provision- } \\
\text { ally registered psychologists, with regular supervision }\end{array}$ \\
\hline Other bias & Low risk & Judgement Comment: The study appears to be free of other sources of bias \\
\hline
\end{tabular}

Hudson 2009

\title{
Study characteristics
}

\begin{tabular}{l} 
Study design: randomised controlled trial \\
Total duration of study: 3 -month follow-up \\
Number of study centres and location: 1 in Australia \\
Study setting: research clinic \\
\hline
\end{tabular}

Participants $\quad \mathbf{N}=\mathbf{1 1 2}($ CBT: $N=60$; active control: $N=52)$

Mean age (SD): CBT: $10.2(2.4)(n=51)$; active control: $0.2(2.7)(n=44)$

Age range: 7 to 16

Gender: CBT: $37.3 \%$ female $(n=51)$; active control: $52.3 \%$ female $(n=44)$

Inclusion criteria: primary anxiety disorder (based on ADIS-C/P)

Exclusion criteria: mental retardation, psychoses, and concurrent psychological treatment

\author{
Interventions \\ CBT \\ Intervention: Cool Kids \\ Concomitant/excluded medications: allowed if stable
}


Hudson 2009 (Continued)

Delivery format: child and parent; group

Therapist contact time: 20 hours ( $10 \times 2$ hours)

Who delivers the intervention: clinical psychologists or postgraduate students

\section{Active control}

Intervention: group support and attention. Aim to provide supportive environment, activities to help express and understand emotions and build relationships between and within families. Therapists instructed not to provide CBT interpretations or strategies.

Concomitant/excluded medications: allowed if stable

Delivery format: child and parent; group

Therapist contact time: 20 hours ( $10 \times 2$ hours)

Who delivers the intervention: clinical psychologists or postgraduate students

Remission of primary anxiety disorder diagnosis post-treatment and 3-month follow-up: ADIS-C/P
Remission of all anxiety disorder diagnoses post-treatment and 3-month follow-up: ADIS-C/P
Reduction in anxiety symptoms (child report) post-treatment and 3-month follow-up: SCAS-C
Reduction in anxiety symptoms (parent report-mother) post-treatment and 3-month follow-up:
SCAS-P

Notes Active control = attention control

\section{Risk of bias}

\begin{tabular}{|c|c|c|}
\hline Bias & Authors' judgement & Support for judgement \\
\hline $\begin{array}{l}\text { Random sequence genera- } \\
\text { tion (selection bias) }\end{array}$ & Low risk & $\begin{array}{l}\text { Judgement Comment: Used a schedule from a random number generator to } \\
\text { assign each group }\end{array}$ \\
\hline $\begin{array}{l}\text { Allocation concealment } \\
\text { (selection bias) }\end{array}$ & Unclear risk & $\begin{array}{l}\text { Judgement Comment: Performed by principal investigator. No detail on at- } \\
\text { tempt to conceal allocation }\end{array}$ \\
\hline $\begin{array}{l}\text { Blinding of participants } \\
\text { and personnel (perfor- } \\
\text { mance bias) } \\
\text { All outcomes }\end{array}$ & Low risk & $\begin{array}{l}\text { Judgement Comment: Appropriate methods to conceal differences between } \\
\text { control and CBT; participants told } 1 \text { treatment new to the clinic, once assign- } \\
\text { ment informed about their condition }\end{array}$ \\
\hline $\begin{array}{l}\text { Blinding of outcome as- } \\
\text { sessment (detection bias) } \\
\text { All outcomes }\end{array}$ & Unclear risk & $\begin{array}{l}\text { Judgement Comment: Reliability of ADIS outcomes assessed, but no detail on } \\
\text { blinding of assessors/double raters }\end{array}$ \\
\hline $\begin{array}{l}\text { Incomplete outcome data } \\
\text { (attrition bias) } \\
\text { All outcomes }\end{array}$ & Low risk & $\begin{array}{l}\text { Judgement Comment: Attrition detailed. Appropriate methods for handling } \\
\text { missing data. Completer analyses reported, and ITT also performed for com- } \\
\text { parison. }\end{array}$ \\
\hline $\begin{array}{l}\text { Selective reporting (re- } \\
\text { porting bias) }\end{array}$ & Low risk & Judgement Comment: Outcomes for all measures reported \\
\hline $\begin{array}{l}\text { Other source of bias - } \\
\text { Therapy integrity }\end{array}$ & Low risk & $\begin{array}{l}\text { Judgement Comment: Therapists qualified and supervised. Therapy adher- } \\
\text { ence assessed and reported. Detailed procedure for managing } 1 \text { instance of } \\
\text { protocol breach }\end{array}$ \\
\hline
\end{tabular}


Hudson 2009 (Continued)

Other bias Low risk_ Judgement Comment: The study appears to be free of other sources of bias

Ingul 2013

\section{Study characteristics}

\begin{tabular}{ll}
\hline Methods & Study design: randomised controlled trial \\
Total duration of study: non-controlled 12-month follow-up \\
Number of study centres and location: Norway \\
Study setting: recruitment through school screening \\
\hline N $=\mathbf{1 2 8}$ (CBT-individual: $\mathrm{N}=36 ; \mathrm{CBT}$-group: $\mathrm{N}=58$; active control: $\mathrm{N}=34)$ \\
Mean age (SD): $14.5(1.01)(\mathrm{n}=57)$ \\
Age range: 13 to 16 \\
Gender: 32 (56.1\%) female ( $\mathrm{n}=57)$ \\
Inclusion criteria: primary problem social phobia diagnosis (based on ADIS-C) \\
Exclusion criteria: mental retardation or psychosis; being treated elsewhere for mental health condi- \\
tions
\end{tabular}

\section{CBT-individual}

Intervention: Clark and Wells manual, adapted for adolescents

Delivery format: child only; individual

Therapist contact time: 10 hours $(12 \times 50$ minutes $)$

Who delivers the intervention: clinical psychologist and co-therapists

\section{CBT-group}

Intervention: based on CAT Project Manual for CBT of Anxious Adolescents, with some play elements from SET-C

Delivery format: child only; group

Therapist contact time: 15 hours ( $10 \times 90$ minutes)

Who delivers the intervention: clinical psychologist and co-therapists

\section{Active control}

Intervention: attentional placebo. No active components in CBT. Social activity, social interaction, and social support

Delivery format: child only; group

Therapist contact time: 15 hours ( $10 \times 90$ minutes)

Who delivers the intervention: experienced milieu therapists 
Ingul 2013 (Continued)

\section{Reduction in depressive symptoms post-treatment: $C D I$}

\begin{tabular}{|c|c|c|}
\hline Notes & \multicolumn{2}{|c|}{$\begin{array}{l}\text { Active control = attention control } \\
\text { Follow-up diagnostic assessment, but only CBT groups }\end{array}$} \\
\hline \multicolumn{3}{|l|}{ Risk of bias } \\
\hline Bias & Authors' judgement & Support for judgement \\
\hline $\begin{array}{l}\text { Random sequence genera- } \\
\text { tion (selection bias) }\end{array}$ & Low risk & Judgement Comment: Pre-assigned random schedule \\
\hline $\begin{array}{l}\text { Allocation concealment } \\
\text { (selection bias) }\end{array}$ & Unclear risk & Judgement Comment: State performed at each site, but no further detail \\
\hline $\begin{array}{l}\text { Blinding of participants } \\
\text { and personnel (perfor- } \\
\text { mance bias) } \\
\text { All outcomes }\end{array}$ & Unclear risk & Judgement Comment: No detail given \\
\hline $\begin{array}{l}\text { Blinding of outcome as- } \\
\text { sessment (detection bias) } \\
\text { All outcomes }\end{array}$ & Low risk & Judgement Comment: Assessors blind \\
\hline $\begin{array}{l}\text { Incomplete outcome data } \\
\text { (attrition bias) } \\
\text { All outcomes }\end{array}$ & Unclear risk & $\begin{array}{l}\text { Judgement Comment: High dropout rate and large amount of missing data } \\
\text { and related to treatment group, although ITT analysis reported to confirm } \\
\text { completer analysis }\end{array}$ \\
\hline $\begin{array}{l}\text { Selective reporting (re- } \\
\text { porting bias) }\end{array}$ & Low risk & Judgement Comment: Outcomes for measures reported \\
\hline $\begin{array}{l}\text { Other source of bias - } \\
\text { Therapy integrity }\end{array}$ & Unclear risk & $\begin{array}{l}\text { Judgement Comment: Therapists appropriately qualified. Adherence and } \\
\text { competence assessed and reported in } 2 \text { CBT groups, but not the control. }\end{array}$ \\
\hline Other bias & Low risk & Judgement Comment: The study appears to be free of other sources of bias \\
\hline
\end{tabular}

Ishikawa 2019

\section{Study characteristics}

Methods Study design: randomised controlled trial

Total duration of study: non-controlled 3- and 6-month follow-up

Number of study centres and location: Japan

Study setting: clinic

Date of study: recruitment 2012 to 2015; follow-up complete 2017

Participants $\quad \mathbf{N}=\mathbf{5 1}(\mathrm{CBT}: \mathrm{N}=26$; waitlist: $\mathrm{N}=25)$

Mean age (SD): $10.9(2.0)$

Age range: 8 to 15

Gender: 29 (56.9\%) female 
Inclusion criteria: (a) age 7 to 15, (b) attend the programme with their parents, (c) have an anxiety disorder (based on ADIS), (d) did not fulfil criteria for PTSD, disruptive behavioural disorders, substance abuse, mental retardation, pervasive developmental disorder, or a psychotic disorder, and (e) agree to discontinue other forms of therapy for the duration of the study

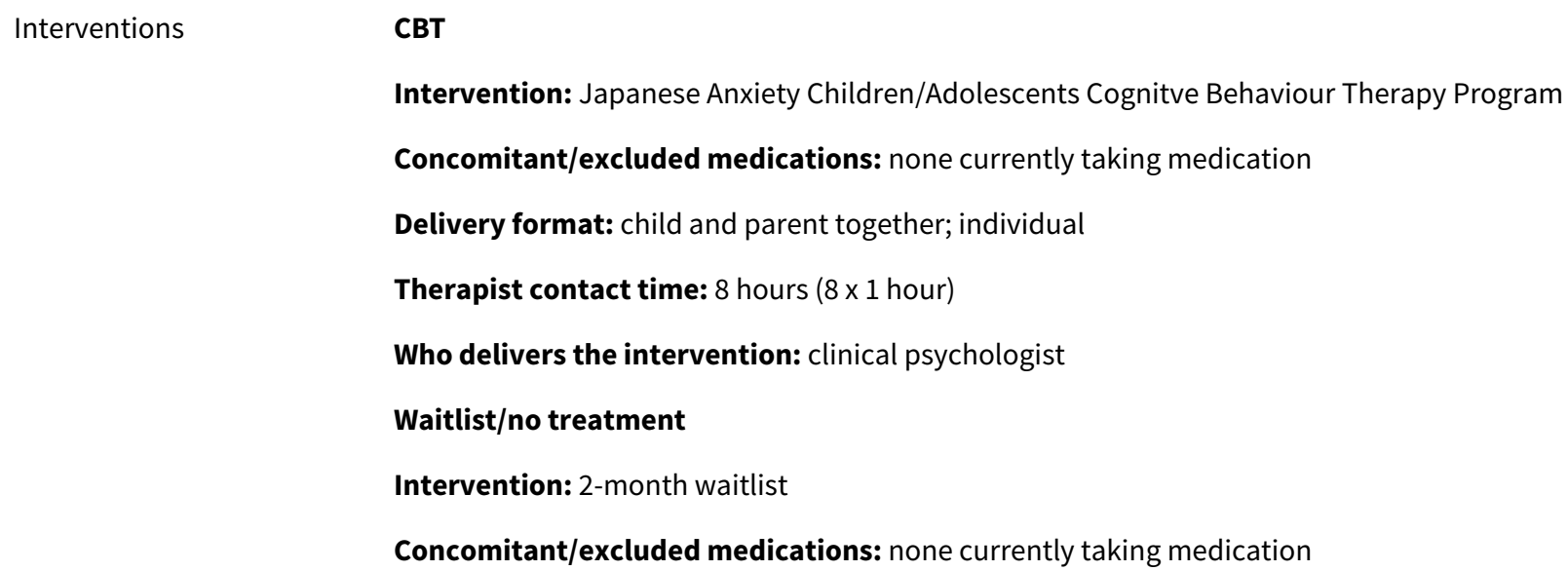

Remission of all anxiety disorder diagnoses post-treatment: ADIS-C/P (parent and child interviewed together)

Reduction in anxiety symptoms (child report) post-treatment: SCAS-C

Reduction in anxiety symptoms (parent report) post-treatment: SCAS-P

Reduction in depressive symptoms post-treatment: CDI

Notes 4 participants met diagnostic criteria for an anxiety disorder, but primary disorder was a depressive disorder. Included in all analyses apart from remission of primary anxiety disorder diagnosis

\section{Risk of bias}

\begin{tabular}{|c|c|c|}
\hline Bias & Authors' judgement & Support for judgement \\
\hline $\begin{array}{l}\text { Random sequence genera- } \\
\text { tion (selection bias) }\end{array}$ & Low risk & Judgement Comment: Random number generator \\
\hline $\begin{array}{l}\text { Allocation concealment } \\
\text { (selection bias) }\end{array}$ & Low risk & Judgement Comment: Independent person \\
\hline $\begin{array}{l}\text { Blinding of participants } \\
\text { and personnel (perfor- } \\
\text { mance bias) } \\
\text { All outcomes }\end{array}$ & Low risk & $\begin{array}{l}\text { Judgement Comment: Even though blinding was not possible, we judged that } \\
\text { it was unlikely this led to a departure from the intended intervention }\end{array}$ \\
\hline $\begin{array}{l}\text { Blinding of outcome as- } \\
\text { sessment (detection bias) } \\
\text { All outcomes }\end{array}$ & Low risk & Judgement Comment: Blind assessors \\
\hline $\begin{array}{l}\text { Incomplete outcome data } \\
\text { (attrition bias) } \\
\text { All outcomes }\end{array}$ & Low risk & $\begin{array}{l}\text { Judgement Comment: Report ITT and completer analyses. Only } 1 \text { dropout in } \\
\text { each condition }\end{array}$ \\
\hline
\end{tabular}


Ishikawa 2019 (Continued)

Selective reporting (re- Low risk_ Judgement Comment: Outcomes for measures reported porting bias)

Other source of bias - Low risk

Judgement Comment: Trained therapists. Adherence assessed and reported.

Therapy integrity

Other bias High risk

Judgement Comment: Difference in timing for post-assessment for CBT vs waitlist - shorter time period for waitlist (70 days vs 122). Included children with depressive disorders as primary diagnoses

\section{Kendall 1994}

\section{Study characteristics}

\begin{tabular}{ll}
\hline Methods & Study design: randomised controlled trial \\
Total duration of study: non-controlled long-term follow-up \\
Number of study centres and location: 1 in the USA \\
Study setting: university clinic \\
\hline
\end{tabular}

Participants $\quad \mathbf{N}=\mathbf{6 0}(\mathrm{CBT}: \mathrm{N}=30$; waitlist: $\mathrm{N}=30)$

Age range: 9 to 13

Gender: $40 \%$ female $(n=47)$

Inclusion criteria: primary anxiety disorder diagnosis (i.e. over-anxious disorder, separation anxiety disorder, avoidant disorder) based on ADIS-C/P

Exclusion criteria: primary diagnosis of specific phobia, IQ below 80, disabling physical condition, displayed psychotic symptoms, currently using anti-anxiety medications

Intervention: Coping Cat

Concomitant/excluded medications: anti-anxiety medication excluded

Delivery format: child only; individual

Therapist contact time: 13 to 20 hours (16 to $20 \times 50$ - to 60 -minute sessions over 16 weeks)

Who delivers the intervention: doctoral students

Waitlist/no treatment

Intervention: 8-week waitlist

Concomitant/excluded medications: anti-anxiety medication excluded

Outcomes Remission of primary anxiety disorder diagnosis post-treatment: ADIS-P

Reduction in anxiety symptoms (child report) post-treatment: RCMAS

Reduction in anxiety symptoms (parent report) post-treatment: STAIC-trait-parent

Reduction in depressive symptoms post-treatment: CDI 
Kendall 1994 (Continued)

Notes

\section{Risk of bias}

\begin{tabular}{|c|c|c|}
\hline Bias & Authors' judgement & Support for judgement \\
\hline $\begin{array}{l}\text { Random sequence genera- } \\
\text { tion (selection bias) }\end{array}$ & Unclear risk & Judgement Comment: No detail given \\
\hline $\begin{array}{l}\text { Allocation concealment } \\
\text { (selection bias) }\end{array}$ & Unclear risk & Judgement Comment: No detail given \\
\hline $\begin{array}{l}\text { Blinding of participants } \\
\text { and personnel (perfor- } \\
\text { mance bias) } \\
\text { All outcomes }\end{array}$ & Low risk & $\begin{array}{l}\text { Judgement Comment: Even though blinding was not possible, we judged that } \\
\text { it was unlikely this led to a departure from the intended intervention }\end{array}$ \\
\hline $\begin{array}{l}\text { Blinding of outcome as- } \\
\text { sessment (detection bias) } \\
\text { All outcomes }\end{array}$ & Unclear risk & $\begin{array}{l}\text { Judgement Comment: Specify observational data rated by blind assessors, } \\
\text { but no detail about diagnostic assessments }\end{array}$ \\
\hline $\begin{array}{l}\text { Incomplete outcome data } \\
\text { (attrition bias) } \\
\text { All outcomes }\end{array}$ & Unclear risk & $\begin{array}{l}\text { Judgement Comment: Number of dropouts detailed, and dropouts vs remain- } \\
\text { ers compared, but analyses only include completers; insufficient detail on how } \\
\text { missing data were managed or comparison with ITT }\end{array}$ \\
\hline $\begin{array}{l}\text { Selective reporting (re- } \\
\text { porting bias) }\end{array}$ & Low risk & Judgement Comment: Outcomes for measures reported \\
\hline $\begin{array}{l}\text { Other source of bias - } \\
\text { Therapy integrity }\end{array}$ & Low risk & $\begin{array}{l}\text { Judgement Comment: Therapists appropriately qualified, integrity assessed } \\
\text { and reported }\end{array}$ \\
\hline Other bias & High risk & $\begin{array}{l}\text { Judgement Comment: Endpoint different for waitlist (8 weeks) and CBT group } \\
\text { (16 weeks) }\end{array}$ \\
\hline
\end{tabular}

Kendall 1997

\section{Study characteristics}

Study design: randomised controlled trial
Total duration of study: non-controlled long-term follow-up
Number of study centres and location: 1 in the USA
Study setting: university clinic
N $=\mathbf{1 1 8}$ (CBT: $\mathrm{N}=69 ;$ waitlist: $\mathrm{N}=49)$
Age range: 9 to 13
Gender: $38 \%$ female $(\mathrm{n}=94)$
Inclusion criteria: age 9 to $13 ;$ primary anxiety disorder (overanxious disorder, separation anxiety dis-
order, avoidant disorder) based on ADIS, NB: diagnosis based on parent report when discrepancy with
child report


Exclusion criteria: primary specific phobia diagnosis, psychotic symptoms, taking anti-anxiety medication

\begin{tabular}{|c|c|c|}
\hline Interventions & \multicolumn{2}{|c|}{$\begin{array}{l}\text { CBT } \\
\text { Intervention: Coping Cat } \\
\text { Concomitant/excluded medications: anti-anxiety medication excluded } \\
\text { Delivery format: child-only sessions and parent meeting; individual } \\
\text { Therapist contact time: } 18 \text { hours (18 x } 1 \text { hour) } \\
\text { Who delivers the intervention: doctoral students } \\
\text { Waitlist/no treatment } \\
\text { Intervention: } 8 \text {-week waitlist } \\
\text { Concomitant/excluded medications: anti-anxiety medication excluded }\end{array}$} \\
\hline Outcomes & \multicolumn{2}{|c|}{$\begin{array}{l}\text { Remission of primary anxiety disorder diagnosis post-treatment: ADIS-P } \\
\text { Reduction in anxiety symptoms (child report) post-treatment: RCMAS } \\
\text { Reduction in anxiety symptoms (parent report) post-treatment: } C B C L-A n x i e t y \\
\text { Reduction in depressive symptoms post-treatment: } C D I\end{array}$} \\
\hline \multicolumn{3}{|l|}{ Notes } \\
\hline \multicolumn{3}{|l|}{ Risk of bias } \\
\hline Bias & Authors' judgement & Support for judgement \\
\hline $\begin{array}{l}\text { Random sequence genera- } \\
\text { tion (selection bias) }\end{array}$ & Unclear risk & Judgement Comment: No detail given \\
\hline $\begin{array}{l}\text { Allocation concealment } \\
\text { (selection bias) }\end{array}$ & Unclear risk & Judgement Comment: No detail given \\
\hline $\begin{array}{l}\text { Blinding of participants } \\
\text { and personnel (perfor- } \\
\text { mance bias) } \\
\text { All outcomes }\end{array}$ & Low risk & $\begin{array}{l}\text { Judgement Comment: Even though blinding was not possible, we judged that } \\
\text { it was unlikely this led to a departure from the intended intervention }\end{array}$ \\
\hline $\begin{array}{l}\text { Blinding of outcome as- } \\
\text { sessment (detection bias) } \\
\text { All outcomes }\end{array}$ & Unclear risk & Judgement Comment: No detail given \\
\hline $\begin{array}{l}\text { Incomplete outcome data } \\
\text { (attrition bias) } \\
\text { All outcomes }\end{array}$ & Unclear risk & $\begin{array}{l}\text { Judgement Comment: Dropout numbers given and reported that no there was } \\
\text { no difference between dropouts and completers on pretreatment characteris- } \\
\text { tics, but reasons for dropouts not reported. No detail on missing data for indi- } \\
\text { vidual measures or how missing data for questionnaires were managed }\end{array}$ \\
\hline $\begin{array}{l}\text { Selective reporting (re- } \\
\text { porting bias) }\end{array}$ & Low risk & Judgement Comment: Outcomes reported for all measures \\
\hline $\begin{array}{l}\text { Other source of bias - } \\
\text { Therapy integrity }\end{array}$ & Low risk & $\begin{array}{l}\text { Judgement Comment: Therapists appropriately qualified. Integrity indepen- } \\
\text { dently assessed. }\end{array}$ \\
\hline
\end{tabular}


Kendall 1997 (Continued) $\begin{array}{ll}\text { Other bias } \quad \text { High risk } & \begin{array}{l}\text { Judgement Comment: Time of endpoint (post-assessment) differed between } \\ \text { waitlist and treatment group }\end{array}\end{array}$

\section{Study characteristics}

\begin{tabular}{l} 
Study design: randomised controlled trial \\
Total duration of study: non-controlled long-term follow-up \\
Number of study centres and location: 1 in the USA \\
Study setting: university clinic \\
Date of study: referred 2000 to 2006 \\
\hline
\end{tabular}

Participants

$\mathbf{N}=\mathbf{1 6 1}$ (CBT-individual: $\mathrm{N}=55$; CBT-family: $\mathrm{N}=56$; active control: $\mathrm{N}=50$ )

Age range: 7 to 14

Gender: $71(44 \%)$ female

Inclusion criteria: age 7 to 14; primary anxiety disorder (based on ADIS-C/P), at least 1 parent speaks English

Exclusion criteria: psychotic symptoms, mental retardation, a disabling medical condition, the child's participation in concurrent treatment, or the child's taking anti-anxiety or anti-depressant medications

Intervention: Coping Cat

Concomitant/excluded medications: anti-anxiety medication excluded

Delivery format: child (14 sessions), parent (2 sessions); individual

Therapist contact time: 16 hours ( $16 \times 1$ hour)

Who delivers the intervention: master's level therapists with 2 to 3 years of experience at the clinic and doctoral-level psychologist

\section{CBT}

Intervention: family CBT for anxious children manual (Howard, Chu, Krain, Marrs-Garcia, \& Kendall, 2000) and used the Coping Cat Workbook

Concomitant/excluded medications: anti-anxiety medication excluded

Delivery format: child and parent together, 2 sessions child and parent separate; individual

Therapist contact time: 16 hours ( $16 \times 1$ hour)

Who delivers the intervention: master's level therapists with 2 to 3 years of experience at the CAADC and doctoral-level psychologist

\section{Active control}

Intervention: manual for family education, support, and attention for anxious children and used a workbook to add child interest/involvement 
Concomitant/excluded medications: anti-anxiety medication excluded

\begin{tabular}{|c|c|}
\hline \multirow[t]{5}{*}{ Outcomes } & Remission of primary anxiety disorder diagnosis post-treatment: ADIS-C/P \\
\hline & Remission of all anxiety disorder diagnoses post-treatment: $A D I S-C / P$ \\
\hline & Reduction in anxiety symptoms (child report) post-treatment: MASC-C \\
\hline & 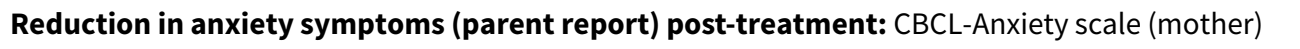 \\
\hline & Reduction in depressive symptoms post-treatment: $C D I$ \\
\hline Notes & $\begin{array}{l}\text { Include 1-year follow-up, but control group who still met criteria offered treatment after post-treat- } \\
\text { ment, so did not include follow-up here } \\
\text { Active control = attention control }\end{array}$ \\
\hline
\end{tabular}

\title{
Risk of bias
}

\begin{tabular}{|c|c|c|}
\hline Bias & Authors' judgement & Support for judgement \\
\hline $\begin{array}{l}\text { Random sequence genera- } \\
\text { tion (selection bias) }\end{array}$ & Low risk & $\begin{array}{l}\text { Judgement Comment: The co-ordinator used a predetermined schedule (ran- } \\
\text { dom number generated) }\end{array}$ \\
\hline $\begin{array}{l}\text { Allocation concealment } \\
\text { (selection bias) }\end{array}$ & Low risk & $\begin{array}{l}\text { Judgement Comment: Key to blind held by co-ordinator, not primary investi- } \\
\text { gator or therapist, but no further detail given }\end{array}$ \\
\hline $\begin{array}{l}\text { Blinding of participants } \\
\text { and personnel (perfor- } \\
\text { mance bias) } \\
\text { All outcomes }\end{array}$ & Unclear risk & Judgement Comment: No detail given regarding blinding of participants \\
\hline $\begin{array}{l}\text { Blinding of outcome as- } \\
\text { sessment (detection bias) } \\
\text { All outcomes }\end{array}$ & Low risk & $\begin{array}{l}\text { Judgement Comment: Independent assessors and families asked not to dis- } \\
\text { cuss treatment }\end{array}$ \\
\hline $\begin{array}{l}\text { Incomplete outcome data } \\
\text { (attrition bias) } \\
\text { All outcomes }\end{array}$ & Low risk & $\begin{array}{l}\text { Judgement Comment: Attrition detailed, and reasons for dropouts given. ITT } \\
\text { analysis. Missing data for measures handled appropriately (last observation } \\
\text { carried forward). }\end{array}$ \\
\hline $\begin{array}{l}\text { Selective reporting (re- } \\
\text { porting bias) }\end{array}$ & Low risk & Judgement Comment: Outcomes reported for all measures \\
\hline $\begin{array}{l}\text { Other source of bias - } \\
\text { Therapy integrity }\end{array}$ & Unclear risk & $\begin{array}{l}\text { Judgement Comment: Therapists trained and supervised, and integrity as- } \\
\text { sessed. Evidence of CBT bleeding in control group could have influenced out- } \\
\text { comes. }\end{array}$ \\
\hline Other bias & Low risk & Judgement Comment: The study appears to be free of other sources of bias \\
\hline
\end{tabular}

\section{Study characteristics}

Methods

\author{
Study design: randomised controlled trial \\ Total duration of study: 6 months (post-treatment assessment) \\ Number of study centres and location: Australia
}


Kennedy 2009 (Continued)

Study setting: university

N $=\mathbf{7 1}$ (CBT: N = 35; waitlist: $\mathrm{N}=36)$
Mean age (SD): 47.07 months (7.05 months)
Age range: 36 to 58 months
Gender: CBT: $58 \%$ female; waitlist: $51 \%$ female
Inclusion criteria: child aged 36 to 58 months, high on a laboratory measure of behavioural inhibition
(BI), at least 1 parent met DSM-IV criteria for diagnosis of an anxiety disorder (NB: not inclusion criteria,
but all children meet diagnostic criteria for anxiety disorder based on ADIS-P)
Exclusion criteria: diagnosed developmental disorder or a severe language delay in the child, or par-
ents who were unable to complete questionnaires in English

Interventions

CBT

Intervention: parent intervention - modified Cool Kids Program

Delivery format: parent only; group

Therapist contact time: 12 hours ( $8 \times 90$ minutes)

Who delivers the intervention: clinical psychologist

Waitlist/no treatment

Intervention: 6-month waitlist

$\begin{array}{ll}\text { Outcomes } & \text { Remission of all anxiety disorder diagnoses post-treatment: ADIS-P } \\ & \text { Reduction in anxiety symptoms (parent report) post-treatment: PAS-R (mother report) }\end{array}$

Post-treatment assessment point is 6 months.

\section{Risk of bias}

\begin{tabular}{lll}
\hline Bias & Authors' judgement & Support for judgement \\
\hline $\begin{array}{l}\text { Random sequence genera- } \\
\text { tion (selection bias) }\end{array}$ & Low risk & Judgement Comment: Toss of coin \\
\hline $\begin{array}{l}\text { Allocation concealment } \\
\text { (selection bias) }\end{array}$ & Unclear risk & Judgement Comment: No detail given \\
\hline $\begin{array}{l}\text { Blinding of participants } \\
\text { and personnel (perfor- } \\
\text { mance bias) }\end{array}$ & Low risk & $\begin{array}{l}\text { Judgement Comment: Even though blinding was not possible, we judged that } \\
\text { All outcomes }\end{array}$ \\
\hline $\begin{array}{l}\text { Blinding of outcome as- } \\
\text { sessment (detection bias) } \\
\text { All outcomes }\end{array}$ & Unclear risk & $\begin{array}{l}\text { Judgement Comment: ADIS assessors trained, and reliability assessed, but no } \\
\text { detail as to whether blind or not }\end{array}$ \\
\hline $\begin{array}{l}\text { Incomplete outcome data } \\
\text { (attrition bias) } \\
\text { All outcomes }\end{array}$ & Low risk & $\begin{array}{l}\text { Judgement Comment: Attrition detailed, and although reasons not given, } \\
\text { small numbers and across both groups }\end{array}$ \\
\hline
\end{tabular}


Kennedy 2009 (Continued)

Selective reporting (re- Low risk Judgement Comment: Outcomes reported for all measures porting bias)

Other source of bias -

Therapy integrity
Unclear risk

Other bias

Low risk

Judgement Comment: Therapy delivered by qualified and experienced therapist, but integrity not assessed/reported

Khanna 2010

\section{Study characteristics}

\begin{tabular}{|c|c|}
\hline Methods & $\begin{array}{l}\text { Study design: randomised controlled trial } \\
\text { Study setting: university } \\
\text { Date of study: } 2006 \text { to } 2008\end{array}$ \\
\hline \multirow[t]{4}{*}{ Participants } & $\begin{array}{l}\mathbf{N}=49 \text { randomised (CBT: } N=17 \text {; computerised CBT: 16; active control: } N=16) \text { (NB: } N=33 \text { included } \\
\text { here) }\end{array}$ \\
\hline & Age range: 7 to 13 \\
\hline & $\begin{array}{l}\text { Inclusion criteria: primary anxiety disorder (separation anxiety, social phobia, GAD, specific phobia, or } \\
\text { panic disorder) (based on ADIS-parent and child interviewed together) }\end{array}$ \\
\hline & $\begin{array}{l}\text { Exclusion criteria: } I Q<80 \text {, psychotic symptoms, use of anti-anxiety or depression medication or ongo- } \\
\text { ing psychotherapy }\end{array}$ \\
\hline
\end{tabular}

Interventions

CBT

Intervention: individual CBT - Coping Cat

Concomitant/excluded medications: use of anti-anxiety or depression medication not allowed

Delivery format: child only, with parent included in 2 sessions; individual

Therapist contact time: 10 hours $(12 \times 50$ minutes $)$

Who delivers the intervention: school psychologists, psychology doctoral candidates, clinical psychologist

\section{Active control}

Intervention: computer-assisted education support \& attention. Education, support, and attention manual (as in Kendal and colleagues, 2008) and child use of computer age-appropriate games

Concomitant/excluded medications: use of anti-anxiety or depression medication not allowed

Delivery format: child only; individual

Therapist contact time: 10 hours ( $12 \times 30$ minutes of support, 20 minutes child use computer)

Who delivers the intervention: school psychologists, psychology doctoral candidates, clinical psychologist

Outcomes

Remission of primary anxiety disorder diagnosis post-treatment: ADIS (parent and child interviewed together)

\section{Reduction in anxiety symptoms (child report) post-treatment: MASC-C}




\section{Reduction in depressive symptoms post-treatment: $C D I$}

Improvement in global functioning post-treatment: CGAS

\begin{tabular}{ll}
\hline Notes & Did not include "Camp Cope-A lot" arm (computer-assisted CBT) \\
Active control = attention control \\
\hline
\end{tabular}

\section{Risk of bias}

\begin{tabular}{|c|c|c|}
\hline Bias & Authors' judgement & Support for judgement \\
\hline $\begin{array}{l}\text { Random sequence genera- } \\
\text { tion (selection bias) }\end{array}$ & Low risk & Judgement Comment: Random number-generating program \\
\hline $\begin{array}{l}\text { Allocation concealment } \\
\text { (selection bias) }\end{array}$ & Low risk & Judgement Comment: Computer generated, so unlikely to foresee assignment \\
\hline $\begin{array}{l}\text { Blinding of participants } \\
\text { and personnel (perfor- } \\
\text { mance bias) } \\
\text { All outcomes }\end{array}$ & Unclear risk & $\begin{array}{l}\text { Judgement Comment: No evidence of blinding participants in individual CBT } \\
\text { vs control, but as control computer program potentially not feasible }\end{array}$ \\
\hline $\begin{array}{l}\text { Blinding of outcome as- } \\
\text { sessment (detection bias) } \\
\text { All outcomes }\end{array}$ & Low risk & Judgement Comment: Assessors blind to treatment condition \\
\hline $\begin{array}{l}\text { Incomplete outcome data } \\
\text { (attrition bias) } \\
\text { All outcomes }\end{array}$ & Low risk & $\begin{array}{l}\text { Judgement Comment: Attrition detailed and ITT analysis reported, with ap- } \\
\text { propriate imputation for missing data. } 2 \text { participants in control group with- } \\
\text { drawn due to worsening of symptoms. }\end{array}$ \\
\hline $\begin{array}{l}\text { Selective reporting (re- } \\
\text { porting bias) }\end{array}$ & Low risk & Judgement Comment: Outcomes reported \\
\hline $\begin{array}{l}\text { Other source of bias - } \\
\text { Therapy integrity }\end{array}$ & Low risk & $\begin{array}{l}\text { Judgement Comment: Therapist trained and supervised. Adherence assessed } \\
\text { and reported. }\end{array}$ \\
\hline Other bias & Low risk & Judgement Comment: The study appears to be free of other sources of bias \\
\hline
\end{tabular}

\section{Kidd 2018}

\section{Study characteristics}

\begin{tabular}{ll}
\hline Methods & Study design: randomised controlled trial \\
& Total duration of study: non-controlled 6-month follow-up \\
& Number of study centres and location: 1 in Australia \\
& Study setting: university clinic \\
\hline Participants & $\mathbf{4 9}$ (CBT: $\mathrm{N}=26 ;$ waitlist: $\mathrm{N}=23)$ \\
Mean age (SD): $14.23(1.76)$ \\
Age range: 12 to 18 \\
Gender: $9(18.4 \%)$ female
\end{tabular}


Kidd 2018 (Continued)

Inclusion criteria: meet diagnostic criteria for an anxiety disorder, with CSR 4+ (based on ADIS-P); able to demonstrate a clinical diagnosis of ASD, Asperger's disorder, or pervasive developmental disorder - not otherwise specified (PDD-NOS); aged 12 to 18; verbal IQ above 70; ability to read English; not taking any psychotropic medication or taking a stable dose of psychiatric medication and, if taking medication, agreement for this to be maintained throughout the study

Exclusion criteria: participated in psychotherapy, social skills training, or behavioural intervention over the course of the study; the family were attending child-related health professional services or parenting classes; taking psychiatric medication or changed his or her dosage during the intervention period; family was not able to participate in the intervention program

Interventions

CBT

Intervention: Cool Kids, ASD adaptation

Concomitant/excluded medications: stable dose, and maintain stable dose during study allowed

Delivery format: combination of child alone, parent alone, and child and parent together; group

Therapist contact time: 24 hours ( $12 \times 2$ hours)

Who delivers the intervention: psychology graduate trainee Master's or PhD students

Waitlist/no treatment

Intervention: 4.5-month waitlist

Concomitant/excluded medications: stable dose, and maintain stable dose during study allowed

\begin{tabular}{|c|c|c|}
\hline Outcomes & \multicolumn{2}{|c|}{$\begin{array}{l}\text { Remission of primary anxiety disorder diagnosis post-treatment: ADIS-P } \\
\text { Reduction in anxiety symptoms (child report) post-treatment: SCAS-C } \\
\text { Reduction in anxiety symptoms (parent report) post-treatment: SCAS-P } \\
\text { Reduction in depressive symptoms post-treatment: SMFQ-C }\end{array}$} \\
\hline Notes & Sample with ASD & \\
\hline \multicolumn{3}{|l|}{ Risk of bias } \\
\hline Bias & Authors' judgement & Support for judgement \\
\hline $\begin{array}{l}\text { Random sequence genera- } \\
\text { tion (selection bias) }\end{array}$ & Low risk & Judgement Comment: Randomisation software \\
\hline $\begin{array}{l}\text { Allocation concealment } \\
\text { (selection bias) }\end{array}$ & Low risk & Judgement Comment: Computer generated, so unlikely to foresee assignment \\
\hline $\begin{array}{l}\text { Blinding of participants } \\
\text { and personnel (perfor- } \\
\text { mance bias) } \\
\text { All outcomes }\end{array}$ & Low risk & $\begin{array}{l}\text { Judgement Comment: Even though blinding was not possible, we judged that } \\
\text { it was unlikely this led to a departure from the intended intervention }\end{array}$ \\
\hline $\begin{array}{l}\text { Blinding of outcome as- } \\
\text { sessment (detection bias) } \\
\text { All outcomes }\end{array}$ & Low risk & Judgement Comment: Blind assessors \\
\hline $\begin{array}{l}\text { Incomplete outcome data } \\
\text { (attrition bias) } \\
\text { All outcomes }\end{array}$ & Low risk & Judgement Comment:Missing data detailed and managed appropriately \\
\hline
\end{tabular}


Kidd 2018 (Continued)

Selective reporting (re- Low risk Judgement Comment:Outcomes reported for all measures
porting bias)

\begin{tabular}{lll}
\hline $\begin{array}{l}\text { Other source of bias - } \\
\text { Therapy integrity }\end{array}$ & Low risk & Judgement Comment: Independent integrity assessment \\
\hline Other bias & Low risk & Judgement Comment: The study appears to be free of other sources of bias \\
\hline
\end{tabular}

Last 1998

\section{Study characteristics}

\begin{tabular}{ll}
\hline Methods & Study design: randomised controlled trial \\
Study setting: university clinic \\
Date of study: September 1993 through April 1995 \\
\hline $\mathbf{N}=\mathbf{5 6}$ (CBT: N = 32; active control: N = 24) \\
Mean age (SD): CBT: 11.67 (3.00); active control: 12.40 (2.79) \\
Age range: 6 to 17 \\
Gender: CBT: 13 (56\%) female; active control: 15 (62\%) female \\
Inclusion criteria: (1) anxiety-based school refusal; (2) current enrolment in elementary, middle, or \\
high school; (3) current DSM-lll-R anxiety disorder diagnosis (based on K-SADS-P); (4) at least 10\% ab- \\
senteeism from classes for at least 1 month before intake; (5) no current diagnosis of major depression; \\
and (6) no current use of psychiatric medication
\end{tabular}

Interventions

CBT

Intervention: modified adult treatment for treatment of anxiety-based school refusal. In vivo exposure and coping self statements

Concomitant/excluded medications: psychiatric medication excluded

Delivery format: child and parent; individual

Therapist contact time: 12 hours ( $12 \times 1$ hour)

Who delivers the intervention: Therapist. Involvement of school-based contact

\section{Active control}

Intervention: educational support therapy - modification of control therapy used previously: educational presentations and supportive psychotherapy

Concomitant/excluded medications: psychiatric medication excluded

Delivery format: child only?; individual

Therapist contact time: 12 hours ( $12 \times 1$ hour)

Who delivers the intervention: Therapist. Involvement of school-based contact

Outcomes

Remission of primary anxiety disorder diagnosis post-treatment: K-SADS (parent and child, abbreviated form)

Reduction in anxiety symptoms (child report) post-treatment: STAIC-modified 
Last 1998 (Continued)

Reduction in depressive symptoms post-treatment: $C D I$

\begin{tabular}{|c|c|c|}
\hline Notes & Active control $=$ attent & n control \\
\hline \multicolumn{3}{|l|}{ Risk of bias } \\
\hline Bias & Authors' judgement & Support for judgement \\
\hline $\begin{array}{l}\text { Random sequence genera- } \\
\text { tion (selection bias) }\end{array}$ & Unclear risk & Judgement Comment: No detail given \\
\hline $\begin{array}{l}\text { Allocation concealment } \\
\text { (selection bias) }\end{array}$ & Unclear risk & Judgement Comment: No detail given \\
\hline $\begin{array}{l}\text { Blinding of participants } \\
\text { and personnel (perfor- } \\
\text { mance bias) } \\
\text { All outcomes }\end{array}$ & Unclear risk & Judgement Comment: No detail given \\
\hline $\begin{array}{l}\text { Blinding of outcome as- } \\
\text { sessment (detection bias) } \\
\text { All outcomes }\end{array}$ & Unclear risk & $\begin{array}{l}\text { Judgement Comment: Assessor blind to initial diagnosis, but no detail given } \\
\text { regarding whether blind to condition }\end{array}$ \\
\hline $\begin{array}{l}\text { Incomplete outcome data } \\
\text { (attrition bias) } \\
\text { All outcomes }\end{array}$ & High risk & $\begin{array}{l}\text { Judgement Comment: Attrition reported, but relatively high dropout rate and } \\
\text { greater in CBT group, with only completer analysis reported }\end{array}$ \\
\hline $\begin{array}{l}\text { Selective reporting (re- } \\
\text { porting bias) }\end{array}$ & Low risk & Judgement Comment: Outcomes reported \\
\hline $\begin{array}{l}\text { Other source of bias - } \\
\text { Therapy integrity }\end{array}$ & Low risk & $\begin{array}{l}\text { Judgement Comment: Treatment fidelity assessed and reported as highly } \\
\text { credible }\end{array}$ \\
\hline Other bias & Low risk & Judgement Comment: The study appears to be free of other sources of bias \\
\hline
\end{tabular}

Lau 2010

\section{Study characteristics}

Study design: randomised controlled trial
Total duration of study: non-controlled 3- and 6-month follow-up
Number of study centres and location: 1 in Hong Kong
Study setting: community clinic

Participants

$\mathbf{N}=\mathbf{4 5}$ randomised (NB: we only include those who met diagnostic criteria for an anxiety disorder at baseline: $\mathrm{N}=38$; $\mathrm{CBT}: \mathrm{N}=21$; waitlist: $\mathrm{N}=17$ )

\section{Age range: 6 to 11}

Inclusion criteria: diagnosed with anxiety problems/disorders (we only include participants that meet diagnostic criteria based on K-SADS)

Exclusion criteria: only specific phobia, severe hyperactivity symptoms

Interventions CBT


Lau 2010 (Continued)

Intervention: Anxiety Group treatment programme - adaptation of Coping Cat

Delivery format: child, with parents invited to observe and meet with co-therapists at the beginning of some sessions; group

Therapist contact time: 18 hours ( $9 \times 2$ hours)

Waitlist/no treatment

Intervention: 13-week waitlist

\begin{tabular}{ll}
\hline Outcomes & Remission of primary anxiety disorder diagnosis post-treatment: K-SADS \\
& Reduction in anxiety symptoms (child report) post-treatment: SCAS-C (modified, only 4 subscales) \\
Reduction in anxiety symptoms (parent report) post-treatment: SCAS-P (modified, only 4 sub- \\
scales)
\end{tabular}

Notes

\section{Risk of bias}

\begin{tabular}{|c|c|c|}
\hline Bias & Authors' judgement & Support for judgement \\
\hline $\begin{array}{l}\text { Random sequence genera- } \\
\text { tion (selection bias) }\end{array}$ & Unclear risk & Judgement Comment: No detail given \\
\hline $\begin{array}{l}\text { Allocation concealment } \\
\text { (selection bias) }\end{array}$ & Unclear risk & Judgement Comment: No detail given \\
\hline $\begin{array}{l}\text { Blinding of participants } \\
\text { and personnel (perfor- } \\
\text { mance bias) } \\
\text { All outcomes }\end{array}$ & Low risk & $\begin{array}{l}\text { Judgement Comment: Even though blinding was not possible, we judged that } \\
\text { it was unlikely this led to a departure from the intended intervention }\end{array}$ \\
\hline $\begin{array}{l}\text { Blinding of outcome as- } \\
\text { sessment (detection bias) } \\
\text { All outcomes }\end{array}$ & Low risk & $\begin{array}{l}\text { Judgement Comment: Independent coder blind to treatment condition rated } \\
\text { subset of interviews }\end{array}$ \\
\hline $\begin{array}{l}\text { Incomplete outcome data } \\
\text { (attrition bias) } \\
\text { All outcomes }\end{array}$ & Low risk & Judgement Comment:No missing data \\
\hline $\begin{array}{l}\text { Selective reporting (re- } \\
\text { porting bias) }\end{array}$ & Low risk & Judgement Comment: Report outcomes for all measures \\
\hline $\begin{array}{l}\text { Other source of bias - } \\
\text { Therapy integrity }\end{array}$ & Low risk & Judgement Comment: Trained therapists, adherence assessed and reported \\
\hline Other bias & Low risk & Judgement Comment: The study appears to be free of other sources of bias \\
\hline
\end{tabular}

Lau 2017

\section{Study characteristics}

Methods

Study design: randomised controlled trial

Total duration of study: 6 months (post-treatment assessment); non-controlled 12-month follow-up 
Lau 2017 (Continued)

Number of study centres and location: 1 in Australia

Study setting: recruited through child care centres and preschools; intervention delivery in university setting

N $=\mathbf{7 2}$ (CBT: N= 39; waitlist: N=33)
Mean age (SD): 52.1 months
Age range: 36 to 65 months
Gender: 34 (47.2\%) female
Inclusion criteria: (1) age 36 to 66 months at the time of recruitment; (2) minimum of 30 (1.15 SD
above the norm) on the child's score of social approach on the Short Temperament Scale for Children;
(3) a minimum of 30 on at least 1 parent's self-reported scores on the Depression Anxiety Stress Scales;
(4) no known diagnosis of any severe developmental disorders; and (5) parents who were able to com-
plete questionnaires in English (NB: all children met diagnostic criteria for an anxiety disorder (based
on ADIS-P), but this was not an inclusion criterion)

Interventions CBT

Intervention: parent and child intervention. Parent: Cool Little Kids. Child: Social Skills Facilitated Play programme

Delivery format: parent sessions and child sessions; group

Therapist contact time: 18 hours total ( 6 ×90 minutes - parent; $6 \times 90$ minutes - child)

Who delivers the intervention: clinical psychologist (parent), graduate or final-year students (child)

\section{Waitlist/no treatment}

Intervention: 6-month waitlist

\begin{tabular}{ll}
\hline Outcomes & Remission of all anxiety disorder diagnoses post-treatment: ADIS-P \\
& Reduction in anxiety symptoms (parent report-mother) post-treatment: Preschool Anxiety Scale-
\end{tabular}

Revised (PAS-R) - mother report

Notes Post-treatment assessment point 6 months

\section{Risk of bias}

\begin{tabular}{|c|c|c|}
\hline Bias & Authors' judgement & Support for judgement \\
\hline $\begin{array}{l}\text { Random sequence genera- } \\
\text { tion (selection bias) }\end{array}$ & Low risk & Judgement Comment: Coin toss \\
\hline $\begin{array}{l}\text { Allocation concealment } \\
\text { (selection bias) }\end{array}$ & Low risk & $\begin{array}{l}\text { Judgement Comment: A coin toss by a research assistant who was blind to } \\
\text { baseline assessment details was used to allocate families to the } 2 \text { conditions }\end{array}$ \\
\hline $\begin{array}{l}\text { Blinding of participants } \\
\text { and personnel (perfor- } \\
\text { mance bias) } \\
\text { All outcomes }\end{array}$ & Low risk & $\begin{array}{l}\text { Judgement Comment: Even though blinding was not possible, we judged that } \\
\text { it was unlikely this led to a departure from the intended intervention }\end{array}$ \\
\hline $\begin{array}{l}\text { Blinding of outcome as- } \\
\text { sessment (detection bias) } \\
\text { All outcomes }\end{array}$ & Low risk & Judgement Comment: Interviewers blind to treatment condition \\
\hline
\end{tabular}


Lau 2017 (Continued)

Incomplete outcome data Low risk Judgement Comment: Dropouts and missing questionnaire data detailed. (attrition bias) Only 1 dropout. Used appropriate imputation to manage missing item data. All outcomes
Mother report low rate missing data

\begin{tabular}{lll}
\hline $\begin{array}{l}\text { Selective reporting (re- } \\
\text { porting bias) }\end{array}$ & Low risk & Judgement Comment: Report all outcomes \\
\hline $\begin{array}{l}\text { Other source of bias - } \\
\text { Therapy integrity }\end{array}$ & Unclear risk & $\begin{array}{l}\text { Judgement Comment: Therapist qualifications detailed, but no further detail } \\
\text { given on assessing/monitoring competence/adherence }\end{array}$ \\
\hline
\end{tabular}

Other bias Low risk Judgement Comment: The study appears to be free of other sources of bias

Lebowitz 2019

\section{Study characteristics}

\begin{tabular}{ll}
\hline Study design: randomised controlled trial \\
Number of study centres and location: 1 in the USA \\
Study setting: clinic \\
\hline $\mathbf{N}=\mathbf{1 2 4}$ (CBT: $\mathrm{N}=60 ;$ control: $\mathrm{N}=64)$ \\
Mean age (SD): $9.4(2.41)$ \\
Age range: 7 to 12 \\
Gender: 66 (53\%) female \\
Inclusion criteria: primary DSM-5 anxiety disorder diagnosis (based on ADIS-C/P); aged 7 to 14 years; \\
residing with mother at least $50 \%$ of the time; fluent in English; medication-free or on a stable dose of \\
anti-depressant or stimulant when child and parent agreed to refrain from changes during the study \\
period; parental informed permission and consent; child assent \\
Exclusion criteria: drug or alcohol abuse; psychotic symptoms; autism spectrum disorder; any comor- \\
bid disorder more impairing than the most severe anxiety disorder; concurrent psychotherapy or med- \\
ication, apart from stable dose of anti-depressant or stimulant; serious suicidal intent or risk
\end{tabular}

Interventions

\section{CBT}

Intervention: manual used in Silverman 2009. Psychoeducation, exposure hierarchy, cognitive restructuring

Concomitant/excluded medications: stable dose of anti-depressant or stimulant allowed

Delivery format: child only (NB: therapist met with parent to provide information on child's therapy); individual

Therapist contact time: 12 hours total ( $12 \times 1$ hour)

Who delivers the intervention: doctoral- and postdoctoral-level psychology students

\section{Active control}

Intervention: SPACE (Supportive Parenting for Anxious Childhood Emotions) manual. Family modifications mapped out and target accommodation selected for modification, modules for problem-solving difficulties related to child's responses to reduced accommodation

Concomitant/excluded medications: stable dose of anti-depressant or stimulant allowed 
Delivery format: parent only; individual

Therapist contact time: 12 hours total ( $12 \times 1$ hour)

Who delivers the intervention: doctoral- and postdoctoral-level psychology students

\begin{tabular}{|c|c|c|}
\hline Outcomes & \multicolumn{2}{|c|}{$\begin{array}{l}\text { Remission of all anxiety disorder diagnoses post-treatment: ADIS-C/P } \\
\text { Reduction in anxiety symptoms (child report) post-treatment: SCARED-C } \\
\text { Reduction in anxiety symptoms (parent report) post-treatment: SCARED-P }\end{array}$} \\
\hline Notes & \multicolumn{2}{|c|}{ Active control = alternative treatment } \\
\hline \multicolumn{3}{|l|}{ Risk of bias } \\
\hline Bias & Authors' judgement & Support for judgement \\
\hline $\begin{array}{l}\text { Random sequence genera- } \\
\text { tion (selection bias) }\end{array}$ & Low risk & Judgement Comment: Computerised randomisation algorithm \\
\hline $\begin{array}{l}\text { Allocation concealment } \\
\text { (selection bias) }\end{array}$ & Low risk & Judgement Comment: Computer generated, so unlikely to foresee assignment \\
\hline $\begin{array}{l}\text { Blinding of participants } \\
\text { and personnel (perfor- } \\
\text { mance bias) } \\
\text { All outcomes }\end{array}$ & Low risk & $\begin{array}{l}\text { Judgement Comment: Even though blinding was not possible, we judged that } \\
\text { it was unlikely this led to a departure from the intended intervention }\end{array}$ \\
\hline $\begin{array}{l}\text { Blinding of outcome as- } \\
\text { sessment (detection bias) } \\
\text { All outcomes }\end{array}$ & Low risk & $\begin{array}{l}\text { Judgement Comment: Independent evaluators masked to treatment assign- } \\
\text { ment }\end{array}$ \\
\hline $\begin{array}{l}\text { Incomplete outcome data } \\
\text { (attrition bias) } \\
\text { All outcomes }\end{array}$ & Low risk & $\begin{array}{l}\text { Judgement Comment: Focus on completer analysis justified. Report remission } \\
\text { rates, comparison between completers and non-completers and missing data } \\
\text { bias }\end{array}$ \\
\hline $\begin{array}{l}\text { Selective reporting (re- } \\
\text { porting bias) }\end{array}$ & Low risk & Judgement Comment: Outcomes reported for all measures \\
\hline $\begin{array}{l}\text { Other source of bias - } \\
\text { Therapy integrity }\end{array}$ & Low risk & Judgement Comment: Trained therapists, regular supervision, fidelity checks \\
\hline Other bias & Low risk & Judgement Comment: The study appears to be free of other sources of bias \\
\hline
\end{tabular}

Leutgeb 2012

\section{Study characteristics}

Methods

Study design: randomised controlled trial

Number of study centres and location: 1 in Austria

Study setting: university

Participants $\quad \mathbf{N}=\mathbf{3 0}(\mathrm{CBT}: \mathrm{N}=16$; waitlist: $\mathrm{N}=14)$

Mean age (SD): CBT: 137.3 (15) months; waitlist: 138.5 (21.0) months 
Leutgeb 2012 (Continued)

\section{Age range: 8 to 14}

Gender: 100\% female

Inclusion criteria: aged 8 to 14 , spider phobia (based on DIPS-K), non-medicated, right-handed, female

Exclusion criteria: any other mental disorder

Intervention: Single Session CBT (OST)

Concomitant/excluded medications: not allowed

Delivery format: child only; individual

Therapist contact time: 4 hours total

\section{Waitlist/no treatment}

Intervention: 1-week waitlist

Concomitant/excluded medications: not allowed

\begin{tabular}{|c|c|c|}
\hline Outcomes & \multicolumn{2}{|c|}{ Reduction in anxiety symptoms (child report) post-treatment: STAIC-C (trait) } \\
\hline \multicolumn{3}{|l|}{ Notes } \\
\hline \multicolumn{3}{|l|}{ Risk of bias } \\
\hline Bias & Authors' judgement & Support for judgement \\
\hline $\begin{array}{l}\text { Random sequence genera- } \\
\text { tion (selection bias) }\end{array}$ & Unclear risk & Judgement Comment: Not detailed \\
\hline $\begin{array}{l}\text { Allocation concealment } \\
\text { (selection bias) }\end{array}$ & Unclear risk & Judgement Comment: Not detailed \\
\hline $\begin{array}{l}\text { Blinding of participants } \\
\text { and personnel (perfor- } \\
\text { mance bias) } \\
\text { All outcomes }\end{array}$ & Low risk & $\begin{array}{l}\text { Judgement Comment: Even though blinding was not possible, we judged that } \\
\text { it was unlikely this led to a departure from the intended intervention }\end{array}$ \\
\hline $\begin{array}{l}\text { Blinding of outcome as- } \\
\text { sessment (detection bias) } \\
\text { All outcomes }\end{array}$ & Low risk & $\begin{array}{l}\text { Judgement Comment: Not detailed, but no post-treatment diagnostic assess- } \\
\text { ment used }\end{array}$ \\
\hline $\begin{array}{l}\text { Incomplete outcome data } \\
\text { (attrition bias) } \\
\text { All outcomes }\end{array}$ & Low risk & Judgement Comment: No missing data \\
\hline $\begin{array}{l}\text { Selective reporting (re- } \\
\text { porting bias) }\end{array}$ & Low risk & Judgement Comment: Relevant outcomes reported \\
\hline $\begin{array}{l}\text { Other source of bias - } \\
\text { Therapy integrity }\end{array}$ & Unclear risk & Judgement Comment: No detail given \\
\hline Other bias & Low risk & Judgement Comment: The study appears to be free of other sources of bias \\
\hline
\end{tabular}




\section{Study characteristics}

\begin{tabular}{l} 
Methods \\
Study design: randomised controlled trial \\
Total duration of study: non-controlled 9-month follow-up \\
Number of study centres and location: 2 schools in the USA \\
Study setting: schools \\
\hline $\mathbf{N}=\mathbf{4 2}$ (CBT: $\mathrm{N}=21 ;$ waitlist: $\mathrm{N}=21)$ \\
Mean age (SD): $14.8(.81)(\mathrm{n}=35)$ \\
Age range: 13 to 17 ( $\mathrm{n}=35)$ \\
Gender: 26 (74.3\%) female $(\mathrm{n}=35)$ \\
Inclusion criteria: met diagnostic criteria for DSM-IV social anxiety disorder (based on ADIS-C/P) \\
Exclusion criteria: diagnosis of substance use disorder, oppositional defiant disorder, conduct disor- \\
der, or major depression that was of greater severity than social phobia; experiencing psychotic symp- \\
toms or current suicidal or homicidal thoughts; or had a current major life event requiring immediate \\
attention (e.g. a parent dying)
\end{tabular}

Interventions

CBT

Intervention: SASS (Skills for Social and Academic Success)

Concomitant/excluded medications: current pharmacological treatment excluded

Delivery format: child sessions, plus parent meetings; group plus individual meetings

Therapist contact time: 17.5 hours total ( $12 \times 40 \mathrm{~min}$ - group, $2 \times 15 \mathrm{~min}$ - individual, $4 \times 90 \mathrm{~min}$ - group social events, $2 \times 45 \mathrm{~min}$ - parent, $2 \times 45 \mathrm{~min}$ - teachers)

Who delivers the intervention: clinical psychologist and clinical psychology graduate student

Waitlist/no treatment

Intervention: waitlist

Concomitant/excluded medications: current pharmacological treatment excluded

\begin{tabular}{ll}
\hline Outcomes & Remission of primary anxiety disorder diagnosis post-treatment: ADIS-C/P \\
Reduction in anxiety symptoms (child report) post-treatment: SPAI-C (child) \\
Reduction in anxiety symptoms (parent report) post-treatment: SAS-A (parent) \\
Reduction in depressive symptoms post-treatment: CDI \\
Improvement in global functioning post-treatment: CGAS
\end{tabular}


Masia Warner 2005 (Continued)

Random sequence genera- Unclear risk Judgement Comment: No detail given tion (selection bias)

Allocation concealment Unclear risk Judgement Comment: No detail given
(selection bias)

$\begin{aligned} & \text { Blinding of participants } \\ & \text { and personnel (perfor- } \\ & \text { mance bias) }\end{aligned}$
$\begin{aligned} & \text { All outcomes } \\ & \text { it was unlikely this led to a departure from the intended intervention }\end{aligned}$

\begin{tabular}{lll}
\hline $\begin{array}{l}\text { Blinding of outcome as- } \\
\text { sessment (detection bias) } \\
\text { All outcomes }\end{array}$ & Low risk & Judgement Comment: Independent blind assessors \\
\hline $\begin{array}{l}\text { Incomplete outcome data } \\
\begin{array}{l}\text { (attrition bias) } \\
\text { All outcomes }\end{array}\end{array}$ & Low risk & $\begin{array}{l}\text { Judgement Comment: Dropouts detailed. Report running completer and ITT } \\
\text { analyses, and no differences in results }\end{array}$ \\
\hline
\end{tabular}

\begin{tabular}{lll}
\hline $\begin{array}{l}\text { Selective reporting (re- } \\
\text { porting bias) }\end{array}$ & Low risk & Judgement Comment: Outcomes reported for all measures \\
\hline $\begin{array}{l}\text { Other source of bias - } \\
\text { Therapy integrity }\end{array}$ & Unclear risk & $\begin{array}{l}\text { Judgement Comment: Qualified therapists, but no detail provided on assess- } \\
\text { ment of competence or adherence }\end{array}$ \\
\hline Other bias & Low risk & Judgement Comment: The study appears to be free of other sources of bias \\
\hline
\end{tabular}

\section{Study characteristics}

\begin{tabular}{|c|c|}
\hline Methods & $\begin{array}{l}\text { Study design: randomised controlled trial } \\
\text { Total duration of study: } 6 \text {-month follow-up } \\
\text { Number of study centres and location: } 2 \text { schools in the USA } \\
\text { Study setting: schools } \\
\text { Withdrawals: } 1 \text { participant died after allocation and is not included in number randomised }\end{array}$ \\
\hline Participants & $\begin{array}{l}\mathbf{N}=\mathbf{3 6}(\mathrm{CBT}: \mathrm{N}=19 \text {; active control: } \mathrm{N}=17) \\
\text { Mean age (SD): } 15.1(0.6) \\
\text { Age range: } 14 \text { to } 16 \\
\text { Gender: } 30(83.3 \%) \text { female } \\
\text { Inclusion criteria: primary social anxiety disorder (based on ADIS-C/P) } \\
\text { Excluded criteria: } 1 \text { ) current psychological or psychopharmacological treatment, 2) current diagnosis } \\
\text { of substance use or conduct disorder, 3) concurrent mental disorder of greater severity than social pho- } \\
\text { bia, and 4) psychotic symptoms or current suicidal or homicidal ideation }\end{array}$ \\
\hline Interventions & $\begin{array}{l}\text { СВТ } \\
\text { Intervention: Skills for Academic and Social Success (SASS) }\end{array}$ \\
\hline
\end{tabular}


Concomitant/excluded medications: current psychopharmacological treatment excluded

Delivery format: child-only sessions, plus parent sessions; group, plus 4 individual

Therapist contact time: 17.5 hours total ( $12 \times 40 \mathrm{~min}$ - group, $2 \times 15 \mathrm{~min}$ - individual, $4 \times 90 \mathrm{~min}$ - group social events, $2 \times 45 \mathrm{~min}$ - parent, $2 \times 45$ min teachers)

Who delivers the intervention: clinical psychologists and clinical psychology graduate

\section{Active control}

Intervention: identical professional attention and format as SASS. Psychoeducation or general relaxation techniques, support targeting social anxiety symptoms

Concomitant/excluded medications: current psychopharmacological treatment excluded

Delivery format: child-only sessions, plus parent sessions; group, plus 4 individual

Therapist contact time: 17.5 hours total ( $12 \times 40 \mathrm{~min}$ - group, $2 \times 15 \mathrm{~min}$ - individual, $4 \times 90 \mathrm{~min}$ - group social events, 2 × $45 \mathrm{~min}$ - parent, $2 \times 45 \mathrm{~min}$ - teachers)

Who delivers the intervention: clinical psychologists and clinical psychology graduates

Remission of primary anxiety disorder diagnosis post-treatment and 6-month follow-up: ADIS-C/P
Remission of all anxiety disorder diagnoses post-treatment and 6-month follow-up: ADIS-C/P
Reduction in anxiety symptoms (child report) post-treatment and 6-month follow-up: SPAI-C
Reduction in anxiety symptoms (parent report) post-treatment and 6-month follow-up: SAS-A
Reduction in depressive symptoms post-treatment: BDI-II
Improvement in global functioning post-treatment: CGAS

Notes Remission data reported in Warwick 2016.

Anxiety symptom measures are social anxiety symptom measures.

Active control $=$ attention control

\section{Risk of bias}

\begin{tabular}{lll}
\hline Bias & Authors' judgement & Support for judgement \\
\hline $\begin{array}{l}\text { Random sequence genera- } \\
\text { tion (selection bias) }\end{array}$ & Unclear risk & Judgement Comment: No detail given \\
\hline $\begin{array}{l}\text { Allocation concealment } \\
\text { (selection bias) }\end{array}$ & Unclear risk & Judgement Comment: No detail given \\
\hline $\begin{array}{l}\text { Blinding of participants } \\
\text { and personnel (perfor- } \\
\text { mance bias) } \\
\text { All outcomes }\end{array}$ & Unclear risk & $\begin{array}{l}\text { Judgement Comment: Students did not know students in other condition to } \\
\text { prevent contamination, but no detail provided regarding whether participants } \\
\text { were blind to receiving 'intervention' or 'control' }\end{array}$ \\
\hline
\end{tabular}

Blinding of outcome as- Low risk Judgement Comment: Independent blind assessors
sessment (detection bias)
All outcomes

\begin{tabular}{|c|c|c|}
\hline $\begin{array}{l}\text { Incomplete outcome data } \\
\text { (attrition bias) }\end{array}$ & Low risk & $\begin{array}{l}\text { Judgement Comment: Dropouts detailed, completer and ITT analyses report- } \\
\text { ed, with appropriate imputation }\end{array}$ \\
\hline
\end{tabular}


Masia Warner 2007 (Continued)

All outcomes

Selective reporting (re- Low risk Judgement Comment: Outcomes reported for all measures
porting bias)

\begin{tabular}{lll}
\hline $\begin{array}{l}\text { Other source of bias - } \\
\text { Therapy integrity }\end{array}$ & Low risk & $\begin{array}{l}\text { Judgement Comment: Qualified therapists. Measures to ensure treatment in- } \\
\text { tegrity }\end{array}$ \\
\hline Other bias & Low risk & Judgement Comment: The study appears to be free of other sources of bias \\
\hline
\end{tabular}

Masia Warner 2011

\section{Study characteristics}

\begin{tabular}{ll}
\hline Study design: randomised controlled trial \\
Total duration of study: non-controlled 3-month follow-up \\
Number of study centres and location: USA \\
Study setting: paediatric primary care and speciality physicians \\
\hline N $=\mathbf{4 0}$ (CBT: $\mathrm{N}=20 ;$ waitlist: $\mathrm{N}=20)$ \\
Mean age (SD): $12.4(2.6)$ \\
Age range: 8 to 16 \\
Gender: 26 (65\%) female \\
Inclusion criteria: age 8 to 16 with functional physical complaints (primarily gastrointestinal symp- \\
toms) and a principal anxiety disorder (based on ADIS-C/P), psychiatric medication allowed if receiving \\
for 6 months and stable during intervention phase
\end{tabular}

Interventions CBT

Intervention: TAPS (Treatment of Anxiety and Physical Symptoms). Jointly addresses anxiety and physical symptoms through identifying contexts where symptoms occur and interact

Concomitant/excluded medications: children receiving psychiatric medication for more than 6 months and stable medication during intervention phase allowed

Delivery format: child-only sessions, and parent meetings; individual

Therapist contact time: 11 to 14 hours ( $12 \times 45$ to 60 minutes (child), $3 \times 45$ minutes (parent))

Who delivers the intervention: PhD level clinical psychologists trained in CBT

\section{Waitlist/no treatment}

Intervention: 8-week waitlist

Concomitant/excluded medications: children receiving psychiatric medication for more than 6 months and stable medication during intervention phase allowed

\section{Outcomes \\ Remission of primary anxiety disorder diagnosis post-treatment: ADIS-C/P \\ Improvement in global functioning post-treatment: CGAS}

\section{Notes}


Masia Warner 2011 (Continued)

Risk of bias

\begin{tabular}{|c|c|c|}
\hline Bias & Authors' judgement & Support for judgement \\
\hline $\begin{array}{l}\text { Random sequence genera- } \\
\text { tion (selection bias) }\end{array}$ & Low risk & Judgement Comment: Table of random numbers \\
\hline $\begin{array}{l}\text { Allocation concealment } \\
\text { (selection bias) }\end{array}$ & Unclear risk & Judgement Comment: Predetermined, but no further detail given \\
\hline $\begin{array}{l}\text { Blinding of participants } \\
\text { and personnel (perfor- } \\
\text { mance bias) } \\
\text { All outcomes }\end{array}$ & Low risk & $\begin{array}{l}\text { Judgement Comment: Even though blinding was not possible, we judged that } \\
\text { it was unlikely this led to a departure from the intended intervention }\end{array}$ \\
\hline $\begin{array}{l}\text { Blinding of outcome as- } \\
\text { sessment (detection bias) } \\
\text { All outcomes }\end{array}$ & Low risk & Judgement Comment: Blind assessors \\
\hline $\begin{array}{l}\text { Incomplete outcome data } \\
\text { (attrition bias) } \\
\text { All outcomes }\end{array}$ & Low risk & Judgement Comment: 1 dropout in control group. $\mathrm{n}$ reported for outcomes. \\
\hline $\begin{array}{l}\text { Selective reporting (re- } \\
\text { porting bias) }\end{array}$ & Low risk & Judgement Comment: Outcomes reported for all measures \\
\hline $\begin{array}{l}\text { Other source of bias - } \\
\text { Therapy integrity }\end{array}$ & Unclear risk & $\begin{array}{l}\text { Judgement Comment: Therapist clinical psychologist with training in CBT, no } \\
\text { detail given regarding supervision or adherence }\end{array}$ \\
\hline Other bias & High risk & $\begin{array}{l}\text { Judgement Comment: Timing post-treatment assessment differs across } \\
\text { groups }\end{array}$ \\
\hline
\end{tabular}

Masia Warner 2016

\section{Study characteristics}

\begin{tabular}{ll}
\hline Study design: randomised controlled trial \\
Total duration of study: recruitment over 3 academic years. 5 months follow-up \\
Number of study centres and location: 3 schools in the USA \\
Study setting: schools \\
Withdrawals: 2 removed (in P-SASS) - irregular school attendance, medical emergency (NB: relate to \\
inclusion criteria, so not included in number randomised) \\
N = 136 (CBT (C-SASS): $N=46 ;$ CBT (P-SASS): N = 47; active control: N = 43) \\
Mean age (SD): 15.42 (0.81) \\
Age range: 13 to 17 \\
Gender: 92 (67.7\%) female \\
Inclusion criteria: diagnosis of social anxiety disorder (based on ADIS-C/P); if other disorders present, \\
SAD had to be of equal or greater severity
\end{tabular}


Exclusion criteria: require immediate care, unreliable school attendance, receiving CBT

Interventions

CBT (C-SASS)

Intervention: Skills for Academic and Social Success delivered by school counsellors (C-SASS)

Concomitant/excluded medications: anxiety medication allowed if stable

Delivery format: child-only sessions, plus 2 parent sessions; group, plus 2 brief individual

Therapist contact time: 16 hours $-12 \times 40$ minutes (group), $2 \times 15$ minutes (individual), $2 \times 45$ minutes (parent), $4 \times 90$ minutes social activities

Who delivers the intervention: Master's-level school counsellors

CBT (P-SASS)

Intervention: Skills for Academic and Social Success delivered by psychologists (P-SASS)

Concomitant/excluded medications: anxiety medication allowed if stable

Delivery format: child-only sessions, plus 2 parent sessions; group, plus 2 brief individual

Therapist contact time: 16 hours $-12 \times 40$ minutes (group), $2 \times 15$ minutes (individual), $2 \times 45$ minutes (parent), $4 \times 90$ minutes social activities

Who delivers the intervention: doctoral-level clinical psychologists

\section{Active control}

Intervention: Skills for Life - non-specific counselling programme

Concomitant/excluded medications: anxiety medication allowed if stable

Delivery format: child-only sessions, plus 1 parent session; group, plus 1 brief individual

Therapist contact time: 9 hours ( $12 \times 40$ minutes (group), $1 \times 15$ minutes (individual), $1 \times 45$ minutes (parent))

Who delivers the intervention: Master's-level counsellors

\section{Outcomes}

Remission of primary anxiety disorder diagnosis post-treatment and 5-month follow-up: ADIS-C/P

Reduction in anxiety symptoms (child report) post-treatment and 5-month follow-up: SPAI-C

Reduction in anxiety symptoms (parent report) post-treatment and 5-month follow-up: SPAI-P

\begin{tabular}{ll}
\hline Notes & Active control = attention control \\
Social anxiety symptom measures
\end{tabular}

\section{Risk of bias}

\begin{tabular}{lll}
\hline Bias & Authors' judgement & Support for judgement \\
\hline $\begin{array}{l}\text { Random sequence genera- } \\
\text { tion (selection bias) }\end{array}$ & Low risk & Judgement Comment: Independent randomisation \\
\hline $\begin{array}{l}\text { Allocation concealment } \\
\text { (selection bias) }\end{array}$ & Low risk & Judgement Comment: Independent \\
\hline
\end{tabular}

Blinding of participants Unclear risk Judgement Comment: No detail given
and personnel (perfor-
mance bias)


Masia Warner 2016 (Continued)

All outcomes

Blinding of outcome as- Low risk $\quad$ Judgement Comment: Blinded independent assessors
sessment (detection bias)

All outcomes

\begin{tabular}{lll}
\hline $\begin{array}{l}\text { Incomplete outcome data } \\
\text { (attrition bias) } \\
\text { All outcomes }\end{array}$ & Low risk & $\begin{array}{l}\text { Judgement Comment: Dropouts detailed across groups, and examined differ- } \\
\text { ences between dropouts and completers, and completer analysis vs ITT }\end{array}$ \\
\hline $\begin{array}{l}\text { Selective reporting (re- } \\
\text { porting bias) }\end{array}$ & Low risk & Judgement Comment: Outcomes reported for all measures \\
\hline $\begin{array}{l}\text { Other source of bias - } \\
\text { Therapy integrity }\end{array}$ & Low risk & $\begin{array}{l}\text { Judgement Comment: Therapist qualifications detailed, and treatment adher- } \\
\text { ence assessed and reported (NB: contamination in } 42 \% \text { of control sessions, } \\
\text { but with } 1 \text { exception was mild, so assume low risk of bias) }\end{array}$ \\
\hline $\begin{array}{l}\text { Other bias } \\
\text { Unclear risk }\end{array}$ & $\begin{array}{l}\text { Judgement Comment: Amount of therapist contact time and social events not } \\
\text { equal across conditions }\end{array}$ \\
\hline
\end{tabular}

McConachie 2014

\section{Study characteristics}

Study design: randomised controlled trial
Total duration of study: assessed 3 months (post-treatment), 6 months, and 9 months
Number of study centres and location: the UK
Study setting: referrals from CAMHS teams in England
N = $\mathbf{3 2}$ (CBT: $\mathrm{N}=17$; waitlist: $\mathrm{N}=15)$
Mean age (SD): CBT: 11.7 (1.4); waitlist: 11.8 (1.3)
Age range: 9 to 13
Gender: CBT: 2 (11.8\%); waitlist: 2 (13.3)
Inclusion criteria: child had a confirmed diagnosis of ASD, met criteria for at least 1 anxiety disorder
(based on ADIS-P), aged 9 years to 13 years 11 months with intellectual ability in the average range (full
scale IQ $>69)$. Parent(s) also had to be willing to attend group therapy in parallel with their child.
Exclusion criteria: children with untreated ADHD or oppositional behaviour that was considered likely
to disrupt a small-group setting

Intervention: Exploring Feelings, with minor adjustments for UK setting

Concomitant/excluded medications: clinician asked to avoid alteration in medication

Delivery format: child and parent separate sessions; group

Therapist contact time: 28 hours total $(7 \times 2$ hours - child; $7 \times 2$ hours - parent), parent and child sessions run same time

Who delivers the intervention: trainee psychologists, no prior CBT qualification 
McConachie 2014 (Continued)

\section{Waitlist/no treatment}

Intervention: delayed treatment, 9 months wait (assessment 3 months, 6 months, 9 months)

Concomitant/excluded medications: clinician asked to avoid alteration in medication

\begin{tabular}{ll}
\hline Outcomes & Remission of primary anxiety disorder diagnosis post-treatment: ADIS-P \\
& Remission of all anxiety disorder diagnoses post-treatment: ADIS-P \\
& Reduction in anxiety symptoms (child report) post-treatment: SCAS-C \\
& Reduction in anxiety symptoms (parent report) post-treatment: SCAS-P \\
\hline Notes & $\begin{array}{l}\text { 6- and 9-month follow-up anxiety symptoms outcomes reported, but only change scores. } \\
\text { Sample with ASD }\end{array}$
\end{tabular}

\section{Risk of bias}

\begin{tabular}{|c|c|c|}
\hline Bias & Authors' judgement & Support for judgement \\
\hline $\begin{array}{l}\text { Random sequence genera- } \\
\text { tion (selection bias) }\end{array}$ & Low risk & $\begin{array}{l}\text { Judgement Comment: Random permuted blocks of variable length (www.ran- } \\
\text { domization.com) }\end{array}$ \\
\hline $\begin{array}{l}\text { Allocation concealment } \\
\text { (selection bias) }\end{array}$ & Low risk & Judgement Comment: Measures to ensure concealed allocation \\
\hline $\begin{array}{l}\text { Blinding of participants } \\
\text { and personnel (perfor- } \\
\text { mance bias) } \\
\text { All outcomes }\end{array}$ & Low risk & $\begin{array}{l}\text { Judgement Comment: Even though blinding was not possible, we judged that } \\
\text { it was unlikely this led to a departure from the intended intervention }\end{array}$ \\
\hline $\begin{array}{l}\text { Blinding of outcome as- } \\
\text { sessment (detection bias) } \\
\text { All outcomes }\end{array}$ & Low risk & Judgement Comment: Blind assessors \\
\hline $\begin{array}{l}\text { Incomplete outcome data } \\
\text { (attrition bias) } \\
\text { All outcomes }\end{array}$ & Low risk & $\begin{array}{l}\text { Judgement Comment: Attrition detailed. Only } 1 \text { dropout. Use last observation } \\
\text { carried forward for this case for questionnaires }\end{array}$ \\
\hline $\begin{array}{l}\text { Selective reporting (re- } \\
\text { porting bias) }\end{array}$ & Low risk & Judgement Comment: Outcomes reported for all measures \\
\hline $\begin{array}{l}\text { Other source of bias - } \\
\text { Therapy integrity }\end{array}$ & Low risk & $\begin{array}{l}\text { Judgement Comment: Therapists trained and supervised, and fidelity as- } \\
\text { sessed and reported }\end{array}$ \\
\hline Other bias & Low risk & Judgement Comment: The study appears to be free of other sources of bias \\
\hline
\end{tabular}

McNally Keehn 2013

\section{Study characteristics}

Methods Study design: randomised controlled trial

Total duration of study: non-controlled 2-month follow-up

Number of study centres and location: 1 in the USA 
McNally Keehn 2013 (Continued)

Study setting: university clinic

Date of study: June to September 2009; post- and follow-up assessments completed by April 2010

Participants 22 (CBT: $N=12$; waitlist: $N=10)$
Mean age (SD): 11.26 (1.53)
Age range: 8 to 14
Gender: 1 (4.5\%) female
Inclusion criteria: a) diagnosis of ASD based on the Autism Diagnostic Observation Schedule (ADOS),
Autism Diagnostic Interview-Revised (ADI-R), and expert clinical judgement based on DSM-IV criteria,
(b) diagnosis of separation anxiety disorder, social phobia, or GAD (based on ADIS-P), C) IQ $>70, d)$ age 7
to 14 , and e) English as the primary language

Interventions

CBT

Intervention: Modified Coping Cat to accommodate learning styles of ASD

Concomitant/excluded medications: usual medication allowed

Delivery format: child sessions, plus 2 parent sessions; individual

Therapist contact time: 16 to 24 hours ( $16 \times 60$ to 90 minutes)

Who delivers the intervention: lead author

Waitlist/no treatment

Intervention: 16-week waitlist

Concomitant/excluded medications: usual medication allowed

\begin{tabular}{|c|c|c|}
\hline Outcomes & \multicolumn{2}{|c|}{$\begin{array}{l}\text { Remission of primary anxiety disorder diagnosis post-treatment: ADIS-P } \\
\text { Reduction in anxiety symptoms (child report) post-treatment: SCAS-C } \\
\text { Reduction in anxiety symptoms (parent report) post-treatment: SCAS-P }\end{array}$} \\
\hline Notes & \multicolumn{2}{|c|}{$\begin{array}{l}\text { Author provided data on remission for separation anxiety disorder, social phobia, and GAD, not able to } \\
\text { use. }\end{array}$} \\
\hline \multicolumn{3}{|l|}{ Risk of bias } \\
\hline Bias & Authors' judgement & Support for judgement \\
\hline $\begin{array}{l}\text { Random sequence genera- } \\
\text { tion (selection bias) }\end{array}$ & Low risk & Judgement Comment: Block randomised, stratified \\
\hline $\begin{array}{l}\text { Allocation concealment } \\
\text { (selection bias) }\end{array}$ & Unclear risk & Judgement Comment: No detail given \\
\hline $\begin{array}{l}\text { Blinding of participants } \\
\text { and personnel (perfor- } \\
\text { mance bias) } \\
\text { All outcomes }\end{array}$ & Low risk & $\begin{array}{l}\text { Judgement Comment: Even though blinding was not possible, we judged that } \\
\text { it was unlikely this led to a departure from the intended intervention }\end{array}$ \\
\hline $\begin{array}{l}\text { Blinding of outcome as- } \\
\text { sessment (detection bias) }\end{array}$ & Low risk & Judgement Comment: Blind assessors \\
\hline
\end{tabular}


McNally Keehn 2013 (Continued)

All outcomes

Incomplete outcome data Low risk Judgement Comment: Detailed no dropouts to post-treatment
(attrition bias)

All outcomes

\begin{tabular}{lll}
\hline $\begin{array}{l}\text { Selective reporting (re- } \\
\text { porting bias) }\end{array}$ & Low risk & Judgement Comment: Outcomes reported for all measures \\
\hline $\begin{array}{l}\text { Other source of bias - } \\
\text { Therapy integrity }\end{array}$ & Low risk & Judgement Comment: Adherence rated and reported \\
\hline Other bias & Low risk & Judgement Comment: The study appears to be free of other sources of bias \\
\hline
\end{tabular}

Melfsen 2011

\section{Study characteristics}

\begin{tabular}{l} 
Study design: randomised controlled trial \\
Total duration of study: 10 -month follow-up for treatment group (6 months post-treatment) \\
Number of study centres and location: 1 in Germany \\
Study setting: clinic \\
Date of study: recruitment 2004 to 2006 \\
\hline
\end{tabular}

Participants $\quad \mathbf{N}=\mathbf{4 4}(\mathrm{CBT}: \mathrm{N}=21$; waitlist: $\mathrm{N}=23)$

Mean age (SD): CBT: 10.60 (1.64); waitlist: 10.76 (1.90)

Age range: 8 to 14

Gender: $21(47.8 \%)$ female

Inclusion criteria: a) DSM-IV criteria for social phobia (based on DIPS-K), (b) child experienced social phobia for at least 6 months, (c) social phobia was considered to be the child's main current problem, (d) child age 8 to 13 years, (e) child and parent agreed not to start any additional treatment during the trial

Exclusion criteria: psychotic symptoms, current suicidal or self-harming behaviour, or current involvement in other psychosocial or psychopharmacological treatment for phobia and anxiety problems

\section{Interventions $\quad$ CBT}

Intervention: psychoeducation, cognitive restructuring, behavioural experiments, relapse

Concomitant/excluded medications: psychopharmacological treatment not allowed

Delivery format: child and parent sessions; individual

Therapist contact time: 20 hours ( $20 \times 50$ minutes, $4 \times$ parent sessions)

Who delivers the intervention: advanced doctoral-level graduate students

\section{Waitlist/no treatment}

Intervention: 4-month waitlist 
Concomitant/excluded medications: psychopharmacological treatment not allowed

\begin{tabular}{ll}
\hline Outcomes & Remission of primary anxiety disorder diagnosis post-treatment: DIPS-K \\
Remission of all anxiety disorder diagnoses post-treatment: DIPS-K \\
Reduction in anxiety symptoms (child report) post-treatment: SPAIK-C \\
Reduction in depressive symptoms post-treatment: CDI \\
Improvement in global functioning post-treatment: K-GAS \\
\hline Notes & Refer to follow-up but do not report follow-up data
\end{tabular}

\section{Risk of bias}

\begin{tabular}{|c|c|c|}
\hline Bias & Authors' judgement & Support for judgement \\
\hline $\begin{array}{l}\text { Random sequence genera- } \\
\text { tion (selection bias) }\end{array}$ & Low risk & $\begin{array}{l}\text { Judgement Comment: Web-based computerised randomisation plan genera- } \\
\text { tor }\end{array}$ \\
\hline $\begin{array}{l}\text { Allocation concealment } \\
\text { (selection bias) }\end{array}$ & Low risk & $\begin{array}{l}\text { Judgement Comment: Independent research assistant placed participants on } \\
\text { randomisation list in next available slot }\end{array}$ \\
\hline $\begin{array}{l}\text { Blinding of participants } \\
\text { and personnel (perfor- } \\
\text { mance bias) } \\
\text { All outcomes }\end{array}$ & Low risk & $\begin{array}{l}\text { Judgement Comment: Even though blinding was not possible, we judged that } \\
\text { it was unlikely this led to a departure from the intended intervention }\end{array}$ \\
\hline $\begin{array}{l}\text { Blinding of outcome as- } \\
\text { sessment (detection bias) } \\
\text { All outcomes }\end{array}$ & Low risk & $\begin{array}{l}\text { Judgement Comment: Assessor not blind, but video recordings of assessments } \\
\text { viewed and rated by blind expert }\end{array}$ \\
\hline $\begin{array}{l}\text { Incomplete outcome data } \\
\text { (attrition bias) } \\
\text { All outcomes }\end{array}$ & Low risk & $\begin{array}{l}\text { Judgement Comment: Attrition detailed and use appropriate imputation, and } \\
\text { report ITT }\end{array}$ \\
\hline $\begin{array}{l}\text { Selective reporting (re- } \\
\text { porting bias) }\end{array}$ & Low risk & $\begin{array}{l}\text { Judgement Comment: Relevant outcomes reported. Not report follow up data } \\
\text { for intervention group, but the follow-up h as no comparison group so would } \\
\text { not be eligible for inclusion here }\end{array}$ \\
\hline $\begin{array}{l}\text { Other source of bias - } \\
\text { Therapy integrity }\end{array}$ & Low risk & $\begin{array}{l}\text { Judgement Comment: Therapy delivered by advanced doctoral graduates, ad- } \\
\text { herence assessed }\end{array}$ \\
\hline Other bias & Low risk & Judgement Comment: The study appears to be free of other sources of bias \\
\hline
\end{tabular}

Muris 2002

\section{Study characteristics}

\begin{tabular}{ll}
\hline Methods & Study design: randomised controlled trial \\
& Number of study centres and location: 2 primary schools in the Netherlands \\
& Study setting: primary schools \\
\hline Participants & $\begin{array}{l}\mathbf{N}=\mathbf{2 0}(\mathrm{CBT}: \mathrm{N}=10 ; \text { control: } \mathrm{N}=10)(\mathrm{NB}: \text { study also includes } 10 \text { participants (not randomly) assigned to } \\
\text { no treatment control, not included here) }\end{array}$ \\
\hline
\end{tabular}


Muris 2002 (Continued)

Mean age (SD): $10(0.8)$

Age range: 9 to 12

Gender: 13 (65\%) female

Inclusion criteria: elevated RCADS score; generalised anxiety disorder, separation anxiety disorder, or social phobia diagnosis (based on DISC)

CBT
Interventions
Delivery format: child only; group
Therapist contact time: 6 hours $(12 \times 30$ minutes $)$
Who delivers the intervention: clinical psychology student
Active control
Intervention: Emotional Disclosure
Delivery format: child only; group
Therapist contact time: 6 hours $(12 \times 30$ minutes $)$
Who delivers the intervention: clinical psychology student

Outcomes Reduction in anxiety symptoms (child report) post-treatment: RCADS-C Anxiety Scale

Reduction in depressive symptoms post-treatment: RCADS-C-Depression Scale

Notes

Participants randomised to CBT vs active control. Study also includes no treatment control, but nonrandomised allocation so not included here.

Active control $=$ attention control

\section{Risk of bias}

\begin{tabular}{lll}
\hline Bias & Authors' judgement & Support for judgement \\
\hline $\begin{array}{l}\text { Random sequence genera- } \\
\text { tion (selection bias) }\end{array}$ & Unclear risk & Judgement Comment: No detail given \\
\hline $\begin{array}{l}\text { Allocation concealment } \\
\text { (selection bias) }\end{array}$ & Unclear risk & Judgement Comment: No detail given \\
\hline $\begin{array}{l}\text { Blinding of participants } \\
\text { and personnel (perfor- } \\
\text { mance bias) }\end{array}$ & Unclear risk & Judgement Comment: No detail given \\
$\begin{array}{l}\text { All outcomes } \\
\text { Blinding of outcome as- } \\
\text { sessment (detection bias) } \\
\begin{array}{l}\text { All outcomes } \\
\text { Incomplete outcome data }\end{array}\end{array}$ & Low risk & \\
\hline $\begin{array}{l}\text { (attrition bias) } \\
\text { All outcomes }\end{array}$ & & $\begin{array}{l}\text { Judgement Comment: Questionnaires administered by psychologist not in- } \\
\text { volved in treatment }\end{array}$ \\
\hline
\end{tabular}


Muris 2002 (Continued)

Selective reporting (re- Low risk Judgement Comment: Outcomes reported for measures
porting bias)

\begin{tabular}{|c|c|c|}
\hline $\begin{array}{l}\text { Other source of bias - } \\
\text { Therapy integrity }\end{array}$ & Unclear risk & $\begin{array}{l}\text { Judgement Comment: Therapist supervised, but no detail given on maintain- } \\
\text { ing adherence }\end{array}$ \\
\hline
\end{tabular}

\begin{tabular}{ll}
\hline Other bias Low risk Judgement Comment: The study appears to be free of other sources of bias \\
\hline
\end{tabular}

Murphy 2017

\section{Study characteristics}

\begin{tabular}{|c|c|}
\hline Methods & $\begin{array}{l}\text { Study design: randomised controlled trial } \\
\text { Total duration of study: } 12 \text {-week follow-up } \\
\text { Number of study centres and location: } 3 \text { CAMHS clinics in the UK } \\
\text { Study setting: clinics } \\
\text { Date of study: recruitment April } 2011 \text { to April } 2013\end{array}$ \\
\hline Participants & $\begin{array}{l}\text { N = } 36 \text { (CBT: } N=17 \text {; active control: } N=19) \\
\text { Mean age (SD): CBT: } 14.94 \text { (1.63); active control: } 15.56 \text { (1.91) } \\
\text { Age range: } 12 \text { to } 18 \\
\text { Gender: } 14 \text { (38.9\%) female } \\
\text { Inclusion criteria: ASD and anxiety disorder diagnoses (separation anxiety, social anxiety, specific } \\
\text { phobia, and/or generalised anxiety) based on ADOS, ADI-R, ADIS-C/P); attend mainstream school } \\
\text { Exclusion criteria: primary diagnosis OCD, agoraphobia with/without panic disorder, PTSD, psychosis, } \\
\text { depression, substance abuse; receipt of current psychological therapy from another source }\end{array}$ \\
\hline Interventions & $\begin{array}{l}\text { CBT } \\
\text { Intervention: Multimodal Anxiety and Social Skill Intervention for adolescents with ASD (MASSI). CBT } \\
\text { for anxiety, plus strategies targeting social skills deficits } \\
\text { Concomitant/excluded medications: not alter medication doses during intervention } \\
\text { Delivery format: child only, parents included at end of all sessions; individual and group } \\
\text { Therapist contact time: } 10 \text { hours (plus group sessions) ( } 12 \times 50 \text { minutes individual sessions) } \\
\text { Who delivers the intervention: NHS clinicians } \\
\text { Active control } \\
\text { Intervention: counselling offered in the NHS dealt with anxiety as and when the client raised it, in a } \\
\text { supportive manner, but with no focus on physical symptoms or cognitions } \\
\text { Concomitant/excluded medications: not alter medication doses during intervention } \\
\text { Delivery format: child only, parents included at end of all sessions; individual and group } \\
\text { Therapist contact time: } 10 \text { hours (plus group sessions) (12 x } 50 \text { minutes individual sessions) } \\
\text { Who delivers the intervention: NHS clinicians }\end{array}$ \\
\hline
\end{tabular}


Murphy 2017 (Continued)

Outcomes

Reduction in anxiety symptoms (parent report) post-treatment and follow-up (< 6 months): CASIASD Anxiety Scale

\begin{tabular}{ll}
\hline Notes & Diagnostic assessment post-treatment but not required remission data \\
Sample with ASD & \\
Active control = alternative treatment (counselling)
\end{tabular}

\section{Risk of bias}

\begin{tabular}{lll}
\hline Bias & Authors' judgement & Support for judgement \\
\hline $\begin{array}{l}\text { Random sequence genera- } \\
\text { tion (selection bias) }\end{array}$ & Low risk & $\begin{array}{l}\text { Judgement Comment: Predetermined random sequence by an independent } \\
\text { statistician }\end{array}$ \\
\hline $\begin{array}{l}\text { Allocation concealment } \\
\text { (selection bias) }\end{array}$ & Low risk & $\begin{array}{l}\text { Judgement Comment: Randomisation was managed by a statistician uncon- } \\
\text { nected with the study who prepared sequentially numbered envelopes con- } \\
\text { taining allocation status }\end{array}$ \\
\hline
\end{tabular}

Blinding of participants Unclear risk Judgement Comment: No detail given regarding blinding of participants
and personnel (perfor-

mance bias)

All outcomes

\begin{tabular}{lll}
\hline $\begin{array}{l}\text { Blinding of outcome as- } \\
\text { sessment (detection bias) } \\
\text { All outcomes }\end{array}$ & Low risk & Judgement Comment: Blind assessors \\
\hline $\begin{array}{l}\text { Incomplete outcome data } \\
\begin{array}{l}\text { (attrition bias) } \\
\text { All outcomes }\end{array}\end{array}$ & Low risk & $\begin{array}{l}\text { Judgement Comment: Attrition detailed. ITT analyses reported, and method } \\
\text { for managing missing data. }\end{array}$ \\
\hline $\begin{array}{l}\text { Selective reporting (re- } \\
\text { porting bias) }\end{array}$ & Low risk & Judgement Comment: Outcomes reported \\
\hline $\begin{array}{l}\text { Other source of bias - } \\
\text { Therapy integrity }\end{array}$ & Low risk & Judgement Comment: Qualified therapists. Fidelity assessed and reported. \\
\hline \begin{tabular}{l} 
Other bias \\
\hline
\end{tabular} & Low risk & Judgement Comment: The study appears to be free of other sources of bias \\
\hline
\end{tabular}

\section{O'Brien 2007}

\section{Study characteristics}

\begin{tabular}{ll}
\hline Study design: randomised controlled trial \\
Total duration of study: 10 weeks; end of 10 weeks TAU group offered CBT \\
Number of study centres and location: 1 in Ireland \\
Study setting: clinic \\
\hline Participants \\
$\mathbf{N}=\mathbf{1 4}$ (CBT: $N=7 ;$ TAU: $N=7)$ \\
Mean age (SD): CBT: $13.8(0.5) ;$ TAU: $12.5(1.0)$
\end{tabular}


O'Brien 2007 (Continued)

\section{Age range: 7 to 15}

Gender: 7 (50\%) female

Inclusion criteria: diagnosis of DSM-IV-R anxiety disorder (based on ADIS)

Exclusion criteria: children with intellectual disabilities, pervasive developmental disorder, or recent history of psychotic symptoms

Interventions

\section{CBT}

Intervention: group CBT: Friends for Youth

Concomitant/excluded medications: stable dose allowed

Delivery format: child sessions and parent sessions; group

Therapist contact time: 19.5 hours ( $10 \times 90$ minutes - child; $3 \times 90$ minutes - parent)

Who delivers the intervention: mental health professionals, and supervised by main author

TAU

Intervention: 10 weeks TAU. Received maximum of 4 reviews with primary clinician - review of progress, medication, and supportive approach

Concomitant/excluded medications: stable dose allowed

Delivery format: individual

Therapist contact time: maximum of 4 sessions

Who delivers the intervention: primary clinician

\begin{tabular}{|c|c|c|}
\hline Outcomes & \multicolumn{2}{|c|}{ Reduction in depressive symptoms post-treatment: CDI } \\
\hline Notes & \multicolumn{2}{|c|}{ Anxiety symptoms outcomes only reported after both groups received CBT intervention. } \\
\hline \multicolumn{3}{|l|}{ Risk of bias } \\
\hline Bias & Authors' judgement & Support for judgement \\
\hline $\begin{array}{l}\text { Random sequence genera- } \\
\text { tion (selection bias) }\end{array}$ & Unclear risk & $\begin{array}{l}\text { Judgement Comment: Sequence generation lacks detail, and unclear how age } \\
\text { managed in the process }\end{array}$ \\
\hline $\begin{array}{l}\text { Allocation concealment } \\
\text { (selection bias) }\end{array}$ & Unclear risk & $\begin{array}{l}\text { Judgement Comment: Refers to person doing randomisation being blind, but } \\
\text { further detail is lacking }\end{array}$ \\
\hline $\begin{array}{l}\text { Blinding of participants } \\
\text { and personnel (perfor- } \\
\text { mance bias) } \\
\text { All outcomes }\end{array}$ & Low risk & $\begin{array}{l}\text { Judgement Comment: Even though blinding was not possible, we judged that } \\
\text { it was unlikely this led to a departure from the intended intervention }\end{array}$ \\
\hline $\begin{array}{l}\text { Blinding of outcome as- } \\
\text { sessment (detection bias) } \\
\text { All outcomes }\end{array}$ & Low risk & $\begin{array}{l}\text { Judgement Comment: Not detailed, but no post-treatment diagnostic assess- } \\
\text { ment }\end{array}$ \\
\hline $\begin{array}{l}\text { Incomplete outcome data } \\
\text { (attrition bias) } \\
\text { All outcomes }\end{array}$ & Low risk & Judgement Comment: Data reported \\
\hline
\end{tabular}


O'Brien 2007 (Continued)

Selective reporting (re- Unclear risk Judgement Comment: Did not report outcomes for all measures at post-asporting bias)

\begin{tabular}{lll}
\hline $\begin{array}{l}\text { Other source of bias - } \\
\text { Therapy integrity }\end{array}$ & Low risk & $\begin{array}{l}\text { Judgement Comment: Experienced supervisors. Sessions recorded to moni- } \\
\text { tor and ensure adherence, although lacking detail on how adherence was as- } \\
\text { sessed }\end{array}$ \\
\hline Other bias & Low risk & Judgement Comment: The study appears to be free of other sources of bias \\
\hline
\end{tabular}

Olivares 2005

\section{Study characteristics}

\begin{tabular}{ll}
\hline Methods & Study design: randomised controlled trial \\
Total duration of study: 6 -month follow-up \\
Number of study centres and location: Spain \\
Study setting: schools (screening) \\
\hline N $=\mathbf{3 4}$ (CBT: $\mathrm{N}=17 ;$ waitlist: $\mathrm{N}=17)$ \\
Mean age (SD): 15.03 (0.86) \\
Age range: 14 to 17 \\
Gender: $60 \%$ female \\
Inclusion criteria: diagnosis of social phobia (based on ADIS-C)
\end{tabular}

Interventions

CBT

Intervention: IAFS (multicomponent programme: Intervention in Adolescents with Social Phobia)

Delivery format: child only; group

Therapist contact time: 18 hours $(12 \times 90$ minutes)

\section{Waitlist/no treatment}

Intervention: waitlist

\begin{tabular}{|c|c|c|}
\hline Outcomes & \multicolumn{2}{|c|}{$\begin{array}{l}\text { Remission of primary anxiety disorder diagnosis post-treatment and 6-month follow-up: ADIS-C } \\
\text { Remission of all anxiety disorder diagnoses post-treatment and 6-month follow-up: ADIS-C } \\
\text { Reduction in anxiety symptoms (child report) post-treatment and 6-month follow-up: SPAI-C }\end{array}$} \\
\hline Notes & \multicolumn{2}{|c|}{ Anxiety symptoms measure - social anxiety symptoms } \\
\hline \multicolumn{3}{|l|}{ Risk of bias } \\
\hline Bias & Authors' judgement & Support for judgement \\
\hline $\begin{array}{l}\text { Random sequence genera- } \\
\text { tion (selection bias) }\end{array}$ & Unclear risk & Judgement Comment: No detail given \\
\hline
\end{tabular}


Olivares 2005 (Continued)

$\begin{aligned} & \text { Allocation concealment } \\ & \text { (selection bias) }\end{aligned}$
Unclear risk Judgement Comment: No detail given

\begin{tabular}{ll}
\hline $\begin{array}{l}\text { Blinding of participants } \\
\text { and personnel (perfor- } \\
\text { mance bias) }\end{array}$ & Low risk \\
ill was unlikely this led to a departure from the intended intervention
\end{tabular}

mance bias)

All outcomes

\begin{tabular}{|c|c|c|}
\hline $\begin{array}{l}\text { Blinding of outcome as- } \\
\text { sessment (detection bias) }\end{array}$ & Low risk & $\begin{array}{l}\text { Judgement Comment: Independent assessor carried out diagnostic interview } \\
\text { and evaluation }\end{array}$ \\
\hline
\end{tabular}

All outcomes

\begin{tabular}{lll}
\hline $\begin{array}{l}\text { Incomplete outcome data } \\
\text { (attrition bias) } \\
\text { All outcomes }\end{array}$ & Low risk & Judgement Comment: No missing data \\
\hline $\begin{array}{l}\text { Selective reporting (re- } \\
\text { porting bias) }\end{array}$ & Low risk & Judgement Comment: Outcomes reported for all measures \\
\hline $\begin{array}{l}\text { Other source of bias - } \\
\text { Therapy integrity }\end{array}$ & Unclear risk & Judgement Comment: Lacks detail \\
\hline Other bias & Low risk & Judgement Comment: The study appears to be free of other sources of bias \\
\hline
\end{tabular}

\section{Olivares 2014}

\section{Study characteristics}

\begin{tabular}{|c|c|}
\hline Methods & $\begin{array}{l}\text { Study design: randomised controlled trial } \\
\text { Number of study centres and location: } 9 \text { high schools in Spain } \\
\text { Study setting: schools }\end{array}$ \\
\hline Participants & $\begin{array}{l}\mathbf{N}=117 \text { (CBT (expert): } \mathrm{N}=39 ; \mathrm{CBT} \text { (inexperienced): } \mathrm{N}=39 \text {; waitlist: } \mathrm{N}=39 \text { ) } \\
\text { Mean age (SD): } 15.42 \text { (0.97) } \\
\text { Age range: } 14 \text { to } 17 \\
\text { Gender: } 61.8 \% \text { female } \\
\text { Inclusion criteria: generalised social phobia (based on ADIS-C) } \\
\text { Exclusion criteria: depression, borderline personality disorder, narcissistic disorder, paranoid disor- } \\
\text { der, long history of substance abuse, aggressive behaviour, missing } 3 \text { consecutive treatment sessions, } \\
\text { and have not previously received psychological treatment }\end{array}$ \\
\hline Interventions & $\begin{array}{l}\text { CBT (expert) } \\
\text { Intervention: IAFS (multicomponent programme: Intervention in Adolescents with Social Phobia) } \\
\text { Delivery format: child only; group } \\
\text { Therapist contact time: } 18 \text { hours ( } 12 \times 90 \text { minutes) } \\
\text { Who delivers the intervention: clinical psychologists (degree in psychology) with more than } 2 \text { years' } \\
\text { experience treating individual cases and applying psychological treatment to groups with social anxi- } \\
\text { ety }\end{array}$ \\
\hline
\end{tabular}




\section{CBT (inexperienced)}

Intervention: IAFS (multicomponent programme: Intervention in Adolescents with Social Phobia)

Delivery format: child only; group

Therapist contact time: 18 hours ( $12 \times 90$ minutes)

Who delivers the intervention: psychologists (degree in psychology) with no experience in applying psychological treatment

\section{Waitlist/no treatment}

Intervention: waitlist

\begin{tabular}{ll}
\hline Outcomes & Remission of primary anxiety disorder diagnosis post-treatment: ADIS-C \\
& Reduction in anxiety symptoms (child report) post-treatment: SAS-A \\
& Reduction in anxiety symptoms (parent report) post-treatment: SAS-P \\
\hline Notes & Anxiety symptoms measures - social anxiety symptoms
\end{tabular}

\section{Risk of bias}

\begin{tabular}{|c|c|c|}
\hline Bias & Authors' judgement & Support for judgement \\
\hline $\begin{array}{l}\text { Random sequence genera- } \\
\text { tion (selection bias) }\end{array}$ & Unclear risk & Judgement Comment: No detail given \\
\hline $\begin{array}{l}\text { Allocation concealment } \\
\text { (selection bias) }\end{array}$ & Unclear risk & Judgement Comment: No detail given \\
\hline $\begin{array}{l}\text { Blinding of participants } \\
\text { and personnel (perfor- } \\
\text { mance bias) } \\
\text { All outcomes }\end{array}$ & Low risk & $\begin{array}{l}\text { Judgement Comment: Even though blinding was not possible, we judged that } \\
\text { it was unlikely this led to a departure from the intended intervention }\end{array}$ \\
\hline $\begin{array}{l}\text { Blinding of outcome as- } \\
\text { sessment (detection bias) } \\
\text { All outcomes }\end{array}$ & Unclear risk & $\begin{array}{l}\text { Judgement Comment: Evaluators trained, and therapist competence masked, } \\
\text { but does not specify if blind to waitlist vs treatment }\end{array}$ \\
\hline $\begin{array}{l}\text { Incomplete outcome data } \\
\text { (attrition bias) } \\
\text { All outcomes }\end{array}$ & Unclear risk & $\begin{array}{l}\text { Judgement Comment: Attrition given, but only reports completer analyses. No } \\
\text { mention of how missing item data were managed }\end{array}$ \\
\hline $\begin{array}{l}\text { Selective reporting (re- } \\
\text { porting bias) }\end{array}$ & Low risk & Judgement Comment: Outcomes reported for all measures \\
\hline $\begin{array}{l}\text { Other source of bias - } \\
\text { Therapy integrity }\end{array}$ & Low risk & Judgement Comment: Fidelity measured \\
\hline Other bias & Low risk & Judgement Comment: The study appears to be free of other sources of bias \\
\hline
\end{tabular}

\section{Olivares 2019}

\section{Study characteristics}


Olivares 2019 (Continued)

Study design: randomised controlled trial
Total duration of study: non-controlled follow-up
Number of study centres and location: 7 schools in Spain
Study setting: high schools
N = 108 randomised (CBT-IAFS: $N=36 ;$ CBT-IAFS without social skills training: $N=36 ;$ waitlist: $N=36)$
Mean age: 15.4 (1.11)
Age range: 14 to 17
Gender: $63 \%$ female
Inclusion criteria: met diagnostic criteria for generalised social phobia (based on ADIS-C)
Exclusion criteria: no written parental consent, met diagnostic criteria for severe psychopathology
(e.g. depression or narcissistic disorder), long history of substance abuse, aggressive behaviour, miss-
ing 3 consecutive treatment sessions, and have not previously received psychological or psychiatric
treatment

CBT
Interventions
Delivery format: child only; group
Therapist contact time: 18 hours (12 x 90 minutes)
Who delivers the intervention: psychology graduates
CBT
Intervention: IAFS without social skills training
Delivery format: child only; group
Therapist contact time: 18 hours (12 x 90 minutes)
Who delivers the intervention: psychology graduates
Waitlist/no treatment
Intervention: waitlist

\begin{tabular}{lll}
\hline Outcomes & Remission of primary anxiety disorder diagnosis post-treatment: ADIS-C \\
\hline Notes & Symptom measures only reported pre-post change. \\
\hline Risk of bias & \\
\hline Bias & Authors' judgement & Support for judgement \\
\hline $\begin{array}{lll}\text { Random sequence genera- } \\
\text { tion (selection bias) }\end{array}$ & Unclear risk & Judgement Comment: No detail \\
\hline $\begin{array}{l}\text { Allocation concealment } \\
\text { (selection bias) }\end{array}$ & Unclear risk & Judgement Comment: No detail \\
\hline
\end{tabular}


Olivares 2019 (Continued)

Blinding of participants Low risk_Judgement Comment: Even though blinding was not possible, we judged that and personnel (perforit was unlikely this led to a departure from the intended intervention mance bias)

All outcomes

\begin{tabular}{|c|c|c|}
\hline $\begin{array}{l}\text { Blinding of outcome as- } \\
\text { sessment (detection bias) } \\
\text { All outcomes }\end{array}$ & Low risk & Judgement Comment: Independent assessors \\
\hline $\begin{array}{l}\text { Incomplete outcome data } \\
\text { (attrition bias) } \\
\text { All outcomes }\end{array}$ & Unclear risk & Judgement Comment: High number of dropouts in 1 CBT group \\
\hline $\begin{array}{l}\text { Selective reporting (re- } \\
\text { porting bias) }\end{array}$ & Low risk & Judgement Comment: Outcomes reported for relevant measure \\
\hline $\begin{array}{l}\text { Other source of bias - } \\
\text { Therapy integrity }\end{array}$ & Unclear risk & Judgement Comment: Experienced therapists, no further detail \\
\hline Other bias & Low risk & Judgement Comment: The study appears to be free of other sources of bias \\
\hline
\end{tabular}

\section{Ollendick 2009}

\section{Study characteristics}

Methods

Study design: randomised controlled trial

Total duration of study: 6 -month follow-up

Number of study centres and location: 2; Sweden and the USA

Study setting: clinics

Date of study: 2001 to 2006

Participants $\quad 196$ (CBT: $N=85$; active control: $N=70 ;$ waitlist: $N=41)$
Mean age (SD): Virginia site: $10.9(1.7)$; Stockholm site: 11.1 (1.9)
Age range: 7 to 16
Gender: $106(43.3 \%)$
Inclusion criteria: age 7 to 16 ; specific phobia diagnosis, present for 6 months with CSR $4+$ (based on
ADIS-C/P); discontinue other psychotherapy or anti-anxiety medication for duration of study
Exclusion criteria: primary major depression, pervasive developmental disorder, drug or alcohol
abuse, psychotic symptoms
CBT
Intervention: One Session Treatment (OST) (follow Ost, but flexibly implemented)
Concomitant/excluded medications: anti-anxiety treatment not allowed
Delivery format: child only; individual
Therapist contact time: 3 hours 45 minutes (pretreatment interview 45 minutes, treatment 3 hours)
Who delivers the intervention: clinicians with at minimum a Master's degree, trained


Ollendick 2009 (Continued)

\section{Active control}

Intervention: education support therapy

Concomitant/excluded medications: anti-anxiety treatment not allowed

Delivery format: child only; individual

Therapist contact time: 3 hours

Who delivers the intervention: clinicians with at minimum a Master's degree, trained

\section{Waitlist/no treatment}

Intervention: 4-week waitlist

Concomitant/excluded medications: anti-anxiety treatment not allowed

Outcomes

Remission of primary anxiety disorder diagnosis post-treatment: ADIS-C/P (mini version to assess pretreatment diagnoses)

Reduction in anxiety symptoms (child report) post-treatment: MASC-C

Reduction in depressive symptoms post-treatment: CDI

Notes

Author confirmed diagnosis free $=$ free from primary disorder

Reported no adverse events during treatment

Active control $=$ attention control

Symptom measures reported for each site separately.

Follow-up includes waitlist participants re-randomised to OST vs active control

CBT vs waitlist and CBT vs active control (did not divide CBT group as included in separate comparisons)

\section{Risk of bias}

\begin{tabular}{lll}
\hline Bias & Authors' judgement & Support for judgement \\
\hline $\begin{array}{l}\text { Random sequence genera- } \\
\text { tion (selection bias) }\end{array}$ & Low risk & $\begin{array}{l}\text { Judgement Comment: Computerised randomisation procedures or random } \\
\text { number generators }\end{array}$ \\
\hline $\begin{array}{l}\text { Allocation concealment } \\
\text { (selection bias) }\end{array}$ & Low risk & $\begin{array}{l}\text { Judgement Comment: Randomisation assignments were kept in opaque en- } \\
\text { velopes that remained unopened until the time of assignment }\end{array}$ \\
\hline $\begin{array}{l}\text { Blinding of participants } \\
\text { and personnel (perfor- } \\
\text { mance bias) }\end{array}$ & Unclear risk & $\begin{array}{l}\text { Judgement Comment: Did not specify whether participants were blind to 'ac- } \\
\text { tive' vs 'control' treatment }\end{array}$ \\
\hline $\begin{array}{l}\text { Blinding of outcome as- } \\
\text { sessment (detection bias) } \\
\begin{array}{l}\text { All outcomes } \\
\text { Incomplete outcome data }\end{array}\end{array}$ & Low risk & Judgement Comment: Blind assessors \\
$\begin{array}{l}\text { (attrition bias) } \\
\text { All outcomes }\end{array}$ & & \\
\hline
\end{tabular}


Ollendick 2009 (Continued)

Selective reporting (re- Low risk Judgement Comment: Outcomes reported for all measures porting bias)

$\begin{array}{ll}\begin{array}{l}\text { Other source of bias - Low risk } \\ \text { Therapy integrity }\end{array} & \begin{array}{l}\text { Judgement Comment: Therapists trained, supervised, and adherence and } \\ \text { competence monitored }\end{array}\end{array}$

\begin{tabular}{ll}
\hline Other bias Low risk Judgement Comment: The study appears to be free of other sources of bias \\
\hline
\end{tabular}

Ost 2001

\section{Study characteristics}

\begin{tabular}{ll}
\hline Methods & Study design: randomised controlled trial \\
Number of study centres and location: Sweden \\
Study setting: referrals from child psychiatric services and school services \\
\hline $\mathbf{N}=\mathbf{6 0}(\mathrm{CBT}(\mathrm{child}): \mathrm{N}=21 ; \mathrm{CBT}$ (child + parent) $\mathrm{N}=20 ;$ waitlist: $\mathrm{N}=19)$ \\
Mean age (SD): $11.7(2.8)$ \\
Age range: 7 to 17 \\
Gender: $37(61 \%)$ female \\
Inclusion criteria: aged 7 to 17, primary specific phobia, with CSR 4+ and present for minimum of 1 \\
year (based on ADIS-C/P), be motivated for treatment \\
$\begin{array}{l}\text { Exclusion criteria: } \text { primary depression, drug or substance abuse, developmental disorder, psychotic } \\
\text { symptoms }\end{array}$
\end{tabular}

\section{CBT (child)}

Intervention: One Session Treatment (OST), adjusted to developmental age

Delivery format: child only; individual

Therapist contact time: 3 hours 45 minutes (pretreatment interview - 45 minutes, treatment - 3 hours)

Who delivers the intervention: experienced clinical psychologist, experienced in treating specific phobias with CBT

\section{CBT (child + parent)}

Intervention: One Session Treatment (OST), adjusted to developmental age. Same as child condition, only parent present too

Delivery format: child and parent; individual

Therapist contact time: 3 hours 45 minutes (pretreatment interview - 45 minutes, treatment - 3 hours)

Who delivers the intervention: experienced clinical psychologist, experienced in treating specific phobias with CBT

\section{Waitlist/no treatment}

Intervention: 4-week waitlist 
Ost 2001 (Continued)

\section{Reduction in anxiety symptoms (child report) post-treatment: RCMAS}

Reduction in depressive symptoms post-treatment: CDI

Notes Author provided remission of all diagnoses for those with pretreatment diagnoses only.

\section{Risk of bias}

\begin{tabular}{|c|c|c|}
\hline Bias & Authors' judgement & Support for judgement \\
\hline $\begin{array}{l}\text { Random sequence genera- } \\
\text { tion (selection bias) }\end{array}$ & Unclear risk & Judgement Comment: No detail given \\
\hline $\begin{array}{l}\text { Allocation concealment } \\
\text { (selection bias) }\end{array}$ & Unclear risk & Judgement Comment: No detail given \\
\hline $\begin{array}{l}\text { Blinding of participants } \\
\text { and personnel (perfor- } \\
\text { mance bias) } \\
\text { All outcomes }\end{array}$ & Low risk & $\begin{array}{l}\text { Judgement Comment: Even though blinding was not possible, we judged that } \\
\text { it was unlikely this led to a departure from the intended intervention }\end{array}$ \\
\hline $\begin{array}{l}\text { Blinding of outcome as- } \\
\text { sessment (detection bias) } \\
\text { All outcomes }\end{array}$ & Low risk & Judgement Comment: Assessors blind to condition \\
\hline $\begin{array}{l}\text { Incomplete outcome data } \\
\text { (attrition bias) } \\
\text { All outcomes }\end{array}$ & Low risk & $\begin{array}{l}\text { Judgement Comment: Not clear if there were dropouts/missing data/ap- } \\
\text { proach to managing missing data, but author provided data }\end{array}$ \\
\hline $\begin{array}{l}\text { Selective reporting (re- } \\
\text { porting bias) }\end{array}$ & Low risk & Judgement Comment: Outcomes reported for measures \\
\hline $\begin{array}{l}\text { Other source of bias - } \\
\text { Therapy integrity }\end{array}$ & Unclear risk & $\begin{array}{l}\text { Judgement Comment: Experienced therapists, but no detail given on assess- } \\
\text { ing adherence }\end{array}$ \\
\hline Other bias & Low risk & Judgement Comment: The study appears to be free of other sources of bias \\
\hline
\end{tabular}

Perrin 2019

\section{Study characteristics}

Methods Study design: randomised controlled trial

Total duration of study: 10-week treatment period; 3-month non-controlled follow-up

Number of study centres and location: referrals from 11 sites in 1 NHS Trust in the UK

Study setting: referral from CAMHS services and specialist anxiety clinician at NHS Trust

Participants $\quad \mathbf{N}=\mathbf{4 0}(\mathrm{CBT}: \mathrm{N}=20$; waitlist: $\mathrm{N}=20)$

Mean age (SD): CBT: 13.2 (2.4); waitlist: $13.6(2.8)$

Age range: 10 to 18

Gender: $25(62.5 \%)$ female 
Perrin 2019 (Continued)

Inclusion criteria: (1) aged 10 to 18 years; (2) referred for treatment of anxiety; (3) a current, primary diagnosis of DSM-IV GAD (Based on ADIS-C/P); (4) no other psychiatric problems in need of more urgent treatment (including self-injurious thoughts/behaviours or substance use/abuse); (5) no concurrent psychological or pharmacological treatment for any disorder; and (6) the absence of moderate to severe learning difficulties as evidenced in the medical or school records or as reported by the referrer/parent at the pretrial screening

CBT
Interventions
Concomitant/excluded medications: concurrent pharmacological treatment
lowed
Delivery format: child only, parent invited to join for 1 session and to join end
Therapist contact time: 10 sessions
Who delivers the intervention: doctoral-level clinical psychologists
Waitlist
Intervention: 10 -week waitlist
Concomitant/excluded medications: concurrent pharmacological treatment
lowed
Remission of primary anxiety disorder diagnosis post-treatment: ADIS-C/P
Remission of all anxiety disorder diagnoses post-treatment: ADIS-C/P
Reduction in anxiety symptoms (child report) post-treatment: SCARED-C
Reduction in anxiety symptoms (parent report) post-treatment: SCARED-P
Reduction in depressive symptoms post-treatment: MFQ-C
Improvement in global functioning post-treatment: CGAS

Notes

\section{Risk of bias}

\begin{tabular}{lll}
\hline Bias & Authors' judgement & Support for judgement \\
\hline $\begin{array}{l}\text { Random sequence genera- } \\
\text { tion (selection bias) }\end{array}$ & Low risk & Judgement Comment: Online random allocation program \\
\hline $\begin{array}{l}\text { Allocation concealment } \\
\text { (selection bias) }\end{array}$ & Low risk & $\begin{array}{l}\text { Judgement Comment: Online random allocation program created by CTU, al- } \\
\text { location after initial assessment }\end{array}$ \\
\hline $\begin{array}{l}\text { Blinding of participants } \\
\text { and personnel (perfor- } \\
\text { mance bias) }\end{array}$ & Low risk & $\begin{array}{l}\text { Judgement Comment: Even though blinding was not possible, we judged that } \\
\text { All outcomes }\end{array}$ \\
\hline $\begin{array}{l}\text { Blinding of outcome as- } \\
\text { sessment (detection bias) } \\
\text { All outcomes }\end{array}$ & Low risk & Judgement Comment: Independent blind assessors \\
\hline $\begin{array}{l}\text { Incomplete outcome data } \\
\text { (attrition bias) }\end{array}$ & Low risk & \\
\hline
\end{tabular}


Perrin 2019 (Continued)

All outcomes

Selective reporting (re- Low risk Judgement Comment: Outcomes reported for all measures
porting bias)

\begin{tabular}{lll}
\hline $\begin{array}{l}\text { Other source of bias - } \\
\text { Therapy integrity }\end{array}$ & Low risk & Judgement Comment: Trained and supervisor therapist. Adherence assessed \\
\hline Other bias & Low risk & Judgement Comment: The study appears to be free of other sources of bias \\
\hline
\end{tabular}

Pincus 2010

\section{Study characteristics}

\begin{tabular}{ll}
\hline Methods & Study design: randomised controlled trial \\
& Number of study centres and location: 1 in the USA \\
& Study setting: university research clinic \\
\hline N $=\mathbf{2 6}(\mathrm{CBT}: \mathrm{N}=13 ;$ active control: $\mathrm{N}=13)$ \\
Mean age (SD): $15.75(1.10)$ \\
Age range: 14 to 17 \\
Gender: 19 (73.1\%) female \\
Inclusion criteria: panic disorder diagnosis, with or without agoraphobia, CSR 4+ (based on ADIS-C/P)
\end{tabular}

Interventions

CBT

Intervention: Panic Treatment Programme (PCT-A) downward extension of Panic Control Treatment

Concomitant/excluded medications: stabilisation period prior to assessment required

Delivery format: child sessions, with parent included in some sessions; individual

Therapist contact time: 9 hours ( $11 \times 50$ minutes)

\section{Active control}

Intervention: attention control. 8-week self-monitoring, meet with therapist every other week to discuss self-monitoring panic and anxiety

Concomitant/excluded medications: stabilisation period prior to assessment required

Delivery format: child only; individual

Therapist contact time: 2 hours $(4 \times 30$ minutes)

\begin{tabular}{ll}
\hline Outcomes & Reduction in anxiety symptoms (child report) post-treatment: MASC-C \\
& Reduction in depressive symptoms post-treatment: CDI \\
\hline Notes & Use mini-ADIS post-treatment. Remission outcomes not reported/provided. \\
& Active control = attention control \\
\hline
\end{tabular}

\section{Risk of bias}


Pincus 2010 (Continued)

\begin{tabular}{|c|c|c|}
\hline Bias & Authors' judgement & Support for judgement \\
\hline $\begin{array}{l}\text { Random sequence genera- } \\
\text { tion (selection bias) }\end{array}$ & Unclear risk & Judgement Comment: No detail given \\
\hline $\begin{array}{l}\text { Allocation concealment } \\
\text { (selection bias) }\end{array}$ & Unclear risk & Judgement Comment: No detail given \\
\hline $\begin{array}{l}\text { Blinding of participants } \\
\text { and personnel (perfor- } \\
\text { mance bias) } \\
\text { All outcomes }\end{array}$ & Low risk & $\begin{array}{l}\text { Judgement Comment: Even though blinding was not possible, we judged that } \\
\text { it was unlikely this led to a departure from the intended intervention }\end{array}$ \\
\hline $\begin{array}{l}\text { Blinding of outcome as- } \\
\text { sessment (detection bias) } \\
\text { All outcomes }\end{array}$ & Unclear risk & Judgement Comment: Not specified \\
\hline $\begin{array}{l}\text { Incomplete outcome data } \\
\text { (attrition bias) } \\
\text { All outcomes }\end{array}$ & Unclear risk & $\begin{array}{l}\text { Judgement Comment: Attrition and how missing data managed detailed, but } \\
\text { high rate of missing data ( } 24 \% \text { for questionnaires) }\end{array}$ \\
\hline $\begin{array}{l}\text { Selective reporting (re- } \\
\text { porting bias) }\end{array}$ & Low risk & Judgement Comment: Outcomes reported \\
\hline $\begin{array}{l}\text { Other source of bias - } \\
\text { Therapy integrity }\end{array}$ & Low risk & $\begin{array}{l}\text { Judgement Comment: Adherence assessed by independent raters and report- } \\
\text { ed }\end{array}$ \\
\hline Other bias & High risk & $\begin{array}{l}\text { Judgement Comment: Time point for post-treatment assessment varies across } \\
\text { conditions }\end{array}$ \\
\hline
\end{tabular}

Rapee 2005

\section{Study characteristics}

$\begin{array}{ll}\text { Methods } & \text { Study design: randomised controlled trial } \\ \text { Total duration of study: } 11 \text {-year follow-up } \\ \text { Number of study centres and location: Australia } \\ \text { Study setting: screening in } 95 \text { preschools } \\ \text { N = 146 randomised (NB: we included } \mathrm{N}=111 \text { who met diagnostic criteria for an anxiety disorder at } \\ \text { baseline. CBT: } \mathrm{N}=60 ; \text { no intervention: } \mathrm{N}=51 \text { ) } \\ \text { Mean age (SD): CBT: } 47.23 \text { (5.08); no intervention: } 45.71 \text { (4.15) } \\ \text { Age range: } 36 \text { to } 62 \text { months } \\ \text { Gender: } 64 \text { (57.7\%) female } \\ \text { Inclusion criteria: inhibited children (score }>1.5 \text { SD above mean) and met for behavioural inhibition } \\ \text { on the lab assessment. We only included children who met diagnostic criteria in the review. }\end{array}$

Interventions CBT

Intervention: parent education. Nature of anxiety, parent management, exposure, cognitive restructuring for parent, apply cognitive principles with child 
Delivery format: parent only, group

Therapist contact time: 9 hours ( $6 \times 90$ minutes)

Who delivers the intervention: clinical psychologist

\title{
No treatment
}

Intervention: monitor condition. No intervention

\begin{tabular}{ll}
\hline Outcomes & Remission of all anxiety disorder diagnoses post-treatment: ADIS-P \\
& Reduction in anxiety symptoms (parent report) post-treatment: Preschool Anxiety Scale
\end{tabular}

Notes We only included children who met diagnostic criteria for an anxiety disorder at baseline.

\section{Risk of bias}

\begin{tabular}{|c|c|c|}
\hline Bias & Authors' judgement & Support for judgement \\
\hline $\begin{array}{l}\text { Random sequence genera- } \\
\text { tion (selection bias) }\end{array}$ & Unclear risk & Judgement Comment: No detail given \\
\hline $\begin{array}{l}\text { Allocation concealment } \\
\text { (selection bias) }\end{array}$ & Unclear risk & Judgement Comment: No detail given \\
\hline $\begin{array}{l}\text { Blinding of participants } \\
\text { and personnel (perfor- } \\
\text { mance bias) } \\
\text { All outcomes }\end{array}$ & Low risk & $\begin{array}{l}\text { Judgement Comment: Even though blinding was not possible, we judged that } \\
\text { it was unlikely this led to a departure from the intended intervention }\end{array}$ \\
\hline $\begin{array}{l}\text { Blinding of outcome as- } \\
\text { sessment (detection bias) } \\
\text { All outcomes }\end{array}$ & Low risk & Judgement Comment: Blind assessors \\
\hline $\begin{array}{l}\text { Incomplete outcome data } \\
\text { (attrition bias) } \\
\text { All outcomes }\end{array}$ & Low risk & Judgement Comment: Author provided data \\
\hline $\begin{array}{l}\text { Selective reporting (re- } \\
\text { porting bias) }\end{array}$ & Low risk & Judgement Comment: Outcomes reported/provided \\
\hline $\begin{array}{l}\text { Other source of bias - } \\
\text { Therapy integrity }\end{array}$ & Unclear risk & $\begin{array}{l}\text { Judgement Comment: Experienced therapist delivered intervention. No fur- } \\
\text { ther detail }\end{array}$ \\
\hline Other bias & Low risk & Judgement Comment: The study appears to be free of other sources of bias \\
\hline
\end{tabular}

Rapee 2006

\section{Study characteristics}

Methods

\author{
Study design: randomised controlled trial \\ Number of study centres and location: 1 in Australia
}

Study setting: university clinic

Date of study: recruitment 1999 to 2002 
Rapee 2006 (Continued)

Participants
$\mathbf{N}=\mathbf{2 6 7}$ randomised (NB: we included $\mathrm{N}=177$, randomised to $C B T: \mathrm{N}=90$; waitlist: $\mathrm{N}=87$ )

Mean age (SD): CBT: 113.7 months (20.4); waitlist: 114.1 months (19.1)

Age range: 6 to 12

Gender: CBT: 52.3\% female; waitlist: $29.9 \%$ female

Inclusion criteria: year 1 to 6 at school (age 6 to 12), primary anxiety disorder (based on ADIS-C/P), parent able to read standard English newspaper

\begin{tabular}{|c|c|}
\hline Interventions & CBT \\
\hline & Intervention: Cool Kids \\
\hline & Concomitant/excluded medications: stable dose for month before allowed \\
\hline & Delivery format: child and parent sessions, group \\
\hline & Therapist contact time: 18 hours ( $9 \times 2$ hours) \\
\hline & Who delivers the intervention: graduate students in clinical psychology \\
\hline & Waitlist/no treatment \\
\hline & Intervention: 3-month waitlist \\
\hline & Concomitant/excluded medications: stable dose for month before allowed \\
\hline Outcomes & Remission of primary anxiety disorder diagnosis post-treatment: $A D I S-C / P$ \\
\hline & Remission of all anxiety disorder diagnoses post-treatment: $A D I S-C / P$ \\
\hline & Reduction in anxiety symptoms (child report) post-treatment: SCAS-C \\
\hline & Reduction in anxiety symptoms (parent report) post-treatment: SCAS-P \\
\hline Notes & $\begin{array}{l}\text { Trial included "bibliotherapy" arm (parent-only condition with no face-to-face contact, so not includ- } \\
\text { ed). }\end{array}$ \\
\hline & Report no adverse events during treatment \\
\hline
\end{tabular}

\section{Risk of bias}

\begin{tabular}{lll}
\hline Bias & Authors' judgement & Support for judgement \\
\hline $\begin{array}{l}\text { Random sequence genera- } \\
\text { tion (selection bias) }\end{array}$ & Low risk & Judgement Comment: Block randomisation, random number schedule \\
\hline $\begin{array}{l}\text { Allocation concealment } \\
\text { (selection bias) }\end{array}$ & Low risk & $\begin{array}{l}\text { Judgement Comment: Random number schedule known only to study co-ordi- } \\
\text { nator }\end{array}$ \\
\hline $\begin{array}{l}\text { Blinding of participants } \\
\begin{array}{l}\text { and personnel (perfor- } \\
\text { mance bias) } \\
\text { All outcomes }\end{array}\end{array}$ & Low risk & $\begin{array}{l}\text { Judgement Comment: Even though blinding was not possible, we judged that } \\
\text { it was unlikely this led to a departure from the intended intervention }\end{array}$ \\
\hline
\end{tabular}

Blinding of outcome as- Low risk Judgement Comment: Blind to treatment condition
sessment (detection bias)

All outcomes 
Rapee 2006 (Continued)

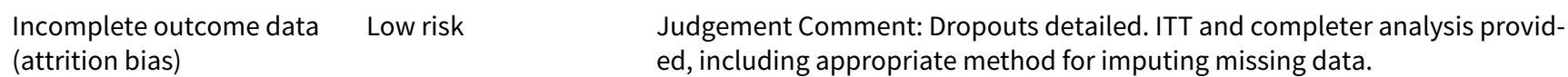
(attrition bias) ed, including appropriate method for imputing missing data.

All outcomes

Selective reporting (re- Low risk Judgement Comment: Outcomes reported for all measures
porting bias)

\begin{tabular}{lll}
\hline $\begin{array}{l}\text { Other source of bias - } \\
\text { Therapy integrity }\end{array}$ & Unclear risk Judgement Comment: Experienced therapist. No detail on adherence \\
\hline Other bias & Low risk & Judgement Comment: The study appears to be free of other sources of bias \\
\hline
\end{tabular}

Reaven 2012

\section{Study characteristics}

\begin{tabular}{ll}
\hline Methods & Study design: randomised controlled trial \\
Total duration of study: 4-month intervention period \\
Number of study centres and location: 1 in the USA \\
Study setting: research clinic \\
\hline N $=\mathbf{5 0}$ (CBT: $\mathrm{N}=24 ; \mathrm{TAU}: \mathrm{N}=26)$ \\
Mean age (SD): CBT: 125.75 months (21.47); TAU: 125.00 months (20.45) \\
Age range: 7 to 14 \\
Gender: 48 (96\%) female \\
Inclusion criteria: age 7 to 14 ; diagnosis of an ASD (determined by expert clinical psychologists based \\
upon review of a recent ADOS and the Social Communication Questionnaire); speaking in full, com- \\
plex sentences, and as reflected in recent standardised cognitive assessment, and clinically significant \\
symptoms of anxiety (NB: include ADIS-P and participants met diagnostic criteria) \\
Excluded criteria: primary psychiatric symptoms reflective of another condition such as depression or \\
psychosis; child does not demonstrate "group readiness" in the first 3 sessions of Facing Your Fears; 1 \\
parent could not commit to attending $80 \%$ of sessions
\end{tabular}

Interventions CBT

Intervention: Facing Your Fears. Adapted CBT programme for children with ASD

Concomitant/excluded medications: medications allowed

Delivery format: child and parent together, group

Therapist contact time: 18 hours $(12 \times 90$ minutes)

Who delivers the intervention: clinical psychologist and co-therapists

\section{TAU}

Intervention: maintained current interventions and allowed to pursue new interventions for 4 months

Concomitant/excluded medications: medications allowed 
Reaven 2012 (Continued)

Remission of all anxiety disorder diagnoses post-treatment: ADIS-P

\begin{tabular}{|c|c|c|}
\hline Notes & $\begin{array}{l}\text { Use data published in } \\
\text { Sample with ASD }\end{array}$ & Jarwick 2016 review \\
\hline \multicolumn{3}{|l|}{ Risk of bias } \\
\hline Bias & Authors' judgement & Support for judgement \\
\hline $\begin{array}{l}\text { Random sequence genera- } \\
\text { tion (selection bias) }\end{array}$ & Low risk & Judgement Comment: Computer generated \\
\hline $\begin{array}{l}\text { Allocation concealment } \\
\text { (selection bias) }\end{array}$ & Low risk & Judgement Comment: Computer generated, so unlikely to foresee assignment \\
\hline $\begin{array}{l}\text { Blinding of participants } \\
\text { and personnel (perfor- } \\
\text { mance bias) } \\
\text { All outcomes }\end{array}$ & Low risk & $\begin{array}{l}\text { Judgement Comment: Even though blinding was not possible, we judged that } \\
\text { it was unlikely this led to a departure from the intended intervention }\end{array}$ \\
\hline $\begin{array}{l}\text { Blinding of outcome as- } \\
\text { sessment (detection bias) } \\
\text { All outcomes }\end{array}$ & Low risk & Judgement Comment: Blind assessors \\
\hline $\begin{array}{l}\text { Incomplete outcome data } \\
\text { (attrition bias) } \\
\text { All outcomes }\end{array}$ & Low risk & $\begin{array}{l}\text { Judgement Comment: Attrition detailed, report ITT. Diagnostic outcomes only } \\
\text { relevant outcome }\end{array}$ \\
\hline $\begin{array}{l}\text { Selective reporting (re- } \\
\text { porting bias) }\end{array}$ & Low risk & Judgement Comment: Outcomes reported/available for relevant measure \\
\hline $\begin{array}{l}\text { Other source of bias - } \\
\text { Therapy integrity }\end{array}$ & Low risk & $\begin{array}{l}\text { Judgement Comment: Trained therapists, regular supervision, adherence as- } \\
\text { sessed and reported }\end{array}$ \\
\hline Other bias & Low risk & Judgement Comment: The study appears to be free of other sources of bias \\
\hline
\end{tabular}

Reigada 2015

\section{Study characteristics}

\begin{tabular}{ll} 
Methods & Study design: randomised controlled trial \\
& Total duration of study: 3 -month follow-up \\
& Number of study centres and location: 1 in the USA \\
& Study setting: university clinic \\
\hline N $=22$ (CBT: $N=11$; active control: $N=11)$ \\
Mean age (SD): $13.2(2.1)$ \\
Age range: 9 to 17 \\
Gender: $13(59.1 \%)$ female
\end{tabular}


Inclusion criteria: confirmed inflammatory bowel disease (IBD) diagnosis and met diagnostic criteria for a DSM-IV anxiety disorder (based on ADIS-C/P)

Exclusion criteria: current suicidal thoughts requiring immediate clinical attention; current use of a psychotropic medication prescribed within the last 2 months; current substance use disorder, conduct disorder, or pervasive developmental disorder; schizophrenia or other psychosis; a history of or current bipolar disorder; or a principal diagnosis of post-traumatic stress disorder

Interventions

\section{CBT}

Intervention: Treatment of Anxiety and Physical symptoms related to IBD (TAPS + IBD)

Concomitant/excluded medications: current use of a psychotropic medication prescribed within the last 2 months excluded

Delivery format: child sessions and 3 parent sessions; individual

Therapist contact time: 16 hours ( $13 \times 1$ hour; $3 \times 1$-hour parent sessions)

Who delivers the intervention: clinical psychology doctoral students

\section{Active control}

Intervention: non-directive supportive therapy. Offered social and emotional support through the use of non-directive techniques (e.g. reflection, summarisation), empathetic communication, and supportive statements

Concomitant/excluded medications: current use of a psychotropic medication prescribed within the last 2 months excluded

Delivery format: child sessions and parent sessions; individual

Therapist contact time: 16 hours ( $13 \times 1$ hour; $3 \times 1$-hour parent sessions)

Who delivers the intervention: clinical psychology doctoral students

\begin{tabular}{|c|c|c|}
\hline Outcomes & \multicolumn{2}{|c|}{$\begin{array}{l}\text { Remission of primary anxiety disorder diagnosis post-treatment and 3-month follow-up: ADIS-C/P } \\
\text { Remission of all anxiety disorder diagnoses post-treatment and 3-month follow-up: ADIS-C/P } \\
\text { Reduction in anxiety symptoms (child report) post-treatment and 3-month follow-up: IBD-Specific } \\
\text { Anxiety Scale }\end{array}$} \\
\hline Notes & \multicolumn{2}{|c|}{$\begin{array}{l}\text { Anxiety symptom measure measure of anxiety related to IBD symptoms } \\
\text { Active control = alternative treatment }\end{array}$} \\
\hline \multicolumn{3}{|l|}{ Risk of bias } \\
\hline Bias & Authors' judgement & Support for judgement \\
\hline $\begin{array}{l}\text { Random sequence genera- } \\
\text { tion (selection bias) }\end{array}$ & Low risk & Judgement Comment: Random number charts \\
\hline $\begin{array}{l}\text { Allocation concealment } \\
\text { (selection bias) }\end{array}$ & Unclear risk & Judgement Comment: No detail given \\
\hline $\begin{array}{l}\text { Blinding of participants } \\
\text { and personnel (perfor- } \\
\text { mance bias) } \\
\text { All outcomes }\end{array}$ & Unclear risk & Judgement Comment: No detail on participant blinding \\
\hline
\end{tabular}


Reigada 2015 (Continued)

Blinding of outcome as- Low risk_ Judgement Comment: Blind independent evaluators sessment (detection bias)

All outcomes

\begin{tabular}{lll}
\hline $\begin{array}{l}\text { Incomplete outcome data } \\
\text { (attrition bias) } \\
\text { All outcomes }\end{array}$ & Low risk & $\begin{array}{l}\text { Judgement Comment: Attrition and missing data detailed. Detailed method of } \\
\text { managing missing data to report ITT analyses }\end{array}$ \\
\hline $\begin{array}{l}\text { Selective reporting (re- } \\
\text { porting bias) }\end{array}$ & Low risk & Judgement Comment: Outcomes reported for all measures \\
\hline $\begin{array}{l}\text { Other source of bias - } \\
\text { Therapy integrity }\end{array}$ & Low risk & $\begin{array}{l}\text { Judgement Comment: Therapists trained and supervised. Adherence and con- } \\
\text { tamination in control assessed and reported }\end{array}$ \\
\hline Other bias & Low risk & Judgement Comment: The study appears to be free of other sources of bias \\
\hline
\end{tabular}

Rosa Alcazar 2009

\section{Study characteristics}

\begin{tabular}{|c|c|}
\hline Methods & $\begin{array}{l}\text { Study design: randomised controlled trial } \\
\text { Total duration of study: } 6 \text { - and 12-month follow-up } \\
\text { Number of study centres and location: Spain } \\
\text { Study setting: schools (screening) }\end{array}$ \\
\hline Participants & $\begin{array}{l}\text { N = } 77 \text { (CBT: } N=20 \text {; active control (psychoeducation): } N=19 ; \text { active control: } N=18 \text {; waitlist: } N=20 \text { ) } \\
\text { Mean age (SD): } 14.87(0.80) \\
\text { Age range: } 14 \text { to } 17 \\
\text { Gender: } 75 \% \text { female } \\
\text { Inclusion criteria: generalised social phobia diagnosis (based on ADIS-C), parental consent }\end{array}$ \\
\hline Interventions & $\begin{array}{l}\text { CBT } \\
\text { Intervention: IAFS (Intervención en Adolescentes con Fobia Social) } \\
\text { Delivery format: child only; group } \\
\text { Therapist contact time: } 18 \text { hours ( } 12 \times 90 \text { minutes) } \\
\text { Who delivers the intervention: therapists } \\
\text { Active control } \\
\text { Intervention: psychoeducation } \\
\text { Delivery format: child only; group } \\
\text { Therapist contact time: } 18 \text { hours ( } 12 \text { × } 90 \text { minutes) } \\
\text { Who delivers the intervention: therapists } \\
\text { Active control } \\
\text { Intervention: health education }\end{array}$ \\
\hline
\end{tabular}


Rosa Alcazar 2009 (Continued)

\section{Concomitant/excluded medications:}

Delivery format: child only; group

Therapist contact time: 18 hours ( $12 \times 90$ minutes)

Who delivers the intervention: therapists

Waitlist/no treatment

Intervention: waitlist

\begin{tabular}{ll}
\hline Outcomes & Remission of primary anxiety disorder diagnosis post-treatment, 6-month and 12-month fol- \\
low-up: ADIS-C & Remission of all anxiety disorder diagnoses post-treatment, 6-month and 12-month follow-up: \\
ADIS-C & Reduction in anxiety symptoms (child report) post-treatment, 6-month and 12-month follow-up: \\
SPAIC & Reduction in anxiety symptoms (parent report) post-treatment, 6-month and 12-month fol- \\
low-up: SAS-P & Active controls = attention controls \\
Comparisons: CBT (split CBT group) vs active control, CBT (split CBT group) vs active control, CBT (total \\
CBT group) vs waitlist \\
Anxiety symptoms measures social anxiety symptom measures
\end{tabular}

\section{Risk of bias}

\begin{tabular}{lll}
\hline Bias & Authors' judgement & Support for judgement \\
\hline $\begin{array}{l}\text { Random sequence genera- } \\
\text { tion (selection bias) }\end{array}$ & Unclear risk & Judgement Comment: Lack of detail \\
\hline $\begin{array}{l}\text { Allocation concealment } \\
\text { (selection bias) }\end{array}$ & Unclear risk & Judgement Comment: No detail \\
\hline $\begin{array}{l}\text { Blinding of participants } \\
\text { and personnel (perfor- } \\
\text { mance bias) }\end{array}$ & Unclear risk & Judgement Comment: No detail \\
All outcomes & \\
\hline
\end{tabular}

\begin{tabular}{|c|c|c|}
\hline $\begin{array}{l}\text { Blinding of outcome as- } \\
\text { sessment (detection bias) } \\
\text { All outcomes }\end{array}$ & Unclear risk & Judgement Comment: Lack of detail \\
\hline $\begin{array}{l}\text { Incomplete outcome data } \\
\text { (attrition bias) } \\
\text { All outcomes }\end{array}$ & Low risk & Judgement Comment: Author provided data \\
\hline $\begin{array}{l}\text { Selective reporting (re- } \\
\text { porting bias) }\end{array}$ & Low risk & Judgement Comment: Outcomes reported/provided for all relevant measures \\
\hline $\begin{array}{l}\text { Other source of bias - } \\
\text { Therapy integrity }\end{array}$ & Unclear risk & Judgement Comment: Lack of detail \\
\hline Other bias & Low risk & Judgement Comment: The study appears to be free of other sources of bias \\
\hline
\end{tabular}


Salari 2018

\section{Study characteristics}

\begin{tabular}{|c|c|}
\hline Methods & $\begin{array}{l}\text { Study design: randomised controlled trial } \\
\text { Number of study centres and location: } 1 \text { in Iran } \\
\text { Study setting: university-affiliated outpatient clinic }\end{array}$ \\
\hline Participants & $\begin{array}{l}\text { N = } 42 \text { (CBT: } N=20 \text {; waitlist: } \mathrm{N}=22 \text { ) } \\
\text { Mean age (SD): CBT: } 8.5 \text { (2.5); waitlist: } 8.1 \text { (1.9) } \\
\text { Age range: } 6 \text { to } 12 \\
\text { Gender: CBT: } 47.4 \% \text { female; waitlist: } 21.4 \% \text { female } \\
\text { Inclusion criteria: DSM-IV criteria for primary diagnosis of GAD, separation anxiety disorder, social } \\
\text { phobia, or specific phobia (based on K-SADS), } 1 \text { SSRI (Selective Serotonin Reuptake Inhibitor) medicine } \\
\text { at a stable dose for at least } 8 \text { weeks before baseline } \\
\text { Excluded criteria: } 1 \text { ) diagnosed as major psychiatric/neurologic disorders; } 2 \text { ) change in medication } \\
\text { during the study; } 3 \text { ) parents missed more than } 2 \text { of } 6 \text { sessions of treatment }\end{array}$ \\
\hline
\end{tabular}

\section{CBT}

Intervention: parent training - group CBT "Friends for life" (parent-training element, translated version)

Concomitant/excluded medications: a stable dose of SSRI for at least 8 weeks before baseline allowed

Delivery format: parent only; group

Therapist contact time: 12 hours $(6 \times 2$ hours)

Who delivers the intervention child psychiatrist and co-led by a fellow of child psychiatry

\section{Waitlist/no treatment}

Intervention: 6-week waitlist

Concomitant/excluded medications: a stable dose of SSRI for at least 8 weeks before baseline

\begin{tabular}{|c|c|c|}
\hline Outcomes & \multicolumn{2}{|c|}{$\begin{array}{l}\text { Reduction in anxiety symptoms (child report) post-treatment: RCMAS } \\
\text { Reduction in depressive symptoms post-treatment: } C D I \\
\text { Improvement in global functioning post-treatment: CGAS }\end{array}$} \\
\hline Notes & Author confirmed no c & agnostic assessment post-treatment. \\
\hline \multicolumn{3}{|l|}{ Risk of bias } \\
\hline Bias & Authors' judgement & Support for judgement \\
\hline $\begin{array}{l}\text { Random sequence genera- } \\
\text { tion (selection bias) }\end{array}$ & Unclear risk & Judgement Comment: No information provided \\
\hline $\begin{array}{l}\text { Allocation concealment } \\
\text { (selection bias) }\end{array}$ & Unclear risk & Judgement Comment: No information provided \\
\hline
\end{tabular}




\section{Salari 2018 (Continued)}

Blinding of participants Low risk_ Judgement Comment: Even though blinding was not possible, we judged that and personnel (performance bias)

All outcomes

\begin{tabular}{|c|c|c|}
\hline $\begin{array}{l}\text { Blinding of outcome as- } \\
\text { sessment (detection bias) } \\
\text { All outcomes }\end{array}$ & Low risk & $\begin{array}{l}\text { Judgement Comment: No information provided, but no post-treatment diag- } \\
\text { nostic assessment used }\end{array}$ \\
\hline $\begin{array}{l}\text { Incomplete outcome data } \\
\text { (attrition bias) } \\
\text { All outcomes }\end{array}$ & Low risk & $\begin{array}{l}\text { Judgement Comment: Did not include participants who missed more than } 2 \\
\text { sessions, which is a potential source of bias. } 25 \% \text { dropout in CBT group, but } \\
\text { author provided data }\end{array}$ \\
\hline $\begin{array}{l}\text { Selective reporting (re- } \\
\text { porting bias) }\end{array}$ & Low risk & $\begin{array}{l}\text { Judgement Comment: Did not report diagnostic outcomes, but author con- } \\
\text { firmed no diagnostic assessment post-treatment }\end{array}$ \\
\hline $\begin{array}{l}\text { Other source of bias - } \\
\text { Therapy integrity }\end{array}$ & Unclear risk & $\begin{array}{l}\text { Judgement Comment: Treatment delivery by psychiatrist. Refer to adherence } \\
\text { ratings, but no further detail reported }\end{array}$ \\
\hline
\end{tabular}

Other bias Low risk Judgement Comment: The study appears to be free of other sources of bias

Salum 2018

\section{Study characteristics}

\begin{tabular}{|c|c|}
\hline Methods & $\begin{array}{l}\text { Study design: factorial randomised controlled trial } \\
\text { Number of study centres and location: Brazil } \\
\text { Date of study: recruitment from July } 2011 \text { to September } 2012 \\
\text { Withdrawals: } N=96 \text { originally randomised, but } 17 \text { withdrew before first session and were not aware of } \\
\text { allocation - not included }\end{array}$ \\
\hline Participants & $\begin{array}{l}\mathbf{N}=79 \text { (CBT + Attention Bias Modification } \mathrm{N}=21 \text {; } \mathrm{CBT}+\text { Attention Control Training: } \mathrm{N}=21 \text {; active control } \\
\text { therapy + Attention Bias Modification: } \mathrm{N}=17 \text {; active control therapy + Attention Control Training: } \mathrm{N}= \\
\text { 20) } \\
\text { Age range: } 7 \text { to } 11 \\
\text { Inclusion criteria: primary diagnosis of generalised anxiety disorder, separation anxiety disorder, or } \\
\text { social anxiety disorder (based on K-SADS) } \\
\text { Exclusion criteria: (i) other psychiatric disorder judged by the clinician to cause more impairment } \\
\text { or distress than GAD, separation anxiety disorder, or social anxiety disorder; (ii) any history of mental } \\
\text { health treatment; (iii) IQ lower than } 70\end{array}$ \\
\hline Interventions & $\begin{array}{l}\text { CBT (CBT + Attention Bias Modification) } \\
\text { Intervention: Friends for Life (group CBT) + Attention Bias Modification (160 trials) } \\
\text { Delivery format: child and parent sessions; group } \\
\text { Therapist contact time: } 15 \text { hours ( } 10 \times 90 \text { minutes) } \\
\text { Who delivers the intervention: trained psychologists } \\
\text { CBT (CBT + Attention Control Training) }\end{array}$ \\
\hline
\end{tabular}


Intervention: Friends for Life (group CBT) + Attention Control Training (160 trials)

Delivery format: child and parent sessions; group

Therapist contact time: 15 hours ( $10 \times 90$ minutes)

Who delivers the intervention: trained psychologists

\section{Active control (control therapy + Attention Bias Modification)}

Intervention: control therapy - educational tasks and psychoeducation + Attention Bias Modification (160 trials)

Delivery format: child and parent sessions; group

Therapist contact time: 15 hours ( $10 \times 90$ minutes)

Who delivers the intervention: trained psychologists

\section{Active control (control therapy + Attention Control Training)}

Intervention: control therapy - educational tasks and psychoeducation + Attention Control Training (160 trials)

Delivery format: child and parent sessions; group

Therapist contact time: 15 hours ( $10 \times 90$ minutes)

Who delivers the intervention: trained psychologists

$\begin{array}{ll}\text { Outcomes } & \text { Reduction in anxiety symptoms (child report) post-treatment: SCAS-C } \\ \text { Reduction in anxiety symptoms (parent report) post-treatment: SCAS-P } \\ \text { Reduction in depressive symptoms post-treatment: CDI } \\ \text { Comparisons: } \\ \text { Notes } \quad \text { CBT + Attention Bias Modification vs active control therapy + Attention Bias Modification } \\ \text { - CBT + Attention Control Training vs active control therapy + Attention Control Training } \\ \text { Active control therapy + Attention Bias Modification = alternative treatment } \\ \text { Active control therapy + Attention Control Training = attention control }\end{array}$

Risk of bias

\begin{tabular}{|c|c|c|}
\hline Bias & Authors' judgement & Support for judgement \\
\hline $\begin{array}{l}\text { Random sequence genera- } \\
\text { tion (selection bias) }\end{array}$ & Low risk & $\begin{array}{l}\text { Judgement Comment: Computer-generated numbers used to allocate partici- } \\
\text { pants to each group }\end{array}$ \\
\hline $\begin{array}{l}\text { Allocation concealment } \\
\text { (selection bias) }\end{array}$ & Low risk & $\begin{array}{l}\text { Quote: "Allocation to groups was concealed, so therapists did not know the } \\
\text { participant's group allocation until the delivery of the first therapy session." } \\
\text { Judgement Comment: Independent }\end{array}$ \\
\hline $\begin{array}{l}\text { Blinding of participants } \\
\text { and personnel (perfor- } \\
\text { mance bias) } \\
\text { All outcomes }\end{array}$ & Low risk & Judgement Comment: Participants and investigators were blind \\
\hline $\begin{array}{l}\text { Blinding of outcome as- } \\
\text { sessment (detection bias) }\end{array}$ & Low risk & Judgement Comment: Independent assessors \\
\hline
\end{tabular}


Salum 2018 (Continued)

All outcomes

\begin{tabular}{lll}
\hline $\begin{array}{l}\text { Incomplete outcome data } \\
\text { (attrition bias) } \\
\text { All outcomes }\end{array}$ & High risk & $\begin{array}{l}\text { Judgement Comment: Attrition detailed, and ITT reported. However, there } \\
\text { was a large number of dropouts (23/79), and dropouts were related to CBT + } \\
\text { Attention Bias Modification. }\end{array}$ \\
\hline $\begin{array}{l}\text { Selective reporting (re- } \\
\text { porting bias) }\end{array}$ & Low risk & Judgement Comment: Outcomes reported \\
\hline $\begin{array}{l}\text { Other source of bias - } \\
\text { Therapy integrity }\end{array}$ & Low risk & Judgement Comment: Trained therapists. Fidelity monitored in supervision. \\
\hline Other bias & Low risk & Judgement Comment: The study appears to be free of other sources of bias \\
\hline
\end{tabular}

\section{Sanchez Garcia 2009}

\section{Study characteristics}

\begin{tabular}{ll}
\hline Methods & Study design: randomised controlled trial \\
Number of study centres and location: screening in 17 schools in Spain \\
Study setting: schools \\
Withdrawals: 7 excluded because they failed to attend 3 consecutive sessions (NB: group not reported) \\
\hline Participants $\mathbf{8 2}$ (included here: $\mathrm{N}=53$, randomised to: CBT: $\mathrm{N}=28$; waitlist: $\mathrm{N}=25)$ \\
Age range: 10 to 14 \\
Inclusion criteria: generalised social phobia diagnosis (based on ADIS-C) \\
Exclusion criteria: failed to attend 3 consecutive treatment sessions
\end{tabular}

Intervention: Intervention in Adolescents with Social Phobia (IAFS), version for 10- to 14-year-olds. Education, training in social skills, exposure, cognitive restructuring

Delivery format: child only; group

Therapist contact time: 18 hours $(12 \times 90$ minutes)

\section{Waitlist/no treatment}

Intervention: waitlist

\begin{tabular}{ll}
\hline Outcomes & Remission of primary anxiety disorder diagnosis post-treatment: ADIS-C \\
& Remission of all anxiety disorder diagnoses post-treatment: ADIS-C \\
& Reduction in anxiety symptoms (child report) post-treatment: SPAI -C \\
\hline Notes & Study includes third arm (IAFS without cognitive restructuring) not used in the review. \\
& Anxiety symptoms measure = social anxiety symptoms measure
\end{tabular}

Risk of bias 
Sanchez Garcia 2009 (Continued)

\begin{tabular}{|c|c|c|}
\hline Bias & Authors' judgement & Support for judgement \\
\hline $\begin{array}{l}\text { Random sequence genera- } \\
\text { tion (selection bias) }\end{array}$ & Unclear risk & Judgement Comment: No information given \\
\hline $\begin{array}{l}\text { Allocation concealment } \\
\text { (selection bias) }\end{array}$ & Unclear risk & Judgement Comment: No information given \\
\hline $\begin{array}{l}\text { Blinding of participants } \\
\text { and personnel (perfor- } \\
\text { mance bias) } \\
\text { All outcomes }\end{array}$ & Low risk & $\begin{array}{l}\text { Judgement Comment: Even though blinding was not possible, we judged that } \\
\text { it was unlikely this led to a departure from the intended intervention }\end{array}$ \\
\hline $\begin{array}{l}\text { Blinding of outcome as- } \\
\text { sessment (detection bias) } \\
\text { All outcomes }\end{array}$ & Unclear risk & Judgement Comment: No information given \\
\hline $\begin{array}{l}\text { Incomplete outcome data } \\
\text { (attrition bias) } \\
\text { All outcomes }\end{array}$ & Unclear risk & $\begin{array}{l}\text { Judgement Comment: Dropouts (beyond those excluded) and missing data } \\
\text { not detailed }\end{array}$ \\
\hline $\begin{array}{l}\text { Selective reporting (re- } \\
\text { porting bias) }\end{array}$ & Low risk & Judgement Comment: Author provided diagnostic outcomes data \\
\hline $\begin{array}{l}\text { Other source of bias - } \\
\text { Therapy integrity }\end{array}$ & Unclear risk & Judgement Comment: No information given \\
\hline Other bias & Unclear risk & $\begin{array}{l}\text { Judgement Comment: Participants who missed treatment sessions were ex- } \\
\text { cluded }\end{array}$ \\
\hline
\end{tabular}

\section{Santucci 2013}

\section{Study characteristics}

\begin{tabular}{ll} 
Methods & Study design: randomised controlled trial \\
Number of study centres and location: USA \\
Study setting: university research clinic (conducted over 3 consecutive summers) \\
\hline $\mathbf{N}=\mathbf{2 9}$ (CBT: $\mathrm{N}=15 ;$ waitlist: $\mathrm{N}=14)$ \\
Mean age (SD): 9.18 (1.58) \\
Age range: 7 to 12 \\
Gender: $100 \%$ female \\
Inclusion criteria: diagnosis of separation anxiety disorder (based on ADIS-C/P); female \\
Exclusion criteria: bipolar or psychotic disorder; anxiety or mood symptoms were primarily due to a \\
medical/physical condition \\
CBT \\
Intervention: Child Anxiety Multi-Day Program (CMAP) for SAD. Psychoeducation, somatic anxiety \\
management, cognitive restructuring, problem solving, graduated exposure. Conducted over 7 days, \\
cumulating in sleepover. Parent sessions incorporate "Building confidence"
\end{tabular}


Concomitant/excluded medications: consistent dose of psychotropic medication allowed

Delivery format: child sessions and parent sessions; parent present in some of child sessions; group

Therapist contact time: 38 hours ( $4 \times 5$ hours; $1 \times 3$ hours; $1 \times 15$ hours (sleepover))

Who delivers the intervention: treatment developers and doctoral/PhD candidate assistants

\section{Waitlist/no treatment}

Intervention: 8-week waitlist

Concomitant/excluded medications: consistent dose of psychotropic medication allowed

\begin{tabular}{|c|c|c|}
\hline Outcomes & \multicolumn{2}{|c|}{$\begin{array}{l}\text { Remission of primary anxiety disorder diagnosis post-treatment: ADIS-C/P } \\
\text { Reduction in anxiety symptoms (child report) post-treatment: SCAS-C } \\
\text { Reduction in anxiety symptoms (parent report) post-treatment: SCAS-P } \\
\text { Improvement in global functioning post-treatment: CGAS }\end{array}$} \\
\hline \multicolumn{3}{|l|}{ Notes } \\
\hline \multicolumn{3}{|l|}{ Risk of bias } \\
\hline Bias & Authors' judgement & Support for judgement \\
\hline $\begin{array}{l}\text { Random sequence genera- } \\
\text { tion (selection bias) }\end{array}$ & Unclear risk & Judgement Comment: No information given \\
\hline $\begin{array}{l}\text { Allocation concealment } \\
\text { (selection bias) }\end{array}$ & Unclear risk & Judgement Comment: No information given \\
\hline $\begin{array}{l}\text { Blinding of participants } \\
\text { and personnel (perfor- } \\
\text { mance bias) } \\
\text { All outcomes }\end{array}$ & Low risk & $\begin{array}{l}\text { Judgement Comment: Even though blinding was not possible, we judged that } \\
\text { it was unlikely this led to a departure from the intended intervention }\end{array}$ \\
\hline $\begin{array}{l}\text { Blinding of outcome as- } \\
\text { sessment (detection bias) } \\
\text { All outcomes }\end{array}$ & Low risk & Judgement Comment: Independent assessors \\
\hline $\begin{array}{l}\text { Incomplete outcome data } \\
\text { (attrition bias) } \\
\text { All outcomes }\end{array}$ & Low risk & $\begin{array}{l}\text { Judgement Comment: Attrition detailed. Small amount of missing data, and } \\
\text { method for imputing data for missing values detailed and appropriate }\end{array}$ \\
\hline $\begin{array}{l}\text { Selective reporting (re- } \\
\text { porting bias) }\end{array}$ & Low risk & Judgement Comment: Outcomes reported \\
\hline $\begin{array}{l}\text { Other source of bias - } \\
\text { Therapy integrity }\end{array}$ & Unclear risk & $\begin{array}{l}\text { Judgement Comment: Therapists trained, and supervision used to monitor/re- } \\
\text { view protocol, but no specific detail on adherence }\end{array}$ \\
\hline Other bias & Low risk & Judgement Comment: The study appears to be free of other sources of bias \\
\hline
\end{tabular}

\section{Study characteristics}


Schneider 2011 (Continued)

Methods
Study design: randomised controlled trial

Number of study centres and location: 1 in Germany

Study setting: university clinic

Date of study: 2004 to 2009

Participants $\quad \mathbf{N 3}$ (CBT: $\mathrm{N}=21$; waitlist: $\mathrm{N}=22)$
Mean age (SD): CBT: 6.29 (1.01); waitlist: 6.18 (0.73)
Age range: 5 to 7
Gender: 25 (58.1\%) female
Inclusion criteria: primary diagnosis of separation anxiety disorder according to the DSM-IV-TR (based
on Kinder-DIPS); knowledge of the local language; age 5 to 7 years; written parental informed consent
and verbal child assent
Exclusion criteria: taking psychotropic medication

Interventions

CBT

Intervention: Separation Anxiety Family Therapy (TAFF) - disorder-specific CBT

Concomitant/excluded medications: psychotropic medication excluded

Delivery format: child-only sessions, parent-only sessions, and family sessions; individual

Therapist contact time: 13 hours, $20 \mathrm{~min}$ (4 x $50 \mathrm{~min}$ - child, 4 × $50 \mathrm{~min}$ - parent, 8 × $50 \mathrm{~min}$ - family/parent) over 12 weeks

Who delivers the intervention: child psychiatrist and co-led by a fellow of child psychiatry

\section{Waitlist/no treatment}

Intervention: 12-week waitlist

Concomitant/excluded medications: psychotropic medication excluded

Outcomes

Remission of primary anxiety disorder diagnosis post-treatment: ADIS-C/P

Reduction in anxiety symptoms (child report) post-treatment: RCMAS-C

Reduction in anxiety symptoms (parent-mother report) post-treatment: RCMAS-P

Notes Diagnostic post-treatment assessment $=4$-week follow-up assessment

\section{Risk of bias}

\begin{tabular}{lll}
\hline Bias & Authors' judgement & Support for judgement \\
\hline $\begin{array}{l}\text { Random sequence genera- } \\
\text { tion (selection bias) }\end{array}$ & Low risk & $\begin{array}{l}\text { Judgement Comment: Randomisation conducted by statistician using a com- } \\
\text { puterised permuted block design }\end{array}$ \\
\hline $\begin{array}{l}\text { Allocation concealment } \\
\text { (selection bias) }\end{array}$ & Low risk & $\begin{array}{l}\text { Judgement Comment: Assignments concealed until the time of group assign- } \\
\text { ment }\end{array}$ \\
\hline $\begin{array}{l}\text { Blinding of participants } \\
\begin{array}{l}\text { and personnel (perfor- } \\
\text { mance bias) }\end{array}\end{array}$ & Low risk & $\begin{array}{l}\text { Judgement Comment: Even though blinding was not possible, we judged that } \\
\text { it was unlikely this led to a departure from the intended intervention }\end{array}$
\end{tabular}


Schneider 2011 (Continued)

All outcomes

Blinding of outcome as- Low risk Judgement Comment: Blind assessors
sessment (detection bias)
All outcomes

All outcomes

\begin{tabular}{lll}
\hline $\begin{array}{l}\text { Incomplete outcome data } \\
\text { (attrition bias) } \\
\text { All outcomes }\end{array}$ & Low risk & $\begin{array}{l}\text { Judgement Comment: Attrition detailed and clear. Appropriate methods used } \\
\text { for managing missing data. }\end{array}$ \\
\hline $\begin{array}{l}\text { Selective reporting (re- } \\
\text { porting bias) }\end{array}$ & Low risk & Judgement Comment: Outcomes reported \\
\hline $\begin{array}{l}\text { Other source of bias - } \\
\text { Therapy integrity }\end{array}$ & Low risk & $\begin{array}{l}\text { Judgement Comment: Therapists trained and supervised. Adherence assessed } \\
\text { and reported. }\end{array}$ \\
\hline \begin{tabular}{l} 
Other bias \\
\hline
\end{tabular} & Low risk & Judgement Comment: The study appears to be free of other sources of bias \\
\hline
\end{tabular}

\section{Sciberras 2018}

\section{Study characteristics}

\begin{tabular}{ll}
\hline Study design: randomised controlled trial \\
Total duration of study: 5 months \\
Number of study centres and location: 1 in Australia \\
Study setting: Attention Deficit Hyperactivity Disorder (ADHD) assessment clinic \\
\hline N = 12 (CBT: $N=6$; usual care: $N=6)$ \\
Mean age (SD): CBT: 10.4 (1.3); usual care: 11.6 (0.6) \\
Age range: 8 to 12 \\
Gender: 1 (8.3\%) female \\
Inclusion criteria: meet criteria for ADHD and generalised, social, or separation anxiety disorder \\
(based on ADIS-P); 8 to 12 years of age \\
Exclusion criteria: receiving specialist help to manage anxiety \\
\hline
\end{tabular}

Intervention: Cool Kids CBT; adaptations to make acceptable for children with ADHD

Concomitant/excluded medications: medication for anxiety allowed if stable dose for 6 weeks prior to enrolment

Delivery format: child and parent together and child and parent alone; individual

Therapist contact time: 10 hours $(8 \times 1$ hour, $2 \times 1$ hour)

Who delivers the intervention: trained facilitators

\section{TAU}

Intervention: continued to receive treatment as usual for ADHD 
Concomitant/excluded medications: medication for anxiety allowed if stable dose for 6 weeks prior to enrolment

\begin{tabular}{|c|c|c|}
\hline Outcomes & \multicolumn{2}{|c|}{$\begin{array}{l}\text { Remission of all anxiety disorder diagnoses post-treatment: ADIS-P } \\
\text { Reduction in anxiety symptoms (child report) post-treatment: SCAS-C } \\
\text { Reduction in anxiety symptoms (parent report) post-treatment: SCAS-P }\end{array}$} \\
\hline Notes & \multicolumn{2}{|c|}{ Post-treatment assessment $=5$ months postrandomisation, approximately 6 weeks post-treatment } \\
\hline \multicolumn{3}{|l|}{ Risk of bias } \\
\hline Bias & Authors' judgement & Support for judgement \\
\hline $\begin{array}{l}\text { Random sequence genera- } \\
\text { tion (selection bias) }\end{array}$ & Low risk & $\begin{array}{l}\text { Judgement Comment: An independent statistician generated a randomisation } \\
\text { schedule using a computerised random number sequence }\end{array}$ \\
\hline $\begin{array}{l}\text { Allocation concealment } \\
\text { (selection bias) }\end{array}$ & Low risk & $\begin{array}{l}\text { Judgement Comment: Independent statistician. Allocation was concealed } \\
\text { from families and researchers using sealed, opaque envelopes until parent } \\
\text { consent was obtained. }\end{array}$ \\
\hline $\begin{array}{l}\text { Blinding of participants } \\
\text { and personnel (perfor- } \\
\text { mance bias) } \\
\text { All outcomes }\end{array}$ & Low risk & $\begin{array}{l}\text { Judgement Comment: Even though blinding was not possible, we judged that } \\
\text { it was unlikely this led to a departure from the intended intervention }\end{array}$ \\
\hline $\begin{array}{l}\text { Blinding of outcome as- } \\
\text { sessment (detection bias) } \\
\text { All outcomes }\end{array}$ & Low risk & Judgement Comment: Blind assessors \\
\hline $\begin{array}{l}\text { Incomplete outcome data } \\
\text { (attrition bias) } \\
\text { All outcomes }\end{array}$ & Low risk & Judgement Comment: No dropouts \\
\hline $\begin{array}{l}\text { Selective reporting (re- } \\
\text { porting bias) }\end{array}$ & Low risk & Judgement Comment: Outcomes reported for all measures \\
\hline $\begin{array}{l}\text { Other source of bias - } \\
\text { Therapy integrity }\end{array}$ & Unclear risk & $\begin{array}{l}\text { Judgement Comment: Trained facilitators delivered treatment. No further de- } \\
\text { tails }\end{array}$ \\
\hline Other bias & Low risk & Judgement Comment: The study appears to be free of other sources of bias \\
\hline
\end{tabular}

Shahnavaz 2016

\section{Study characteristics}

Methods Study design: randomised controlled trial

Total duration of study: 1-year follow-up

Number of study centres and location: 2 in Sweden

Study setting: dental clinics

Date of study: recruitment January 2013 to March 2014

Participants $\quad \mathbf{N}=\mathbf{3 0}(\mathrm{CBT}: \mathrm{N}=13 ;$ TAU: $\mathrm{N}=17)$


Shahnavaz 2016 (Continued)

\section{Mean age (SD): $10(3.1)$}

Age range: 7 to 18

Gender: 22 (73.3\%) female

Inclusion criteria: 1) patient and all primary caregivers agreed to participate; 2) patient had a principal diagnosis of specific phobia (dental anxiety or intraoral injection phobia) according to the DSM-IVTR (adapted DAWBA); 3) no other psychiatric or developmentally related diagnoses considered to be the primary diagnosis, 4) patient was not receiving concurrent psychological treatments and did not have appointments for psychological examination elsewhere; and 5) patient needed dental care but not emergency care; and practical requirements

Intervention: manual developed by research team. Behavioural analyses, psychoeducation, parent education, exposure to dental procedures both in vivo and in films, relaxation techniques, procedural pain management information, and cognitive restructuring

Delivery format: child and parent; individual

Therapist contact time: 10 hours ( $10 \times 1$ hour)

Who delivers the intervention: psychologists, $C B T$ training

\section{TAU}

Intervention: tell-show-do, distraction, premedication with midazolam, nitrous oxide sedation, general anaesthesia

Outcomes Remission of primary anxiety disorder diagnosis post-treatment and 12-month follow-up: adapted DAWBA

Reduction in anxiety symptoms (child report) post-treatment and 12-month follow-up: Child Survey Fear Schedule: Dental Subscale

Reduction in anxiety symptoms (parent report) post-treatment and 12-month follow-up: Child Survey Fear Schedule: Dental Subscale

Notes Anxiety symptoms measure dental phobia symptoms measure

No adverse events reported in CBT group.

\section{Risk of bias}

\begin{tabular}{lll}
\hline Bias & Authors' judgement & Support for judgement \\
\hline $\begin{array}{l}\text { Random sequence genera- } \\
\text { tion (selection bias) }\end{array}$ & Low risk & Judgement Comment: True randomisation list, generated at www.random.org \\
\hline $\begin{array}{l}\text { Allocation concealment } \\
\text { (selection bias) }\end{array}$ & Low risk & $\begin{array}{l}\text { Judgement Comment: External person. Allocation after eligibility assessed, } \\
\text { agreed to be included }\end{array}$ \\
\hline $\begin{array}{l}\text { Blinding of participants } \\
\text { and personnel (perfor- } \\
\text { mance bias) } \\
\text { All outcomes }\end{array}$ & Low risk & $\begin{array}{l}\text { Judgement Comment: Even though blinding was not possible, we judged that } \\
\text { it was unlikely this led to a departure from the intended intervention }\end{array}$ \\
\hline
\end{tabular}

Blinding of outcome as- Low risk

Judgement Comment: Blind assessors

sessment (detection bias)

All outcomes 
Shahnavaz 2016 (Continued)

Incomplete outcome data Low risk Judgement Comment: Attrition detailed and reasons provided for small num(attrition bias) ber of dropouts. Outcomes reported for completers

All outcomes

Selective reporting (re- Low risk Judgement Comment: Outcomes reported
porting bias)

\begin{tabular}{lll}
\hline $\begin{array}{l}\text { Other source of bias - } \\
\text { Therapy integrity }\end{array}$ & Low risk & $\begin{array}{l}\text { Judgement Comment: Trained therapists with regular supervision where ad- } \\
\text { herence and competence monitored }\end{array}$ \\
\hline Other bias & Low risk & Judgement Comment: The study appears to be free of other sources of bias \\
\hline
\end{tabular}

Sharma 2017

\section{Study characteristics}

\begin{tabular}{|c|c|}
\hline Methods & $\begin{array}{l}\text { Study design: randomised controlled trial } \\
\text { Number of study centres and location: } 1 \text { in India } \\
\text { Study setting: university clinic }\end{array}$ \\
\hline Participants & $\begin{array}{l}\mathbf{N}=63 \text { randomised (NB: } N=61 \text { aged } 10 \text { to } 18 \text { included here: } \mathrm{CBT}: \mathrm{N}=30 ; \mathrm{TAU}: \mathrm{N}=31 \text { ) } \\
\text { Age range: } 10 \text { to } 18 \\
\text { Inclusion criteria: aged } 10 \text { to } 19 \text { (NB: } 19 \text {-year-olds not included in analysis in review); current ICD di- } \\
\text { agnosis of anxiety disorder (mini-KID); current International Headache Society diagnosis of primary } \\
\text { headaches; basic reading and writing; no significant medication change in last } 4 \text { weeks; no history of } \\
\text { organic disorder; willingness to be randomised } \\
\text { Exclusion criteria: depression excluded on basis CDI }\end{array}$ \\
\hline Interventions & $\begin{array}{l}\text { CBT } \\
\text { Intervention: transdiagnostic CBT } \\
\text { Delivery format: child only; group } \\
\text { Therapist contact time: } 24 \text { hours ( } 12 \times 2 \text { hours) } \\
\text { TAU } \\
\text { Intervention: continued receiving TAU - pharmacotherapy, visit psychiatrist for medication review }\end{array}$ \\
\hline Outcomes & $\begin{array}{l}\text { Remission of primary anxiety disorder diagnosis post-treatment: mini-KID } \\
\text { Remission of all anxiety disorder diagnoses post-treatment: mini-KID } \\
\text { Reduction in anxiety symptoms (child report) post-treatment: STAIC-trait } \\
\text { Improvement in global functioning post-treatment: CGAS }\end{array}$ \\
\hline Notes & Author provided data on under 19s. \\
\hline Risk of bias & \\
\hline Bias & Authors' judgement Support for judgement \\
\hline
\end{tabular}


Sharma 2017 (Continued)
Random sequence genera- Low risk
Judgement Comment: Computer-generated list tion (selection bias)

\begin{tabular}{lll}
\hline $\begin{array}{l}\text { Allocation concealment } \\
\text { (selection bias) }\end{array}$ & Low risk & Judgement Comment: Computer generated, so unlikely to foresee assignment \\
\hline $\begin{array}{l}\text { Blinding of participants } \\
\text { and personnel (perfor- }\end{array}$ & Low risk & $\begin{array}{l}\text { Judgement Comment: Even though blinding was not possible, we judged that } \\
\text { it was unlikely this led to a departure from the intended intervention }\end{array}$
\end{tabular}

$$
\text { mance bias) }
$$

All outcomes

\begin{tabular}{lll}
\hline $\begin{array}{l}\text { Blinding of outcome as- } \\
\text { sessment (detection bias) } \\
\text { All outcomes }\end{array}$ & Unclear risk & Judgement Comment: Not detailed \\
\hline $\begin{array}{l}\text { Incomplete outcome data } \\
\text { (attrition bias) } \\
\text { All outcomes }\end{array}$ & Low risk & Judgement Comment: Data presented \\
\hline $\begin{array}{l}\text { Selective reporting (re- } \\
\text { porting bias) }\end{array}$ & Low risk & Judgement Comment: Outcomes reported/provided \\
\hline $\begin{array}{l}\text { Other source of bias - } \\
\text { Therapy integrity }\end{array}$ & Unclear risk & $\begin{array}{l}\text { Judgement Comment: Review of treatment content by committee, but no fur- } \\
\text { ther detail }\end{array}$ \\
\hline \begin{tabular}{l} 
Other bias \\
\hline
\end{tabular} & Low risk & Judgement Comment: The study appears to be free of other sources of bias \\
\hline
\end{tabular}

\section{Shortt 2001}

\section{Study characteristics}

\begin{tabular}{|c|c|}
\hline Methods & $\begin{array}{l}\text { Study design: randomised controlled trial } \\
\text { Total duration of study: non-controlled 12-month follow-up } \\
\text { Number of study centres and location: } 1 \text { in Australia } \\
\text { Study setting: university clinic }\end{array}$ \\
\hline Participants & $\begin{array}{l}\mathbf{N}=\mathbf{7 1} \text { (CBT: } \mathrm{N}=54 \text {; waitlist: } \mathrm{N}=17 \text { ) } \\
\text { Mean age (SD): CBT: } 7.83 \text { (1.33); waitlist: } 7.88(1.32) \\
\text { Age range: } 6 \text { to } 10 \\
\text { Gender: CBT: } 57 \% \text { female; waitlist: } 65 \% \text { female } \\
\text { Inclusion criteria: a principal diagnosis of Generalised Anxiety Disorder, Separation Anxiety Disorder, } \\
\text { or Social Phobia (determined using the DISCAP) } \\
\text { Exclusion criteria: all children with intellectual or severe physical impairment, or who were currently } \\
\text { involved in psychosocial or psychopharmacological interventions }\end{array}$ \\
\hline Interventions & $\begin{array}{l}\text { CBT } \\
\text { Intervention: FRIENDS. Children work through workbook, parents booklet with parent strategies } \\
\text { Concomitant/excluded medications: medications not allowed }\end{array}$ \\
\hline
\end{tabular}


Delivery format: child-only and parent-only sessions, with parent and child together for some sessions; group

Therapist contact time: 15 hours (10 x 1 hour (child sessions), $10 \times 30$ min (parent sessions))

Who delivers the intervention: clinical doctoral candidates

\section{Waitlist/no treatment}

Intervention: 10 -week waitlist

Concomitant/excluded medications: medications not allowed

Outcomes Remission of all anxiety disorder diagnoses post-treatment: DISCAP

Reduction in anxiety symptoms (child report) post-treatment: RCMAS

Notes

\section{Risk of bias}

\begin{tabular}{|c|c|c|}
\hline Bias & Authors' judgement & Support for judgement \\
\hline $\begin{array}{l}\text { Random sequence genera- } \\
\text { tion (selection bias) }\end{array}$ & Unclear risk & Judgement Comment: 3:1 ratio, but no further detail \\
\hline $\begin{array}{l}\text { Allocation concealment } \\
\text { (selection bias) }\end{array}$ & Unclear risk & Judgement Comment: No detail \\
\hline $\begin{array}{l}\text { Blinding of participants } \\
\text { and personnel (perfor- } \\
\text { mance bias) } \\
\text { All outcomes }\end{array}$ & Low risk & $\begin{array}{l}\text { Judgement Comment: Even though blinding was not possible, we judged that } \\
\text { it was unlikely this led to a departure from the intended intervention }\end{array}$ \\
\hline $\begin{array}{l}\text { Blinding of outcome as- } \\
\text { sessment (detection bias) } \\
\text { All outcomes }\end{array}$ & Low risk & Judgement Comment: Blind assessors \\
\hline $\begin{array}{l}\text { Incomplete outcome data } \\
\text { (attrition bias) } \\
\text { All outcomes }\end{array}$ & Low risk & $\begin{array}{l}\text { Judgement Comment: Attrition reported. Completer and ITT analyses for diag- } \\
\text { nostic outcomes. Missing data managed appropriately. }\end{array}$ \\
\hline $\begin{array}{l}\text { Selective reporting (re- } \\
\text { porting bias) }\end{array}$ & Low risk & Judgement Comment: Outcomes for relevant measures reported \\
\hline $\begin{array}{l}\text { Other source of bias - } \\
\text { Therapy integrity }\end{array}$ & Low risk & Judgement Comment: Integrity assessed and reported \\
\hline Other bias & Low risk & Judgement Comment: The study appears to be free of other sources of bias \\
\hline
\end{tabular}

Silk 2018

\section{Study characteristics}

Methods Study design: randomised controlled trial

Total duration of study: 12 -month follow-up 
Silk 2018 (Continued)

Number of study centres and location: 1 in the USA

Participants $\quad \mathbf{1 3 3}$ (CBT: N = 90; active control: N = 43)
Mean age (SD): CBT: 10.943 (1.395); waitlist: 10.981 (1.633)
Age range: 9 to 14
Gender: CBT: 50 (55.6\%) female; active control: 24 (55.8\%) female
Inclusion criteria: meet criteria for current Generalised Anxiety Disorder, Separation Anxiety Disorder,
and/or Social Anxiety Disorder (Based on K-SADS)
Exclusion criteria: IQ below 70, psychoactive medications; acute suicidality or risk for harm to self or
others; presence of metal objects in the body; current primary diagnosis of major depressive disorder,
OCD, PTSD, conduct disorder, substance abuse or dependence, or ADHD. Anxious youth were also ex-
cluded if they had evidence of an autism spectrum disorder or a lifetime diagnosis of bipolar disorder,
psychotic depression, schizophrenia, or schizoaffective disorder.

Interventions

CBT

Intervention: Coping Cat. Anxiety management sessions; exposure sessions

Concomitant/excluded medications: psychoactive medication not allowed

Delivery format: child only; 2 parent sessions; individual

Therapist contact time: 16 sessions

Who delivers the intervention: Master's and doctoral-level therapists

\section{Active control}

Intervention: Child Centred Therapy (CCT). Manualised non-directive, supportive psychotherapy based on humanistic principles such as unconditional positive regard, empathy, and therapist genuineness

Concomitant/excluded medications: psychoactive medication not allowed

Delivery format: child only; 2 parent sessions; individual

Therapist contact time: 16 sessions

Who delivers the intervention: Master's and doctoral-level therapists

Reduction in anxiety symptoms (child report) post-treatment and 12-month follow-up: SCARED-C Reduction in anxiety symptoms (parent report) post-treatment and 12-month follow-up: SCARED$P$

Notes Active control = alternative treatment

\section{Risk of bias}

\begin{tabular}{lll}
\hline Bias & Authors' judgement & Support for judgement \\
\hline $\begin{array}{l}\text { Random sequence genera- } \\
\text { tion (selection bias) }\end{array}$ & Unclear risk & Judgement Comment: No detail \\
\hline $\begin{array}{l}\text { Allocation concealment } \\
\text { (selection bias) }\end{array}$ & Unclear risk & Judgement Comment: No detail \\
\hline
\end{tabular}


Silk 2018 (Continued)

Blinding of participants Unclear risk Judgement Comment: No detail blinding of participants and personnel (performance bias)

All outcomes

\begin{tabular}{|c|c|c|}
\hline $\begin{array}{l}\text { Blinding of outcome as- } \\
\text { sessment (detection bias) } \\
\text { All outcomes }\end{array}$ & Low risk & Judgement Comment: Independent evaluators \\
\hline $\begin{array}{l}\text { Incomplete outcome data } \\
\text { (attrition bias) } \\
\text { All outcomes }\end{array}$ & Low risk & $\begin{array}{l}\text { Judgement Comment: Attrition detailed, and missing data managed appropri- } \\
\text { ately }\end{array}$ \\
\hline $\begin{array}{l}\text { Selective reporting (re- } \\
\text { porting bias) }\end{array}$ & Low risk & Judgement Comment: Outcomes reported for all measures \\
\hline $\begin{array}{l}\text { Other source of bias - } \\
\text { Therapy integrity }\end{array}$ & Low risk & Judgement Comment: Trained therapists, and integrity assessed and reported \\
\hline Other bias & Low risk & Judgement Comment: The study appears to be free of other sources of bias \\
\hline
\end{tabular}

Silverman 1999a

Study characteristics

\begin{tabular}{|c|c|}
\hline Methods & $\begin{array}{l}\text { Study design: randomised controlled trial } \\
\text { Total duration of study: } 12 \text {-month follow-up } \\
\text { Number of study centres and location: } 1 \text { in the USA } \\
\text { Study setting: university clinic }\end{array}$ \\
\hline Participants & $\begin{array}{l}\text { N = } 104 \text { randomised (NB: } N=64 \text { included here, randomised to: } C B T: N=41 \text {; active control: } N=23 \text { ) } \\
\text { Mean age (SD): CBT: } 10.3 \text { (2.6); active control: } 9.7 \text { (2.9) } \\
\text { Age range: } 6 \text { to } 16 \\
\text { Gender: CBT: } 20 \text { (48.8\%) female; active control: } 14 \text { (60.9\%) female } \\
\text { Inclusion criteria: primary phobic disorder (simple phobia-majority; social phobia or agoraphobia) } \\
\text { (ADIS-C/P) } \\
\text { Exclusion criteria: pervasive developmental disorders, psychotic symptoms, current involvement in } \\
\text { psychosocial or psychopharmacological treatment }\end{array}$ \\
\hline Interventions & $\begin{array}{l}\text { CBT } \\
\text { Intervention: exposure + self control. Faciliate graded exposure, using cognitive self-control proce- } \\
\text { dures. Taught cognitive strategies and devised fear hierarchy, perform in- and out-of-session exposure } \\
\text { Concomitant/excluded medications: psychopharmacological treatment not allowed } \\
\text { Delivery format: child sessions and parent sessions, and part of session together; individual } \\
\text { Therapist contact time: } 13 \text { hours } 20 \mathrm{~min}(10 \times 80 \mathrm{~min}) \\
\text { Who delivers the intervention: postdoctoral fellows }\end{array}$ \\
\hline
\end{tabular}


Silverman 1999a (Continued)

\section{Active control}

Intervention: educational support. Education about phobias and treatment. No information on therapeutic strategies or implementing these

Concomitant/excluded medications: psychopharmacological treatment not allowed

Delivery format: child sessions and parent sessions, and part of session together; individual

Therapist contact time: 13 hours $20 \mathrm{~min}(10 \times 80 \mathrm{~min})$

Who delivers the intervention: postdoctoral fellows

\begin{tabular}{ll}
\hline Outcomes & Remission of primary anxiety disorder diagnosis post-treatment: ADIS-C/P \\
& Reduction in anxiety symptoms (child report) post-treatment and 6-month and 12-month fol- \\
low-up: RCMAS-C \\
Reduction in anxiety symptoms (parent report) post-treatment and 6-month and 12-month fol- \\
low-up: RCMAS-P
\end{tabular}

Reduction in depressive symptoms post-treatment: CDI

Notes Study includes Contingency Management condition - behavioural intervention not included here.
Active control $=$ attention control

\section{Risk of bias}

\begin{tabular}{|c|c|c|}
\hline Bias & Authors' judgement & Support for judgement \\
\hline $\begin{array}{l}\text { Random sequence genera- } \\
\text { tion (selection bias) }\end{array}$ & Unclear risk & Judgement Comment: Not detailed \\
\hline $\begin{array}{l}\text { Allocation concealment } \\
\text { (selection bias) }\end{array}$ & Unclear risk & Judgement Comment: Not detailed \\
\hline $\begin{array}{l}\text { Blinding of participants } \\
\text { and personnel (perfor- } \\
\text { mance bias) } \\
\text { All outcomes }\end{array}$ & Unclear risk & Judgement Comment: Not detailed \\
\hline $\begin{array}{l}\text { Blinding of outcome as- } \\
\text { sessment (detection bias) } \\
\text { All outcomes }\end{array}$ & Unclear risk & Judgement Comment: Not detailed \\
\hline $\begin{array}{l}\text { Incomplete outcome data } \\
\text { (attrition bias) } \\
\text { All outcomes }\end{array}$ & Unclear risk & $\begin{array}{l}\text { Judgement Comment: Overall attrition detailed. Missing data for measures } \\
\text { and procedure for managing missing data not detailed. It appears that com- } \\
\text { pleter analysis was reported only. }\end{array}$ \\
\hline $\begin{array}{l}\text { Selective reporting (re- } \\
\text { porting bias) }\end{array}$ & Low risk & Judgement Comment: Outcomes reported \\
\hline $\begin{array}{l}\text { Other source of bias - } \\
\text { Therapy integrity }\end{array}$ & Low risk & Judgement Comment: Therapists trained and supervised. Integrity assessed. \\
\hline Other bias & Low risk & Judgement Comment: The study appears to be free of other sources of bias \\
\hline
\end{tabular}


Silverman 1999b

\section{Study characteristics}

\begin{tabular}{l} 
Study design: randomised controlled trial \\
Total duration of study: non-controlled 12-month follow-up \\
Number of study centres and location: 1 in the USA \\
Study setting: university clinic \\
\hline N $=\mathbf{5 6}$ (CBT: $\mathrm{N}=37$; waitlist: $\mathrm{N}=19)$ \\
Mean age (SD): 9.96 \\
Age range: 6 to 16 \\
Gender: CBT: 17 (46\%) female; waitlist 5 (26\%) female \\
Inclusion criteria: primary diagnosis of social phobia, overanxious disorder, or GAD (based on ADIS-C/ \\
P) \\
Exclusion criteria: pervasive developmental disorder, psychotic symptoms, current psychological or \\
psychopharmacological treatment
\end{tabular}

\section{CBT}

Intervention: group CBT - parent and child. Contigency management, exposure, child self-talk

Concomitant/excluded medications: psychopharmacological treatment not allowed

Delivery format: child sessions and parent sessions, and parent joined child sessions; group

Therapist contact time:? sessions 40 minutes each +15 minutes together

Who delivers the intervention: psychologist and doctoral student

Waitlist/no treatment

Intervention: 8- to 10-week waitlist

Concomitant/excluded medications: psychopharmacological treatment not allowed

\begin{tabular}{ll}
\hline Outcomes & Remission of primary anxiety disorder diagnosis post-treatment: $A D I S-C / P$ \\
& Reduction in anxiety symptoms (child report) post-treatment: RCMAS-C \\
Reduction in anxiety symptoms (parent report) post-treatment: $R C M A S-P$ \\
Reduction in depressive symptoms post-treatment: $C D I$
\end{tabular}

Notes

\section{Risk of bias}

\begin{tabular}{lll}
\hline Bias & Authors' judgement & Support for judgement \\
\hline $\begin{array}{l}\text { Random sequence genera- } \\
\text { tion (selection bias) }\end{array}$ & Unclear risk & Judgement Comment: Not detailed \\
\hline
\end{tabular}

Allocation concealment Unclear risk Judgement Comment: Not detailed
(selection bias)


Silverman 1999b (Continued)

$\begin{array}{ll}\text { Blinding of participants } & \text { Low risk } \\ \text { and personnel (perfor- } & \text { Judgement Comment: Even though blinding was not possible, we judged that }\end{array}$
mance bias)

All outcomes

\begin{tabular}{|c|c|c|}
\hline $\begin{array}{l}\text { Blinding of outcome as- } \\
\text { sessment (detection bias) } \\
\text { All outcomes }\end{array}$ & Low risk & $\begin{array}{l}\text { Judgement Comment: Subsample rated by independent evaluators, although } \\
\text { blinding of assessors not specified }\end{array}$ \\
\hline $\begin{array}{l}\text { Incomplete outcome data } \\
\text { (attrition bias) } \\
\text { All outcomes }\end{array}$ & Unclear risk & $\begin{array}{l}\text { Judgement Comment: Attrition detailed. } 27 \% \text { dropout. Completers compared } \\
\text { to non-completers on baseline characteristics. It appears that only completer } \\
\text { analysis was reported, and does not specify comparison to ITT. }\end{array}$ \\
\hline $\begin{array}{l}\text { Selective reporting (re- } \\
\text { porting bias) }\end{array}$ & Low risk & Judgement Comment: Outcomes reported \\
\hline $\begin{array}{l}\text { Other source of bias - } \\
\text { Therapy integrity }\end{array}$ & Low risk & Judgement Comment: Integrity assessed and reported \\
\hline Other bias & Low risk & Judgement Comment: The study appears to be free of other sources of bias \\
\hline
\end{tabular}

Simon 2011

\section{Study characteristics}

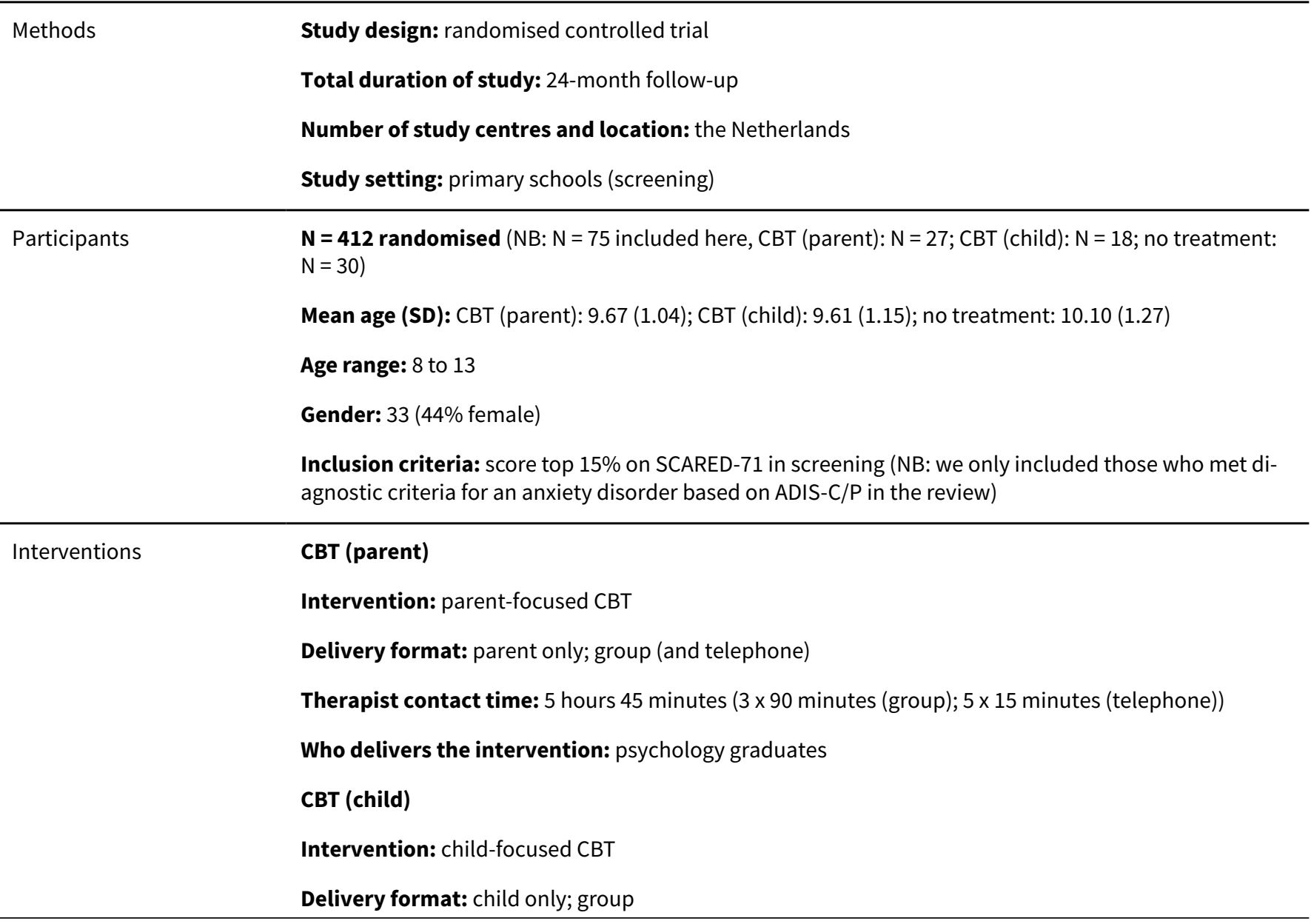


Therapist contact time: 12 hours $(8 \times 90$ minutes $)$

Who delivers the intervention: psychology graduates

\title{
Waitlist/no treatment
}

Intervention: no intervention

\begin{tabular}{ll}
\hline Outcomes & Remission of all anxiety disorder diagnoses post-treatment and 24-month follow-up: ADIS-C/P \\
& Reduction in anxiety symptoms (child report) post-treatment and 24-month follow-up: SCARED-C
\end{tabular}

Notes We only included participants who met diagnostic criteria for anxiety disorder at baseline.

Post-treament assessment $=12$ months

\begin{tabular}{|c|c|c|}
\hline \multicolumn{3}{|l|}{ Risk of bias } \\
\hline Bias & Authors' judgement & Support for judgement \\
\hline $\begin{array}{l}\text { Random sequence genera- } \\
\text { tion (selection bias) }\end{array}$ & Unclear risk & Judgement Comment: Not detailed \\
\hline $\begin{array}{l}\text { Allocation concealment } \\
\text { (selection bias) }\end{array}$ & Unclear risk & Judgement Comment: Not detailed \\
\hline $\begin{array}{l}\text { Blinding of participants } \\
\text { and personnel (perfor- } \\
\text { mance bias) } \\
\text { All outcomes }\end{array}$ & Low risk & $\begin{array}{l}\text { Judgement Comment: Even though blinding was not possible, we judged that } \\
\text { it was unlikely this led to a departure from the intended intervention }\end{array}$ \\
\hline $\begin{array}{l}\text { Blinding of outcome as- } \\
\text { sessment (detection bias) } \\
\text { All outcomes }\end{array}$ & Unclear risk & Judgement Comment: Not detailed \\
\hline $\begin{array}{l}\text { Incomplete outcome data } \\
\text { (attrition bias) } \\
\text { All outcomes }\end{array}$ & Low risk & Judgement Comment: Detail provided on missing data \\
\hline $\begin{array}{l}\text { Selective reporting (re- } \\
\text { porting bias) }\end{array}$ & Low risk & Judgement Comment: Data on outcomes provided by author \\
\hline $\begin{array}{l}\text { Other source of bias - } \\
\text { Therapy integrity }\end{array}$ & Low risk & $\begin{array}{l}\text { Judgement Comment: Trained and supervised, integrity assessed and report- } \\
\text { ed }\end{array}$ \\
\hline Other bias & Low risk & Judgement Comment: The study appears to be free of other sources of bias \\
\hline
\end{tabular}

Smith 2014

\section{Study characteristics}

Methods

\author{
Study design: randomised controlled trial \\ Total duration of study: non-controlled 3-month follow-up \\ Number of study centres and location: 1 in the USA
}

Study setting: university clinic 
Smith 2014 (Continued)

$\begin{array}{ll}\text { Participants } & \mathbf{N}=\mathbf{3 1}(\mathrm{CBT}: \mathrm{N}=18 ; \text { waitlist: } \mathrm{N}=13) \\ & \text { Mean age (SD): } 9.80(1.78) \\ & \text { Age range: } 7 \text { to } 13\end{array}$

Gender: 12 (38.7\%)

Inclusion criteria: met DSM-IV diagnostic criteria for 1 or more of the following: separation anxiety disorder, social anxiety disorder, specific phobia, and/or generalised anxiety disorder

Exclusion criteria: comorbid pervasive developmental disorder, traumatic brain injury, organic brain damage, psychotic symptoms, or engagement in concurrent psychotherapy for anxiety

Intervention: individualised parent intervention. Includes psychoeducation and strategies for responding adaptively to child anxiety, and demonstrates CBT techniques for parents to teach their children

Concomitant/excluded medications: concurrent pharmacological treatment for anxiety required stable dosages 1 month prior to and throughout participation

Delivery format: parent only; individual

Therapist contact time: 10 hours ( $10 \times 1$ hour)

Who delivers the intervention: advanced doctoral psychology student

\section{Waitlist}

Intervention: 10-week waitlist

Concomitant/excluded medications: concurrent pharmacological treatment for anxiety required stable dosages 1 month prior to and throughout participation

\section{Outcomes}

Reduction in anxiety symptoms (child report) post-treatment: MASC-C

Reduction in anxiety symptoms (parent report) post-treatment: MASC-P

\section{Risk of bias}

\begin{tabular}{lll}
\hline Bias & Authors' judgement & Support for judgement \\
\hline $\begin{array}{l}\text { Random sequence genera- } \\
\text { tion (selection bias) }\end{array}$ & Unclear risk & Judgement Comment: Not detailed \\
\hline $\begin{array}{l}\text { Allocation concealment } \\
\text { (selection bias) }\end{array}$ & Unclear risk & Judgement Comment: Not detailed \\
\hline $\begin{array}{l}\text { Blinding of participants } \\
\text { and personnel (perfor- } \\
\text { mance bias) }\end{array}$ & Low risk & Judgement Comment: Even though blinding was not possible, we judged that \\
$\begin{array}{l}\text { All outcomes } \\
\text { Blinding of outcome as- } \\
\text { sessment (detection bias) } \\
\text { All outcomes }\end{array}$ & Low risk & \\
\hline
\end{tabular}


Smith 2014 (Continued)

Incomplete outcome data Unclear risk Judgement Comment: High rate of missing questionnaire data
(attrition bias)

All outcomes

\begin{tabular}{lll}
\hline $\begin{array}{l}\text { Selective reporting (re- } \\
\text { porting bias) }\end{array}$ & Low risk & Judgement Comment: Outcomes for measures reported \\
\hline $\begin{array}{l}\text { Other source of bias - } \\
\text { Therapy integrity }\end{array}$ & Low risk & $\begin{array}{l}\text { Judgement Comment: Trained therapists. Regular supervision, fidelity moni- } \\
\text { tored }\end{array}$ \\
\hline Other bias & Low risk & Judgement Comment: The study appears to be free of other sources of bias \\
\hline
\end{tabular}

Southam Gerow 2010

\title{
Study characteristics
}

\begin{tabular}{ll}
\hline Methods & Study design: randomised controlled trial \\
& Number of study centres and location: USA \\
& Study setting: recruited from 6 mental health clinics \\
\hline Participants $=\mathbf{4 8}$ (CBT: $\mathrm{N}=24 ;$ TAU: $\mathrm{N}=24)$ \\
Mean age (SD): 10.9 (2.1) \\
Age range: 8 to 15 \\
Gender: $56.2 \%$ female \\
Inclusion criteria: primary diagnosis of GAD, separation anxiety disorder, social phobia, specific pho- \\
bia (based on DISC); anxiety the treatment priority \\
Exclusion criteria: a) pervasive developmental disorder, (b) a psychotic disorder, or (c) mental retar- \\
dation
\end{tabular}

Interventions

\author{
CBT \\ Intervention: Coping Cat
}

Concomitant/excluded medications: medication use was tracked during treatment

Delivery format: child only; individual

Therapist contact time: 16 to 20 sessions

Who delivers the intervention: social workers/doctoral-level psychologists/Master's-level psychologists/other (e.g. marriage and family therapists)

\section{TAU}

Intervention: usual care. Therapists used treatment procedures regularly used and believed to be effective in clinical practice.

Concomitant/excluded medications: medication use was tracked during treatment

Delivery format: child only; individual

Who delivers the intervention: social workers/doctoral-level psychologists/Master's-level psychologists/other (e.g. marriage and family therapists) 
Southam Gerow 2010 (Continued)

Outcomes Remission of primary anxiety disorder diagnosis post-treatment: DISC

Reduction in anxiety symptoms (child report) post-treatment: STAIC-Trait

Reduction in anxiety symptoms (parent report) post-treatment: STAIC-Trait

Notes

\section{Risk of bias}

\begin{tabular}{|c|c|c|}
\hline Bias & Authors' judgement & Support for judgement \\
\hline $\begin{array}{l}\text { Random sequence genera- } \\
\text { tion (selection bias) }\end{array}$ & Low risk & Judgement Comment: Block randomised \\
\hline $\begin{array}{l}\text { Allocation concealment } \\
\text { (selection bias) }\end{array}$ & Unclear risk & Judgement Comment: No detail given \\
\hline $\begin{array}{l}\text { Blinding of participants } \\
\text { and personnel (perfor- } \\
\text { mance bias) } \\
\text { All outcomes }\end{array}$ & Unclear risk & Judgement Comment: No detail given \\
\hline $\begin{array}{l}\text { Blinding of outcome as- } \\
\text { sessment (detection bias) } \\
\text { All outcomes }\end{array}$ & Low risk & Judgement Comment: Blind assessors \\
\hline $\begin{array}{l}\text { Incomplete outcome data } \\
\text { (attrition bias) } \\
\text { All outcomes }\end{array}$ & Low risk & $\begin{array}{l}\text { Judgement Comment: Attrition fully described. Appropriate method used for } \\
\text { managing missing data - diagnostic outcomes report for completers and using } \\
\text { ITT, and explored if any differences between those with and without missing } \\
\text { data. }\end{array}$ \\
\hline $\begin{array}{l}\text { Selective reporting (re- } \\
\text { porting bias) }\end{array}$ & Low risk & Judgement Comment: Outcomes for measures reported \\
\hline $\begin{array}{l}\text { Other source of bias - } \\
\text { Therapy integrity }\end{array}$ & Low risk & $\begin{array}{l}\text { Judgement Comment: Therapists trained and supervised. Adherence as- } \\
\text { sessed. }\end{array}$ \\
\hline Other bias & Low risk & Judgement Comment: The study appears to be free of other sources of bias \\
\hline
\end{tabular}

Spence 2000

\section{Study characteristics}

\begin{tabular}{ll}
\hline Sethods & Study design: randomised controlled trial \\
& Total duration of study: non-controlled 6- and 12-month follow-up \\
& Number of study centres and location: 1 in Australia \\
& Study setting: university clinic \\
\hline Participants & $\mathbf{N}=\mathbf{5 1}$ (CBT (child + parent): $\mathrm{N}=17 ; \mathrm{CBT}$ (child only): $\mathrm{N}=19 ;$ waitlist: $\mathrm{N}=15)$ \\
& $\begin{array}{l}\text { Mean age (SD): } \mathrm{CBT} \text { (child + parent): } 10.94 \text { (1.92), } \mathrm{n}=17 ; \mathrm{CBT} \text { (child only): } 11 \text { (2.45), } \mathrm{n}=19 ; \text { waitlist: } 9.93 \\
(1.77), \mathrm{n}=14\end{array}$
\end{tabular}


Spence 2000 (Continued)

\section{Age range: 7 to 14}

Gender: CBT (child + parent): 7 (41.2\%) female, $n=17$; CBT (child only): 9 (47.4\%), $n=19$; waitlist: 3 (21.4\%) female, $n=14$

Inclusion criteria: social phobia principal diagnosis (based on ADIS-C/P)

Exclusion criteria: not report any avoidance or worry related to any social situation on the SWQ-PU, severe learning difficulty, medication for a psychological disorder

CBT (child + parent)

Intervention: social skills training, relaxation techniques, social problem-solving, positive self-instruction, cognitive challenging and graded exposure. Parent observes child sessions and attends parent training sessions.

Concomitant/excluded medications: medications excluded

Delivery format: child-only sessions (parent observes), plus parent-only sessions; group

Therapist contact time: 24 hours (child: $12 \times 1$ hour $+12 \times 30$ min; parents: 6 hours)

Who delivers the intervention: psychologists

CBT (child only)

Intervention: social skills training, relaxation techniques, social problem-solving, positive self-instruction, cognitive challenging and graded exposure

Concomitant/excluded medications: medications excluded

Delivery format: child only; group

Therapist contact time: 18 hours $(12 \times 1$ hour $+12 \times 30 \mathrm{~min})$

Who delivers the intervention: psychologists

Waitlist/no treatment

Intervention: 12 -week waitlist

Concomitant/excluded medications: medications excluded

Outcomes

Remission of primary anxiety disorder diagnosis post-treatment: ADIS-C/P

Remission of all anxiety disorder diagnosis post-treatment: ADIS-C/P

Reduction in anxiety symptoms (child report) post-treatment: RCMAS

Notes

\section{Risk of bias}

\begin{tabular}{lll}
\hline Bias & Authors' judgement & Support for judgement \\
\hline $\begin{array}{l}\text { Random sequence genera- } \\
\text { tion (selection bias) }\end{array}$ & Unclear risk & Judgement Comment: No detail given \\
\hline $\begin{array}{l}\text { Allocation concealment } \\
\text { (selection bias) }\end{array}$ & Unclear risk & Judgement Comment: No detail given \\
\hline $\begin{array}{l}\text { Blinding of participants } \\
\text { and personnel (perfor- } \\
\text { mance bias) }\end{array}$ & Low risk & $\begin{array}{l}\text { Judgement Comment: Even though blinding was not possible, we judged that } \\
\text { it was unlikely this led to a departure from the intended intervention }\end{array}$ \\
\hline
\end{tabular}


Spence 2000 (Continued)

All outcomes

\begin{tabular}{|c|c|c|}
\hline $\begin{array}{l}\text { Blinding of outcome as- } \\
\text { sessment (detection bias) }\end{array}$ & Low risk & $\begin{array}{l}\text { Judgement Comment: Blind assessor not specified for post-treatment, but in } \\
\text { dependent rater assessed subset of interviews }\end{array}$ \\
\hline
\end{tabular}

All outcomes

\begin{tabular}{|c|c|c|}
\hline $\begin{array}{l}\text { Incomplete outcome data } \\
\text { (attrition bias) } \\
\text { All outcomes }\end{array}$ & Low risk & Judgement Comment: Data provided by author \\
\hline $\begin{array}{l}\text { Selective reporting (re- } \\
\text { porting bias) }\end{array}$ & Low risk & Judgement Comment: Outcomes for all measures reported \\
\hline $\begin{array}{l}\text { Other source of bias - } \\
\text { Therapy integrity }\end{array}$ & Unclear risk & $\begin{array}{l}\text { Judgement Comment: Qualified therapists, but no further detail on assess- } \\
\text { ing/monitoring adherence/competence }\end{array}$ \\
\hline Other bias & Low risk & Judgement Comment: The study appears to be free of other sources of bias \\
\hline
\end{tabular}

Spence 2006

\section{Study characteristics}

\begin{tabular}{|c|c|}
\hline Methods & $\begin{array}{l}\text { Study design: randomised controlled trial } \\
\text { Total duration of study: } 12 \text {-month non-controlled follow-up } \\
\text { Number of study centres and location: } 1 \text { in Australia } \\
\text { Study setting: university clinic }\end{array}$ \\
\hline Participants & $\begin{array}{l}\text { N = } 72 \text { randomised (NB: } N=45 \text { included here, randomised to CBT: } N=22 \text {; waitlist: } N=23 \text { ) } \\
\text { Mean age (SD): CBT: } 10.26 \text { (2.05); waitlist: } 9.70 \text { (1.73) } \\
\text { Age range: } 7 \text { to } 14 \\
\text { Gender: } C B T: 40.90 \% \text { female; waitlist: } 43.50 \% \text { female } \\
\text { Inclusion criteria: primary diagnosis of an anxiety disorder (based on ADIS-P), aged } 7 \text { to } 14 \text {, ability to } \\
\text { read and write English, and access to internet at home } \\
\text { Exclusion criteria: no significant differences in demographic or clinical variables }\end{array}$ \\
\hline Interventions & $\begin{array}{l}\text { CBT } \\
\text { Intervention: clinic group. Components included identifying physiological symptoms of anxiety; pro- } \\
\text { gressive muscle relaxation, guided imagery, and deep breathing; coping self-talk and cognitive restruc- } \\
\text { turing; graded exposure; problem solving; and self-evaluation and reward. Parent component included } \\
\text { psychoeducation about (a) child anxiety, (b) contingency management, and (c) ways of assisting their } \\
\text { child with relaxation training, cognitive restructuring, graded exposure, and problem solving. } \\
\text { Concomitant/excluded medications: not allowed } \\
\text { Delivery format: child only and parent only; group } \\
\text { Therapist contact time: } 16 \text { hours (10 x } 1 \text { hour (child), } 6 \times 1 \text { hour (parent)) } \\
\text { Who delivers the intervention: psychologists } \\
\text { Waitlist/no treatment }\end{array}$ \\
\hline
\end{tabular}


Intervention: 10-week waitlist

Concomitant/excluded medications: not allowed

\begin{tabular}{|c|c|c|}
\hline Outcomes & \multicolumn{2}{|c|}{$\begin{array}{l}\text { Remission of primary anxiety disorder diagnosis post-treatment: ADIS-P } \\
\text { Remission of all anxiety disorder diagnosis post-treatment: ADIS-P } \\
\text { Reduction in anxiety symptoms (child report) post-treatment: SCAS-C } \\
\text { Reduction in anxiety symptoms (child report) post-treatment: SCAS-P }\end{array}$} \\
\hline Notes & \multicolumn{2}{|c|}{ Study included "NET" condition (online delivery) which we did not include in the review. } \\
\hline \multicolumn{3}{|l|}{ Risk of bias } \\
\hline Bias & Authors' judgement & Support for judgement \\
\hline $\begin{array}{l}\text { Random sequence genera- } \\
\text { tion (selection bias) }\end{array}$ & Low risk & Judgement Comment: A coin was tossed \\
\hline $\begin{array}{l}\text { Allocation concealment } \\
\text { (selection bias) }\end{array}$ & Unclear risk & $\begin{array}{l}\text { Judgement Comment: Assigned in advance, but no further detail provided, } \\
\text { e.g. if independent }\end{array}$ \\
\hline $\begin{array}{l}\text { Blinding of participants } \\
\text { and personnel (perfor- } \\
\text { mance bias) } \\
\text { All outcomes }\end{array}$ & Low risk & $\begin{array}{l}\text { Judgement Comment: Even though blinding was not possible, we judged that } \\
\text { it was unlikely this led to a departure from the intended intervention }\end{array}$ \\
\hline $\begin{array}{l}\text { Blinding of outcome as- } \\
\text { sessment (detection bias) } \\
\text { All outcomes }\end{array}$ & Unclear risk & $\begin{array}{l}\text { Judgement Comment: Interrater reliability assessed, with rater blind to origi- } \\
\text { nal diagnoses, but does not specify if assessors were blind to condition }\end{array}$ \\
\hline $\begin{array}{l}\text { Incomplete outcome data } \\
\text { (attrition bias) } \\
\text { All outcomes }\end{array}$ & Low risk & Judgement Comment: Report ITT and completer analyses. Attrition detailed. \\
\hline $\begin{array}{l}\text { Selective reporting (re- } \\
\text { porting bias) }\end{array}$ & Low risk & Judgement Comment: Outcomes reported \\
\hline $\begin{array}{l}\text { Other source of bias - } \\
\text { Therapy integrity }\end{array}$ & Low risk & Judgement Comment: Independent assessment of integrity \\
\hline Other bias & Low risk & Judgement Comment: The study appears to be free of other sources of bias \\
\hline
\end{tabular}

Spence 2011

\title{
Study characteristics
}

Methods

\author{
Study design: randomised controlled trial \\ Total duration of study: 12-month non-controlled follow-up \\ Number of study centres and location: 3 in Australia
}

Study setting: 3 university clinics

Participants $\quad \mathbf{N}=\mathbf{1 1 5}$ randomised (NB: $\mathrm{N}=71$ included here: $\mathrm{CBT}: \mathrm{N}=44$; waitlist: $\mathrm{N}=27$ )


Spence 2011 (Continued)

\section{Age range: 12 to 18}

Inclusion criteria: primary diagnosis of separation anxiety disorder, social phobia, GAD, or specific phobia (ADIS-C/P); aged 12 to 18; access to computer and internet at home; able to read and write English at age-appropriate level

Exclusion criteria: primary diagnosis of panic disorder, OCD, PTSD, mood disorder with CSR 6+, pervasive developmental disorder, learning disorder, significant behavioural, substance abuse, suicidal ideation or current self-harm

Intervention: BRAVE CLINIC. Manualised individual CBT for adolescent anxiety, adapted from child version (equivalent to online programme in content, number, and length sessions)

Delivery format: child only and parent only; individual

Therapist contact time: 15 hours ( $10 \times 1$ hour (child), $5 \times 1$ hour (parent))

Who delivers the intervention: psychologists

\section{Waitlist/no treatment}

Intervention: 12-week waitlist

\begin{tabular}{ll}
\hline Outcomes & Remission of primary anxiety disorder diagnosis post-treatment: ADIS-P \\
Remission of all anxiety disorder diagnosis post-treatment: ADIS-P \\
Reduction in anxiety symptoms (child report) post-treatment: SCAS-C \\
Reduction in anxiety symptoms (child report) post-treatment: SCAS-P \\
Improvement in global functioning post-treatment: CGAS
\end{tabular}

Notes Study included "NET" condition (online CBT) which we did not include in the review.

\section{Risk of bias}

\begin{tabular}{|c|c|c|}
\hline Bias & Authors' judgement & Support for judgement \\
\hline $\begin{array}{l}\text { Random sequence genera- } \\
\text { tion (selection bias) }\end{array}$ & Low risk & Judgement Comment: Computer-generated sequence \\
\hline $\begin{array}{l}\text { Allocation concealment } \\
\text { (selection bias) }\end{array}$ & Low risk & Judgement Comment: In advance, implemented by independent researcher \\
\hline $\begin{array}{l}\text { Blinding of participants } \\
\text { and personnel (perfor- } \\
\text { mance bias) } \\
\text { All outcomes }\end{array}$ & Low risk & $\begin{array}{l}\text { Judgement Comment: Even though blinding was not possible, we judged that } \\
\text { it was unlikely this led to a departure from the intended intervention }\end{array}$ \\
\hline $\begin{array}{l}\text { Blinding of outcome as- } \\
\text { sessment (detection bias) } \\
\text { All outcomes }\end{array}$ & Low risk & Judgement Comment: Blind assessors \\
\hline $\begin{array}{l}\text { Incomplete outcome data } \\
\text { (attrition bias) } \\
\text { All outcomes }\end{array}$ & Low risk & $\begin{array}{l}\text { Judgement Comment: Attrition reported. Reported completer and ITT analy- } \\
\text { ses for diagnostic outcomes, and ITT for continuous. Examined and reported } \\
\text { impact of missing data }\end{array}$ \\
\hline
\end{tabular}


Spence 2011 (Continued)

Selective reporting (re- Low risk Judgement Comment: Outcomes reported
porting bias)

\begin{tabular}{|c|c|c|}
\hline $\begin{array}{l}\text { Other source of bias - } \\
\text { Therapy integrity }\end{array}$ & Low risk & $\begin{array}{l}\text { Judgement Comment: Trained therapists, regular supervision, fidelity as- } \\
\text { sessed and reported }\end{array}$ \\
\hline
\end{tabular}

Other bias Low risk Judgement Comment: The study appears to be free of other sources of bias

Storch 2013

\section{Study characteristics}

Study design: randomised controlled trial
Methods
Number of study centres and location: 1 in the USA
Study setting: university clinic
N $=\mathbf{4 5}$ (CBT: $\mathrm{N}=24:$ TAU: $\mathrm{N}=21)$
Mean age (SD): 8.89 (13.4)
Age range: 7 to 11
Gender: 9 (20\%) female
Inclusion criteria: diagnosis of autistic disorder, Asperger's syndrome, or pervasive developmental
disorder-not otherwise specified; primary diagnosis of separation anxiety disorder, social phobia, gen-
eralised anxiety disorder, or obsessive-compulsive disorder (based on ADIS-C/P)
Exclusion criteria: full-scale or verbal comprehension IQ less than 70 on a standardised test; concur-
rent participation in psychosocial interventions (excluding occupational/speech therapy, and social
skills training groups that did not include homework and were less than 90 minutes per week, given
that treatment efficacy for anxiety was limited, and parents are often reluctant to give up services); ac-
tive suicidality or engagement in suicidal behaviours within 6 months before enrolment; or bipolar or
psychotic disorder

Interventions $\quad$ CBT

Intervention: Behavioral Interventions for Anxiety in Children with Autism (BIACA)

Concomitant/excluded medications: stable dose before screening allowed

Delivery format: child only and parent only; individual

Therapist contact time: 16 to 24 hours ( $16 \times 60$ to 90 minutes)

Who delivers the intervention: doctoral students or postdoctoral fellows in clinical or school psychology

\section{TAU}

Intervention: free to begin, continue, change, or refrain from any psychosocial or pharmacological treatment

Concomitant/excluded medications: any allowed 
Storch 2013 (Continued)

Remission of all anxiety disorder diagnosis post-treatment: ADIS-C/P

Reduction in anxiety symptoms (parent report) post-treatment: MASC-P

\begin{tabular}{ll}
\hline Notes & Sample with ASD \\
& Remission for all anxiety diagnoses reported in Warwick 2016. \\
\hline
\end{tabular}

Risk of bias

\begin{tabular}{|c|c|c|}
\hline Bias & Authors' judgement & Support for judgement \\
\hline $\begin{array}{l}\text { Random sequence genera- } \\
\text { tion (selection bias) }\end{array}$ & Low risk & Judgement Comment: Computer-generated algorithm \\
\hline $\begin{array}{l}\text { Allocation concealment } \\
\text { (selection bias) }\end{array}$ & Low risk & Judgement Comment: Computer generated, so unlikely to foresee assignment \\
\hline $\begin{array}{l}\text { Blinding of participants } \\
\text { and personnel (perfor- } \\
\text { mance bias) } \\
\text { All outcomes }\end{array}$ & Low risk & $\begin{array}{l}\text { Judgement Comment: Even though blinding was not possible, we judged that } \\
\text { it was unlikely this led to a departure from the intended intervention }\end{array}$ \\
\hline $\begin{array}{l}\text { Blinding of outcome as- } \\
\text { sessment (detection bias) } \\
\text { All outcomes }\end{array}$ & Low risk & Judgement Comment: Blind assessors \\
\hline $\begin{array}{l}\text { Incomplete outcome data } \\
\text { (attrition bias) } \\
\text { All outcomes }\end{array}$ & Low risk & $\begin{array}{l}\text { Judgement Comment: Attrition and missing data detailed. Small amount of } \\
\text { missing data, and appropriate methods used to manage missing data }\end{array}$ \\
\hline $\begin{array}{l}\text { Selective reporting (re- } \\
\text { porting bias) }\end{array}$ & Low risk & Judgement Comment: Outcomes reported \\
\hline $\begin{array}{l}\text { Other source of bias - } \\
\text { Therapy integrity }\end{array}$ & Low risk & Judgement Comment: Therapists trained. Fidelity assessed and reported. \\
\hline Other bias & Low risk & Judgement Comment: The study appears to be free of other sources of bias \\
\hline
\end{tabular}

Storch 2015

\section{Study characteristics}

\begin{tabular}{ll}
\hline Methods & Study design: randomised controlled trial \\
& Total duration of study: 1 -month non-controlled follow-up \\
& Number of study centres and location: 1 in the USA \\
& Study setting: university clinic \\
\hline Participants & N $=\mathbf{3 1}$ (CBT: $\mathrm{N}=16: \mathrm{TAU}: \mathrm{N}=15)$ \\
& Mean age (SD): $12.74(1.34)$ \\
& Age range: 12 to 16 \\
Gender: $6(19.4 \%)$ female
\end{tabular}


Inclusion criteria: met criteria for (1) a diagnosis of autism, Asperger's syndrome, or Pervasive Developmental Disorder- Not Otherwise Specified

; (2) a co-occurring anxiety diagnosis of separation anxiety disorder, generalised anxiety disorder, Obsessive Compulsive Disorder, or social phobia (based on ADIS-C/P); (3) a score of 13 on the Pediatric Anxiety Rating Scale (PARS); and (4) an IQ 80 evaluated by the Wechsler Abbreviated Scale of Intelligence or review of standardised testing occurring in the prior 2 years

Exclusion criteria: (1) initiation of new medications within 6 weeks (anti-psychotics, Attention Deficit Hyperactivity Disorder medications) or 12 weeks (anti-depressants) before study enrolment; (2) concurrent participation in psychotherapy, social skills training over 90 min per week, or other programmatic behavioural interventions (e.g. applied behavioural analysis); (3) active suicidality; (4) lifetime history of mania, psychosis, or substance abuse

Intervention: developmentally modified version of the Behavioral Interventions for Anxiety in Children with Autism (BIACA) treatment manual. A minimum of 3 sessions were spent developing coping skills (e.g. behavioural activation, cognitive restructuring) with at least 8 sessions of exposure therapy.

Concomitant/excluded medications: any stable medication allowed

Delivery format: child sessions, with parent included in most sessions; individual

Therapist contact time: 16 to 24 hours ( $16 \times 60$ to 90 minutes)

Who delivers the intervention: postdoctoral fellows or doctoral students in clinical or school psychology with at least 1 year of experience in CBT for childhood anxiety

TAU

Intervention: participants could seek any psychosocial or pharmacological treatment that they chose, or not seek treatment, for 16 weeks

Concomitant/excluded medications: continued with pre-existing medications or therapy or were able to initiate dosage changes or new medications with the prescribing provider

\section{Outcomes Remission of primary anxiety disorder diagnosis post-treatment: ADIS-C/P \\ Remission of all anxiety disorder diagnoses post-treatment: ADIS-C/P \\ Reduction in anxiety symptoms (parent report) post-treatment: MASC-P}

Notes

Remission from all anxiety disorders diagnoses provided in Warwick 2016.

Sample with ASD

\section{Risk of bias}

\begin{tabular}{|c|c|c|}
\hline Bias & Authors' judgement & Support for judgement \\
\hline $\begin{array}{l}\text { Random sequence genera- } \\
\text { tion (selection bias) }\end{array}$ & Low risk & Judgement Comment: Computer generated \\
\hline $\begin{array}{l}\text { Allocation concealment } \\
\text { (selection bias) }\end{array}$ & Low risk & Judgement Comment: Computer generated, so unlikely to foresee assignment \\
\hline $\begin{array}{l}\text { Blinding of participants } \\
\text { and personnel (perfor- } \\
\text { mance bias) } \\
\text { All outcomes }\end{array}$ & Low risk & $\begin{array}{l}\text { Judgement Comment: Even though blinding was not possible, we judged that } \\
\text { it was unlikely this led to a departure from the intended intervention }\end{array}$ \\
\hline
\end{tabular}


Storch 2015 (Continued)

Blinding of outcome as- $\quad$ Low risk Judgement Comment: Blind assessors
sessment (detection bias)

All outcomes

\begin{tabular}{|c|c|c|}
\hline $\begin{array}{l}\text { Incomplete outcome data } \\
\text { (attrition bias) } \\
\text { All outcomes }\end{array}$ & Low risk & $\begin{array}{l}\text { Judgement Comment: No dropouts to post-treatment assessment. Missing da- } \\
\text { ta managed appropriately. }\end{array}$ \\
\hline $\begin{array}{l}\text { Selective reporting (re- } \\
\text { porting bias) }\end{array}$ & Low risk & Judgement Comment: Outcomes reported/available \\
\hline $\begin{array}{l}\text { Other source of bias - } \\
\text { Therapy integrity }\end{array}$ & Low risk & $\begin{array}{l}\text { Judgement Comment: Therapists trained and supervised, and adherence rat- } \\
\text { ed and reported }\end{array}$ \\
\hline Other bias & Low risk & Judgement Comment: The study appears to be free of other sources of bias \\
\hline
\end{tabular}

Thirlwall 2013

\section{Study characteristics}

Methods

Study design: randomised controlled trial

Total duration of study: non-controlled 6-month follow-up and 3- to 5-year follow-up

Number of study centres and location: 1 in the UK

Study setting: university and NHS clinic

Date of study: recruitment April 2008 to December 2010

\begin{tabular}{ll}
\hline Participants & $\mathbf{N} 194$ (CBT (full): $\mathrm{N}=64:$ CBT (brief): $\mathrm{N}=61 ;$ waitlist: $\mathrm{N}=69)$ \\
& Age range: 7 to 12 \\
& Gender: CBT (full): $30(46.9 \%)$ female; CBT (brief): $30(49.2 \%)$ female; waitlist: $34(49.3 \%)$ female \\
& Inclusion criteria: age 7 to 12, primary diagnosis of GAD, social phobia, separation anxiety disorder, \\
& panic disorder/agoraphobia, or specific phobia (based on ADIS-C/P), no significant physical or intellec- \\
& tual impairment, if prescription of psychotropic medication, stable dose (parent and child), primary \\
& carer not have current anxiety disorder or other serious mental health disorder, parent not have signifi- \\
& cant intellectual impairment
\end{tabular}

Interventions

\section{CBT (full)}

Intervention: guided parent-delivered CBT treatment. Parents given self-help book and receive full guidance, some face-to-face and some on phone.

Concomitant/excluded medications: stable dose allowed

Delivery format: parent only; individual

Therapist contact time: 5 hours 20 minutes

Who delivers the intervention: some clinical experience or novice (some experience delivering CBT or working with clinical populations)

\section{CBT (brief)}

Intervention: guided parent-delivered CBT treatment. Parents given self-help book and receive brief guidance, some face-to-face and some on phone. 
Thirlwall 2013 (Continued)

Concomitant/excluded medications: stable dose allowed

Delivery format: parent only; individual

Therapist contact time: 2 hours 40 minutes

Who delivers the intervention: some clinical experience or novice (some experience delivering CBT or working with clinical populations)

\section{Waitlist/no treatment}

Intervention: 12-week waitlist

Concomitant/excluded medications: stable dose allowed

$\begin{array}{ll}\text { Outcomes } & \text { Remission of primary anxiety disorder diagnosis post-treatment: ADIS-C/P } \\ \text { Remission of all anxiety disorder diagnoses post-treatment: ADIS-C/P } \\ \text { Reduction in anxiety symptoms (child report) post-treatment: SCAS-C } \\ \text { Reduction in anxiety symptoms (parent report) post-treatment: SCAS-P } \\ \text { Reduction in depressive symptoms post treatment: SMFQ-child }\end{array}$

Notes

\section{Risk of bias}

\begin{tabular}{lll}
\hline Bias & Authors' judgement & Support for judgement \\
\hline $\begin{array}{l}\text { Random sequence genera- } \\
\text { tion (selection bias) }\end{array}$ & Low risk & $\begin{array}{l}\text { Judgement Comment: Centralised telephone randomisation service at the } \\
\text { Centre for Statistics in Medicine, University of Oxford }\end{array}$ \\
\hline $\begin{array}{l}\text { Allocation concealment } \\
\text { (selection bias) }\end{array}$ & Low risk & Judgement Comment: Centralised \\
\hline
\end{tabular}

$\begin{array}{ll}\begin{array}{l}\text { Blinding of participants } \\ \text { and personnel (perfor- }\end{array} & \text { Jow risk } \\ \text { it was unlikely this led to a departure from the intended intervention }\end{array}$
mance bias)

All outcomes

\begin{tabular}{|c|c|c|}
\hline $\begin{array}{l}\text { Blinding of outcome as- } \\
\text { sessment (detection bias) } \\
\text { All outcomes }\end{array}$ & Low risk & Judgement Comment: Blind assessors \\
\hline $\begin{array}{l}\text { Incomplete outcome data } \\
\text { (attrition bias) } \\
\text { All outcomes }\end{array}$ & Low risk & $\begin{array}{l}\text { Judgement Comment: Attrition and missing data detailed. Sensitivity analyses } \\
\text { used to examine impact of missing data. }\end{array}$ \\
\hline $\begin{array}{l}\text { Selective reporting (re- } \\
\text { porting bias) }\end{array}$ & Low risk & Judgement Comment: Outcomes reported for all measures \\
\hline $\begin{array}{l}\text { Other source of bias - } \\
\text { Therapy integrity }\end{array}$ & Low risk & $\begin{array}{l}\text { Judgement Comment: Therapists attend training and regular supervision. Ad- } \\
\text { herence assessed and reported. }\end{array}$ \\
\hline Other bias & Low risk & Judgement Comment: The study appears to be free of other sources of bias \\
\hline
\end{tabular}


Villabo 2018

\section{Study characteristics}

Study design: randomised controlled trial
Total duration of study: 2-year non-controlled follow-up
Number of study centres and location: 5 clinics in Norway
Study setting: community clinics
Date of study: recruitment September 2008 through October 2011

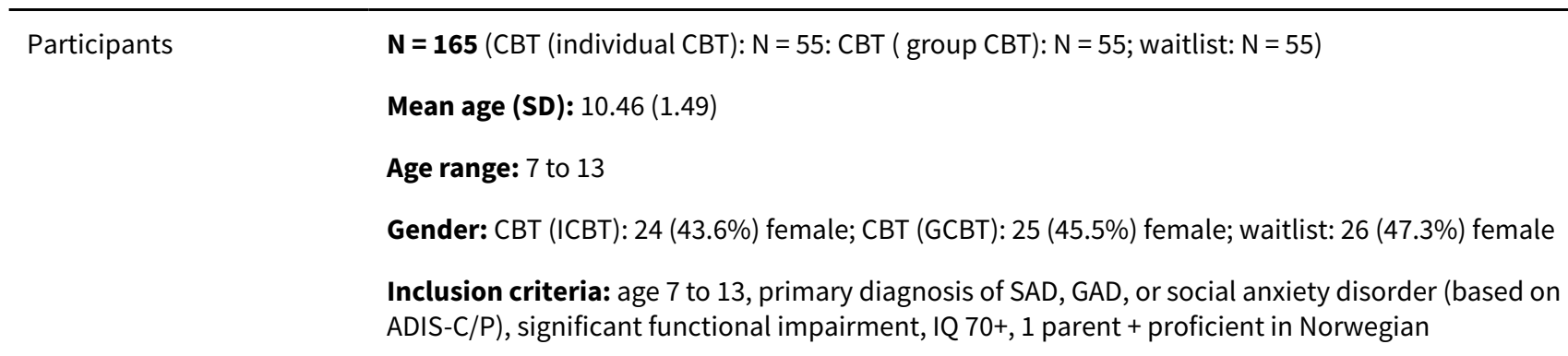

Exclusion criteria: (a) a mental health disorder with a higher treatment priority, (b) pervasive developmental disorder(s), (c) psychosis, or (d) current use of anxiolytic medication

\section{CBT (individual CBT)}

Intervention: Norwegian translation of Coping Cat

Concomitant/excluded medications: current use of anxiolytic medication not allowed

Delivery format: child only, and 2 parent only; individual

Therapist contact time: 14 sessions

Who delivers the intervention: community therapists (psychologists, social workers, psychiatry residents)

\section{CBT (group CBT)}

Intervention: Norwegian translation of Coping Cat

Concomitant/excluded medications: current use of anxiolytic medication not allowed

Delivery format: child only, and 2 parent only; group

Therapist contact time: 14 sessions

Who delivers the intervention: community therapists (psychologists, social workers, psychiatry residents)

\section{Waitlist/no treatment}

Intervention: 12-week waitlist

Concomitant/excluded medications: current use of anxiolytic medication not allowed

Remission of all anxiety disorder diagnoses post-treatment: ADIS-C/P

Reduction in anxiety symptoms (child report) post-treatment: MASC-C

Reduction in anxiety symptoms (parent report) post-treatment: MASC-P 
Villabo 2018 (Continued)

Improvement in global functioning post-treatment: CGAS

\section{Notes}

\section{Risk of bias}

\begin{tabular}{|c|c|c|}
\hline Bias & Authors' judgement & Support for judgement \\
\hline $\begin{array}{l}\text { Random sequence genera- } \\
\text { tion (selection bias) }\end{array}$ & Low risk & Judgement Comment: Computer-generated permuted blocking procedure \\
\hline $\begin{array}{l}\text { Allocation concealment } \\
\text { (selection bias) }\end{array}$ & Low risk & Judgement Comment: Computer generated, so unlikely to foresee assignment \\
\hline $\begin{array}{l}\text { Blinding of participants } \\
\text { and personnel (perfor- } \\
\text { mance bias) } \\
\text { All outcomes }\end{array}$ & Low risk & $\begin{array}{l}\text { Judgement Comment: Even though blinding was not possible, we judged that } \\
\text { it was unlikely this led to a departure from the intended intervention }\end{array}$ \\
\hline $\begin{array}{l}\text { Blinding of outcome as- } \\
\text { sessment (detection bias) } \\
\text { All outcomes }\end{array}$ & Low risk & Judgement Comment: Blind assessors \\
\hline $\begin{array}{l}\text { Incomplete outcome data } \\
\text { (attrition bias) } \\
\text { All outcomes }\end{array}$ & Low risk & $\begin{array}{l}\text { Judgement Comment: Attrition reported. Appropriate methods used to man- } \\
\text { age missing data. }\end{array}$ \\
\hline $\begin{array}{l}\text { Selective reporting (re- } \\
\text { porting bias) }\end{array}$ & Low risk & Judgement Comment: Outcomes reported for all measures \\
\hline $\begin{array}{l}\text { Other source of bias - } \\
\text { Therapy integrity }\end{array}$ & Low risk & $\begin{array}{l}\text { Judgement Comment: Therapists with a range of experience. Adherence as- } \\
\text { sessed and reported. }\end{array}$ \\
\hline Other bias & Low risk & Judgement Comment: The study appears to be free of other sources of bias \\
\hline
\end{tabular}

Walkup 2008

\section{Study characteristics}

Methods

Study design: randomised controlled trial

Total duration of study: long-term follow-up (6 years) to date

Number of study centres and location: 6 in the USA

Study setting: university clinics

Date of study: 2002 to 2007

Participants

$\mathbf{N}=488$ (CBT alone: $\mathrm{N}=139: \mathrm{CBT}+$ sertraline: $\mathrm{N}=140$; sertraline alone: $\mathrm{N}=133$; drug placebo: $\mathrm{N}=76$ )

Mean age (SD): CBT alone: 10.5 (2.9); CBT + sertraline: 10.7 (2.8); sertraline alone: 10.8 (2.8); drug placebo: $10.6(2.8)$

\section{Age range: 7 to 17}

Gender: CBT alone: 72 (51.8\%) female; CBT + sertraline: 72 (51.4\%) female; sertraline alone: 61 (45.9\%) female; drug placebo: 37 (48.7\%) female 
Inclusion criteria: aged 7 to 17 , primary diagnosis of separation or generalised or social phobia, substantial impairment (based on ADIS-C/P), IQ 80+

Exclusion criteria: unstable medical condition, were refusing to attend school because of anxiety, or had tried but had no response to 2 adequate trials of SSRIs or an adequate trial of CBT. Girls who were pregnant or sexually active and were not using an effective method of birth control were also excluded. Children who were receiving psychoactive medications other than stable doses of stimulants and who had psychiatric diagnoses that made participation in the study clinically inappropriate (i.e. current major depressive or substance use disorder; unmedicated ADHD, combined type; or a lifetime history of bipolar, psychotic, or pervasive developmental disorders) or who presented an acute risk to themselves or others were also excluded.

\section{CBT alone}

Intervention: based on Coping Cat

Concomitant/excluded medications: receiving psychoactive medications other than stable doses of stimulants excluded

Delivery format: child only, parent check-in, and 2 parent only

Therapist contact time: 14 hours (14 x 1 hour)

Who delivers the intervention: experienced psychotherapists certified in the Coping Cat protocol

CBT + sertraline

Intervention: based on Coping Cat + sertraline (Zoloft)

Concomitant/excluded medications: receiving psychoactive medications other than stable doses of stimulants excluded

Delivery format: child only, parent check-in, and 2 parent only

Therapist contact time: 18 to 22 hours: 14 hours ( $14 \times 1$ hour) +4 to 8 hours ( $8 \times 30$ to 60 minutes)

Who delivers the intervention: experienced psychotherapists certified in the Coping Cat protocol. Psychiatrists and nurse clinicians with experience in medicating children with anxiety disorders

\section{Sertraline alone}

Intervention: sertraline (Zoloft)

Concomitant/excluded medications: receiving psychoactive medications other than stable doses of stimulants excluded

Therapist contact time: 4 to 8 hours ( $8 \times 30$ to 60 minutes)

Who delivers the intervention: psychiatrists and nurse clinicians with experience in medicating children with anxiety disorders

\section{Drug placebo}

Intervention: drug placebo

Concomitant/excluded medications: receiving psychoactive medications other than stable doses of stimulants excluded

Therapist contact time: 4 to 8 hours $(8 \times 30$ to 60 minutes)

Who delivers the intervention: psychiatrists and nurse clinicians with experience in medicating children with anxiety disorders 
Improvement in global functioning post-treatment: CGAS

Reduction in depressive symptoms post-treatment: MFQ-C

$\begin{array}{ll}\text { Notes } & \text { Adverse events reported. } \\ & \text { Long-term remission outcomes reported elsewhere. }\end{array}$

\section{Risk of bias}

\begin{tabular}{|c|c|c|}
\hline Bias & Authors' judgement & Support for judgement \\
\hline $\begin{array}{l}\text { Random sequence genera- } \\
\text { tion (selection bias) }\end{array}$ & Low risk & Judgement Comment: Computer generated \\
\hline $\begin{array}{l}\text { Allocation concealment } \\
\text { (selection bias) }\end{array}$ & Low risk & Judgement Comment: Managed central pharmacy \\
\hline $\begin{array}{l}\text { Blinding of participants } \\
\text { and personnel (perfor- } \\
\text { mance bias) } \\
\text { All outcomes }\end{array}$ & Low risk & Judgement Comment: Drug placebo kept secret from participants \\
\hline $\begin{array}{l}\text { Blinding of outcome as- } \\
\text { sessment (detection bias) } \\
\text { All outcomes }\end{array}$ & Low risk & Judgement Comment: Independent blind assessors \\
\hline $\begin{array}{l}\text { Incomplete outcome data } \\
\text { (attrition bias) } \\
\text { All outcomes }\end{array}$ & Low risk & $\begin{array}{l}\text { Judgement Comment: Dropouts clear, and procedures for managing clear and } \\
\text { robust }\end{array}$ \\
\hline $\begin{array}{l}\text { Selective reporting (re- } \\
\text { porting bias) }\end{array}$ & Low risk & Judgement Comment: Outcomes reported for all measures \\
\hline $\begin{array}{l}\text { Other source of bias - } \\
\text { Therapy integrity }\end{array}$ & Low risk & $\begin{array}{l}\text { Judgement Comment: Experienced therapists. Regular supervision. Fidelity } \\
\text { assessed. }\end{array}$ \\
\hline Other bias & Low risk & Judgement Comment: The study appears to be free of other sources of bias \\
\hline
\end{tabular}

\section{Waters 2009}

\section{Study characteristics}

\begin{tabular}{ll}
\hline Methods & Study design: randomised controlled trial \\
Number of study centres and location: 1 in Australia \\
Study setting: university clinic \\
\hline
\end{tabular}

Participants

$\mathbf{N}=\mathbf{8 0}(\mathrm{CBT}$ (parent + child): $\mathrm{N}=31 ; \mathrm{CBT}$ (parent only): $38 ;$ waitlist: $\mathrm{N}=11)$

Mean age (SD): CBT (parent + child): 6.89 (1.2); CBT (parent only): 6.68 (1.20); waitlist: 6.68 (1.20)

Age range: 4 to 8 
Gender: CBT (parent + child): 17 (54.8\%) female; CBT (parent only): 20 (64.5\%) female; waitlist: 5 (45.5\%) female

Inclusion criteria: specific phobia, social phobia, GAD, and/or separation anxiety disorder diagnosis (based on ADIS-P)

Exclusion criteria: comorbid externalising disorder, pervasive developmental disorder, organic brain damage, or psychosis. Children involved in psychological or pharmacological treatment for anxiety disorders were excluded.

Interventions

CBT (parent + child)

Intervention: Take Action. Children: psychoeducation, training in relaxation, identifying anxious selftalk, coping strategies, exposure, problem solving, social skills. Parent sessions: provided with child workbook, cover: psychoeducation, parent strategies for managing anxiety, coverage of take action steps children complete each week, positive parent coping, communication and problem-solving skills

Concomitant/excluded medications: not allowed

Delivery format: child sessions and parent sessions; group

Therapist contact time: 20 hours (10 1 hour (child); $10 \times 1$ hour (parent))

Who delivers the intervention: psychologists trained in CBT for child anxiety

CBT (parent only)

Intervention: same as parent sessions in parent + child above

Concomitant/excluded medications: not allowed

Delivery format: parent sessions; group

Therapist contact time: 10 hours $(10 \times 1$ hour (parent))

Who delivers the intervention: psychologists trained in CBT for child anxiety

Waitlist/no treatment

Intervention: approximately 10-week wait

Concomitant/excluded medications: not allowed

Outcomes Remission of primary anxiety disorder diagnosis post-treatment: ADIS-P

Remission of all anxiety disorder diagnoses post-treatment: ADIS-P

Notes

Anxiety symptoms measure not reported for waitlist, so not able to use in review.

\section{Risk of bias}

\begin{tabular}{lll}
\hline Bias & Authors' judgement & Support for judgement \\
\hline $\begin{array}{l}\text { Random sequence genera- } \\
\text { tion (selection bias) }\end{array}$ & Unclear risk & Judgement Comment: No detail given \\
\hline $\begin{array}{l}\text { Allocation concealment } \\
\text { (selection bias) }\end{array}$ & Unclear risk & Judgement Comment: No detail given \\
\hline $\begin{array}{l}\text { Blinding of participants } \\
\text { and personnel (perfor- } \\
\text { mance bias) }\end{array}$ & Low risk & \\
All outcomes & & $\begin{array}{l}\text { Judgement Comment: Even though blinding was not possible, we judged that } \\
\text { it was unlikely this led to a departure from the intended intervention }\end{array}$ \\
\hline \hline
\end{tabular}


Waters 2009 (Continued)

Blinding of outcome as- Low risk Judgement Comment: Blind ADIS assessors sessment (detection bias)

All outcomes

\begin{tabular}{lll}
\hline $\begin{array}{l}\text { Incomplete outcome data } \\
\text { (attrition bias) } \\
\text { All outcomes }\end{array}$ & Low risk & $\begin{array}{l}\text { Judgement Comment: Report dropouts. High number of dropouts, but report } \\
\text { completer analyses and ITT and same conclusions }\end{array}$ \\
\hline $\begin{array}{l}\text { Selective reporting (re- } \\
\text { porting bias) }\end{array}$ & Unclear risk & $\begin{array}{l}\text { Judgement Comment: Did not report parent-reported child anxiety symptoms } \\
\text { for waitlist }\end{array}$ \\
\hline $\begin{array}{l}\text { Other source of bias - } \\
\text { Therapy integrity }\end{array}$ & Low risk & $\begin{array}{l}\text { Judgement Comment: Trained therapists, regular supervision. Therapist } \\
\text { checklist used to maintain integrity. }\end{array}$ \\
\hline Other bias & Low risk & Judgement Comment: The study appears to be free of other sources of bias
\end{tabular}

Wergeland 2014

\title{
Study characteristics
}

Methods

\author{
Study design: randomised controlled trial
}

Total duration of study: non-controlled 1-year follow-up

Number of study centres and location: 7 public child and adolescent mental health outpatient clinics in Norway

\section{Study setting: CAMHS clinics}

Date of study: 2008 to 2010

N $=\mathbf{1 8 2}(\mathrm{CBT}$ (iCBT): N =77; CBT (GCBT): N =67; waitlist: N=38)
Mean age (SD): $11.5(2.1)$
Age range: 8 to 15
Gender: $96(52.7 \%)$ female
Inclusion criteria: principal anxiety disorder SAD, SOP, GAD (based on ADIS-C/P)
Exclusion criteria: pervasive developmental disorder, psychotic disorder, and/or mental retardation

Interventions

\section{CBT (iCBT)}

Intervention: individual CBT - Friends

Concomitant/excluded medications: stable dose allowed

Delivery format: child only, child + parent for 2 sessions and part of 8 sessions; individual

Therapist contact time: 10 hours ( $10 \times 1$ hour)

Who delivers the intervention: clinical psychologists, clinical pedagogues, clinical social worker

\section{CBT (GCBT)}

Intervention: group CBT - Friends

Concomitant/excluded medications: stable dose allowed 
Wergeland 2014 (Continued)

Delivery format: child only, child + parent for 2 sessions and part of 8 sessions; group

Therapist contact time: 15 hours ( $10 \times 90$ minutes)

Who delivers the intervention: clinical psychologists, clinical pedagogues, clinical social worker

Waitlist/no treatment

Intervention: 10-week waitlist

Concomitant/excluded medications: stable dose allowed

\begin{tabular}{|c|c|c|}
\hline Outcomes & \multicolumn{2}{|c|}{$\begin{array}{l}\text { Remission of primary anxiety disorder diagnosis post-treatment: ADIS-C/P } \\
\text { Reduction in anxiety symptoms (child report) post-treatment: SCAS-C } \\
\text { Reduction in anxiety symptoms (parent report) post-treatment: SCAS-P } \\
\text { Reduction in depressive symptoms post-treatment: MFQ-child }\end{array}$} \\
\hline \multicolumn{3}{|l|}{ Notes } \\
\hline \multicolumn{3}{|l|}{ Risk of bias } \\
\hline Bias & Authors' judgement & Support for judgement \\
\hline $\begin{array}{l}\text { Random sequence genera- } \\
\text { tion (selection bias) }\end{array}$ & Unclear risk & Judgement Comment: Block randomisation, no further detail \\
\hline $\begin{array}{l}\text { Allocation concealment } \\
\text { (selection bias) }\end{array}$ & Unclear risk & Judgement Comment: Block randomisation, no further detail \\
\hline $\begin{array}{l}\text { Blinding of participants } \\
\text { and personnel (perfor- } \\
\text { mance bias) } \\
\text { All outcomes }\end{array}$ & Low risk & $\begin{array}{l}\text { Judgement Comment: Even though blinding was not possible, we judged that } \\
\text { it was unlikely this led to a departure from the intended intervention }\end{array}$ \\
\hline $\begin{array}{l}\text { Blinding of outcome as- } \\
\text { sessment (detection bias) } \\
\text { All outcomes }\end{array}$ & Unclear risk & Judgement Comment: Assessor not blind to treatment approach \\
\hline $\begin{array}{l}\text { Incomplete outcome data } \\
\text { (attrition bias) } \\
\text { All outcomes }\end{array}$ & Low risk & $\begin{array}{l}\text { Judgement Comment: Appropriate methods used for managing missing data. } \\
\text { Report ITT, and also conducted completer analyses }\end{array}$ \\
\hline $\begin{array}{l}\text { Selective reporting (re- } \\
\text { porting bias) }\end{array}$ & Low risk & Judgement Comment: Outcomes reported \\
\hline $\begin{array}{l}\text { Other source of bias - } \\
\text { Therapy integrity }\end{array}$ & Low risk & Judgement Comment: Adherence and competence assessed and reported \\
\hline Other bias & Low risk & Judgement Comment: The study appears to be free of other sources of bias \\
\hline
\end{tabular}

White 2013

\section{Study characteristics}

Methods Study design: randomised controlled trial


White 2013 (Continued)

Number of study centres and location: 1 in the USA

Study setting: university clinic

Date of study: recruitment October 2008 to October 2010

\begin{tabular}{|c|c|c|}
\hline \multirow[t]{6}{*}{ Participants } & \multicolumn{2}{|c|}{$\mathbf{N}=\mathbf{3 0}$ (CBT: $\mathbf{N}=15 ;$ waitlist: $\mathbf{N}=15)$} \\
\hline & \multicolumn{2}{|c|}{ Mean age (SD): 15 years } \\
\hline & \multicolumn{2}{|l|}{ Age range: 12 to 17} \\
\hline & \multicolumn{2}{|c|}{ Gender: $7(23 \%)$ female } \\
\hline & \multirow{2}{*}{\multicolumn{2}{|c|}{$\begin{array}{l}\text { Inclusion criteria: age } 12 \text { to } 17 \text {, ASD diagnosis supported by ADOS, meet criteria for social phobia GAD, } \\
\text { specific phobia, or separation anxiety disorder (based on ADIS-C/P) } \\
\text { Exclusion criteria: primary OCD, panic disorder, or panic disorder with agoraphobia or serious behav- } \\
\text { iour problems }\end{array}$}} \\
\hline & & \\
\hline \multirow[t]{9}{*}{ Interventions } & \multicolumn{2}{|l|}{ CBT } \\
\hline & \multicolumn{2}{|c|}{$\begin{array}{l}\text { Intervention: Multimodal Anxiety and Social Skills Intervention (MASSI). Individual, group, and parent } \\
\text { education. Select appropriate modules from manual }\end{array}$} \\
\hline & \multicolumn{2}{|c|}{$\begin{array}{l}\text { Concomitant/excluded medications: concomitant psychiatric medication was permitted if the dose } \\
\text { was stable for at least } 4 \text { weeks with no planned changes for the duration of the randomised trial }\end{array}$} \\
\hline & \multicolumn{2}{|c|}{ Delivery format: child only, parent attend for part of session; individual and group } \\
\hline & \multicolumn{2}{|c|}{ Therapist contact time: 21 to 23 hours ( $13 \times 60$ to 70 minutes (individual), $7 \times 75$ minutes (group)) } \\
\hline & \multicolumn{2}{|c|}{ Who delivers the intervention: principal investigator and doctoral students } \\
\hline & \multicolumn{2}{|l|}{ Waitlist/no treatment } \\
\hline & \multicolumn{2}{|c|}{ Intervention: 14-week waitlist } \\
\hline & \multicolumn{2}{|c|}{$\begin{array}{l}\text { Concomitant/excluded medications: concomitant psychiatric medication was permitted if the dose } \\
\text { was stable for at least } 4 \text { weeks with no planned changes for the duration of the trial }\end{array}$} \\
\hline \multirow[t]{2}{*}{ Outcomes } & \multicolumn{2}{|c|}{ Reduction in anxiety symptoms (parent report) post-treatment: CASI-Anxiety Scale } \\
\hline & \multicolumn{2}{|c|}{ Improvement in global functioning post-treatment: DD-CGAS } \\
\hline Notes & \multicolumn{2}{|l|}{ Sample with ASD } \\
\hline \multicolumn{3}{|l|}{ Risk of bias } \\
\hline Bias & Authors' judgement & Support for judgement \\
\hline $\begin{array}{l}\text { Random sequence genera- } \\
\text { tion (selection bias) }\end{array}$ & Low risk & Judgement Comment: Names drawn from a box \\
\hline $\begin{array}{l}\text { Allocation concealment } \\
\text { (selection bias) }\end{array}$ & Low risk & $\begin{array}{l}\text { Judgement Comment: Done by person unaffiliated with study. Baseline mea- } \\
\text { sures complete prior to allocation }\end{array}$ \\
\hline $\begin{array}{l}\text { Blinding of participants } \\
\text { and personnel (perfor- } \\
\text { mance bias) } \\
\text { All outcomes }\end{array}$ & Low risk & $\begin{array}{l}\text { Judgement Comment: Even though blinding was not possible, we judged that } \\
\text { it was unlikely this led to a departure from the intended intervention }\end{array}$ \\
\hline
\end{tabular}


White 2013 (Continued)

Blinding of outcome as- Low risk Judgement Comment: Blind assessors for appropriate measures sessment (detection bias)

All outcomes

\begin{tabular}{lll}
$\begin{array}{l}\text { Incomplete outcome data } \\
\text { (attrition bias) } \\
\text { All outcomes }\end{array}$ & Low risk & Judgement Comment: Use appropriate methods for managing missing data \\
\hline $\begin{array}{l}\text { Selective reporting (re- } \\
\text { porting bias) }\end{array}$ & Low risk & Judgement Comment: Outcomes reported \\
\hline $\begin{array}{l}\text { Other source of bias - } \\
\text { Therapy integrity }\end{array}$ & Low risk & $\begin{array}{l}\text { Judgement Comment: Trained and supervised therapists. Sessions recorded } \\
\text { and reviewed. Fidelity assessed independently. }\end{array}$ \\
\hline Other bias & Low risk & Judgement Comment: The study appears to be free of other sources of bias
\end{tabular}

Wood 2009

\section{Study characteristics}

$\begin{array}{ll}\text { Methods } & \text { Study design: randomised controlled trial } \\ & \text { Number of study centres and location: } 1 \text { in the USA } \\ & \mathbf{N}=\mathbf{4 0} \text { (CBT: } \mathrm{N}=17 \text {; waitlist: } \mathrm{N}=23) \\ & \text { Mean age (SD): CBT: } 9.18(1.42) \text {; waitlist: } 9.22(1.57) \\ \text { Participants } & \text { Gender: CBT: } 5 \text { (29\%) female; waitlist: } 8 \text { (35\%) female } \\ \text { Inclusion criteria: } 1) \text { autism/Asperger's or Pervasive Developmental Disorder - Not Otherwise Speci- } \\ \text { fied (PDD-NOA) diagnosis; 2) separation anxiety disorder, social phobia, or OCD (based on ADIS-C/P); 3) } \\ \text { no psychiatric medication or stable dose for at least 1 month and maintained throughout study } \\ \text { Exclusion criteria: GAD diagnosis only, IQ less than 70, current psychotherapy or social skills training, } \\ \text { family therapy, or parent coaching }\end{array}$

Interventions

CBT

Intervention: Building confidence CBT

Concomitant/excluded medications: stable dose allowed

Delivery format: child only, and parent/family with child; individual

Therapist contact time: 24 hours ( $16 \times 90$ minutes)

Who delivers the intervention: doctoral students

Waitlist/no treatment

Intervention: 3-month waitlist

Concomitant/excluded medications: stable dose allowed

Outcomes Reduction in anxiety symptoms (child report) post-treatment: MASC-C

Reduction in anxiety symptoms (parent report) post-treatment: MASC-P 
Wood 2009 (Continued)

$\begin{array}{ll}\text { Notes } & \text { Only report remission from } 4 \text { entry diagnoses } \\ & \text { Sample with ASD }\end{array}$

Sample with ASD

\section{Risk of bias}

\begin{tabular}{|c|c|c|}
\hline Bias & Authors' judgement & Support for judgement \\
\hline $\begin{array}{l}\text { Random sequence genera- } \\
\text { tion (selection bias) }\end{array}$ & Low risk & Judgement Comment: Computer randomisation program \\
\hline $\begin{array}{l}\text { Allocation concealment } \\
\text { (selection bias) }\end{array}$ & Low risk & Judgement Comment: Concealed from investigators \\
\hline $\begin{array}{l}\text { Blinding of participants } \\
\text { and personnel (perfor- } \\
\text { mance bias) } \\
\text { All outcomes }\end{array}$ & Low risk & $\begin{array}{l}\text { Judgement Comment: Even though blinding was not possible, we judged that } \\
\text { it was unlikely this led to a departure from the intended intervention }\end{array}$ \\
\hline $\begin{array}{l}\text { Blinding of outcome as- } \\
\text { sessment (detection bias) } \\
\text { All outcomes }\end{array}$ & Low risk & Judgement Comment: Blinded assessors \\
\hline $\begin{array}{l}\text { Incomplete outcome data } \\
\text { (attrition bias) } \\
\text { All outcomes }\end{array}$ & Low risk & $\begin{array}{l}\text { Judgement Comment: Dropouts reported. ITT and completer analyses report- } \\
\text { ed }\end{array}$ \\
\hline $\begin{array}{l}\text { Selective reporting (re- } \\
\text { porting bias) }\end{array}$ & Low risk & Judgement Comment: Outcomes for planned measures reported \\
\hline $\begin{array}{l}\text { Other source of bias - } \\
\text { Therapy integrity }\end{array}$ & Low risk & $\begin{array}{l}\text { Judgement Comment: Therapists trained and supervised and adherence mea- } \\
\text { sures }\end{array}$ \\
\hline Other bias & Low risk & Judgement Comment: The study appears to be free of other sources of bias \\
\hline
\end{tabular}

Wood 2015

\section{Study characteristics}

\begin{tabular}{l} 
Study design: randomised controlled trial \\
Number of study centres and location: 2 in the USA \\
Study setting: 2 university clinics \\
\hline
\end{tabular}

Participants $\quad \mathbf{N}=\mathbf{3 3}(\mathrm{CBT}: \mathrm{N}=19$; waitlist: $\mathrm{N}=14)$

Mean age (SD): CBT: 12.3 (1.14); waitlist: 12.2 (0.98)

Age range: 11 to 15

Gender: CBT: 6 (32\%) female; waitlist: 4 (29\%) female

Inclusion criteria: criteria for ASD and 1 anxiety disorder (based on ADIS-C/P) 
Wood 2015 (Continued)

Exclusion criteria: receiving CBT, social skills training with homework or applied behavioural analysis, had initiated an anti-depressant or anti-psychotic within 12 or 6 weeks, respectively, prior to treatment, suicide risk, bipolar, schizophrenia, or substance abuse

\begin{tabular}{|c|c|c|}
\hline Interventions & \multicolumn{2}{|c|}{$\begin{array}{l}\text { CBT } \\
\text { Intervention: Behavioral Interventions for Anxiety in Children with Autism (BIACA) } \\
\text { Concomitant/excluded medications: stable dose allowed } \\
\text { Delivery format: child only, parent only, altogether; individual } \\
\text { Therapist contact time: } 24 \text { hours ( } 16 \text { x } 90 \text { minutes) } \\
\text { Who delivers the intervention: postdoctoral clinical fellow or doctoral students } \\
\text { Waitlist/no treatment } \\
\text { Intervention: } 16 \text {-week waitlist } \\
\text { Concomitant/excluded medications: stable dose allowed }\end{array}$} \\
\hline Outcomes & \multicolumn{2}{|c|}{$\begin{array}{l}\text { Remission of primary anxiety disorder diagnosis post-treatment: } A D I S-C / P \\
\text { Reduction in anxiety symptoms (parent report) post-treatment: MASC-P }\end{array}$} \\
\hline Notes & Sample with ASD & \\
\hline \multicolumn{3}{|l|}{ Risk of bias } \\
\hline Bias & Authors' judgement & Support for judgement \\
\hline $\begin{array}{l}\text { Random sequence genera- } \\
\text { tion (selection bias) }\end{array}$ & Low risk & Judgement Comment: Computer randomisation \\
\hline $\begin{array}{l}\text { Allocation concealment } \\
\text { (selection bias) }\end{array}$ & Low risk & Judgement Comment: Concealed from investigators \\
\hline $\begin{array}{l}\text { Blinding of participants } \\
\text { and personnel (perfor- } \\
\text { mance bias) } \\
\text { All outcomes }\end{array}$ & Low risk & $\begin{array}{l}\text { Judgement Comment: Even though blinding was not possible, we judged that } \\
\text { it was unlikely this led to a departure from the intended intervention }\end{array}$ \\
\hline $\begin{array}{l}\text { Blinding of outcome as- } \\
\text { sessment (detection bias) } \\
\text { All outcomes }\end{array}$ & Low risk & Judgement Comment: Independent evaluators \\
\hline $\begin{array}{l}\text { Incomplete outcome data } \\
\text { (attrition bias) } \\
\text { All outcomes }\end{array}$ & Low risk & $\begin{array}{l}\text { Judgement Comment: Dropouts detailed. Imputed missing data, but small } \\
\text { amount of missing data }\end{array}$ \\
\hline $\begin{array}{l}\text { Selective reporting (re- } \\
\text { porting bias) }\end{array}$ & Low risk & Judgement Comment: Outcomes reported for all measures \\
\hline $\begin{array}{l}\text { Other source of bias - } \\
\text { Therapy integrity }\end{array}$ & Low risk & Judgement Comment: Trained, supervised, adherence rated and reported \\
\hline Other bias & Low risk & Judgement Comment: The study appears to be free of other sources of bias \\
\hline
\end{tabular}

ADI-R: Autism Diagnostic Interview-Revised 
ADIS-C/P: Anxiety Disorder Interview Schedule for Children-Child and Parent interviews ADOS: Autism Diagnostic Observation Schedule

ASD: Autism Spectrum Disorder

BDI-II: Beck Depression Inventory-II

CAMHS: Child and Adolescent Mental Health Services

CASI: Child and Adolescent Symptom Inventory

CBCL: Child Behaviour Checklist

CBT: cognitive behaviour therapy

CDI: Children's Depression Inventory

CGAS: Children's Global Assessment Scale

CSR: Clinical Severity Rating

CTU: Clinical Trials Unit

DAWBA: Development and Wellbeing Assessment

DD-CGAS: Developmental Disabled Children's Global Assessment Scale

DIPS-K: Diagnostischen Interviews bei psychischen Störungen im Kindes- und Jugendalter

DISC: Diagnostic Interview Schedule for Children

DISCAP: Diagnostic Interview Schedule for Children, Adolescents, and Parents

DSM-IV: Diagnostic and Statistical Manual of Mental Disorders-4th edition

DSM-5: Diagnostic and Statistical Manual of Mental Disorders-5th edition

ICD: International Classification of Disease

IQ: intelligence quotient

ITT: intention-to-treat

K-GAS: Children's Global Assessment Scale (German Version)

K-SADS: Kiddie Schedule for Affective Disorders and Schizophrenia

MASC-C/P: Multidimensional Anxiety Scale for Children-Child/Parent report

MFQ-C: Mood and Feelings Questionnaire-Child report

MINI-KID: Mini International Neuropsychiatric Interview for Children and Adolescents

PAS-R: Preschool Anxiety Scale-Revised

RCADS: Revised Children's Anxiety and Depression Scale

RCMAS: Revised Children's Manifest Anxiety Scale

SAS-A: Social Anxiety Scale for Adolescents

SAS-C/P: Social Anxiety Scale for Children-Child/Parent report

SET-C: Social Effectiveness Therapy for Children

SCAS-C/P: Spence Children's Anxiety Scale-Child/Parent report

SCARED-C/P: Screen for Child Anxiety Related Emotional Disorders-Child/Parent report

SD: standard deviation

SMFQ-C: Short Mood and Feelings Questionnaire-Child report

SPAI-C: Social Phobia and Anxiety Inventory for Children

SPAIK-C: Social Phobia and Anxiety Inventory for Children (German Version)

STAIC: State-Trait Anxiety Inventory for Children

TAU: treatment as usual

Characteristics of excluded studies [ordered by study ID]

\begin{tabular}{ll}
\hline Study & Reason for exclusion \\
\hline Asbrand & Data not analysable \\
\hline Asbrand 2019 & Data not analysable \\
\hline Baer 2005 & Data not analysable \\
\hline Beidel 2000 & Not manualised/modular CBT \\
\hline Beidel 2007 & Not manualised/modular CBT \\
\hline Bergman 2013 & Not manualised/modular CBT \\
\hline Bernstein 2005 & Participants did not all meet diagnostic criteria for an anxiety disorder. \\
\hline
\end{tabular}




\begin{tabular}{|c|c|}
\hline Study & Reason for exclusion \\
\hline Bodden 2008a & Not an RCT \\
\hline Clementi 2019 & No non-CBT control/comparator \\
\hline Cobham 2012 & Not an RCT \\
\hline Cotton 2019 & Not manualised/modular CBT \\
\hline Ebrahiminejad 2016 & Not manualised/modular CBT \\
\hline Flatt 2010 & Data not analysable \\
\hline Gil Bernal 2009 & $\begin{array}{l}\text { No baseline structured diagnostic assessment/participants did not meet diagnostic criteria for anx- } \\
\text { iety disorder }\end{array}$ \\
\hline Ginsburg 2019b & Participants did not all meet diagnostic criteria for an anxiety disorder. \\
\hline Hayward 2000 & Not an RCT \\
\hline Jansen 2012 & $\begin{array}{l}\text { No baseline structured diagnostic assessment/participants did not meet diagnostic criteria for anx- } \\
\text { iety disorder }\end{array}$ \\
\hline Kerns 2016 & $\begin{array}{l}\text { No baseline structured diagnostic assessment/participants did not meet diagnostic criteria for anx- } \\
\text { iety disorder }\end{array}$ \\
\hline Kujawa 2019 & Not an RCT \\
\hline Lyneham 2006 & No direct contact with parent/child/not face-to-face \\
\hline Mendlowitz 1999 & Not an RCT \\
\hline Nauta 2003 & Not an RCT \\
\hline NCT00576719 & Data not analysable \\
\hline Oerbeck 2014 & Not manualised/modular CBT \\
\hline Ollendick 2018 & Not manualised/modular CBT \\
\hline Ost 2015 & Not manualised/modular CBT \\
\hline Ozyurt 2018 & Not manualised/modular CBT \\
\hline Pereira 2014 & Participants did not all meet diagnostic criteria for an anxiety disorder. \\
\hline Rudy 2017 & Not manualised/modular CBT \\
\hline Salzer 2018 & Adults ( $\geq 19$ years) (includes 20-year-olds) \\
\hline Sevi Tok 2016 & $\begin{array}{l}\text { No baseline structured diagnostic assessment/participants did not meet diagnostic criteria for anx- } \\
\text { iety disorder }\end{array}$ \\
\hline Storch 2019 & Not manualised/modular CBT \\
\hline
\end{tabular}




\begin{tabular}{ll}
\hline Study & Reason for exclusion \\
\hline Sung 2011 & $\begin{array}{l}\text { No baseline structured diagnostic assessment/participants did not meet diagnostic criteria for anx- } \\
\text { iety disorder }\end{array}$ \\
\hline Weisz 2012 & Participants did not all meet diagnostic criteria for an anxiety disorder. \\
\hline
\end{tabular}

CBT: cognitive behavioural therapy

$\mathrm{RCT}$ : randomised controlled trial

\section{Characteristics of studies awaiting classification [ordered by study ID]}

\section{ACTRN12613000217707}

\begin{tabular}{ll}
\hline Methods & RCT \\
\hline Participants & Inclusion criteria: age 12 to 19, clinical levels of anxiety acquired brain injury \\
\hline Interventions & CBT \\
\hline Outcomes & Primary: SCAS, SCARED, ADIS \\
\hline Notes & \\
\hline
\end{tabular}

\section{ACTRN12615000730505}

\begin{tabular}{ll}
\hline Methods & RCT. CBT vs waitlist \\
\hline Participants & Inclusion criteria: anxiety disorder, sleep problems \\
& Age: 13 to 17 \\
\hline Interventions & Cognitive behaviour therapy for adolescents with anxiety and sleep-related problems \\
\hline Outcomes & Primary: ADIS (anxiety). Insomnia (Insomnia Severity Index) \\
\hline Notes & Status: not yet recruiting \\
\hline
\end{tabular}

\section{ACTRN12619000619145}

\begin{tabular}{ll}
\hline Methods & RCT \\
\hline Participants & Target: $\mathrm{N}=80$ \\
& Inclusion criteria: age 10 to 14; diagnosed with diabetes, clinical or subclinical anxiety \\
\hline Interventions & CBT \\
& Waitlist \\
\hline Outcomes & Primary: YAM-5 \\
\hline Notes & Not yet recruiting \\
\hline
\end{tabular}


DRKS00012823

\begin{tabular}{ll}
\hline Methods & RCT \\
\hline Participants & Target: $\mathrm{N}=420$ \\
& Inclusion criteria: depressive disorder or anxiety disorder; age 6 to 18
\end{tabular}

Interventions CBT

Psychodynamic psychotherapy

Outcomes Primary: number of psychiatric diagnoses

\section{Notes}

\section{IRCT20181006041250N}

\begin{tabular}{ll}
\hline Methods & RCT \\
\hline Participants & $\begin{array}{l}\text { Inclusion criteria: female; age 8 to 12; anxiety symptoms (SCAS cut-off), mild to moderate anxiety } \\
\text { disorder not requiring drug therapy }\end{array}$ \\
\hline Interventions & CBT: group, based on Coping Cat \\
& Control: group, storytelling \\
\hline Outcomes & SCAS, SDQ, CDI \\
\hline Notes & Complete \\
\hline
\end{tabular}

\section{ISRCTN59518816}

\begin{tabular}{ll}
\hline Methods & RCT \\
\hline Participants & Target: $\mathrm{N}=228$ \\
& $\begin{array}{l}\text { Inclusion criteria: aged } 8 \text { to 12; Attention Deficit Hyperactivity Disorder; anxiety disorder (gener- } \\
\text { alised anxiety disorder, social anxiety disorder, separation anxiety disorder) }\end{array}$ \\
\hline Interventions & CBT \\
\hline Outcomes & Treatment As Usual \\
\hline Notes & Remission of anxiety disorder (GAD, social anxiety disorder, separation anxiety disorder) \\
\hline
\end{tabular}

\section{JPRN-UMIN000008724}

Methods RCT




\begin{tabular}{|c|c|}
\hline \multicolumn{2}{|c|}{ JPRN-UMIN000008724 (Continued) } \\
\hline Participants & $\begin{array}{l}\text { Inclusion criteria: age } 7 \text { to } 18 \text {; anxiety disorder (based on ADIS); discontinue other psychotherapy } \\
\text { for the trial }\end{array}$ \\
\hline \multirow[t]{2}{*}{ Interventions } & CBT \\
\hline & Control (no treatment) \\
\hline Outcomes & Primary: ADIS \\
\hline \multicolumn{2}{|l|}{ Notes } \\
\hline \multicolumn{2}{|c|}{ JPRN-UMIN000032275 } \\
\hline Methods & $\mathrm{RCT}$ \\
\hline Participants & $\begin{array}{l}\text { Inclusion criteria: age } 9 \text { to } 15 \text {; clinical or subclinical diagnosis of anxiety disorder or depressive dis- } \\
\text { order }\end{array}$ \\
\hline \multirow[t]{2}{*}{ Interventions } & CBT \\
\hline & Control: no treatment \\
\hline Outcomes & Primary: ADIS \\
\hline Notes & \\
\hline
\end{tabular}

\section{JPRN-UMIN000033218}

\begin{tabular}{ll}
\hline Methods & RCT \\
\hline Participants & Target: $\mathrm{N}=60$ \\
& $\begin{array}{l}\text { Inclusion criteria: age } 10 \text { to } 16 ; \text { IQ in average range; Autism Spectrum Disorder diagnosis; clinical } \\
\text { level of anxiety (semi-structured interview) }\end{array}$ \\
\hline Interventions & CBT \\
\hline Outcomes & Active control \\
\hline Notes & Primary: anxiety symptoms \\
\hline
\end{tabular}

\section{NCT02810171}

\begin{tabular}{ll}
\hline Methods & RCT \\
\hline Participants & Target: $\mathrm{N}=280$ \\
& $\begin{array}{l}\text { Inclusion: age } 7 \text { to } 17 \text {; clinically significant anxiety as determined by structured clinical interview; } \\
\text { anxiety primary concern }\end{array}$ \\
\hline
\end{tabular}




\section{NCT02810171 (Continued)}

Interventions CBT

Relaxation Therapy

\begin{tabular}{ll}
\hline Outcomes & Primary: brain function/structure \\
& Secondary: Pediatric Anxiety Rating Scale \\
\hline Notes & Started December 2016 \\
\hline
\end{tabular}

\section{NCT02977962}

\begin{tabular}{ll}
\hline Methods & RCT \\
\hline Participants & Target: $\mathrm{N}=44$ \\
& $\begin{array}{l}\text { Inclusion criteria: age } 16 \text { to } 21 ; \text { ASD; diagnosis of } 1 \text { of the following anxiety disorders: separation } \\
\text { anxiety disorder, specific phobia, panic disorder, generalised anxiety disorder, social phobia, or ob- } \\
\text { sessive compulsive disorder, and minimum score of } 14 \text { on the Hamilton Anxiety Scale }\end{array}$
\end{tabular}

\begin{tabular}{ll}
\hline Interventions & CBT
\end{tabular}

TAU

\begin{tabular}{ll}
\hline Outcomes & Primary: Hamilton Anxiety Scale (change from baseline) \\
\hline Notes & Not all participants would be eligible. \\
\hline
\end{tabular}

\section{NCT03412227}

\begin{tabular}{ll}
\hline Methods & RCT \\
\hline Participants & Target: $\mathrm{N}=200$ \\
& Inclusion criteria: age 9 to 17 ; primary DSM-5 anxiety disorder or depressive disorder \\
\hline Interventions & Individual Behavioral Activation Therapy (IBAT) \\
& The PASCET Program for Youth Depressive Disorders \\
& Coping Cat (CBT) \\
& Waitlist
\end{tabular}

Outcomes Primary: Clinical Global Impression Severity (CGIS) scale (change)

Notes

\section{NCT03899948}

\begin{tabular}{ll}
\hline Methods & RCT \\
\hline Participants & Target N $=135$ \\
\hline
\end{tabular}

Cognitive behavioural therapy for anxiety disorders in children and adolescents (Review)

Copyright $\odot 2020$ The Cochrane Collaboration. Published by John Wiley \& Sons, Ltd. 
NCT03899948 (Continued)

Inclusion criteria: attend elementary school, elevated anxiety symptoms and Clinical Severity Rating $3+$ on ADIS

\begin{tabular}{ll}
\hline Interventions & Teacher Anxiety Program for Elementary Students (TAPES) \\
& Teacher Anxiety Training (TAT)
\end{tabular}

Teacher Anxiety Training (TAT)

Outcomes Secondary: change on modified ADIS

Notes Anxiety diagnosis not required.

ADIS: Anxiety Disorder Interview Schedule for Children

CBT: cognitive behavioural therapy

CDI: Children's Depression Inventory

IQ: intelligence quotient

RCT: randomised controlled trial

SCARED: Screen for Child Anxiety Related Emotional Disorders

SCAS: Spence Children's Anxiety Scale

SDQ: Strengths and Difficulties Questionnaire

YAM-5: Youth Anxiety Scale for DSM-5

Characteristics of ongoing studies [ordered by study ID]

\section{ACTRN12616001552471} $\begin{array}{ll}\text { Study name } & \text { Effectiveness of school group-based acceptance and commitment therapy for children with anxi- } \\ \text { ety: a randomised controlled trial }\end{array}$

\begin{tabular}{|c|c|}
\hline Methods & $\mathrm{RCT}$ \\
\hline \multirow[t]{2}{*}{ Participants } & Target $\mathrm{N}=130$ \\
\hline & $\begin{array}{l}\text { Inclusion criteria: } 1 \text {. Aged between } 7 \text { and } 17 \text { years. } 2 \text {. Meet criteria for a primary diagnosis of an anx- } \\
\text { iety disorder. } 3 \text {. Available/able to attend The Children's Hospital at Westmead for pretreatment, } \\
\text { immediate post-treatment, and } 6 \text {-month post-treatment assessments as well as attending a min- } \\
\text { imum of } 80 \% \text { of therapy sessions. } 4 \text {. Have a parent/caregiver who is willing to attend and partici- } \\
\text { pate in the assessment as well as } 2 \text { parent sessions }\end{array}$ \\
\hline Interventions & $\begin{array}{l}\text { Proactive (Acceptance and Commitment Therapy group-based manualised therapy programme } \\
\text { delivered by school counsellors) vs waitlist ( } 10 \text { weeks) }\end{array}$ \\
\hline \multirow[t]{3}{*}{ Outcomes } & Primary outcomes: \\
\hline & $\begin{array}{l}\text { Change in score on the Pediatric Anxiety Rating Scale (PARS) - clinician, child, and parent rated } \\
\text { (pre-, post-treatment and } 6 \text { months post-treatment) }\end{array}$ \\
\hline & $\begin{array}{l}\text { Change in the Spence Anxiety Scale for Children (SCAS) - child and parent rated (pre-, post-treat- } \\
\text { ment and } 6 \text { months post-treatment) }\end{array}$ \\
\hline Starting date & 28 September 2016 \\
\hline \multirow[t]{4}{*}{ Contact information } & Dr Karen Hancock \\
\hline & SCHN, CHW Department of Psychological Medicine Locked Bag 4001 Westmead NSW 2145 \\
\hline & Australia \\
\hline & karen.hancock@health.nsw.gov.au \\
\hline Notes & www.who.int/trialsearch/Trial2.aspx?TrialID=ACTRN12616001552471 \\
\hline
\end{tabular}

Cognitive behavioural therapy for anxiety disorders in children and adolescents (Review) 
NCT02725619

\begin{tabular}{|c|c|}
\hline Study name & Neural mechanisms of CBT for anxiety in children with autism: randomized controlled trial \\
\hline Methods & $\mathrm{RCT}$ \\
\hline \multirow[t]{2}{*}{ Participants } & Target $\mathrm{N}=150$ \\
\hline & $\begin{array}{l}\text { Inclusion criteria: aged } 8 \text { to } 14 \text {; diagnosis of autism spectrum disorder; diagnosis of anxiety disor- } \\
\text { der and clinically significant level of anxiety; full-scale IQ and verbal IQ > 70; unmedicated or on sta- } \\
\text { ble medication with no planned changes for duration of study; able to meet MRI safety (e.g. no met- } \\
\text { al medical implants) and data quality requirements (e.g. able to keep head still during scanning) }\end{array}$ \\
\hline
\end{tabular}

\begin{tabular}{ll}
\hline Outcomes & Primary outcomes: \\
& $\begin{array}{l}\text { Pediatric Anxiety Rating Scale (PARS) (change from baseline - midpoint; change from baseline - } \\
\text { endpoint) } \\
\text { Clinical Global Impression-Improvement (CGI-I) scale (change from baseline - midpoint; change } \\
\text { from baseline - endpoint) } \\
\text { Blood oxygenation level dependent signal (BOLD) during down-regulation versus passive viewing } \\
\text { of affective images (change from baseline - midpoint; change from baseline - endpoint) }\end{array}$ \\
\hline Starting date & April 2016 \\
\hline Contact information & $\begin{array}{l}\text { Denis Sukhodolsky } \\
\text { Yale Child Study Center } \\
\text { New Haven, Connecticut, USA } \\
\text { denis.sukhodolsky@yale.edu }\end{array}$ \\
\hline clinicaltrials.gov/show/nct02725619
\end{tabular}

\section{NCT02908321}

\begin{tabular}{ll}
\hline Study name & $\begin{array}{l}\text { Cognitive behavioural therapy for anxiety disorder in children with co-morbid autism spectrum dis- } \\
\text { order }\end{array}$ \\
\hline Methods & RCT \\
\hline Participants & Target N = 50 \\
& $\begin{array}{l}\text { Inclusion criteria: aged } 7 \text { to 14; diagnosed with Autism Spectrum Disorder and experiencing anxiety } \\
\text { symptoms impairing their quality of life }\end{array}$ \\
& Exclusion criteria: IQ under 70; not meeting diagnostic criteria for primary anxiety diagnosis on \\
& ADIS; active psychosis; untreated Attention Deficit Hyperactivity Disorder; families not able to fol- \\
low programme
\end{tabular}


NCT02908321 (Continued)

\begin{tabular}{ll} 
Interventions & CBT (Cool Kids ASD) vs waitlist \\
\hline Outcomes & Primary outcomes: ADIS (baseline, 14 weeks, 3 months) \\
\hline Starting date & August 2016 \\
\hline Contact information & Tina R Kilburn \\
& Centre for Child and Adolescent Psychiatry \\
& Risskov, Region Of Midtjylland, Denmark, 8240 \\
& tinakilb@rm.dk \\
\hline Notes & clinicaltrials.gov/show/nct02908321
\end{tabular}

\section{NCT03279471}

\begin{tabular}{|c|c|}
\hline Study name & Specifying and treating the anxiety phenotype in autism spectrum disorder \\
\hline Methods & $\mathrm{RCT}$ \\
\hline \multirow[t]{2}{*}{ Participants } & Target $\mathrm{N}=132$ \\
\hline & $\begin{array}{l}\text { Inclusion criteria: age } 8 \text { to 14; meets criteria for a diagnosis of ASD; clinically significant anxiety } \\
\text { symptoms (minimum score of } 8 \text { on the Pediatric Anxiety Rating Scale); clinically significant anxiety } \\
\text { symptoms as defined by qualifying for a diagnosis on } 1 \text { or more non-phobia items on the ADIS; full- } \\
\text { scale and verbal comprehension IQ greater than 50; anxiety symptoms are considered the prima- } \\
\text { ry mental health problem (i.e. most impairing/distressing); stable medication, non-psychotherapy } \\
\text { and psychosocial regimen }\end{array}$ \\
\hline Interventions & CBT vs drug (sertraline) vs pill placebo \\
\hline Outcomes & $\begin{array}{l}\text { Primary outcome: change in Pediatric Anxiety Rating Scale (pretreatment, post-treatment, 3- } \\
\text { month follow-up) }\end{array}$ \\
\hline Starting date & 1 November 2017 \\
\hline \multirow[t]{4}{*}{ Contact information } & Brianna Health, PhD \\
\hline & UC Davis MIND Institute \\
\hline & Sacramento, California, USA, 95817 \\
\hline & bheath@ucdavis.edu \\
\hline Notes & clinicaltrials.gov/ct2/show/nct03279471 \\
\hline
\end{tabular}

\section{NCT03585010}

\begin{tabular}{ll}
\hline Study name & Brain response associated with parent-based treatment for childhood anxiety disorders \\
\hline Methods & RCT \\
\hline Participants & Target $\mathrm{N}=226$ \\
\hline
\end{tabular}


NCT03585010 (Continued)

Inclusion criteria: prepubertal; clinical diagnosis of primary anxiety disorder; must not have anoth-

er mental illness more impairing than the most impairing anxiety disorder; IQ of at least 80

\begin{tabular}{ll}
\hline Interventions & Supportive Parenting for Anxious Child Emotions vs CBT vs parent educational support \\
\hline Outcomes & Primary outcome: Pediatric Anxiety Rating Scale (PARS) (12 weeks) \\
& $\begin{array}{l}\text { Secondary outcome: Multidimensional Anxiety Scale for Children (MASC) parent and child report } \\
(12 \text { weeks) }\end{array}$ \\
\hline Starting date & 1 September 2018 \\
\hline Contact information & Eli R Lebowitz, PhD \\
& Yale University Child Study Center \\
New Haven, Connecticut, USA, 06519 \\
eli.lebowitz@yale.edu \\
\hline clinicaltrials.gov/ct2/show/NCT03585010
\end{tabular}

ADIS: Anxiety Disorder Interview Schedule for Children

CBT: cognitive behavioural therapy

IQ: intelligence quotient

MRI: magnetic resonance imaging

RCT: randomised controlled trial

\section{DATA AND ANALYSES}

\section{Comparison 1. CBT versus waitlist/no treatment}

\begin{tabular}{|c|c|c|c|c|}
\hline Outcome or subgroup title & $\begin{array}{l}\text { No. of } \\
\text { studies }\end{array}$ & $\begin{array}{l}\text { No. of } \\
\text { partici- } \\
\text { pants }\end{array}$ & Statistical method & Effect size \\
\hline $\begin{array}{l}\text { 1.1 Remission of primary anxiety diagnosis } \\
\text { post-treatment (ITT) }\end{array}$ & 39 & 2697 & Odds Ratio (IV, Random, $95 \% \mathrm{CI}$ ) & $5.45[3.90,7.60]$ \\
\hline 1.1.1 Child focused & 19 & 1184 & Odds Ratio (IV, Random, 95\% CI) & $\begin{array}{l}10.42[5.84 \\
18.58]\end{array}$ \\
\hline 1.1.2 Child and parent & 19 & 1142 & Odds Ratio (IV, Random, 95\% CI) & $4.08[2.72,6.11]$ \\
\hline 1.1.3 Parent only & 4 & 371 & Odds Ratio (IV, Random, 95\% Cl) & $2.83[1.12,7.16]$ \\
\hline $\begin{array}{l}\text { 1.2 Subgroup analysis: remission of prima- } \\
\text { ry anxiety diagnosis post-treatment (ITT) } \\
\text { (individual vs group) }\end{array}$ & 39 & & Odds Ratio (IV, Random, $95 \% \mathrm{Cl}$ ) & Subtotals only \\
\hline 1.2.1 Individual focused & 17 & 1165 & Odds Ratio (IV, Random, 95\% Cl) & $4.53[2.55,8.03]$ \\
\hline 1.2.2 Group focused & 25 & 1532 & Odds Ratio (IV, Random, 95\% CI) & $6.25[4.45,8.78]$ \\
\hline
\end{tabular}




\begin{tabular}{|c|c|c|c|c|}
\hline Outcome or subgroup title & $\begin{array}{l}\text { No. of } \\
\text { studies }\end{array}$ & $\begin{array}{l}\text { No. of } \\
\text { partici- } \\
\text { pants }\end{array}$ & Statistical method & Effect size \\
\hline $\begin{array}{l}1.3 \text { Subgroup analysis: remission of prima- } \\
\text { ry anxiety diagnosis post-treatment (ITT) } \\
\text { (amount of therapist contact time) }\end{array}$ & 39 & & Odds Ratio (IV, Random, 95\% CI) & Subtotals only \\
\hline 1.3.1 Therapist contact ( $<10$ hours) & 6 & 513 & Odds Ratio (IV, Random, 95\% CI) & $6.12[2.08,18.00]$ \\
\hline $\begin{array}{l}\text { 1.3.2 Therapist contact ( } \geq 10 \text { and }<20 \\
\text { hours) }\end{array}$ & 23 & 1622 & Odds Ratio (IV, Random, 95\% CI) & $5.85[3.95,8.64]$ \\
\hline 1.3.3 Therapist contact ( $\geq 20$ hours) & 13 & 562 & Odds Ratio (IV, Random, 95\% CI) & $4.44[2.26,8.72]$ \\
\hline $\begin{array}{l}\text { 1.4 Subgroup analysis: remission of prima- } \\
\text { ry anxiety diagnosis post-treatment (ITT) } \\
\text { (age) }\end{array}$ & 39 & & Odds Ratio (IV, Random, 95\% CI) & Subtotals only \\
\hline $1.4 .1 \leq 12$ years & 11 & 789 & Odds Ratio (IV, Random, 95\% CI) & $3.93[2.20,7.02]$ \\
\hline 1.4.2 Mixed age range ( $<12$ and $\geq 12$ years) & 21 & 1447 & Odds Ratio (IV, Random, 95\% Cl) & $6.43[3.90,10.58]$ \\
\hline $1.4 .3 \geq 12$ years & 7 & 461 & Odds Ratio (IV, Random, 95\% CI) & $5.28[2.83,9.86]$ \\
\hline $\begin{array}{l}1.5 \text { Subgroup analysis: remission of prima- } \\
\text { ry anxiety diagnosis post-treatment (ITT) } \\
\text { (ASD vs non-ASD) }\end{array}$ & 39 & & Odds Ratio (IV, Random, 95\% CI) & Subtotals only \\
\hline 1.5.1 Sample with ASD & 4 & 136 & Odds Ratio (IV, Random, 95\% CI) & $1.79[0.39,8.16]$ \\
\hline 1.5.2 Not sample with ASD & 35 & 2561 & Odds Ratio (IV, Random, 95\% CI) & $5.88[4.19,8.24]$ \\
\hline $\begin{array}{l}\text { 1.6 Remission of primary anxiety diagnosis } \\
\text { post-treatment (completers) }\end{array}$ & 38 & 2406 & Odds Ratio (IV, Random, 95\% CI) & $\begin{array}{l}11.55[8.41 \\
15.86]\end{array}$ \\
\hline $\begin{array}{l}1.7 \text { Acceptability (number of participants } \\
\text { lost to post-treatment assessment) }\end{array}$ & 45 & 3158 & Odds Ratio (IV, Random, 95\% CI) & $1.09[0.85,1.41]$ \\
\hline $\begin{array}{l}1.8 \text { Remission of all anxiety diagnoses post- } \\
\text { treatment (ITT) }\end{array}$ & 28 & 2075 & Odds Ratio (IV, Random, 95\% CI) & $4.43[2.89,6.78]$ \\
\hline 1.8.1 Child focused & 11 & 564 & Odds Ratio (IV, Random, 95\% CI) & $8.52[2.97,24.39]$ \\
\hline 1.8.2 Child and parent & 15 & 916 & Odds Ratio (IV, Random, 95\% CI) & $5.19[3.26,8.27]$ \\
\hline 1.8.3 Parent only & 7 & 595 & Odds Ratio (IV, Random, 95\% CI) & $1.89[0.98,3.65]$ \\
\hline $\begin{array}{l}1.9 \text { Subgroup analysis: remission of all anx- } \\
\text { iety diagnoses post-treatment (ITT) (indi- } \\
\text { vidual vs group) }\end{array}$ & 28 & & Odds Ratio (IV, Random, 95\% CI) & Subtotals only \\
\hline 1.9.1 Individual focused & 9 & 671 & Odds Ratio (IV, Random, 95\% CI) & $3.72[1.70,8.12]$ \\
\hline 1.9.2 Group focused & 20 & 1404 & Odds Ratio (IV, Random, 95\% CI) & $4.92[2.95,8.20]$ \\
\hline
\end{tabular}




\begin{tabular}{|c|c|c|c|c|}
\hline Outcome or subgroup title & $\begin{array}{l}\text { No. of } \\
\text { studies }\end{array}$ & $\begin{array}{l}\text { No. of } \\
\text { partici- } \\
\text { pants }\end{array}$ & Statistical method & Effect size \\
\hline $\begin{array}{l}\text { 1.10 Subgroup analysis: remission of all } \\
\text { anxiety diagnoses post-treatment (ITT) } \\
\text { (amount of therapist contact time) }\end{array}$ & 28 & & Odds Ratio (IV, Random, 95\% Cl) & Subtotals only \\
\hline 1.10.1 Therapist contact $(<10$ hours) & 5 & 461 & Odds Ratio (IV, Random, 95\% CI) & $1.42[0.68,2.96]$ \\
\hline $\begin{array}{l}1.10 .2 \text { Therapist contact ( } \geq 10 \text { and }<20 \\
\text { hours) }\end{array}$ & 17 & 1123 & Odds Ratio (IV, Random, 95\% CI) & $6.59[3.62,12.01]$ \\
\hline 1.10.3 Therapist contact ( $\geq 20$ hours) & 9 & 491 & Odds Ratio (IV, Random, 95\% Cl) & $5.03[2.55,9.93]$ \\
\hline $\begin{array}{l}\text { 1.11 Subgroup analysis: remission of all } \\
\text { anxiety diagnoses post-treatment (ITT) } \\
\text { (age) }\end{array}$ & 28 & & Odds Ratio (IV, Random, 95\% CI) & Subtotals only \\
\hline $1.11 .1 \leq 12$ years & 10 & 950 & Odds Ratio (IV, Random, 95\% CI) & $3.59[1.97,6.56]$ \\
\hline $\begin{array}{l}1.11 .2 \text { Mixed age range }(<12 \text { years and } \geq 12 \\
\text { years) }\end{array}$ & 15 & 980 & Odds Ratio (IV, Random, 95\% Cl) & $4.87[2.58,9.21]$ \\
\hline $1.11 .3 \geq 12$ years & 3 & 145 & Odds Ratio (IV, Random, 95\% Cl) & $8.48[0.79,91.08]$ \\
\hline $\begin{array}{l}1.12 \text { Remission of all anxiety diagnoses } \\
\text { post-treatment (completers) }\end{array}$ & 27 & 1871 & Odds Ratio (IV, Random, 95\% Cl) & $9.13[5.78,14.41]$ \\
\hline $\begin{array}{l}1.13 \text { Reduction in anxiety symptoms (child } \\
\text { report) post-treatment }\end{array}$ & 45 & 2831 & $\begin{array}{l}\text { Std. Mean Difference (IV, Random, 95\% } \\
\mathrm{Cl} \text { ) }\end{array}$ & $\begin{array}{l}-0.67[-0.88 \\
-0.47]\end{array}$ \\
\hline 1.13.1 Child focused & 24 & 1239 & $\begin{array}{l}\text { Std. Mean Difference (IV, Random, 95\% } \\
\mathrm{Cl} \text { ) }\end{array}$ & $\begin{array}{l}-1.04[-1.41 \\
-0.67]\end{array}$ \\
\hline 1.13.2 Child and parent & 20 & 1285 & $\begin{array}{l}\text { Std. Mean Difference (IV, Random, 95\% } \\
\mathrm{CI} \text { ) }\end{array}$ & $\begin{array}{l}-0.45[-0.67 \\
-0.23]\end{array}$ \\
\hline 1.13.3 Parent only & 5 & 307 & $\begin{array}{l}\text { Std. Mean Difference (IV, Random, 95\% } \\
\mathrm{Cl} \text { ) }\end{array}$ & $0.04[-0.38,0.46]$ \\
\hline $\begin{array}{l}\text { 1.14 Sensitivity analysis: reduction in anx- } \\
\text { iety symptoms (child report) post-treat- } \\
\text { ment (broad anxiety measures) }\end{array}$ & 38 & 2459 & $\begin{array}{l}\text { Std. Mean Difference (IV, Random, 95\% } \\
\mathrm{Cl} \text { ) }\end{array}$ & $\begin{array}{l}-0.41[-0.57 \\
-0.25]\end{array}$ \\
\hline
\end{tabular}

\begin{tabular}{|c|c|c|c|}
\hline $\begin{array}{l}1.15 \text { Subgroup analysis: reduction in anx } \\
\text { iety symptoms (child report) post-treat- }\end{array}$ & 45 & $\begin{array}{l}\text { Std. Mean Difference (IV, Random, 95\% } \\
\mathrm{CI} \text { ) }\end{array}$ & Subtotals only \\
\hline
\end{tabular}
ment (individual vs group)

\begin{tabular}{|c|c|c|c|c|}
\hline 1.15.1 Individual focused & 21 & 1203 & $\begin{array}{l}\text { Std. Mean Difference (IV, Random, 95\% } \\
\mathrm{Cl} \text { ) }\end{array}$ & $\begin{array}{l}-0.39[-0.64 \\
-0.15]\end{array}$ \\
\hline 1.15.2 Group focused & 27 & 1628 & $\begin{array}{l}\text { Std. Mean Difference (IV, Random, 95\% } \\
\mathrm{CI} \text { ) }\end{array}$ & $\begin{array}{l}-0.91[-1.22 \\
-0.60]\end{array}$ \\
\hline $\begin{array}{l}1.16 \text { Subgroup analysis: reduction in anx- } \\
\text { iety symptoms (child report) post-treat- } \\
\text { ment (amount of therapist contact time) }\end{array}$ & 45 & & $\begin{array}{l}\text { Std. Mean Difference (IV, Random, 95\% } \\
\mathrm{Cl} \text { ) }\end{array}$ & Subtotals only \\
\hline
\end{tabular}




\begin{tabular}{|c|c|c|c|c|}
\hline Outcome or subgroup title & $\begin{array}{l}\text { No. of } \\
\text { studies }\end{array}$ & $\begin{array}{l}\text { No. of } \\
\text { partici- } \\
\text { pants }\end{array}$ & Statistical method & Effect size \\
\hline 1.16.1 Therapist contact ( $<10$ hours) & 9 & 587 & $\begin{array}{l}\text { Std. Mean Difference (IV, Random, 95\% } \\
\mathrm{CI} \text { ) }\end{array}$ & $-0.36[-0.75,0.03]$ \\
\hline $\begin{array}{l}1.16 .2 \text { Therapist contact ( } \geq 10 \text { and }<20 \\
\text { hours) }\end{array}$ & 29 & 1840 & $\begin{array}{l}\text { Std. Mean Difference (IV, Random, 95\% } \\
\mathrm{Cl} \text { ) }\end{array}$ & $\begin{array}{l}-0.81[-1.09 \\
-0.52]\end{array}$ \\
\hline 1.16.3 Therapist contact ( $\geq 20$ hours) & 10 & 404 & $\begin{array}{l}\text { Std. Mean Difference (IV, Random, 95\% } \\
\mathrm{CI} \text { ) }\end{array}$ & $\begin{array}{l}-0.62[-1.06 \\
-0.18]\end{array}$ \\
\hline $\begin{array}{l}1.17 \text { Subgroup analysis: reduction in anx- } \\
\text { iety symptoms (child report) post-treat- } \\
\text { ment (age) }\end{array}$ & 45 & & $\begin{array}{l}\text { Std. Mean Difference (IV, Random, 95\% } \\
\mathrm{CI} \text { ) }\end{array}$ & Subtotals only \\
\hline $1.17 .1 \leq 12$ years & 11 & 663 & $\begin{array}{l}\text { Std. Mean Difference (IV, Random, 95\% } \\
\mathrm{CI} \text { ) }\end{array}$ & $-0.23[-0.54,0.08]$ \\
\hline $\begin{array}{l}\text { 1.17.2 Mixed age range }(<12 \text { years and } \geq 12 \\
\text { years) }\end{array}$ & 28 & 1834 & $\begin{array}{l}\text { Std. Mean Difference (IV, Random, 95\% } \\
\mathrm{Cl} \text { ) }\end{array}$ & $\begin{array}{l}-0.62[-0.83 \\
-0.41]\end{array}$ \\
\hline $1.17 .3 \geq 12$ years & 6 & 334 & $\begin{array}{l}\text { Std. Mean Difference (IV, Random, 95\% } \\
\mathrm{Cl} \text { ) }\end{array}$ & $\begin{array}{l}-1.78[-3.01 \\
-0.56]\end{array}$ \\
\hline $\begin{array}{l}1.18 \text { Subgroup analysis: reduction in anx- } \\
\text { iety symptoms (child report) post-treat- } \\
\text { ment (ASD vs non-ASD) }\end{array}$ & 45 & & $\begin{array}{l}\text { Std. Mean Difference (IV, Random, 95\% } \\
\mathrm{CI} \text { ) }\end{array}$ & Subtotals only \\
\hline 1.18.1 Sample with ASD & 5 & 181 & $\begin{array}{l}\text { Std. Mean Difference (IV, Random, 95\% } \\
\mathrm{Cl} \text { ) }\end{array}$ & $-0.61[-1.54,0.33]$ \\
\hline 1.18.2 Not sample with ASD & 40 & 2650 & $\begin{array}{l}\text { Std. Mean Difference (IV, Random, 95\% } \\
\mathrm{Cl} \text { ) }\end{array}$ & $\begin{array}{l}-0.68[-0.90 \\
-0.47]\end{array}$ \\
\hline $\begin{array}{l}\text { 1.19 Reduction in anxiety symptoms (par- } \\
\text { ent report) post-treatment }\end{array}$ & 35 & 2137 & $\begin{array}{l}\text { Std. Mean Difference (IV, Random, 95\% } \\
\mathrm{Cl} \text { ) }\end{array}$ & $\begin{array}{l}-0.70[-0.90 \\
-0.51]\end{array}$ \\
\hline 1.19.1 Child focused & 13 & 734 & $\begin{array}{l}\text { Std. Mean Difference (IV, Random, 95\% } \\
\mathrm{CI} \text { ) }\end{array}$ & $\begin{array}{l}-0.87[-1.21 \\
-0.53]\end{array}$ \\
\hline 1.19.2 Child and parent & 17 & 1031 & $\begin{array}{l}\text { Std. Mean Difference (IV, Random, 95\% } \\
\mathrm{CI})\end{array}$ & $\begin{array}{l}-0.69[-0.98 \\
-0.39]\end{array}$ \\
\hline 1.19.3 Parent only & 5 & 372 & $\begin{array}{l}\text { Std. Mean Difference (IV, Random, 95\% } \\
\mathrm{Cl} \text { ) }\end{array}$ & $-0.37[-0.77,0.04]$ \\
\hline $\begin{array}{l}1.20 \text { Sensitivity analysis: reduction in anx- } \\
\text { iety symptoms (parent report) post-treat- } \\
\text { ment (broad anxiety measures only) }\end{array}$ & 32 & 1952 & $\begin{array}{l}\text { Std. Mean Difference (IV, Random, 95\% } \\
\mathrm{Cl} \text { ) }\end{array}$ & $\begin{array}{l}-0.59[-0.77 \\
-0.41]\end{array}$ \\
\hline $\begin{array}{l}1.21 \text { Subgroup analysis: reduction in anx- } \\
\text { iety symptoms (parent report) post-treat- } \\
\text { ment (individual vs group) }\end{array}$ & 35 & & $\begin{array}{l}\text { Std. Mean Difference (IV, Random, 95\% } \\
\mathrm{CI} \text { ) }\end{array}$ & Subtotals only \\
\hline 1.21.1 Individual focused & 17 & 858 & $\begin{array}{l}\text { Std. Mean Difference (IV, Random, 95\% } \\
\mathrm{Cl} \text { ) }\end{array}$ & $\begin{array}{l}-0.43[-0.65 \\
-0.21]\end{array}$ \\
\hline
\end{tabular}




\begin{tabular}{|c|c|c|c|c|}
\hline Outcome or subgroup title & $\begin{array}{l}\text { No. of } \\
\text { studies }\end{array}$ & $\begin{array}{l}\text { No. of } \\
\text { partici- } \\
\text { pants }\end{array}$ & Statistical method & Effect size \\
\hline 1.21.2 Group focused & 21 & 1279 & $\begin{array}{l}\text { Std. Mean Difference (IV, Random, 95\% } \\
\mathrm{CI})\end{array}$ & $\begin{array}{l}-0.92[-1.21 \\
-0.62]\end{array}$ \\
\hline $\begin{array}{l}1.22 \text { Subgroup analysis: reduction in anx- } \\
\text { iety symptoms (parent report) post-treat- } \\
\text { ment (amount of therapist contact time) }\end{array}$ & 35 & & $\begin{array}{l}\text { Std. Mean Difference (IV, Random, 95\% } \\
\mathrm{Cl} \text { ) }\end{array}$ & Subtotals only \\
\hline 1.22.1 Therapist contact ( $<10$ hours) & 4 & 336 & $\begin{array}{l}\text { Std. Mean Difference (IV, Random, 95\% } \\
\mathrm{CI} \text { ) }\end{array}$ & $-0.36[-0.81,0.10]$ \\
\hline $\begin{array}{l}\text { 1.22.2 Therapist contact ( } \geq 10 \text { and }<20 \\
\text { hours) }\end{array}$ & 22 & 1402 & $\begin{array}{l}\text { Std. Mean Difference (IV, Random, 95\% } \\
\mathrm{CI} \text { ) }\end{array}$ & $\begin{array}{l}-0.66[-0.89 \\
-0.43]\end{array}$ \\
\hline 1.22.3 Therapist contact ( $\geq 20$ hours) & 10 & 399 & $\begin{array}{l}\text { Std. Mean Difference (IV, Random, 95\% } \\
\mathrm{CI} \text { ) }\end{array}$ & $\begin{array}{l}-1.03[-1.55 \\
-0.52]\end{array}$ \\
\hline $\begin{array}{l}1.23 \text { Subgroup analysis: reduction in anx- } \\
\text { iety symptoms (parent report) post-treat- } \\
\text { ment (age) }\end{array}$ & 35 & & $\begin{array}{l}\text { Std. Mean Difference (IV, Random, 95\% } \\
\mathrm{CI} \text { ) }\end{array}$ & Subtotals only \\
\hline $1.23 .1 \leq 12$ years & 11 & 750 & $\begin{array}{l}\text { Std. Mean Difference (IV, Random, 95\% } \\
\mathrm{CI} \text { ) }\end{array}$ & $\begin{array}{l}-0.40[-0.63 \\
-0.17]\end{array}$ \\
\hline $\begin{array}{l}\text { 1.23.2 Mixed age range }(<12 \text { years and } \geq 12 \\
\text { years) }\end{array}$ & 18 & 1057 & $\begin{array}{l}\text { Std. Mean Difference (IV, Random, 95\% } \\
\mathrm{CI} \text { ) }\end{array}$ & $\begin{array}{l}-0.76[-1.04 \\
-0.49]\end{array}$ \\
\hline $1.23 .3 \geq 12$ years & 6 & 330 & $\begin{array}{l}\text { Std. Mean Difference (IV, Random, 95\% } \\
\mathrm{Cl} \text { ) }\end{array}$ & $\begin{array}{l}-1.07[-1.78 \\
-0.37]\end{array}$ \\
\hline $\begin{array}{l}1.24 \text { Subgroup analysis: reduction in anx- } \\
\text { iety symptoms (parent report) post-treat- } \\
\text { ment (ASD vs non-ASD) }\end{array}$ & 35 & & $\begin{array}{l}\text { Std. Mean Difference (IV, Random, 95\% } \\
\mathrm{CI} \text { ) }\end{array}$ & Subtotals only \\
\hline 1.24.1 Sample with ASD & 7 & 244 & $\begin{array}{l}\text { Std. Mean Difference (IV, Random, 95\% } \\
\mathrm{CI} \text { ) }\end{array}$ & $\begin{array}{l}-1.12[-1.91 \\
-0.34]\end{array}$ \\
\hline 1.24.2 Not sample with ASD & 28 & 1893 & $\begin{array}{l}\text { Std. Mean Difference (IV, Random, 95\% } \\
\mathrm{CI})\end{array}$ & $\begin{array}{l}-0.63[-0.82 \\
-0.44]\end{array}$ \\
\hline $\begin{array}{l}1.25 \text { Reduction in depressive symptoms } \\
\text { post-treatment }\end{array}$ & 17 & 1157 & $\begin{array}{l}\text { Std. Mean Difference (IV, Random, 95\% } \\
\mathrm{Cl} \text { ) }\end{array}$ & $\begin{array}{l}-0.34[-0.51 \\
-0.17]\end{array}$ \\
\hline $\begin{array}{l}1.26 \text { Improvement in global functioning } \\
\text { post-treatment }\end{array}$ & 11 & 557 & $\begin{array}{l}\text { Std. Mean Difference (IV, Random, 95\% } \\
\mathrm{Cl} \text { ) }\end{array}$ & $1.03[0.68,1.38]$ \\
\hline $\begin{array}{l}1.27 \text { Remission of primary anxiety diagno- } \\
\text { sis at follow-up (ITT) }\end{array}$ & 5 & & Odds Ratio (IV, Random, 95\% Cl) & Subtotals only \\
\hline 1.27.1 Follow-up ( $\leq 6$ months) & 4 & 153 & Odds Ratio (IV, Random, 95\% Cl) & $\begin{array}{l}10.94[2.33 \\
51.41]\end{array}$ \\
\hline 1.27.2 Follow-up ( $>6$ and $\leq 12$ months) & 3 & 166 & Odds Ratio (IV, Random, 95\% CI) & $2.80[0.24,33.19]$ \\
\hline
\end{tabular}




\begin{tabular}{|c|c|c|c|c|}
\hline Outcome or subgroup title & $\begin{array}{l}\text { No. of } \\
\text { studies }\end{array}$ & $\begin{array}{l}\text { No. of } \\
\text { partici- } \\
\text { pants }\end{array}$ & Statistical method & Effect size \\
\hline $\begin{array}{l}1.28 \text { Remission of primary anxiety diagno- } \\
\text { sis at follow-up (completers) }\end{array}$ & 5 & & Odds Ratio (IV, Random, 95\% Cl) & Subtotals only \\
\hline 1.28.1 Follow-up ( $\leq 6$ months) & 4 & 151 & Odds Ratio (IV, Random, 95\% Cl) & $\begin{array}{l}11.46[2.40 \\
54.67]\end{array}$ \\
\hline 1.28.2 Follow-up ( $>6$ and $\leq 12$ months) & 3 & 161 & Odds Ratio (IV, Random, 95\% CI) & $4.04[0.77,21.20]$ \\
\hline $\begin{array}{l}\text { 1.29 Remission of all anxiety diagnoses at } \\
\text { follow-up (ITT) }\end{array}$ & 5 & & Odds Ratio (IV, Random, 95\% Cl) & Subtotals only \\
\hline 1.29.1 Follow-up ( $\leq 6$ months) & 3 & 130 & Odds Ratio (IV, Random, 95\% Cl) & $8.25[0.69,98.10]$ \\
\hline 1.29.2 Follow-up (> 6 and $\leq 12$ months) & 3 & 166 & Odds Ratio (IV, Random, 95\% Cl) & $3.75[0.25,56.88]$ \\
\hline 1.29.3 Follow-up (> 12 months) & 2 & 131 & Odds Ratio (IV, Random, 95\% Cl) & $0.48[0.17,1.38]$ \\
\hline $\begin{array}{l}\text { 1.30 Remission of all anxiety diagnoses at } \\
\text { follow-up (completers) }\end{array}$ & 5 & & Odds Ratio (IV, Random, 95\% Cl) & Subtotals only \\
\hline 1.30.1 Follow-up ( $\leq 6$ months) & 3 & 128 & Odds Ratio (IV, Random, 95\% Cl) & $\begin{array}{l}8.68[0.72 \\
104.46]\end{array}$ \\
\hline 1.30.2 Follow-up ( $>6$ and $\leq 12$ months) & 3 & 161 & Odds Ratio (IV, Random, 95\% Cl) & $5.01[0.70,35.87]$ \\
\hline 1.30.3 Follow-up (> 12 months) & 2 & 114 & Odds Ratio (IV, Random, 95\% Cl) & $1.25[0.36,4.34]$ \\
\hline $\begin{array}{l}1.31 \text { Reduction in anxiety symptoms (child } \\
\text { report) at follow-up }\end{array}$ & 5 & & $\begin{array}{l}\text { Std. Mean Difference (IV, Random, 95\% } \\
\mathrm{Cl} \text { ) }\end{array}$ & Subtotals only \\
\hline 1.31.1 Follow-up ( $\leq 6$ months) & 5 & 179 & $\begin{array}{l}\text { Std. Mean Difference (IV, Random, 95\% } \\
\mathrm{CI})\end{array}$ & $\begin{array}{l}-1.92[-2.95 \\
-0.89]\end{array}$ \\
\hline $\begin{array}{l}\text { 1.32 Sensitivity analysis: remission of pri- } \\
\text { mary anxiety diagnosis post-treatment } \\
\text { (ITT) (only studies where sequence genera- } \\
\text { tion is clear) }\end{array}$ & 20 & 1476 & Odds Ratio (IV, Random, 95\% Cl) & $4.84[3.03,7.73]$ \\
\hline $\begin{array}{l}\text { 1.33 Sensitivity analysis: remission of pri- } \\
\text { mary anxiety diagnosis post-treatment } \\
\text { (ITT) (only studies where blind assessors } \\
\text { used) }\end{array}$ & 31 & 2037 & Odds Ratio (IV, Random, 95\% Cl) & $5.54[3.76,8.18]$ \\
\hline
\end{tabular}


Analysis 1.1. Comparison 1: CBT versus waitlist/no treatment, Outcome 1: Remission of primary anxiety diagnosis post-treatment (ITT)

\begin{tabular}{|c|c|c|c|c|c|c|c|c|}
\hline \multirow{3}{*}{$\frac{\text { Study or Subgroup }}{\text { 1.1.1 Child focused }}$} & \multicolumn{2}{|c|}{ CBT } & \multicolumn{2}{|c|}{ Wait list / no treatment } & \multirow{2}{*}{ Weight } & \multirow{2}{*}{$\begin{array}{c}\text { Odds Ratio } \\
\text { IV, Random, 95\% CI }\end{array}$} & \multirow{2}{*}{\multicolumn{2}{|c|}{$\begin{array}{c}\text { Odds Ratio } \\
\text { IV, Random, } 95 \% \text { CI }\end{array}$}} \\
\hline & \multirow{2}{*}{ Events } & \multirow{2}{*}{ Total } & \multirow{2}{*}{ Events } & \multirow{2}{*}{ Total } & & & & \\
\hline & & & & & & & & \\
\hline Dadds 1997 & 21 & 23 & 28 & 33 & $2.1 \%$ & $1.88[0.33,10.63]$ & & \\
\hline Flannery Schroeder 2000 & 9 & 18 & 2 & 7 & $1.9 \%$ & $2.50[0.38,16.42]$ & & \\
\hline Flannery Schroeder 2000 & 6 & 13 & 1 & 7 & $1.4 \%$ & $5.14[0.48,55.64]$ & & \\
\hline Gallagher 2004 & 5 & 12 & 0 & 11 & $1.0 \%$ & $16.87[0.81,351.79]$ & & \\
\hline Kendall 1994 & 17 & 40 & 1 & 20 & $1.6 \%$ & $14.04[1.71,115.41]$ & & \\
\hline Kendall 1997 & 32 & 69 & 17 & 49 & $3.8 \%$ & $1.63[0.77,3.46]$ & & $\leftarrow$ \\
\hline Lau 2010 & 14 & 21 & 0 & 17 & $1.0 \%$ & $67.67[3.56,1287.81]$ & & \\
\hline Masia Warner 2005 & 12 & 21 & 5 & 21 & $2.7 \%$ & $4.27[1.13,16.05]$ & & \\
\hline Masia Warner 2011 & 9 & 20 & 1 & 20 & $1.6 \%$ & $15.55[1.73,139.65]$ & & \\
\hline McNally Keehn 2013 & 7 & 12 & 0 & 10 & $1.0 \%$ & $28.64[1.37,600.41]$ & & \\
\hline Olivares 2005 & 10 & 17 & 0 & 17 & $1.0 \%$ & $49.00[2.53,948.62]$ & & \\
\hline Olivares 2014 & 11 & 39 & 2 & 19 & $2.2 \%$ & $3.34[0.66,16.92]$ & & \\
\hline Olivares 2014 & 20 & 39 & 2 & 20 & $2.3 \%$ & $9.47[1.93,46.46]$ & & \\
\hline Olivares 2019 & 8 & 36 & 0 & 18 & $1.0 \%$ & $11.04[0.60,202.89]$ & & \\
\hline Olivares 2019 & 13 & 36 & 0 & 18 & $1.0 \%$ & $21.26[1.18,381.56]$ & & \\
\hline Ollendick 2009 & 47 & 85 & 1 & 41 & $1.7 \%$ & $49.47[6.50,376.64]$ & & \\
\hline Ost 2001 & 18 & 21 & 0 & 10 & $1.0 \%$ & $111.00[5.21,2363.64]$ & & \\
\hline Perrin 2019 & 16 & 20 & 0 & 20 & $1.0 \%$ & $150.33[7.54,2997.83]$ & & \\
\hline Rosa Alcazar 2009 & 12 & 20 & 0 & 20 & $1.0 \%$ & $60.29[3.20,1137.79]$ & & \\
\hline Sanchez Garcia 2009 & 21 & 28 & 0 & 25 & $1.0 \%$ & $146.20[7.89,2709.82]$ & & \\
\hline Spence 2000 & 11 & 19 & 1 & 7 & $1.5 \%$ & $8.25[0.82,82.67]$ & & \\
\hline Villabo 2018 & 29 & 55 & 4 & 27 & $3.0 \%$ & $6.41[1.96,21.01]$ & & \\
\hline Villabo 2018 & 36 & 55 & 4 & 28 & $2.9 \%$ & $11.37[3.44,37.58]$ & & \\
\hline Subtotal (95\% CI) & & 719 & & 465 & $38.7 \%$ & $10.42[5.84,18.58]$ & & \\
\hline Total events: & 384 & & 69 & & & & & \\
\hline Heterogeneity: $\mathrm{Tau}^{2}=0.8$ & $\mathrm{i}^{2}=43.41$, & If $=22(\mathrm{P}$ & $0.004) ; I^{2}=4$ & & & & & \\
\hline Test for overall effect: $Z=$ & $(\mathrm{P}<0.000$ & & & & & & & \\
\hline 1.1.2 Child and parent & & & & & & & & \\
\hline Arendt 2016 & 37 & 56 & 6 & 53 & $3.3 \%$ & $15.25[5.53,42.05]$ & & \\
\hline Cornacchio 2019 & 1 & 14 & 1 & 15 & $1.1 \%$ & $1.08[0.06,19.05]$ & & \\
\hline Hirshfeld Becker 2010 & 22 & 34 & 13 & 30 & $3.3 \%$ & $2.40[0.87,6.57]$ & & \\
\hline Holmes 2014 & 9 & 20 & 3 & 22 & $2.4 \%$ & $5.18[1.15,23.29]$ & & \\
\hline Ishikawa 2019 & 10 & 23 & 4 & 24 & $2.7 \%$ & $3.85[0.99,14.89]$ & & \\
\hline Kidd 2018 & 18 & 26 & 10 & 23 & $3.0 \%$ & $2.92[0.91,9.44]$ & & \\
\hline McConachie 2014 & 0 & 17 & 1 & 15 & $0.9 \%$ & $0.28[0.01,7.31]$ & & \\
\hline Melfsen 2011 & 7 & 21 & 2 & 23 & $2.1 \%$ & $5.25[0.95,29.05]$ & & \\
\hline Ost 2001 & 12 & 20 & 0 & 9 & $1.0 \%$ & $27.94[1.43,546.98]$ & & \\
\hline Rapee 2006 & 44 & 90 & 17 & 87 & $4.0 \%$ & $3.94[2.01,7.71]$ & & $\rightarrow$ \\
\hline Santucci 2013 & 7 & 15 & 1 & 14 & $1.5 \%$ & $11.38[1.17,110.42]$ & & \\
\hline Schneider 2011 & 16 & 21 & 4 & 22 & $2.5 \%$ & $14.40[3.29,63.08]$ & & \\
\hline Silverman 1999b & 16 & 37 & 5 & 19 & $2.9 \%$ & $2.13[0.64,7.16]$ & & \\
\hline Spence 2000 & 14 & 17 & 1 & 8 & $1.3 \%$ & $32.67[2.85,374.13]$ & & \\
\hline Spence 2006 & 13 & 22 & 3 & 23 & $2.5 \%$ & $9.63[2.19,42.37]$ & & - \\
\hline Spence 2011 & 13 & 44 & 4 & 27 & $2.9 \%$ & $2.41[0.70,8.36]$ & & 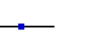 \\
\hline Waters 2009 & 17 & 31 & 1 & 5 & $1.5 \%$ & $4.86[0.49,48.57]$ & & \\
\hline Wergeland 2014 & 24 & 67 & 3 & 19 & $2.7 \%$ & $2.98[0.79,11.26]$ & & \\
\hline Wergeland 2014 & 27 & 77 & 3 & 19 & $2.7 \%$ & $2.88[0.77,10.77]$ & & \\
\hline Wood 2015 & 6 & 19 & 6 & 14 & $2.5 \%$ & $0.62[0.15,2.58]$ & $=$ & - \\
\hline Subtotal (95\% CI) & & 671 & & 471 & $46.6 \%$ & $4.08[2.72,6.11]$ & & $\gamma$ \\
\hline Total events: & 313 & & 88 & & & & & \\
\hline Heterogeneity: $\mathrm{Tau}^{2}=0.2$ & $\mathrm{i}^{2}=29.75$ & If $=19(\mathrm{P}$ & $0.06) ; I^{2}=36$ & & & & & \\
\hline Test for overall effect: $Z=$ & $(\mathrm{P}<0.000$ & & & & & & & \\
\hline 1.1.3 Parent only & & & & & & & & \\
\hline Cartwright Hatton 2011 & 21 & 37 & 5 & 33 & $3.0 \%$ & $7.35[2.32,23.27]$ & & $\longrightarrow$ \\
\hline Cobham 2017 & 20 & 33 & 6 & 30 & $3.1 \%$ & $6.15[1.98,19.14]$ & & $\longrightarrow$ \\
\hline
\end{tabular}




\section{Analysis 1.1. (Continued)}

Cartwright Hatton 2011

Cobham 2017

Thirlwall 2013

Thirlwall 2013

Waters 2009

Subtotal (95\% CI)

Total events:

Heterogeneity: $\mathrm{Tau}^{2}=0.74 ; \mathrm{Chi}^{2}=13.17, \mathrm{df}=4(\mathrm{P}=0.01) ; \mathrm{I}^{2}=70 \%$

Test for overall effect: $\mathrm{Z}=2.20(\mathrm{P}=0.03)$

\section{Total (95\% CI)}

1623

Total events:

802

\section{7}

33

61

64

38

233

Heterogeneity: $\mathrm{Tau}^{2}=0.61 ; \mathrm{Chi}^{2}=97.73, \mathrm{df}=47(\mathrm{P}<0.0001) ; \mathrm{I}^{2}=52 \%$

Test for overall effect: $\mathrm{Z}=9.96(\mathrm{P}<0.00001)$

Test for subgroup differences: $\mathrm{Chi}^{2}=8.57, \mathrm{df}=2(\mathrm{P}=0.01), \mathrm{I}^{2}=76.7 \%$

191

$7.35[2.32,23.27]$

$6.15[1.98,19.14]$

$0.88[0.35,2.16]$

$1.40[0.58,3.35]$

$6.18[0.66,58.03]$

$2.83[1.12,7.16]$

$1074 \quad 100.0 \%$

$5.45[3.90,7.60]$

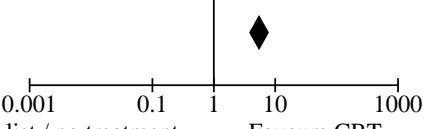

Favours Wait list / no treatment

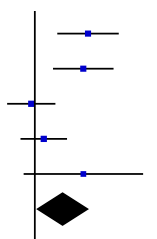

Favours CBT 
Analysis 1.2. Comparison 1: CBT versus waitlist/no treatment, Outcome 2: Subgroup analysis: remission of primary anxiety diagnosis post-treatment (ITT) (individual vs group)

\begin{tabular}{|c|c|c|c|c|c|c|c|}
\hline & \multicolumn{2}{|c|}{ CBT } & \multicolumn{2}{|c|}{ Wait list / no treatment } & \multicolumn{2}{|r|}{ Odds Ratio } & Odds Ratio \\
\hline Study or Subgroup & Events & Total & Events & Total & Weight & IV, Random, 95\% CI & IV, Random, 95\% CI \\
\hline
\end{tabular}

\begin{tabular}{lrrrrr}
\hline 1.2.1 Individual focused & & & & 7 & $4.6 \%$ \\
Flannery Schroeder 2000 & 9 & 18 & 2 & 30 & $7.2 \%$ \\
Hirshfeld Becker 2010 & 22 & 34 & 13 & 24 & $6.1 \%$ \\
Ishikawa 2019 & 10 & 23 & 4 & 20 & $4.1 \%$ \\
Kendall 1994 & 17 & 40 & 1 & 49 & $8.0 \%$ \\
Kendall 1997 & 32 & 69 & 17 & 20 & $3.9 \%$ \\
Masia Warner 2011 & 9 & 20 & 1 & 10 & $2.6 \%$ \\
McNally Keehn 2013 & 7 & 12 & 0 & 23 & $5.1 \%$ \\
Melfsen 2011 & 7 & 21 & 2 & 41 & $4.3 \%$ \\
Ollendick 2009 & 47 & 85 & 1 & 9 & $2.7 \%$ \\
Ost 2001 & 12 & 20 & 0 & 10 & $2.6 \%$ \\
Ost 2001 & 18 & 21 & 0 & 20 & $2.6 \%$ \\
Perrin 2019 & 16 & 20 & 0 & 27 & $5.7 \%$ \\
Schneider 2011 & 16 & 21 & 4 & 35 & $7.6 \%$ \\
Spence 2011 & 13 & 44 & 4 & 34 & $7.5 \%$ \\
Thirlwall 2013 & 25 & 64 & 11 & 27 & $6.6 \%$ \\
Thirlwall 2013 & 18 & 61 & 11 & 19 & $6.2 \%$ \\
Villabo 2018 & 29 & 55 & 4 & 14 & $5.9 \%$ \\
Wergeland 2014 & 27 & 77 & 3 & $\mathbf{4 4 1}$ & $\mathbf{1 0 0 . 0 \%}$ \\
Wood 2015 & 6 & 19 & 6 & & \\
Subtotal (95\% CI) & & $\mathbf{7 2 4}$ & & 84 & \\
Total ev & 340 & & & & \\
\end{tabular}

Total events: $\quad 340 \quad 84$

Heterogeneity: $\mathrm{Tau}^{2}=0.92 ; \mathrm{Chi}^{2}=51.71, \mathrm{df}=18(\mathrm{P}<0.0001) ; \mathrm{I}^{2}=65 \%$

Test for overall effect: $\mathrm{Z}=5.16(\mathrm{P}<0.00001)$

1.2.2 Group focused

Arendt 2016

Cartwright Hatton 2011

Cobham 2017

Cornacchio 2019

Dadds 1997

Flannery Schroeder 2000

Gallagher 2004

Holmes 2014

Kidd 2018

Lau 2010

Masia Warner 2005

McConachie 2014

Olivares 2005

Olivares 2014

Olivares 2014

Olivares 2019

Olivares 2019

Rapee 2006

Rosa Alcazar 2009

Sanchez Garcia 2009

Santucci 2013

Silverman 1999b

Spence 2000

Spence 2000

Spence 2006

Villabo 2018

Waters 2009

Waters 2009

Wergeland 2014

Subtotal (95\% CI)

Total events:

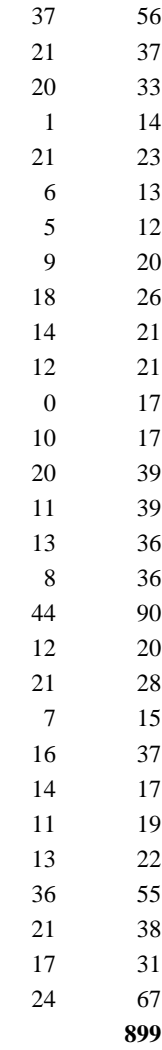

Heterogeneity: $\mathrm{Tau}^{2}=0.15 ; \mathrm{Chi}^{2}=34.41$,

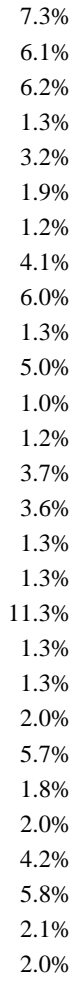

$2.50[0.38,16.42]$

$2.40[0.87,6.57]$

$3.85[0.99,14.89]$

$14.04[1.71,115.41]$

$1.63[0.77,3.46]$

$15.55[1.73,139.65]$

$28.64[1.37,600.41]$

$5.25[0.95,29.05]$

49.47 [6.50, 376.64]

$27.94[1.43,546.98]$

111.00 [5.21, 2363.64]

150.33 [7.54, 2997.83]

$14.40[3.29,63.08]$

$2.41[0.70,8.36]$

$1.40[0.58,3.35]$

$0.88[0.35,2.16]$

$6.41[1.96,21.01]$

$2.88[0.77,10.77]$

$0.62[0.15,2.58]$

$4.53[2.55,8.03]$

$15.25[5.53,42.05]$

7.35 [2.32, 23.27]

$6.15[1.98,19.14]$

$1.08[0.06,19.05]$

$1.88[0.33,10.63]$

$5.14[0.48,55.64]$

$16.87[0.81,351.79]$

$5.18[1.15,23.29]$

$2.92[0.91,9.44]$

67.67 [3.56, 1287.81]

$4.27[1.13,16.05]$

$0.28[0.01,7.31]$

$49.00[2.53,948.62]$

$9.47[1.93,46.46]$

$3.34[0.66,16.92]$

$21.26[1.18,381.56]$

$11.04[0.60,202.89]$

$3.94[2.01,7.71]$

$60.29[3.20,1137.79]$

$146.20[7.89,2709.82]$

$11.38[1.17,110.42]$

$2.13[0.64,7.16]$

32.67 [2.85, 374.13]

8.25 [0.82, 82.67]

$9.63[2.19,42.37]$

$11.37[3.44,37.58]$

$6.18[0.66,58.03]$

$4.86[0.49,48.57]$

$2.98[0.79,11.26]$

$6.25[4.45,8.78]$
Random, $95 \%$ CI

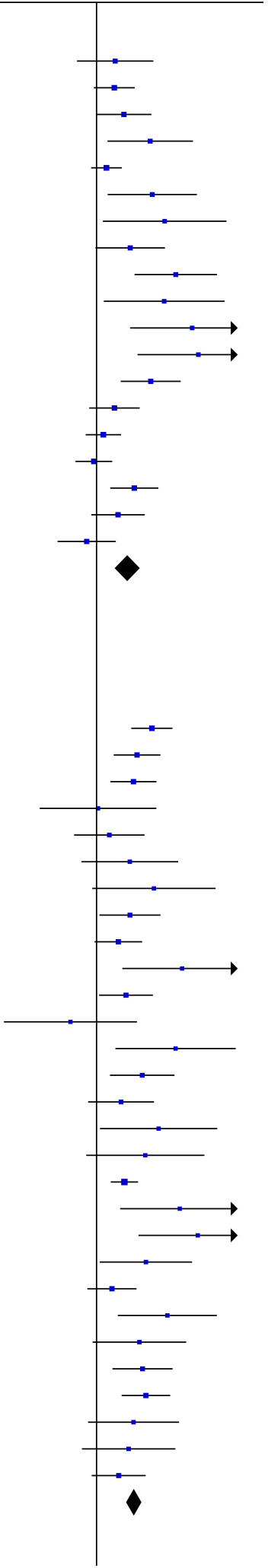




\section{Analysis 1.2. (Continued)}

Total events: $\quad 462 \quad 107$

Heterogeneity: $\mathrm{Tau}^{2}=0.15 ; \mathrm{Chi}^{2}=34.41, \mathrm{df}=28(\mathrm{P}=0.19) ; \mathrm{I}^{2}=19 \%$

Test for overall effect: $\mathrm{Z}=10.55(\mathrm{P}<0.00001)$

Test for subgroup differences: $\mathrm{Chi}^{2}=0.90, \mathrm{df}=1(\mathrm{P}=0.34), \mathrm{I}^{2}=0 \%$

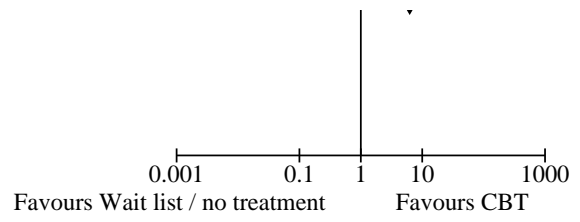


Analysis 1.3. Comparison 1: CBT versus waitlist/no treatment, Outcome 3: Subgroup analysis: remission of primary anxiety diagnosis post-treatment (ITT) (amount of therapist contact time)

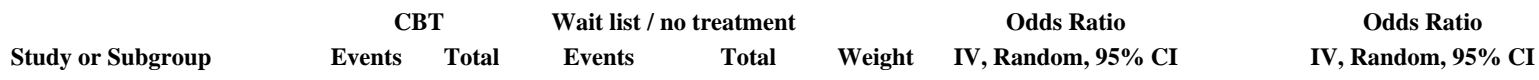

1.3.1 Therapist contact $(<10$ hours $)$

Cobham 2017

Gallagher 2004

Ishikawa 2019

Ollendick 2009

Ost 2001

Ost 2001

Thirlwall 2013

Thirlwall 2013

Subtotal $(95 \%$ CI $)$

Total events:

Heterogeneity: $\mathrm{Tau}^{2}=1.55 ; \mathrm{Chi}^{2}=27.73, \mathrm{df}=7(\mathrm{P}=0.0002) ; \mathrm{I}^{2}=75 \%$

Test for overall effect: $\mathrm{Z}=3.29(\mathrm{P}=0.0010)$

1.3.2 Therapist contact (\#10 and < 20 hours)

Dadds 1997

Flannery Schroeder 2000

Holmes 2014

Kendall 1994

Kendall 1997

Lau 2010

Masia Warner 2005

Masia Warner 2011

Olivares 2005

Olivares 2014

Olivares 2014

Olivares 2019

Olivares 2019

Perrin 2019

Rapee 2006

Rosa Alcazar 2009

Sanchez Garcia 2009

Schneider 2011

Silverman 1999 b

Spence 2000

Spence 2006

Spence 2011

Villabo 2018

Villabo 2018

Waters 2009

Wergeland 2014

Wergeland 2014

Subtotal (95\% CI)

$\begin{array}{rr}21 & 23 \\ 9 & 18 \\ 9 & 20 \\ 17 & 40 \\ 32 & 69 \\ 14 & 21 \\ 12 & 21 \\ 9 & 20 \\ 10 & 17 \\ 20 & 39 \\ 11 & 39 \\ 13 & 36 \\ 8 & 36 \\ 16 & 20 \\ 44 & 90 \\ 12 & 20 \\ 21 & 28 \\ 16 & 21 \\ 16 & 37 \\ 11 & 19 \\ 13 & 22 \\ 13 & 44 \\ 36 & 55 \\ 29 & 55 \\ 21 & 38 \\ 24 & 67 \\ 27 & 77 \\ & 992 \\ 84 & \end{array}$

Total events:

484

$\begin{array}{rr}33 & 6 \\ 12 & 0 \\ 23 & 4 \\ 85 & 1 \\ 20 & 0 \\ 21 & 0 \\ 61 & 11 \\ 64 & 11 \\ \mathbf{3 1 9} & \end{array}$

$30 \quad 16.0 \%$

$11 \quad 7.7 \%$

$24 \quad 14.9 \%$

$41 \quad 11.5 \%$

$9 \quad 7.8 \%$

$10 \quad 7.6 \%$

$34 \quad 17.1 \%$

$35 \quad 17.3 \%$

$194100.0 \%$

33

Heterogeneity: $\mathrm{Tau}^{2}=0.36 ; \mathrm{Chi}^{2}=42.26, \mathrm{df}=26(\mathrm{P}=0.02) ; \mathrm{I}^{2}=38 \%$

Test for overall effect: $\mathrm{Z}=8.85(\mathrm{P}<0.00001)$

1.3.3 Therapist contact (\# 20 hours)

Arendt 2016

Cartwright Hatton 2011

Cornacchio 2019

Flannery Schroeder 2000

Hirshfeld Becker 2010

Kidd 2018

McConachie 2014

McNally Keehn 2013

Melfsen 2011

Santucci 2013

23
18
20
40
69
21
21
20
17
39
39
36
36
20
90
20
28
21
37
19
22
44
55
55
38
67
77
992

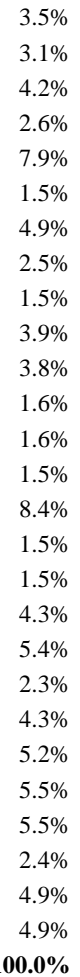

$1.88[0.33,10.63]$

$2.50[0.38,16.42]$

$5.18[1.15,23.29]$

$14.04[1.71,115.41]$

$1.63[0.77,3.46]$

$67.67[3.56,1287.81]$

$4.27[1.13,16.05]$

$15.55[1.73,139.65]$

49.00 [2.53, 948.62]

$9.47[1.93,46.46]$

$3.34[0.66,16.92]$

$21.26[1.18,381.56]$

$11.04[0.60,202.89]$

$150.33[7.54,2997.83]$

$3.94[2.01,7.71]$

$60.29[3.20,1137.79]$

$146.20[7.89,2709.82]$

$14.40[3.29,63.08]$

$2.13[0.64,7.16]$

$8.25[0.82,82.67]$

$9.63[2.19,42.37]$

$2.41[0.70,8.36]$

11.37 [3.44, 37.58]

$6.41[1.96,21.01]$

$6.18[0.66,58.03]$

$2.98[0.79,11.26]$

$2.88[0.77,10.77]$

$5.85[3.95,8.64]$ 


\section{Analysis 1.3. (Continued)}

$\begin{array}{lrrrrr}\text { Melfsen 2011 } & 7 & 21 & 2 & 23 & 8.2 \% \\ \text { Santucci 2013 } & 7 & 15 & 1 & 14 & 5.9 \% \\ \text { Spence 2000 } & 14 & 17 & 1 & 8 & 5.3 \% \\ \text { Waters 2009 } & 17 & 31 & 1 & 5 & 5.8 \% \\ \text { Wood 2015 } & 6 & 19 & 6 & 14 & 9.8 \% \\ \text { Subtotal (95\% CI) } & & \mathbf{3 1 2} & & \mathbf{2 5 0} & \mathbf{1 0 0 . 0 \%}\end{array}$

$5.25[0.95,29.05]$

$11.38[1.17,110.42]$

$32.67[2.85,374.13]$

$4.86[0.49,48.57]$

$0.62[0.15,2.58]$

$4.44[2.26,8.72]$

Total events:

163

48

Heterogeneity: $\mathrm{Tau}^{2}=0.68 ; \mathrm{Chi}^{2}=24.07, \mathrm{df}=12(\mathrm{P}=0.02) ; \mathrm{I}^{2}=50 \%$

Test for overall effect: $\mathrm{Z}=4.34(\mathrm{P}<0.0001)$

Test for subgroup differences: $\mathrm{Chi}^{2}=0.52, \mathrm{df}=2(\mathrm{P}=0.77), \mathrm{I}^{2}=0 \%$ 
Analysis 1.4. Comparison 1: CBT versus waitlist/no treatment, Outcome 4: Subgroup analysis: remission of primary anxiety diagnosis post-treatment (ITT) (age)

\begin{tabular}{|c|c|c|c|c|c|c|c|}
\hline Study or Subgroup & \multicolumn{2}{|c|}{ CBT } & \multicolumn{2}{|c|}{ Wait list / no treatment } & Weight & $\begin{array}{c}\text { Odds Ratio } \\
\text { IV, Random, 95\% CI }\end{array}$ & $\begin{array}{c}\text { Odds Ratio } \\
\text { IV, Random, } 95 \% \mathrm{Cl}\end{array}$ \\
\hline \multicolumn{8}{|l|}{ 1.4.1 \# 12 years } \\
\hline Cartwright Hatton 2011 & 21 & 37 & 5 & 33 & $10.3 \%$ & $7.35[2.32,23.27]$ & \\
\hline Cornacchio 2019 & 1 & 14 & 1 & 15 & $3.3 \%$ & $1.08[0.06,19.05]$ & \\
\hline Gallagher 2004 & 5 & 12 & 0 & 11 & $3.0 \%$ & $16.87[0.81,351.79]$ & \\
\hline Hirshfeld Becker 2010 & 22 & 34 & 13 & 30 & $11.3 \%$ & $2.40[0.87,6.57]$ & - \\
\hline Holmes 2014 & 9 & 20 & 3 & 22 & $8.0 \%$ & $5.18[1.15,23.29]$ & 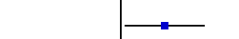 \\
\hline Lau 2010 & 14 & 21 & 0 & 17 & $3.2 \%$ & $67.67[3.56,1287.81]$ & \\
\hline Rapee 2006 & 44 & 90 & 17 & 87 & $14.0 \%$ & $3.94[2.01,7.71]$ & $\rightarrow$ \\
\hline Santucci 2013 & 7 & 15 & 1 & 14 & $4.7 \%$ & $11.38[1.17,110.42]$ & \\
\hline Schneider 2011 & 16 & 21 & 4 & 22 & $8.1 \%$ & $14.40[3.29,63.08]$ & \\
\hline Thirlwall 2013 & 18 & 61 & 11 & 34 & $12.1 \%$ & $0.88[0.35,2.16]$ & - \\
\hline Thirlwall 2013 & 25 & 64 & 11 & 35 & $12.4 \%$ & $1.40[0.58,3.35]$ & $f=-$ \\
\hline Waters 2009 & 21 & 38 & 1 & 6 & $4.8 \%$ & $6.18[0.66,58.03]$ & \\
\hline Waters 2009 & 17 & 31 & 1 & 5 & $4.6 \%$ & $4.86[0.49,48.57]$ & \\
\hline Subtotal $(95 \%$ CI $)$ & & 458 & & 331 & $100.0 \%$ & $3.93[2.20,7.02]$ & \\
\hline Total events: & 220 & & 68 & & & & \\
\hline \multicolumn{8}{|c|}{ Heterogeneity: $\mathrm{Tau}^{2}=0.51 ; \mathrm{Chi}^{2}=25.87, \mathrm{df}=12(\mathrm{P}=0.01) ; \mathrm{I}^{2}=54 \%$} \\
\hline \multicolumn{8}{|c|}{ Test for overall effect: $\mathrm{Z}=4.62(\mathrm{P}<0.00001)$} \\
\hline \multicolumn{8}{|c|}{ 1.4.2 Mixed age range $(<12$ and \# 12 years) } \\
\hline Arendt 2016 & 37 & 56 & 6 & 53 & $5.9 \%$ & $15.25[5.53,42.05]$ & \\
\hline Cobham 2017 & 20 & 33 & 6 & 30 & $5.5 \%$ & $6.15[1.98,19.14]$ & \\
\hline Dadds 1997 & 21 & 23 & 28 & 33 & $4.0 \%$ & $1.88[0.33,10.63]$ & \\
\hline Flannery Schroeder 2000 & 9 & 18 & 2 & 7 & $3.7 \%$ & $2.50[0.38,16.42]$ & - \\
\hline Flannery Schroeder 2000 & 6 & 13 & 1 & 7 & $2.8 \%$ & $5.14[0.48,55.64]$ & \\
\hline Ishikawa 2019 & 10 & 23 & 4 & 24 & $4.9 \%$ & $3.85[0.99,14.89]$ & \\
\hline Kendall 1994 & 17 & 40 & 1 & 20 & $3.2 \%$ & $14.04[1.71,115.41]$ & \\
\hline Kendall 1997 & 32 & 69 & 17 & 49 & $6.6 \%$ & $1.63[0.77,3.46]$ & - \\
\hline Masia Warner 2011 & 9 & 20 & 1 & 20 & $3.1 \%$ & $15.55[1.73,139.65]$ & \\
\hline McConachie 2014 & 0 & 17 & 1 & 15 & $1.8 \%$ & $0.28[0.01,7.31]$ & \\
\hline McNally Keehn 2013 & 7 & 12 & 0 & 10 & $2.0 \%$ & $28.64[1.37,600.41]$ & \\
\hline Melfsen 2011 & 7 & 21 & 2 & 23 & $4.0 \%$ & $5.25[0.95,29.05]$ & \\
\hline Ollendick 2009 & 47 & 85 & 1 & 41 & $3.4 \%$ & $49.47[6.50,376.64]$ & \\
\hline Ost 2001 & 18 & 21 & 0 & 10 & $2.0 \%$ & $111.00[5.21,2363.64]$ & \\
\hline Ost 2001 & 12 & 20 & 0 & 9 & $2.1 \%$ & $27.94[1.43,546.98]$ & \\
\hline Perrin 2019 & 16 & 20 & 0 & 20 & $2.0 \%$ & $150.33[7.54,2997.83]$ & \\
\hline Sanchez Garcia 2009 & 21 & 28 & 0 & 25 & $2.1 \%$ & $146.20[7.89,2709.82]$ & \\
\hline Silverman 1999b & 16 & 37 & 5 & 19 & $5.3 \%$ & $2.13[0.64,7.16]$ & \\
\hline Spence 2000 & 11 & 19 & 1 & 7 & $2.9 \%$ & $8.25[0.82,82.67]$ & \\
\hline Spence 2000 & 14 & 17 & 1 & 8 & $2.7 \%$ & $32.67[2.85,374.13]$ & \\
\hline Spence 2006 & 13 & 22 & 3 & 23 & $4.6 \%$ & $9.63[2.19,42.37]$ & $\longrightarrow$ \\
\hline Villabo 2018 & 29 & 55 & 4 & 27 & $5.4 \%$ & $6.41[1.96,21.01]$ & $\longrightarrow$ \\
\hline Villabo 2018 & 36 & 55 & 4 & 28 & $5.3 \%$ & $11.37[3.44,37.58]$ & $\longrightarrow$ \\
\hline Wergeland 2014 & 27 & 77 & 3 & 19 & $5.0 \%$ & $2.88[0.77,10.77]$ & 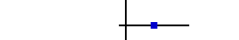 \\
\hline Wergeland 2014 & 24 & 67 & 3 & 19 & $5.0 \%$ & $2.98[0.79,11.26]$ & 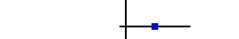 \\
\hline Wood 2015 & 6 & 19 & 6 & 14 & $4.7 \%$ & $0.62[0.15,2.58]$ & - \\
\hline Subtotal (95\% CI) & & 887 & & 560 & $100.0 \%$ & $6.43[3.90,10.58]$ & \\
\hline Total events: & 465 & & 100 & & & & \\
\hline \multicolumn{8}{|c|}{ Heterogeneity: $\mathrm{Tau}^{2}=0.84 ; \mathrm{Chi}^{2}=58.15, \mathrm{df}=25(\mathrm{P}=0.0002) ; \mathrm{I}^{2}=57 \%$} \\
\hline \multicolumn{8}{|c|}{ Test for overall effect: $\mathrm{Z}=7.31(\mathrm{P}<0.00001)$} \\
\hline \multicolumn{8}{|l|}{ 1.4.3 \# 12 years } \\
\hline Kidd 2018 & 18 & 26 & 10 & 23 & $20.9 \%$ & $2.92[0.91,9.44]$ & $\rightarrow$ \\
\hline Masia Warner 2005 & 12 & 21 & 5 & 21 & $17.3 \%$ & $4.27[1.13,16.05]$ & $\longrightarrow$ \\
\hline Olivares 2005 & 10 & 17 & 0 & 17 & $4.2 \%$ & $49.00[2.53,948.62]$ & $\longrightarrow$ \\
\hline Olivares 2014 & 11 & 39 & 2 & 19 & $12.5 \%$ & $3.34[0.66,16.92]$ & $\longrightarrow$ \\
\hline Olivares 2014 & 20 & 39 & 2 & 20 & $12.9 \%$ & $9.47[1.93,46.46]$ & $\longrightarrow$ \\
\hline Olivares 2019 & 8 & 36 & 0 & 18 & $4.4 \%$ & $11.04[0.60,202.89]$ & \\
\hline
\end{tabular}




\section{Analysis 1.4. (Continued)}

Olivares 2014

Olivares 2019

Olivares 2019

Rosa Alcazar 2009

Spence 2011

Subtotal $(95 \%$ CI $)$

Total events:

Heterogeneity: $\mathrm{Tau}^{2}=0.13 ; \mathrm{Chi}^{2}=9.32, \mathrm{df}=8(\mathrm{P}=0.32) ; \mathrm{I}^{2}=14 \%$

Test for overall effect: $\mathrm{Z}=5.22(\mathrm{P}<0.00001)$

Test for subgroup differences: $\mathrm{Chi}^{2}=1.59, \mathrm{df}=2(\mathrm{P}=0.45), \mathrm{I}^{2}=0 \%$

$\begin{array}{rr}12.9 \% & 9.47[1.93,46.46] \\ 4.4 \% & 11.04[0.60,202.89] \\ 4.4 \% & 21.26[1.18,381.56] \\ 4.3 \% & 60.29[3.20,1137.79] \\ 19.1 \% & 2.41[0.70,8.36] \\ \mathbf{1 0 0 . 0 \%} & \mathbf{5 . 2 8}[\mathbf{2 . 8 3}, \mathbf{9 . 8 6}]\end{array}$

$5.28[2.83,9.86]$

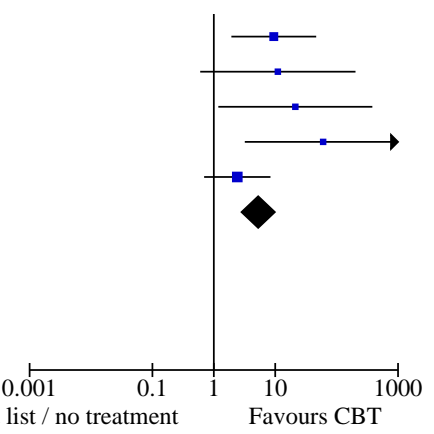


Analysis 1.5. Comparison 1: CBT versus waitlist/no treatment, Outcome 5: Subgroup analysis: remission of primary anxiety diagnosis post-treatment (ITT) (ASD vs non-ASD)

\begin{tabular}{|c|c|c|c|c|c|c|}
\hline & \multicolumn{2}{|c|}{ CBT } & Wait list / no treatment & \multicolumn{2}{|r|}{ Odds Ratio } & Odds Ratio \\
\hline Study or Subgroup & Events & Total & Events & Weight & IV, Random, $95 \%$ CI & IV, Random, $95 \%$ CI \\
\hline
\end{tabular}

1.5.1 Sample with ASD

Events

Total

Weight

Kidd 2018

McConachie 2014

McNally Keehn 2013

Wood 2015

Subtotal $(95 \%$ CI $)$

Total events:

\section{$36.4 \%$}

$14.6 \%$

$16.2 \%$

$14 \quad 32.8 \%$

$62 \quad 100.0 \%$

17

Heterogeneity: $\mathrm{Tau}^{2}=1.28 ; \mathrm{Chi}^{2}=7.24, \mathrm{df}=3(\mathrm{P}=0.06) ; \mathrm{I}^{2}=59 \%$

Test for overall effect: $\mathrm{Z}=0.76(\mathrm{P}=0.45)$

1.5.2 Not sample with ASD

Arendt 2016

Cartwright Hatton 2011

Cobham 2017

Cornacchio 2019

Dadds 1997

Flannery Schroeder 2000

Flannery Schroeder 2000

Gallagher 2004

Hirshfeld Becker 2010

Holmes 2014

Ishikawa 2019

Kendall 1994

Kendall 1997

Lau 2010

Masia Warner 2005

Masia Warner 2011

Melfsen 2011

Olivares 2005

Olivares 2014

Olivares 2014

Olivares 2019

Olivares 2019

Ollendick 2009

Ost 2001

Ost 2001

Perrin 2019

Rapee 2006

Rosa Alcazar 2009

Sanchez Garcia 2009

Santucci 2013

Schneider 2011

Silverman 1999b

Spence 2000

Spence 2000

Spence 2006

Spence 2011

Thirlwall 2013

Thirlwall 2013

Villabo 2018

Villabo 2018

Waters 2009

Waters 2009

Wergeland 2014

Wergeland 2014

Subtotal (95\% CI)

Total events:

771
$2.92[0.91,9.44]$

$0.28[0.01,7.31]$

$28.64[1.37,600.41]$

$0.62[0.15,2.58]$

$1.79[0.39,8.16]$
$15.25[5.53,42.05]$

7.35 [2.32, 23.27]

$6.15[1.98,19.14]$

$1.08[0.06,19.05]$

$1.88[0.33,10.63]$

$2.50[0.38,16.42]$

$5.14[0.48,55.64]$

$16.87[0.81,351.79]$

$2.40[0.87,6.57]$

5.18 [1.15, 23.29]

$3.85[0.99,14.89]$

$14.04[1.71,115.41]$

$1.63[0.77,3.46]$

$67.67[3.56,1287.81]$

$4.27[1.13,16.05]$

$15.55[1.73,139.65]$

$5.25[0.95,29.05]$

$49.00[2.53,948.62]$

$3.34[0.66,16.92]$

$9.47[1.93,46.46]$

$11.04[0.60,202.89]$

$21.26[1.18,381.56]$

$49.47[6.50,376.64]$

$27.94[1.43,546.98]$

$111.00[5.21,2363.64]$

150.33 [7.54, 2997.83]

$3.94[2.01,7.71]$

$60.29[3.20,1137.79]$

146.20 [7.89, 2709.82]

$11.38[1.17,110.42]$

$14.40[3.29,63.08]$

$2.13[0.64,7.16]$

$32.67[2.85,374.13]$

$8.25[0.82,82.67]$

$9.63[2.19,42.37]$

$2.41[0.70,8.36]$

$1.40[0.58,3.35]$

$0.88[0.35,2.16]$

$11.37[3.44,37.58]$

$6.41[1.96,21.01]$

$4.86[0.49,48.57]$

$6.18[0.66,58.03]$

$2.88[0.77,10.77]$

$2.98[0.79,11.26]$

$5.88[4.19,8.24]$
IV, Random, $95 \%$ CI 


\section{Analysis 1.5. (Continued)}

$\begin{array}{lcc}\text { Total events: } & 771 & 174 \\ \text { Heterogeneity: } \mathrm{Tau}^{2}=0.55 ; \mathrm{Chi}^{2}=85.92, \mathrm{df}=43(\mathrm{P}=0.0001) ; \mathrm{I}^{2}=50 \%\end{array}$

Test for overall effect: $\mathrm{Z}=10.28(\mathrm{P}<0.00001)$

Test for subgroup differences: $\mathrm{Chi}^{2}=2.25, \mathrm{df}=1(\mathrm{P}=0.13), \mathrm{I}^{2}=55.5 \%$

0.001

Favours Wait list / no treatment

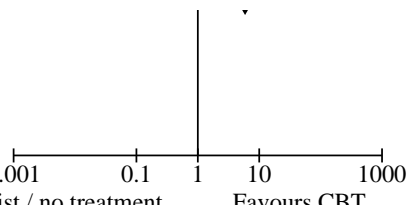

Favours CBT 
Analysis 1.6. Comparison 1: CBT versus waitlist/no treatment, Outcome 6: Remission of primary anxiety diagnosis post-treatment (completers)

\begin{tabular}{|c|c|c|c|c|c|c|c|c|}
\hline \multirow[b]{2}{*}{ Study or Subgroup } & \multicolumn{2}{|c|}{ CBT } & \multicolumn{2}{|c|}{ Wait list / no treatment } & \multirow[b]{2}{*}{ Weight } & \multirow{2}{*}{$\begin{array}{c}\text { Odds Ratio } \\
\text { IV, Random, } \mathbf{9 5 \%} \text { CI }\end{array}$} & \multirow{2}{*}{\multicolumn{2}{|c|}{$\begin{array}{c}\text { Odds Ratio } \\
\text { IV, Random, } 95 \% \text { CI }\end{array}$}} \\
\hline & Events & Total & Events & Total & & & & \\
\hline Arendt 2016 & 37 & 56 & 4 & 51 & $4.2 \%$ & $22.88[7.17,73.07]$ & & $\longrightarrow$ \\
\hline Cartwright Hatton 2011 & 21 & 37 & 5 & 33 & $4.2 \%$ & $7.35[2.32,23.27]$ & & $\longrightarrow$ \\
\hline Cobham 2017 & 20 & 31 & 5 & 29 & $4.0 \%$ & $8.73[2.60,29.33]$ & & $\longrightarrow$ \\
\hline Cornacchio 2019 & 1 & 14 & 0 & 14 & $0.8 \%$ & $3.22[0.12,86.09]$ & & \\
\hline Dadds 1997 & 21 & 22 & 27 & 32 & $1.7 \%$ & $3.89[0.42,35.86]$ & & \\
\hline Flannery Schroeder 2000 & 6 & 12 & 0 & 6 & $1.0 \%$ & $13.00[0.60,281.46]$ & & \\
\hline Flannery Schroeder 2000 & 9 & 13 & 1 & 6 & $1.4 \%$ & $11.25[0.97,130.22]$ & & \\
\hline Gallagher 2004 & 5 & 12 & 0 & 11 & $1.0 \%$ & $16.87[0.81,351.79]$ & & \\
\hline Hirshfeld Becker 2010 & 22 & 29 & 11 & 28 & $4.3 \%$ & $4.86[1.55,15.18]$ & & $\longrightarrow$ \\
\hline Holmes 2014 & 9 & 17 & 0 & 19 & $1.0 \%$ & $43.59[2.27,837.56]$ & & \\
\hline Ishikawa 2019 & 10 & 22 & 3 & 23 & $3.1 \%$ & $5.56[1.27,24.29]$ & & \\
\hline Kendall 1994 & 17 & 27 & 1 & 20 & $1.8 \%$ & $32.30[3.74,279.31]$ & & \\
\hline Kendall 1997 & 32 & 60 & 2 & 34 & $3.0 \%$ & $18.29[4.02,83.27]$ & & \\
\hline Kidd 2018 & 18 & 25 & 6 & 19 & $3.6 \%$ & $5.57[1.51,20.51]$ & & $\longrightarrow$ \\
\hline Lau 2010 & 14 & 21 & 0 & 17 & $1.0 \%$ & $67.67[3.56,1287.81]$ & & \\
\hline Masia Warner 2005 & 12 & 18 & 1 & 17 & $1.6 \%$ & $32.00[3.39,302.22]$ & & \\
\hline Masia Warner 2011 & 9 & 20 & 0 & 19 & $1.0 \%$ & $32.22[1.71,606.80]$ & & \\
\hline McNally Keehn 2013 & 7 & 12 & 0 & 10 & $1.0 \%$ & $28.64[1.37,600.41]$ & & \\
\hline Melfsen 2011 & 7 & 15 & 0 & 21 & $1.0 \%$ & $37.94[1.94,740.15]$ & & \\
\hline Olivares 2005 & 10 & 17 & 0 & 17 & $1.0 \%$ & $49.00[2.53,948.62]$ & & \\
\hline Olivares 2014 & 11 & 37 & 0 & 17 & $1.1 \%$ & $15.19[0.84,274.68]$ & & \\
\hline Olivares 2014 & 20 & 38 & 0 & 18 & $1.1 \%$ & $41.00[2.31,729.27]$ & & \\
\hline Olivares 2019 & 13 & 34 & 0 & 18 & $1.1 \%$ & $23.23[1.29,418.15]$ & & \\
\hline Olivares 2019 & 8 & 24 & 0 & 18 & $1.0 \%$ & $19.06[1.02,356.38]$ & & \\
\hline Ollendick 2009 & 47 & 85 & 1 & 41 & $1.9 \%$ & $49.47[6.50,376.64]$ & & \\
\hline Ost 2001 & 12 & 20 & 0 & 9 & $1.0 \%$ & $27.94[1.43,546.98]$ & & \\
\hline Ost 2001 & 18 & 21 & 0 & 10 & $1.0 \%$ & $111.00[5.21,2363.64]$ & & \\
\hline Perrin 2019 & 16 & 18 & 0 & 20 & $0.9 \%$ & $270.60[12.13,6035.59]$ & & \\
\hline Rapee 2006 & 44 & 72 & 5 & 75 & $4.8 \%$ & $22.00[7.90,61.23]$ & & $\Longrightarrow$ \\
\hline Rosa Alcazar 2009 & 12 & 20 & 0 & 20 & $1.0 \%$ & $60.29[3.20,1137.79]$ & & \\
\hline Sanchez Garcia 2009 & 21 & 28 & 0 & 25 & $1.0 \%$ & $146.20[7.89,2709.82]$ & & \\
\hline Santucci 2013 & 7 & 14 & 0 & 13 & $1.0 \%$ & $27.00[1.35,541.57]$ & & \\
\hline Schneider 2011 & 16 & 18 & 3 & 21 & $2.1 \%$ & $48.00[7.10,324.71]$ & & \\
\hline Silverman 1999b & 16 & 25 & 2 & 16 & $2.6 \%$ & $12.44[2.29,67.56]$ & & \\
\hline Spence 2000 & 11 & 19 & 1 & 7 & $1.6 \%$ & $8.25[0.82,82.67]$ & & \\
\hline Spence 2000 & 14 & 16 & 0 & 7 & $0.9 \%$ & $87.00[3.68,2054.22]$ & & \\
\hline Spence 2006 & 13 & 20 & 3 & 23 & $3.0 \%$ & $12.38[2.70,56.73]$ & & \\
\hline Spence 2011 & 13 & 40 & 1 & 24 & $1.8 \%$ & $11.07[1.34,91.22]$ & & \\
\hline Thirlwall 2013 & 18 & 46 & 8 & 31 & $4.9 \%$ & $1.85[0.68,5.02]$ & & 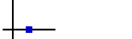 \\
\hline Thirlwall 2013 & 25 & 50 & 8 & 32 & $5.0 \%$ & $3.00[1.13,7.94]$ & & $\longrightarrow$ \\
\hline Villabo 2018 & 36 & 55 & 4 & 28 & $4.0 \%$ & $11.37[3.44,37.58]$ & & $\longrightarrow$ \\
\hline Villabo 2018 & 29 & 55 & 4 & 27 & $4.1 \%$ & $6.41[1.96,21.01]$ & & $\longrightarrow$ \\
\hline Waters 2009 & 21 & 25 & 1 & 6 & $1.5 \%$ & $26.25[2.38,288.94]$ & & \\
\hline Waters 2009 & 17 & 23 & 1 & 5 & $1.5 \%$ & $11.33[1.05,122.55]$ & & \\
\hline Wergeland 2014 & 27 & 64 & 2 & 18 & $2.9 \%$ & $5.84[1.24,27.54]$ & & \\
\hline Wergeland 2014 & 24 & 59 & 2 & 18 & $2.9 \%$ & $5.49[1.15,26.08]$ & & \\
\hline Wood 2015 & 6 & 16 & 3 & 11 & $2.6 \%$ & $1.60[0.30,8.49]$ & &. \\
\hline Total $(95 \%$ CI $)$ & & 1422 & & 984 & $100.0 \%$ & $11.55[8.41,15.86]$ & & 0 \\
\hline Total events: & 802 & & 115 & & & & & \\
\hline Heterogeneity: $\mathrm{Tau}^{2}=0.2$ & $=61.53$ & $\mathrm{df}=46(\mathrm{P}$ & $0.06) ; I^{2}=25 \%$ & & & 0.001 & 0.1 & 10 \\
\hline Test for overall effect: $Z=$ & $1(\mathrm{P}<0.00$ & & & & & Favours Wait list / & treatment & Favou \\
\hline
\end{tabular}

Test for subgroup differences: Not applicable 
Analysis 1.7. Comparison 1: CBT versus waitlist/no treatment, Outcome 7: Acceptability (number of participants lost to post-treatment assessment)

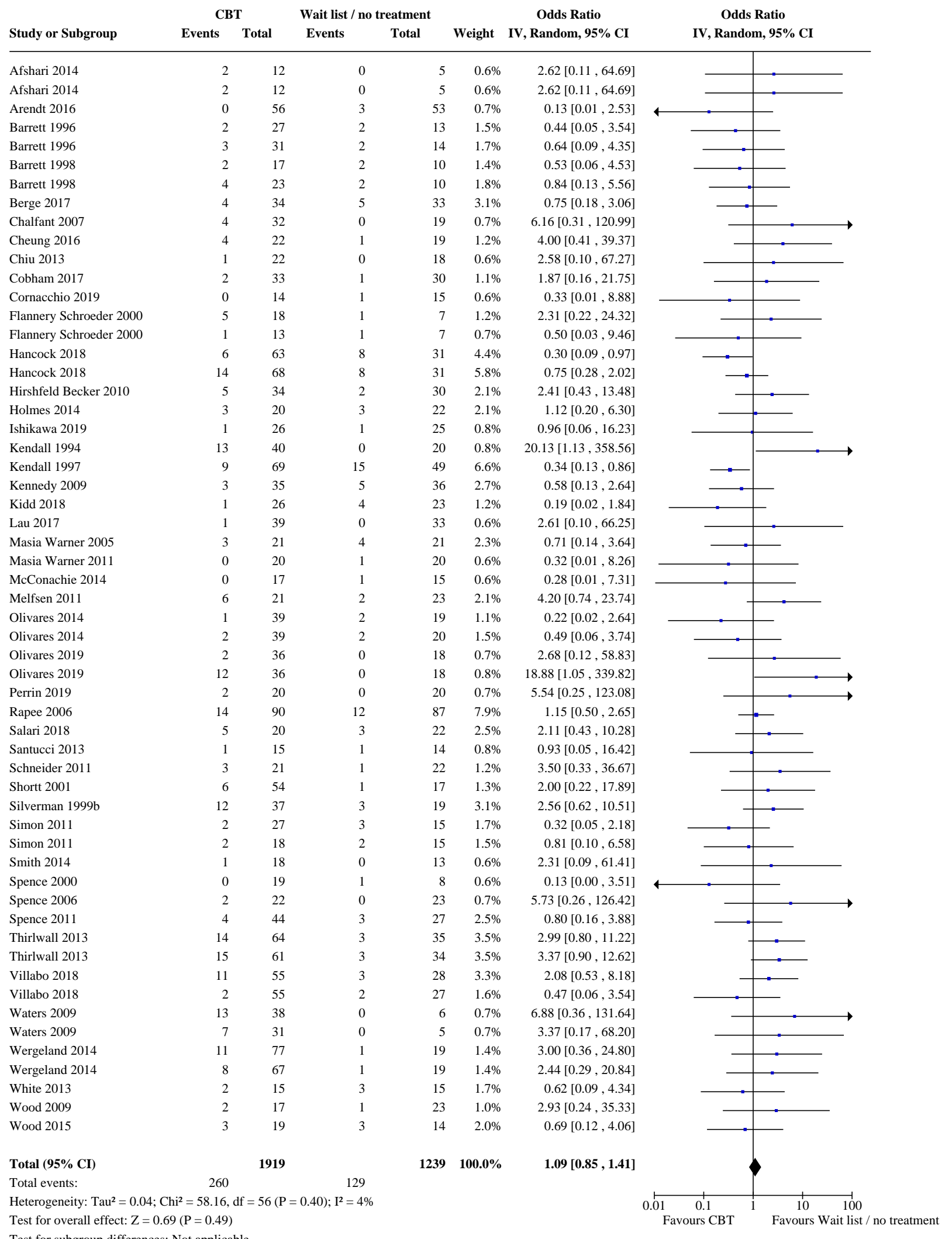




\section{Analysis 1.7. (Continued)}

Iest for overall ettect: $\angle=U .6 Y(P=U .4 Y)$

ravours CB I

Favours Wait list / no treatment 
Analysis 1.8. Comparison 1: CBT versus waitlist/no treatment, Outcome 8: Remission of all anxiety diagnoses posttreatment (ITT)

\begin{tabular}{|c|c|c|c|c|c|c|c|}
\hline & \multicolumn{2}{|c|}{ CBT } & Wait list & eatment & \multicolumn{2}{|r|}{ Odds Ratio } & Odds Ratio \\
\hline Study or Subgroup & Events & Total & Events & Total & Weight & andom, $95 \%$ CI & IV, Random, 95\% CI \\
\hline
\end{tabular}

\subsubsection{Child focused}

Barrett 1996

Barrett 1998

Chiu 2013

Dadds 1997

Olivares 2005

Perrin 2019

Rosa Alcazar 2009

Sanchez Garcia 2009

Simon 2011

Spence 2000

Villabo 2018

Villabo 2018

Subtotal (95\% CI)

Total events:

Heterogeneity: $\mathrm{Tau}^{2}=2.27 ; \mathrm{Chi}^{2}=37.22, \mathrm{df}=11(\mathrm{P}=0.0001) ; \mathrm{I}^{2}=70 \%$

Test for overall effect: $\mathrm{Z}=3.99(\mathrm{P}<0.0001)$

\subsubsection{Child and parent}

Barrett 1996

Barrett 1998

Chalfant 2007

Hirshfeld Becker 2010

Ishikawa 2019

Lau 2017

McConachie 2014

Melfsen 2011

Rapee 2006

Shortt 2001

Spence 2000

Spence 2006

Spence 2011

Waters 2009

Subtotal $(\mathbf{9 5 \%}$ CI)

Total events:

Heterogeneity: $\mathrm{Tau}^{2}=0.20 ; \mathrm{Chi}^{2}=18.86, \mathrm{df}=14(\mathrm{P}=0.17) ; \mathrm{I}^{2}=26 \%$

Test for overall effect: $\mathrm{Z}=6.93(\mathrm{P}<0.00001)$

\subsubsection{Parent only}

Cartwright Hatton 2011

Cobham 2017

Kennedy 2009

Rapee 2005

Simon 2011

Thirlwall 2013

Thirlwall 2013

Waters 2009

Subtotal (95\% CI)

Total events:

$\begin{array}{rr}12 & 37 \\ 12 & 33 \\ 15 & 35 \\ 30 & 60 \\ 20 & 27 \\ 17 & 64 \\ 7 & 61 \\ 15 & 38 \\ & \mathbf{3 5 5}\end{array}$

Heterogeneity: $\mathrm{Tau}^{2}=0.43 ; \mathrm{Chi}^{2}=14.42, \mathrm{df}=7(\mathrm{P}=0.04) ; \mathrm{I}^{2}=51 \%$

Test for overall effect: $\mathrm{Z}=1.91(\mathrm{P}=0.06)$

\section{Total $(95 \%$ CI)}

1213

Total events:

568

165

Heterogeneity: $\mathrm{Tau}^{2}=0.85 ; \mathrm{Chi}^{2}=84.49, \mathrm{df}=34(\mathrm{P}<0.00001) ; \mathrm{I}^{2}=60 \%$ Test for overall effect: $\mathrm{Z}=6.85(\mathrm{P}<0.00001)$
$1.71[0.46,6.39]$

$1.30[0.29,5.76]$

$105.00[9.93,1110.02]$

$1.78[0.47,6.69]$

31.32 [1.62, 604.04]

150.33 [7.54, 2997.83]

$33.87[1.80,636.88]$

77.61 [4.29, 1404.91]

$0.40[0.07,2.45]$

$8.25[0.82,82.67]$

$16.06[2.03,127.27]$

$16.79[3.62,77.84]$

8.52 [2.97, 24.39]
$8.94[3.10,25.79]$

$6.30[1.52,26.08]$

$3.60[0.70,18.56]$

$63.96[3.54,1155.34]$

$3.29[1.12,9.68]$

$2.09[0.35,12.59]$

34.13 [1.94, 600.96]

$0.28[0.01,7.31]$

$4.20[0.74,23.74]$

$3.94[2.01,7.71]$

$11.79[2.44,56.85]$

$32.67[2.85,374.13]$

$10.50[1.97,56.00]$

$1.48[0.41,5.37]$

$9.11[0.46,179.00]$

$5.19[3.26,8.27]$ 


\section{Analysis 1.8. (Continued)}

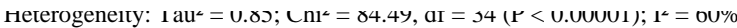
Test for overall effect: $\mathrm{Z}=6.85(\mathrm{P}<0.00001)$

\begin{tabular}{ccccc}
0.001 & 0.1 & 1 & 10 & 1000 \\
Favours Wait list / no treatment & & \multicolumn{2}{c}{ Favours CBT }
\end{tabular}

\section{Analysis 1.9. Comparison 1: CBT versus waitlist/no treatment, Outcome 9: Subgroup analysis: remission of all anxiety diagnoses post-treatment (ITT) (individual vs group)}

\begin{tabular}{|c|c|c|c|c|c|}
\hline \multirow[b]{2}{*}{ Study or Subgroup } & \multicolumn{2}{|c|}{ CBT } & \multicolumn{2}{|c|}{ Wait list / no treatment } & \multirow[b]{2}{*}{ Weight } \\
\hline & Events & Total & Events & Total & \\
\hline \multicolumn{6}{|l|}{ 1.9.1 Individual focused } \\
\hline Barrett 1996 & 16 & 31 & 5 & 13 & $10.3 \%$ \\
\hline Barrett 1996 & 21 & 27 & 5 & 14 & $9.9 \%$ \\
\hline Chiu 2013 & 21 & 22 & 3 & 18 & $6.3 \%$ \\
\hline Hirshfeld Becker 2010 & 17 & 34 & 7 & 30 & $11.5 \%$ \\
\hline Ishikawa 2019 & 4 & 26 & 2 & 25 & $8.3 \%$ \\
\hline Melfsen 2011 & 6 & 21 & 2 & 23 & $8.5 \%$ \\
\hline Perrin 2019 & 16 & 20 & 0 & 20 & $4.7 \%$ \\
\hline Spence 2011 & 9 & 44 & 4 & 27 & $10.5 \%$ \\
\hline Thirlwall 2013 & 17 & 64 & 7 & 35 & $11.9 \%$ \\
\hline Thirlwall 2013 & 7 & 61 & 6 & 34 & $11.0 \%$ \\
\hline Villabo 2018 & 21 & 55 & 1 & 27 & $7.2 \%$ \\
\hline Subtotal $(95 \%$ CI) & & 405 & & 266 & $100.0 \%$ \\
\hline Total events: & 155 & & 42 & & \\
\hline
\end{tabular}

Odds Ratio

IV, Random, $95 \%$ CI

$1.71[0.46,6.39]$

$6.30[1.52,26.08]$

$105.00[9.93,1110.02]$

$3.29[1.12,9.68]$

$2.09[0.35,12.59]$

$4.20[0.74,23.74]$

$150.33[7.54,2997.83]$

$1.48[0.41,5.37]$

$1.45[0.53,3.92]$

$0.60[0.19,1.97]$

$16.06[2.03,127.27]$

$3.72[1.70,8.12]$

Heterogeneity: $\mathrm{Tau}^{2}=1.08 ; \mathrm{Chi}^{2}=29.80, \mathrm{df}=10(\mathrm{P}=0.0009) ; \mathrm{I}^{2}=66 \%$

Test for overall effect: $\mathrm{Z}=3.29(\mathrm{P}=0.0010)$

\subsubsection{Group focused}

Arendt 2016

Barrett 1998

Barrett 1998

Cartwright Hatton 2011

Chalfant 2007

Cobham 2017

Dadds 1997

Kennedy 2009

Lau 2017

McConachie 2014

Olivares 2005

Rapee 2005

Rapee 2006

Rosa Alcazar 2009

Sanchez Garcia 2009

Shortt 2001

Simon 2011

Simon 2011

Spence 2000

Spence 2000

Spence 2006

Villabo 2018

Waters 2009

Waters 2009

Subtotal (95\% CI)

Total events:

$\begin{array}{rr}27 & 56 \\ 13 & 23 \\ 12 & 17 \\ 12 & 37 \\ 20 & 32 \\ 12 & 33 \\ 19 & 23 \\ 15 & 35 \\ 13 & 39 \\ 0 & 17 \\ 8 & 17 \\ 30 & 60 \\ 44 & 90 \\ 9 & 20 \\ 17 & 28 \\ 33 & 54 \\ 13 & 18 \\ 20 & 27 \\ 11 & 19 \\ 14 & 17 \\ 11 & 22 \\ 31 & 55 \\ 15 & 38 \\ 14 & 31 \\ & \mathbf{8 0 8}\end{array}$

Heterogeneity: $\mathrm{Tau}^{2}=0.76 ; \mathrm{Chi}^{2}=52.14, \mathrm{df}=23(\mathrm{P}=0.0005) ; \mathrm{I}^{2}=56 \%$

Test for overall effect: $\mathrm{Z}=6.11(\mathrm{P}<0.00001)$

Test for subgroup differences: $\mathrm{Chi}^{2}=0.35, \mathrm{df}=1(\mathrm{P}=0.56), \mathrm{I}^{2}=0 \%$

$\begin{array}{rr}53 & 6.5 \% \\ 10 & 5.1 \% \\ 10 & 4.7 \% \\ 33 & 4.8 \% \\ 19 & 2.3 \% \\ 30 & 4.8 \% \\ 33 & 5.6 \% \\ 36 & 6.5 \% \\ 33 & 2.3 \% \\ 15 & 1.9 \% \\ 17 & 2.2 \% \\ 51 & 7.5 \% \\ 87 & 7.7 \% \\ 20 & 2.3 \% \\ 25 & 2.3 \% \\ 17 & 4.8 \% \\ 15 & 4.2 \% \\ 15 & 3.4 \% \\ 7 & 3.2 \% \\ 8 & 2.9 \% \\ 23 & 4.6 \% \\ 28 & 5.0 \% \\ 6 & 3.3 \% \\ 5 & 2.2 \% \\ \mathbf{5 9 6} & \mathbf{1 0 0 . 0 \%}\end{array}$

$8.94[3.10,25.79]$

$1.30[0.29,5.76]$

$3.60[0.70,18.56]$

$7.44[1.52,36.37]$

$63.96[3.54,1155.34]$

$8.00[1.61,39.64]$

$2.63[0.94,7.37]$

$34.13[1.94,600.96]$

$0.28[0.01,7.31]$

$31.32[1.62,604.04]$

$1.68[0.79,3.60]$

$3.94[2.01,7.71]$

$33.87[1.80,636.88]$

$77.61[4.29,1404.91]$

$11.79[2.44,56.85]$

$0.40[0.07,2.45]$

$0.20[0.02,1.85]$

8.25 [0.82, 82.67]

$32.67[2.85,374.13]$

10.50 [1.97, 56.00]

$16.79[3.62,77.84]$

$3.26[0.35,30.73]$

$9.11[0.46,179.00]$

$4.92[2.95,8.20]$
$1.78[0.47,6.69]$
Odds Ratio

IV, Random, $95 \%$ CI 


\section{Analysis 1.10. Comparison 1: CBT versus waitlist/no treatment, Outcome 10: Subgroup analysis: remission of all anxiety diagnoses post-treatment (ITT) (amount of therapist contact time)}

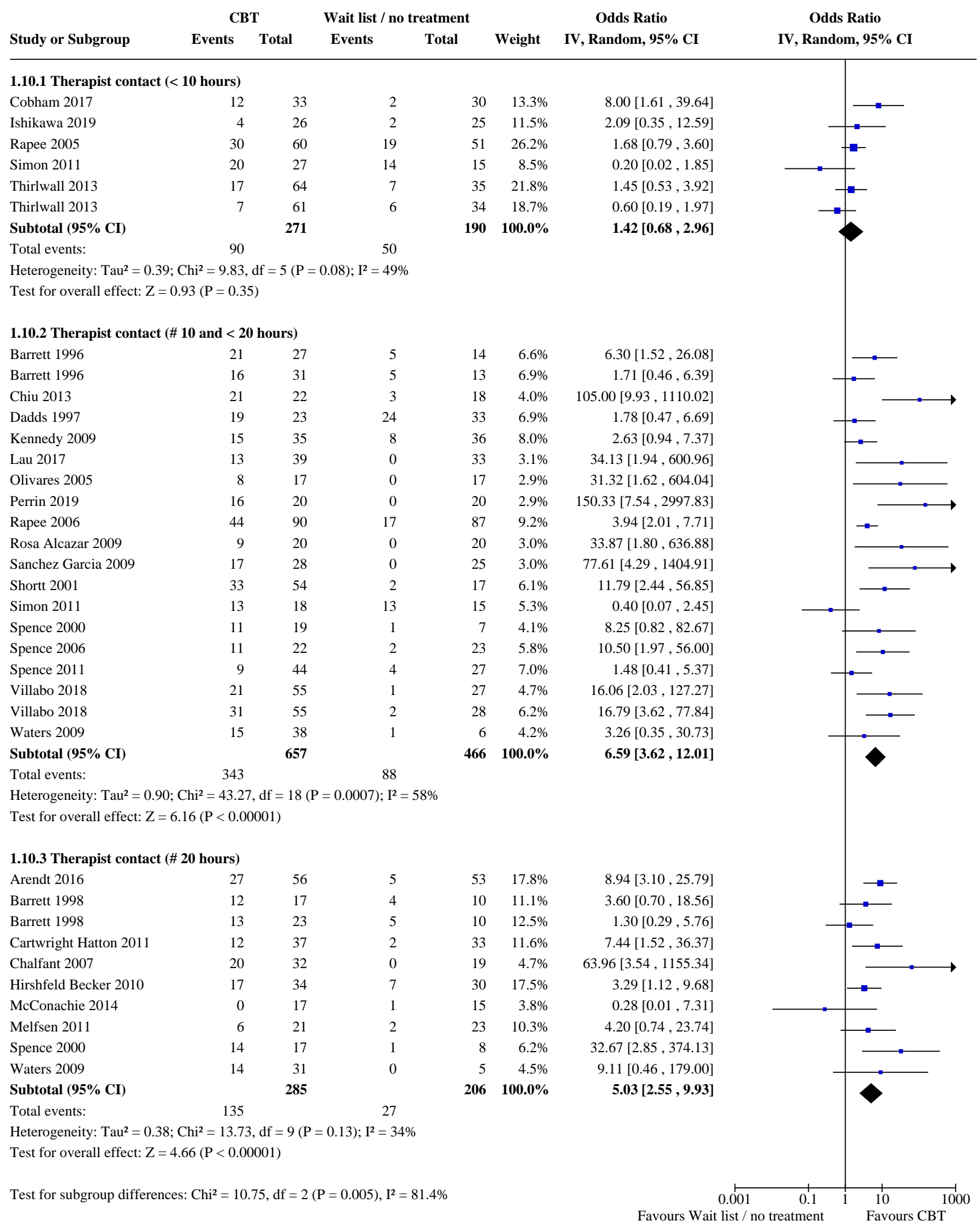


Analysis 1.11. Comparison 1: CBT versus waitlist/no treatment, Outcome 11: Subgroup analysis: remission of all anxiety diagnoses post-treatment (ITT) (age)

\begin{tabular}{lrrr} 
Study or Subgroup & CBT & \multicolumn{2}{c}{ Wait list / no t } \\
Events
\end{tabular}

Odds Ratio

Odds Ratio

$\begin{array}{lcc}\text { Total events: } & 238 & 72 \\ \text { Heterogeneity: } \mathrm{Tau}^{2}=0.59 ; \mathrm{Chi}^{2}=28.19, \mathrm{df}=11(\mathrm{P}=0.003) ; \mathrm{I}^{2}=61 \%\end{array}$

Test for overall effect: $\mathrm{Z}=4.17(\mathrm{P}<0.0001)$

1.11.2 Mixed age range ( $<12$ years and \# 12 years)

$\begin{array}{lrrrrr}\text { Arendt 2016 } & 27 & 56 & 5 & 53 & 7.2 \% \\ \text { Barrett 1996 } & 16 & 31 & 5 & 13 & 6.5 \% \\ \text { Barrett 1996 } & 21 & 27 & 5 & 14 & 6.2 \% \\ \text { Barrett 1998 } & 12 & 17 & 4 & 10 & 5.6 \% \\ \text { Barrett 1998 } & 13 & 23 & 5 & 10 & 6.0 \% \\ \text { Chalfant 2007 } & 20 & 32 & 0 & 19 & 3.1 \% \\ \text { Cobham 2017 } & 12 & 33 & 2 & 30 & 5.7 \% \\ \text { Dadds 1997 } & 19 & 23 & 24 & 33 & 6.5 \% \\ \text { Ishikawa 2019 } & 4 & 26 & 2 & 25 & 5.2 \% \\ \text { McConachie 2014 } & 0 & 17 & 1 & 15 & 2.7 \% \\ \text { Melfsen 2011 } & 6 & 21 & 2 & 23 & 5.4 \% \\ \text { Perrin 2019 } & 16 & 20 & 0 & 20 & 3.0 \% \\ \text { Sanchez Garcia 2009 } & 17 & 28 & 0 & 25 & 3.1 \% \\ \text { Simon 2011 } & 13 & 18 & 13 & 15 & 5.2 \% \\ \text { Simon 2011 } & 20 & 27 & 14 & 15 & 4.3 \% \\ \text { Spence 2000 } & 11 & 19 & 1 & 7 & 4.1 \% \\ \text { Spence 2000 } & 14 & 17 & 1 & 8 & 3.9 \% \\ \text { Spence 2006 } & 11 & 22 & 2 & 23 & 5.5 \% \\ \text { Villabo 2018 } & 31 & 55 & 2 & 28 & 5.9 \% \\ \text { Villabo 2018 } & 21 & 55 & 1 & 27 & 4.6 \% \\ \text { Subtotal (95\% CI) } & & \mathbf{5 6 7} & & \mathbf{4 1 3} & \mathbf{1 0 0 . 0 \%}\end{array}$

$8.94[3.10,25.79]$

$1.71[0.46,6.39]$

$6.30[1.52,26.08]$

$3.60[0.70,18.56]$

$1.30[0.29,5.76]$

$63.96[3.54,1155.34]$

$8.00[1.61,39.64]$

$1.78[0.47,6.69]$

$2.09[0.35,12.59]$

$0.28[0.01,7.31]$

$4.20[0.74,23.74]$

150.33 [7.54, 2997.83]

$77.61[4.29,1404.91]$

$0.40[0.07,2.45]$

$0.20[0.02,1.85]$

$8.25[0.82,82.67]$

$32.67[2.85,374.13]$

$10.50[1.97,56.00]$

$16.79[3.62,77.84]$

$16.06[2.03,127.27]$

$4.87[2.58,9.21]$

IV, Random, $95 \%$ CI

Total events:

304

89

Heterogeneity: $\mathrm{Tau}^{2}=1.17 ; \mathrm{Chi}^{2}=47.24, \mathrm{df}=19(\mathrm{P}=0.0003) ; \mathrm{I}^{2}=60 \%$

Test for overall effect: $\mathrm{Z}=4.87(\mathrm{P}<0.00001)$

\begin{tabular}{|c|c|c|c|}
\hline \multicolumn{4}{|l|}{ 1.11.3 \# 12 years } \\
\hline Olivares 2005 & 8 & 17 & 0 \\
\hline Rosa Alcazar 2009 & 9 & 20 & 0 \\
\hline Spence 2011 & 9 & 44 & 4 \\
\hline Subtotal $(95 \%$ CI) & & 81 & \\
\hline Total events: & 26 & & 4 \\
\hline \multicolumn{4}{|c|}{ Heterogeneity: $\mathrm{Tau}^{2}=2.94 ; \mathrm{Chi}^{2}=6.12, \mathrm{df}=2(\mathrm{P}=0.05) ; \mathrm{I}^{2}=67 \%$} \\
\hline Test for overall effe & $=0.0$ & & \\
\hline
\end{tabular}


Analysis 1.12. Comparison 1: CBT versus waitlist/no treatment, Outcome 12: Remission of all anxiety diagnoses post-treatment (completers)

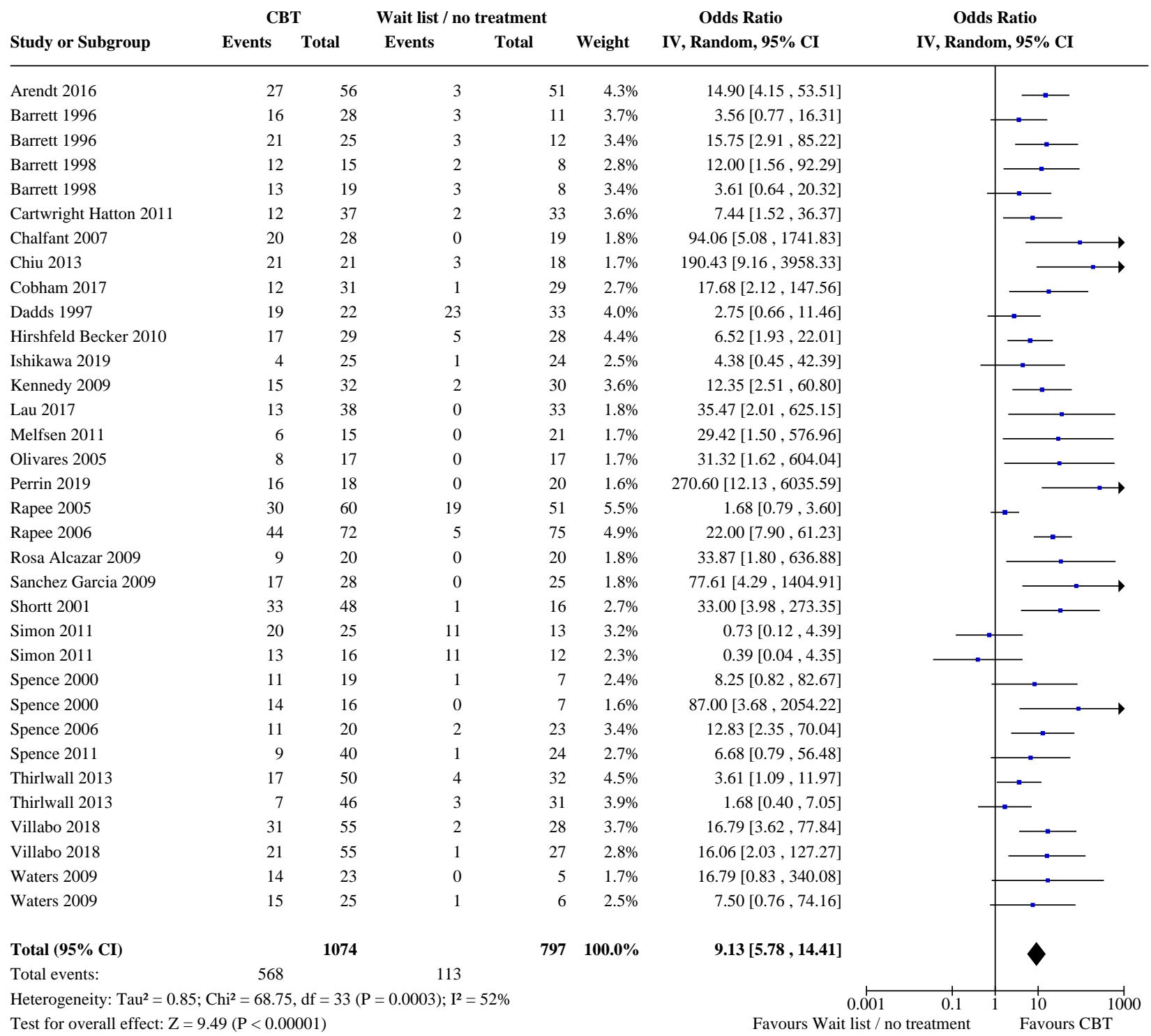


Analysis 1.13. Comparison 1: CBT versus waitlist/no treatment, Outcome 13: Reduction in anxiety symptoms (child report) post-treatment

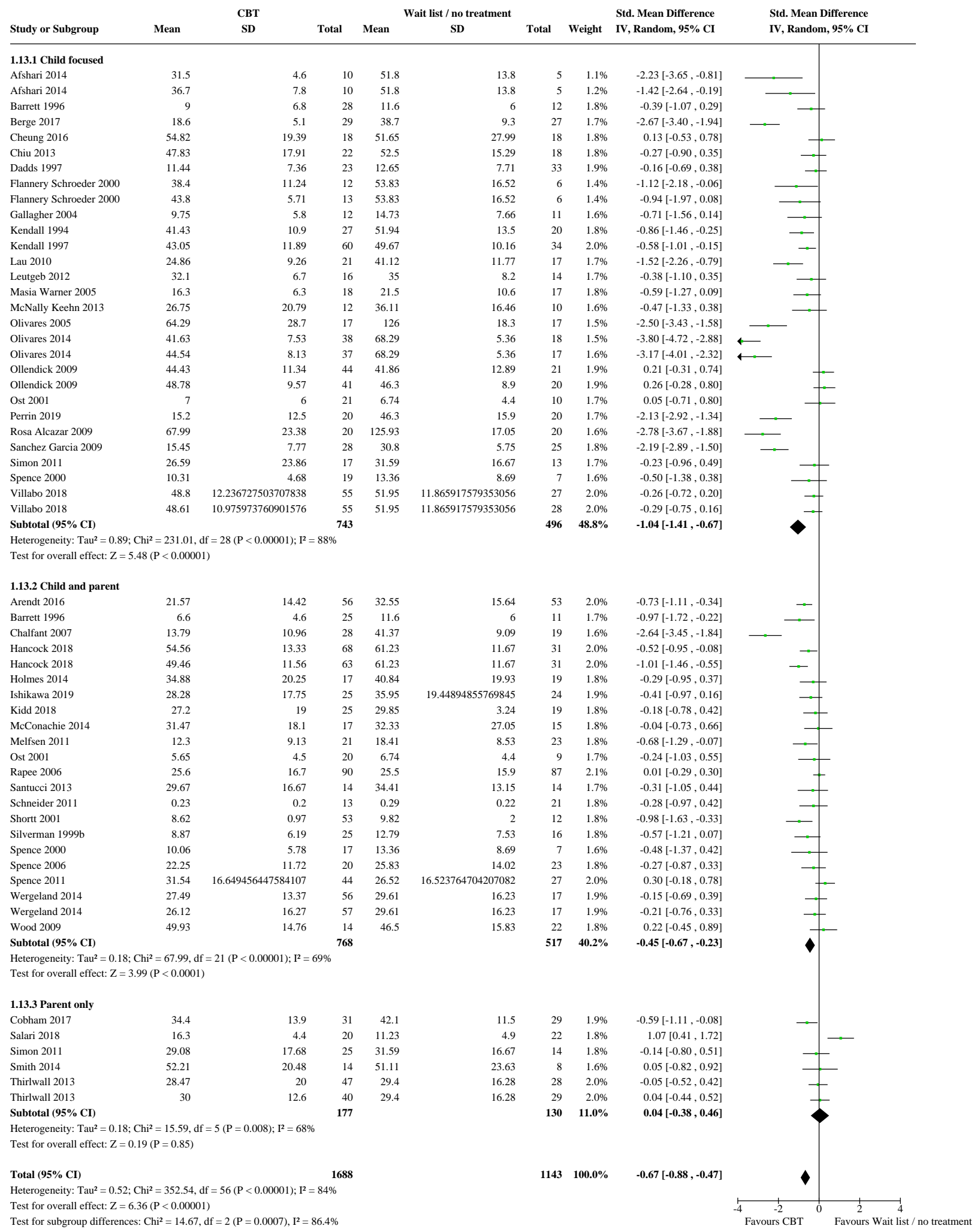


Analysis 1.14. Comparison 1: CBT versus waitlist/no treatment, Outcome 14: Sensitivity analysis: reduction in anxiety symptoms (child report) post-treatment (broad anxiety measures)

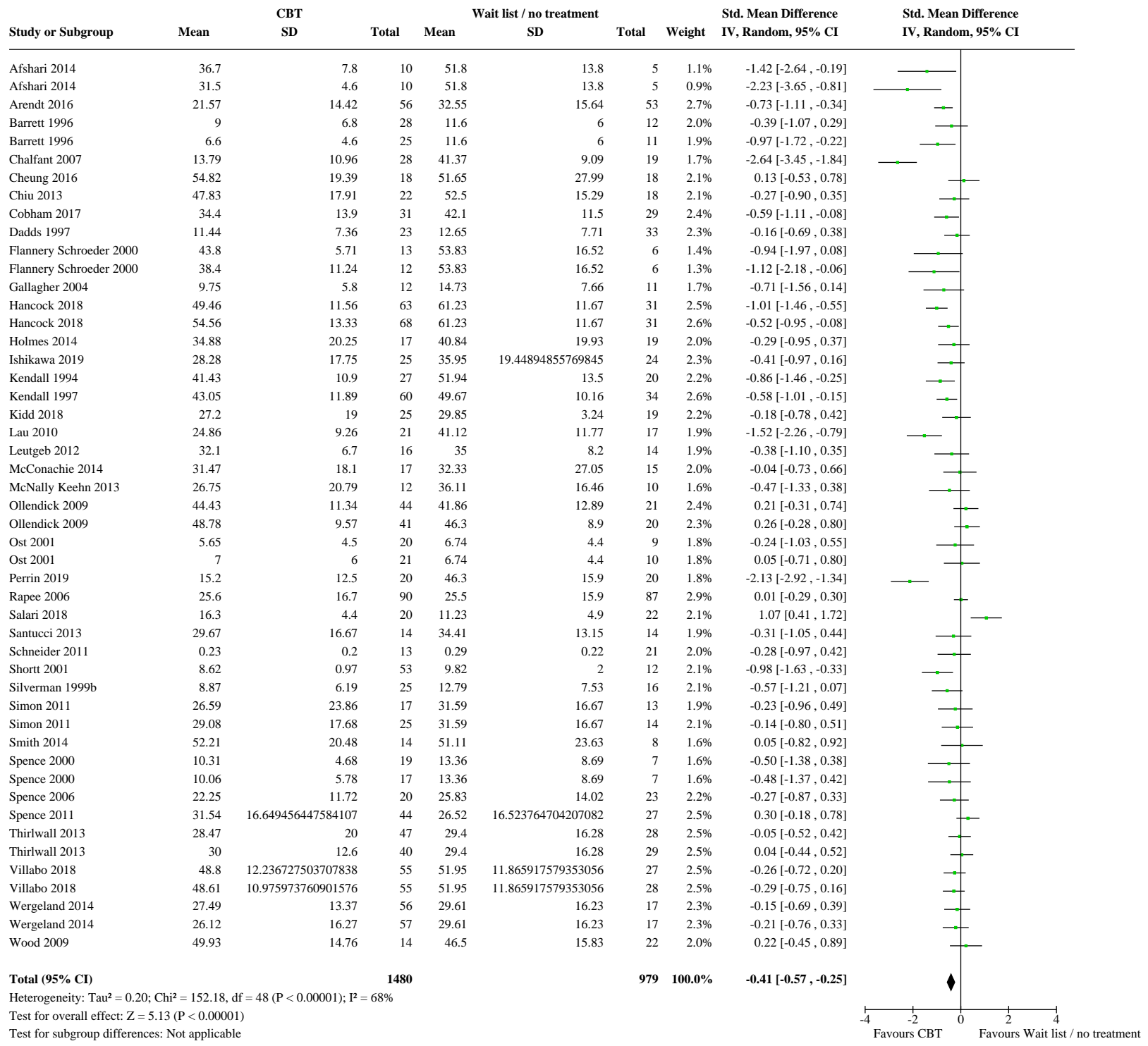


Analysis 1.15. Comparison 1: CBT versus waitlist/no treatment, Outcome 15: Subgroup analysis: reduction in anxiety symptoms (child report) post-treatment (individual vs group)

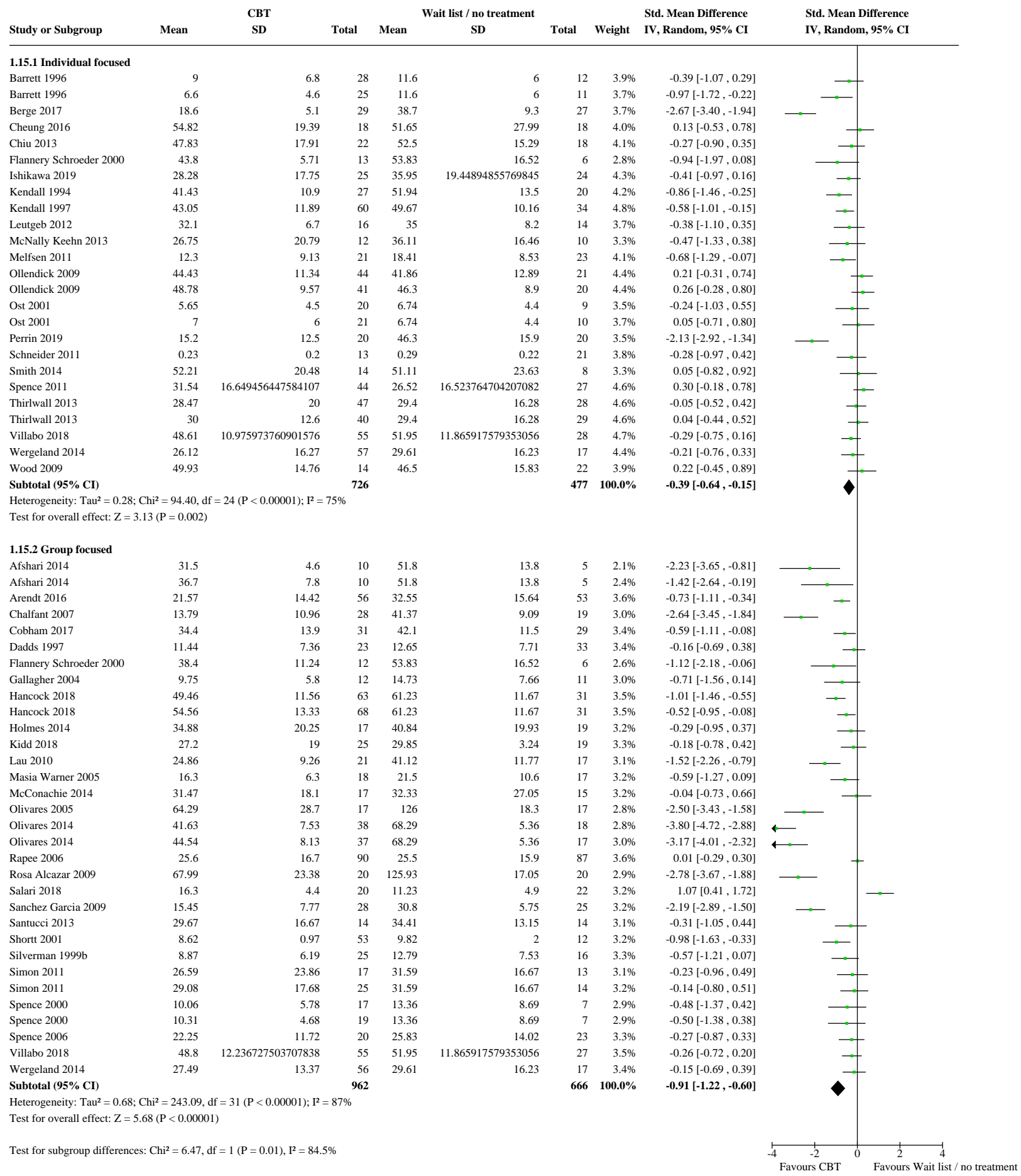


Analysis 1.16. Comparison 1: CBT versus waitlist/no treatment, Outcome 16: Subgroup analysis: reduction in anxiety symptoms (child report) post-treatment (amount of therapist contact time)

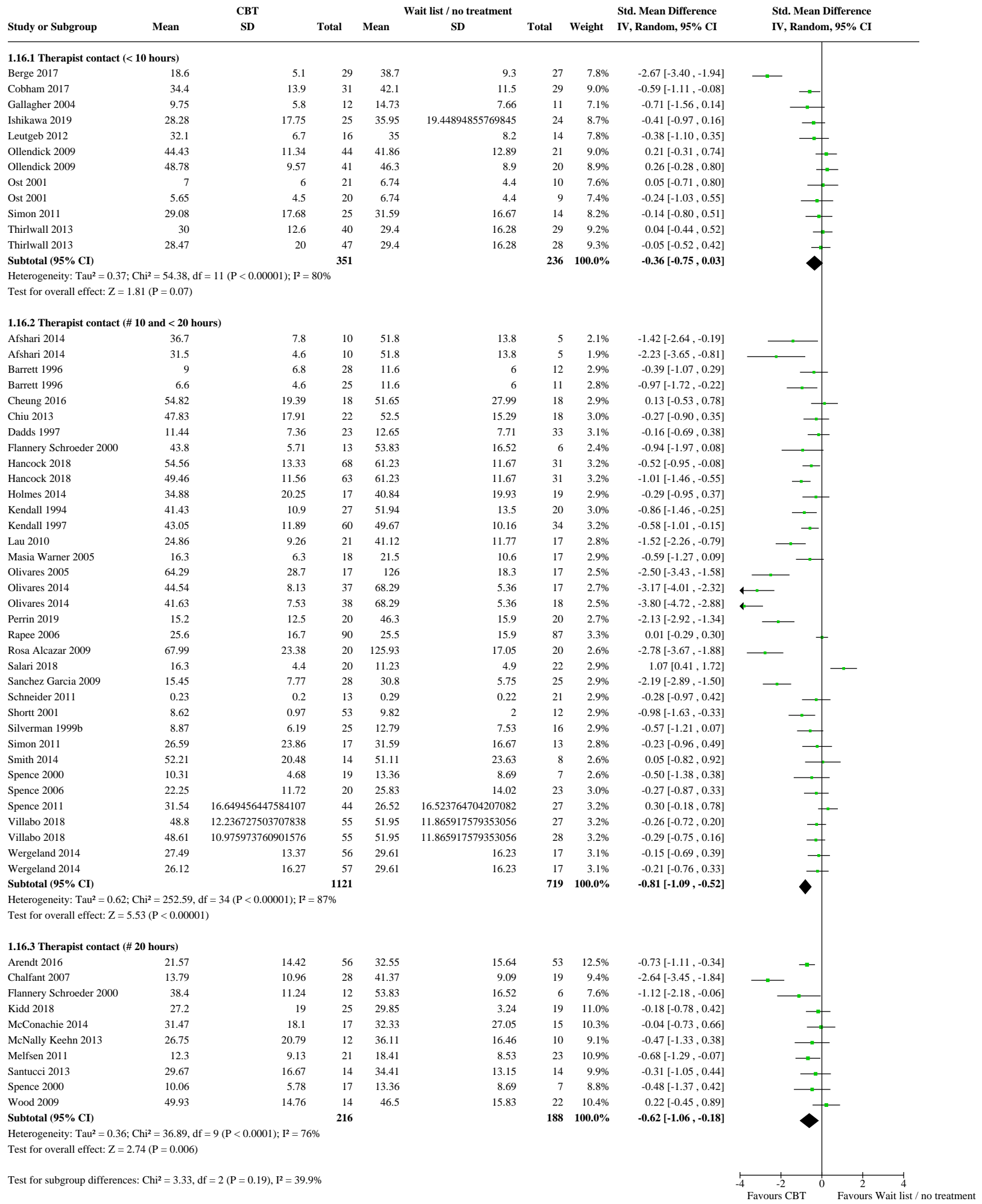


Analysis 1.17. Comparison 1: CBT versus waitlist/no treatment, Outcome 17: Subgroup analysis: reduction in anxiety symptoms (child report) post-treatment (age)

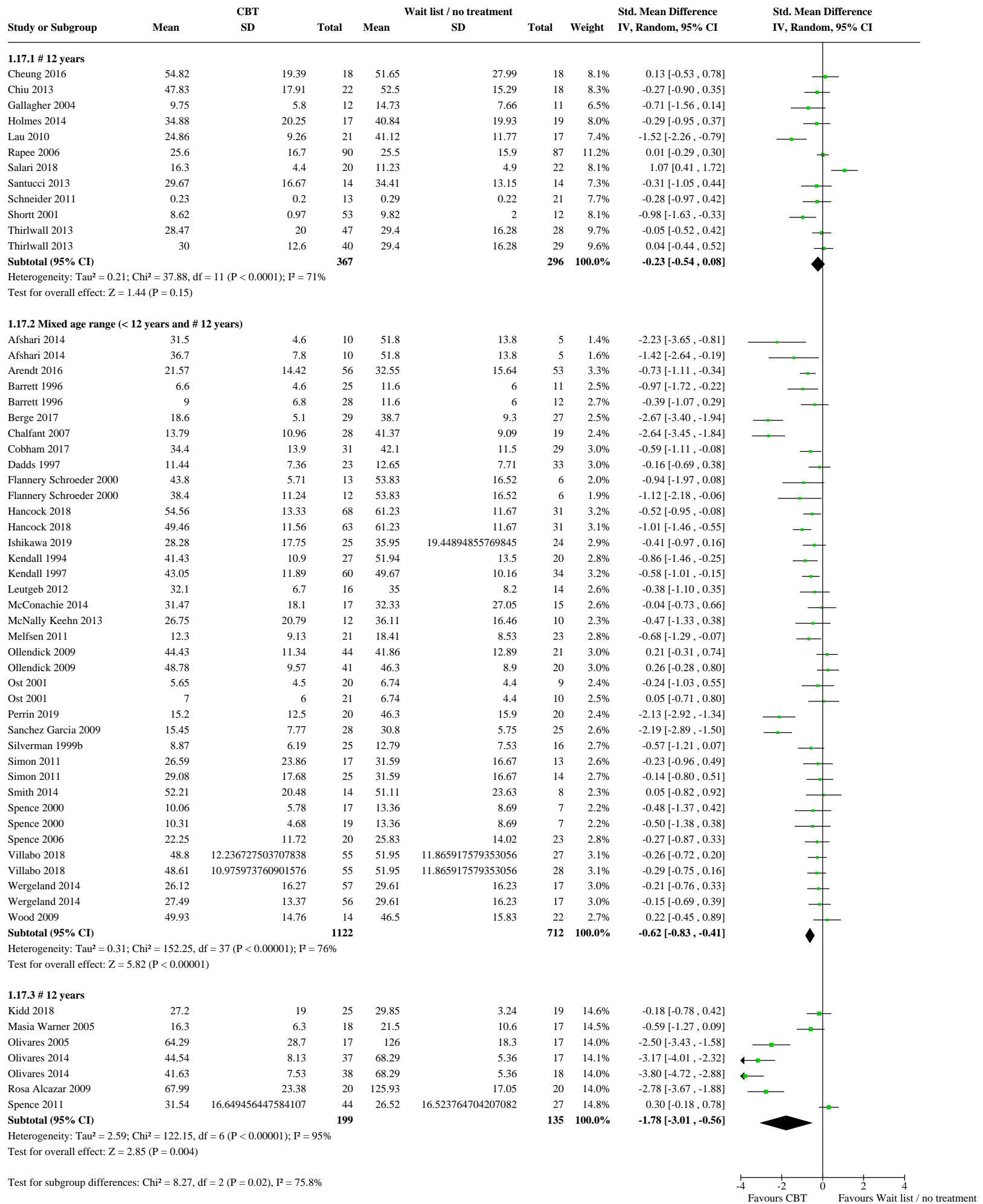


Analysis 1.18. Comparison 1: CBT versus waitlist/no treatment, Outcome 18: Subgroup analysis: reduction in anxiety symptoms (child report) post-treatment (ASD vs non-ASD)

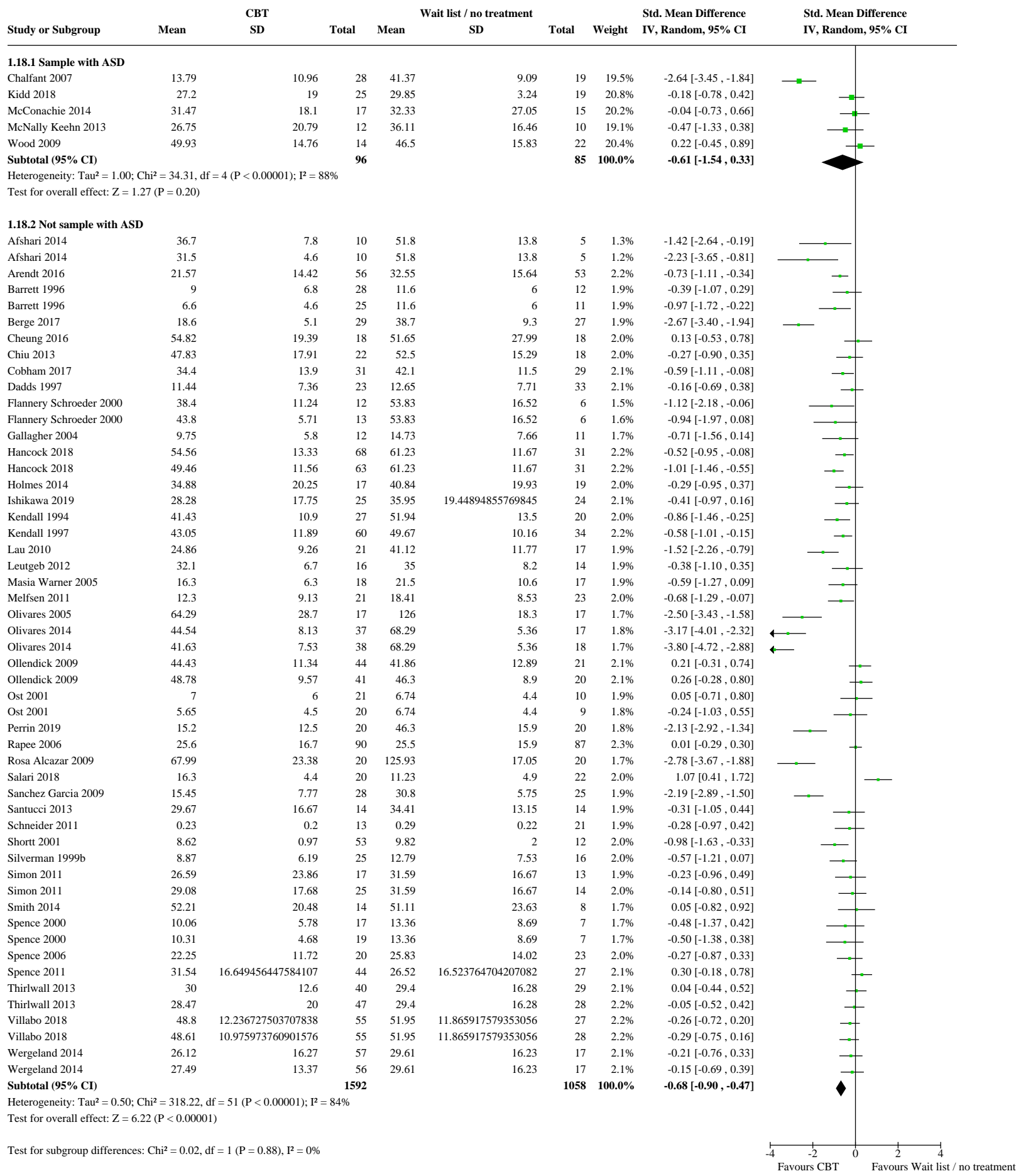


Analysis 1.19. Comparison 1: CBT versus waitlist/no treatment, Outcome 19: Reduction in anxiety symptoms (parent report) post-treatment

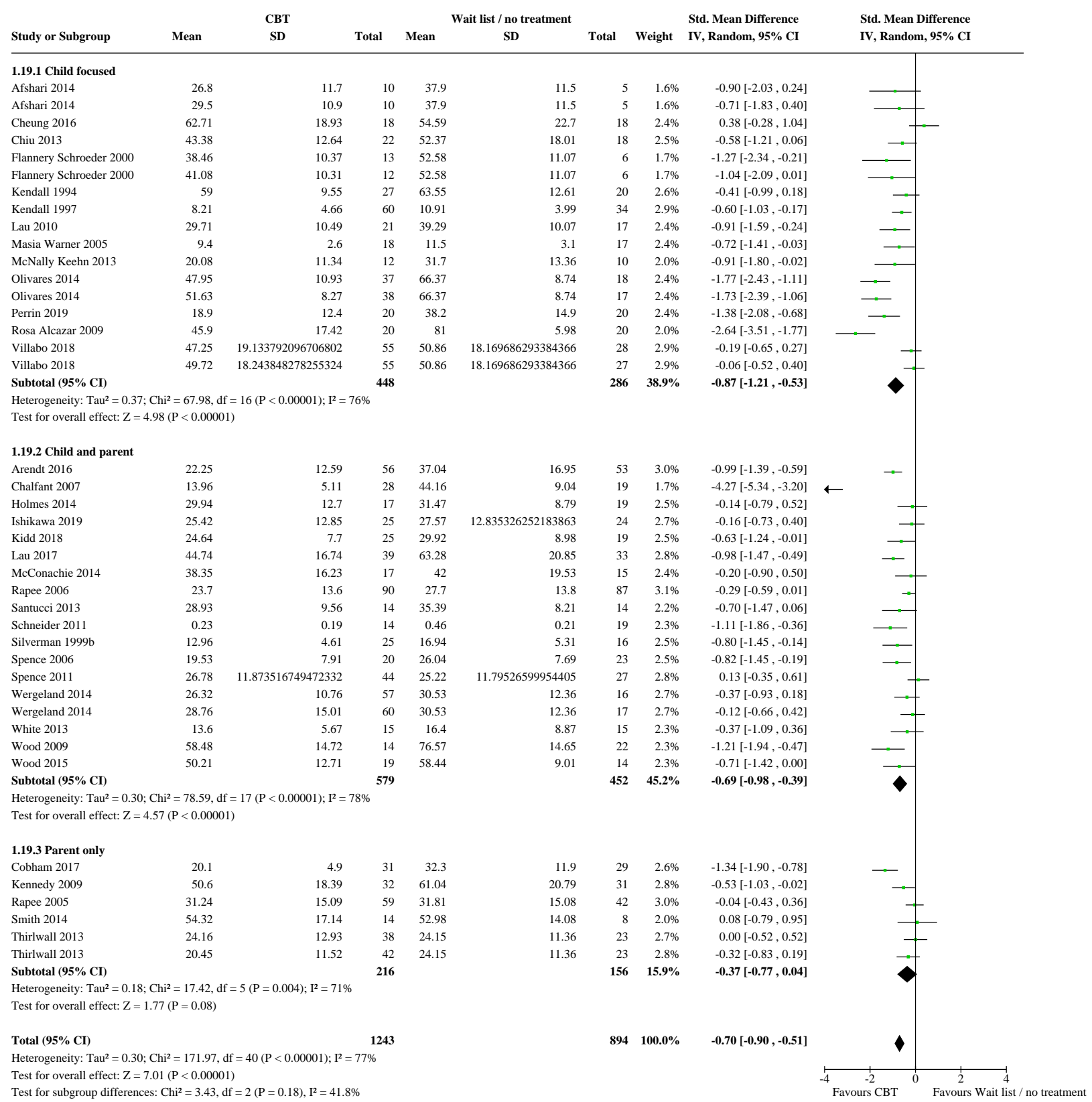


Analysis 1.20. Comparison 1: CBT versus waitlist/no treatment, Outcome 20: Sensitivity analysis: reduction in anxiety symptoms (parent report) post-treatment (broad anxiety measures only)

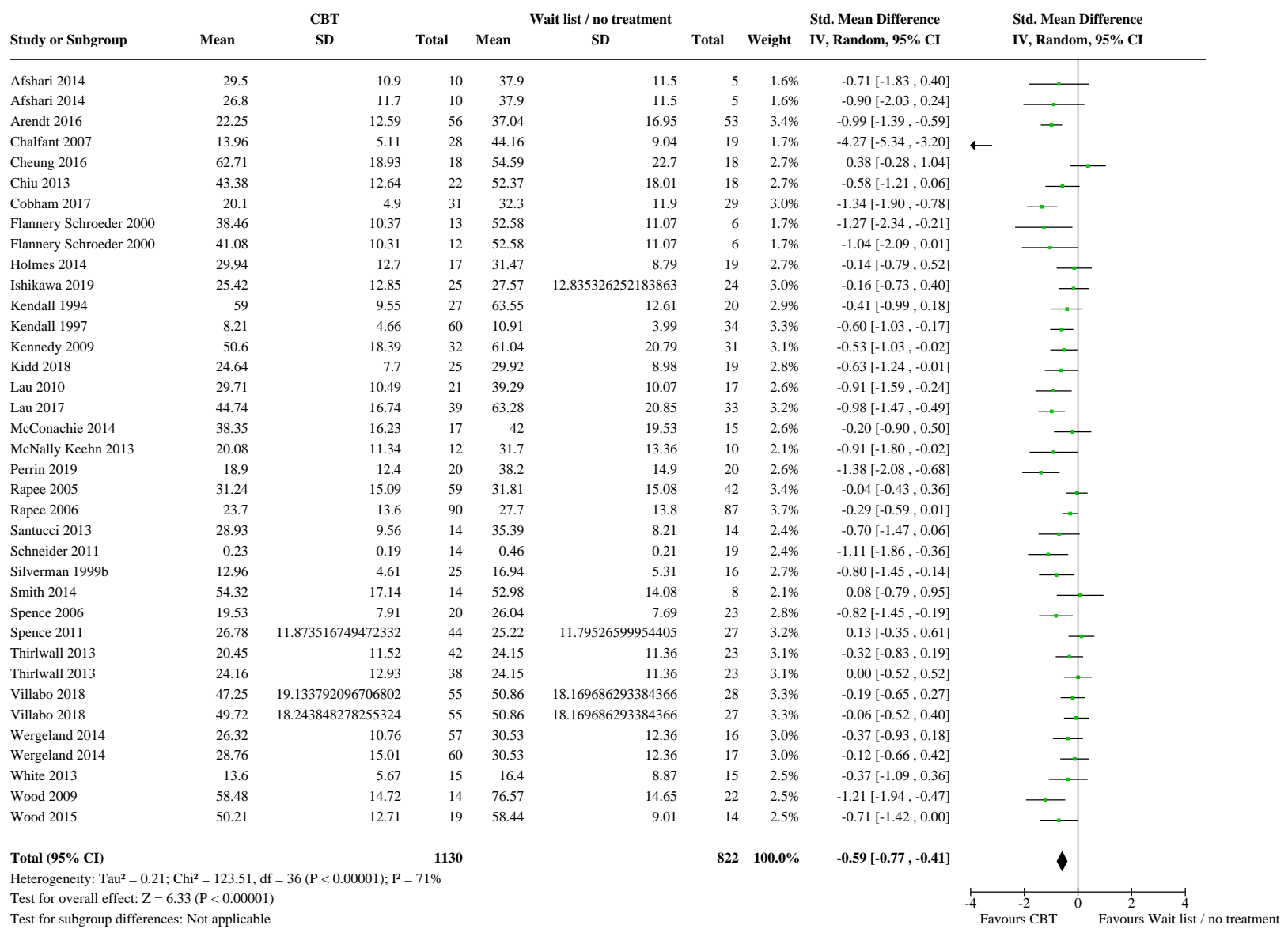


Analysis 1.21. Comparison 1: CBT versus waitlist/no treatment, Outcome 21: Subgroup analysis: reduction in anxiety symptoms (parent report) post-treatment (individual vs group)

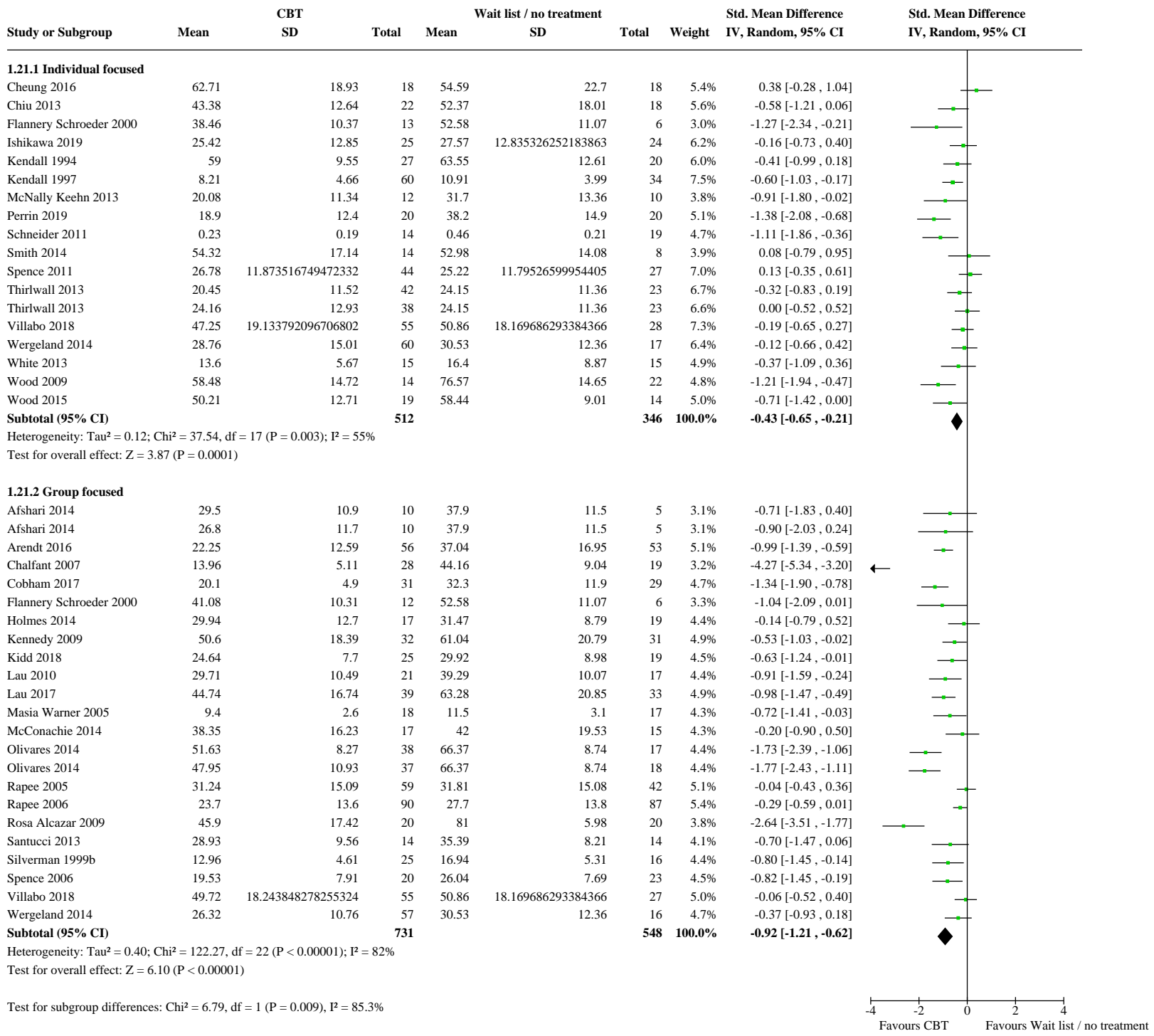


Analysis 1.22. Comparison 1: CBT versus waitlist/no treatment, Outcome 22: Subgroup analysis: reduction in anxiety symptoms (parent report) post-treatment (amount of therapist contact time)

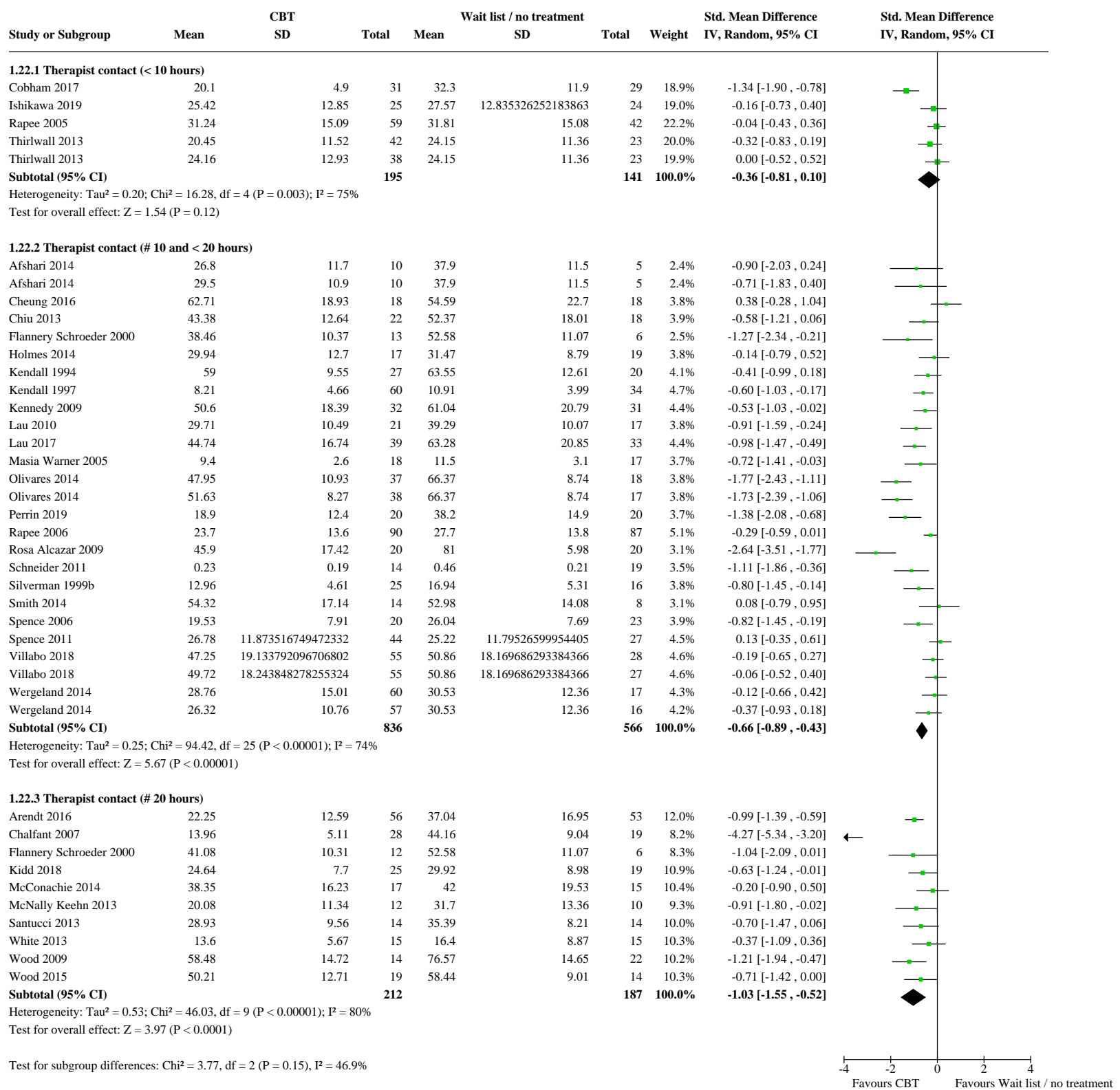


Analysis 1.23. Comparison 1: CBT versus waitlist/no treatment, Outcome 23: Subgroup analysis: reduction in anxiety symptoms (parent report) post-treatment (age)

\begin{tabular}{|c|c|c|c|c|c|c|c|c|c|c|}
\hline \multirow{2}{*}{$\begin{array}{l}\text { Study or Subgroup } \\
1.23 .1 \text { \# } 12 \text { years }\end{array}$} & Mean & \multicolumn{2}{|l|}{ CBT } & \multicolumn{3}{|c|}{ Wait list / no treatment } & Weight & $\begin{array}{l}\text { Std. Mean Difference } \\
\text { IV, Random, } 95 \% \text { CI }\end{array}$ & \multicolumn{2}{|c|}{$\begin{array}{l}\text { Std. Mean Difference } \\
\text { IV, Random, } 95 \% \text { CI }\end{array}$} \\
\hline & & & & Mean & SD & Total & & & & \\
\hline Cheung 2016 & 62.71 & 18.93 & 18 & 54.59 & 22.7 & 18 & $7.0 \%$ & $0.38[-0.28,1.04]$ & & - \\
\hline Chiu 2013 & 43.38 & 12.64 & 22 & 52.37 & 18.01 & 18 & $7.2 \%$ & $-0.58[-1.21,0.06]$ & & \\
\hline Holmes 2014 & 29.94 & 12.7 & 17 & 31.47 & 8.79 & 19 & $7.0 \%$ & $-0.14[-0.79,0.52]$ & & - \\
\hline Kennedy 2009 & 50.6 & 18.39 & 32 & 61.04 & 20.79 & 31 & $9.2 \%$ & $-0.53[-1.03,-0.02]$ & & \\
\hline Lau 2010 & 29.71 & 10.49 & 21 & 39.29 & 10.07 & 17 & $6.8 \%$ & $-0.91[-1.59,-0.24]$ & & \\
\hline Lau 2017 & 44.74 & 16.74 & 39 & 63.28 & 20.85 & 33 & $9.3 \%$ & $-0.98[-1.47,-0.49]$ & & \\
\hline Rapee 2005 & 31.24 & 15.09 & 59 & 31.81 & 15.08 & 42 & $11.0 \%$ & $-0.04[-0.43,0.36]$ & & \\
\hline Rapee 2006 & 23.7 & 13.6 & 90 & 27.7 & 13.8 & 87 & $12.8 \%$ & $-0.29[-0.59,0.01]$ & & \\
\hline Santucci 2013 & 28.93 & 9.56 & 14 & 35.39 & 8.21 & 14 & $5.8 \%$ & $-0.70[-1.47,0.06]$ & & \\
\hline Schneider 2011 & 0.23 & 0.19 & 14 & 0.46 & 0.21 & 19 & $6.0 \%$ & $-1.11[-1.86,-0.36]$ & & \\
\hline Thirlwall 2013 & 20.45 & 11.52 & 42 & 24.15 & 11.36 & 23 & $9.0 \%$ & $-0.32[-0.83,0.19]$ & & \\
\hline Thirlwall 2013 & 24.16 & 12.93 & 38 & 24.15 & 11.36 & 23 & $8.9 \%$ & $0.00[-0.52,0.52]$ & & \\
\hline Subtotal (95\% CI) & & & 406 & & & 344 & $100.0 \%$ & $-0.40[-0.63,-0.17]$ & 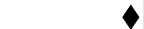 & \\
\hline $\begin{array}{l}\text { Heterogeneity: } \mathrm{Tau}^{2}=0.08 \\
\text { Test for overall effect: } Z=\end{array}$ & 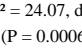 & $=11(\mathrm{P}=0.01) ; \mathrm{I}^{2}=54 \%$ & & & & & & & & \\
\hline 1.23.2 Mixed age range ( & vears and \# & 2 years) & & & & & & & & \\
\hline Afshari 2014 & 29.5 & 10.9 & 10 & 37.9 & 11.5 & 5 & $3.2 \%$ & $-0.71[-1.83,0.40]$ & & \\
\hline Afshari 2014 & 26.8 & 11.7 & 10 & 37.9 & 11.5 & 5 & $3.1 \%$ & $-0.90[-2.03,0.24]$ & & \\
\hline Arendt 2016 & 22.25 & 12.59 & 56 & 37.04 & 16.95 & 53 & $5.7 \%$ & $-0.99[-1.39,-0.59]$ & & \\
\hline Chalfant 2007 & 13.96 & 5.11 & 28 & 44.16 & 9.04 & 19 & $3.3 \%$ & $-4.27[-5.34,-3.20]$ & $\leftarrow$ & \\
\hline Cobham 2017 & 20.1 & 4.9 & 31 & 32.3 & 11.9 & 29 & $5.1 \%$ & $-1.34[-1.90,-0.78]$ & & \\
\hline Flannery Schroeder 2000 & 41.08 & 10.31 & 12 & 52.58 & 11.07 & 6 & $3.4 \%$ & $-1.04[-2.09,0.01]$ & & \\
\hline Flannery Schroeder 2000 & 38.46 & 10.37 & 13 & 52.58 & 11.07 & 6 & $3.3 \%$ & $-1.27[-2.34,-0.21]$ & & \\
\hline Ishikawa 2019 & 25.42 & 12.85 & 25 & 27.57 & 12.835326252183863 & 24 & $5.1 \%$ & $-0.16[-0.73,0.40]$ & & \\
\hline Kendall 1994 & 59 & 9.55 & 27 & 63.55 & 12.61 & 20 & $5.0 \%$ & $-0.41[-0.99,0.18]$ & & \\
\hline Kendall 1997 & 8.21 & 4.66 & 60 & 10.91 & 3.99 & 34 & $5.6 \%$ & $-0.60[-1.03,-0.17]$ & - & \\
\hline McConachie 2014 & 38.35 & 16.23 & 17 & 42 & 19.53 & 15 & $4.6 \%$ & $-0.20[-0.90,0.50]$ & & \\
\hline McNally Keehn 2013 & 20.08 & 11.34 & 12 & 31.7 & 13.36 & 10 & $3.9 \%$ & $-0.91[-1.80,-0.02]$ & & \\
\hline Perrin 2019 & 18.9 & 12.4 & 20 & 38.2 & 14.9 & 20 & $4.6 \%$ & $-1.38[-2.08,-0.68]$ & & \\
\hline Silverman 1999b & 12.96 & 4.61 & 25 & 16.94 & 5.31 & 16 & $4.8 \%$ & $-0.80[-1.45,-0.14]$ & 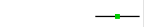 & \\
\hline Smith 2014 & 54.32 & 17.14 & 14 & 52.98 & 14.08 & 8 & $4.0 \%$ & $0.08[-0.79,0.95]$ & & - \\
\hline Spence 2006 & 19.53 & 7.91 & 20 & 26.04 & 7.69 & 23 & $4.9 \%$ & $-0.82[-1.45,-0.19]$ & & \\
\hline Villabo 2018 & 47.25 & 19.133792096706802 & 55 & 50.86 & 18.169686293384366 & 28 & $5.5 \%$ & $-0.19[-0.65,0.27]$ & & \\
\hline Villabo 2018 & 49.72 & 18.243848278255324 & 55 & 50.86 & 18.169686293384366 & 27 & $5.5 \%$ & $-0.06[-0.52,0.40]$ & & \\
\hline Wergeland 2014 & 26.32 & 10.76 & 57 & 30.53 & 12.36 & 16 & $5.1 \%$ & $-0.37[-0.93,0.18]$ & & \\
\hline Wergeland 2014 & 28.76 & 15.01 & 60 & 30.53 & 12.36 & 17 & $5.2 \%$ & $-0.12[-0.66,0.42]$ & & \\
\hline Wood 2009 & 58.48 & 14.72 & 14 & 76.57 & 14.65 & 22 & $4.5 \%$ & $-1.21[-1.94,-0.47]$ & - & \\
\hline Wood 2015 & 50.21 & 12.71 & 19 & 58.44 & 9.01 & 14 & $4.5 \%$ & $-0.71[-1.42,0.00]$ & & \\
\hline Subtotal (95\% CI) & & & 640 & & & 417 & $100.0 \%$ & $-0.76[-1.04,-0.49]$ & & \\
\hline Heterogeneity: $\mathrm{Tau}^{2}=0.31$ & ${ }^{2}=84.16, \mathrm{~d}$ & $=21(\mathrm{P}<0.00001) ; \mathrm{I}^{2}=75$ & & & & & & & & \\
\hline Test for overall effect: $Z=$ & $(\mathrm{P}<0.0000$ & & & & & & & & & \\
\hline 1.23.3 \# 12 years & & & & & & & & & & \\
\hline Kidd 2018 & 24.64 & 7.7 & 25 & 29.92 & 8.98 & 19 & $14.6 \%$ & $-0.63[-1.24,-0.01]$ & 一 & \\
\hline Masia Warner 2005 & 9.4 & 2.6 & 18 & 11.5 & 3.1 & 17 & $14.2 \%$ & $-0.72[-1.41,-0.03]$ & & \\
\hline Olivares 2014 & 51.63 & 8.27 & 38 & 66.37 & 8.74 & 17 & $14.4 \%$ & $-1.73[-2.39,-1.06]$ & -- & \\
\hline Olivares 2014 & 47.95 & 10.93 & 37 & 66.37 & 8.74 & 18 & $14.4 \%$ & $-1.77[-2.43,-1.11]$ & $\multimap-$ & \\
\hline Rosa Alcazar 2009 & 45.9 & 17.42 & 20 & 81 & 5.98 & 20 & $13.1 \%$ & $-2.64[-3.51,-1.77]$ & & \\
\hline Spence 2011 & 26.78 & 11.873516749472332 & 44 & 25.22 & 11.79526599954405 & 27 & $15.3 \%$ & $0.13[-0.35,0.61]$ & & \\
\hline White 2013 & 13.6 & 5.67 & 15 & 16.4 & 8.87 & 15 & $14.0 \%$ & $-0.37[-1.09,0.36]$ & & \\
\hline Subtotal (95\% CI) & & & 197 & & & 133 & $100.0 \%$ & $-1.07[-1.78,-0.37]$ & & \\
\hline Heterogeneity: $\mathrm{Tau}^{2}=0.78$ & ${ }^{2}=48.84, \mathrm{~d}$ & $=6(\mathrm{P}<0.00001) ; \mathrm{I}^{2}=88^{\circ}$ & & & & & & & & \\
\hline Test for overall effect: $Z=$ & $(\mathrm{P}=0.003)$ & & & & & & & & & \\
\hline Test for subgroup differenc & $\mathrm{Ch}^{2}=5.87$, & $\mathrm{f}=2(\mathrm{P}=0.05), \mathrm{I}^{2}=65.9 \%$ & & & & & & & $\begin{array}{l}1 \\
-2 \\
\end{array}$ & 2 \\
\hline
\end{tabular}


Analysis 1.24. Comparison 1: CBT versus waitlist/no treatment, Outcome 24: Subgroup analysis: reduction in anxiety symptoms (parent report) post-treatment (ASD vs non-ASD)

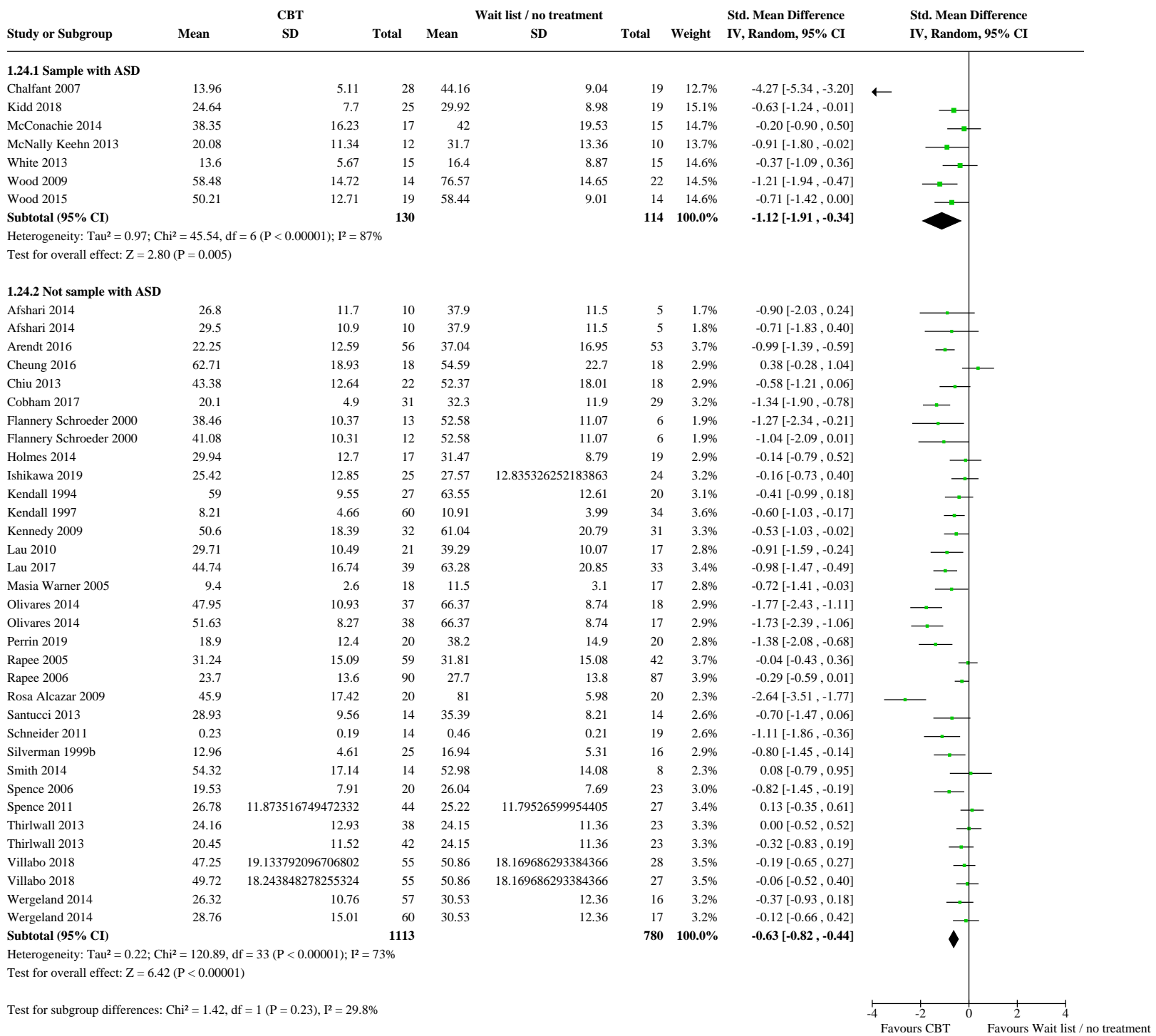


Analysis 1.25. Comparison 1: CBT versus waitlist/no treatment, Outcome 25: Reduction in depressive symptoms post-treatment

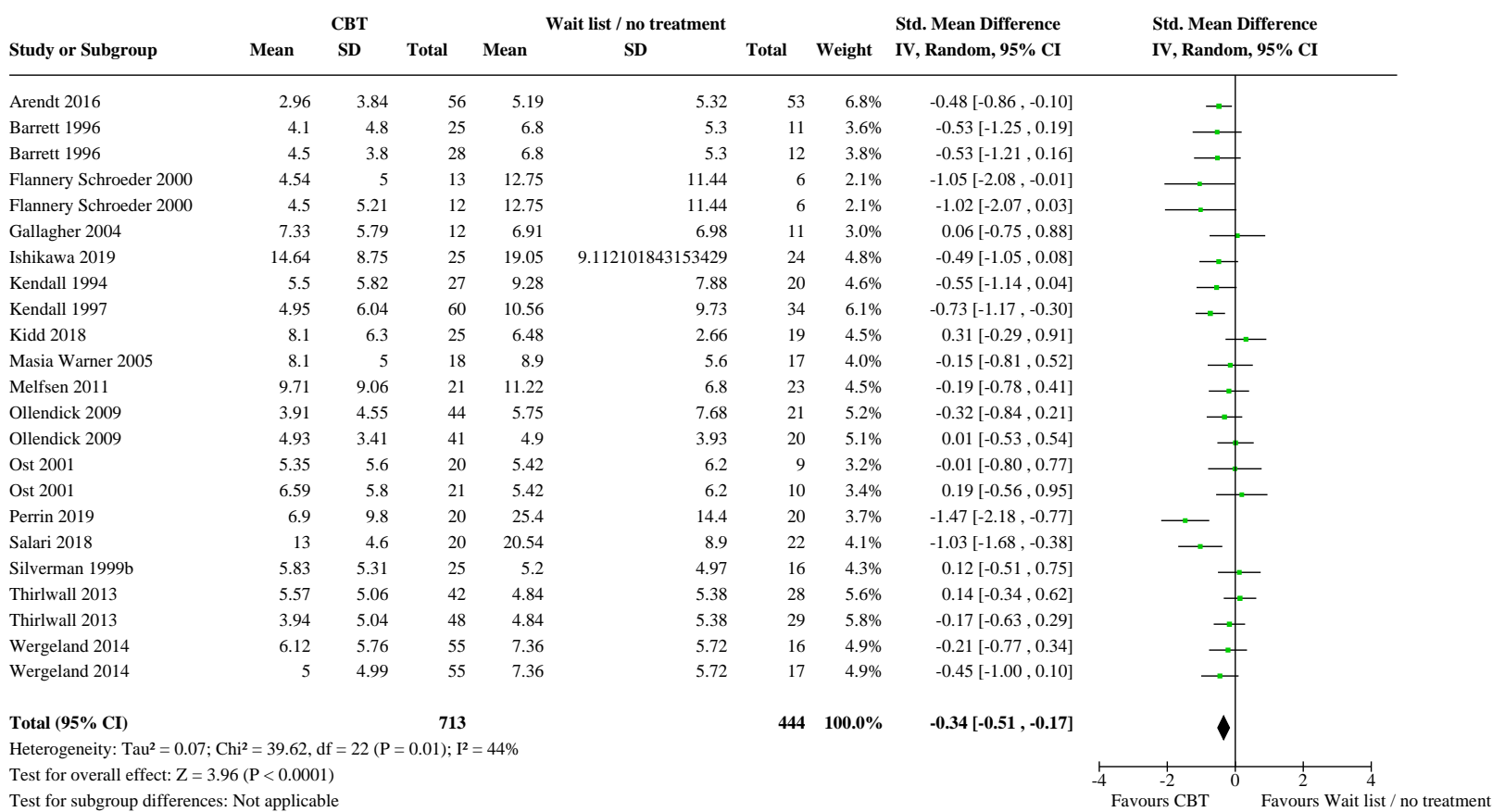

Analysis 1.26. Comparison 1: CBT versus waitlist/no treatment, Outcome 26: Improvement in global functioning post-treatment

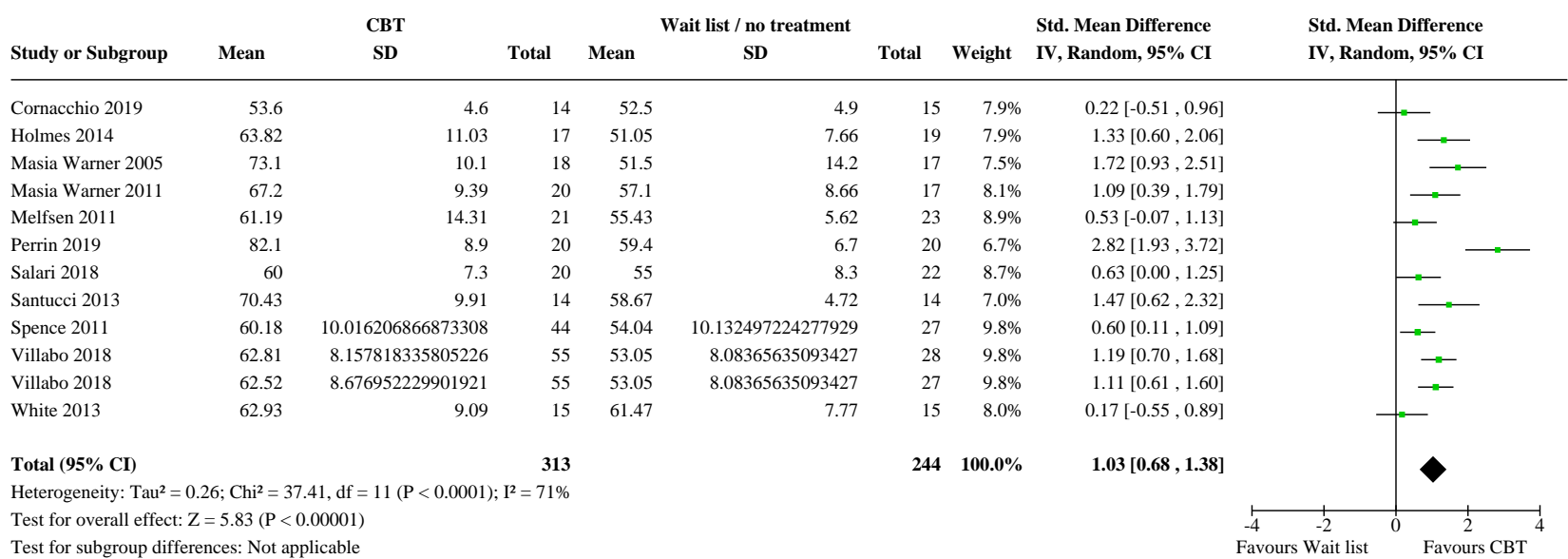


Analysis 1.27. Comparison 1: CBT versus waitlist/no treatment, Outcome 27: Remission of primary anxiety diagnosis at follow-up (ITT)

\begin{tabular}{|c|c|c|c|c|c|c|}
\hline & \multicolumn{2}{|c|}{ CBT } & Wait list / no treatment & \multicolumn{2}{|r|}{ Odds Ratio } & Odds Ratio \\
\hline Study or Subgroup & Events & Total & Events & Weight & IV, Random, 95\% CI & IV, Random, 95\% CI \\
\hline
\end{tabular}

1.27.1 Follow-up (\# 6 months)

\section{Dadds 1997}

Gallagher 2004

Olivares 2005

Rosa Alcazar 2009

Subtotal (95\% CI)

Total events:

Test for overall effect: $\mathrm{Z}=3.03(\mathrm{P}=0.002)$

1.27.2 Follow-up (> 6 and \# 12 months)

Cartwright Hatton 2011

Dadds 1997

Rosa Alcazar 2009

Subtotal $(95 \%$ CI $)$

$20 \quad 37$

$18 \quad 23$

1120

Total events:

49

Heterogeneity: $\mathrm{Tau}^{2}=3.80 ; \mathrm{Chi}^{2}=11.68, \mathrm{df}=2(\mathrm{P}=0.003) ; \mathrm{I}^{2}=83 \%$

Test for overall effect: $\mathrm{Z}=0.82(\mathrm{P}=0.41)$

$$
\begin{aligned}
& 35.9 \% \\
& 25.8 \% \\
& 19.0 \% \\
& 19.3 \%
\end{aligned}
$$

$100.0 \%$
$2.33[0.43,12.76]$

$10.00[0.96,104.49]$

$49.00[2.53,948.62]$

$49.63[2.64,933.26]$

$10.94[2.33,51.41]$
$39.0 \%$
$34.7 \%$
$26.3 \%$
$\mathbf{1 0 0 . 0 \%}$
$3.68[1.32,10.25]$

$0.23[0.04,1.32]$

$49.63[2.64,933.26]$

$2.80[0.24,33.19]$

V, Random, $95 \%$ CI

\begin{tabular}{|c|c|c|c|c|c|}
\hline & & & Wait list / no treatment & Odds Ratio & Odds Ratio \\
\hline Study or Subgroup & Events & Total & Events & IV, Random, 95\% CI & IV, Random, $95 \%$ CI \\
\hline
\end{tabular}

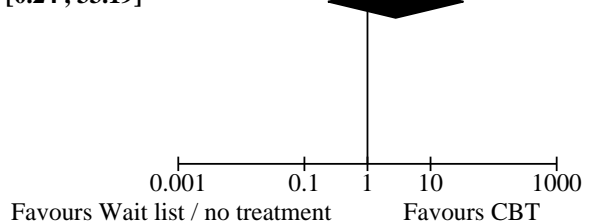

\section{Analysis 1.28. Comparison 1: CBT versus waitlist/no treatment, Outcome 28: Remission of primary anxiety diagnosis at follow-up (completers)}

1.28.1 Follow-up (\# 6 months)

\section{Dadds 1997}

Gallagher 2004

Olivares 2005

Rosa Alcazar 2009

Subtotal $(95 \%$ CI $)$

Total events:

Heterogeneity: $\mathrm{Tau}^{2}=1.03 ; \mathrm{Chi}^{2}=5.06, \mathrm{df}=3(\mathrm{P}=0.17) ; \mathrm{I}^{2}=41 \%$

Test for overall effect: $\mathrm{Z}=3.06(\mathrm{P}=0.002)$

1.28.2 Follow-up (> 6 and \# 12 months)

Cartwright Hatton 2011

Dadds 1997

Rosa Alcazar 2009

Subtotal (95\% CI)

Total events:

$\begin{array}{ll}20 & 37 \\ 18 & 20 \\ 11 & 20 \\ & \mathbf{7 7}\end{array}$

37
20
20
77

49

Heterogeneity: $\mathrm{Tau}^{2}=1.26 ; \mathrm{Chi}^{2}=4.94, \mathrm{df}=2(\mathrm{P}=0.08) ; \mathrm{I}^{2}=60 \%$

Test for overall effect: $\mathrm{Z}=1.65(\mathrm{P}=0.10)$
$32 \quad 35.7 \%$

$11 \quad 25.8 \%$

$17 \quad 19.1 \%$

$20 \quad 19.4 \%$

$80 \quad \mathbf{1 0 0 . 0} \%$
$2.42[0.44,13.27]$ $10.00[0.96,104.49]$ $56.54[2.88,1109.18]$ $49.63[2.64,933.26]$

$11.46[2.40,54.67]$

IV, Random, $95 \%$ CI

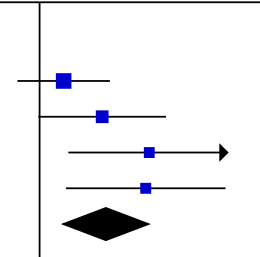


Analysis 1.29. Comparison 1: CBT versus waitlist/no treatment, Outcome 29: Remission of all anxiety diagnoses at follow-up (ITT)

\begin{tabular}{|c|c|c|c|c|c|}
\hline & $\mathrm{C}$ & & Wait list / no treatment & Odds Ratio & Odds Ratio \\
\hline Study or Subgroup & Events & Total & Events & Weight IV, Random, $95 \%$ CI & IV, Random, 95\% CI \\
\hline
\end{tabular}

1.29.1 Follow-up (\# 6 months)

\section{Dadds 1997}

Olivares 2005

Rosa Alcazar 2009

Subtotal $(95 \%$ CI $)$

Total events:

Heterogeneity: $\mathrm{Tau}^{2}=3.28 ; \mathrm{Chi}^{2}=6.50, \mathrm{df}=2(\mathrm{P}=0.04) ; \mathrm{I}^{2}=69 \%$

Test for overall effect: $\mathrm{Z}=1.67(\mathrm{P}=0.09)$

1.29.2 Follow-up (> 6 and \# 12 months)

Cartwright Hatton 201

Dadds 1997

Rosa Alcazar 2009

Subtotal $(95 \%$ CI $)$

Total events:

$\begin{array}{rr}17 & 37 \\ 16 & 23 \\ 9 & 20 \\ & \mathbf{8 0}\end{array}$

37
23
20
$\mathbf{8 0}$

3
29
0

32

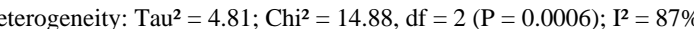

Test for overall effect: $\mathrm{Z}=0.95(\mathrm{P}=0.34)$

1.29.3 Follow-up (> 12 months)

Dadds 1997

Simon 2011

Simon 2011

Subtotal $(95 \%$ CI $)$

$\begin{array}{ll}18 & 23 \\ 14 & 18 \\ 23 & 27 \\ & \mathbf{6 8}\end{array}$

28
14
14

Total events: $\quad 55 \quad 56$

Heterogeneity: $\mathrm{Tau}^{2}=0.00 ; \mathrm{Chi}^{2}=0.50, \mathrm{df}=2(\mathrm{P}=0.78) ; \mathrm{I}^{2}=0 \%$

Test for overall effect: $\mathrm{Z}=1.36(\mathrm{P}=0.17)$

$42.4 \%$
$28.7 \%$
$28.9 \%$
$\mathbf{1 0 0 . 0 \%}$

$31.32[1.62,604.04]$

$33.87[1.80,636.88]$

$8.25[0.69,98.10]$
$1.28[0.33,5.00]$
$8.50[2.20,32.84]$

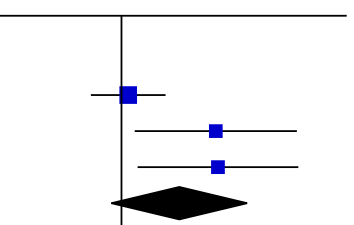

$0.64[0.16,2.54]$

$0.25[0.02,2.53]$

$0.41[0.04,4.06]$

$0.48[0.17,1.38]$

]

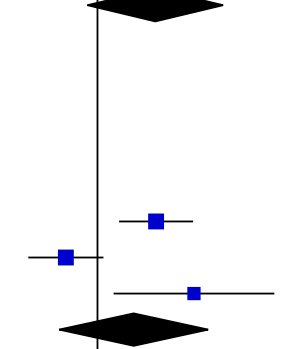

$\begin{array}{rr}33 & 58.4 \% \\ 15 & 20.6 \% \\ 15 & 21.0 \% \\ \mathbf{6 3} & \mathbf{1 0 0 . 0 \%}\end{array}$
$0.32[0.08,1.24]$
$33.87[1.80,636.88]$
$3.75[0.25,56.88]$
$20-27.3 \%$
$86 \quad \mathbf{1 0 0 . 0 \%}$

$\stackrel{\longmapsto}{0.001}$

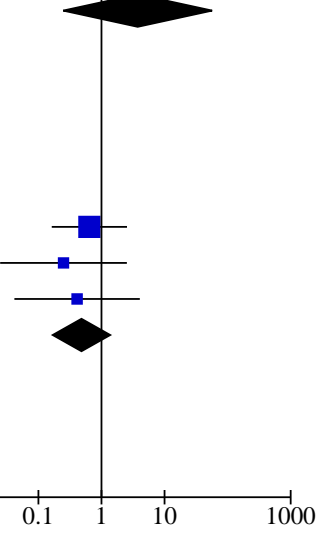

Favours Wait list / no treatment 
Analysis 1.30. Comparison 1: CBT versus waitlist/no treatment, Outcome 30: Remission of all anxiety diagnoses at follow-up (completers)

\begin{tabular}{|c|c|c|c|c|c|c|c|}
\hline & \multicolumn{2}{|c|}{ CBT } & Wait list & eatment & \multicolumn{2}{|r|}{ Odds Ratio } & Odds Ratio \\
\hline Study or Subgroup & Events & Total & Events & Total & Weight & IV, 1 & IV, Random, 95\% CI \\
\hline
\end{tabular}

1.30.1 Follow-up (\# 6 months)

\section{Dadds 1997}

Olivares 2005

Rosa Alcazar 2009

Subtotal $(\mathbf{9 5 \%}$ CI $)$

Total events:

Heterogeneity: $\mathrm{Tau}^{2}=3.32 ; \mathrm{Chi}^{2}=6.54, \mathrm{df}=2(\mathrm{P}=0.04) ; \mathrm{I}^{2}=69 \%$

Test for overall effect: $\mathrm{Z}=1.70(\mathrm{P}=0.09)$

1.30.2 Follow-up (> 6 and \# 12 months)

Cartwright Hatton 2011

Dadds 1997

Rosa Alcazar 2009

Subtotal $(95 \%$ CI $)$

Total events:

$\begin{array}{rr}17 & 37 \\ 17 & 20 \\ 9 & 20 \\ & 77\end{array}$

$37 \quad 3$

77

Heterogeneity: $\mathrm{Tau}^{2}=2.06 ; \mathrm{Chi}^{2}=6.81, \mathrm{df}=2(\mathrm{P}=0.03) ; \mathrm{I}^{2}=71 \%$

Test for overall effect: $\mathrm{Z}=1.61(\mathrm{P}=0.11)$

\subsubsection{Follow-up (> 12 months)}

Dadds 1997

Simon 2011

Simon 2011

Subtotal (95\% CI)

Total events:

$\begin{array}{ll}18 & 20 \\ 14 & 16 \\ 23 & 25 \\ & \mathbf{6 1}\end{array}$
23
11
12

55

46

Heterogeneity: $\mathrm{Tau}^{2}=0.00 ; \mathrm{Chi}^{2}=0.57, \mathrm{df}=2(\mathrm{P}=0.75) ; \mathrm{I}^{2}=0 \%$

Test for overall effect: $\mathrm{Z}=0.34(\mathrm{P}=0.73)$

$\begin{array}{rr}42.3 \% & 1.33[0.34,5.21] \\ 28.7 \% & 35.00[1.80,680.54] \\ 29.0 \% & 33.87[1.80,636.88] \\ \mathbf{1 0 0 . 0 \%} & \mathbf{8 . 6 8}[\mathbf{0 . 7 2}, \mathbf{1 0 4 . 4 6}]\end{array}$

$8.68[0.72,104.46]$
$8.50[2.20,32.84]$

$0.84[0.17,4.22]$

$33.87[1.80,636.88]$

$5.01[0.70,35.87]$
$1.96[0.34,11.28]$

$0.64[0.05,7.96]$

$0.96[0.08,11.67]$

$1.25[0.36,4.34]$
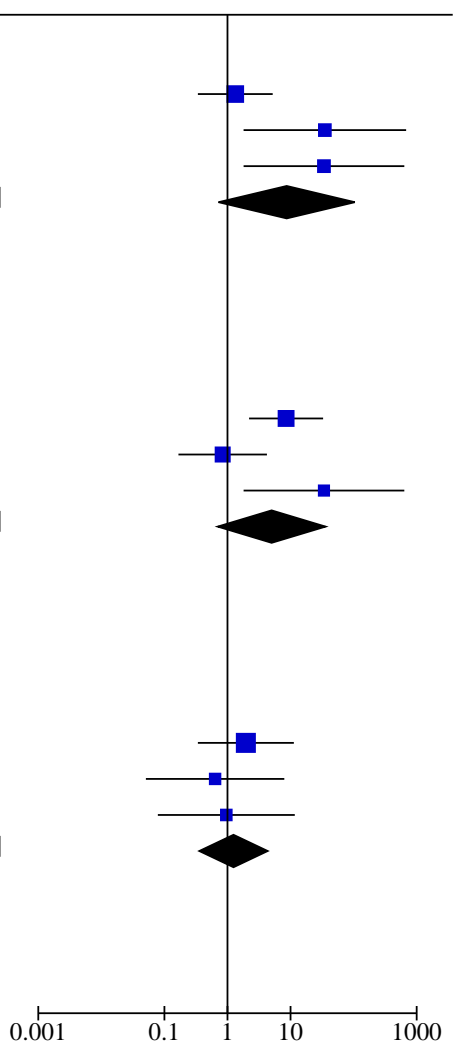

Favours Wait list / no treatment Favours CBT

Analysis 1.31. Comparison 1: CBT versus waitlist/no treatment, Outcome 31: Reduction in anxiety symptoms (child report) at follow-up

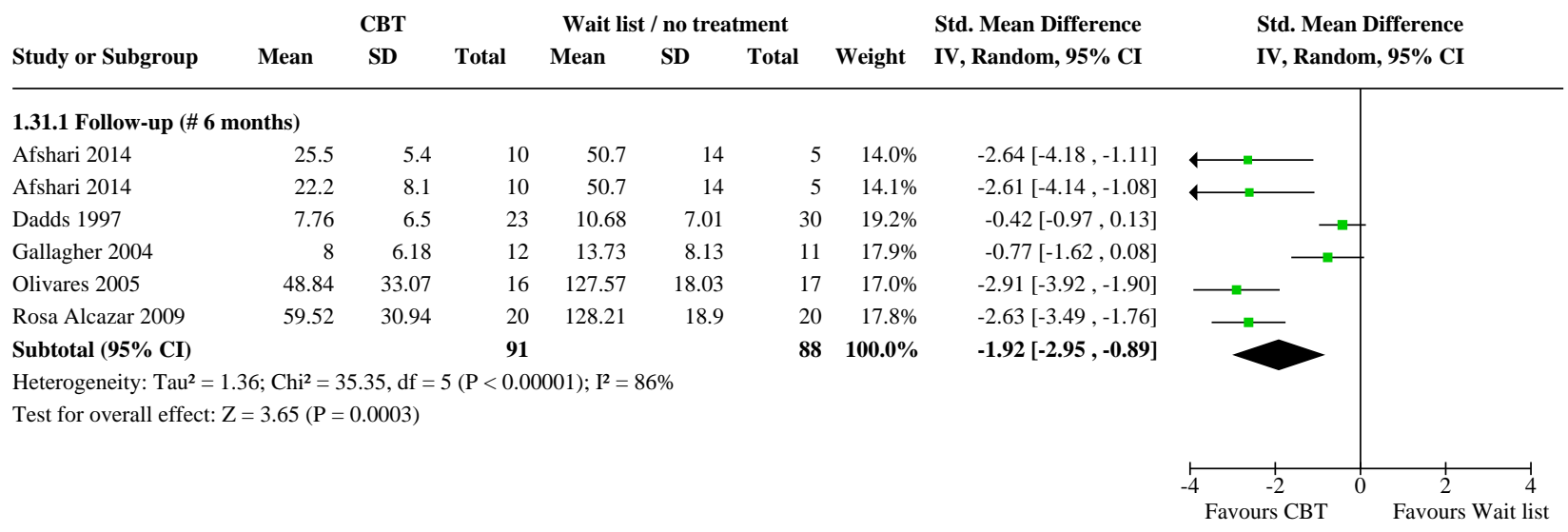


Analysis 1.32. Comparison 1: CBT versus waitlist/no treatment, Outcome 32: Sensitivity analysis: remission of primary anxiety diagnosis post-treatment (ITT) (only studies where sequence generation is clear)

\begin{tabular}{|c|c|c|c|c|c|c|c|c|}
\hline \multirow[b]{2}{*}{ Study or Subgroup } & \multicolumn{2}{|c|}{ CBT } & \multicolumn{2}{|c|}{ Wait list / no treatment } & \multirow[b]{2}{*}{ Weight } & \multirow{2}{*}{$\begin{array}{c}\text { Odds Ratio } \\
\text { IV, Random, } 95 \% \text { CI }\end{array}$} & \multirow{2}{*}{\multicolumn{2}{|c|}{$\begin{array}{c}\text { Odds Ratio } \\
\text { IV, Random, } 95 \% \text { CI }\end{array}$}} \\
\hline & Events & Total & Events & Total & & & & \\
\hline Arendt 2016 & 37 & 56 & 6 & 53 & $5.8 \%$ & $15.25[5.53,42.05]$ & & $\longrightarrow$ \\
\hline Cartwright Hatton 2011 & 21 & 37 & 5 & 33 & $5.3 \%$ & $7.35[2.32,23.27]$ & & $\longrightarrow$ \\
\hline Cobham 2017 & 20 & 33 & 6 & 30 & $5.4 \%$ & $6.15[1.98,19.14]$ & & $\longrightarrow$ \\
\hline Hirshfeld Becker 2010 & 22 & 34 & 13 & 30 & $5.8 \%$ & $2.40[0.87,6.57]$ & & \\
\hline Holmes 2014 & 9 & 20 & 3 & 22 & $4.4 \%$ & $5.18[1.15,23.29]$ & & \\
\hline Ishikawa 2019 & 10 & 23 & 4 & 24 & $4.8 \%$ & $3.85[0.99,14.89]$ & & \\
\hline Kidd 2018 & 18 & 26 & 10 & 23 & $5.3 \%$ & $2.92[0.91,9.44]$ & & \\
\hline Masia Warner 2011 & 9 & 20 & 1 & 20 & $2.9 \%$ & $15.55[1.73,139.65]$ & & \\
\hline McConachie 2014 & 0 & 17 & 1 & 15 & $1.6 \%$ & $0.28[0.01,7.31]$ & & \\
\hline McNally Keehn 2013 & 7 & 12 & 0 & 10 & $1.8 \%$ & $28.64[1.37,600.41]$ & & \\
\hline Melfsen 2011 & 7 & 21 & 2 & 23 & $3.8 \%$ & $5.25[0.95,29.05]$ & & \\
\hline Ollendick 2009 & 47 & 85 & 1 & 41 & $3.2 \%$ & $49.47[6.50,376.64]$ & & \\
\hline Perrin 2019 & 16 & 20 & 0 & 20 & $1.9 \%$ & $150.33[7.54,2997.83]$ & & \\
\hline Rapee 2006 & 44 & 90 & 17 & 87 & $6.8 \%$ & $3.94[2.01,7.71]$ & & \\
\hline Schneider 2011 & 16 & 21 & 4 & 22 & $4.4 \%$ & $14.40[3.29,63.08]$ & & \\
\hline Spence 2006 & 13 & 22 & 3 & 23 & $4.4 \%$ & $9.63[2.19,42.37]$ & & \\
\hline Spence 2011 & 13 & 44 & 4 & 27 & $5.1 \%$ & $2.41[0.70,8.36]$ & & \\
\hline Thirlwall 2013 & 18 & 61 & 11 & 34 & $6.1 \%$ & $0.88[0.35,2.16]$ & & \\
\hline Thirlwall 2013 & 25 & 64 & 11 & 35 & $6.2 \%$ & $1.40[0.58,3.35]$ & & - \\
\hline Villabo 2018 & 29 & 55 & 4 & 27 & $5.2 \%$ & $6.41[1.96,21.01]$ & & $\longrightarrow$ \\
\hline Villabo 2018 & 36 & 55 & 4 & 28 & $5.2 \%$ & $11.37[3.44,37.58]$ & & \\
\hline Wood 2015 & 6 & 19 & 6 & 14 & $4.5 \%$ & $0.62[0.15,2.58]$ & 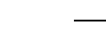 & - \\
\hline Total $(95 \%$ CI) & & 835 & & 641 & $100.0 \%$ & $4.84[3.03,7.73]$ & & \\
\hline Total events: & 423 & & 116 & & & & & \\
\hline Heterogeneity: $\mathrm{Tau}^{2}=0$. & $\mathrm{hi}^{2}=58.30$ &, $\mathrm{df}=21$ & $<0.0001) ; \mathrm{I}^{2}=$ & & & 0.001 & 0.1 & 10 \\
\hline Test for overall effect: $Z$ & $51(\mathrm{P}<0.00$ & $001)$ & & & & Favours Wait list / $\mathrm{n}$ & treatment & Favours \\
\hline
\end{tabular}


Analysis 1.33. Comparison 1: CBT versus waitlist/no treatment, Outcome 33: Sensitivity analysis: remission of primary anxiety diagnosis post-treatment (ITT) (only studies where blind assessors used)

\begin{tabular}{|c|c|c|c|c|c|c|}
\hline & \multicolumn{2}{|c|}{ CBT } & Wait list / no treatment & \multicolumn{2}{|r|}{ Odds Ratio } & Odds Ratio \\
\hline Study or Subgroup & Events & Total & Events & Weight & IV, Random, 95\% CI & IV, Random, 95\% CI \\
\hline
\end{tabular}

\section{Arendt 2016}

Cartwright Hatton 2011

Cobham 2017

Cornacchio 2019

Dadds 1997

Gallagher 2004

Hirshfeld Becker 2010

Holmes 2014

Ishikawa 2019

Kidd 2018

Lau 2010

Masia Warner 2005

Masia Warner 2011

McConachie 2014

McNally Keehn 2013

Melfsen 2011

Olivares 2005

Olivares 2019

Olivares 2019

Ollendick 2009

Ost 2001

Ost 2001

Perrin 2019

Rapee 2006

Santucci 2013

Schneider 2011

Silverman 1999b

Spence 2000

Spence 2000

Spence 2011

Thirlwall 2013

Thirlwall 2013

Villabo 2018

Villabo 2018

Waters 2009

Waters 2009

Wood 2015

Total (95\% CI)

Total events:

Heterogeneity: $\mathrm{Tau}^{2}=0.66 ; \mathrm{Chi}^{2}=78.27, \mathrm{df}=36(\mathrm{P}<0.0001) ; \mathrm{I}^{2}=54 \%$

Test for overall effect: $\mathrm{Z}=8.63$ ( $\mathrm{P}<0.00001)$

Test for subgroup differences: Not applicable
$15.25[5.53,42.05]$

$7.35[2.32,23.27]$

$6.15[1.98,19.14]$

$1.08[0.06,19.05]$

$1.88[0.33,10.63]$

$16.87[0.81,351.79]$

$2.40[0.87,6.57]$

5.18 [1.15, 23.29]

$3.85[0.99,14.89]$

$2.92[0.91,9.44]$

67.67 [3.56, 1287.81]

$4.27[1.13,16.05]$

$15.55[1.73,139.65]$

$0.28[0.01,7.31]$

$28.64[1.37,600.41]$

$5.25[0.95,29.05]$

$49.00[2.53,948.62]$

$11.04[0.60,202.89]$

21.26 [1.18, 381.56]

$49.47[6.50,376.64]$

$111.00[5.21,2363.64]$

$27.94[1.43,546.98]$

150.33 [7.54, 2997.83]

$3.94[2.01,7.71]$

11.38 [1.17, 110.42]

$14.40[3.29,63.08]$

$2.13[0.64,7.16]$

$32.67[2.85,374.13]$

8.25 [0.82, 82.67]

$2.41[0.70,8.36]$

$1.40[0.58,3.35]$

$0.88[0.35,2.16]$

$6.41[1.96,21.01]$

$11.37[3.44,37.58]$

$6.18[0.66,58.03]$

$4.86[0.49,48.57]$

$0.62[0.15,2.58]$

5.54 [3.76, 8.18]

$846100.0 \%$ 


\begin{tabular}{|c|c|c|c|c|}
\hline Outcome or subgroup title & $\begin{array}{l}\text { No. of } \\
\text { studies }\end{array}$ & $\begin{array}{l}\text { No. of } \\
\text { partici- } \\
\text { pants }\end{array}$ & Statistical method & Effect size \\
\hline 2.1.2 Child and parent & 5 & 172 & Odds Ratio (IV, Random, 95\% CI) & $\begin{array}{l}8.56[3.10 \\
23.66]\end{array}$ \\
\hline $\begin{array}{l}\text { 2.2 Subgroup analysis: remission of primary anx- } \\
\text { iety diagnosis post-treatment (ITT) (ASD vs non- } \\
\text { ASD) }\end{array}$ & 8 & & Odds Ratio (IV, Random, 95\% CI) & Subtotals only \\
\hline 2.2.1 Sample with ASD & 4 & 142 & Odds Ratio (IV, Random, 95\% CI) & $\begin{array}{l}11.25[3.11 \\
40.79]\end{array}$ \\
\hline 2.2.2 Not sample with ASD & 4 & 345 & Odds Ratio (IV, Random, 95\% CI) & $1.14[0.29,4.47]$ \\
\hline $\begin{array}{l}2.3 \text { Remission of primary anxiety diagnosis post- } \\
\text { treatment (completers) }\end{array}$ & 8 & 440 & Odds Ratio (IV, Random, 95\% CI) & $\begin{array}{l}4.87[1.51 \\
15.72]\end{array}$ \\
\hline $\begin{array}{l}\text { 2.4 Acceptability (number of participants lost to } \\
\text { post-treatment assessment) }\end{array}$ & 8 & 441 & Odds Ratio (IV, Random, 95\% CI) & $1.37[0.73,2.56]$ \\
\hline $\begin{array}{l}\text { 2.5 Remission of all anxiety diagnoses post-treat- } \\
\text { ment (ITT) }\end{array}$ & 5 & 203 & Odds Ratio (IV, Random, 95\% CI) & $2.74[1.16,6.46]$ \\
\hline $\begin{array}{l}\text { 2.6 Remission of all anxiety diagnoses post-treat- } \\
\text { ment (completers) }\end{array}$ & 5 & 201 & Odds Ratio (IV, Random, 95\% CI) & $2.78[1.18,6.55]$ \\
\hline $\begin{array}{l}\text { 2.7 Reduction in anxiety symptoms (child report) } \\
\text { post-treatment }\end{array}$ & 6 & 214 & $\begin{array}{l}\text { Std. Mean Difference (IV, Ran- } \\
\text { dom, } 95 \% \mathrm{CI} \text { ) }\end{array}$ & $\begin{array}{l}-0.15[-0.78 \\
0.48]\end{array}$ \\
\hline $\begin{array}{l}\text { 2.8 Sensitivity analysis: reduction in anxiety symp- } \\
\text { toms (child report) post-treatment (broad anxiety } \\
\text { measures only) }\end{array}$ & 5 & 187 & $\begin{array}{l}\text { Std. Mean Difference (IV, Ran- } \\
\text { dom, } 95 \% \mathrm{CI} \text { ) }\end{array}$ & $\begin{array}{l}0.07[-0.54 \\
0.68]\end{array}$ \\
\hline $\begin{array}{l}\text { 2.9 Reduction in anxiety symptoms (parent report) } \\
\text { post-treatment }\end{array}$ & 7 & 228 & $\begin{array}{l}\text { Std. Mean Difference (IV, Ran- } \\
\text { dom, } 95 \% \mathrm{CI} \text { ) }\end{array}$ & $\begin{array}{l}-0.32[-0.70 \\
0.06]\end{array}$ \\
\hline $\begin{array}{l}\text { 2.10 Sensitivity analysis: reduction in anxiety symp- } \\
\text { toms (parent report) post-treatment (broad anxiety } \\
\text { measures only) }\end{array}$ & 6 & 202 & $\begin{array}{l}\text { Std. Mean Difference (IV, Ran- } \\
\text { dom, } 95 \% \mathrm{CI} \text { ) }\end{array}$ & $\begin{array}{l}-0.16[-0.44 \\
0.11]\end{array}$ \\
\hline
\end{tabular}




\section{Analysis 2.1. Comparison 2: CBT versus treatment as usual, Outcome} 1: Remission of primary anxiety diagnosis post-treatment (ITT)

Study or Subgroup
CBT

TAU
Odds Ratio

Odds Ratio
IV, Random, $95 \%$ CI

\subsubsection{Child focused}

Ginsburg 2019a

Sharma 2017

Southam Gerow 2010

Subtotal (95\% CI)

Total events:

Total Events

Heterogeneity: $\mathrm{Tau}^{2}=0.63 ; \mathrm{Chi}^{2}=4.93, \mathrm{df}=2(\mathrm{P}=0.09) ; \mathrm{I}^{2}=59 \%$

Test for overall effect: $\mathrm{Z}=0.82(\mathrm{P}=0.41)$

\subsubsection{Child and parent}

$\begin{array}{rrrrr}49 & 140 & 33 & 66 & 18.3 \% \\ 30 & 30 & 27 & 31 & 9.3 \% \\ 12 & 24 & 19 & 24 & 16.0 \% \\ & \mathbf{1 9 4} & & \mathbf{1 2 1} & \mathbf{4 3 . 7 \%}\end{array}$

$0.54[0.30,0.98]$

$9.98[0.51,193.95]$

$0.26[0.07,0.94]$

$0.61[0.18,2.01]$

\section{Fujii 2013}

Reaven 2012

Shahnavaz 2016

Storch 2013

Storch 2015

Subtotal (95\% CI)

Total events:

Heterogeneity: $\mathrm{Tau}^{2}=0.00 ; \mathrm{Chi}^{2}=1.11, \mathrm{df}=4(\mathrm{P}=0.89) ; \mathrm{I}^{2}=0 \%$

Test for overall effect: $\mathrm{Z}=4.14(\mathrm{P}<0.0001)$

\section{Total $(95 \%$ CI $)$}

281

Total events: 124

\section{0}

624

$7 \quad 13$

924

$6 \quad 16$

87

Test for overall effect: $\mathrm{Z}=1.80(\mathrm{P}=0.07)$

Test for subgroup differences: $\mathrm{Chi}^{2}=10.90, \mathrm{df}=1(\mathrm{P}=0.0010), \mathrm{I}^{2}=90.8 \%$
$5.00[0.42,59.66]$ $18.62[0.99,351.22]$

$5.44[1.04,28.53]$

$12.00[1.37,105.28]$

$19.19[0.97,378.28]$

8.56 [3.10, 23.66]

(1)

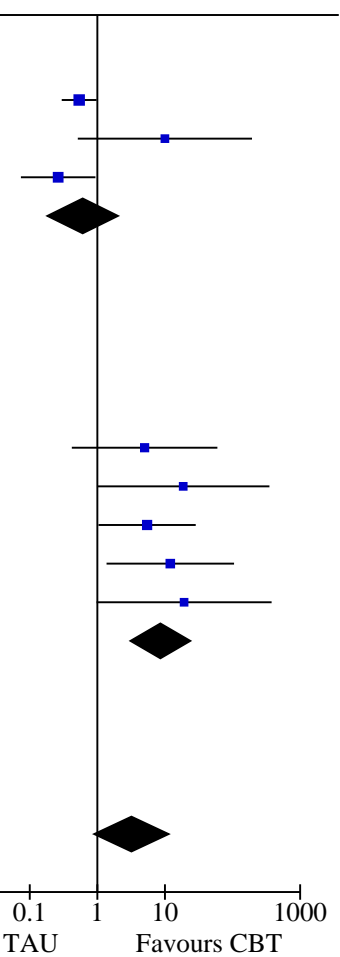


Analysis 2.2. Comparison 2: CBT versus treatment as usual, Outcome 2: Subgroup analysis: remission of primary anxiety diagnosis post-treatment (ITT) (ASD vs non-ASD)
CBT
TAU
Odds Ratio
Odds Ratio

Study or Subgroup Events Total

Events Total

Weight IV, Random, 95\% CI

IV, Random, 95\% CI

2.2.1 Sample with ASD

Fujii 2013

Reaven 2012

Storch 2013

Storch 2015

Subtotal (95\% CI)

Total events:

$\begin{array}{ll}5 & 10 \\ 6 & 24 \\ 9 & 24 \\ 6 & 16 \\ & 74\end{array}$

$5.00[0.42,59.66]$

$18.62[0.99,351.22]$

$12.00[1.37,105.28]$

$\begin{array}{lll}1 & 21 \quad 35.2 \%\end{array}$

19.19 [0.97, 378.28]

26

$15 \quad 18.7 \%$

11.25 [3.11, 40.79]

Heterogeneity: $\mathrm{Tau}^{2}=0.00 ; \mathrm{Chi}^{2}=0.65, \mathrm{df}=3(\mathrm{P}=0.88) ; \mathrm{I}^{2}=0 \%$

Test for overall effect: $\mathrm{Z}=3.68(\mathrm{P}=0.0002)$

2.2.2 Not sample with ASD

Ginsburg 2019a

Shahnavaz 2016

Sharma 2017

Southam Gerow 2010

Subtotal $(95 \%$ CI $)$

$\begin{array}{rr}49 & 140 \\ 7 & 13 \\ 30 & 30 \\ 12 & 24 \\ & \mathbf{2 0 7}\end{array}$

$\begin{array}{rrr}33 & 66 & 34.5 \% \\ 3 & 17 & 24.0 \% \\ 27 & 31 & 13.5 \% \\ 19 & 24 & 28.0 \% \\ & \mathbf{1 3 8} & \mathbf{1 0 0 . 0 \%}\end{array}$

$0.54[0.30,0.98]$

$5.44[1.04,28.53]$

$9.98[0.51,193.95]$

$0.26[0.07,0.94]$

Total events:

98

82

$1.14[0.29,4.47]$

Heterogeneity: $\mathrm{Tau}^{2}=1.32 ; \mathrm{Chi}^{2}=11.92, \mathrm{df}=3(\mathrm{P}=0.008) ; \mathrm{I}^{2}=75 \%$

Test for overall effect: $\mathrm{Z}=0.18(\mathrm{P}=0.85)$

Test for subgroup differences: $\mathrm{Chi}^{2}=5.71, \mathrm{df}=1(\mathrm{P}=0.02), \mathrm{I}^{2}=82.5 \%$

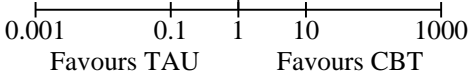

Analysis 2.3. Comparison 2: CBT versus treatment as usual, Outcome 3: Remission of primary anxiety diagnosis post-treatment (completers)

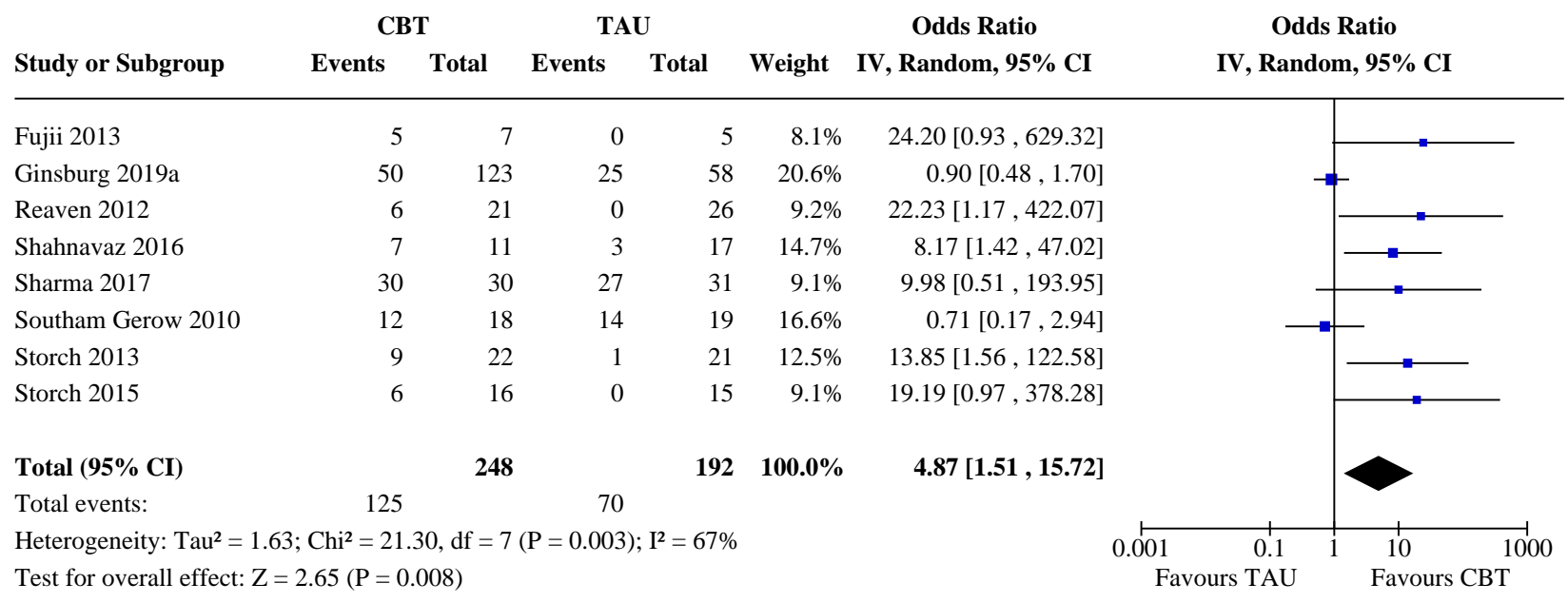


Analysis 2.4. Comparison 2: CBT versus treatment as usual, Outcome 4: Acceptability (number of participants lost to post-treatment assessment)

\begin{tabular}{|c|c|c|c|c|c|c|c|}
\hline & \multicolumn{2}{|c|}{ CBT } & \multicolumn{2}{|c|}{ TAU } & \multicolumn{2}{|r|}{ Odds Ratio } & Odds Ratio \\
\hline Study or Subgroup & Events & Total & Events & Total & Weight & IV, Random, $95 \%$ CI & IV, Random, 95\% CI \\
\hline
\end{tabular}

\begin{tabular}{lrrrrrr}
\hline Fujii 2013 & 3 & 10 & 1 & 6 & $6.1 \%$ & $2.14[0.17,27.10]$ \\
Ginsburg 2012 & 1 & 17 & 2 & 15 & $6.2 \%$ & $0.41[0.03,5.00]$ \\
Ginsburg 2019a & 18 & 140 & 8 & 66 & $49.5 \%$ & $1.07[0.44,2.60]$ \\
O'Brien 2007 & 1 & 7 & 1 & 7 & $4.4 \%$ & $1.00[0.05,19.96]$ \\
Reaven 2012 & 3 & 24 & 0 & 26 & $4.3 \%$ & $8.63[0.42,176.32]$ \\
Shahnavaz 2016 & 2 & 13 & 0 & 17 & $4.0 \%$ & $7.61[0.33,173.38]$ \\
Southam Gerow 2010 & 6 & 24 & 5 & 24 & $21.5 \%$ & $1.27[0.33,4.89]$ \\
Storch 2013 & 2 & 24 & 0 & 21 & $4.1 \%$ & $4.78[0.22,105.36]$
\end{tabular}

Total (95\% CI)

259

$182 \quad 100.0 \%$

$1.37[0.73,2.56]$

Total events:

36

17

Heterogeneity: $\mathrm{Tau}^{2}=0.00 ; \mathrm{Chi}^{2}=4.58, \mathrm{df}=7(\mathrm{P}=0.71) ; \mathrm{I}^{2}=0 \%$

Test for overall effect: $\mathrm{Z}=0.98(\mathrm{P}=0.33)$

Test for subgroup differences: Not applicable

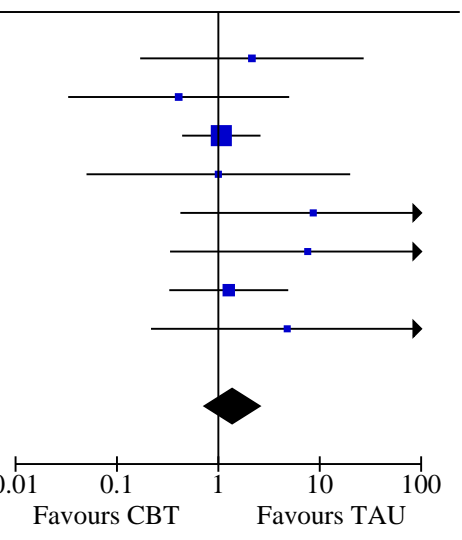

Analysis 2.5. Comparison 2: CBT versus treatment as usual, Outcome 5: Remission of all anxiety diagnoses post-treatment (ITT)

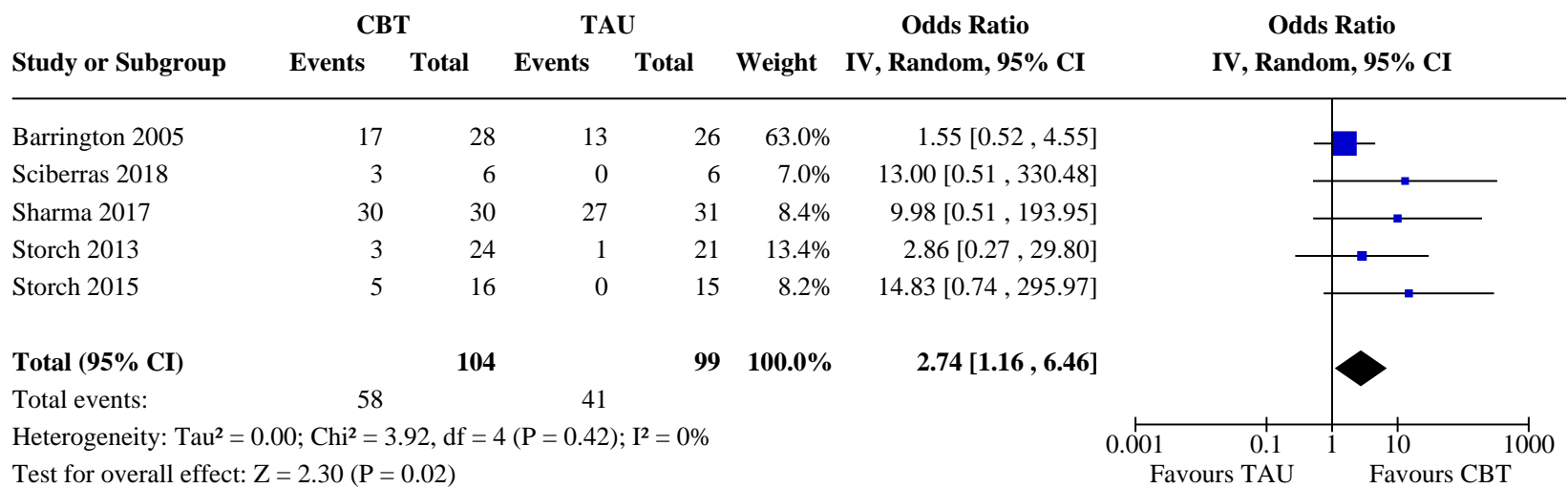

Test for overall effect: $\mathrm{Z}=2.30(\mathrm{P}=0.02)$

Test for subgroup differences: Not applicable 
Analysis 2.6. Comparison 2: CBT versus treatment as usual, Outcome 6: Remission of all anxiety diagnoses post-treatment (completers)

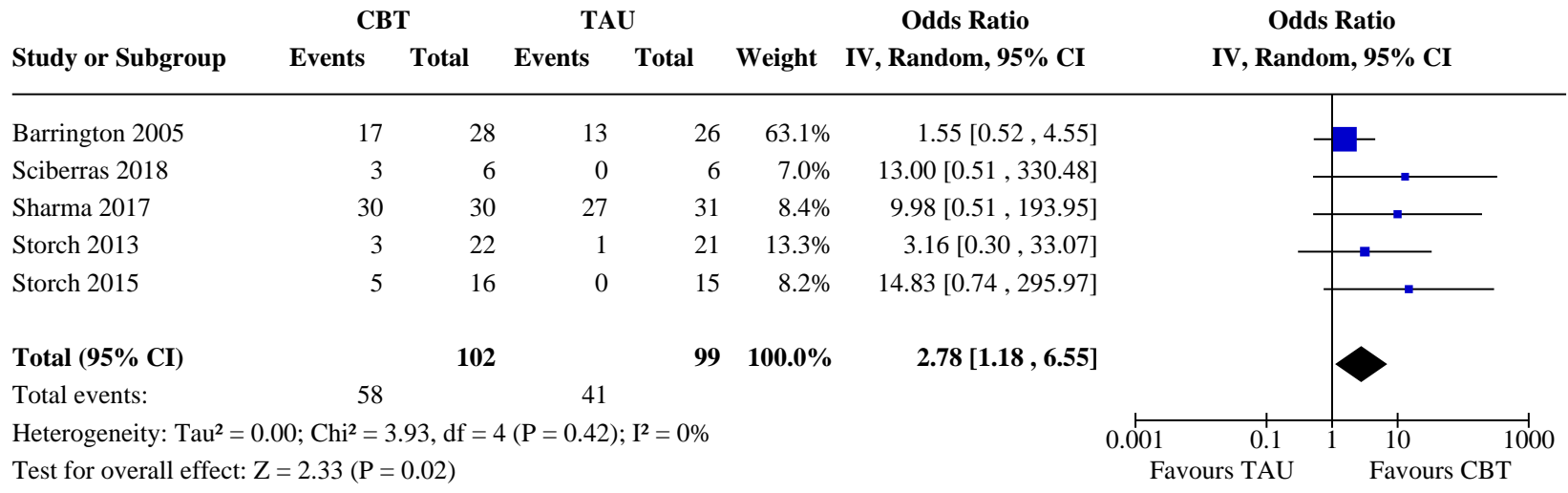

Test for subgroup differences: Not applicable

Analysis 2.7. Comparison 2: CBT versus treatment as usual, Outcome 7: Reduction in anxiety symptoms (child report) post-treatment

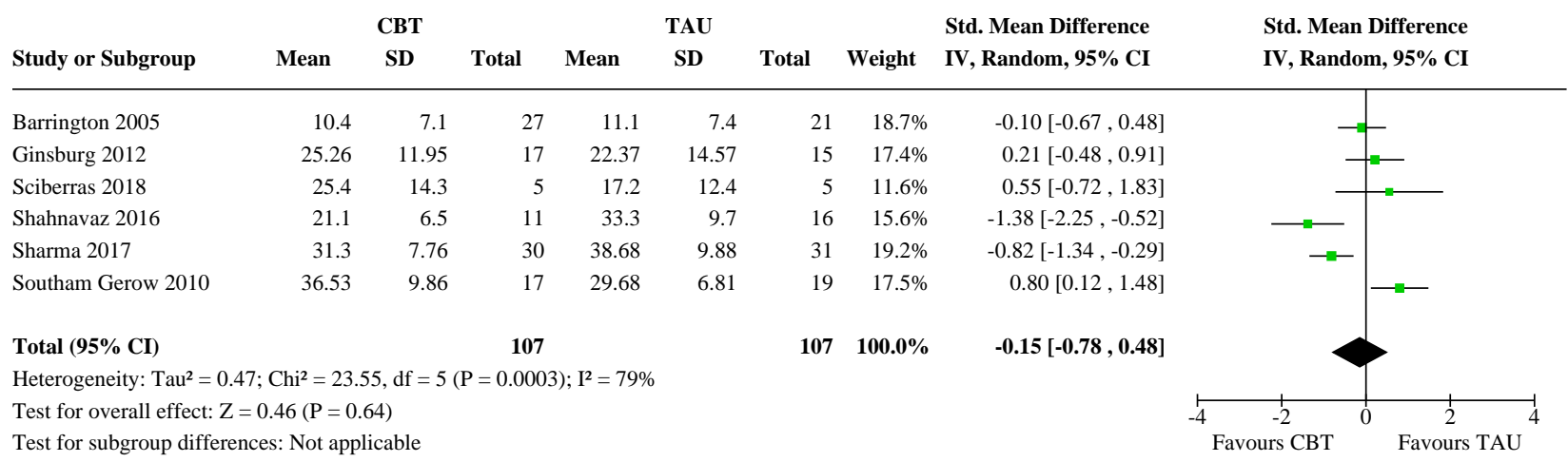

Analysis 2.8. Comparison 2: CBT versus treatment as usual, Outcome 8: Sensitivity analysis: reduction in anxiety symptoms (child report) post-treatment (broad anxiety measures only)

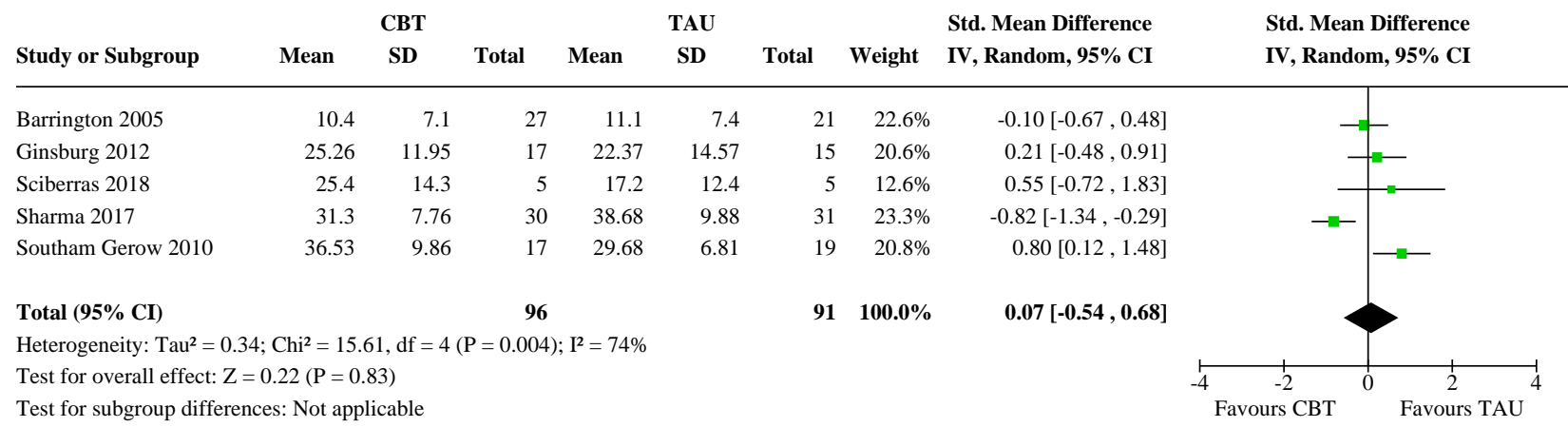




\section{Analysis 2.9. Comparison 2: CBT versus treatment as usual, Outcome}

9: Reduction in anxiety symptoms (parent report) post-treatment

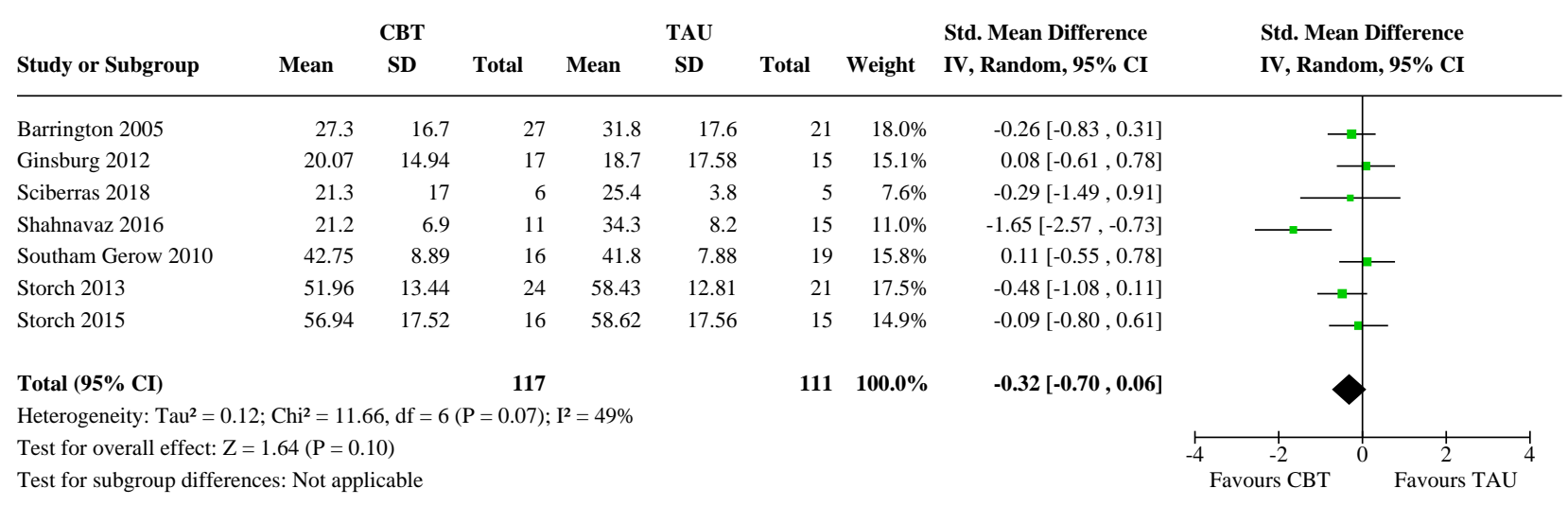

Analysis 2.10. Comparison 2: CBT versus treatment as usual, Outcome 10: Sensitivity analysis: reduction in anxiety symptoms (parent report) post-treatment (broad anxiety measures only)

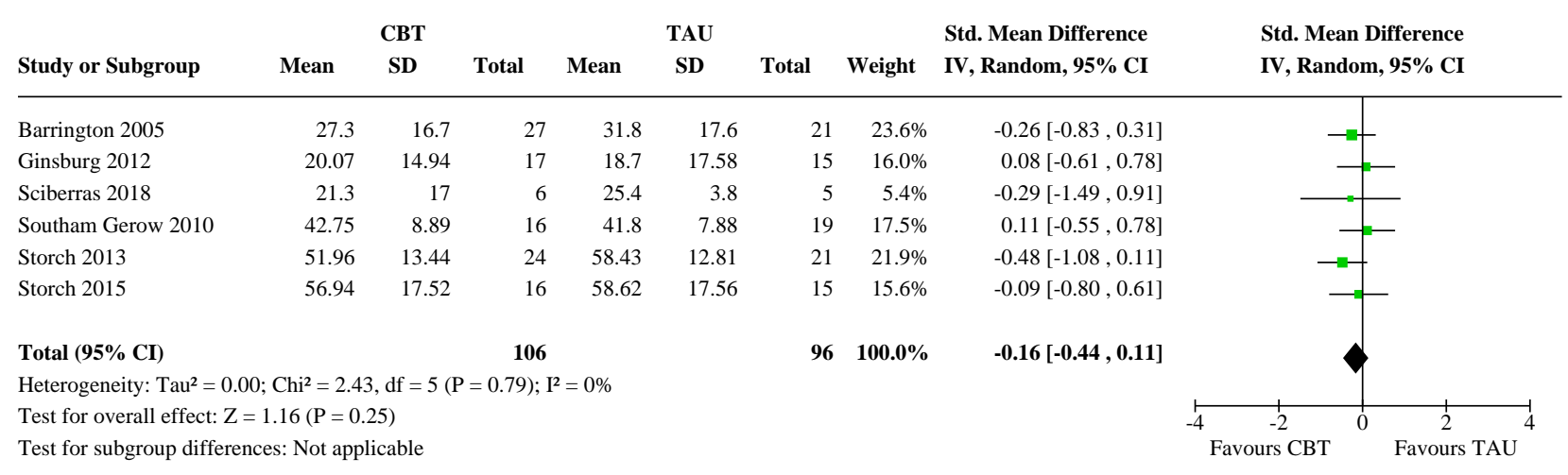

\section{Comparison 3. CBT versus attention control}

\begin{tabular}{|c|c|c|c|c|}
\hline Outcome or subgroup title & $\begin{array}{l}\text { No. of } \\
\text { studies }\end{array}$ & $\begin{array}{l}\text { No. of } \\
\text { partici- } \\
\text { pants }\end{array}$ & Statistical method & Effect size \\
\hline $\begin{array}{l}\text { 3.1 Remission of primary anxiety diagnosis } \\
\text { post-treatment (ITT) }\end{array}$ & 10 & 822 & Odds Ratio (IV, Random, 95\% Cl) & $2.28[1.33,3.89]$ \\
\hline 3.1.1 Child focused & 7 & 509 & Odds Ratio (IV, Random, 95\% CI) & $3.58[1.92,6.65]$ \\
\hline 3.1.2 Child and parent & 4 & 313 & Odds Ratio (IV, Random, 95\% CI) & $1.12[0.65,1.92]$ \\
\hline $\begin{array}{l}\text { 3.2 Subgroup analysis: remission of primary } \\
\text { anxiety diagnosis post-treatment (ITT) (individ- } \\
\text { ual vs group) }\end{array}$ & 10 & & Odds Ratio (IV, Random, 95\% CI) & Subtotals only \\
\hline 3.2.1 Individual focused & 5 & 469 & Odds Ratio (IV, Random, 95\% CI) & $2.04[1.06,3.91]$ \\
\hline 3.2.2 Group focused & 5 & 353 & Odds Ratio (IV, Random, 95\% Cl) & $3.10[1.14,8.45]$ \\
\hline
\end{tabular}




\begin{tabular}{|c|c|c|c|c|}
\hline Outcome or subgroup title & $\begin{array}{l}\text { No. of } \\
\text { studies }\end{array}$ & $\begin{array}{l}\text { No. of } \\
\text { partici- } \\
\text { pants }\end{array}$ & Statistical method & Effect size \\
\hline $\begin{array}{l}\text { 3.3 Remission of primary anxiety diagnosis } \\
\text { post-treatment (completers) }\end{array}$ & 10 & 752 & Odds Ratio (IV, Random, 95\% CI) & $3.88[2.52,5.97]$ \\
\hline $\begin{array}{l}\text { 3.4 Acceptability (number of participants lost } \\
\text { to post-treatment assessment) }\end{array}$ & 12 & 797 & Odds Ratio (IV, Random, 95\% CI) & $1.00[0.68,1.49]$ \\
\hline $\begin{array}{l}\text { 3.5 Remission of all anxiety diagnoses post- } \\
\text { treatment (ITT) }\end{array}$ & 5 & 378 & Odds Ratio (IV, Random, 95\% Cl) & $2.75[1.22,6.17]$ \\
\hline $\begin{array}{l}\text { 3.6 Remission of all anxiety diagnoses post- } \\
\text { treatment (completers) }\end{array}$ & 5 & 359 & Odds Ratio (IV, Random, 95\% Cl) & $3.88[1.58,9.51]$ \\
\hline $\begin{array}{l}\text { 3.7 Reduction in anxiety symptoms (child re- } \\
\text { port) post-treatment }\end{array}$ & 15 & 978 & $\begin{array}{l}\text { Std. Mean Difference (IV, Random, } \\
95 \% \mathrm{CI})\end{array}$ & $\begin{array}{l}-0.31[-0.51 \\
-0.11]\end{array}$ \\
\hline $\begin{array}{l}\text { 3.8 Sensitivity analysis: reduction in anxiety } \\
\text { symptoms (child report) post-treatment (broad } \\
\text { anxiety measures only) }\end{array}$ & 11 & 685 & $\begin{array}{l}\text { Std. Mean Difference (IV, Random, } \\
95 \% \mathrm{CI})\end{array}$ & $\begin{array}{l}-0.18[-0.34 \\
-0.02]\end{array}$ \\
\hline $\begin{array}{l}\text { 3.9 Reduction in anxiety symptoms (parent re- } \\
\text { port) post-treatment }\end{array}$ & 8 & 638 & $\begin{array}{l}\text { Std. Mean Difference (IV, Random, } \\
95 \% \mathrm{CI})\end{array}$ & $\begin{array}{l}-0.25[-0.61 \\
0.11]\end{array}$ \\
\hline $\begin{array}{l}\text { 3.10 Sensitivity analysis: reduction in anxi- } \\
\text { ety symptoms (parent report) post-treatment } \\
\text { (broad anxiety measures only) }\end{array}$ & 4 & 345 & $\begin{array}{l}\text { Std. Mean Difference (IV, Random, } \\
95 \% \mathrm{CI})\end{array}$ & $\begin{array}{l}-0.04[-0.26 \\
0.18]\end{array}$ \\
\hline $\begin{array}{l}3.11 \text { Reduction in depressive symptoms post- } \\
\text { treatment }\end{array}$ & 10 & 613 & $\begin{array}{l}\text { Std. Mean Difference (IV, Random, } \\
95 \% \mathrm{CI})\end{array}$ & $\begin{array}{l}-0.18[-0.45 \\
0.09]\end{array}$ \\
\hline $\begin{array}{l}\text { 3.12 Remission of primary anxiety diagnosis at } \\
\text { follow-up (ITT) }\end{array}$ & 4 & & Odds Ratio (IV, Random, 95\% Cl) & Subtotals only \\
\hline 3.12.1 Follow-up ( $\leq 6$ months) & 4 & 341 & Odds Ratio (IV, Random, 95\% Cl) & $3.06[1.22,7.65]$ \\
\hline $\begin{array}{l}3.13 \text { Remission of primary anxiety diagnosis at } \\
\text { follow-up (completers) }\end{array}$ & 4 & & Odds Ratio (IV, Random, 95\% Cl) & Subtotals only \\
\hline 3.13.1 Follow-up ( $\leq 6$ months) & 4 & 302 & Odds Ratio (IV, Random, 95\% Cl) & $\begin{array}{l}6.52[2.58 \\
16.43]\end{array}$ \\
\hline $\begin{array}{l}\text { 3.14 Remission of all anxiety diagnoses at fol- } \\
\text { low-up (ITT) }\end{array}$ & 3 & & Odds Ratio (IV, Random, 95\% Cl) & Subtotals only \\
\hline 3.14.1 Follow-up ( $\leq 6$ months) & 3 & 205 & Odds Ratio (IV, Random, 95\% CI) & $\begin{array}{l}5.44[1.00 \\
29.60]\end{array}$ \\
\hline $\begin{array}{l}\text { 3.15 Remission of all anxiety diagnoses at fol- } \\
\text { low-up (completers) }\end{array}$ & 3 & & Odds Ratio (IV, Random, 95\% Cl) & Subtotals only \\
\hline 3.15.1 Follow-up ( $\leq 6$ months) & 3 & 182 & Odds Ratio (IV, Random, 95\% Cl) & $\begin{array}{l}11.19[1.94 \\
64.51]\end{array}$ \\
\hline $\begin{array}{l}\text { 3.16 Reduction in anxiety symptoms (child re- } \\
\text { port) at follow-up }\end{array}$ & 6 & & $\begin{array}{l}\text { Std. Mean Difference (IV, Random, } \\
95 \% \mathrm{CI})\end{array}$ & Subtotals only \\
\hline
\end{tabular}




\begin{tabular}{|c|c|c|c|c|}
\hline Outcome or subgroup title & $\begin{array}{l}\text { No. of } \\
\text { studies }\end{array}$ & $\begin{array}{l}\text { No. of } \\
\text { partici- } \\
\text { pants }\end{array}$ & Statistical method & Effect size \\
\hline 3.16.1 Follow-up ( $\leq 6$ months) & 6 & 434 & $\begin{array}{l}\text { Std. Mean Difference (IV, Random, } \\
95 \% \mathrm{CI})\end{array}$ & $\begin{array}{l}-0.44[-0.79 \\
-0.08]\end{array}$ \\
\hline $\begin{array}{l}\text { 3.17 Reduction in anxiety symptoms (parent re- } \\
\text { port) at follow-up }\end{array}$ & 6 & & $\begin{array}{l}\text { Std. Mean Difference (IV, Random, } \\
95 \% \mathrm{Cl})\end{array}$ & Subtotals only \\
\hline 3.17.1 Follow-up ( $\leq 6$ months) & 6 & 434 & $\begin{array}{l}\text { Std. Mean Difference (IV, Random, } \\
95 \% \mathrm{Cl})\end{array}$ & $\begin{array}{l}-0.63[-1.33, \\
0.08]\end{array}$ \\
\hline
\end{tabular}

\section{Analysis 3.1. Comparison 3: $C B T$ versus attention control, Outcome 1: Remission of primary anxiety diagnosis post-treatment (ITT)}

\begin{tabular}{lccccccc} 
& \multicolumn{2}{c}{ CBT } & \multicolumn{2}{c}{ Attention control } & \multicolumn{2}{c}{ Odds Ratio } & Odds Ratio \\
Study or Subgroup & Events & Total & Events & Total & Weight & IV, Random, 95\% CI & IV, Random, 95\% CI
\end{tabular}

\begin{tabular}{|c|c|c|c|c|c|c|}
\hline \multicolumn{7}{|l|}{ 3.1.1 Child focused } \\
\hline Ginsburg 2002 & 3 & 6 & 2 & 6 & $3.9 \%$ & $2.00[0.19,20.61]$ \\
\hline Kendall 2008 & 32 & 55 & 8 & 25 & $9.9 \%$ & $2.96[1.09,8.01]$ \\
\hline Khanna 2010 & 12 & 17 & 6 & 16 & $7.1 \%$ & $4.00[0.93,17.11]$ \\
\hline Masia Warner 2007 & 11 & 19 & 2 & 17 & $5.8 \%$ & $10.31[1.82,58.39]$ \\
\hline Masia Warner 2016 & 9 & 46 & 4 & 21 & $7.9 \%$ & $1.03[0.28,3.83]$ \\
\hline Masia Warner 2016 & 12 & 47 & 4 & 22 & $8.2 \%$ & $1.54[0.43,5.47]$ \\
\hline Ollendick 2009 & 47 & 85 & 16 & 70 & $11.9 \%$ & $4.17[2.07,8.43]$ \\
\hline Rosa Alcazar 2009 & 6 & 10 & 0 & 19 & $2.6 \%$ & $56.33[2.66,1193.81]$ \\
\hline Rosa Alcazar 2009 & 6 & 10 & 0 & 18 & $2.6 \%$ & $53.44[2.52,1134.56]$ \\
\hline Subtotal (95\% CI) & & 295 & & 214 & $59.9 \%$ & $3.58[1.92,6.65]$ \\
\hline
\end{tabular}

Total events: $\quad 138$

Heterogeneity: $\mathrm{Tau}^{2}=0.32 ; \mathrm{Chi}^{2}=13.26, \mathrm{df}=8(\mathrm{P}=0.10) ; \mathrm{I}^{2}=40 \%$

Test for overall effect: $\mathrm{Z}=4.03(\mathrm{P}<0.0001)$

\subsubsection{Child and parent}

$\begin{array}{lrrrrrr}\text { Hudson 2009 } & 23 & 60 & 19 & 52 & 11.5 \% & 1.08[0.50,2.33] \\ \text { Kendall 2008 } & 32 & 56 & 9 & 25 & 10.0 \% & 2.37[0.90,6.27] \\ \text { Last 1998 } & 13 & 32 & 13 & 24 & 9.4 \% & 0.58[0.20,1.69] \\ \text { Silverman 1999a } & 28 & 41 & 16 & 23 & 9.2 \% & 0.94[0.31,2.85] \\ \text { Subtotal (95\% CI) } & & \mathbf{1 8 9} & & \mathbf{1 2 4} & \mathbf{4 0 . 1 \%} & \mathbf{1 . 1 2}[\mathbf{0 . 6 5 , \mathbf { 1 . 9 2 } ]} \\ \text { Total events. } & 96 & & 57 & & & \end{array}$

Total events:

Heterogeneity: $\mathrm{Tau}^{2}=0.07 ; \mathrm{Chi}^{2}=3.85, \mathrm{df}=3(\mathrm{P}=0.28) ; \mathrm{I}^{2}=22 \%$

Test for overall effect: $\mathrm{Z}=0.40(\mathrm{P}=0.69)$

Total $(95 \%$ CI)

Total events:

234

484

Heterogeneity: $\mathrm{Tau}^{2}=0.50 ; \mathrm{Chi}^{2}=28.77, \mathrm{df}=12(\mathrm{P}=0.004) ; \mathrm{I}^{2}=58 \%$

Test for overall effect: $\mathrm{Z}=3.00(\mathrm{P}=0.003)$

Test for subgroup differences: $\mathrm{Chi}^{2}=7.65, \mathrm{df}=1(\mathrm{P}=0.006), \mathrm{I}^{2}=86.9 \%$
IV, Random, $95 \%$ CI

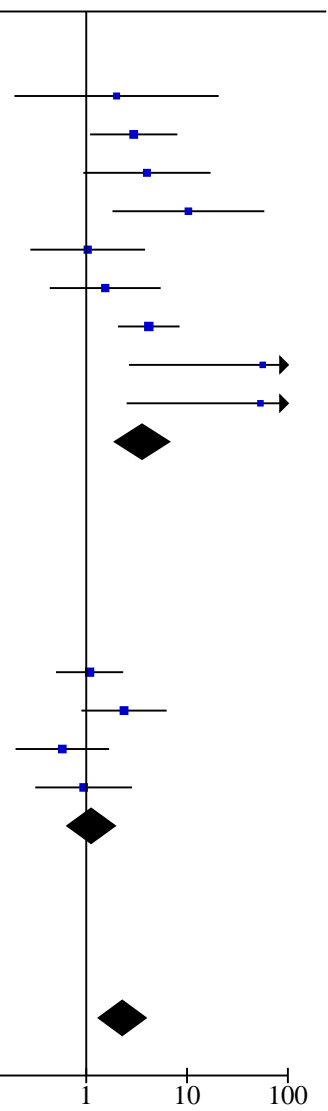

Favours Attention control Favours CBT 
Analysis 3.2. Comparison 3: CBT versus attention control, Outcome 2: Subgroup analysis: remission of primary anxiety diagnosis post-treatment (ITT) (individual vs group)

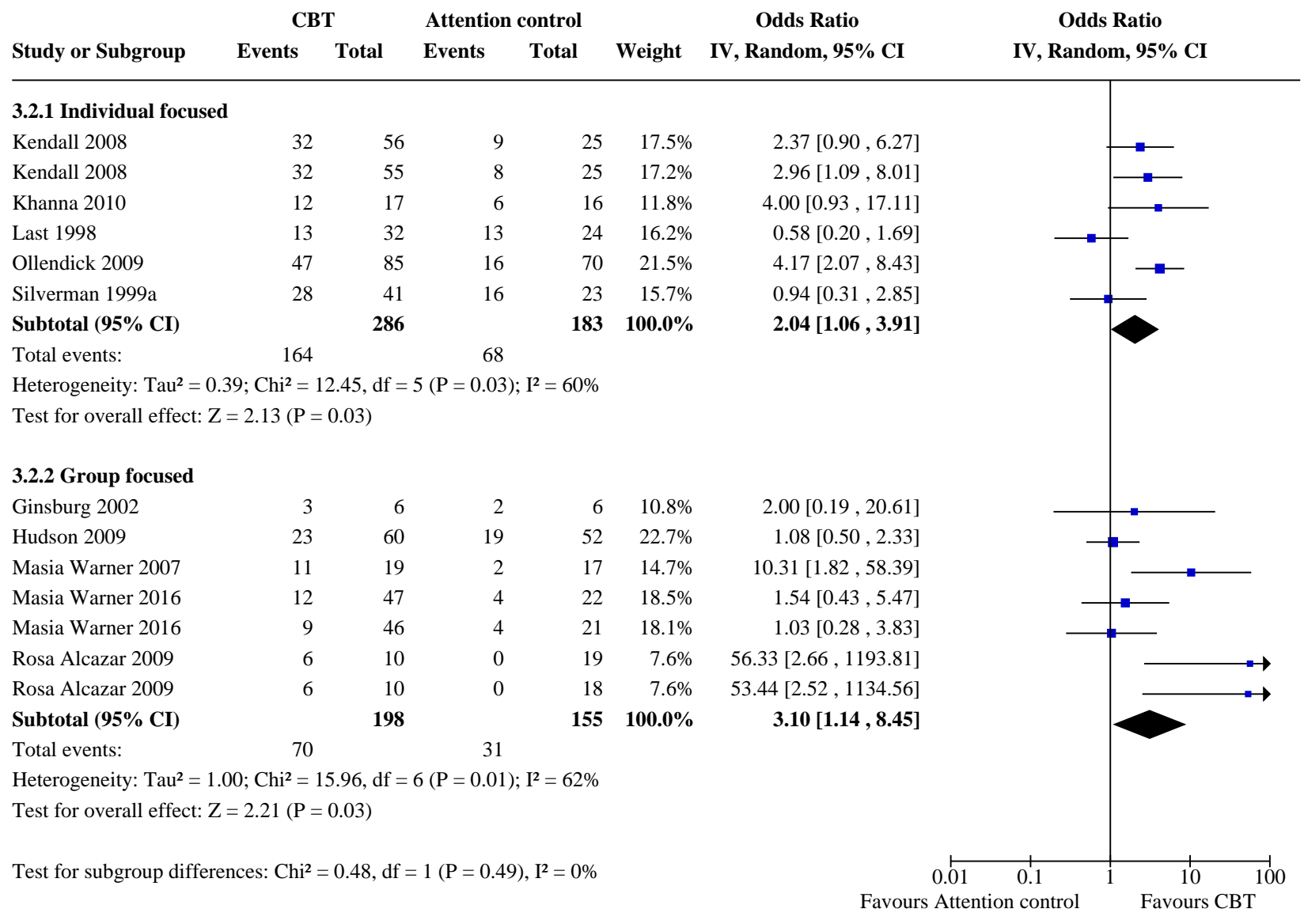


Analysis 3.3. Comparison 3: CBT versus attention control, Outcome 3: Remission of primary anxiety diagnosis post-treatment (completers)

\begin{tabular}{lcccccc} 
& \multicolumn{2}{c}{ CBT } & \multicolumn{2}{c}{ Attention control } & Odds Ratio & Odds Ratio \\
Study or Subgroup & Events & Total & Events $\quad$ Total & Weight & IV, Random, 95\% CI & IV, Random, 95\% CI
\end{tabular}

\begin{tabular}{lrrrrrr}
\hline Ginsburg 2002 & 3 & 4 & 1 & 5 & $1.8 \%$ & $12.00[0.51,280.09]$ \\
Hudson 2009 & 23 & 53 & 13 & 46 & $15.8 \%$ & $1.95[0.84,4.51]$ \\
Kendall 2008 & 32 & 55 & 8 & 25 & $12.7 \%$ & $2.96[1.09,8.01]$ \\
Kendall 2008 & 32 & 56 & 9 & 25 & $13.1 \%$ & $2.37[0.90,6.27]$ \\
Khanna 2010 & 12 & 16 & 3 & 13 & $5.4 \%$ & $10.00[1.80,55.63]$ \\
Last 1998 & 13 & 20 & 10 & 21 & $9.1 \%$ & $2.04[0.58,7.17]$ \\
Masia Warner 2007 & 11 & 18 & 0 & 15 & $2.0 \%$ & $47.53[2.46,919.68]$ \\
Masia Warner 2016 & 9 & 43 & 1 & 19 & $3.7 \%$ & $4.76[0.56,40.64]$ \\
Masia Warner 2016 & 12 & 39 & 2 & 19 & $6.0 \%$ & $3.78[0.75,19.00]$ \\
Ollendick 2009 & 47 & 85 & 16 & 70 & $19.3 \%$ & $4.17[2.07,8.43]$ \\
Rosa Alcazar 2009 & 6 & 10 & 0 & 19 & $1.9 \%$ & $56.33[2.66,1193.81]$ \\
Rosa Alcazar 2009 & 6 & 10 & 0 & 18 & $1.9 \%$ & $53.44[2.52,1134.56]$ \\
Silverman 1999a & 28 & 32 & 9 & 16 & $7.3 \%$ & $5.44[1.29,22.98]$ \\
& & & & & & \\
Total (95\% CI) & & $\mathbf{4 4 1}$ & & $\mathbf{3 1 1}$ & $\mathbf{1 0 0 . 0 \%}$ & $\mathbf{3 . 8 8}[\mathbf{2 . 5 2}, \mathbf{5 . 9 7}]$ \\
Total events: & 234 & & 72 & & &
\end{tabular}

Heterogeneity: $\mathrm{Tau}^{2}=0.12 ; \mathrm{Chi}^{2}=15.20, \mathrm{df}=12(\mathrm{P}=0.23) ; \mathrm{I}^{2}=21 \%$

Test for overall effect: $\mathrm{Z}=6.17(\mathrm{P}<0.00001)$

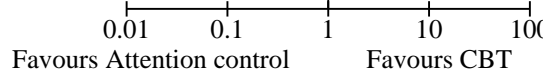

Test for subgroup differences: Not applicable

Analysis 3.4. Comparison 3: CBT versus attention control, Outcome 4: Acceptability (number of participants lost to post-treatment assessment)

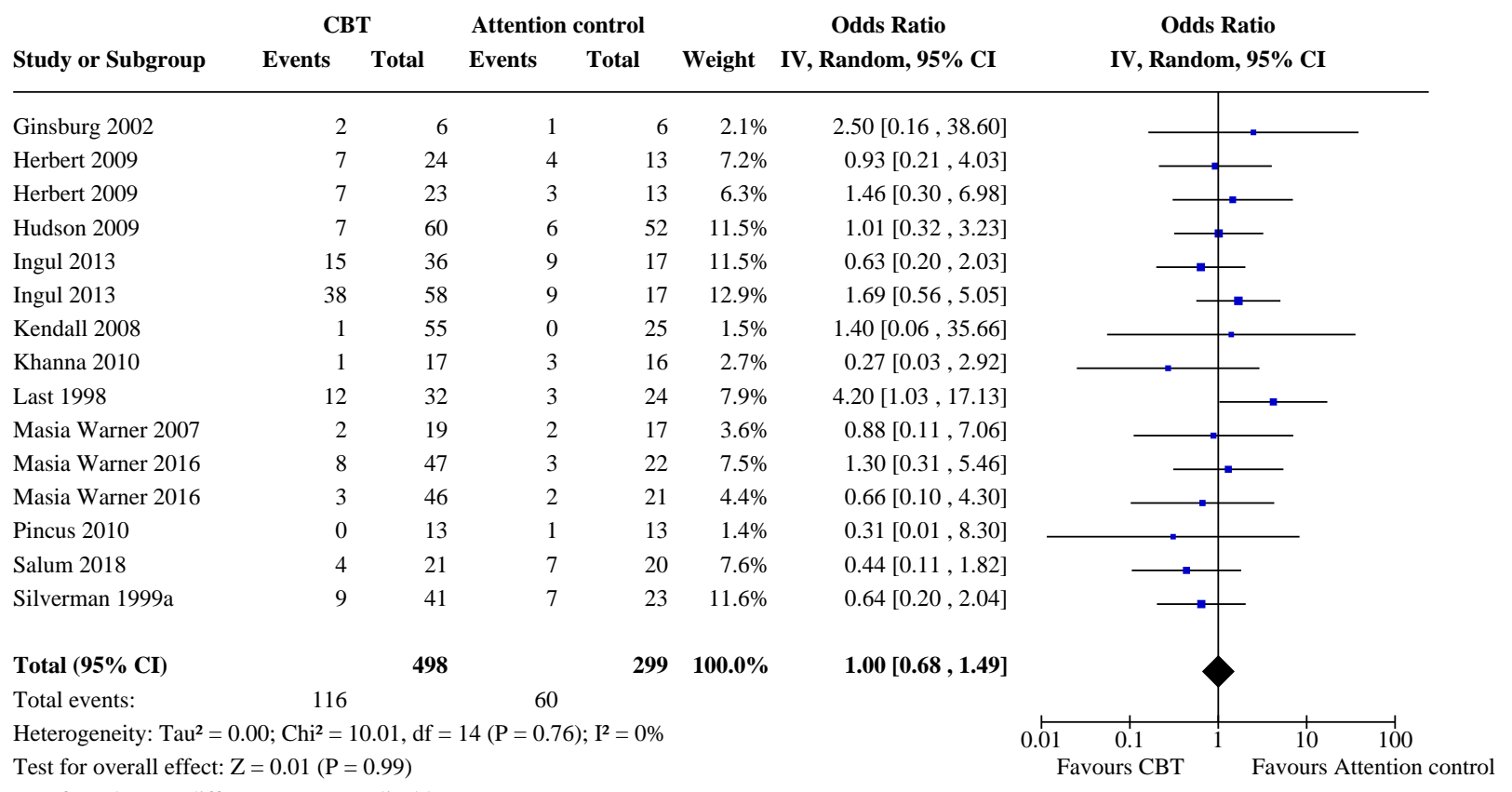


Analysis 3.5. Comparison 3: CBT versus attention control, Outcome 5: Remission of all anxiety diagnoses post-treatment (ITT)

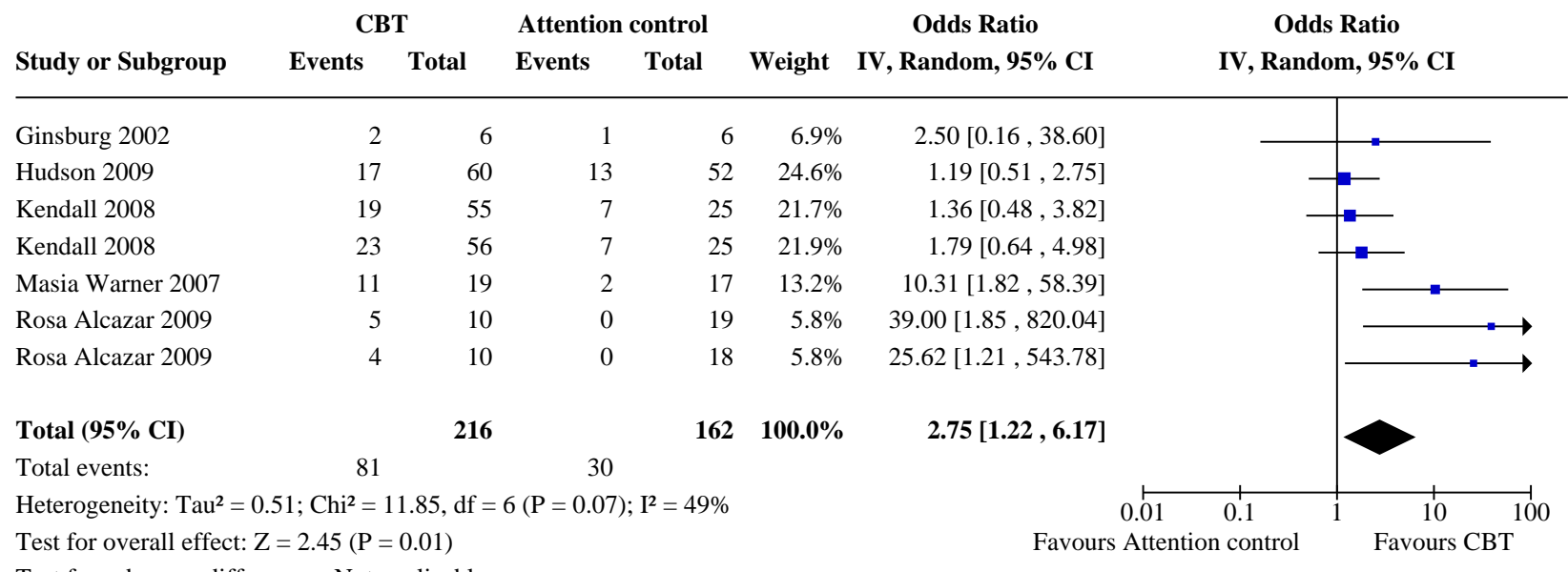

Test for subgroup differences: Not applicable

\section{Analysis 3.6. Comparison 3: CBT versus attention control, Outcome 6: Remission of all anxiety diagnoses post-treatment (completers)}

\begin{tabular}{|c|c|c|c|c|c|c|}
\hline & \multicolumn{2}{|c|}{ CBT } & Attention control & \multicolumn{2}{|r|}{ Odds Ratio } & Odds Ratio \\
\hline Study or Subgroup & Events & Total & Events & Weight & IV, Random, 95\% CI & IV, Random, $95 \%$ CI \\
\hline
\end{tabular}

\begin{tabular}{lrrrrrr}
\hline Ginsburg 2002 & 2 & 4 & 0 & 5 & $5.9 \%$ & $11.00[0.37,324.52]$ \\
Hudson 2009 & 17 & 53 & 7 & 46 & $24.7 \%$ & $2.63[0.98,7.08]$ \\
Kendall 2008 & 19 & 55 & 7 & 25 & $24.0 \%$ & $1.36[0.48,3.82]$ \\
Kendall 2008 & 23 & 56 & 7 & 25 & $24.2 \%$ & $1.79[0.64,4.98]$ \\
Masia Warner 2007 & 11 & 18 & 0 & 15 & $7.3 \%$ & $47.53[2.46,919.68]$ \\
Rosa Alcazar 2009 & 4 & 10 & 0 & 18 & $6.9 \%$ & $25.62[1.21,543.78]$ \\
Rosa Alcazar 2009 & 5 & 10 & 0 & 19 & $7.0 \%$ & $39.00[1.85,820.04]$ \\
& & & & & & \\
Total (95\% CI) & 81 & $\mathbf{2 0 6}$ & & $\mathbf{1 5 3}$ & $\mathbf{1 0 0 . 0 \%}$ & $\mathbf{3 . 8 8}[\mathbf{1 . 5 8 , 9 . 5 1}]$ \\
Total events: & & & &
\end{tabular}

Test for overall effect: $\mathrm{Z}=2.96(\mathrm{P}=0.003)$

Favours Attention control $\quad$ Favours CBT

Test for subgroup differences: Not applicable 
Analysis 3.7. Comparison 3: $C B T$ versus attention control, Outcome 7: Reduction in anxiety symptoms (child report) post-treatment

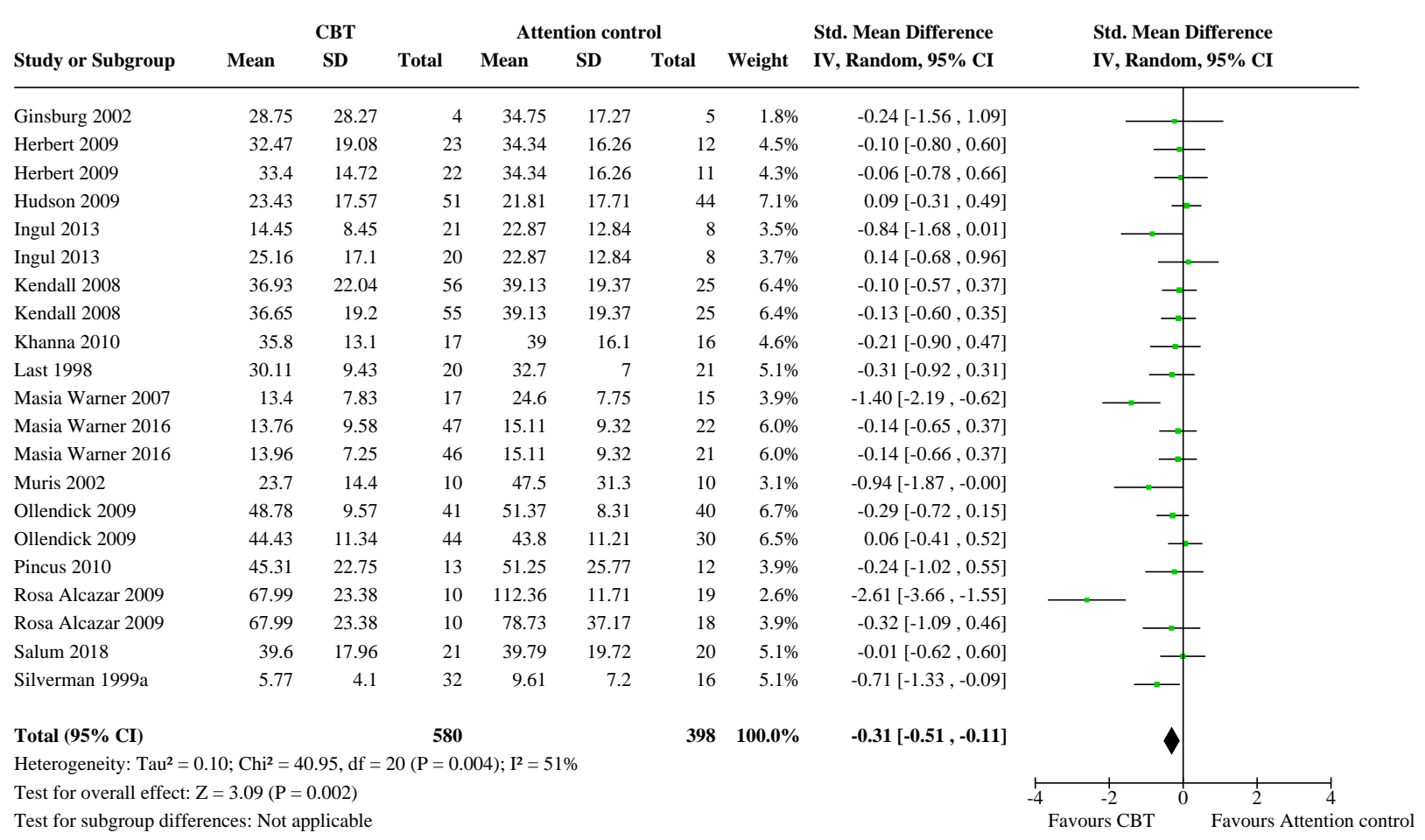

Analysis 3.8. Comparison 3: CBT versus attention control, Outcome 8: Sensitivity analysis: reduction in anxiety symptoms (child report) post-treatment (broad anxiety measures only)

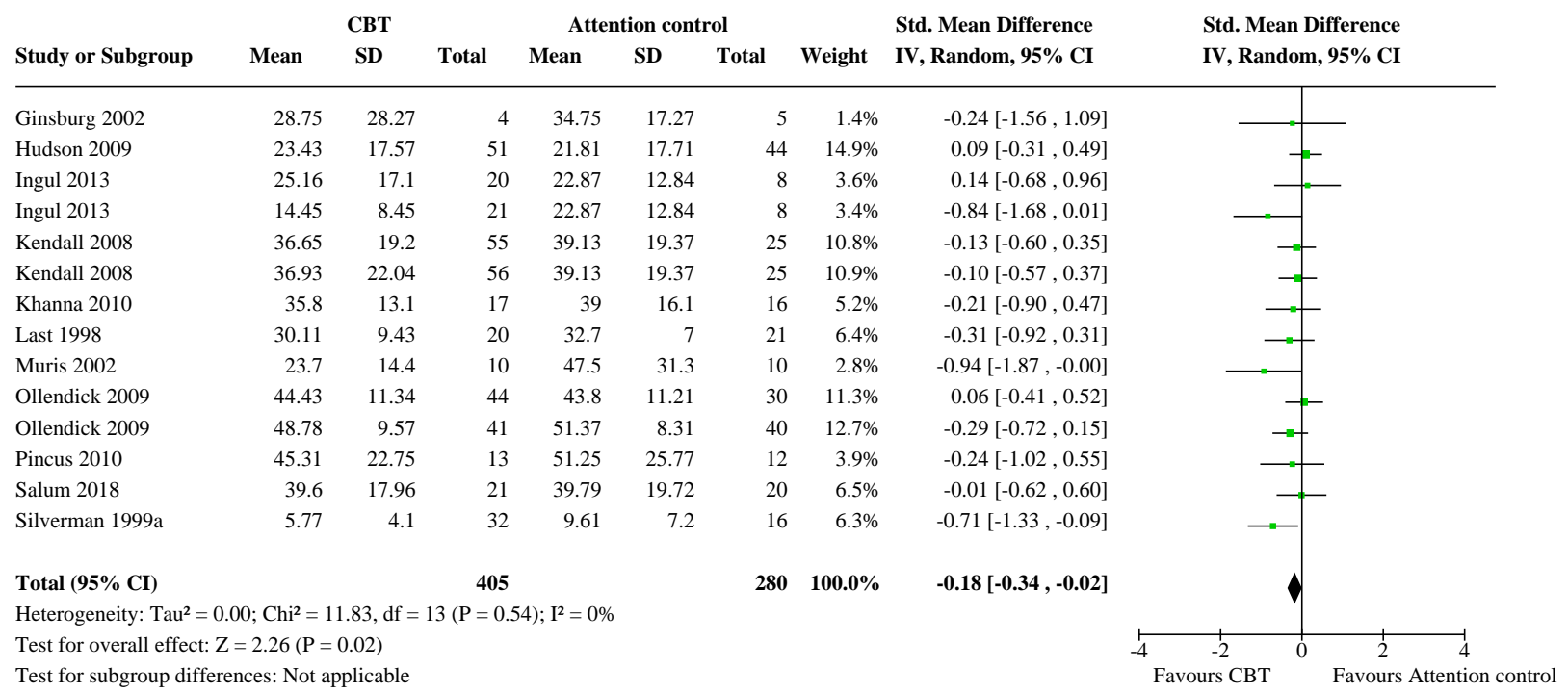


Analysis 3.9. Comparison 3: $C B T$ versus attention control, Outcome 9: Reduction in anxiety symptoms (parent report) post-treatment

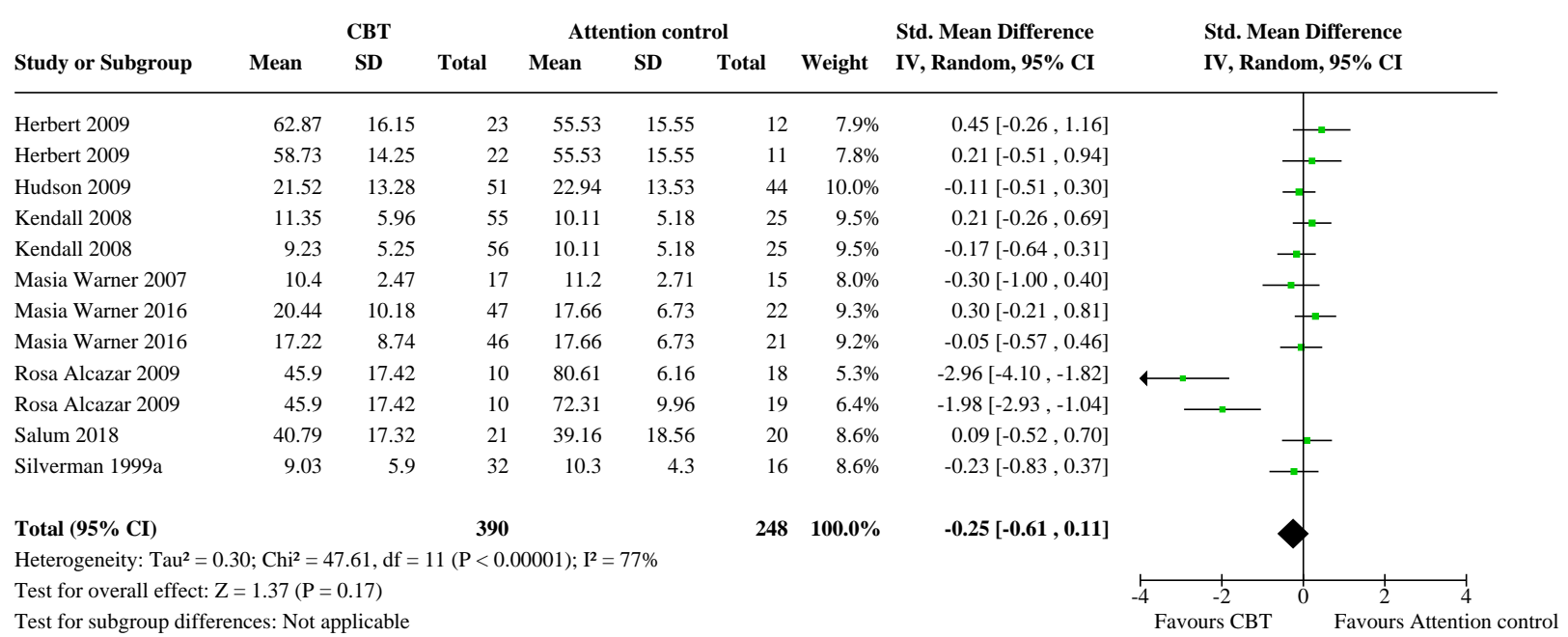

Analysis 3.10. Comparison 3: CBT versus attention control, Outcome 10: Sensitivity analysis: reduction in anxiety symptoms (parent report) post-treatment (broad anxiety measures only)

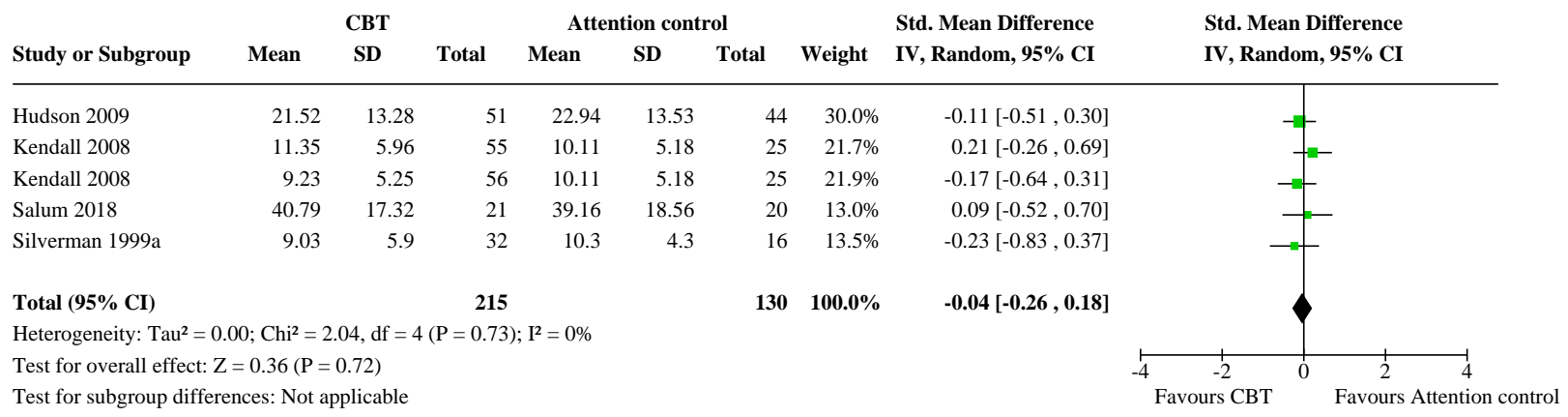

Analysis 3.11. Comparison 3: CBT versus attention control, Outcome 11: Reduction in depressive symptoms post-treatment

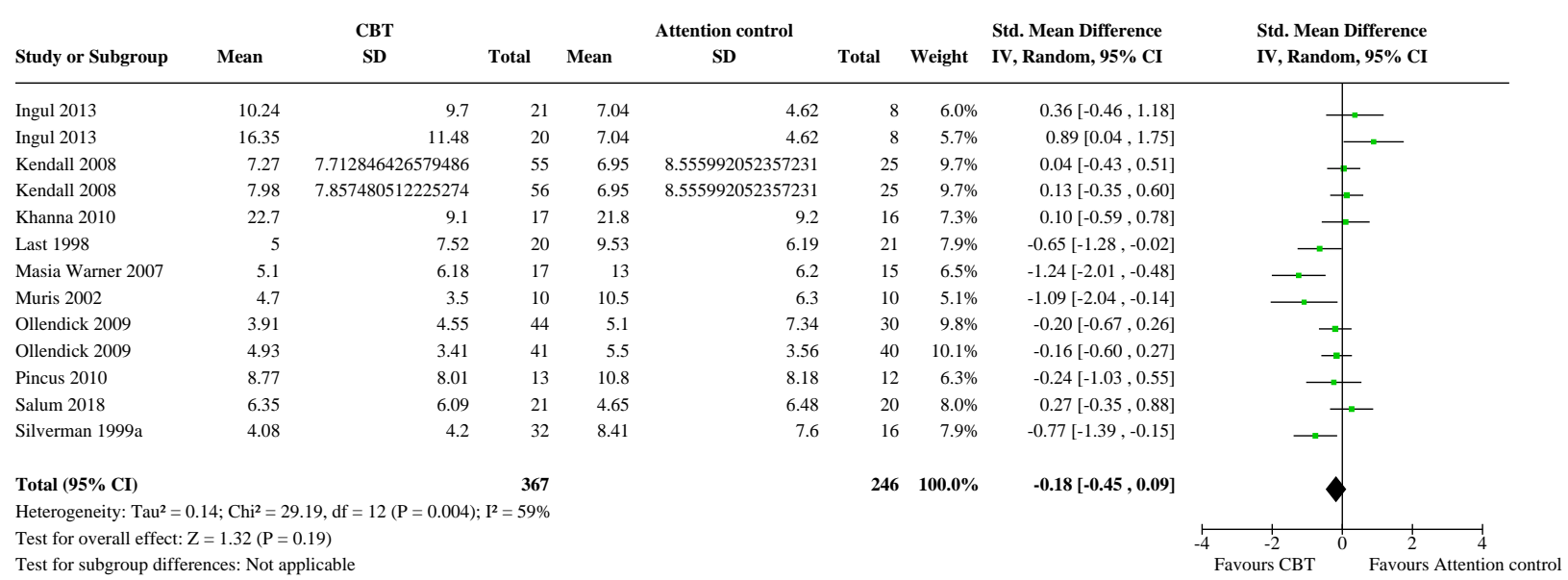




\section{Analysis 3.12. Comparison 3: CBT versus attention control, Outcome} 12: Remission of primary anxiety diagnosis at follow-up (ITT)

\begin{tabular}{|c|c|c|c|c|c|}
\hline & CBT & Attention control & & Odds Ratio & Odds Ratio \\
\hline Study or Subgroup & Events & Events & Weight & IV, Random, $95 \%$ CI & IV, Random, 95\% CI \\
\hline
\end{tabular}

\subsubsection{Follow-up (\# 6 months)} Hudson 2009

Masia Warner 2007

Masia Warner 2016

Masia Warner 2016

Rosa Alcazar 2009

Rosa Alcazar 2009

Subtotal (95\% CI)

Total events:

35

11

13

17

6

60
19
47
46
10
10
192

87

Heterogeneity: $\mathrm{Tau}^{2}=0.71 ; \mathrm{Chi}^{2}=12.62, \mathrm{df}=5(\mathrm{P}=0.03) ; \mathrm{I}^{2}=60 \%$

Test for overall effect: $\mathrm{Z}=2.39(\mathrm{P}=0.02)$

$\begin{array}{rrr}28 & 52 & 25.6 \% \\ 3 & 17 & 16.5 \% \\ 5 & 22 & 20.4 \% \\ 5 & 21 & 20.6 \% \\ 1 & 19 & 10.0 \% \\ 0 & 18 & 7.0 \% \\ & \mathbf{1 4 9} & \mathbf{1 0 0 . 0 \%}\end{array}$

$1.20[0.57,2.54]$
$6.42[1.37,30.05]$
$1.30[0.40,4.25]$
$1.88[0.58,6.04]$
$27.00[2.50,291.19]$
$37.00[1.76,779.34]$
$\mathbf{3 . 0 6}[\mathbf{1 . 2 2}, \mathbf{7 . 6 5}]$

IV, Random, $95 \%$ CI

Analysis 3.13. Comparison 3: CBT versus attention control, Outcome 13: Remission of primary anxiety diagnosis at follow-up (completers)

\begin{tabular}{lccccccc}
\multicolumn{2}{c}{ CBT } & \multicolumn{2}{c}{ Attention control } & \multicolumn{2}{c}{ Odds Ratio } & Odds Ratio \\
Study or Subgroup & Events & Total & Events & Total & Weight & IV, Random, 95\% CI & IV, Random, 95\% CI
\end{tabular}

\begin{tabular}{lrrrrrr}
\hline 3.13.1 Follow-up (\# 6 months) & & & & & & \\
Hudson 2009 & 35 & 51 & 20 & 44 & $30.5 \%$ & $2.63[1.14,6.07]$ \\
Masia Warner 2007 & 11 & 15 & 1 & 15 & $11.4 \%$ & $38.50[3.75,395.41]$ \\
Masia Warner 2016 & 17 & 43 & 3 & 19 & $21.4 \%$ & $3.49[0.88,13.81]$ \\
Masia Warner 2016 & 13 & 39 & 2 & 19 & $18.2 \%$ & $4.25[0.85,21.25]$ \\
Rosa Alcazar 2009 & 6 & 10 & 1 & 19 & $11.0 \%$ & $27.00[2.50,291.19]$ \\
Rosa Alcazar 2009 & 5 & 10 & 0 & 18 & $7.5 \%$ & $37.00[1.76,779.34]$ \\
Subtotal (95\% CI) & & $\mathbf{1 6 8}$ & & $\mathbf{1 3 4}$ & $\mathbf{1 0 0 . 0 \%}$ & $\mathbf{6 . 5 2}[\mathbf{2 . 5 8 , \mathbf { 1 6 . 4 3 } ]}$ \\
Total events: & 87 & & 27 & & &
\end{tabular}

Heterogeneity: $\mathrm{Tau}^{2}=0.55 ; \mathrm{Chi}^{2}=9.01, \mathrm{df}=5(\mathrm{P}=0.11) ; \mathrm{I}^{2}=44 \%$

Test for overall effect: $\mathrm{Z}=3.97(\mathrm{P}<0.0001)$

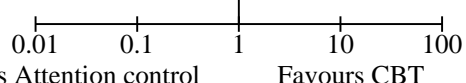


Analysis 3.14. Comparison 3: CBT versus attention control, Outcome 14: Remission of all anxiety diagnoses at follow-up (ITT)

\begin{tabular}{|c|c|c|c|c|c|c|c|}
\hline & \multicolumn{2}{|c|}{ CBT } & Attenti & control & \multicolumn{2}{|r|}{ Odds Ratio } & Odds Ratio \\
\hline Study or Subgroup & Events & Total & Events & Total & Weight & IV, Random, $95 \%$ CI & IV, Random, 95\% CI \\
\hline
\end{tabular}

3.14.1 Follow-up (\# 6 months)

Hudson 2009

Masia Warner 2007

Rosa Alcazar 2009

Rosa Alcazar 2009

Subtotal $(95 \%$ CI $)$

Total events:

$10-60$

$60 \quad 21$

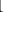

$17 \quad 29.5 \%$

$4 \quad 10$

19
10

0

$18 \quad 17.2 \%$

$5 \quad 10$

99

44

0

$19 \quad 17.2 \%$

$106 \quad 100.0 \%$

$25.62[1.21,543.78]$

$39.00[1.85,820.04]$

$5.44[1.00,29.60]$

Heterogeneity: $\mathrm{Tau}^{2}=1.92 ; \mathrm{Chi}^{2}=10.54, \mathrm{df}=3(\mathrm{P}=0.01) ; \mathrm{I}^{2}=72 \%$

Test for overall effect: $\mathrm{Z}=1.96(\mathrm{P}=0.05)$

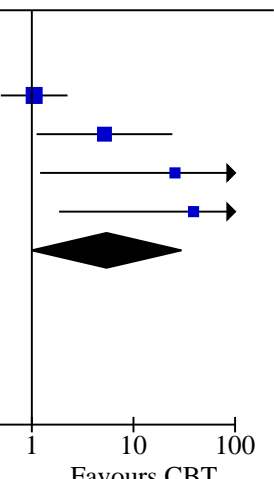

Favours CBT

\section{Analysis 3.15. Comparison 3: CBT versus attention control, Outcome 15: Remission of all anxiety diagnoses at follow-up (completers)}

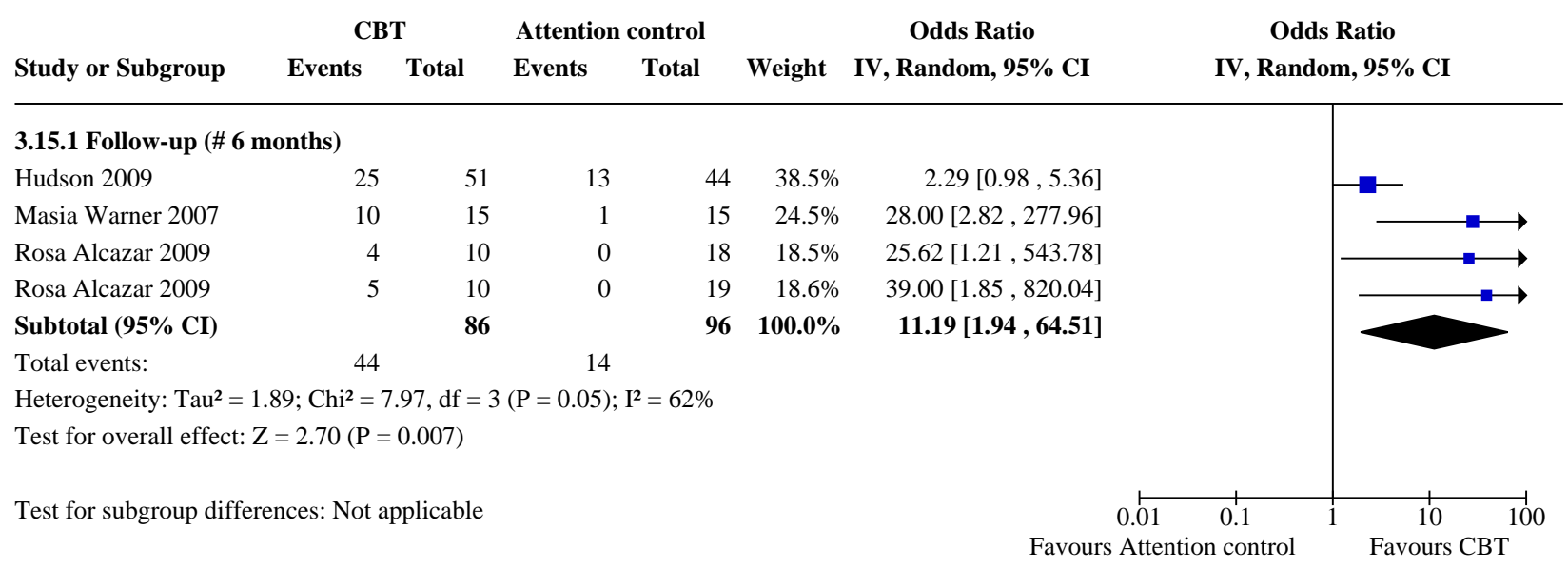




\section{Analysis 3.16. Comparison 3: CBT versus attention control, Outcome} 16: Reduction in anxiety symptoms (child report) at follow-up

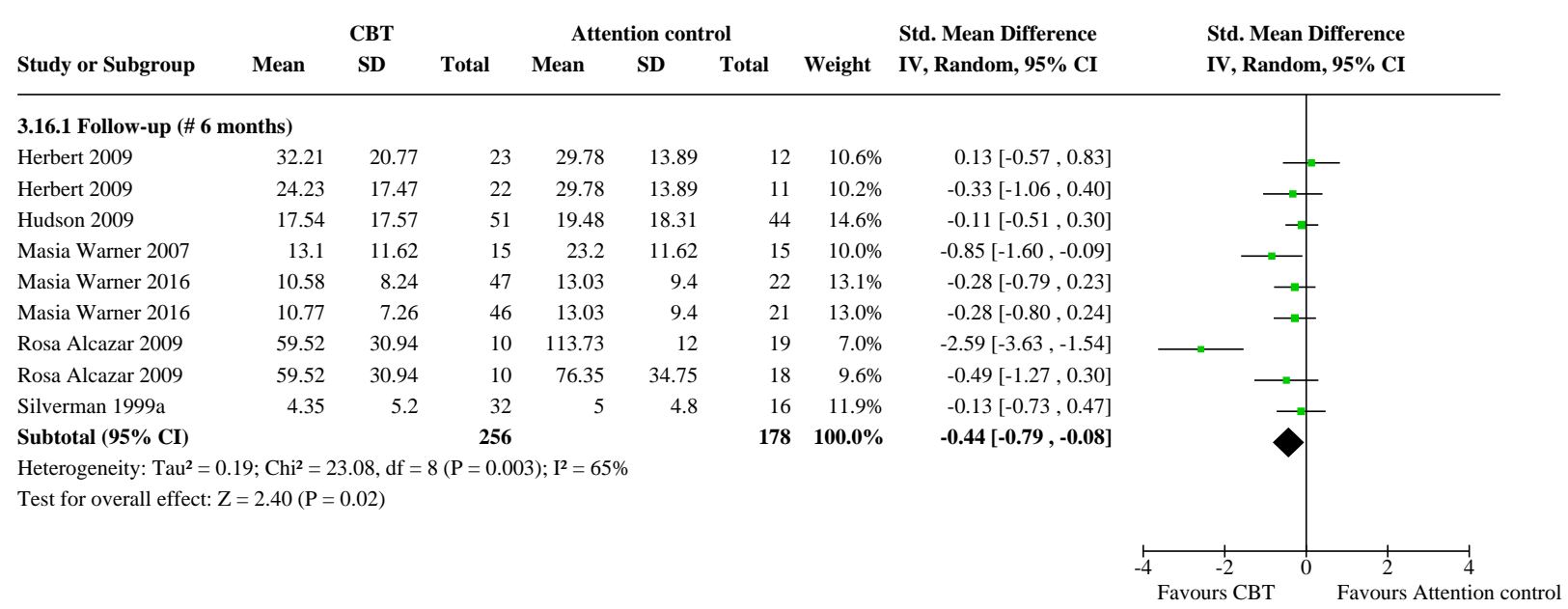

\section{Analysis 3.17. Comparison 3: CBT versus attention control, Outcome 17: Reduction in anxiety symptoms (parent report) at follow-up}

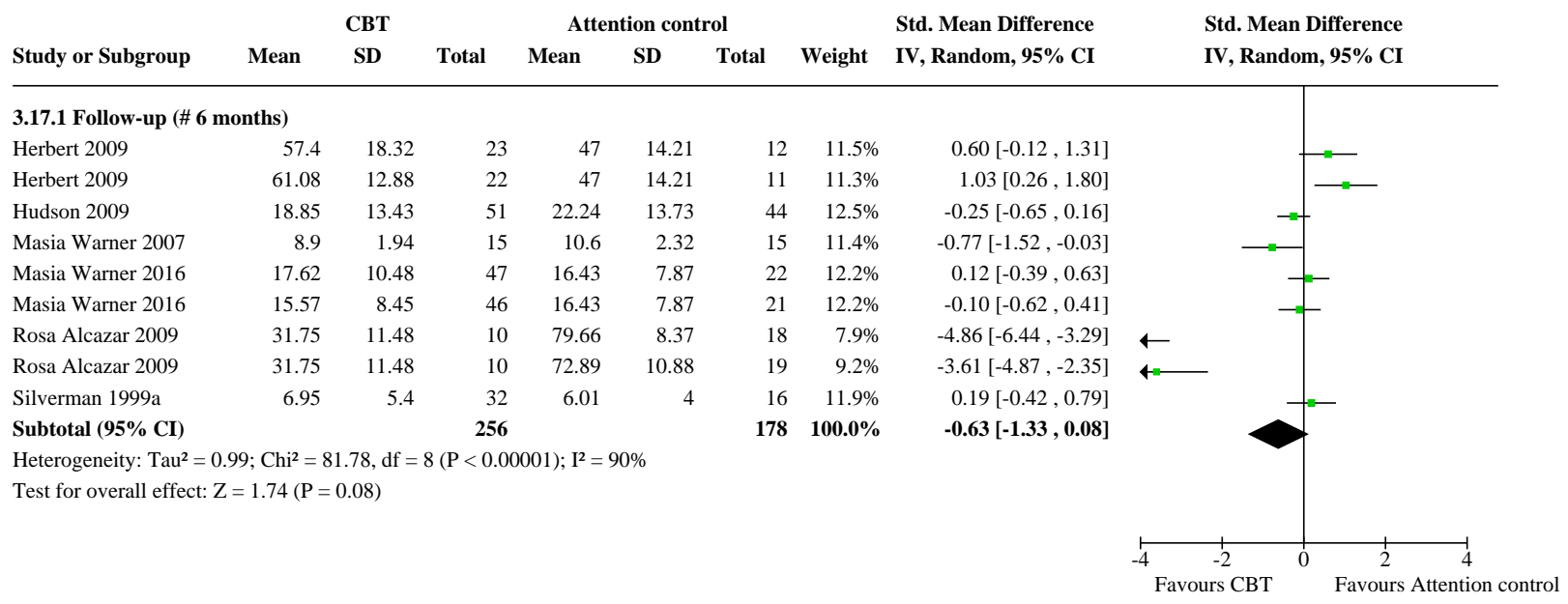

\section{Comparison 4. CBT versus alternative treatment}

\begin{tabular}{lllll}
\hline Outcome or subgroup title & $\begin{array}{l}\text { No. of } \\
\text { studies }\end{array}$ & $\begin{array}{l}\text { No. of } \\
\text { partici- } \\
\text { pants }\end{array}$ & Statistical method & Effect size \\
\hline $\begin{array}{l}4.1 \text { Acceptability (number of participants lost to post- } \\
\text { treatment assessment) }\end{array}$ & 7 & 515 & $\begin{array}{l}\text { Odds Ratio (IV, Random, 95\% } \\
\text { Cl) }\end{array}$ & $\begin{array}{l}1.58 \text { [0.61, } \\
4.13]\end{array}$ \\
\hline $\begin{array}{l}\text { 4.2 Remission of all anxiety diagnoses post-treatment } \\
\text { (ITT) }\end{array}$ & 4 & 401 & $\begin{array}{l}\text { Odds Ratio (IV, Random, 95\% } \\
\text { Cl) }\end{array}$ & 0.89 [0.35, \\
\hline $\begin{array}{l}\text { 4.3 Remission of all anxiety diagnoses post-treatment } \\
\text { (completers) }\end{array}$ & 4 & 348 & Odds Ratio (IV, Random, 95\% & $\begin{array}{l}1.52 \text { [0.70, } \\
\text { CI) }\end{array}$ \\
\hline
\end{tabular}




\begin{tabular}{lllll}
\hline Outcome or subgroup title & $\begin{array}{l}\text { No. of } \\
\text { studies }\end{array}$ & $\begin{array}{l}\text { No. of } \\
\text { partici- } \\
\text { pants }\end{array}$ & Statistical method & Effect size \\
\hline $\begin{array}{l}\text { 4.4 Reduction in anxiety symptoms (child report) } \\
\text { post-treatment }\end{array}$ & 6 & 399 & $\begin{array}{l}\text { Std. Mean Difference (IV, Ran- } \\
\text { dom, 95\% Cl) }\end{array}$ & $\begin{array}{l}-0.09[-0.40, \\
0.21]\end{array}$ \\
\hline $\begin{array}{l}\text { 4.5 Sensitivity analysis: reduction in anxiety symp- } \\
\text { toms (child report) post-treatment (broad anxiety } \\
\text { measures only) }\end{array}$ & 5 & 378 & Std. Mean Difference (IV, Ran- & $-0.00[-0.21$, \\
\hline $\begin{array}{l}\text { 4.6 Reduction in anxiety symptoms (parent report) } \\
\text { post-treatment }\end{array}$ & 6 & 423 & $\begin{array}{l}\text { Std. Mean Difference (IV, Ran- } \\
\text { dom, 95\% CI) }\end{array}$ & $\begin{array}{l}-0.13 \text { [-0.33, } \\
0.06]\end{array}$ \\
\hline
\end{tabular}

\section{Analysis 4.1. Comparison 4: CBT versus alternative treatment, Outcome 1: Acceptability (number of participants lost to post-treatment assessment)}

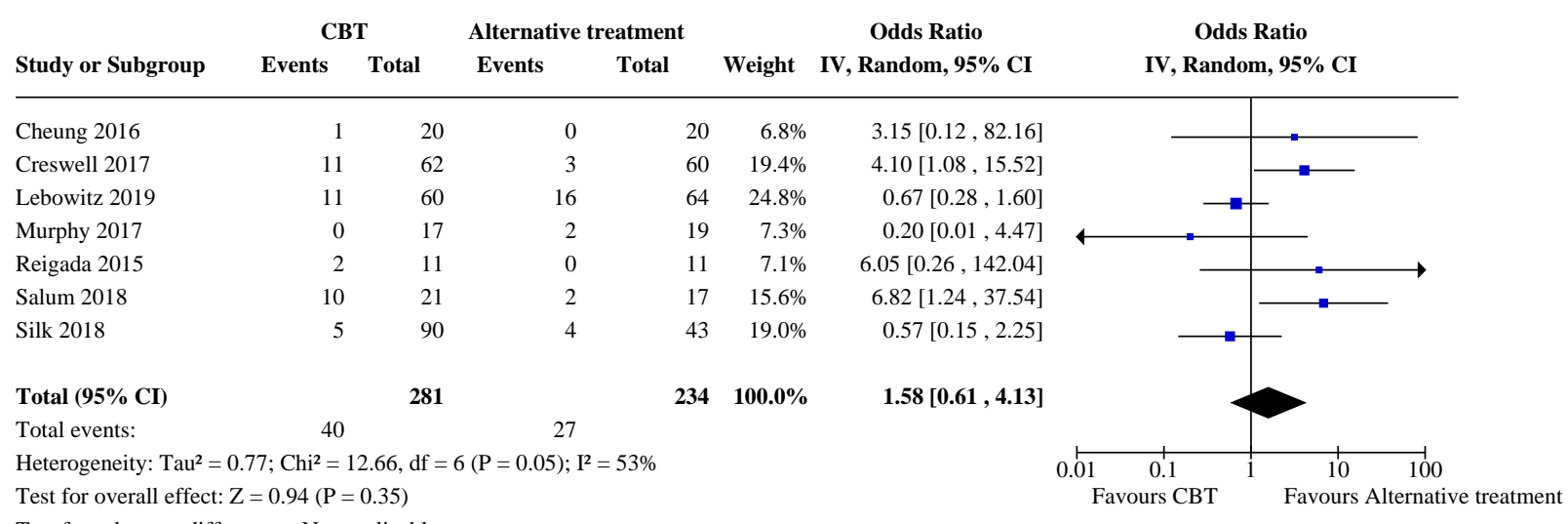

Test for subgroup differences: Not applicable

\section{Analysis 4.2. Comparison 4: CBT versus alternative treatment, Outcome 2: Remission of all anxiety diagnoses post-treatment (ITT)}

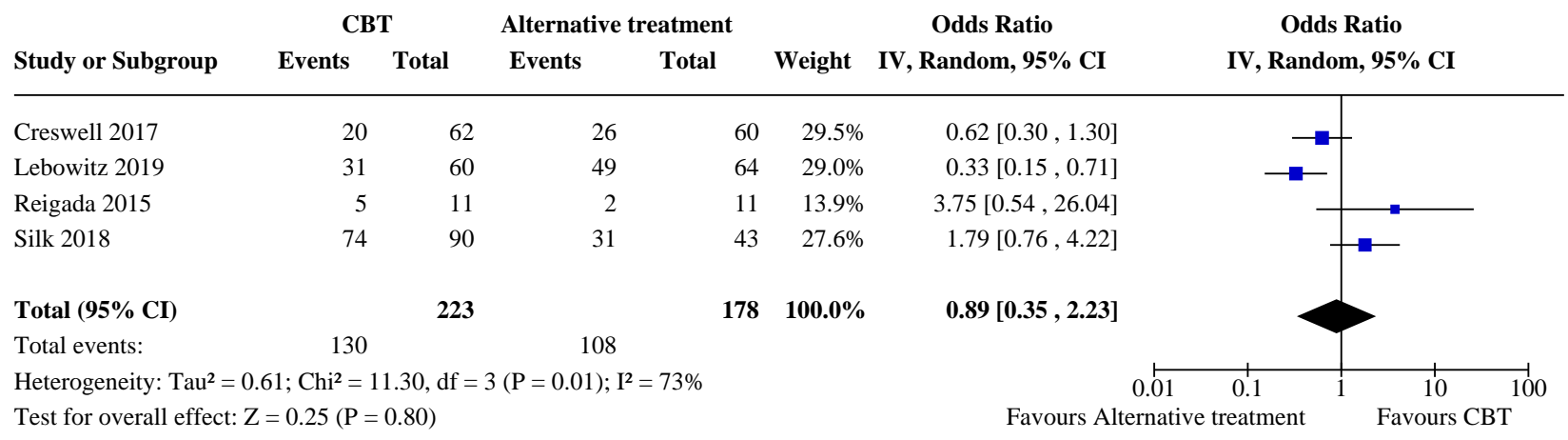


Analysis 4.3. Comparison 4: CBT versus alternative treatment, Outcome 3: Remission of all anxiety diagnoses post-treatment (completers)

\begin{tabular}{lccccccc}
\multicolumn{4}{c}{ CBT } & \multicolumn{2}{c}{ Alternative treatment } & & \multicolumn{2}{c}{ Odds Ratio } & Odds Ratio \\
Study or Subgroup & Events & Total & Events & Total & Weight & IV, Random, 95\% CI & IV, Random, 95\% CI
\end{tabular}

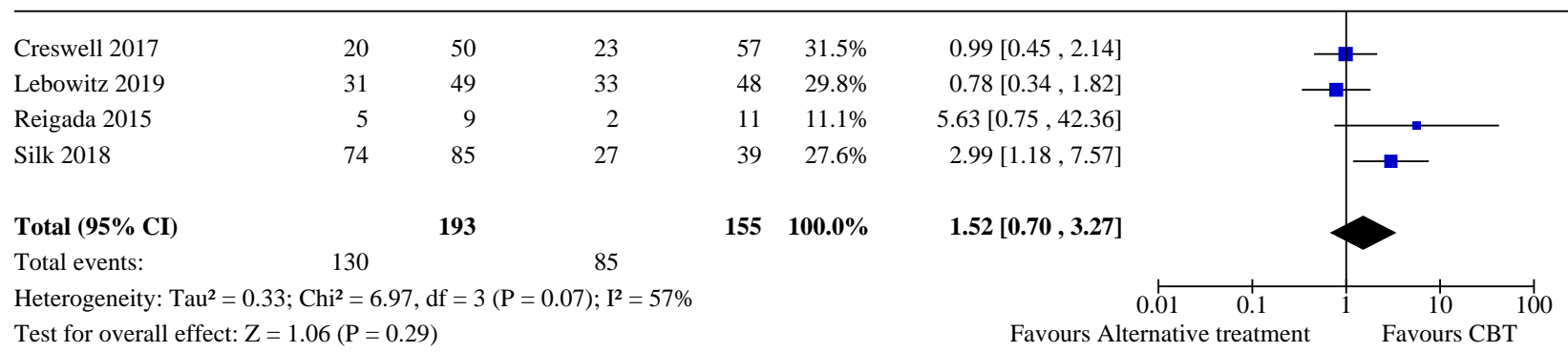

Test for subgroup differences: Not applicable

\section{Analysis 4.4. Comparison 4: CBT versus alternative treatment, Outcome 4: Reduction in anxiety symptoms (child report) post-treatment}

\begin{tabular}{|c|c|c|c|c|c|c|c|c|c|}
\hline \multirow[b]{2}{*}{ Study or Subgroup } & \multirow[b]{2}{*}{ Mean } & \multicolumn{2}{|l|}{ CBT } & \multicolumn{3}{|c|}{ Alternative treatment } & \multirow[b]{2}{*}{ Weight } & \multirow{2}{*}{$\begin{array}{l}\text { Std. Mean Difference } \\
\text { IV, Random, 95\% CI }\end{array}$} & \multirow{2}{*}{$\begin{array}{l}\text { Std. Mean Difference } \\
\text { IV, Random, } 95 \% \text { CI }\end{array}$} \\
\hline & & SD & Total & Mean & SD & Total & & & \\
\hline Cheung 2016 & 50.63 & 29.32 & 19 & 45.4 & 25.46 & 20 & $14.0 \%$ & $0.19[-0.44,0.82]$ & - \\
\hline Creswell 2017 & 31.49 & 24.02 & 39 & 25.1 & 19 & 52 & $21.0 \%$ & $0.30[-0.12,0.72]$ & - \\
\hline Lebowitz 2019 & 19.63 & 14.05 & 49 & 22.12 & 13.9 & 48 & $21.8 \%$ & $-0.18[-0.58,0.22]$ & \\
\hline Reigada 2015 & 5.55 & 12.57 & 10 & 31.04 & 23.95 & 11 & $7.9 \%$ & $-1.26[-2.22,-0.31]$ & - \\
\hline Salum 2018 & 36.58 & 23.6 & 21 & 37.21 & 18.68 & 17 & $13.7 \%$ & $-0.03[-0.67,0.61]$ & \\
\hline Silk 2018 & 17 & 15.38 & 79 & 19.65 & 14.48 & 34 & $21.6 \%$ & $-0.17[-0.58,0.23]$ & \\
\hline Total $(95 \% \mathrm{CI})$ & & & 217 & & & 182 & $100.0 \%$ & $-0.09[-0.40,0.21]$ & \\
\hline \multicolumn{10}{|c|}{ Heterogeneity: $\mathrm{Tau}^{2}=0.07 ; \mathrm{Chi}^{2}=10.13, \mathrm{df}=5(\mathrm{P}=0.07) ; \mathrm{I}^{2}=51 \%$} \\
\hline \multicolumn{9}{|c|}{ Test for overall effect: $\mathrm{Z}=0.58(\mathrm{P}=0.56)$} & $\begin{array}{l}1 \\
-2\end{array}$ \\
\hline \multicolumn{9}{|c|}{ Test for subgroup differences: Not applicable } & ours CBT \\
\hline
\end{tabular}

Analysis 4.5. Comparison 4: CBT versus alternative treatment, Outcome 5: Sensitivity analysis: reduction in anxiety symptoms (child report) post-treatment (broad anxiety measures only)

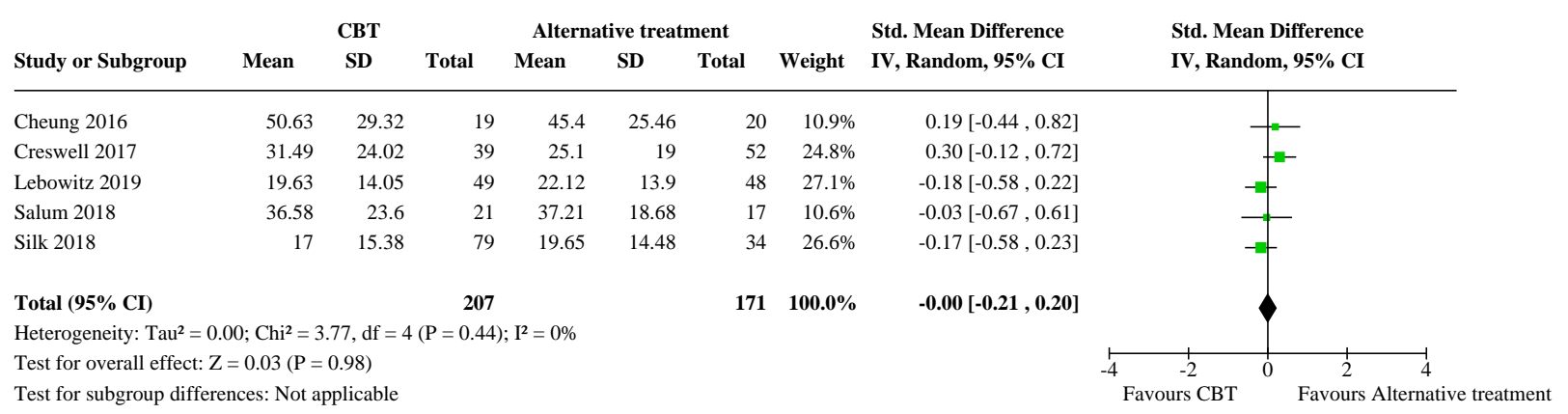




\section{Analysis 4.6. Comparison 4: CBT versus alternative treatment, Outcome} 6: Reduction in anxiety symptoms (parent report) post-treatment

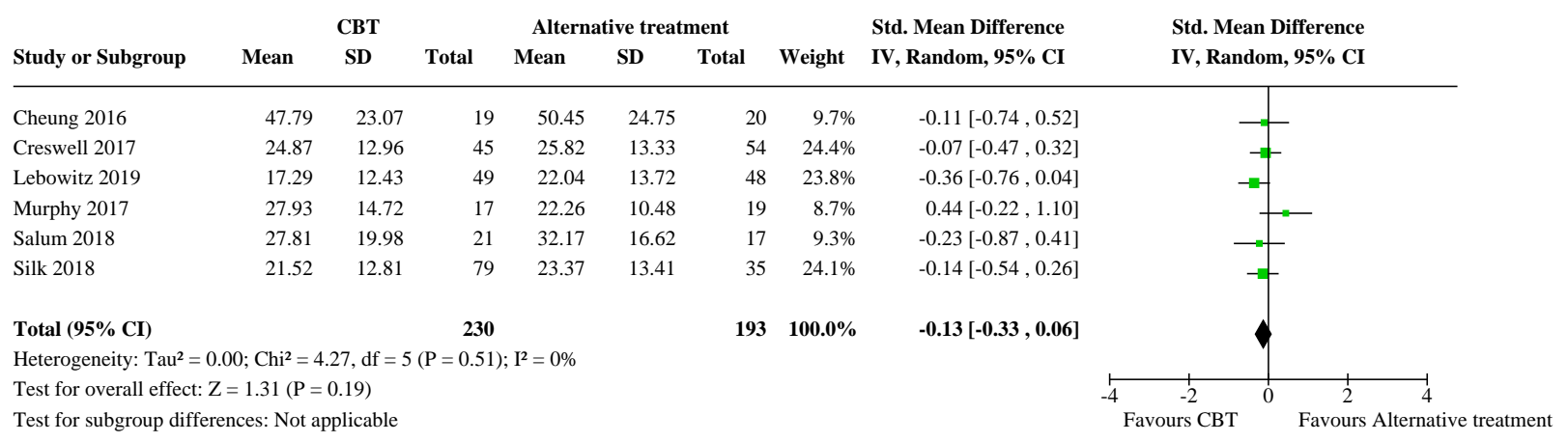

\section{ADDITIONAL TABLES}




\begin{tabular}{|c|c|c|c|c|c|c|c|c|c|c|c|c|}
\hline Study & Comparison 1 & $\begin{array}{l}\text { Com- } \\
\text { pari- } \\
\text { son } 2\end{array}$ & $\begin{array}{l}\text { Com- } \\
\text { pari- } \\
\text { son } 3\end{array}$ & $\begin{array}{l}\text { Broad } \\
\text { vs spe- } \\
\text { cific } \\
\text { anx- } \\
\text { iety } \\
\text { mea- } \\
\text { sure }\end{array}$ & $\begin{array}{l}\text { Delivery } \\
\text { mode }\end{array}$ & $\begin{array}{l}\text { Deliv- } \\
\text { ery } \\
\text { mode }\end{array}$ & $\begin{array}{l}\text { ASD } \\
\text { vs not } \\
\text { ASD }\end{array}$ & $\begin{array}{l}\text { Ther- } \\
\text { apist } \\
\text { con- } \\
\text { tact } \\
\text { time } \\
\text { (hours) }\end{array}$ & Age & Setting & $\begin{array}{l}\text { Disor- } \\
\text { der-spe- } \\
\text { cific }\end{array}$ & $\begin{array}{l}\text { Oth- } \\
\text { er co- } \\
\text { mor- } \\
\text { bid } \\
\text { condi- } \\
\text { tions } \\
\text { (re- } \\
\text { quired) }\end{array}$ \\
\hline Afshari 2014 & CBT vs Wait list / no treatment & $\begin{array}{l}\text { CBT } \\
\text { vs } \\
\text { Wait } \\
\text { list / } \\
\text { no } \\
\text { treat- } \\
\text { ment }\end{array}$ & & Broad & Child focused & Group & $\begin{array}{l}\text { Not } \\
\text { ASD }\end{array}$ & $\begin{array}{l}10 \text { to } \\
20\end{array}$ & $\operatorname{mix}$ & clinic & & \\
\hline Arendt 2016 & CBT vs Wait list / no treatment & & & Broad & Child+parent & Group & $\begin{array}{l}\text { Not } \\
\text { ASD }\end{array}$ & $20+$ & $\operatorname{mix}$ & $\begin{array}{l}\text { clinic (univer- } \\
\text { sity) }\end{array}$ & & \\
\hline Barrett 1996 & CBT vs Wait list / no treatment & $\begin{array}{l}\text { CBT } \\
\text { vs } \\
\text { Wait } \\
\text { list / } \\
\text { no } \\
\text { treat- } \\
\text { ment }\end{array}$ & & Broad & $\begin{array}{l}\text { Child focused } \\
\text { and child } \\
\text { +parent }\end{array}$ & $\begin{array}{l}\text { Indi- } \\
\text { vidual }\end{array}$ & $\begin{array}{l}\text { Not } \\
\text { ASD }\end{array}$ & $\begin{array}{l}10 \text { to } \\
20\end{array}$ & $\operatorname{mix}$ & $\begin{array}{l}\text { clinic (univer- } \\
\text { sity) }\end{array}$ & & \\
\hline Barrett 1998 & CBT vs Wait list / no treatment & $\begin{array}{l}\text { CBT } \\
\text { vs } \\
\text { Wait } \\
\text { list / } \\
\text { no } \\
\text { treat- } \\
\text { ment }\end{array}$ & & & $\begin{array}{l}\text { Child focused } \\
\text { and child } \\
\text { +parent }\end{array}$ & Group & $\begin{array}{l}\text { Not } \\
\text { ASD }\end{array}$ & $20+$ & $\operatorname{mix}$ & $\begin{array}{l}\text { clinic (univer- } \\
\text { sity) }\end{array}$ & & \\
\hline $\begin{array}{l}\text { Barrington } \\
2005\end{array}$ & CBT vs TAU & & & Broad & Child+parent & $\begin{array}{l}\text { Indi- } \\
\text { vidual }\end{array}$ & $\begin{array}{l}\text { Not } \\
\text { ASD }\end{array}$ & $\begin{array}{l}10 \text { to } \\
20\end{array}$ & $\operatorname{mix}$ & $\begin{array}{l}\text { clinic } \\
\text { (CAMHS) }\end{array}$ & & \\
\hline Berge 2017 & CBT vs Wait list / no treatment & & & $\begin{array}{l}\text { Specif- } \\
\text { ic }\end{array}$ & Child focused & $\begin{array}{l}\text { Indi- } \\
\text { vidual }\end{array}$ & $\begin{array}{l}\text { Not } \\
\text { ASD }\end{array}$ & $\begin{array}{l}\text { less } \\
\text { than } \\
10\end{array}$ & $\operatorname{mix}$ & clinic-dental & $\begin{array}{l}\text { Specif- } \\
\text { ic Pho- } \\
\text { bia }\end{array}$ & \\
\hline
\end{tabular}




\begin{tabular}{|c|c|c|c|c|c|c|c|c|c|c|}
\hline $\begin{array}{l}\text { Cartwright } \\
\text { Hatton } 2011\end{array}$ & CBT vs Wait list / no treatment & & & Parent only & Group & $\begin{array}{l}\text { Not } \\
\text { ASD }\end{array}$ & $20+$ & $\leq 12$ & $\begin{array}{l}\text { clinic-univer- } \\
\text { sity hospital }\end{array}$ & \\
\hline $\begin{array}{l}\text { Chalfant } \\
2007\end{array}$ & CBT vs Wait list / no treatment & & Broad & Child+parent & Group & ASD & $20+$ & $\operatorname{mix}$ & clinic & \\
\hline $\begin{array}{l}\text { Cheung } \\
2016\end{array}$ & CBT vs Wait list / no treatment & $\begin{array}{l}\text { CBT } \\
\text { vs Al- } \\
\text { ter- } \\
\text { na- } \\
\text { tive } \\
\text { treat- } \\
\text { ment }\end{array}$ & Broad & Child focused & $\begin{array}{l}\text { Indi- } \\
\text { vidual }\end{array}$ & $\begin{array}{l}\text { Not } \\
\text { ASD }\end{array}$ & $\begin{array}{l}10 \text { to } \\
20\end{array}$ & $\leq 12$ & clinic-CAMHS & \\
\hline Chiu 2013 & CBT vs Wait list / no treatment & & Broad & Child focused & $\begin{array}{l}\text { Indi- } \\
\text { vidual }\end{array}$ & $\begin{array}{l}\text { Not } \\
\text { ASD }\end{array}$ & $\begin{array}{l}10 \text { to } \\
20\end{array}$ & $\leq 12$ & school & \\
\hline $\begin{array}{l}\text { Cobham } \\
2017\end{array}$ & CBT vs Wait list / no treatment & & Broad & Parent only & Group & $\begin{array}{l}\text { Not } \\
\text { ASD }\end{array}$ & $\begin{array}{l}\text { less } \\
\text { than } \\
10\end{array}$ & $\operatorname{mix}$ & $\begin{array}{l}\text { clinic (univer- } \\
\text { sity) }\end{array}$ & \\
\hline $\begin{array}{l}\text { Cornacchio } \\
2019\end{array}$ & CBT vs Wait list / no treatment & & & Child+parent & Group & $\begin{array}{l}\text { Not } \\
\text { ASD }\end{array}$ & $20+$ & $\leq 12$ & clinic & $\begin{array}{l}\text { Selec- } \\
\text { tive } \\
\text { mutism }\end{array}$ \\
\hline $\begin{array}{l}\text { Creswell } \\
2017\end{array}$ & $\mathrm{CBT}$ vs Alertnative treatment & & Broad & Parent only & $\begin{array}{l}\text { Indi- } \\
\text { vidual }\end{array}$ & $\begin{array}{l}\text { Not } \\
\text { ASD }\end{array}$ & $\begin{array}{l}\text { less } \\
\text { than } \\
10\end{array}$ & $\leq 12$ & $\begin{array}{l}\text { clinic } \\
\text { (CAMHS) }\end{array}$ & \\
\hline Dadds 1997 & CBT vs Wait list / no treatment & & Broad & Child focused & Group & $\begin{array}{l}\text { Not } \\
\text { ASD }\end{array}$ & $\begin{array}{l}10 \text { to } \\
20\end{array}$ & $\operatorname{mix}$ & school & \\
\hline $\begin{array}{l}\text { Flannery } \\
\text { Schroeder } \\
2000\end{array}$ & CBT vs Wait list / no treatment & $\begin{array}{l}\text { CBT } \\
\text { vs } \\
\text { Wait } \\
\text { list / } \\
\text { no } \\
\text { treat- } \\
\text { ment }\end{array}$ & Broad & Child focused & $\begin{array}{l}\text { Indi- } \\
\text { vidual } \\
\text { and } \\
\text { group }\end{array}$ & $\begin{array}{l}\text { Not } \\
\text { ASD }\end{array}$ & $\begin{array}{l}10 \text { to } \\
20 \\
\text { and } \\
20+\end{array}$ & $\operatorname{mix}$ & $\begin{array}{l}\text { clinic (univer- } \\
\text { sity) }\end{array}$ & \\
\hline Fujii 2013 & CBT vs TAU & & & Child+ parent & $\begin{array}{l}\text { Indi- } \\
\text { vidual }\end{array}$ & ASD & $20+$ & $\leq 12$ & clinic & \\
\hline
\end{tabular}




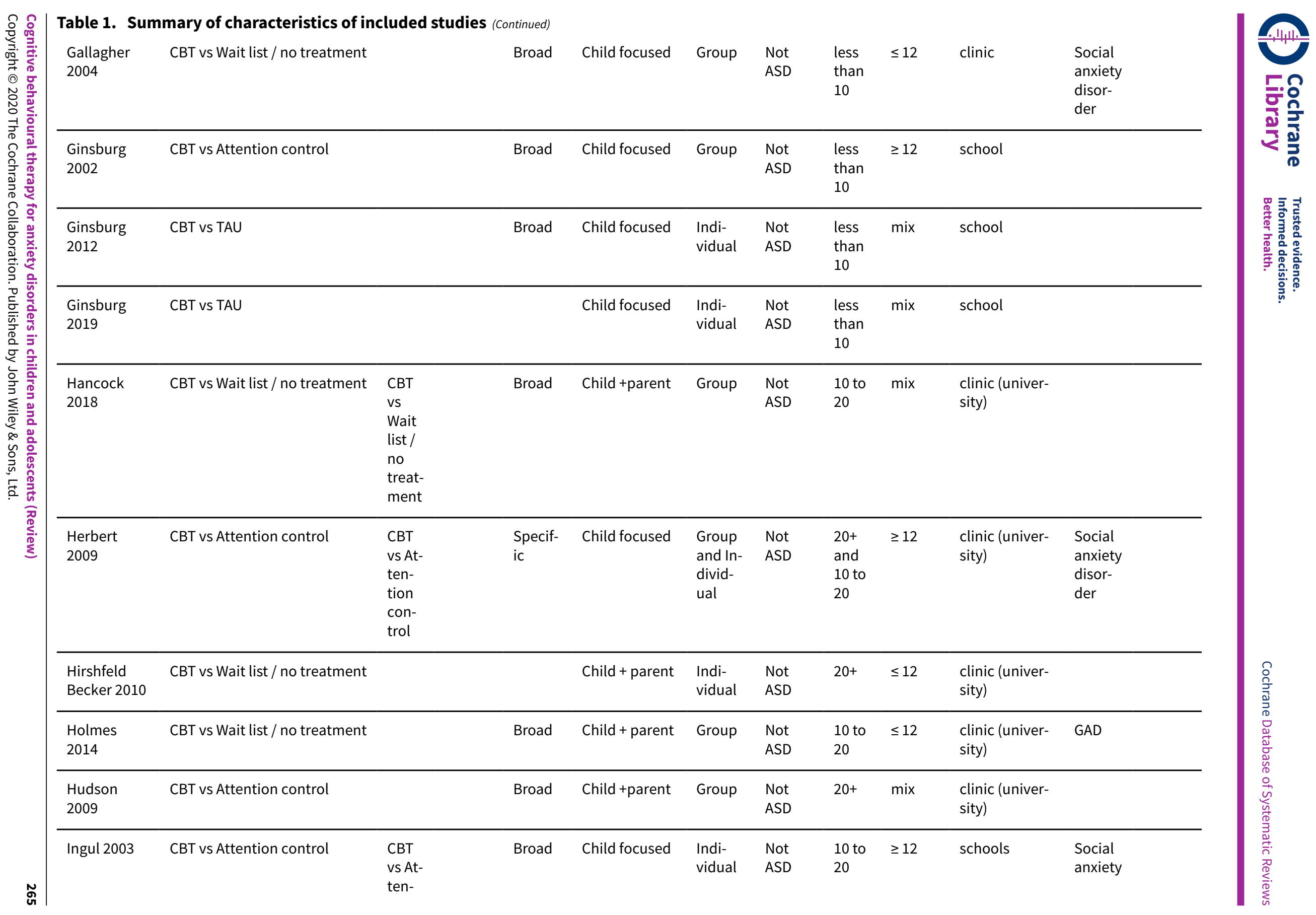




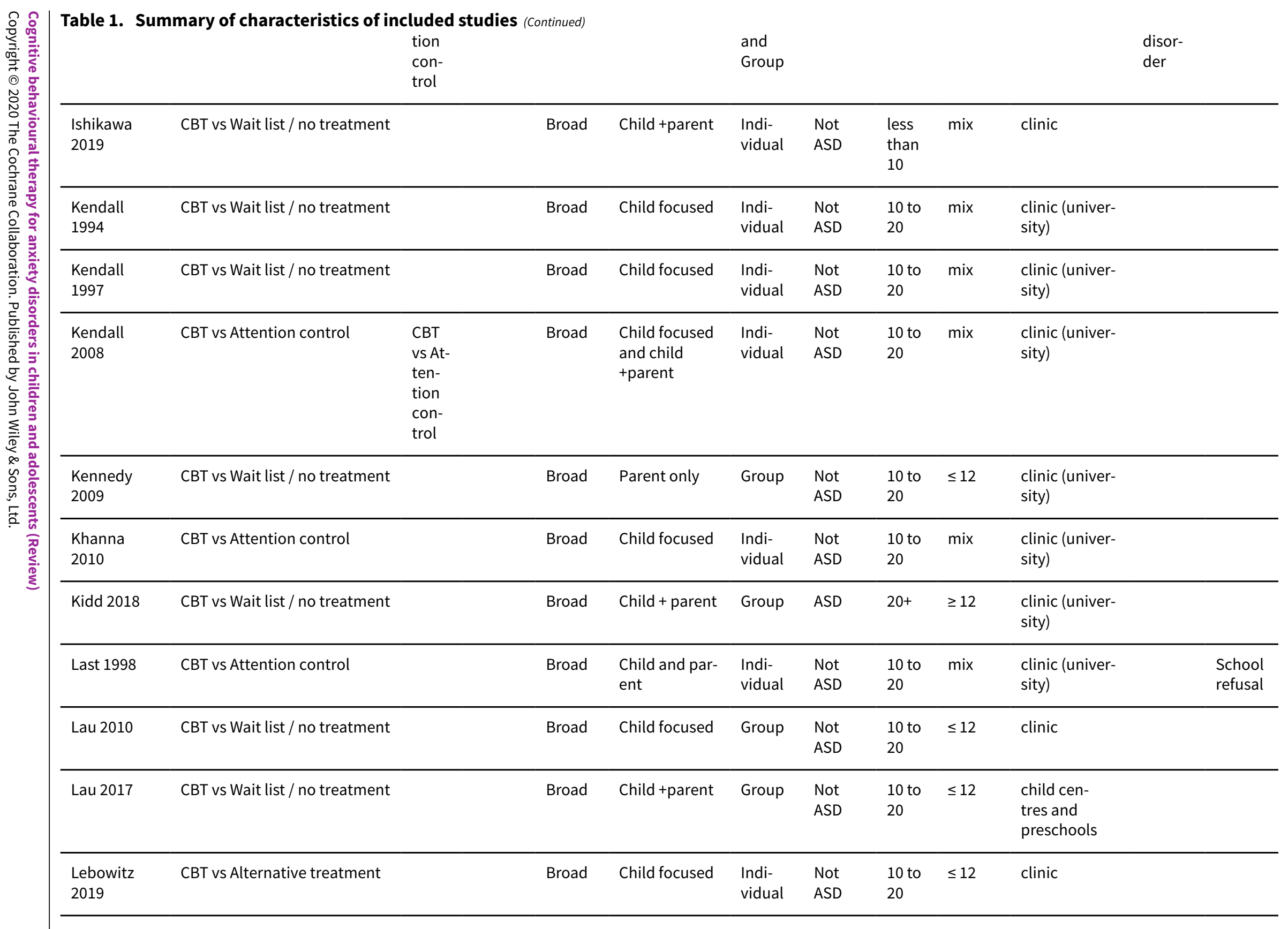




\begin{tabular}{|c|c|c|c|c|c|c|c|c|c|c|c|}
\hline $\begin{array}{l}\text { Leutgeb } \\
2012\end{array}$ & CBT vs Wait list / no treatment & & Broad & Child focused & $\begin{array}{l}\text { Indi- } \\
\text { vidual }\end{array}$ & $\begin{array}{l}\text { Not } \\
\text { ASD }\end{array}$ & $\begin{array}{l}\text { less } \\
\text { than } \\
10\end{array}$ & $\operatorname{mix}$ & university & $\begin{array}{l}\text { Specif- } \\
\text { ic Pho- } \\
\text { bia }\end{array}$ & \\
\hline $\begin{array}{l}\text { Masia Warn- } \\
\text { er } 2005\end{array}$ & CBT vs Wait list / no treatment & & $\begin{array}{l}\text { Specif- } \\
\text { ic }\end{array}$ & Child focused & $\begin{array}{l}\text { Group } \\
\text { fo- } \\
\text { cused }\end{array}$ & $\begin{array}{l}\text { Not } \\
\text { ASD }\end{array}$ & $\begin{array}{l}10 \text { to } \\
20\end{array}$ & $\geq 12$ & School & $\begin{array}{l}\text { Social } \\
\text { anxiety } \\
\text { disor- } \\
\text { der }\end{array}$ & \\
\hline $\begin{array}{l}\text { Masia Warn- } \\
\text { er } 2007\end{array}$ & CBT vs Attention control & & $\begin{array}{l}\text { Specif- } \\
\text { ic }\end{array}$ & Child focused & $\begin{array}{l}\text { Group } \\
\text { fo- } \\
\text { cused }\end{array}$ & $\begin{array}{l}\text { Not } \\
\text { ASD }\end{array}$ & $\begin{array}{l}10 \text { to } \\
20\end{array}$ & $\geq 12$ & School & $\begin{array}{l}\text { Social } \\
\text { anxiety } \\
\text { disor- } \\
\text { der }\end{array}$ & \\
\hline $\begin{array}{l}\text { Masia Warn- } \\
\text { er } 2011\end{array}$ & CBT vs Wait list / no treatment & & & Child focused & $\begin{array}{l}\text { Indi- } \\
\text { vidual }\end{array}$ & $\begin{array}{l}\text { Not } \\
\text { ASD }\end{array}$ & $\begin{array}{l}10 \text { to } \\
20\end{array}$ & $\operatorname{mix}$ & $\begin{array}{l}\text { primary care/ } \\
\text { special clinic }\end{array}$ & & $\begin{array}{l}\text { func- } \\
\text { tional } \\
\text { phys- } \\
\text { ical } \\
\text { com- } \\
\text { plaints }\end{array}$ \\
\hline $\begin{array}{l}\text { Masia Warn- } \\
\text { er } 2016\end{array}$ & CBT vs Attention control & $\begin{array}{l}\text { CBT } \\
\text { vs At- } \\
\text { ten- } \\
\text { tion } \\
\text { con- } \\
\text { trol }\end{array}$ & $\begin{array}{l}\text { Specif- } \\
\text { ic }\end{array}$ & Child focused & $\begin{array}{l}\text { Group } \\
\text { fo- } \\
\text { cused }\end{array}$ & $\begin{array}{l}\text { Not } \\
\text { ASD }\end{array}$ & $\begin{array}{l}10 \text { to } \\
20\end{array}$ & $\geq 12$ & School & $\begin{array}{l}\text { Social } \\
\text { anxiety } \\
\text { disor- } \\
\text { der }\end{array}$ & \\
\hline $\begin{array}{l}\text { McConachie } \\
2014\end{array}$ & CBT vs Wait list / no treatment & & Broad & Child + parent & Group & ASD & $20+$ & $\operatorname{mix}$ & $\begin{array}{l}\text { clinic (univer- } \\
\text { sity) }\end{array}$ & & \\
\hline $\begin{array}{l}\text { McNally } \\
\text { Keehn } 2013\end{array}$ & CBT vs Wait list / no treatment & & Broad & Child focused & $\begin{array}{l}\text { Indi- } \\
\text { vidual }\end{array}$ & ASD & $20+$ & $\operatorname{mix}$ & $\begin{array}{l}\text { clinic (univer- } \\
\text { sity) }\end{array}$ & & \\
\hline $\begin{array}{l}\text { Melfsen } \\
2011\end{array}$ & CBT vs Wait list / no treatment & & $\begin{array}{l}\text { Specif- } \\
\text { ic }\end{array}$ & $\begin{array}{l}\text { Child and par- } \\
\text { ent }\end{array}$ & $\begin{array}{l}\text { Indi- } \\
\text { vidual }\end{array}$ & $\begin{array}{l}\text { Not } \\
\text { ASD }\end{array}$ & $20+$ & $\operatorname{mix}$ & clinic & $\begin{array}{l}\text { Social } \\
\text { anxiety } \\
\text { disor- } \\
\text { der }\end{array}$ & \\
\hline Muris 2002 & CBT vs Attention control & & Broad & Child focused & Group & $\begin{array}{l}\text { Not } \\
\text { ASD }\end{array}$ & $\begin{array}{l}\text { less } \\
\text { than } \\
10\end{array}$ & $\leq 12$ & school & & \\
\hline
\end{tabular}




\begin{tabular}{|c|c|c|c|c|c|c|c|c|c|c|}
\hline $\begin{array}{l}\text { Murphy } \\
2017\end{array}$ & CBT vs Alternative treatment & & Broad & Child focused & $\begin{array}{l}\text { Indi- } \\
\text { vid- } \\
\text { ual fo- } \\
\text { cused }\end{array}$ & ASD & $\begin{array}{l}10 \text { to } \\
20\end{array}$ & $\geq 12$ & CAMHS & \\
\hline $\begin{array}{l}\text { O'Brien } \\
2007\end{array}$ & CBT vs TAU & & & $\begin{array}{l}\text { Child and par- } \\
\text { ent }\end{array}$ & Group & $\begin{array}{l}\text { Not } \\
\text { ASD }\end{array}$ & $\begin{array}{l}10 \text { to } \\
20\end{array}$ & $\operatorname{mix}$ & clinic & \\
\hline $\begin{array}{l}\text { Olivares } \\
2005\end{array}$ & CBT vs Wait list / no treatment & & $\begin{array}{l}\text { Specif- } \\
\text { ic }\end{array}$ & Child focused & Group & $\begin{array}{l}\text { Not } \\
\text { ASD }\end{array}$ & $\begin{array}{l}10 \text { to } \\
20\end{array}$ & $\geq 12$ & school & $\begin{array}{l}\text { Social } \\
\text { anxiety } \\
\text { disor- } \\
\text { der }\end{array}$ \\
\hline $\begin{array}{l}\text { Olivares } \\
2014\end{array}$ & CBT vs Wait list / no treatment & $\begin{array}{l}\text { CBT } \\
\text { vs } \\
\text { Wait } \\
\text { list / } \\
\text { no } \\
\text { treat- } \\
\text { ment }\end{array}$ & $\begin{array}{l}\text { Specif- } \\
\text { ic }\end{array}$ & Child focused & Group & $\begin{array}{l}\text { Not } \\
\text { ASD }\end{array}$ & $\begin{array}{l}10 \text { to } \\
20\end{array}$ & $\geq 12$ & school & $\begin{array}{l}\text { Social } \\
\text { anxiety } \\
\text { disor- } \\
\text { der }\end{array}$ \\
\hline $\begin{array}{l}\text { Olivares } \\
2019\end{array}$ & CBT vs Wait list / no treatment & $\begin{array}{l}\text { CBT } \\
\text { vs } \\
\text { Wait } \\
\text { list / } \\
\text { no } \\
\text { treat- } \\
\text { ment }\end{array}$ & & Child focused & Group & $\begin{array}{l}\text { Not } \\
\text { ASD }\end{array}$ & $\begin{array}{l}10 \text { to } \\
20\end{array}$ & $\geq 12$ & school & $\begin{array}{l}\text { Social } \\
\text { anxiety } \\
\text { disor- } \\
\text { der }\end{array}$ \\
\hline $\begin{array}{l}\text { Ollendick } \\
2009\end{array}$ & CBT vs Wait list / no treatment & $\begin{array}{l}\text { CBT } \\
\text { vs At- } \\
\text { ten- } \\
\text { tion } \\
\text { con- } \\
\text { trol }\end{array}$ & Broad & Child focused & $\begin{array}{l}\text { Indi- } \\
\text { vidual }\end{array}$ & $\begin{array}{l}\text { Not } \\
\text { ASD }\end{array}$ & $\begin{array}{l}\text { less } \\
\text { than } \\
10\end{array}$ & $\operatorname{mix}$ & clinic & $\begin{array}{l}\text { Specif- } \\
\text { ic Pho- } \\
\text { bia }\end{array}$ \\
\hline Ost 2001 & CBT vs Wait list / no treatment & $\begin{array}{l}\text { CBT } \\
\text { vs } \\
\text { Wait } \\
\text { list / } \\
\text { no } \\
\text { treat- } \\
\text { ment }\end{array}$ & Broad & $\begin{array}{l}\text { Child focused } \\
\text { and child } \\
\text { +parent }\end{array}$ & $\begin{array}{l}\text { Indi- } \\
\text { vidual }\end{array}$ & $\begin{array}{l}\text { Not } \\
\text { ASD }\end{array}$ & $\begin{array}{l}\text { less } \\
\text { than } \\
10\end{array}$ & $\operatorname{mix}$ & $\begin{array}{l}\text { referrals from } \\
\text { clinic and } \\
\text { schools }\end{array}$ & $\begin{array}{l}\text { Specif- } \\
\text { ic Pho- } \\
\text { bia }\end{array}$ \\
\hline
\end{tabular}




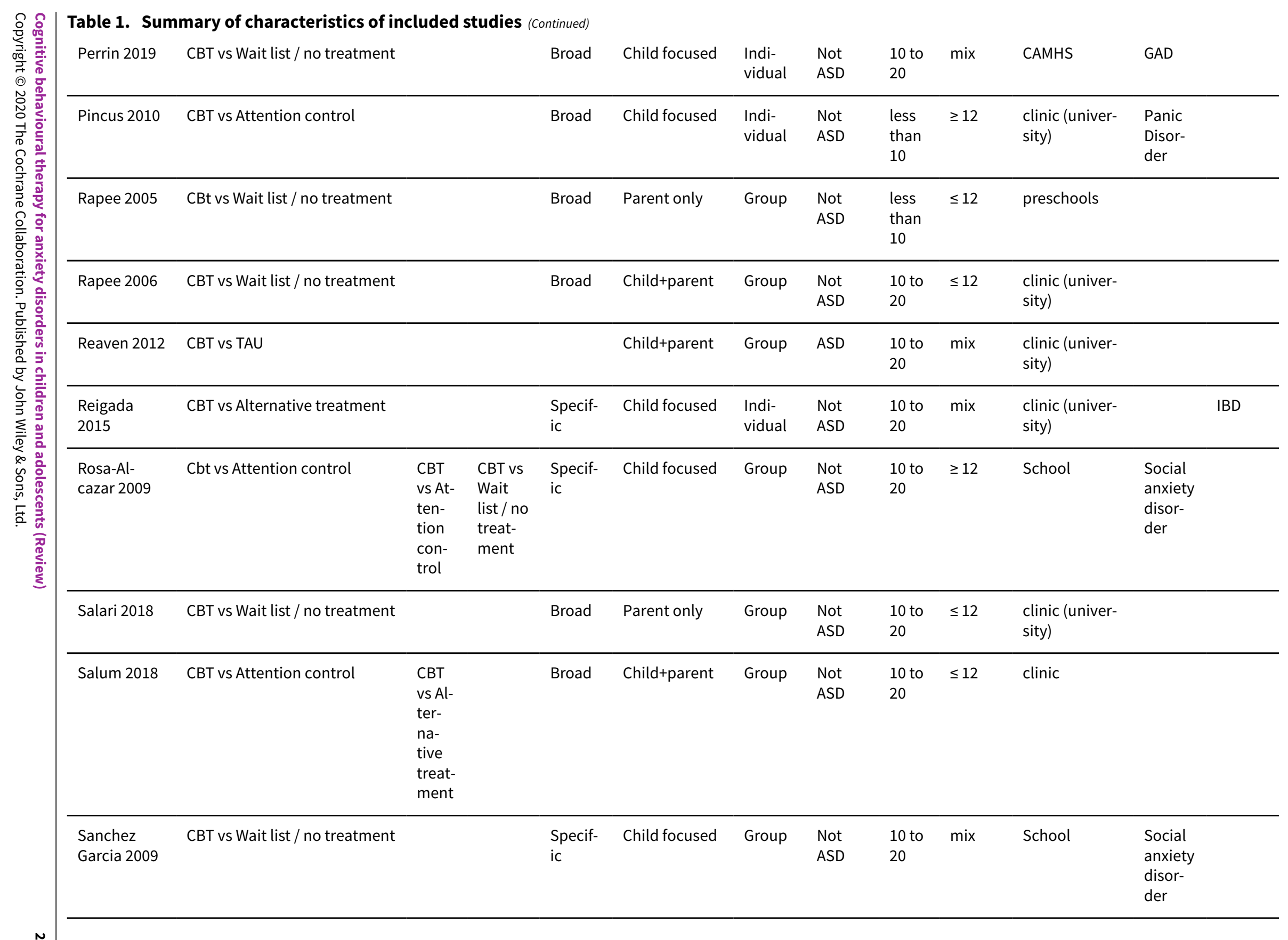




\begin{tabular}{|c|c|c|c|c|c|c|c|c|c|c|c|}
\hline $\begin{array}{l}\text { Santucci } \\
2013\end{array}$ & CBT vs Wait list / no treatment & & Broad & Child + parent & Group & $\begin{array}{l}\text { Not } \\
\text { ASD }\end{array}$ & $20+$ & $\leq 12$ & $\begin{array}{l}\text { clinic (univer- } \\
\text { sity) }\end{array}$ & $\begin{array}{l}\text { Sepa- } \\
\text { ration } \\
\text { anxiety } \\
\text { disor- } \\
\text { der }\end{array}$ & \\
\hline $\begin{array}{l}\text { Schneider } \\
2011\end{array}$ & CBT vs Wait list / no treatment & & Broad & Child + parent & $\begin{array}{l}\text { Indi- } \\
\text { vidual }\end{array}$ & $\begin{array}{l}\text { Not } \\
\text { ASD }\end{array}$ & $\begin{array}{l}10 \text { to } \\
20\end{array}$ & $\leq 12$ & $\begin{array}{l}\text { clinic (univer- } \\
\text { sity) }\end{array}$ & $\begin{array}{l}\text { Sepa- } \\
\text { ration } \\
\text { anxiety } \\
\text { disor- } \\
\text { der }\end{array}$ & \\
\hline $\begin{array}{l}\text { Sciberras } \\
2018\end{array}$ & CBT vs TAU & & Broad & Child+parent & $\begin{array}{l}\text { Indi- } \\
\text { vidual }\end{array}$ & $\begin{array}{l}\text { Not } \\
\text { ASD }\end{array}$ & $\begin{array}{l}10 \text { to } \\
20\end{array}$ & $\leq 12$ & clinic ADHD & & ADHD \\
\hline $\begin{array}{l}\text { Shahnavaz } \\
2016\end{array}$ & CBT vs TAU & & $\begin{array}{l}\text { Specif- } \\
\text { ic }\end{array}$ & Child+parent & $\begin{array}{l}\text { Indi- } \\
\text { vidual }\end{array}$ & $\begin{array}{l}\text { Not } \\
\text { ASD }\end{array}$ & $\begin{array}{l}10 \text { to } \\
20\end{array}$ & $\operatorname{mix}$ & dental clinics & $\begin{array}{l}\text { Specif- } \\
\text { ic Pho- } \\
\text { bia }\end{array}$ & \\
\hline $\begin{array}{l}\text { Sharma } \\
2017\end{array}$ & CBT vs TAU & & Broad & Child focused & Group & $\begin{array}{l}\text { Not } \\
\text { ASD }\end{array}$ & $20+$ & $\operatorname{mix}$ & $\begin{array}{l}\text { clinic (univer- } \\
\text { sity) }\end{array}$ & & Headaches \\
\hline Shortt 2001 & CBT vs Wait list / no treatment & & Broad & Child+parent & Group & $\begin{array}{l}\text { Not } \\
\text { ASD }\end{array}$ & $\begin{array}{l}10 \text { to } \\
20\end{array}$ & $\leq 12$ & $\begin{array}{l}\text { clinic (univer- } \\
\text { sity) }\end{array}$ & & \\
\hline Silk 2018 & CBT vs Alternative treatment & & Broad & Child focused & $\begin{array}{l}\text { Indi- } \\
\text { vidual }\end{array}$ & $\begin{array}{l}\text { Not } \\
\text { ASD }\end{array}$ & $\begin{array}{l}10 \text { to } \\
20\end{array}$ & $\operatorname{mix}$ & & & \\
\hline $\begin{array}{l}\text { Silverman } \\
\text { 1999a }\end{array}$ & CBT vs Attention control & & Broad & Child+parent & $\begin{array}{l}\text { Indi- } \\
\text { vidual }\end{array}$ & $\begin{array}{l}\text { Not } \\
\text { ASD }\end{array}$ & $\begin{array}{l}10 \text { to } \\
20\end{array}$ & $\operatorname{mix}$ & $\begin{array}{l}\text { clinic (univer- } \\
\text { sity) }\end{array}$ & & \\
\hline $\begin{array}{l}\text { Silverman } \\
\text { 1999b }\end{array}$ & CBT vs Wait list / no treatment & & Broad & Child + parent & Group & $\begin{array}{l}\text { Not } \\
\text { ASD }\end{array}$ & $\begin{array}{l}10 \text { to } \\
20\end{array}$ & $\operatorname{mix}$ & $\begin{array}{l}\text { clinic (univer- } \\
\text { sity) }\end{array}$ & & \\
\hline Simon 2011 & CBt vsWait list / no treatment & $\begin{array}{l}\text { CBT } \\
\text { vs } \\
\text { Wait } \\
\text { list / } \\
\text { no } \\
\text { treat- } \\
\text { ment }\end{array}$ & Broad & $\begin{array}{l}\text { Child focused } \\
\text { and parent } \\
\text { only }\end{array}$ & Group & $\begin{array}{l}\text { Not } \\
\text { ASD }\end{array}$ & $\begin{array}{l}\text { less } \\
\text { than } \\
10 \\
\text { and } \\
10 \text { to } \\
20\end{array}$ & $\operatorname{mix}$ & School & & \\
\hline
\end{tabular}




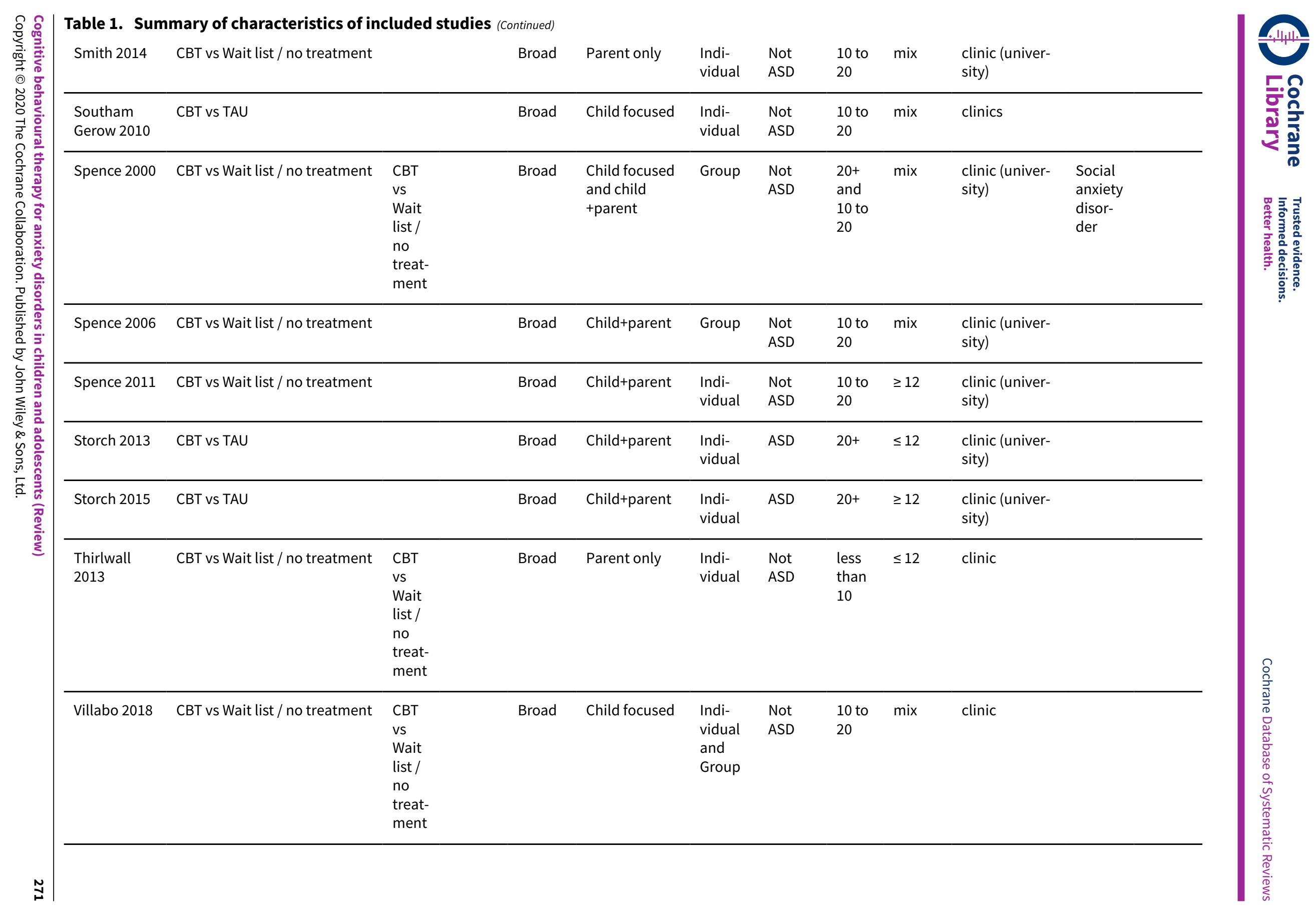




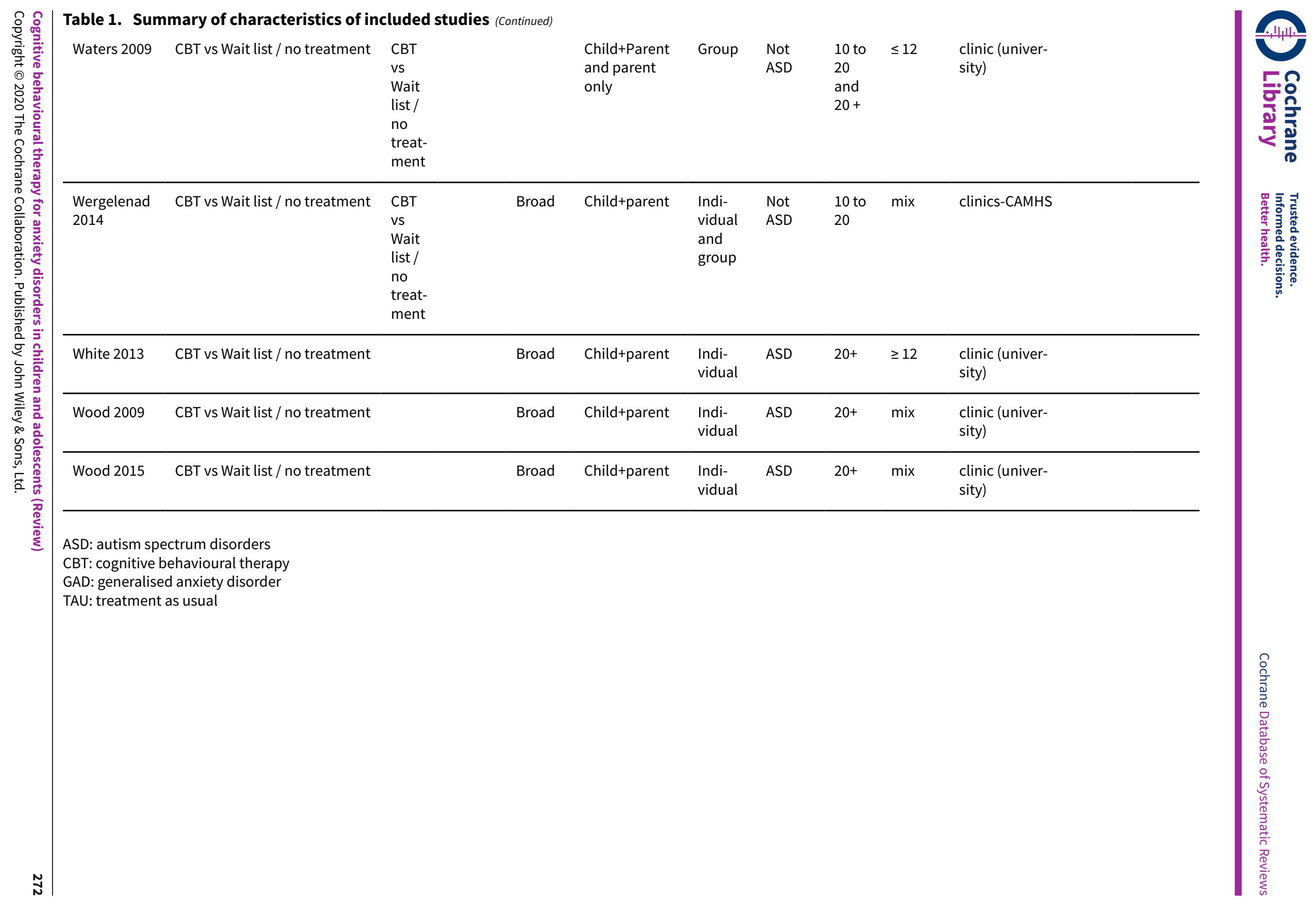




\section{A P PE N D I CES}

\section{Appendix 1. Hierarchy of anxiety symptoms measures}

\section{Broad-based anxiety measures}

1. Spence Children's Anxiety Scale (SCAS) (child-/parent-report versions) or derivatives of the SCAS, including Revised Children's Anxiety and Depression Scale (RCADS) (child- and parent-report versions) Anxiety Scale and the Preschool Anxiety Scale (parent-report)

2. Multidimensional Anxiety Scale for Children (MASC) (child- and parent-report versions)

3. Screen for Child Anxiety Related Disorders (SCARED) (child- and parent-report versions)

4. Revised Children's Manifest Anxiety Scale (RCMAS)

5. State-Trait Anxiety Inventory for Children (STAIC) - Trait Scale

6. Child and Adolescent Symptom Inventory-4 ASD Anxiety Scale

\section{Social anxiety (disorder-specific) measures}

7. Social Phobia and Anxiety Inventory for Children (SPAI-C)

8. Social Anxiety Scale for Adolescents (SAS-A)/Social Anxiety Scale for Children-Revised (SASC-R)

\section{Appendix 2. Cochrane Common Mental Disorders Controlled Trials Register (CCMDCTR) \\ Cochrane Common Mental Disorders Controlled Trials Register (CCMDCTR)}

Cochrane Common Mental Disorders (CCMD) maintains two archived clinical trials registers at its editorial base in York, UK: a references register and a studies-based register. The CCMDCTR-References Register contains over 40,000 reports of RCTs in depression, anxiety and neurosis. Approximately $50 \%$ of these references have been tagged to individual, coded trials. The coded trials are held in the CCMDCTRStudies Register and records are linked between the two registers through the use of unique Study ID tags. Coding of trials is based on the EU-Psi coding manual, using a controlled vocabulary; (please contact the CCMD Information Specialists for further details). Reports of trials for inclusion in the Group's registers are collated from routine (weekly), generic searches of MEDLINE (1950 to 2016), Embase (1974 to 2016) and PsycINFO (1967 to 2016); quarterly searches of the Cochrane Central Register of Controlled Trials (CENTRAL) and review-specific searches of additional databases. Reports of trials are also sourced from international trial registers via the World Health Organization's trials portal (the International Clinical Trials Registry Platform (ICTRP)), pharmaceutical companies, the handsearching of key journals, conference proceedings and other (non-Cochrane) systematic reviews and meta-analyses.

Details of CCMD's generic search strategies (used to identify RCTs) can be found on the Group's website, (cmd.cochrane.org/specialisedregister), with an example of the core MEDLINE search (used to inform the register) listed below. The Group's Specialised Register has fallen out of date with the Editorial Group's move from Bristol to York in the summer of 2016.

\section{Core search strategy used to inform the Cochrane Common Mental Disorders Group's Specialised Register: OVID MEDLINE (to June 2016)}

A weekly search alert based on condition + RCT filter only

1. [MeSH Headings]: eating disorders/ or anorexia nervosa/ or binge-eating disorder/ or bulimia nervosa/ or female athlete triad syndrome/ or pica/ or hyperphagia/ or bulimia/ or self-injurious behavior/ or self mutilation/ or suicide/ or suicidal ideation/ or suicide, attempted/ or mood disorders/ or affective disorders, psychotic/ or bipolar disorder/ or cyclothymic disorder/or depressive disorder/ or depression, postpartum/ or depressive disorder, major/ or depressive disorder, treatment-resistant/ or dysthymic disorder/ or seasonal affective disorder/ or neurotic disorders/ or depression/ or adjustment disorders/ or exp antidepressive agents/or anxiety disorders/ or agoraphobia/ or neurocirculatory asthenia/ or obsessive-compulsive disorder/ or obsessive hoarding/ or panic disorder/ or phobic disorders/ or stress disorders, traumatic/ or combat disorders/ or stress disorders, post-traumatic/ or stress disorders, traumatic, acute/ or anxiety/ or anxiety, castration/ or koro/ or anxiety, separation/ or panic/ or exp anti-anxiety agents/or somatoform disorders/ or body dysmorphic disorders/ or conversion disorder/ or hypochondriasis/ or neurasthenia/ or hysteria/ or munchausen syndrome by proxy/ or 
munchausen syndrome/ or fatigue syndrome, chronic/ or obsessive behavior/ or compulsive behavior/ or behavior, addictive/ or impulse control disorders/ or firesetting behavior/ or gambling/ or trichotillomania/ or stress, psychological/ or burnout, professional/ or sexual dysfunctions, psychological/ or vaginismus/ or Anhedonia/ or Affective Symptoms/ or *Mental Disorders/

2. [Title/ Author Keywords]: (eating disorder ${ }^{\star}$ or anorexia nervosa or bulimi* or binge eat* or (self adj (injur ${ }^{\star}$ or mutilat $\left.^{\star}\right)$ ) or suicide* or suicidal or parasuicid* or mood disorder ${ }^{\star}$ or affective disorder* or bipolar i or bipolar ii or (bipolar and (affective or disorder $\left.{ }^{\star}\right)$ ) or mania or manic or cyclothymic* or depression or depressive or dysthymi* or neurotic or neurosis or adjustment disorder ${ }^{\star}$ or antidepress ${ }^{\star}$ or anxiety disorder ${ }^{\star}$ or agoraphobia or obsess* or compulsi* or panic or phobi* or ptsd or posttrauma* or post trauma* or combat or somatoform or somati\#ation or medical* unexplained or body dysmorphi* or conversion disorder or hypochondria* or neurastheni* or hysteria or munchausen or chronic fatigue ${ }^{\star}$ or gambling or trichotillomania or vaginismus or anhedoni* or affective symptoms or mental disorder ${ }^{\star}$ or mental health).ti,kf.

3. [RCT filter]: (controlled clinical trial.pt. or randomized controlled trial.pt. or (randomi\#ed or randomi\#ation).ab,ti. or randomly.ab. or

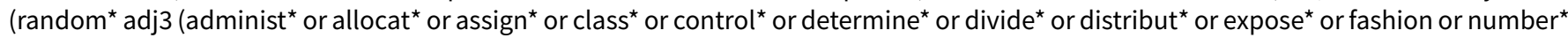
or place ${ }^{\star}$ or recruit ${ }^{\star}$ or subsitut ${ }^{\star}$ or treat $\left.{ }^{\star}\right)$ ).ab. or placebo ${ }^{\star}$.ab,ti. or drug therapy.fs. or trial.ab,ti. or groups.ab. or (control ${ }^{\star}$ adj3 (trial ${ }^{\star}$ or study or studies)).ab,ti. or ((singl* or doubl* or tripl* or trebl*) adj3 (blind* or mask* or dummy*)).mp. or clinical trial, phase ii/ or clinical trial, phase iii/ or clinical trial, phase iv/ or randomized controlled trial/ or pragmatic clinical trial/ or (quasi adj (experimental or random*)).ti,ab. or ((waitlist* or wait* list* or treatment as usual or TAU) adj3 (control or group)).ab.)

4. (1 and 2 and 3 )

Records are screened for reports of RCTs within the scope of the Cochrane Common Mental Disorders Group. Secondary reports of RCTs are tagged to the appropriate study record.

Similar weekly search alerts are also conducted on OVID Embase and PsycINFO, using relevant subject headings (controlled vocabularies) and search syntax, appropriate to each resource.

\section{Search strategy for this review}

The CCMDCTR was searched for this review using the following terms:

\section{CCMDCTR-Studies (all years up to June 2016)}

\#1 (anxiety or anxious or phobi* or agoraphobi* or panic or mutism or "school refusal") and STUDY:CRSTYPE and INSEGMENT

\#2 behavio* or cognitive or counsel* or train* or therap* or psychotherap* and STUDY:CRSTYPE and INSEGMENT

$\# 3$ (child* ${ }^{\star}$ or adolescent ${ }^{\star}$ ) and STUDY:CRSTYPE and INSEGMENT

\#4 (\#1 or \#2 or \#3)

\section{CCMDCTR-References (all years up to June 2016)}

[Outcomes]

\#1 (ADIS-C* or ADIS-P* or DISCAP or SCARED or SCAS or RCADS or RCMAS or MASC or CBCL or STAI-C or SPAI-C or SAS-A) AND INSEGMENT [Condition]

\#2 ("anxiety disorder" or agoraphobi* or "generalized anxiety" or "generalised anxiety" or GAD or (separation near2 anxiety) or (social near2 anxi*) or (social near2 phobi $^{\star}$ ) or phobi* or panic or mute* or mutism or (school near2 refus $\left.{ }^{\star}\right)$ AND INSEGMENT

\#3 ((infant ${ }^{\star}$ or child ${ }^{\star}$ or adolesc ${ }^{\star}$ or pediatric ${ }^{\star}$ or paediatric ${ }^{\star}$ or teen ${ }^{\star}$ or young ${ }^{\star}$ or youth or school ${ }^{\star}$ or preschool ${ }^{\star}$ ) near2 anxi ${ }^{\star}$ ) AND INSEGMENT

\#4 (anxiety. and (autism or autistic or "child development disorder*")) AND INSEGMENT

\#5 (anxiety or panic or phobi* or agoraphobi`):ti AND INSEGMENT

[Intervention]

\#6 ((ACCEPTANCE and COMMITMENT) or "ANXIETY MANAGEMENT" or "BEHAVIOUR MODIFICATION" or "BEHAVIOR MODIFICATION" or "BEHAVIOURAL MODIFICATION" or "BEHAVIORAL MODIFICATION" or BIBLIOTHERAPY or "COGNITIVE BEHAVIOUR THERAPY" or "COGNITIVE BEHAVIOR THERAPY" or "COGNITIVE BEHAVIOURAL THERAPY" or "COGNITIVE BEHAVIORAL THERAPY" or "COGNITIVE THERAPY" or "COGNITIVE TECHNIQUES" or "COGNITIVE RESTRUCTURING" or COUNSELING or (PSYCHOTHERAPY near2 GROUP) or "FAMILY THERAPY" or "GROUP THERAPY" or MINDFULNESS or "PLAY THERAPY" or PSYCHODRAMA or PSYCHOTHERAPY or "RATIONAL EMOTIVE THERAPY" or RELAXATION or "ROLE PLAYING" or "SENSITIVITY TRAINING" or BIBLIOTHERAPY or "SELF CONTROL"):emt,mh,kw,ky AND INSEGMENT

\#7 (CBT or CBGT* or bCBT or b-CBT or GPDCBT or GPD-CBT) AND INSEGMENT

\#8 ((cogniti ${ }^{\star}$ or behavio $\left.{ }^{\star}\right)$ near3 (counsel ${ }^{\star}$ or intervention or therap ${ }^{\star}$ or psychotherap ${ }^{\star}$ or training or treatment or technique ${ }^{\star}$ or restructur $^{\star}$ or defusion)):ti,ab AND INSEGMENT

\#9 ("rational emoti"” or (problem* near2 (focus* or sol*)) or psychoeducat* or "role play" or schema* or "self-control*" or "self control "):ti,ab AND INSEGMENT

\#10 (((psychotherap* or therap*) near3 (commitment or acceptance)) or ((self* or stress $\left.{ }^{\star}\right)$ near3 (control or analysis or direct* or esteem or help or instruct* or manage $\left.\left.{ }^{\star}\right)\right)$ ):ti,ab AND INSEGMENT

\#11 ((attribution ${ }^{\star}$ or reattribution $\left.{ }^{\star}\right)$ near3 (therap* ${ }^{\star}$ or psychotherap $\left.\left.{ }^{\star}\right)\right)$ :ti,ab AND INSEGMENT

\#12 (mindfulness* or "third wave" or experiential or (behavio* near3 (activation or modification)) or (thought* near3 suppress ${ }^{\star}$ ) or rumination):ti,ab AND INSEGMENT

\#13 ("anxiety manag" or "confidence building" or "coping skills" or "exposure therapy" or "exposure task"” or psychoeducat" or psychoeducat* or relaxation or "sensitivity training" or "self talk" or (social near2 (coach* or skill* or effectiveness))):ti,ab AND INSEGMENT \#14 ((controlling or overcoming) near2 (anxiety or panic or phobi* or agoraphobi* or shyness)):ti,ab AND INSEGMENT 
\#15 (child* near2 (cent* or deliver ${ }^{\star}$ or focus*) adj2 (counsel* or intervention* or program* or psychotherap* or therap* or train* or treat $\left.\left.^{\star}\right)\right)$ :ti,ab AND INSEGMENT

\#16 ((parent ${ }^{\star}$ or guardian $\left.{ }^{\star}\right)$ near2 (cent* or deliver $^{\star}$ or focus $^{\star}$ ) adj2 (counsel* or intervention* or program* or psychotherap* or therap ${ }^{\star}$ or train $^{\star}$ or treat $\left.\left.{ }^{\star}\right)\right)$ :ti,ab AND INSEGMENT

\#17 ((individually or group or conjoint or family) near2 (counsel* or intervention* or program* or psychotherap* or therap* or train* or treat $\left.\left.{ }^{\star}\right)\right):$ ti,ab AND INSEGMENT

[Age Group]

\#18 (infant ${ }^{\star}$ or child $^{\star}$ or adolesc ${ }^{\star}$ or paediatr ${ }^{\star}$ or pediatr ${ }^{\star}$ ):emt, mh,kw,ky,so AND INSEGMENT

\#19 (infant ${ }^{\star}$ or child* or boy* or girl* or kids or juvenil ${ }^{\star}$ or minors or paediatric ${ }^{\star}$ or pediatric ${ }^{\star}$ or adolesc ${ }^{\star}$ or preadolesc ${ }^{\star}$ or pre-adolesc ${ }^{\star}$ or pubert ${ }^{\star}$ or pubescen* or prepube* or pre-pube* or teen ${ }^{\star}$ or (young next (survivor ${ }^{\star}$ or offender ${ }^{\star}$ or minorit ${ }^{\star}$ )) or youth ${ }^{\star}$ or school $^{\star}$ or $^{*}$ preschool* $^{*}$ or nurser* or kindergarten):ti,emt,mh,kw,ky AND INSEGMENT

\#20 (\#2 OR \#3 OR \#4 OR \#5) [Condition]

\#21 (\#6 OR \#7 OR \#8 OR \#9 OR \#10 OR \#11 OR \#12 OR \#13 OR \#14 OR \#15 OR \#16 OR \#17) [Intervention]

\#22 (\#18 OR \#19) [Age Group]

\#23 (\#20 AND \#21 AND \#22)

\#24 ((anxi ${ }^{\star}$ or phobi* or agoraphobi* or panic) and (effectiveness or efficacy or evaluat* or intervention or program* or train* or treat* or prevent ${ }^{\star}$ or therapy or psychotherapy or trial or study) and (infant ${ }^{\star}$ or child ${ }^{\star}$ or adolesc or paediatric $^{\star}$ or pediatric ${ }^{\star}$ or teen $^{\star}$ or young ${ }^{\star}$ or youth or school* or preschool $\left.{ }^{\star}\right)$ ):ti AND INSEGMENT

\#25 (\#24 AND \#21)

\#26 (\#1 OR \#23 OR \#25)

\#27 ((OCD or obsessive compulsive or PTSD or posttraumatic stress disorder ${ }^{\star}$ or post-traumatic stress disorder $\left.{ }^{\star}\right)$ not (anxi ${ }^{\star}$ or phobi ${ }^{\star}$ or agoraphobi* or panic)):ti AND INSEGMENT

\#28 (\#26 not \#27)

\#29 ((pregnan* or "post natal" or postnatal ${ }^{\star}$ "post partum" or postpartum) not (adolesc* or teen* or young* or youth)):ti AND INSEGMENT \#30 (\#28 not \#29)

\#31 ("Coping Cat" or "Cool Kids" or "Coping Koala" or "Face your Fear" or "Face your Fears" or "Facing your Fear" or "Facing your Fears" or ("Multimodal Anxiety" and "Social Skills") or (Academic and Skills and "Social Success") or (Intervention near adolesc* near "Social Phobia") or BIACA or IAFS or MASSI or SASS or TAPS) AND INSEGMENT

\#32 (\#30 OR \#31)

\section{Appendix 3. Database search strategies}

In addition to searching the CCMDCTR we ran searches on CENTRAL, Ovid MEDLINE, Embase, and PsycINFO as listed below.

Dates of searches: 21 November 2018 and 10 October 2019.

Cochrane Central Register of Controlled Trials (CENTRAL; issue 10 of 12, 2019) in the Cochrane Library

\#1 ((infant or infants or child ${ }^{\star}$ or adolesc ${ }^{\star}$ or pediatric* or paediatric ${ }^{\star}$ or teen ${ }^{\star}$ or young ${ }^{\star}$ or youth or school* or preschool $\left.{ }^{\star}\right)$ and $\left(\right.$ anxi $^{\star}$ or phobi* or panic)):ti

\#2 ((infant or infants or child ${ }^{\star}$ or adolesc ${ }^{\star}$ or pediatric ${ }^{\star}$ or paediatric ${ }^{\star}$ or teen ${ }^{\star}$ or young ${ }^{\star}$ or youth or school ${ }^{\star}$ or preschool ${ }^{\star}$ ) near/2 $\left(\right.$ anxi $^{\star}$ or phobi* or panic)):ab

\#3 MeSH descriptor: [Anxiety Disorders] this term only

\#4 MeSH descriptor: [Anxiety] this term only and with qualifier(s): [diagnosis - DI, prevention \& control - PC, psychology - PX, therapy - TH]

\#5 MeSH descriptor: [Agoraphobia] this term only

\#6 MeSH descriptor: [Panic Disorder] this term only

\#7 MeSH descriptor: [Anxiety, Separation] this term only

\#8 MeSH descriptor: [Phobic Disorders] explode all trees

\#9 MeSH descriptor: [Phobia, Social] this term only

\#10 (agoraphobi* or "generalized anxiety" or "generalised anxiety" or GAD or "separation anxiety" or (social* NEAR/2 (anxi or fear*)) or phobi $^{\star}$ or mute or mutes or mutism or "school refusal"):ti,ab,kw

\#11 panic:ti,ab,kw

\#12 (anxiety near (autism or autistic)):ti,ab,kw

\#13 MeSH descriptor: [Child Development Disorders, Pervasive] explode all trees

\#14 anxiety:ti,ab,kw 
\#15 (\#13 and \#14)

\#16 (\#3 OR \#4 OR \#5 OR \#6 OR \#7 OR \#8 OR \#9 OR \#10 OR \#11 OR \#12 OR \#15)

\#17 MeSH descriptor: [Cognitive Therapy] explode all trees

\#18 MeSH descriptor: [Psychotherapy, Group] explode all trees

\#19 MeSH descriptor: [Bibliotherapy] this term only

\#20 MeSH descriptor: [Early Intervention (Education)] this term only

\#21 (CBT or $\mathrm{CBGT}^{\star}$ or bCBT or b-CBT or GPDCBT or GPD-CBT):ab

\#22 ((cogniti* or behavio*) NEAR/3 (counsel* or intervention or therap ${ }^{\star}$ or psychotherap ${ }^{\star}$ or training or treatment or technique* or restructur* or defusion)):Ti,AB

\#23 ("rational emotive" or (problem* NEAR/2 (focus* or sol*)) or psychoeducat* or psycho-educat* or "role play" or "role playing" or schema* or self-control* or "self control" or "self controlling"):ti,ab

\#24 (((psychotherap* or therap $\left.{ }^{\star}\right)$ near/3 (commitment or acceptance)) or ((self* or stress $\left.{ }^{\star}\right)$ near/3 (control or analysis or direct* or esteem or help or instruct ${ }^{\star}$ or manage $\left.\left.e^{\star}\right)\right):$ ti,ab

\#25 ((attribution* or reattribution ${ }^{\star}$ ) near/3 (therap* or psychotherap $\left.{ }^{\star}\right)$ ):ti,ab 20

\#26 (mindfulness* or "third wave" or experiential or (behavio* near/3 (activation or modification)) or (thought* near/3 suppress*) or rumination):ti,ab

\#27 ((anxiety near/2 manag*) or "confidence building" or "coping skills" or "exposure therapy" or "exposure task" or "exposure tasks" or psychoeducat* or psycho-educat* or relaxation or "sensitivity training" or "self talk" or (social near/2 (coach* or skill* or effectiveness))):ti,ab

\#28 ((controlling or overcoming) near/2 (anxiety or panic or phobi* or agoraphobi ${ }^{\star}$ or shyness)):ti,ab

\#29 (child* near/2 (deliver ${ }^{\star}$ or focus ${ }^{\star}$ ) near/2 (counsel ${ }^{\star}$ or intervention* or program* or psychotherap* or therap* or train* or treat*)):ti,ab

\#30 ((parent ${ }^{\star}$ or guardian ${ }^{\star}$ ) near/2 (deliver ${ }^{\star}$ or focus ${ }^{\star}$ ) near/2 (counsel ${ }^{\star}$ or intervention* or program* or psychotherap* or therap* or train ${ }^{\star}$ or treat $\left.\left.{ }^{\star}\right)\right): \mathrm{ti}, \mathrm{ab}$

\#31 ((individual* or group or conjoint or family) next (counsel* or intervention* or program* or psychotherap* or therap* or train ${ }^{\star}$ or treat $\left.\left.^{\star}\right)\right): t i, a b, k w$

\#32 ("Coping Cat" or "Cool Kids" or "Coping Koala" or "Facing your Fears" or (Skills near Academic near "Social Success") or (Intervention near Adolescents near "Social Phobia") or ("Multimodal Anxiety" near "Social Skills") or BIACA or IAFS or MASSI or SASS or TAPS):ti,ab

\#33 MeSH descriptor: [Psychotherapy] this term only and with qualifier(s): [methods - MT, standards - ST, statistics \& numerical data - SN] \#34 (\#17 or \#18 or \#19 or \#20 or \#21 or \#22 or \#23 or \#24 or \#25 or \#26 or \#27 or \#28 or \#29 or \#30 or \#31 or \#32 or \#33)

\#35 MeSH descriptor: [Adolescent] this term only 97662

\#36 MeSH descriptor: [Child] explode all trees 1394

\#37 MeSH descriptor: [Infant] this term only

\#38 (infant* or child $^{\star}$ or adolesc ${ }^{\star}$ or paediatr ${ }^{\star}$ or pediatr $^{\star}$ ):kw,so

\#39 (infant* or child* or boy* or girl* or kids or juvenil ${ }^{\star}$ or minors or paediatric ${ }^{\star}$ or pediatric ${ }^{\star}$ or adolesc ${ }^{\star}$ or preadolesc $^{\star}$ or pre-adolesc ${ }^{\star}$

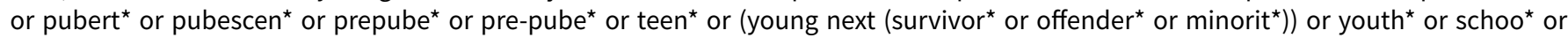

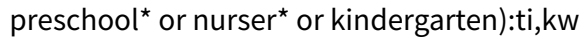

\#40 (\#36 or \#37 or \#38 or \#39 or \#40)

\#41 (\#17 and \#35)

\#41 (\#16 and \#34 and \#40)

\#42 (\#1 or \#2) and \#34

\#43 (\#41 or \#42) 
\#44 ((OCD or "obsessive compulsive" or PTSD or "posttraumatic stress" or "post-traumatic stress") not (anxi ${ }^{\star}$ or phobi or agoraphobi or panic)):ti

\#45 (\#43 not \#44)

\#46 (ADIS-C* or ADIS-P* or DISCAP or SCARED or SCAS or RCADS or RCMAS or MASC or CBCL or STAI-C or SPAI-C or SAS-A):ab

\#47 (\#46 and \#34)

\#48 (\#47 not \#43)

\#49 (\#48 not \#44)

Ovid PsycINFO (all years to October Week 40 2019)

1 clinical trials.sh.

2 (randomi\#ed or randomi\#ation or randomi\#ing).ti,ab,id.

3 (RCT or at random or (random ${ }^{\star}$ adj3 (administ* or allocat ${ }^{\star}$ or assign ${ }^{\star}$ or class ${ }^{\star}$ or control* $^{\star}$ or determine or divide $^{\star}$ or division or distribut ${ }^{\star}$ or expose $e^{\star}$ or fashion or number ${ }^{\star}$ or place ${ }^{\star}$ or recruit ${ }^{\star}$ or split or subsitut ${ }^{\star}$ or treat $\left.\left.{ }^{\star}\right)\right)$ ).ti,ab,id.

4 (control* and (trial or study or group) and (placebo or waitlist* or wait* list* or ((treatment or care) adj2 usual))).ti,ab,id,hw.

5 ((single or double or triple or treble) adj2 (blind* or mask* or dummy)).ti,ab,id.

6 trial.ti.

7 placebo.ti,ab,id,hw.

8 treatment outcome.md.

9 treatment effectiveness evaluation.sh.

10 mental health program evaluation.sh.

11 or/1-10

12 *anxiety/

13 anxiety disorders/ or generalized anxiety disorder/ or panic disorder/ or exp phobias/ or separation anxiety disorder/

14 social anxiety/

15 school refusal/ or school phobia/

16 exp separation anxiety/

17 panic/ or panic attack/ or panic disorder/

18 mutism/ or elective mutism/

19 (agoraphobi ${ }^{\star}$ or generali\#ed anxiety or GAD or separation anxiety or (social ${ }^{\star}$ adj2 (anxi ${ }^{\star}$ or fear ${ }^{\star}$ )) or phobi ${ }^{\star}$ or mute? or mutism or school refusal).ti,ab,id.

20 ((infant? or child ${ }^{\star}$ or adolesc ${ }^{\star}$ or p?ediatric ${ }^{\star}$ or teen* or young* or youth or school? or preschool*) adj2 anxi*).ti,ab,id.

21 over anxious.ti,ab,id.

22 anxiety.ab. $/$ freq $=3$

23 panic.ti,ab,id,hw.

24 (anxiety adj5 (autism or autistic)).ti,ab,id.

25 anxiety.ti,ab,id,tm. and (autism spectrum disorders/ or autistic thinking/ or exp developmental disabilities/)

26 or/12-25

27 ((anxi ${ }^{\star}$ or phobi ${ }^{\star}$ or panic) and (effectiveness or efficacy or evaluat* or intervention or program ${ }^{\star}$ or train $^{\star}$ or treat ${ }^{\star}$ or prevent ${ }^{\star}$ or therapy or psychotherapy or trial or study) and (infant? or child* or adolesc $c^{\star}$ or paediatric ${ }^{\star}$ or pediatric or teen $^{\star}$ or young* or youth or school? or preschool $\left.{ }^{\star}\right)$ ).ti.

28 anxiety management/

29 exp cognitive behavior therapy/

30 cognitive techniques/ or cognitive restructuring/ or cognitive therapy/

31 behavior modification/

32 exp relaxation therapy/

33 exp family therapy/

34 exp group psychotherapy/ or psychodrama/ or role playing/ or role playing games/

35 bibliotherapy/

36 counseling/ or group counseling/ or exp psychotherapeutic counseling/ or school counseling/

37 psychotherapy/

38 psychotherapeutic techniques/ or mindfulness/

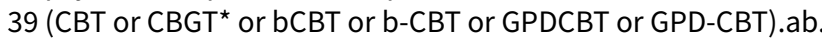

40 ((cogniti ${ }^{\star}$ or behavio*) adj3 (counsel* or intervention or therap* or psychotherap* or training or treatment or technique* or restructur ${ }^{\star}$ or defusion)).ti,ab,id.

41 (rational emoti* or (problem* adj2 (focus* ${ }^{\star}$ or sol $\left.\left.{ }^{\star}\right)\right)$ or psychoeducat* or role play ${ }^{\star}$ or schema* or self-control* or self control*).ti,ab,id,hw. 42 (((psychotherap ${ }^{\star}$ or therap*) adj3 (commitment or acceptance)) or ((self* or stress $\left.{ }^{\star}\right)$ adj3 (control or analysis or direct ${ }^{\star}$ or esteem or help or instruct* or manage*))).ti,ab,id.

43 ((attribution* or reattribution*) adj3 (therap* or psychotherap $\left.\left.{ }^{\star}\right)\right)$.ti,ab,id.

44 (mindfulness* $^{\star}$ or third wave or experiential or (behavio* adj3 (activation or modification)) or (thought* adj3 suppress ${ }^{\star}$ ) or rumination).ti,ab,id. 
45 ((anxiety adj1 manag*) or confidence building or coping skills or exposure therapy or exposure task* or psychoeducat* or psycho-

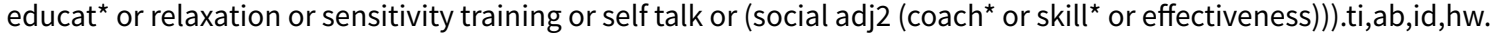

46 ((controlling or overcoming) adj2 (anxiety or panic or phobi* or agoraphobi* or shyness)).ti,ab,id.

47 (child $^{\star}$ adj2 (centred or centered or deliver ${ }^{\star}$ or focus ${ }^{\star}$ ) adj2 (counsel ${ }^{\star}$ or intervention* or program ${ }^{\star}$ or psychotherap* or therap $^{\star}$ or train $^{\star}$ or treat $\left.\left.{ }^{\star}\right)\right)$.ti,ab,id.

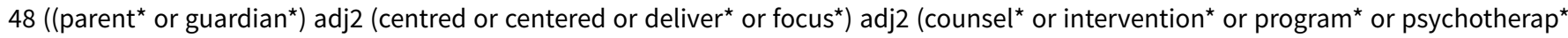
or therap* or train* or treat*)).ti,ab,id.

49 ((individually or group or conjoint or family) adj2 (counsel* or intervention* or program* or psychotherap* or therap* or train* or treat $\left.\left.{ }^{\star}\right)\right) . t i, a b, i d$.

50 (Coping Cat or Cool Kids or Coping Koala or Fac* your Fear? or (Multimodal Anxiety adj Social Skills) or (Skills adj Academic adj Social Success) or (Intervention adj Adolescents adj Social Phobia) or BIACA or IAFS or MASSI or SASS or TAPS).ti,ab,id.

51 or $/ 28-50$

52 pediatrics/

53 child psychiatry/ or child psychopathology/ or child psychology/

54 adolescent psychiatry/ or adolescent psychopathology/or adolescent psychology/

55 (infant? or child* or adolesc* or paediatr $^{\star}$ or pediatr*).hw,jx.

56 (infant $^{\star}$ or child* or boy* or girl ${ }^{\star}$ or kids or juvenil ${ }^{\star}$ or minors or paediatric ${ }^{\star}$ or pediatric ${ }^{\star}$ or adolesc ${ }^{\star}$ or preadolesc ${ }^{\star}$ or pre-adolesc ${ }^{\star}$ or pubert ${ }^{\star}$ or pubescen ${ }^{\star}$ or prepube ${ }^{\star}$ or pre-pube or teen $^{\star}$ or (young adj (survivor ${ }^{\star}$ or offender ${ }^{\star}$ or minorit ) $^{\star}$ or youth* or school? or preschool* or nurser* or kindergarten).ti,id.

57 or $/ 52-56$

58 (ADIS-C* or ADIS-P* or DISCAP or SCARED or SCAS or RCADS or RCMAS or MASC or CBCL or STAI-C or SPAI-C or SAS-A).ab,id,tm.

5911 and 26 and 51 and 57

6011 and 27 and 51

6111 and 51 and 58

6211 and 50

6359 or 60 or 61 or 62

64 ((OCD or obsessive compulsive or PTSD or posttraumatic stress disorder ${ }^{\star}$ or post-traumatic stress disorder $\left.{ }^{\star}\right)$ not $\left(\right.$ anxi $^{\star}$ or phobi ${ }^{\star}$ or agoraphobi* or panic)).ti.

6563 not 64

Ovid MEDLINE Databases (2016 to 10 Oct 2019)

Ovid MEDLINE(R) and Epub Ahead of Print, In-Process \& Other Non-Indexed Citations and Daily

1 controlled clinical trial.pt.

2 randomized controlled trial.pt.

3 (randomi\#ted or randomi\#ation or randomi\#ing).ti,ab,kf.

4 (RCT or "at random" or (random* adj3 (administ* or allocat* or assign* or class* or cluster or control* or determine* or divide* or division

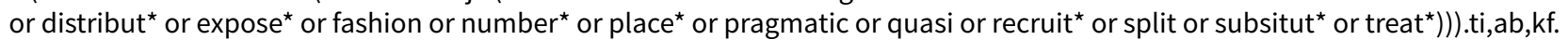

5 (placebo or ((attention or active) adj control $\left.\left.{ }^{\star}\right)\right)$. ti, ab,kf.

6 trial.ab,ti,kf.

7 ((control* or group $^{\star}$ or compar $\left.{ }^{\star}\right) \operatorname{adj5}\left(\left(\left(\right.\right.\right.$ care or treatment $\left.{ }^{\star}\right)$ adj2 (usual or standard or routine)) or TAU or CAU)).ab.

$8\left(\left(\right.\right.$ control $^{\star}$ or group $^{\star}$ or compar $\left.^{\star}\right)$ adj5 (waitlist* or wait $^{\star}$ list $^{\star}$ or waiting or WLC)).ab.

9 or/1-8

10 ANXIETY DISORDERS/

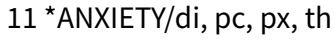

12 AGORAPHOBIA/ Or PANIC DISORDER/ Or ANXIETY, SEPARATION/

13 PHOBIC DISORDERS/ or PHOBIA, SOCIAL/

14 (agoraphobi* or generali\#ed anxiety or GAD or separation anxiety or (social ${ }^{\star}$ adj2 (anxi ${ }^{\star}$ or fear ${ }^{\star}$ )) or phobi ${ }^{\star}$ or mute? or mutism or school refusal).ti,ab,kf.

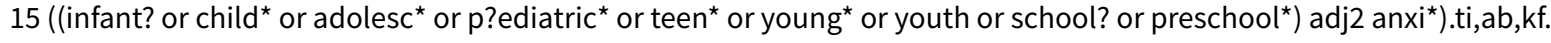

16 anxiety.ab. /freq=3

17 panic.mp.

18 (anxiety adj5 (autism or autistic)).ti,ab,kf.

19 anxiety.mp. and (child development disorders, pervasive/px or autism spectrum disorder/px or autistic disorder/px)

20 or/10-19

21 ((anxi ${ }^{\star}$ or phobi ${ }^{\star}$ or panic) and (effectiveness or efficacy or evaluat* or intervention or program ${ }^{\star}$ or train $^{\star}$ or treat ${ }^{\star}$ or prevent $^{\star}$ or therapy $^{*}$

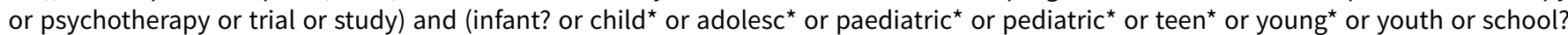
or preschool*)).ti.

22 exp COGNITIVE THERAPY/

23 PSYCHOTHERAPY, GROUP/

24 FAMILY THERAPY/ or PSYCHODRAMA/ or ROLE PLAYING/ or SENSITIVITY TRAINING GROUPS/ 25 BIBLIOTHERAPY/ 
26 "EARLY INTERVENTION (Education)"/

27 (CBT or CBGT* or bCBT or b-CBT or GPDCBT or GPD-CBT).ab.

28 ((cogniti* or behavio*) adj3 (counsel ${ }^{\star}$ or intervention or therap* or psychotherap* or training or treatment or technique* or restructur ${ }^{\star}$ or defusion)).ti,ab,kf.

29 (rational emoti ${ }^{\star}$ or (problem ${ }^{\star}$ adj2 $\left(\right.$ focus $^{\star}$ or sol $\left.\left.{ }^{\star}\right)\right)$ or psychoeducat ${ }^{\star}$ or role play ${ }^{\star}$ or schema ${ }^{\star}$ or self-control ${ }^{\star}$ or self control*).ti,ab,kf. 30 (((psychotherap* or therap $\left.^{\star}\right)$ adj3 (commitment or acceptance)) or ((self* or stress $\left.{ }^{\star}\right)$ adj3 (control or analysis or direct ${ }^{\star}$ or esteem or help or instruct* or manage*))).ti,ab,kf.

31 ((attribution* or reattribution $\left.{ }^{\star}\right)$ adj3 (therap* or psychotherap $\left.\left.{ }^{\star}\right)\right) . t i, a b, k f$.

32 (mindfulness* or third wave or experiential or (behavio* adj3 (activation or modification)) or (thought* adj3 suppress*) or rumination).ti,ab,kf.

33 ((anxiety adj1 manag*) or confidence building or coping skills or exposure therapy or exposure task* or psychoeducat* or psychoeducat ${ }^{\star}$ or relaxation or sensitivity training or self talk or (social adj2 (coach or skill* or effectiveness))).ti,ab,kf,hw.

34 ((controlling or overcoming) adj2 (anxiety or panic or phobi* or agoraphobi* or shyness)).ti,ab,kf.

35 (child* adj2 (deliver ${ }^{\star}$ or focus ${ }^{\star}$ ) adj2 (counsel ${ }^{\star}$ or intervention* or program* or psychotherap* or therap $^{\star}$ or train $^{\star}$ or treat $\left.{ }^{\star}\right)$ ).ti,ab,kf.

36 ((parent ${ }^{\star}$ or guardian $\left.{ }^{\star}\right)$ adj2 (deliver ${ }^{\star}$ or focus ${ }^{\star}$ ) adj2 (counsel ${ }^{\star}$ or intervention* or program ${ }^{\star}$ or psychotherap ${ }^{\star}$ or therap $^{\star}$ or train $^{\star}$ or $^{*}$ treat $\left.\left.^{\star}\right)\right) . \mathrm{ti}, \mathrm{ab}, \mathrm{kf}$.

37 ((individually or group or conjoint or family) adj2 (counsel* or intervention* or program* or psychotherap* or therap* or train ${ }^{\star}$ or treat $\left.\left.^{\star}\right)\right) . \mathrm{ti}, \mathrm{ab}, \mathrm{kf}$

38 (Coping Cat or Cool Kids or Coping Koala or Fac* your Fear? or (Multimodal Anxiety adj Social Skills) or (Skills adj Academic adj Social Success) or (Intervention adj Adolescents adj Social Phobia) or BIACA or IAFS or MASSI or SASS or TAPS).ti,ab,kf.

39 *PSYCHOTHERAPY/mt, st, sn, tu

40 or/22-39

41 ADOLESCENT/ or CHILD/ or CHILD, PRESCHOOL/

42 (infant? or child ${ }^{\star}$ or adolesc ${ }^{\star}$ or paediatr* or pediatr $^{\star}$ ).hw,jn.

43 infant $^{\star}$ or child ${ }^{\star}$ or boy ${ }^{\star}$ or girl ${ }^{\star}$ or kids or juvenil ${ }^{\star}$ or minors or paediatric ${ }^{\star}$ or pediatric ${ }^{\star}$ or adolesc ${ }^{\star}$ or preadolesc or $^{\star}$ pre-adolesc $^{\star}$

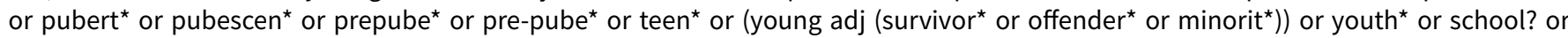
preschool* or nurser ${ }^{\star}$ or kindergarten).ti,kf.

44 (infant? or child* or adolesc $^{\star}$ or paediatr ${ }^{\star}$ or pediatr $^{\star}$ ).ab. /freq=3

45 or/ $41-44$

469 and 20 and 40 and 45

479 and 21 and 40

4846 or 47

49 (ADIS-C* or ADIS-P* or DISCAP or SCARED or SCAS or RCADS or RCMAS or MASC or CBCL or STAI-C or SPAI-C or SAS-A).ab.

509 and 40 and 49

5148 or 50

52 ((OCD or obsessive compulsive or PTSD or posttraumatic stress disorder ${ }^{\star}$ or post-traumatic stress disorder $\left.{ }^{\star}\right)$ not $\left(a n x i^{\star}\right.$ or phobi ${ }^{\star}$ or agoraphobi* or panic)).ti.

$53\left(2016^{\star}\right.$ or $2017^{\star}$ or $\left.2018^{\star}\right) \cdot y r, d p, d t, e p, e z$.

5451 not 52

5553 and 54

Ovid Embase (2016 to 2019 Week 40)

1 randomized controlled trial/

2 randomization.de.

3 controlled clinical trial/ and (Disease Management or Drug Therapy or Prevention or Rehabilitation or Therapy).fs.

4 *clinical trial/

5 placebo.de.

6 placebo.ti,ab.

7 trial.ti.

8 (randomi\#ed or randomi\#ation or randomi\#ing).ti,ab,kw.

9 (RCT or "at random" or (random* adj3 (administ* or allocat* or assign* or class* or control* or determine* or divide* or division or distribut $^{\star}$ or expose* or fashion or number ${ }^{\star}$ or place* or recruit ${ }^{\star}$ or split or subsitut ${ }^{\star}$ or treat $\left.\left.\left.{ }^{\star}\right)\right)\right)$.ti,ab,kw.

10 ((singl\$ or doubl\$ or trebl\$ or tripl\$) adj3 (blind\$ or mask\$ or dummy)).mp.

11 (control ${ }^{\star}$ and (trial or study or group) and (placebo or waitlist* or wait* list $^{\star}$ or ((treatment or care) adj2 usual))).ti,ab,kw,hw.

12 or $/ 1-11$

13 ((animal or nonhuman) not (human and (animal or nonhuman))).de.

1412 not 13

15 anxiety disorder/ or anxiety neurosis/ or generalized anxiety disorder/ or "mixed anxiety and depression"/ or panic/ or exp phobia/ or separation anxiety/

$16^{*}$ anxiety/

17 (agoraphobi* or generali\#ed anxiety or GAD or separation anxiety or (social ${ }^{\star}$ adj2 (anxi ${ }^{\star}$ or fear $\left.{ }^{\star}\right)$ ) or phobi ${ }^{\star}$ or mute? or mutism or school refusal).ti,ab,kw. 
18 ((infant? or child ${ }^{\star}$ or adolesc ${ }^{\star}$ or p? ediatric ${ }^{\star}$ or teen* or young $^{\star}$ or youth or school? or preschool ${ }^{\star}$ ) adj2 anxi $\left.{ }^{\star}\right)$. ti,ab,kw.

19 anxiety.ab. $/$ freq $=3$

20 panic.mp.

21 (anxiety adj5 (autism or autistic)).ti,ab,kw.

22 anxiety.mp. and (autism/ or asperger syndrome/ or "pervasive developmental disorder not otherwise specified"/)

23 school refusal/

24 mutism/ or selective mutism/

25 or/ $15-24$

26 cognitive behavioral therapy/ or cognitive behavioral stress management/

27 exp cognitive therapy/

28 psychotherapy/ or bibliotherapy/ or family therapy/ or group therapy/or mindfulness/ or play therapy/ or psychodrama/ or relaxation training/ or role playing/

29 (CBT or $\mathrm{CBGT}^{\star}$ or bCBT or b-CBT or GPDCBT or GPD-CBT).ab.

30 ((cogniti ${ }^{\star}$ or behavio*) adj3 (counsel* or intervention or therap* or psychotherap* or training or treatment or technique* or restructur ${ }^{\star}$ or defusion)).ti,ab,kw.

31 (rational emoti* or (problem ${ }^{\star}$ adj2 (focus ${ }^{\star}$ or sol $\left.{ }^{\star}\right)$ ) or psychoeducat ${ }^{\star}$ or role play* or schema* or self-control* or self control*).ti,ab,kw. 32 *self control/

33 (((psychotherap* or therap $\left.^{\star}\right)$ adj3 (commitment or acceptance)) or ((self* or stress $\left.{ }^{\star}\right)$ adj3 (control or analysis or direct ${ }^{\star}$ or esteem or help

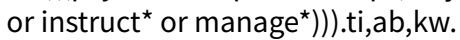

34 ((attribution* or reattribution $\left.{ }^{\star}\right)$ adj3 (therap* or psychotherap $\left.{ }^{\star}\right)$ ).ti,ab,kw.

35 (mindfulness* or third wave or experiential or (behavio* adj3 (activation or modification)) or (thought* adj3 suppress ${ }^{\star}$ ) or rumination).ti,ab,kw.

36 ((anxiety adj1 manag*) or confidence building or coping skills or exposure therapy or exposure task* or psychoeducat* or psycho-

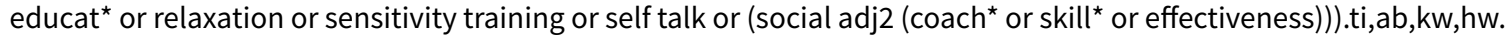

37 ((controlling or overcoming) adj2 (anxiety or panic or phobi* or agoraphobi* or shyness)).ti,ab,kw.

38 (child ${ }^{\star}$ adj2 (centred or centered or deliver ${ }^{\star}$ or focus ${ }^{\star}$ ) adj2 (counsel ${ }^{\star}$ or intervention* or program* or psychotherap* or therap* or train $^{\star}$ or treat*) $)$.ti,ab,kw.

39 ((parent* or guardian*) adj2 (centred or centered or deliver* or focus ${ }^{\star}$ ) adj2 (counsel ${ }^{\star}$ or intervention* or program $^{\star}$ or psychotherap $^{\star}$ or therap* or train* or treat*)).ti,ab,kw.

40 ((individually or group or conjoint or family) adj2 (counsel* or intervention* or program* or psychotherap* or therap* or train* or treat $\left.)^{\star}\right)$.ti,ab,kw.

41 (Coping Cat or Cool Kids or Coping Koala or Fac* your Fear? or (Multimodal Anxiety adj Social Skills) or (Skills adj Academic adj Social Success) or (Intervention adj Adolescents adj Social Phobia) or BIACA or IAFS or MASSI or SASS or TAPS).ti,ab,kw.

42 or $/ 26-41$

43 juvenile/ or exp adolescent/ or exp child/

44 (infant? or child ${ }^{\star}$ or adolesc ${ }^{\star}$ or paediatr ${ }^{\star}$ or pediatr $\left.{ }^{\star}\right) \cdot$.hw,jx.

45 (infant $^{\star}$ or child* or boy ${ }^{\star}$ or girl ${ }^{\star}$ or kids or juvenil ${ }^{\star}$ or minors or paediatric ${ }^{\star}$ or pediatric ${ }^{\star}$ or adolesc or preadolesc $^{\star}$ or pre-adolesc ${ }^{\star}$

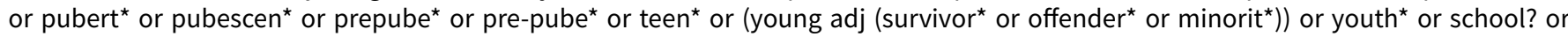
preschool* $^{*}$ or nurser* or kindergarten).ti,kw.

46 or $/ 43-45$

4714 and 25 and 42 and 46

48 ((anxi ${ }^{\star}$ or phobi ${ }^{\star}$ or panic) and (effectiveness or efficacy or evaluat ${ }^{\star}$ or intervention or program ${ }^{\star}$ or train* or treat ${ }^{\star}$ or prevent ${ }^{\star}$ or therapy $^{*}$ or psychotherapy or trial or study) and (infant? or child* or adolesc ${ }^{\star}$ or paediatric ${ }^{\star}$ or pediatric or teen $^{\star}$ or young* or youth or school? or preschool*)).ti.

4914 and 42 and 48

50 (ADIS-C* or ADIS-P* or DISCAP or SCARED or SCAS or RCADS or RCMAS or MASC or CBCL or STAI-C or SPAI-C or SAS-A).ab.

5114 and 42 and 50

5247 or 49 or 51

53 ((OCD or obsessive compulsive or PTSD or posttraumatic stress disorder ${ }^{\star}$ or post-traumatic stress disorder $\left.{ }^{\star}\right)$ not (anxi ${ }^{\star}$ or phobi $^{\star}$ or agoraphobi or panic)).ti.

5452 not 53

$55\left(2016^{\star}\right.$ or $2017^{\star}$ or $\left.2018^{\star}\right) \cdot y r, d p, d c$.

5654 and 55

\section{Appendix 4. Grey literature search strategies}

We ran the searches listed below on Proquest Dissertations \& Theses Global, PQDT Open, Open Access Theses and Dissertations, DART and NDLTD.

Dates of searches: All years to 14 Oct 2019

Proquest Dissertations \& Theses Global search.proquest.com/pqdtglobal/dissertations/ 
ti(adolescent OR adolescents OR teenager OR teenagers OR teen OR teens OR child OR children OR childhood OR kids OR kiddies OR youth OR youths OR young OR pediatric OR paediatric OR school OR schools) AND ti(anxiety OR anxious OR fear OR panic OR phobia OR phobias OR phobic) AND ti(effect OR effects OR effective OR effectiveness OR evaluation OR evaluations OR evaluating OR evaluated OR findings OR intervention OR interventions OR outcome OR outcomes OR prevent OR preventing OR prevention OR program OR programs OR programme OR programmes OR psychotherapy OR psychotherapies OR psychotherapeutic OR results OR study OR studies OR therapy OR therapies OR therapeutic OR treat OR treated OR treating OR treatment OR treatments OR trial OR trials) AND noft(CBT OR cognitive OR behaviour OR behavioural OR behavior OR behavioral) AND noft(RCT OR randomized OR randomised OR randomly OR assignment OR allocation)

PQDT Open - open access dissertations and theses pqdtopen.proquest.com/search.html

ti(anxiety OR anxious OR fear OR panic OR phobia OR phobias OR phobic) AND (RCT OR randomized OR randomised OR randomly)

Open Access Theses and Dissertations oatd.org

title:(anxiety OR anxious OR fear OR panic OR phobia OR phobias OR phobic) AND title:(adolescent OR adolescents OR teenager OR teenagers OR teen OR teens OR child OR children OR childhood OR kids OR kiddies OR youth OR youths OR young OR pediatric OR paediatric OR school OR schools) AND (RCT OR randomized OR randomised OR randomly)

DART - Europe e-theses Portal www.dart-europe.eu/basic-search.php

(anxiety OR anxious OR fear OR panic OR phobia OR phobias OR phobic) AND (RCT OR randomized OR randomised OR randomly) AND (CBT OR cognitive OR behaviour OR behavioural OR behavior OR behavioral) AND (adolescent OR adolescents OR teenager OR teenagers OR teen OR teens OR child OR children OR childhood OR kids OR kiddies OR youth OR youths OR young OR pediatric OR paediatric OR school OR schools)

\section{Networked Digital Library of Theses and Dissertations (NDLTD) search.ndltd.org/}

title:(anxiety OR anxious OR fear OR panic OR phobia OR phobias OR phobic) AND title:(adolescent OR adolescents OR teenager OR teenagers OR teen OR teens OR child OR children OR childhood OR kids OR kiddies OR youth OR youths OR young OR pediatric OR paediatric OR school OR schools) AND (RCT OR randomized OR randomised OR randomly)

Electronic Theses Online Service (EThOS) - British Library ethos.bl.uk/Home.do

Recommend searching EThOS via Proquest Dissertations and Theses Global (as listed above).

\section{WHAT'S NEW}

\begin{tabular}{lll}
\hline Date & Event & Description \\
\hline 13 November 2020 & New search has been performed & $\begin{array}{l}\text { This Cochrane Review supersedes the Cochrane Review 'Cogni- } \\
\text { tive behavioural therapy for anxiety disorders in children and } \\
\text { adolescents' published in 2015 (James 2015). }\end{array}$ \\
& & \\
\hline
\end{tabular}

\section{H I S T O R Y}

Protocol first published: Issue 10, 2018

Review first published: Issue 11, 2020

\section{CONTRIBUTIONSOF AUTHORS}

Anthony James and Tessa Reardon reviewed all papers; Angela Soler reviewed papers in Spanish; and Georgina James and Cathy Creswell reviewed papers where there was any disagreement. Anthony James and Tessa Reardon extracted study data, contacted study authors, and assessed risk of bias, and were responsible for statistical analyses. Anthony James, Tessa Reardon, and Cathy Creswell were jointly responsible for interpreting the data and analyses and drafting the text of the review. All authors contributed to revising and finalising the text.

\section{DECLARATIONSOF INTEREST}

Anthony James: nothing to declare 
Tessa Reardon was funded by a National Institute for Health Research (NIHR) Research Professorship to Cathy Creswell. This study presents independent research partly funded by the NIHR.

Angela Soler: nothing to declare

Georgina James: nothing to declare

Cathy Creswell was funded by an NIHR Research Professorship. This study presents independent research partly funded by the NIHR.

\section{SOURCES OF SUPPORT}

\section{Internal sources}

- Oxford Healthcare NHS Foundation Trust, UK

- Oxford University Department of Psychiatry, Warneford Hospital, Oxford, UK

\section{External sources}

- National Institute for Health Research (NIHR) Research Professorship, UK

Cathy Creswell and Tessa Reardon are funded by an NIHR Research Professorship to Cathy Creswell (NIHR-RP-2014-04-018). This study presents independent research partly funded by the NIHR.

\section{DIFFERENCES BETWEEN PROTOCOLAND REVIEW}

- The criteria used to assess the eligibility of studies for inclusion in this review differed from previous versions of this review (e.g. this review included parent-delivered CBT). We therefore used updated search strategies (see Appendix 2; Appendix 3).

- We removed the 'active control' comparison, dividing this into two separate comparisons: attention controls and alternative treatments.

- As there were a large number of eligible studies $(>80)$, we only included studies that met the defined eligibility criteria and reported (or the author provided) data on at least one of the specified primary or secondary outcomes.

- We removed the requirement for therapists to receive regular supervision. Supervision arrangements varied widely across studies and were not always specified, even where it was suspected to be in place. Rather than specifying supervision as a requirement, we assessed therapy integrity (including therapist competence and adherence) as part of the 'Risk of bias' assessment in all included studies.

- In the protocol, we specified that participants meet diagnostic criteria for an anxiety disorder. In the review, we clarified that these baseline diagnoses were made by reliable and valid structured interviews for Diagnostic and Statistical Manual of Mental Disorders (DSM) or International Classification of Diseases (ICD) child and adolescent anxiety disorders (to be consistent with measures required for diagnostic outcomes).

- Where broad anxiety symptom measures and disorder-specific symptom measures were reported, we prioritised broad anxiety symptom measures. We also conducted sensitivity analyses for child- and parent-reported anxiety symptom measures, removing studies that only reported disorder-specific symptom measures.

- Child Behaviour Checklist - Internalising Scale was not included as an anxiety symptom measure, as it is not designed to assess only anxiety symptoms.

- Subgroup analyses.

We undertook subgroup analyses for the primary outcome (remission of primary anxiety disorder) across comparisons. For the main comparison with the largest number of studies (CBT versus waitlist/no treatment), we also undertook subgroup analyses for the most frequently reported secondary anxiety symptom outcomes (remission of all anxiety diagnoses post-treatment, and reduction in child- and parent-reported anxiety symptoms post-treatment).

CBT interventions were delivered either individually or in a group format, and also varied in terms of the amount of parental involvement (child-focused, child and parent, parent-only). We therefore undertook separate subgroup analyses to examine the differences between individual versus group formats, and child-focused, child-and-parent, and parent-only formats. We also provided a more detailed description of 'child-focused' and 'child-and-parent' interventions than was given in the protocol.

In order to examine the differences between interventions with varying amount of therapist contact time, we planned to use four subgroups; however, we collapsed two of these subgroups due to the small number of studies with $<10$ hours therapist contact time. We therefore examined differences between $<10$ hours, $\geq 10$ hours and $<20$ hours, and $\geq 20$ hours.

In order to evaluate differences between different ages, we undertook subgroup analyses to examine differences between studies with participants $\leq 12$ years, $\geq 12$ years, and mixed age range including participants $<12$ and participants $\geq 12$ years.

As the above subgroup analyses were conducted, meta-regression analyses were not required.

In order to ensure that subgroup analyses were meaningful, we only undertook these analyses where data were available from at least three studies for each subgroup. 
- We made minor changes to the Background to reflect up-to-date evidence and to ensure that the order in which relevant issues/ objectives/results were presented was as consistent as possible throughout the review. 